\title{
Parcours de vie et mémoires de pauvres
}

Changements personnels et sociohistoriques

dans les bidonvilles de Mumbai

Aude Martenot

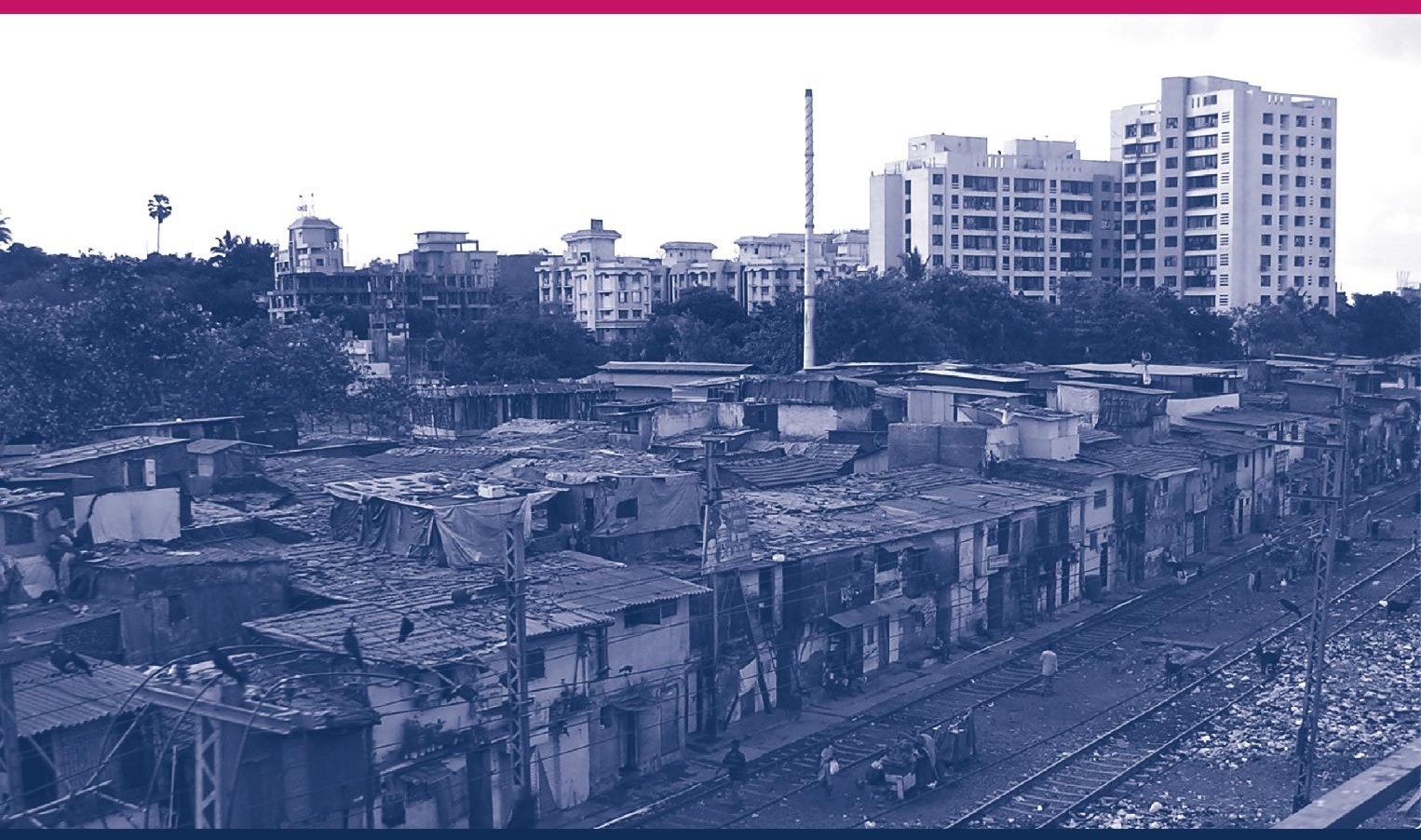

POPULATION, FAMILY, AND SOCIETY

VOL. 33

PETER LANG 


\section{POPULATION, FAMILY, AND SOCIETY \\ VOL. 33}

\section{Aude Martenot}

\section{Parcours de vie et mémoires de pauvres}

Cette thèse s'inscrit dans la perspective du parcours de vie, sous l'angle du regard porté sur les changements personnels et sociohistoriques de l'existence, dans un contexte culturel précis, celui de l'Inde urbaine moderne. Au travers de la récolte de plus de 1250 interviews, réalisées à Mumbai en 2012 et 2014 parmi des adultes âgés de 20 à 86 ans, habitant.e.s de bidonvilles et d'immeubles de classe moyenne inférieure, le contenu et la temporalité des événements vécus considérés comme importants par les répondant.e.s sont analysés. Outre le souci évident d’observer les trajectoires et les moments marquants de la vie, selon le point de vue des personnes elles-mêmes, cette thèse cherche à dépasser l'a priori selon lequel les habitant-e.s des slums seraient vulnérables par évidence, afin de révéler des vulnérabilités insoupçonnées, présentes sous des formes diverses.

Aude Martenot a étudié l'histoire économique et sociale et la socioéconomie à l'Université de Genève. Depuis 2017, elle est titulaire d'un doctorat de I'Université de Genève en socioéconomie, où elle a été chercheuse et a enseigné en tant qu'assistante. Ses domaines de recherche sont la sociologie des parcours de vie, l'étude de la vulnérabilité/de la précarité, la démographie, I'histoire sociale. 
Parcours de vie et mémoires de pauvres 


\section{Population, Family, and Society Population, Famille et Société}

\section{Volume 33}

Edited by / Edité par

Michel Oris

Editorial Board / Comité de lecture

Guy Brunet (Démographie historique, Université Lyon II)

Martin Dribe (Economic History, Lund University)

Georg Fertig (Geschichte, Universitüt Münster)

Vincent Gourdon (Histoire, Université de Paris-Sorbonne)

Matteo Manfredini (Biology and Demography, Universitò degli Studi di Parma)

Jon Mathieu (Geschichte, Universiẗ̈t Luzern)

Muriel Neven (Histoire, Université de Liège)

Emiko Ochiai (Sociology, Kyoto University)

Diego Ramiro Farinas (Demography, Spanish National Research Council)

Gilbert Ritschard (Econométrie, Université de Genève)

Clémentine Rossier (Démographie, Université de Genève)

Paul Servais (Histoire, Université catholique de Louvain-la-Neuve)

Frans van Poppel (Demography, The Hague)

Philippe Wanner (Démographie, Université de Genève)

Eric D. Widmer (Sociologie, Université de Genève)

\section{PETER LANG}

Bern • Berlin • Bruxelles • New York • Oxford • Warszawa • Wien 
Aude Martenot

\section{Parcours de vie et mémoires de pauvres}

Changements personnels et sociohistoriques

dans les bidonville de Mumbai

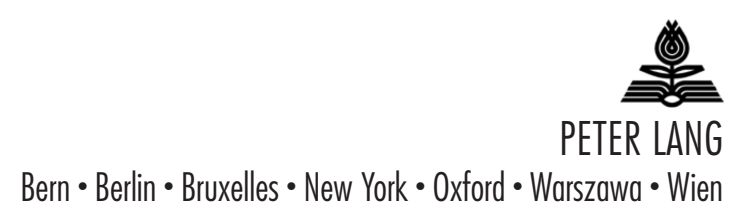




\section{Information bibliographique publiée par «Die Deutsche Nationalbibliothek»}

«Die Deutsche Nationalbibliothek» répertorie cette publication dans la «Deutsche Nationalbibliografie»;

les données bibliographiques détaillées sont disponibles sur Internet sous «http://dnb.d-nb.dè.

Publié avec le soutien du Fonds national suisse de la recherche scientifique.

ISSN 1660-6043

ISBN 978-3-0343-3828-8 Print • E-ISBN 978-3-0343-3829-5 eBook

E-ISBN 978-3-0343-3864-6 ePUB • E-ISBN 978-3-0343-3865-3 MOBI

DOl 10.3726/b15940

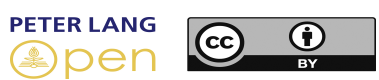

Open Access: This work is licensed under a Creative Commons Attribution

CC-BY 4.0 license. To view a copy of this license, visit https://creativecommons.org/licenses/by/4.0/

Cette publication a fait l'objet d'une évaluation par les pairs.

(C) Aude Martenot 2019

Peter Lang SA

Editions scientifiques internationales

Berne

www.peterlang.com

Cette publication est protégée dans sa totalité par copyright.

Toute utilisation en dehors des strictes limites de la loi sur le copyright est interdite et punissable sans le consentement explicite de la maison d'édition. Ceci s'applique en particulier pour les reproductions, traductions, microfilms, ainsi que le stockage et le traitement sous forme électronique. 


\section{Table des matières}

Table des figures XIII

Table des tableaux XVII

Remerciements XXI

Introduction 1

Chapitre 1. Population, dynamique urbaine et enjeux socioéconomiques de Mumbai. 11

1.1 L'Inde indépendante : capitalisme et urbanisation à grande échelle 12

1.1.1 Ouverture sur l'histoire de l'Inde et de Mumbai...... 13

1.1.2 Une entrée à deux vitesses dans le capitalisme mondial .............................................. 16

1.1.3 Exode rural et urbanisation rampante ......................20

1.2 (Dés)organisation de Mumbai..............................................2 24

1.2.1 Mumbai, une megacity du Sud ..............................25

1.2.2 Les dynamiques spatiales en réponse à la

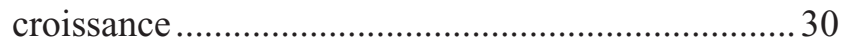

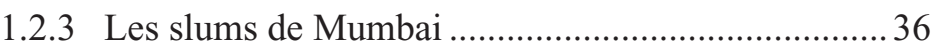

1.3 Structures sociales inégales et discriminations culturelles.... 43

1.3.1 Les transformations récentes de la stratification socioéconomique et sociopolitique.... 44

1.3.2 Religion, castes et classes sociales : une hiérarchisation de la société indienne qui persiste dans la modernité

1.3.3 Condition féminine et patriarcat .................................55

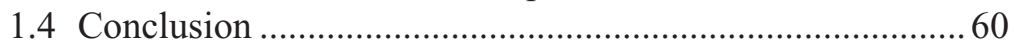

Chapitre 2. Parcours de vie et vulnérabilités en Inde .............................63 63

2.1 L'étude du parcours de vie: un bref état des lieux................ 65

2.2 L'Inde millénaire : puissantes structures d'encadrement des vies et contexte de pauvreté de masse 
2.2.1 La classe et la caste comme déterminants sociaux des individus ............................................... 70

2.2.2 Puissance des institutions informelles normatives : religion et famille ................................. 74

2.2.3 Stratifications d'âge et de genre en Inde .................. 79

2.3 Temporalités individuelles et collectives ............................ 90

2.3.1 Age et cohortes : un ancrage historique partagé dès la naissance ..................................................... 91

2.3.2 Entre histoire et biographies : le concept de génération à la croisée des chemins individuels et collectifs

2.3.3 Les événements de la vie : croisements, transitions et bifurcations

2.4 Vulnérabilités et parcours de vie 101

2.4.1 Aux origines de la vulnérabilité : environnement, développement et pauvreté

2.4.2 "A risk society": vulnérabilités sociétales et protections individuelles

2.4.3 Processus de vulnérabilisation et construction des inégalités à travers le parcours de vie

2.5 Conclusion 125

Chapitre 3. Enquêter la subjectivité des parcours de vie à Mumbai.... 129

3.1 La perception des changements marquants de la vie ....... 130

3.1.1 Se rappeler sa vie: le processus de mémoire........... 131

3.1.2 Penser sa vie : entre normes collectives et réflexion individuelle ............................................ 133

3.1.3 Questionner au travers d'une approche dite «subjective»............................................................. 136

3.1.4 L'enquête Changements et événements au cours de la vie ......................................................... 139

3.2 Design d'enquête et questions de recherche ..................... 140

3.2.1 Trois questions sur la perception du parcours

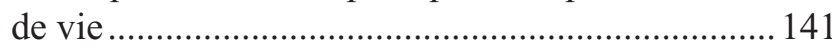

3.2.2 Un échantillon non-aléatoire, structuré selon les «âges de la vie» adulte........................................ 146

3.3 Pratique de la recherche : deux terrains à Mumbai........... 149

3.3.1 Genèse d'une recherche sise dans un environnement atypique 
3.3.2 Collecter des données dans une zone défavorisée.... 156

3.3.3 Diriger une enquête à Mumbai.............................. 159

3.3.4 Particularités et difficultés des terrains .................. 164

3.3.5 Les répondantes et les répondants mumbaikars..... 167

3.4 Transformer les données pour les analyser ...................... 170

3.4.1 Bases de données et variables sociodémographiques.............................................. 171

3.4.2 Interpréter le subjectif pour le rendre quantifiable : typologies et codifications des trois questions ouvertes ................................. 173

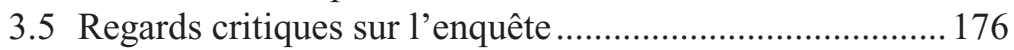

3.5.1 Evaluation des données ......................................... 176

3.5.2 Parler de sa vie : l'enquête comme outil et comme filtre du discours ....................................... 179

Chapitre 4. Temporalités et contenus des souvenirs personnels .......... 185

4.1 Le nombre et la distribution des événements perçus comme marquants à différentes étapes du parcours

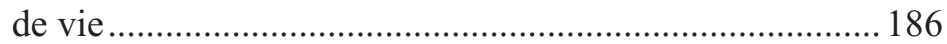

4.1.1 Fréquence des changements selon la position dans le parcours de vie ........................................... 187

4.1.2 La temporalité des grands tournants de l'existence selon les habitants de Mumbai.............. 191

4.1.2.1 La répartition des tournants selon les groupes d'âge ............................................. 191

4.1.2.2 Un faible pic de souvenirs durant l'adolescence et l'entrée à l'âge adulte ...... 192

4.2 La perception des événements de la vie..... 195

4.2.1 Des changements spécifiques aux diverses positions d'âge ........................................................ 196

4.2.1.1 A chaque âge ses tracas............................. 199

4.2.1.2 Les événements de l'année écoulée selon le sexe : des stéréotypes nuancés ...............203

4.2.2 Les grands tournants de la vie.................................206

4.2.2.1 Impacts croisés du lieu de résidence et de la cohorte ...........................................208

4.2.2.2 Les tournants de la vie au prisme du genre 209 
4.3 Evénements récents et tournants : les capitaux à disposition des habitants de Mumbai pour

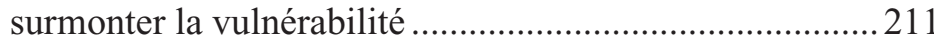

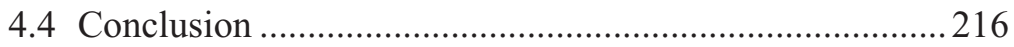

Chapitre 5. Profession, économie, formation: expressions et déterminants des inégalités

5.1 Quitter l'école, quitter l'enfance: l'éducation en tant que palier de la vie et clé de sortie de la pauvreté ............220

5.2 Pauvreté matérielle, financière et humaine .......................227

5.2.1 Le travail, au centre de la survie ........................... 227

5.2.2 Au gré de l'économie: responsabilité globale, vulnérabilités individuelles ................................... 232

5.2.3 Le lieu de vie comme déterminant social .............. 234

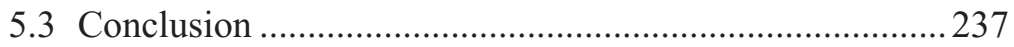

Chapitre 6. La place de la famille dans une société en transition........239

6.1 La famille indienne dans une modernité ambivalente ......240

6.1.1 Les dits et les non-dits des trajectoires familiales indiennes .............................................. 240

6.1.2 Fiançailles et mariages : effilochement des traditions ou transformation liée à la modernité ? ....242

6.1.3 L'impact du décès sur les proches .......................... 248

6.2 Continuité et rupture entre les générations ........................255

6.2.1 Cohabitation et linked lives : de la difficile coexistence de plusieurs générations dans un environnement urbain saturé ...................................25

6.2.2 La perception d'un clivage générationnel ..............261

6.3 Des inégalités de genre contre vents et marées.................267

6.3.1 Sphère privée versus sphère publique ....................2269

6.3.2 La préférence pour un fils .....................................2271

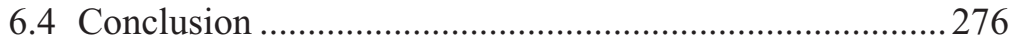

Chapitre 7. La santé : un bien précieux dans un contexte de pauvreté urbaine. 279

7.1 Le fardeau de la santé, de l'enfance à la vieillesse, à travers la stratification socioéconomique 
7.1.1 Des inégalités de santé assignées à la naissance, liées à la classe sociale et au sexe ......... 282

7.1.2 Du Nord vers le Sud : diffusion de la médecine et des inégalités de santé ........................................284

7.1.3 Mégapole du Sud et bidonvilles : des facteurs défavorables pour la santé

7.1.4 La perception de la santé à travers les lieux et les étapes de la vie adulte .............................................28 288

7.1.5 Un effet WIN? ......................................................... 294

7.2 Le phénomène du multiple burden of disease .................... 297

7.2.1 Le contexte de vie comme facteur de différenciation sociale dans les pathologies

7.2.2 En quelques chiffres, la santé des habitants de Mumbai à l'aube du $21^{\text {ème }}$ siècle ..............................300

7.2.3 La montée des maladies chroniques......................... 302

7.2.4 Le cumul des risques ou multiple burden

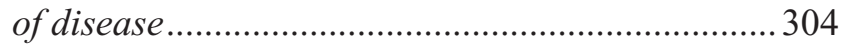

7.2.5 Les événements de santé rapportés par les habitants de Mumbai, selon le multiple burden of disease. 306

7.3 Les raisons pour mentionner un tournant de santé : vulnérabilité économique, émotions et description factuelle.

7.3.1 Trois catégories de raisons au fait de mentionner la santé

7.3.2 Des raisons communes pour parler de sa santé...... 314

7.4 Conclusion

Chapitre 8. Histoire et biographies : souvenirs des vulnérabilités environnementales et sociohistoriques.

8.1 Mémoire collective : construction générationnelle et représentation partagée de l'histoire

8.2 La perception des moments sociohistoriques marquants à Mumbai. 328

8.2.1 Des événements proches localement et temporellement 328

8.2.2 Les médias, au centre des processus mémoriels .... 338 
8.3 Souvenirs de vulnérabilités environnementales et sociales

8.3.1 Précarité matérielle, vulnérabilités environnementales................................................ 343

8.3.2 Montée des violences contre les femmes............... 347

8.4 La nation indienne dans la mondialisation......................... 350

8.4.1 Emeutes et pogroms : le poids de l'histoire politique et religieuse ........................................... 350

8.4.2 L'Inde mondialisée et les attentats terroristes .........353

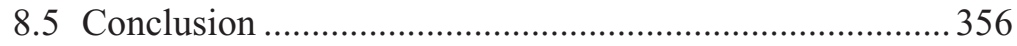

Chapitre 9. Vulnérabilités multiples dans la perception du parcours de vie

9.1 L'incapacité d'être agent de sa vie comme marqueur de vulnérabilité.

9.1.1 Singularité des trajectoires et processus d'agency dans la seconde modernité....

9.1.2 La place de l'individu dans les souvenirs personnels et sociohistoriques des habitants

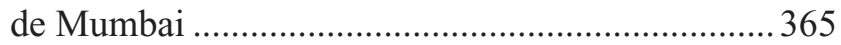

9.1.3 Être l'acteur des changements de sa vie : une capabilité réservée aux moins vulnérables ?....371

9.2 Stress et imprévisibilité dans les tournants de l'existence 375

9.2.1 Vulnérabilité des tournants marquants de la vie : changements, états et carences 376

9.2.2 Les différentes formes de stress présentes dans les raisons de citer un tournant .......................381

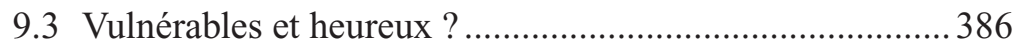

9.3.1 Gains et pertes aux différents âges de la vie .......... 387

9.3.2 La valence des grands changements de la

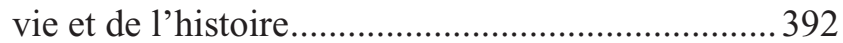

9.4 Conclusion 394

Conclusion 397

Bibliographie 415 
Annexe 1: le questionnaire CEVI en anglais.........................................463

Annexe 2: le questionnaire CEVI en marathi......................................469

Annexe 3: tableau descriptif des capitaux culturels et

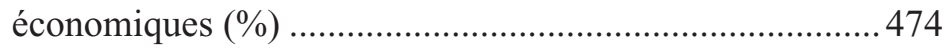

Annexe 4: les changements récents et les tournants concernant

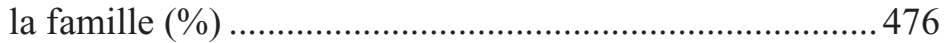

Annexe 5: avoir mentionné un événement de santé ........................... 477 



\section{Table des figures}

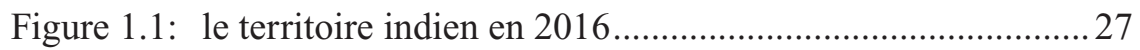

Figure 1.2: carte de la ville et de l'agglomération de Mumbai .............28

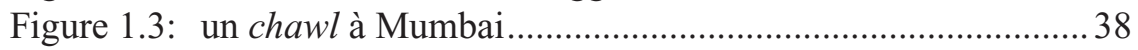

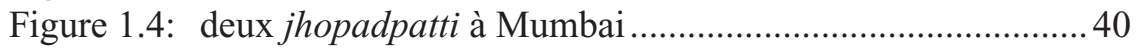

Figure 3.1: l'intérieur d'un slum de Bandra East ................................ 149

Figure 3.2: le quartier de Santa Cruz East et l'exemple d'immeubles SRA .............................................................. 151

Figure 3.3: la zone de bidonvilles de Bandra East et les 20 cliniques de l'ONG CSSC ....................................... 152

Figure 3.4: l'exemple d'une clinique du CSSC ................................... 153

Figure 3.5: l'une des assistantes sociales du CSSC faisant passer le questionnaire à Bandra..................................... 158

Figure 3.6: la répartition (\%) par âge, sexe, niveau d'éducation et religion des répondants interrogés à Santa Cruz........... 170

Figure 4.1: la répartition des changements selon l'âge des répondants au moment de leur survenue (\%).

Figure 4.2: les tournants de la vie (\%) selon l'âge des répondants de Bandra au moment de leur survenue

Figure 4.3: les tournants de la vie (\%) selon l'âge des répondants de Santa Cruz au moment de leur survenue.

Figure 4.4: les changements récents (\%) à Bandra, selon la classe d'âge .................................................................... 213

Figure 4.5: les changements récents (\%) à Santa Cruz, selon la

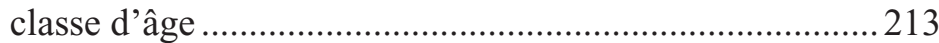

Figure 4.6: les changements récents (\%) à Bandra, selon le sexe .......214

Figure 4.7: les changements récents (\%) à Santa Cruz, selon le sexe ... 214 Figure 4.8: les tournants (\%) à Bandra, selon la classe d'âge .............215

Figure 4.9: les tournants (\%) à Santa Cruz, selon la classe d'âge.......215

Figure 4.10: les tournants (\%) à Bandra, selon le sexe..........................2 215

Figure 4.11: les tournants (\%) à Santa Cruz, selon le sexe ....................215

Figure 5.1 les mentions d'éducation $(n)$ selon l'âge du répondant au moment du tournant et le lieu de résidence. 224 
Figure 5.2: les mentions d'éducation ( $n$ ) selon le sexe et l'âge du répondant au moment du tournant.

Figure 5.3: les individus (\%) ayant mentionné au moins un tournant du domaine éducation, en fonction du lieu de résidence et du niveau d'éducation ...............................225

Figure 5.4: les mentions professionnelles $(n)$ selon l'âge du répondant au moment du tournant et le lieu de résidence.... 231

Figure 5.5: les mentions d'éducation $(n)$ selon le sexe et l'âge du répondant au moment du tournant 232

Figure 6.1: lien de parenté des personnes décédées $(n)$ à Bandra, selon le groupe d'âge du répondant au moment de l'enquête.

Figure 6.2: lien de parenté des personnes décédées $(n)$ à Santa Cruz, selon le groupe d'âge du répondant au moment de l'enquête 250

Figure 6.3: âge au moment de la perte du géniteur à Bandra, selon le groupe d'âge 251

Figure 6.4: âge au moment de la perte du géniteur à Santa Cruz, selon le groupe d'âge

Figure 6.5: qui se marie $(n)$, selon le sexe et le lieu de résidence du répondant. 256

Figure 6.6: qui se marie $(n)$, selon le groupe d'âge ............................. 257

Figure 6.7: qui vient au monde (n), selon le sexe et le lieu de résidence du répondant 258

Figure 6.8: qui vient au monde $(n)$, selon le groupe d'âge.................. 258

Figure 6.9: les écarts générationnels (\%) selon la classe d'âge........... 262 Figure 6.10: les changements récents répartis entre sphères privée et publique (\%), selon le sexe et la classe d'âge à Bandra 270

Figure 6.11: les changements récents répartis entre sphères privée et publique (\%), selon le sexe et la classe d'âge à Santa Cruz. 270

Figure 6.12: les tournants répartis entre sphère privée et sphère publique (\%), selon le sexe et la classe d'âge à Bandra....271

Figure 6.13: les tournants répartis entre sphère privée et sphère publique (\%), selon le sexe et la classe d'âge à Santa Cruz...271

Figure 6.14: les naissances de filles et de fils (\%), selon le groupe d'âge et le lieu de résidence du répondant. 273 
Figure 7.1: les changements récents de santé (\%), selon les groupes d'âge et le pays de résidence.

Figure 7.2: les changements récents de santé $(n)$ concernant l'interviewé, selon le type de maladie et le groupe d'âge à Bandra ..... 307

Figure 7.3: les changements récents de santé $(n)$ concernant l'interviewé, selon le type de maladie et le groupe d'âge à Santa Cruz

Figure 7.4: les tournants de santé $(n)$ concernant l'interviewé, selon le type de maladie et le groupe d'âge à Bandra........307

Figure 7.5: les tournants de santé $(n)$ concernant l'interviewé, selon le type de maladie et le groupe d'âge à Santa Cruz.... 308

Figure 7.6: les changements récents de santé $(n)$ concernant l'interviewé, selon le type de maladie et le sexe à Bandra ....309

Figure 7.7: les changements récents de santé $(n)$ concernant l'interviewé, selon le type de maladie et le sexe à Santa Cruz. 309

Figure 7.8: les tournants de santé $(n)$ concernant l'interviewé, selon le type de maladie et le sexe à Bandra 309

Figure 7.9: les tournants de santé $(n)$ concernant l'interviewé, selon le type de maladie et le sexe à Santa Cruz.

Figure 7.10: les raisons des tournants de santé $(n)$, selon le groupe d'âge, à Bandra

Figure 7.11: les raisons des tournants de santé $(n)$, selon le groupe d'âge, à Santa Cruz.

Figure 7.12: les raisons des tournants de santé $(n)$ à Bandra, selon le sexe

Figure 7.13: les raisons des tournants de santé (n) à Santa Cruz, selon le sexe

Figure 7.14: les raisons des tournants de santé $(n)$, selon le niveau d'éducation

Figure 8.1: les changements sociohistoriques (\%) mentionnés à Bandra, selon l'année de survenue et la classe d'âge du répondant

Figure 8.2: les changements sociohistoriques (\%) mentionnés à Santa Cruz, selon l'année de survenue et la classe d'âge du répondant 
Figure 8.3: les ménages indiens possédant un poste de télévision (en millions)

Figure 9.1: le pourcentage de tournants à Bandra, selon la classe d'âge.....

Figure 9.2: le pourcentage de tournants à Santa Cruz, selon la classe d'âge

Figure 9.3: le pourcentage de tournants, selon le sexe et le lieu de vie

Figure 9.4: le pourcentage de tournants, selon les sept domaines les plus mentionnés

Figure 9.5: les raisons des tournants (\%) à Bandra, selon la classe d'âge

Figure 9.6: les raisons des tournants (\%) à Santa Cruz, selon la classe d'âge

Figure 9.7: les raisons des tournants (\%), selon le sexe et le lieu de résidence.

Figure 9.8: les raisons des tournants (\%), selon les sept domaines les plus mentionnés

Figure 9.9: les raisons (\%) selon le type de tournant

(changement, état, absence d'événement)

Figure 9.10: le rapport des gains et des pertes à travers le parcours de vie 388

Figure 9.11: la répartition des gains et des pertes (\%) selon les groupes d'âge 390 


\section{Table des tableaux}

Tableau 3.1: effectifs de 2012 et 2014, selon la classe d'âge et le sexe

Tableau 3.2: caractéristiques de l'échantillon selon les groupes

d'âge et le lieu de résidence.

Tableau 3.3: nombre moyen de changements cités parmi les individus ayant cité au moins un événement, selon les trois questions de l'enquête

Tableau 4.1:le pourcentage d'individus n'ayant pas ou ayant cité au moins un changement récent, selon l'enquête, le sexe et l'âge. 188

Tableau 4.2:rapports de cotes issus de modèles de régression logistique sur la variable «avoir mentionné au moins un changement récent» 190

Tableau 4.3: rapports de cotes issus de modèles de régression logistique sur la variable «avoir mentionné au moins un tournant situé au cours de l'entrée dans l'âge adulte» ... 193

Tableau 4.4 les domaines et les types de changements récents les plus mentionnés (\%), selon l'âge et le lieu de résidence.

Tableau 4.5: les domaines et les types de changements récents les plus mentionnés (\%), selon le sexe et le lieu de résidence.

Tableau 4.6: les domaines et les types de tournants les plus mentionnés (\%), selon l'âge et le lieu de résidence

Tableau 4.7:les domaines et les types de tournants les plus mentionnés (\%), selon le sexe et le lieu de résidence (\%).....210

Tableau 6.1: les changements récents et les tournants concernant la famille (\%), selon le sexe et le lieu de résidence .......... 268

Tableau 7.1: les sept principaux domaines de tournants (\%), selon le pays 290

Tableau 7.2: rapports de cotes issus de modèles de régression logistique sur la variable «avoir mentionné au moins un événement de santé» 
Tableau 7.3:rapports de cotes issus de modèles de régression logistique sur la variable «avoir mentionné au moins un événement de santé (à soi)», à Bandra .......................... 295

Tableau 8.1:les individus (\%) mentionnant au moins une fois les principaux domaines de changements sociohistoriques, selon le lieu de résidence

Tableau 8.2: les changements sociohistoriques (\%), selon l'âge et le lieu de résidence

Tableau 8.3: les changements sociohistoriques (\%), selon 1 e sexe et le lieu de résidence

Tableau 8.4: rapports de cotes issus de modèles de régression logistique sur la variable «avoir cité une catastrophe naturelle».

Tableau 8.5: rapports de cotes issus de modèles de régression logistique sur la variable «avoir cité l'inondation de 2005 à Mumbai»»

Tableau 8.6 rapports de cotes issus de modèles de régression logistique sur la variable «avoir cité les violences contre les femmes»

Tableau 8.7: rapports de cotes issus de modèles de régression logistique sur la variable «avoir cité des émeutes interreligieuses».

Tableau 8.8:rapports de cotes issus de modèles de régression logistique sur la variable «avoir cité des attentats en Inde»

Tableau 9.1: rapports de cotes issus de modèles de régression logistique sur la variable «avoir cité un changement sociohistorique mettant le répondant au centre»...

Tableau 9.2: rapports de cotes issus de modèles de régression logistique sur la variable «avoir cité un changement récent mettant le répondant au centre» 368

Tableau 9.3: rapports de cotes issus de modèles de régression logistique sur la variable «avoir cité un tournant mettant le répondant au centre»

Tableau 9.4:rapports de cotes issus de modèles de régression logistique sur la variable «avoir cité un changement récent choisi» 
Tableau 9.5: rapports de cotes issus de modèles de régression logistique sur la variable «avoir cité un tournant choisi» ....... 373

Tableau 9.6: résumé des modèles de régression concernant les variables d'individualisation et d'agency.

Tableau 9.7: rapports de cotes issus de régressions logistiques sur la question des changements survenus au cours de la dernière année

Tableau 9.8: rapports de cotes issus de régressions logistiques sur la question des grands tournants de la vie.

Tableau 9.9: rapports de cotes issus de régressions logistiques sur la question des changements sociohistoriques

Tableau 1 annexe 5: rapports de cotes issus de modèles de régression logistique sur la variable «avoir mentionné au moins un changement récent de santé»

Tableau 2 annexe 5: rapports de cotes issus de modèles de régression logistique sur la variable «avoir mentionné au moins un tournant de santé». 478 



\section{Remerciements}

S'il est évident qu'un travail de thèse se construit en grande majorité sur la base d'un effort solitaire de son auteure, fait de longues heures derrières un écran ou le nez dans les livres, il n'en demeure pas moins que de nombreuses personnes s'avèrent nécessaires à son aboutissement. Le présent travail ne déroge pas à cette règle, et je suis heureuse de pouvoir ici remercier les personnes ayant participé à la réussite de mon projet.

A commencer par son principal initiateur, Michel Oris, qui, par son accompagnement, ses relectures et son soutien tout au long des étapes de ce parcours, a su apporter les fondations indispensables à l'élaboration de ce livre. Il est peu probable qu'un directeur ait accordé plus de libertés et d'encouragements que Michel n'en a montré au moment où il a soutenu l'idée que je réalise une thèse sur un sujet aussi éloigné de la Suisse que peut l'être celui des parcours de vie d'habitantes et d'habitants des bidonvilles de Bandra, ou encore lorsque j'ai mis sur pied le projet du second terrain à Santa Cruz. Pour cela comme pour ses autres marques de confiance, je l'en remercie. Je remercie également les membres de mon jury, Claudine Burton-Jeangros, Felix Bühlmann et Guy Elcheroth, pour leurs relectures attentives, leurs remarques avisées et les pertinentes critiques apportées à la première version du manuscrit.

Outre ces indispensables appuis, la réussite d'une thèse - à tout le moins de la mienne - repose également sur les nombreuses, très nombreuses, marques d'amitié, de réconfort et d'approbation reçues durant les moments marquants de mon travail. L'environnement accueillant du CIG, puis des premières années du CIGEV, y est pour beaucoup. Je pense bien sûr à Claire, Greg et Nathalie, toujours prêt·e's à aider et à suivre nos projets...jusqu'en Inde. A Marie, Aude, Isabela, Oana, Myriam, Julia, Alessandra...et à bien d'autres collègues présents à un moment ou un autre au CIG(EV), dans LIVES ou en IDESO, disposés à discuter, à traduire, à rigoler et sans qui les congrès auraient été bien ternes. A Eduardo puis Julia, qui ont eu le courage de partager leur bureau avec moi, ainsi que des discussions - plus ou moins animées - et bien des éclats de rire. A Marthe et Aline, évidemment, avec qui, en plus des mois chaotiques de la fin de thèse - dont le souvenir, pourtant, m'est déjà sympathique grâce à 
leur présence -, j'ai partagé ces cinq années d'assistanat. Enfin, pour son amitié avant tout, mais aussi son écoute, son savoir et ses conseils, à Delphine. Ainsi qu'à toutes et tous, à l'Université et ailleurs, ami·e·s, famille qui ont suivi cette aventure.

Mais surtout, j'aimerais souligner ici que ce travail aurait été irréalisable sans la collaboration des 1255 individus qui ont bien voulu répondre à mes questionnaires, sans la bonne humeur de Dinesh Shenai, sans les enquêtrices et les enquêteurs - notamment de WIN -, sans Anouk qui a patiemment mené à bien le premier terrain à Bandra, sans Camille avec qui j'ai découvert l'Inde. Chacune et chacun ayant rendu possible l'idée un peu folle d'aller questionner les vies des habitantes et des habitants de Mumbai. 
A toutes celles et tous ceux qui, comme le dit Prévert :

(Paroles, 1946 [1976], p. 15)

«soufflent vides les bouteilles que d'autres boiront, qui fabriquent dans les caves les stylos avec lesquels d'autres écriront en plein air que tout va pour le mieux, qui ont le pain quotidien relativement hebdomadaire».

«Ne jamais s'habituer à la violence sans bornes ni aux flagrantes disparités du monde où nous vivons. [...] Essayer de comprendre. Ne jamais détourner les yeux. Et ne jamais, surtout, ne jamais oublier.»

(Arundhati Roy, 1999, p. 139) 



\section{Introduction}

«J'ai préféré me concentrer sur des acteurs plus modestes, souvent invisibles : les gens ordinaires, les femmes, les enfants, les castes répertoriées [...]»

(Butalia, 2002, p. 18)

Depuis la concrétisation du parcours de vie en tant que domaine d'étude à part entière, plusieurs auteurs ont ausculté le contexte sociétal pour comprendre les biographies. L'analyse de la modernisation des sociétés industrielles (puis post-industrielles) a par exemple participé à éclaircir les mutations dans les trajectoires au cours de ces dernières décennies (Kohli, 1986). De même, les institutions ont été considérées comme centrales dans la construction et la définition des trajectoires en Occident (Mayer, 2004). D'autres chercheurs se sont penchés sur les politiques sociales (Vrancken et Thomsin, 2008) ou encore sur le processus de mondialisation (Buchholz et al., 2009; Lalive d'Epinay, 2012) comme source de modélisation des vies humaines. Avec ce dernier constat, il est apparu d'autant plus nécessaire de dépasser les frontières de l'Occident (dans lequel se trouvent enfermées encore la grande majorité des études sur les parcours de vie) pour aller explorer l'alchimie sociale des vies au sein des masses démographiques des pays du Sud.

Comment les individus perçoivent-ils les événements marquants qui orientent leur existence ? La notion associée de changement constitue le levier principal de notre étude. Ce concept est précisément issu du champ d'étude des parcours de vie, qui préconise que les vies sont scandées par des «moments charnières», reliant entre elles les différentes étapes de l'existence (Elder, 1998). Comme l'a souligné Clausen (1995), un des angles d'attaque les plus fructueux pour étudier les vies humaines consiste à se pencher sur les transitions vécues par les individus au cours de leur développement. L'analyse de ces bifurcations permet de saisir les éléments clés qui façonnent les existences (Bessin, Bidart, \& Grossetti, 2010; Bidart, 2006a). C'est l'objet de ce livre, qui se focalise sur les événements et changements marquants de l'existence. Notre travail ambitionne de relever simultanément un autre défi car les «passages» ne permettent de 
saisir l'enchaînement des étapes de la vie qu'à condition d'être compris dans leurs cadres culturel et historique. A cet égard, la recherche a bien établi que les contextes influencent le nombre et le type des événements et changements (Glasner, van der Vaart, \& Belli, 2012; Longo, Bourdon, Charbonneau, Kornig, \& Mora, 2013).

Cadres et contextes, dans le temps et dans l'espace, spécifient profondément notre investigation, qui a été conduite dans un environnement pour le moins particulier. Suivant la proposition de Dale Dannefer (2003) d'élargir l'observation de l'existence humaine à des recherches comparatives internationales et interculturelles, de déborder l'espace atlantique pour bâtir les fondations d'une «géographie globale» des parcours de vie, c'est dans un pays asiatique en émergence, cumulant normes traditionnelles et mutations liées à la modernité et à la globalisation, que nous nous situons, en Inde.

\section{L'Inde et Mumbai comme environnements de recherche}

L'urbanisation est l'un des premiers facteurs de changement, encastré dans les dynamiques démographiques, économiques et sociales. La population indienne est aujourd'hui évaluée à plus de 1.3 milliard d'individus, dont 377 millions résident en ville (Pison, 2015). Dans un pays où la majorité de la population vit encore dans les campagnes, l'armature urbaine indienne est dominée par des mégapoles immenses qui sont parmi les plus grandes au monde. Elles concentrent les forces de production, parmi lesquelles une réserve de travailleurs à bas prix, qui s'entassent dans des quartiers miséreux (Nijman, 2015; Vincent-Geslin, Pedrazzini, Adly, \& Zorro, 2015).

La visibilité des inégalités socioéconomiques par la concentration et l'exposition des masses ne sera sans doute jamais aussi apparente qu'elle peut l'être dans ces megacities indiennes que sont Mumbai, Delhi ou Kolkata (Marchal \& Stébé, 2012; Narayan, 2014). En effet, comme tant d'autres pays marqués par la colonisation occidentale et le sous-développement qui s'en est suivi, le continent indien se transforme avec l'ouverture au marché international des années 1990 (Drèze \& Sen, 2013). La croissance exponentielle des bidonvilles se situe dans cette dynamique historique. L'augmentation du nombre de personnes résidant en milieu urbain, dans des conditions de profond dénuement, manifeste un urbanisme chaotique, 
dérégulé. Ces slums ${ }^{1}$ sont l'un des enjeux premiers du pays, représentant des formes de vie et d'habitat indignes, mais majoritaires dans les mégapoles de l'Inde contemporaine (Subbaraman et al., 2012).

Emblème de ce pays de contrastes dont elle est la capitale économique et financière autant qu'une vitrine de ses inégalités, Mumbai - et ses bidonvilles - ont récemment été mis sous le feu des projecteurs par le film Slumdog Millionnaire, réalisé par Danny Boyle. Plus de la moitié des habitants de la cité vivent dans ces taudis ${ }^{2}$ qui se mêlent aux constructions en dur, à l'ombre de gratte-ciels luxueux, révélant une fracture sociale abyssale. Le manque de régulation du marché du logement, la privatisation et la libéralisation conduisant à une prolifération des emplois informels, les politiques fiscales régressives inaptes à assurer une protection sociale aux plus pauvres, produisent et reproduisent en série des individus précaires. L'inégalité est également entretenue par des politiques discriminatoires, ainsi que des normes socioculturelles parfois très anciennes, de genre, de caste et de classe. Les groupes vulnérables, les femmes en particulier, sont affectés, ne possédant pas d'accès à la propriété, au crédit, à l'éducation, à la santé ou à toute autre forme de ressource.

\section{Subjectivité et simplicité comme méthodes d'enquête}

A l'image de beaucoup de pays du Sud, et plus encore en tant que géant démographique, l'Inde intéresse les scientifiques qui étudient des populations au sens large. Mais comprendre le déroulement des vies humaines implique un travail différent, positionné au plus près des individus. Les deux approches ne s'opposent pas. Au contraire, l'alliance d'une compréhension macro et d'une analyse plus micro est essentielle pour saisir les modifications des parcours de vie et les causes de ces changements.

Dans le cadre de ce livre, tout particulièrement dans l'optique de laisser aussi directement que possible la parole aux actrices et aux acteurs, c'est au travers de la perception des grands moments à la fois personnels et sociohistoriques qui ont marqué leur existence, que le contexte du vécu

1 Le mot anglais slum est un synonyme de «bidonville», apparu à Londres dans les années 1820 pour parler de l'habitat insalubre des familles pauvres. D'origine misérabiliste, il est à présent largement utilisé pour évoquer les taudis en Inde, à l'image du terme de favela désignant les quartiers informels pauvres du Brésil (Marchal \& Stébé, 2012).

2 Terme utilisé ici sans distinction comme un synonyme de bidonville, de slum, d'habitat précaire ou informel. 
a été appréhendé. Ce lien entre vie privée et histoire globale n'est pas une réflexion spontanée de la part de celles et ceux qui ont bien voulu nous répondre, et ce quel que soit le sens de la causalité: que l'être humain se positionne en tant qu'architecte de l'histoire ou jouet de ses contraintes (Mills, 1997 [1959]). S'intéressant aux relations micro-macro à travers la socialisation $^{3}$, Richard Settersten (2002) recommande de se focaliser sur la dimension subjective, en se demandant si les individus ont, ou n'ont pas, conscience de participer à la reproduction des normes sociales, ainsi qu'aux grands événements qui affectent la trame de l'histoire.

Les enquêtes sur la perception des changements ou événements marquants au cours de la vie mettant en lien la biographie et le contexte sociétal restent rares (Bidart et Gosselin [2014]). Plusieurs d'entre elles ont cependant démontré que les personnes interrogées évoquent des événements sociohistoriques parmi les souvenirs qui les ont marqués (des moments forts ayant impacté leur vie, comme des guerres [Crenner, Guérin, \& Houseaux, 2002; Golaz \& Lelièvre, 2012]). Deux modalités d'enquête ont obtenu ce résultat: soit par questionnement différencié, d'un côté le personnel et d'un autre l'historique (Lalive d'Epinay et al., 2012; Lani-Bayle et Mallet, 2006; Oddone et Lynch, 2010), soit au sein d'une même question portant sur les événements en général, laissant à l'individu le soin d'apprécier lui-même ce qu'il souhaite donner comme information (Crenner et al., 2002; Laborde, Lelièvre, \& Vivier, 2012). Il arrive que dans les études où les questions ne sont pas différenciées, les répondants mélangent les informations biographiques et sociohistoriques. C'est pour gérer ce risque que notre enquête suit la première approche et questionne séparément l'appréhension de l'histoire globale, le vécu collectif, d'une part, et d'autre part la mémoire du parcours de vie individuel. Cela nous permet aussi de les situer l'une par rapport à l'autre.

Un premier terrain réalisé en 2012 avec l'appui d'une ONG locale a permis de récolter plus de 600 questionnaires auprès d'une population difficile d'accès : les résidents de bidonvilles situés au centre de Mumbai. Femmes et hommes âgés de 20 à 85 ans ont répondu à quelques questions ouvertes, remarquables par leur simplicité (quels ont été les changements personnels de cette dernière année / quels sont les tournants les plus

3 Soit la transmission de valeurs entre générations (Mayer, 2004), ou le «processus par lequel les individus, en acquérant les compétences nécessaires, s'intègrent à une société, et la manière dont une société se dote d'un type d'individu.» (Martuccelli, 2005, p. 2). 
marquants de votre vie / quels sont les changements sociohistoriques les plus importants). Ce faisant, c'est clairement la voix des pauvres que nous voulions capter et le mythe que la dureté objective de leurs conditions de vie ne leur laisse pas de marge pour la subjectivité, pour le ressenti, que nous voulions briser.

Par la suite, nous avons éprouvé le besoin de réaliser un second terrain d'enquête, hors des taudis, dans un quartier de classe moyenne inférieure (les individus logeant également à Mumbai mais dans des immeubles en dur), surtout à titre comparatif, afin de spécifier les résultats issus du premier terrain, dont l'interprétation aurait été délicate autrement. Jusqu'à un certain point, il permet d'attribuer ressemblances ou différences entre les deux quartiers au contexte culturel ou à un environnement de misère. Ce second échantillon, récolté en 2014 et également constitué de plus de 600 répondants, a aussi permis d'illustrer la situation d'un groupe social en émergence dans l'Inde urbaine moderne.

\section{Questions de recherche autour de la vulnérabilité dans les parcours de vie}

L'objectif premier de cette recherche est donc celui de parvenir à rendre audible la voix des pauvres de Mumbai, d'écouter et d'utiliser la mémoire de leur existence pour en identifier les moments cruciaux, les événements sociohistoriques ayant défini leurs trajectoires. La subjectivité est résolument au centre de ce livre, mise en exergue au travers de trois questions spécifiques : la capacité d'agency (le fait de pouvoir décider, choisir les événements marquant de sa vie) pour des individus pauvres et moins pauvres dans un pays émergent du Sud, les dimensions de stress perceptibles au travers de cette mémoire, ainsi que de bien-être (par l'analyse des moments de malheur ou de bonheur ressentis par les répondants). Ces interrogations traversent le livre avant de se ramasser dans un dernier chapitre spécifique.

La seconde dimension qui se situe en filigrane de ce livre est la mise en application des théories occidentales du parcours de vie dans un monde extra-atlantique. Nous l'avons dit, les recherches dans ce champ d'étude ont souvent porté sur une compréhension institutionnelle de celui-ci, considérant l'impact des institutions formelles sur le déroulement des vies. Dit autrement, en posant des règles et en organisant des institutions à divers moments et dans plusieurs domaines de la vie, l'Etat-providence «à l'occidentale» a construit son rôle de protecteur contre les aléas prévisi- 
bles et imprévisibles de l'existence (chômage, parentalité, maladie, etc.), de réducteur des inégalités et de médiateur des conflits sociaux (Justino, 2007), tout en devenant l'organisateur des trajectoires humaines (Marshall \& Mueller, 2003). En Inde, où de telles structures étatiques restent essentiellement absentes ou inaccessibles, voire sont en train de régresser en termes de prestations - aménagements sanitaires, électricité, transports, télécommunications, santé, éducation, etc. - au profit d'entreprises privées ou à la charge d'ONG (Roy, 2014), ce sont les relations familiales qui servent de support tout au long du parcours de vie et qui sont capitales au quotidien (Heuzé, 1989). Ce processus est récurrent dans les pays du $\mathrm{Sud}^{4}$, où les restrictions budgétaires sont élevées, les capacités étatiques de récolte des impôts faibles et les failles du système économique nombreuses (Justino, 2007). Quels seront les impacts de ces enjeux sur les modes de vie des individus et des familles et sur la perception que nous en restitue leur mémoire?

Finalement, le troisième axe de recherche concerne la confrontation des traditions, qui englobe le système pour ainsi dire unique des castes jusqu'aux pratiques religieuses en passant par le principe de la dot, avec les visions de nouvelles générations qui vivent l'entrée dans la globalisation. Comment les jeunes ressentent-ils, au temps de la communication instantanée et universelle, les normes sociales d'antan ? Comment les personnes âgées perçoivent-elles les nouvelles opportunités qui ne leur ont pas été offertes ? Mais aussi, quelle avancée de la condition féminine en Inde, pays où le rapport de masculinité ${ }^{5}$ déséquilibré et où l'équité entre les genres est un objectif difficile à atteindre ? En résumé, il s'agit d'appliquer à l'Inde urbaine les questions de genre et de générations qui recoupent les enjeux de

4 Tout au long du texte, malgré les imperfections de ces différentes expressions, nous utilisons de manière interchangeable des termes tels que «pays du Sud», «pays moins développés économiquement», "pays en développement» pour désigner les nations qui connaissent encore une transition des multiples modes de vie traditionnels vers un mode de vie considéré comme moderne (s'appuyant sur le modèle de la révolution industrielle européenne). De même, nous attribuons la dénomination de «pays du Nord», «pays les plus développés économiquement» ou «pays occidentaux» aux Etats ayant un fort produit intérieur brut ou un indicateur élevé de développement humain (autrement dit, des pays où la majorité de la population accède à tous ses besoins vitaux, c'est-à-dire avoir accès à un logement décent, à l'eau potable, à la santé, à l'éducation, et à la nourriture).

5 Autrement appelé sex ratio, il s'agit du rapport entre le nombre d'hommes et de femmes au sein d'une population. 
modernisation. Mais il faudra aussi revenir sur l'impact d'être pauvre dans un environnement de pauvreté de masse, d'être femme face aux discriminations et aux violences, d'être circonscrit dès l'enfance à une caste et une classe sociale qui déterminent les avantages de la vie, mais surtout les désavantages (accès à l'éducation, à un travail formel, à un logement décent...).

Outre le souci évident d'observer la vulnérabilité présente dans les moments marquants de la vie, selon le point de vue des personnes ellesmêmes, ce livre cherche donc à dépasser l'a priori selon lequel les habitants des slums seraient vulnérables par évidence, afin de révéler des vulnérabilités insoupçonnées, présentes sous des formes diverses.

La vulnérabilité en tant que sujet de recherche en sciences sociales et humaines a le vent en poupe depuis le début du nouveau millénaire, autant en francophonie qu'ailleurs (Bresson, Géronimi, \& Pottier, 2013; Misztal, 2011). Rassemblant des concepts issus de différents domaines (fragilité pour la gérontologie, précarité et pauvreté pour la sociologie, l'économie et la socioéconomie, dépression ou désordre en psychologie), ce terme repose sur une transdisciplinarité qui lui donne une assise théorique considérable (Spini, Hanappi, Bernardi, Oris, \& Bickel, 2013). C'est sans doute aussi ce qui explique le débat continuel des chercheurs autour de sa définition (Oris, Roberts, Joye, \& Ernst Stähli, 2016), cette imperfection offrant parallèlement l'opportunité d'une réinterprétation permanente, en adéquation avec le sujet étudié. En effet, au sein de sociétés mouvantes, auxquelles la globalisation des marchés tend à imposer des logiques communes malgré des contextes institutionnels divergents, dont les ressortissants tracent leur destinée de manière individuelle dans des espaces de contraintes et d'opportunités, sous le joug d'une incertitude de plus en plus marquée, un besoin croissant se faisait sentir pour l'utilisation d'une notion plurielle, rassembleuse et capable d'adaptation face aux nouvelles formes d'instabilité qui marquent nos vies (Bresson et al., 2013; Spini et al., 2013).

***

Le premier chapitre de ce livre est l'occasion de poser quelques jalons de la situation de l'Inde urbaine d'aujourd'hui. Une première section offre un bref retour sur les événements historiques ayant marqué le $20^{\text {ème }}$ siècle. Après quoi, nous nous penchons sur l'émergence des mégapoles du pays, avec bien entendu au centre la formation du pôle urbain de Mumbai. L'urbanisation de cette ville est décortiquée dans une seconde section, afin d'expliquer au mieux la formation des bidonvilles et leur aspect actuel. Le chapitre s'achève sur une description des structures sociales, des 
inégalités et des discriminations culturelles qui minent la population indienne à l'orée du $21^{\text {ème }}$ siècle.

Les connaissances théoriques nécessaires à l'encadrement de la réflexion autour des résultats empiriques sont exposées dans le chapitre 2. Sans prétention d'exhaustivité, nous revenons sur plusieurs notions phares du parcours de vie, mais également des structures sociales : caractéristiques de classe et de caste assignées à la naissance, stratifications sociales d'âge et de sexe, influence des institutions normatives informelles telles que la famille et la religion. Nous poursuivons avec une focale sur les temporalités qui déterminent les trajectoires de vie : notions d'âge, de cohorte et de génération qui participent à situer l'individu dans son propre parcours ainsi que dans l'histoire. Enfin, une quatrième section met davantage l'accent sur le concept de vulnérabilité, en offrant un panorama des études. A partir de l'observation de la pauvreté dans le monde francophone, nous présentons la théorie de la société du risque avant de passer aux multiples facettes de la vulnérabilité : ressources et capabilités, processus d'avantages et de désavantages cumulatifs, stress et inégalités sociales. Pris ensemble, ce savoir de base amène les outils pour comprendre une situation aussi complexe que celle de la précarité au sein d'un monde en pleines turbulences (l'Inde), dans une ville traversée par la ségrégation spatiale (Mumbai), pour des personnes supportant le poids tant des anciennes normes que des nouvelles injonctions liées à la modernité.

Une présentation des outils méthodologiques, du protocole de l'enquête, ainsi que des questions qui constituent le questionnaire, fournissent matière aux deux premières sections du chapitre 3. Par la suite, un retour sur la genèse du projet ainsi que sur les terrains offrent une compréhension de leur déroulement mais aussi des biais qui ont pu surgir sous les contraintes matérielles rencontrées. Les échantillons finaux sont ensuite exposés, ainsi que les techniques de codification des réponses employées. La dernière section du chapitre est un retour critique sur les réponses obtenues, afin de situer leur qualité en regard des biais méthodologiques évoqués.

Les chapitres 4, 5, 6 et 7 composent la partie empirique de ce livre recouvrant l'analyse des parcours de vie individuels. D'abord en analysant la distribution des événements personnels entre les diverses positions d'âge puis au cours de l'existence, ensuite en revenant sur les contenus des souvenirs, nous posons ici un premier tableau de ce qui constitue les événements marquants de la vie à divers moments du parcours, selon le sexe et le lieu de résidence (chapitre 4). Le chapitre 5 revient plus en détail 
sur les dimensions d'éducation, de profession et de situation économique des individus, afin de comprendre les enjeux liés au niveau de vie des répondants présents dans la perception des événements de l'existence. Le chapitre qui suit se penche sur les évocations familiales (mariages, naissances et décès avant tout), centrales dans les vies des Indiennes et des Indiens. En outre, le chapitre 6 propose de mettre l'emphase sur les dimensions de genre et de générations, afin de saisir les possibles sources de conflits au sein de la population observée. Enfin, une particularité des changements cités par les habitants de Mumbai est le nombre conséquent de mentions de santé. Ces dernières font l'objet du chapitre 7, illustration s'il en est de la condition vulnérable des populations du Sud, marquée par une ancienne (quoique pas si lointaine) présence des maladies infectieuses, ainsi que par une nouvelle montée des risques sanitaires en lien avec la globalisation (manque d'exercice, mauvaise alimentation, progression des maladies chroniques).

Différant pour des raisons évidentes d'avec les chapitres précédents, le huitième fournit une compréhension des vulnérabilités environnementales rencontrées par les habitants de Mumbai, au travers de l'analyse des réponses à la question portant sur les changements sociohistoriques. Sans équivoque, les éléments rapportés par les individus soulignent l'extrême précarité de l'existence face aux aléas naturels (majoritairement les inondations), mais aussi les conséquences du traumatisme que fut la Partition du pays en 1947, qui se perçoivent au travers des évocations des pogroms interreligieux et des attentats qui ont scandés les trois ou quatre dernières décennies, ainsi que la médiatisation des violences affectant avant tout les femmes. Les caractéristiques de lieu de résidence, de sexe et d'âge des répondants sont particulièrement cruciales pour comprendre les impacts de tels ou tels souvenirs sur les mémoires.

Finalement, le chapitre 9 prépare la conclusion en creusant diverses dimensions plus fines de la subjectivité. Dans une première section, les notions d'agency et d'individualisation permettent de sonder l'impact de la modernité sur les souvenirs des cohortes étudiées. Une seconde partie revient sur le stress perceptible dans les tournants de l'existence, ainsi que la forme que celui-ci peut prendre. La troisième et ultime section s'ouvre sur une réflexion à propos de l'évaluation des changements en termes de bien-être, au regard de toutes les vulnérabilités perçues. Les événements marquant les trajectoires des Indiennes et des Indiens interrogés peuvent-ils tout de même être qualifiés d'heureux? 



\title{
Chapitre 1. Population, dynamique urbaine et enjeux socioéconomiques de Mumbai
}

\author{
"The most significant among these is that the cities are trying \\ to achieve economic efficiency through a process of \\ exclusion - exclusion of marginal areas and marginalized \\ segments of population who cannot afford the costs of \\ living in the fast globalizing urban space. This reflected \\ sometimes in higher levels of poverty but more often in \\ increased vulnerability among the city-dwellers."
}

(Kundu \& Mahadevia, 2002, p. 1)

Chacune des trajectoires et chacun des événements qui rythment l'existence se déroulent dans un lieu particulier et à une époque spécifique, dans un contexte sociétal qui les influence, dont il est important de connaître les tenants et aboutissants afin d'ancrer nos interprétations (Settersten, 1999). Ceci s'avère d'autant plus crucial dans un livre qui transcende les distances géographiques, culturelles et économiques. Ce chapitre s'intéresse donc aux évolutions dont est issue la ville de Mumbai actuelle. Il offre aux lecteurs les clés pour accéder à une ville aux multiples facettes, au sein d'un pays en pleine transition et y puiser des outils de compréhension des résultats qui seront discutés ultérieurement.

Sa position de capitale commerciale confère à Mumbai un certain nombre de spécificités qui distinguent son développement économique et urbanistique. La première partie de ce chapitre se chargera d'expliquer la centralité de cette mégapole dans le système socioéconomique indien, et les retombées de cet état de fait sur les populations qui l'habitent. Comment le passage à un capitalisme forcené au moment de l'ouverture économique au début des années 1990 s'est-il répercuté sur les individus et leur niveau de vie ? A l'origine de l'essor de Mumbai, l'exode rural et l'urbanisation monstre qui s'en est suivie ont-ils servi l'avancée du pays dans son entier? Les impacts de la migration sur une mégapole en pleine ascension, qui voit en outre son paysage industriel évoluer vers un monde de services, seront aussi considérés. Mais au préalable, quelques repères de l'histoire indienne permettront d'avoir en tête les moments cruciaux 
de la seconde moitié du $20^{\text {ème }}$ siècle et du début du $21^{\text {ème }}$, un passé mouvementé depuis l'Indépendance en 1947 qui n'est pas sans conséquence.

Par la suite, le déploiement de la ville et les enjeux qui y sont attachés seront analysés. Mumbai, en tant que mégapole d'une nation émergente, subit des transformations liées à ce statut, mais aussi propres à sa qualité de presque-île. La spéculation immobilière dans un espace restreint provoque des dynamiques spatiales de périurbanisation et de concentration de la pauvreté urbaine considérables. Le foisonnement des bidonvilles qui caractérise Mumbai à l'orée du $21^{\text {ème }}$ siècle s'appuie-t-il donc sur une simple exclusion des masses? Le monde des slums recèle davantage de diversité que n'en véhicule l'image médiatique de déchéance qui nous est usuelle (Nijman, 2015).

Dans un troisième temps seront discutées les innombrables inégalités sociales qui existent en Inde aujourd'hui, tant au niveau des opportunités professionnelles sur un marché du travail en mutation que des discriminations de caste et de genre. Actuellement, le secteur de l'informalité est devenu prédominant dans différents domaines (agriculture, services, construction, ...), avec des implications importantes sur la vulnérabilité des travailleurs. Comment se décline cette informalité ? Son incidence est bien évidemment plus grande pour certaines couches de la population, déjà précarisées. Outre cette ligne de fracture qui semble ancienne mais qui a été renouvelée récemment, la société indienne est historiquement découpée en castes hiérarchisées entre elles, une configuration traditionnelle dont les heurts à la modernité interpellent. Dans un registre proche, peut-on parler d'un remplacement des castes par des classes sociales à l'heure du capitalisme et de la globalisation ? Dans la continuité de cette discussion sur les anciennes et nouvelles divisions sociales, une explication du patriarcat qui discrimine encore les Indiennes de la naissance jusqu'à la mort complètera le tableau.

\subsection{L'Inde indépendante : capitalisme et urbanisation à grande échelle}

Suketu Mehta (2006), dans son roman publié au début du $21^{\text {ème }}$ siècle, offre à la postérité l'expression de Maximum city pour décrire la mégapole 
indienne dans laquelle il a grandi. Ce qu'il souhaite démontrer ainsi est éloquent : Mumbai est la ville des extrêmes, de la richesse incontestable comme du dénuement le plus absolu, du logement le plus extravagant aux bidonvilles les plus repoussants, de l'espoir comme de la résignation. Certainement caricatural, ce sentiment de paradoxe n'est pas faux pour autant, et peut en partie être expliqué par l'évolution historique et socioéconomique du complexe urbain. En effet, Mumbai profite d'une position économique stratégique au sein de l'Inde depuis le milieu du $19^{\text {ème }}$ siècle jusqu'à son ouverture à la mondialisation après 1991, tout en présentant toujours de larges défaillances dans la gestion de ses infrastructures. Bien que capitale commerciale du pays et l'un des premiers ports de la planète, son urbanisation éclair ne s'est pas déroulée au bénéfice de tous. Entamons le voyage avec une mise en contexte historique plus générale.

\subsubsection{Ouverture sur l'histoire de l'Inde et de Mumbai}

Aujourd'hui considérée comme un pays émergent, l'Inde possède une histoire mouvante, marquée par des vagues successives d'invasions extérieures en alternance avec de longues périodes de domination. L'Empire Moghol représente l'apogée de l'expansion musulmane en Inde, qui s'installe au nord du territoire en 1572 et en prend petit à petit le contrôle, après un long affrontement avec les hindous. Tout au long de cette période, des conflits intérieurs déchirent également le territoire. En outre, depuis 1498 et l'arrivée de Vasco de Gama à Calicut, mais surtout à partir du $17^{\text {ème }}$ siècle, le pays doit supporter une colonisation européenne de plus en plus invasive (Racine, 2009). Les Portugais furent les premiers à repérer le potentiel du sous-continent indien, ainsi que sa position géographique stratégique. Plusieurs de leurs colonies se sont établi le long de la côte est et ouest de l'Inde. Ce sont eux qui bâtirent Mumbai et lui donnèrent son premier nom, avant de la céder en 1661 aux Britanniques.

Par la suite, les Anglais ${ }^{6}$ vont occuper d'autres points stratégiques du sous-continent, des comptoirs idéaux pour le trafic maritime et terrestre

6 Si différents Etablissements français ont également existé entre la fin du $17^{\text {ème }}$ siècle et 1954 (comme Pondichéry), seule Londres aura vraiment administré l'Inde en tant que nation colonisatrice. Cette mainmise passa au début essentiellement par des échanges marchands contrôlés (par le biais de l'East India Company), avant de se transformer en une gestion coloniale dès 1858 . 
que la compagnie entretient entre l'Asie et l'Europe. Mumbai, Kolkata et Chennai sont de parfaits exemples de ces villes commerciales portuaires. En effet, l'Inde regorge de ressources naturelles mais aussi de grandes plaines fertiles et de terres arables permettant des cultures de tous types (riz, millet, coton, thé, épices, etc.), qu'il s'agit de transporter vers le reste du monde. Les Britanniques transforment formellement l'Inde en possession coloniale en 1858. Un peu plus tard, avec l'ouverture du canal de Suez en 1869, le port de Mumbai devient le premier de l'Inde et prend une place centrale dans le commerce mondial (Saglio, 2001).

En 1947, la colonisation prend fin et l'Inde proclame son indépendance, tout en voyant se fractionner son territoire, à la demande des élites musulmanes (Sen, 2007). Le pays se redéfinit selon les frontières qu'on lui connait aujourd'hui, est reconnu de confession majoritairement hindoue ${ }^{7}$ et Jawaharlal Nehru en devient le premier Premier ministre durant 17 ans. De leur côté, le Pakistan et le Pakistan oriental deviennent un seul Etat, dont la religion principale est l'islam, jusqu'en 1971 lorsque le Pakistan oriental s'émancipe pour devenir le Bangladesh. La Partition de 1947 autant que l'Indépendance de 1971 se sont faites au prix du sang, au travers d'une succession de guerres ouvertes, d'affrontements entre civils, de persécutions et d'attaques des minorités, le tout entraînant la fuite de réfugiés vers un côté ou l'autre des frontières (Heuzé, 2012). Après l'indépendance, les affrontements et les violences ne s'arrêtent pas, exacerbés au contraire par les tensions géographiques et religieuses (Desai, 2000 [1960]). Entre la seconde moitié du vingtième siècle et le début du nouveau millénaire, quatre guerres successives ont lieu entre l'Inde et le Pakistan autour de la question du Cachemire, zone géographique prise en tenaille entre les deux Etats (Racine, 2009).

A l'intérieur des pays, la tension entre les individus de confession musulmane et ceux de confession hindoue est restée forte et s'est cristallisée à de nombreuses reprises dans des pogroms meurtriers. En Inde, ce sont les musulmans, les communautés indigènes ou d'autres minorités religieuses qui subissent des atrocités, pour des raisons religieuses ou parce que le gouvernement veut s'accaparer leurs terres. Ces violences affectent particulièrement les couches inférieures de la population et les femmes. Notamment, un épisode sanglant a marqué la mémoire de l'Inde et fut à l'origine de nombreuses émeutes. En 1984, l'armée assaille le

7 Le recensement national de 2011 relève 69\% d'hindous à Mumbai, ce qui est moins que la moyenne nationale. 
Temple d'Amritsar où se sont retranchés des Sikhs (minorité religieuse). La même année, en représailles, Indira Gandhi, alors Première ministre (1966-1977), est assassinée par ses gardes du corps sikhs. Des pogroms ont alors lieu dans toute l'Inde pour la venger, surtout dans les grandes villes et dans les quartiers pauvres de celles-ci. Autre incident majeur, en 1992 et 1993, la mosquée d'Ayodhya est détruite par des hindous et les hostilités interreligieuses reprennent. L'Inde s'embrase; dans les bidonvilles de Mumbai, à l'instar d'autres grands centres urbains, les émeutes font près de 2’000 morts (Appadurai, 2000; Contractor, 2012).

Ces conflits prennent leurs racines dans une opposition entre majoritaires et communautés minoritaires, qui se trouve fortement exacerbée dès la fin de la colonisation anglaise par la montée d'un nationalisme hindouiste farouche comme réponse anticolonialiste (Chatterjee, 1993). Au fil du temps, ce national-hindouisme (promulguant un imaginaire autour d'une hiérarchie entre les ethnies et les religions et un retour au «droit du sol») projette un discours de rejet des minorités religieuses (Assayag, 2005; Mahadevia, Liu, \& Yuan, 2012). Leurs différences deviennent une source de stigmatisation justifiant les exactions, mises en place par certains acteurs politiques qui bénéficient des turbulences (Appadurai, 2000; Jaffrelot, 2014). Même le parti du Congrès, sous Indira Gandhi puis son fils Rajiv Gandhi (Premier ministre de 1984 à 1989 quand il est assassiné), est absorbé par cette idéologie, issue des couches sociales privilégiées mais intégrée peu à peu par les groupes socialement exclus (Assayag, 2005). A l'heure actuelle, les tensions sont encore fortes, potentiel latent de discorde qui explose parfois en émeutes sanglantes, bien souvent dans les zones où la pauvreté, l'économie informelle et la densité de population forment un terreau favorable (Heuzé, 2009).

Mumbai a été le théâtre de bon nombre de ces pogroms, rassemblant en son sein une population nombreuse, migrante, diverse - $21 \%$ des habitants appartiennent à la minorité musulmane et $10 \%$ aux autres minorités religieuses (sikh, chrétienne ou bouddhiste) selon le recensement indien de 2011 -, avec des différences socioéconomiques importantes. En particulier, les bidonvilles de la cité ont été le théâtre de combats sanglants, qui se répètent de manière chronique jusqu'à nos jours. Depuis les années 1990, une nouvelle forme de violence éclot : les attentats (attaques à la bombe ou massacres à l'arme à feu). Le 11 juillet 2006, la ville compte plus de 200 victimes suite à l'explosion de bombes déposées dans les gares et les trains de la ville; fin novembre 2008, à nouveau une dizaine 
de bombes font près de 150 victimes (incluant celles de la célèbre attaque de l'hôtel Taj Mahal); en 2011 à nouveau trois bombes explosent.

Parallèlement à ces rebondissements historiques, l'Inde connait un développement économique en deux phases, plutôt lent jusqu'aux années 1980 puis rapide avec son entrée sur le marché mondial.

\subsubsection{Une entrée à deux vitesses dans le capitalisme mondial}

Le développement économique de l'Inde a été pénalisé par son passé de nation colonisée, qui lui imposa durant plusieurs siècles une subordination au système commercial de l'Empire colonial anglais. Après l'Indépendance, le pays prend son envol mais reste miné par une pauvreté endurante, une industrialisation archaïque et des flux commerciaux au profit du nord (Bairoch, 1997). Sa population se met à croitre spectaculairement avec la chute de la mortalité, bénéficiaire de l'arrivée des médicaments et du savoir médical occidentaux, mais cette explosion démographique accentue la pression sur un système social et éducatif déjà peu propice aux couches pauvres de la société (Dreze \& Sen, 2013).

\section{Une lente envolée...}

Au cours des décennies 1950 à 1980, l'Inde se détermine selon deux lignes directrices: la première d'ordre politique, le non-alignement soutenu par Nehru, et la seconde économique, un marché mixte, quoique de plus en plus réglementé, ainsi qu'un fort protectionnisme (Racine, 2009). A cette époque, elle fait figure de retardataire parmi les tigres asiatiques, refusant de participer aux échanges mondiaux et se refermant sur son marché intérieur. Jusqu'aux années 1970, l'économie indienne est très majoritairement agraire et sa production manufacturière concerne essentiellement le coton et les textiles, avec quelques larges structures industrielles (Chandavarkar, 1994). La Révolution verte (entre 1970 et 1980) est une réussite partielle dans le domaine de la productivité alimentaire, dans la mesure où la culture de céréales à haut rendement a permis de nourrir une grande partie de la population, alors même que sa croissance effrénée faisait craindre une vague de famine (Guilmoto, 1996). Toutefois, des impacts écologiques et des inégalités sociales accrues furent le revers accablant de cette médaille.

En partie incité par le décollage de ses voisins asiatiques, un virage s'amorce en 1981 (sous la houlette d'Indira Gandhi qui favorise l'ouverture 
au capital étranger [Assayag, 2005]) mais le basculement se profile surtout à partir de 1991, avec la mise en place d'un plan d'ajustement structurel orchestré par le Fond Monétaire International (FMI). L'Inde s'ouvre alors au commerce mondial et à l'investissement étranger (Boquérat, 2002). Cette transition économique va se traduire par un démantèlement du protectionnisme et une désindustrialisation, amenant à la fermeture de nombreuses usines, dont les filatures de coton. Le centre portuaire de Mumbai, qui depuis la fin du $19^{\text {ème }}$ siècle était réputé pour abriter les premières industries cotonnières à la base de son économie, ne fut pas épargné ${ }^{8}$.

L'industrie textile a débuté à Mumbai vers 1850 (Bhowmik \& More, 2001) et demeure le premier domaine de production jusqu'aux années 1980, avec environ 250'000 travailleurs employés dans les mills (Bhowmik \& More, 2001; Heuzé, 2009). Après 1981, le secteur secondaire est miné par la concurrence mondiale (notamment les cotons de Chine) et choisit comme échappatoire de baisser les salaires. Une large grève du textile a lieu pendant 18 mois entre 1982 et 1983, suivie par des centaines de milliers de personnes pour dénoncer des rémunérations trop faibles et des conditions de travail insatisfaisantes. Face au libéralisme montant et à l'absence de soutien du gouvernement, les syndicats ne parviennent pas à s'imposer. Au contraire, les employeurs profitent de la situation pour diminuer drastiquement le nombre de places de travail, en se tournant vers le secteur informel (non protégé) et en délocalisant leur production dans les villes alentours. Après la grève, seuls 123’000 employés sont recensés dans le textile, et à partir de la moitié des années 1990 leur nombre tombe à 80'000 (Bhowmik \& More, 2001; Mahadevia, 2002).

A l'aube du $21^{\text {ème }}$ siècle, les emplois en Inde se répartissent selon une nouvelle configuration. Le secteur primaire est toujours dominant dans le pays, bien que de manière moins flagrante $(55 \%)$. De son côté, l'industrie manufacturière reste faible économiquement et ce sont les services (services aux entreprises, communication, vente, restauration, etc.) qui prennent les rênes de la croissance et proposent désormais la majorité des emplois urbains (Chauvin \& Lemoine, 2005; Mahadevia \& Sarkar, 2012; Racine, 2009). En parallèle, le secteur informel - une réalité que l'on pourrait croire, à tort, ancienne - a pris de nouvelles formes et représente désormais la majorité écrasante des postes de travail (93\%), comprenant la

8 D'autres industries avaient également trouvé leurs marques à Mumbai, de larges groupes industriels à l'image des fameuses industries Tata.

9 Nom commun utilisé en anglais pour parler des fabriques de textile de Mumbai. 
quasi-totalité des emplois dans l'agriculture (Chauvin \& Lemoine, 2005; Racine, 2009) et $80 \%$ des travailleurs du secondaire et du tertiaire (Sakthivel \& Joddar, 2006); nous en analyserons l'impact sur la stratification sociale plus bas dans la section 1.3.1. Dans le même ordre d'idée, une montée du chômage et du sous-emploi a été constatée. En effet, ce sont davantage les secteurs intensifs en capitaux plutôt qu'en travail qui se développent à partir des années 1980, ne permettant pas d'absorber l'augmentation de la population active et la stagnation de la demande de main d'œuvre dans l'agriculture.

De manière globale, l'économie indienne s'envole avec un taux de croissance de 6\% par an entre 1986 et 2000 (Harasty, 2002), en prenant appui sur des secteurs en expansion ainsi que sur une main d'œuvre peu coûteuse, et l'Inde prend véritablement sa place au sein du peloton de tête des pays émergents asiatiques (Dyson, Cassen, \& Visaria, 2004). Une fenêtre d'opportunité démographique et économique débute, où la part des actifs est à son apogée alors que la portion de personnes dépendantes est en recul car la fécondité se réduit et le vieillissement est encore très limité (Guilmoto, 2011).

\section{...et ses conséquences en termes d'économie politique}

Un regard porté généralement sur le territoire semble attester d'une entrée dans un capitalisme mondialisé plutôt profitable au pays, malgré quelques déboires. Néanmoins, lorsque l'on se penche sur les évolutions intérieures, les coûts et les profits de cette intégration n'ont pas été les mêmes pour tout le monde (Harasty, 2002; Heuzé, 2001). L'adoption d'une politique économique néolibérale en 1991, faisant suite à une crise importante de la balance des paiements, a impliqué une réduction du déficit fiscal par une diminution des dépenses étatiques, l'ouverture du marché interne pour une croissance orientée vers les exportations, une réduction du contrôle gouvernemental sur le commerce et une promotion de la participation du secteur privé pour stimuler la compétition et promouvoir l'efficience; en bref, une montée de la pauvreté (Bhagat \& Mohanty, 2009; Heuzé, 2001). La libéralisation grandissante, la montée du secteur privé s'accompagnent d'une décentralisation, d'un amoindrissement de la présence étatique dans l'économie (Chauvin \& Lemoine, 2005), d'une redéfinition des secteurs privés et publics ainsi que des rapports entre grandes et petites entreprises (Kennedy, 2002), qui vont mener à l'augmentation des inégalités humaines et de leur inscription spatiale. La réforme du secteur public qui 
a lieu au travers de déréglementations, de privatisations et de la promotion de partenariats public-privé, a accentué cette tendance (Dupont, 2008).

Ces transformations ont affecté la répartition rural/urbain selon deux vecteurs: un accroissement favorable de l'économie et des emplois dans le monde urbain (les points d'accroche commerciale), et le délitement de l'industrie dans les campagnes, avec pour conséquence l'appauvrissement des populations rurales (Bhagat \& Mohanty, 2009). Une double polarisation géographique s'opère. La première a lieu entre les différents Etats de l'Inde qui gagnent en autonomie face au gouvernement central, propageant rapidement des disparités régionales, en particulier entre les Etats pauvres et ruraux et les Etats urbains et développés (Harasty, 2002). Le deuxième écartement se situe à un niveau inférieur, au sein même des Etats. Il est provoqué par une concentration des pouvoirs et des richesses dans les centres urbains, ce qui crée un écart grandissant avec les zones rurales (Kundu \& Mahadevia, 2002; Mahadevia \& Sarkar, 2012). Suite à un amendement à la Constitution promulgué en 1992, les municipalités urbaines se voient octroyer davantage d'autonomie en termes de planification et de financement (Angueletou, 2007). La dichotomie s'accroit: les villes deviennent des centres où la grande industrie a les mains libres, représentant les moteurs des avancées socioéconomiques du pays et des zones de haute consommation des ressources naturelles, alors que les campagnes restent en bas sur l'échelle du développement (Racine, 2009).

Les auteurs ne se montrent pas tous d'accord quant aux conséquences de la croissance économique sur le paysage socioéconomique indien. D'aucuns soulignent que la pauvreté a régressé depuis les années 90, tout en conservant une importance significative ( $35 \%$ de la population vit avec moins de un dollar par jour) (Chauvin \& Lemoine, 2005). D'autres identifient une crise de la gestion gouvernementale, caractérisée par la montée du pouvoir des élites locales et fédérales, qui mène à un accroissement de la pauvreté, suite principalement à l'augmentation du prix des denrées alimentaires (Assayag, 2005). Par contre, tous se rejoignent pour admettre le creusement des inégalités qui s'en est suivi (Harasty, 2002). En effet, les impacts sociaux positifs de la croissance économique ont été faibles en Inde, et la distribution des revenus s'avère encore plus inégalitaire que durant la période précédente (Roy, 2014). De plus, les salaires réels ont stagné et les finances publiques (le système d'imposition étant lui-même corrompu et inégal) ont été peu utilisées pour renforcer des infrastructures importantes pour la population (Drèze \& Sen, 2013). Ce 
sous-investissement a été mis en exergue par la coupure d'électricité survenue les 30 et 31 juillet 2012. Durant deux jours, 600 millions de personnes se sont trouvées privée d'énergie, une première lacune gestionnaire notable; toutefois, parmi eux se trouvaient 200 millions d'individu qui n'y avaient en fait jamais eu accès, démontrant l'énorme inadéquation structurelle de la répartition des ressources (Drèze \& Sen, 2013).

Simultanément au creusement de l'écart entre zones rurales et urbaines, les inégalités de revenu au sein de ces territoires grandissent, voyant s'opposer une élite qui s'enrichit toujours plus - ces «ultra-riches» qui résident principalement en ville (Banerjee \& Piketty, 2005; Roy, 2014) - à la masse des exclus des banlieues et des campagnes avec, à un niveau intermédiaire, la constitution d'une classe moyenne urbaine (Assayag, 2005). Dans ce contexte de déstabilisation, encore accrue par la montée du chômage dans l'agriculture, les déshérités ruraux migrent vers les villes, dans un exode rural et une urbanisation qui prend une ampleur considérable au vu de la taille de la population du pays (Bhagat \& Mohanty, 2009; Todaro, 1980).

\subsubsection{Exode rural et urbanisation rampante}

En Occident, l'industrialisation et le développement économique ont été les déclencheurs de l'urbanisation. Dans les pays du Sud et particulièrement en Asie, le même schéma a été constaté bien que plus tardivement, soit au cours du $20^{\text {ème }}$ siècle (Bhagat \& Mohanty, 2009; Narayan, 2014). Cependant, dans les pays du Sud, le processus s'est déroulé en quelques décennies seulement, faisant montre d'une rapidité sidérante (Véron, 1987). Plusieurs facteurs appellent ce phénomène éclair de croissance des villes, auxquels l'Inde répond parfaitement: l'ouverture économique à la globalisation qui crée à la fois des opportunités de travail en ville et un appauvrissement des campagnes, puis la migration du monde rural vers le monde urbain qui en découle, enfin, la constitution d'un secteur informel de l'emploi qui permet d'absorber la demande de travail des populations reléguées dans la pauvreté et qui se base sur l'exploitation d'une main d'œuvre extrêmement bon marché. Ainsi, alors qu'en 1901 moins de 11\% des Indiens sont urbains (Bhagat \& Mohanty, 2009), la part de la population indienne qui réside en ville triple au cours du siècle pour atteindre $31 \%$ en 2011 (Narayan, 2014). 


\section{Des campagnes vers les villes}

En Inde, essentiellement, deux facteurs se sont conjugués pour produire cette croissance de la population dans les villes: un accroissement naturel fort (excès des naissances par rapport aux décès) et une migration importante de personnes jeunes, en âge de fonder une famille, ce qui a aussi contribué à l'accroissement naturel (Desai, 1994). La première cause de l'envolée urbaine provient donc du renouvellement naturel; nonobstant, depuis le début du $21^{\text {ème }}$ siècle, la migration prend une importance considérable (Bhagat \& Mohanty, 2009; Narayan, 2014). Par ailleurs, la taille et le nombre de villes s'étendent également avec l'incorporation progressive des zones rurales à leur périphérie (Guilmoto, 2005), ainsi que la reclassification administrative de villages en villes suite à leurs évolutions socioéconomiques et démographiques (Bhagat \& Mohanty, 2009).

L'urbanisation indienne est donc de plus en plus liée à la migration interne. Initialement, cette dernière était de type rural-rural, mais elle s'est progressivement modifiée en un flux rural-urbain. Récemment, des migrations à l'intérieur du système urbain se sont aussi développées, effectuées surtout par des individus de niveau socioéconomique moyen ou supérieur. Le phénomène migratoire s'explique par la recherche d'opportunités et de ressources qui font défaut au point de départ, formant en somme une stratégie d'adaptation (Véron, 2006). Il se produit généralement en parallèle du développement économique et de l'augmentation des secteurs secondaires et tertiaires (Jones \& Visaria, 1997), les migrants cherchant la plupart du temps à fuir une situation de pauvreté. Dans cette idée, la relégation du monde rural, affecté par les changements de productivité des cultures et les inégalités d'accès à la propriété foncière, a poussé les habitants des campagnes vers les cités où ils sont attirés par des perspectives d'emploi (Desai, 1994). Les villes puisent dans cette réserve de main d'œuvre disponible, flexible et peu coûteuse, la sève pour alimenter leur croissance (Bhagat, 2011). Pour illustration, la moitié de la masse ouvrière des usines de textiles de Mumbai du début du $20^{\text {ème }}$ siècle, qui aura largement contribué à offrir à la cité sa place de pivot économique, était issue d'un district rural (Ratnagiri) situé plus au sud sur la côte ouest (Chandavarkar, 1994).

En moyenne, les migrants sont plutôt jeunes, entre 16 et 40 ans, la limite basse correspondant à l'âge de l'entrée sur le marché de l'emploi et de la formation de la famille (Connell, Dasgupta, Laishley, \& Lipton, 1976; Joshi, 1999). Ils ont généralement un niveau d'éducation plus élevé 
que leurs pairs qui restent au village, mais moindre que celui des citadins natifs (Desai, 1994). Les hommes recherchent avant tout un revenu, afin d'assurer la survie de leur famille, mais d'autres causes peuvent être à l'origine de leur départ : les possibilités professionnelles, l'accès à l'éducation, la protection face aux catastrophes naturelles et aux conflits. En ce qui concerne les femmes, la cause majeure de la migration est le mariage, ce qui implique des déplacements de personnes jeunes, sur une courte distance mais pour une longue durée. Entre les années 70 et 90 , elles ont eu plus tendance à migrer que les hommes et, une fois installées en ville, à prendre un travail. Toutefois, cette tendance semble s'inverser depuis le début du $21^{\text {ème }}$ siècle et ce sont à présent davantage les hommes qui arrivent (Bhagat \& Mohanty, 2009), majoritairement pour le travail, la formation ou le mariage (Bhagat, 2011).

Ces dernières décennies, alors que la structure du marché de l'emploi s'est modifiée (Srivastava \& Bhattacharyya, 2003) avec une hausse du chômage, une baisse des salaires et des places de travail dans le secteur organisé, le volume de la migration continue malgré tout de croître. Cela résulte d'une part de l'effet du réseau de connaissances sur place qui encourage à se diriger vers les agglomérations, celles-ci offrant de facto plus de perspectives d'avenir et de mobilité sociale pour les familles pauvres que les campagnes délaissées par le développement (Marius-Gnanou, 2008). D'autre part, les familles moyennes ou aisées viennent à migrer elles aussi, bien que pour des raisons différentes (Kundu \& Sarangi, 2007). Enfin, le surplus de main d'œuvre et les difficultés de logement en ville ont créé une nouvelle forme de migration, à caractère temporaire (Dupont, 2008).

\section{Migration circulaire et exclusion}

Bien que l'emploi au jour le jour soit une pratique répandue depuis longtemps dans divers domaines (comme l'industrie du textile par exemple), et que les migrants aient toujours gardé un lien avec leur communauté d'origine (Desai, 1994; Srivastava \& Echanove, 2015), par solidarité autant que par sécurité, la proportion de migrants temporaires s'est accrue depuis les années 1990 (Chandavarkar, 1994). En effet, les opportunités professionnelles formelles en ville correspondent davantage aux travailleurs qualifiés et semi-qualifiés (Narayan, 2014), il devient difficile pour les pauvres sans qualification de trouver un travail fixe en ville. Par contre, la demande en main d'œuvre peu coûteuse existe toujours et les lois de protection des travailleurs du secteur formel, ainsi que la syndicalisation 
relativement élevée, encouragent les patrons dans l'industrie de construction, la manufacture, les transports ou le commerce à embaucher quotidiennement des journaliers, de manière informelle, soit sans contrat, en fonction de leurs besoins.

La migration de courte durée, ou circulaire, se caractérise par de multiples allées et venues entre le village d'origine et les quartiers urbains, pour des périodes allant de un mois à six mois maximum. Cette forme de migration est davantage observée parmi les groupes socioéconomiques les plus bas, comprenant les individus les moins éduqués (Bhagat, 2011; Deshingkar, 2008). Ce sont avant tout les paysans pauvres sans terre, souvent illettrés et contraints de vendre leur force de travail là où il y a nécessité, qui présentent une propension à migrer comme travailleurs saisonniers et acceptent toute forme d'emploi en ville (Connell et al., 1976; Haberfeld, Menaria, Sahoo, \& Vyas, 1999; Rogaly et al., 2001). Les employeurs montrent une préférence pour cette main d'œuvre qui ne rechigne pas à la tâche et dont le salaire est peu élevé. En 2010, 30\% des travailleurs urbains étaient des temporaires (Bhagat, 2011) ${ }^{10}$.

Ainsi soumise à la précarité du revenu, la population migrante est particulièrement à risque d'être discriminée et exploitée. A la différence de la Chine, aucun processus administratif n'empêche les migrants de s'établir en ville ${ }^{11}$, mais leur vulnérabilité se construit sur une absence de protection face aux mécanismes du marché, une impossibilité d'obtenir des papiers officiels (tels que passeport, ration card $^{12}$, carte d'électeur ou encore permis de conduire [Mahadevia et al., 2012]), un manque d'accès à un logement décent et le sentiment anti-migrant qui est entretenu par la politique de certains partis pro-nationalistes. Leurs origines rurales, avec peu de formation, ainsi que la barrière de la langue et l'exclusion dans le processus de prise de décisions politiques, relèguent les migrants pauvres précaires aux marges de la société citadine. L'urbanisation a contribué à cette exclusion, en termes sociaux et spatiaux (Bhagat, 2011). Outre les conditions de travail défavorables, le rejet ambiant est subit de plein fouet au travers

10 Deux-tiers des travailleurs temporaires se trouvent dans les villes, contre un tiers dans les campagnes (Bhagat, 2011).

11 En Inde, aucun enregistrement n'est obligatoire - ni dans le lieu d'origine ni dans celui de destination - et le libre déplacement des individus est garanti par la Constitution indienne (Bhagat \& Mohanty, 2009; Bhagat, 2011).

12 Carte en deux modalités, l'une pour les personnes se situant au-dessus du seuil de pauvreté, la seconde pour celles en dessous, comprenant l'adresse permanente de l'individu. 
de déplacements forcés de population du centre vers les périphéries, de destructions de baraquements, de violences, etc. (Bhagat, 2011).

Alors que l'exode rural s'annonçait comme une transition d'adaptation vers un système urbain qui promet une augmentation des ressources financières et sanitaires, c'est en fait la part de misère des campagnes qui vient grossir celle des villes, l'une étant en quelque sorte la conséquence de l'autre (Damon, 2014). De plus, le manque chronique d'habitations dans les grandes villes affecte en premier les populations démunies, expliquant le caractère transitoire d'une grande part de cette migration. A la vulnérabilité professionnelle s'ajoute donc celle du logement, les pauvres étant de plus en plus nombreux à squatter des emplacements à proximité de leur lieu de travail éphémère, le temps de gagner quelques roupies (Nangia \& Thorat, 2000). Face à cette situation, Mumbai (dont la configuration géographique empêche l'étalement urbain) se trouve rapidement dépassée.

\section{2 (Dés)organisation de Mumbai}

En 2011, année où la croissance de population urbaine mondiale dépassa celle des campagnes, à peine 8'000 agglomérations ont été comptabilisées à travers l'Inde (malgré une définition plutôt large de celles-ci) ${ }^{13}$. En dépit du phénomène migratoire rural/urbain, environ $70 \%$ des Indiennes et de Indiens vivent toujours à la campagne, bien que la minorité citadine soit numériquement importante (377 millions de personnes sont concernées par cette situation, davantage que la population des Etats-Unis) ${ }^{14}$. Par ailleurs, si la plupart des villes indiennes grandissent rapidement, elles conservent une taille modeste et seule une cinquantaine d'agglomérations atteint le million d'habitants (Bhagat, 2011; Narayan, 2014). A l'heure

13 Les villes sont définies selon les critères de 1961: le statut administratif doit correspondre, la population doit atteindre un minimum de 5'000 personnes, parmi laquelle $75 \%$ des hommes en âge de travailler doivent se situer dans le secteur secondaire et tertiaire, enfin la densité doit être d'au moins 400 habitants par kilomètre carré (Bhagat \& Mohanty, 2009; Narayan, 2014).

14 A noter qu'il est possible que la périurbanisation, ces vastes espaces urbains en bordure de cités, ne soit pas assez prise en compte dans le calcul de l'urbanité indienne (Dupont, 2008). 
actuelle, l'urbanisation indienne suit son cours mais reste plutôt réduite, caractérisée cependant par la présence de grands bassins de population.

Se détachant du lot des million plus cities, quelques très grandes mégapoles sont devenues des centres commerciaux et politiques intégrés dans le système global: elles dominent l'économie nationale tout en abritant quelques-unes des plus grandes concentrations d'individus jamais répertoriées sur la planète (Angueletou, 2007). Ces mega-cities attirent les programmes et les politiques de gestion, à la différence des petites villes indiennes qui rassemblent pourtant davantage de pauvreté, moins d'infrastructures et moins de services de base (Mahadevia, 2001). Un monde urbain à étage s'est de cette manière progressivement constitué, avec une hiérarchisation des villes (selon leur accès au capital, leur développement, leur connectivité) qui se trouvent toujours plus opposées au monde rural (Angueletou, 2007).

\subsubsection{Mumbai, une megacity du Sud}

Mumbai représente un exemple typique de ces villes géantes, développées sous la houlette d'un néo-libéralisme déchaîné, grossissant au travers d'une migration interne tant saisonnière que de longue durée (Dyson et al., 2004; Roy, 2014). Sa taille démographique a fortement cru depuis le milieu du vingtième siècle (passant de 1.5 millions en 1941 à 2.3 millions d'habitants en 1951) suite à la Seconde Guerre mondiale et à l'arrivée de nombreux réfugiés lors de la Partition du pays (Saglio-Yatzimirsky, 2002). Dans les décennies suivantes, l'aura de Mumbai, ancrée dans l'essor industriel puis dans le développement des services et de l'informel, attire de plus en plus de migrants ${ }^{15}$. Ce phénomène, associé à un taux de fécondité encore élevé, conduit la ville à atteindre les 8.6 millions de résidents déjà en 1980. D'après la division population des Nations Unies, le peuplement de la zone urbaine de Mumbai passe de 9.9 millions d'individus en 1991 à plus du double, soit 21.4 millions, en 2016. Cela en fait la deuxième ville la plus grande au monde, confirmant les projections des démographes (Véron, 2007) ${ }^{16}$.

15 En 1988, le nombre de nouveaux migrants par semaine était d'environ 4'000 (Saglio, 2001).

16 D'après la National Family and Household Survey de 2005-06, le taux total de fécondité par femme à Mumbai est passé en dessous du seuil de renouvellement (2.1), soit 
Selon le recensement de 2001, Mumbai compte 43\% de migrants de toutes origines, une majorité de ceux-ci provenant de la campagne pauvre du Maharastra et le reste étant issu d'une migration inter-Etats (Risbud, 2003). Jusque dans les années 70 , cette dernière engageait principalement des individus originaires du Gujarat et des Ghats (zones rurales de falaises au bord de l'océan) au sud du pays (dans le Karnataka et le Tamil Nadu principalement), où sévissent sécheresses et inondations, la déforestation faisant le reste pour forcer au départ une partie de la population rurale. Par la suite, dans les années 80 , le flux s'est transformé pour voir arriver des personnes venant d'Etats du nord plus lointains, comme l'Uttar Pradesh et le Bihar, qui cherchent à échapper à une pauvreté endémique. Parallèlement, moins de migrants arrivent des Etats du Sud et du Gujarat, qui connaissent une remontée économique (Saglio-Yatzimirsky, 2002, 2013). Cette diversité des origines explique la multiplicité des ethnies et des langages qui se trouvent mélangés à Mumbai, où se parlent le Marathi avant tout, mais aussi le Gujarati, l'Urdu, le Hindi et nombre d'autres dialectes (Saglio-Yatzimirsky, 2013).

Capitale du Maharastra située sur la côte ouest de l'Inde (voir figure 1.1), Mumbai est initialement composée de sept îles et d'une grappe de petits villages de pêcheurs, qui se sont progressivement développés afin de soutenir les flux commerciaux coloniaux (Saglio, 2001). Suite à d'importants travaux de réaménagement effectués au $19^{\text {ème }}$ siècle sous l'administration britannique, les sept îles n'en formèrent plus qu'une et Mumbai atteint son aspect actuel de presque-île. Colaba, le centre-ville historique qui héberge un quartier d'affaires, se situe à la pointe sud et la totalité de l'agglomération s'étend sur $63 \mathrm{~km}$ de long (Saglio, 2001). Du fait de la disposition géographique de la ville, les seuls espaces disponibles permettant de s'étendre sont localisés vers le nord, l'est et le sud-est, à l'opposé donc du nœud des affaires et des ambassades. Dans les années 80, la croissance géographique prend de l'ampleur et un second quartier d'affaires se constitue plus au nord, dans la zone suburbaine de Bandra-Kurla, qui

à 1.7 enfants par femme. Ce qui signifie qu'à l'heure actuelle, la croissance de la population de Mumbai provient uniquement du fait que la population est jeune ainsi que de l'arrivée de nouveaux migrants (NFHS-3, IIPS, 2005-06). Par ailleurs, ce taux est sensiblement moindre que celui calculé sur l'entier de l'Etat du Maharastra (2.31), démontrant que l'urbanisation a bien encouragé cette diminution. Toutefois, la baisse n'est pas homogène à travers la ville, et la fécondité s'avère légèrement plus élevée parmi les pauvres (de 0.2 à 0.5 points supplémentaires), qui sont en partie des immigrés des zones rurales. 
semble alors se positionner au centre de l'agglomération tant l'expansion de cette dernière a été conséquente. Les quartiers industriels sont toujours davantage repoussés vers le nord (Saglio, 2001) et une troisième couronne périurbaine voit le jour, regroupant à présent la majorité de la population.

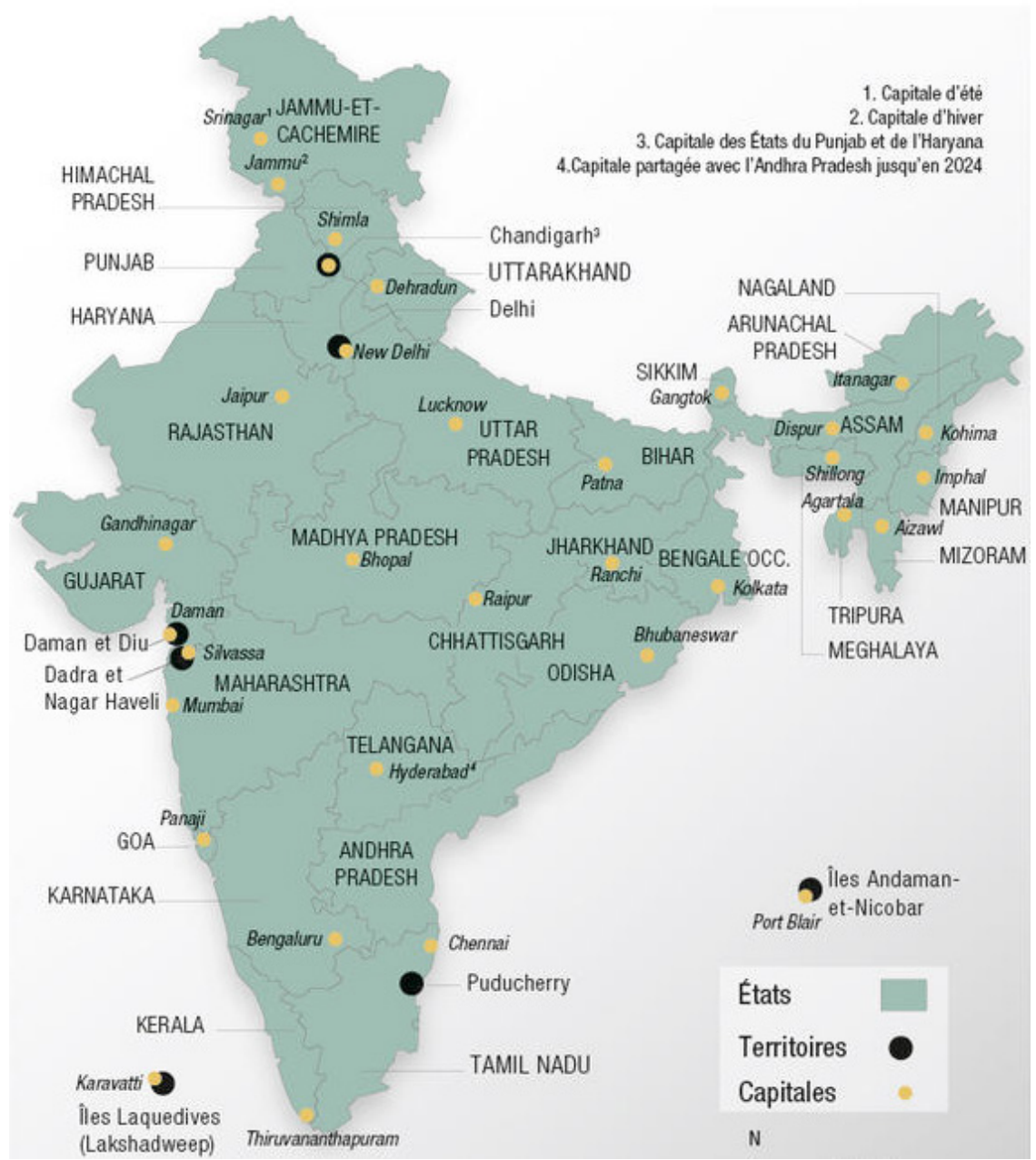

Figure 1.1: le territoire indien en 2016

Source: Dejouhanet, 2016

Le grand Mumbai est composé de quatre municipalités : Mumbai, Thane, Kalyan et Navi Mumbai, elles-mêmes subdivisées en plus petites 
administrations (voir figure 1.2). Dès 1964, le focus du développement urbain porte sur les zones périurbaines afin de décongestionner le centre. Dans les années 80, Navi Mumbai est créée au sud-est de la cité (Saglio, 2001). Toutefois, avec la crise industrielle et le manque d'investissement financier dans les décennies suivantes, le projet n'aboutit pas au résultat escompté et Navi Mumbai reste essentiellement une cité-dortoir (Angueletou, 2007). Il faut attendre le début du $21^{1 \text { ème }}$ siècle pour voir cette zone décoller, avec le développement immobilier, les commerces et l'industrie informatique, ainsi qu'un meilleur réseau de transport (Bhagat \& Jones, 2015).

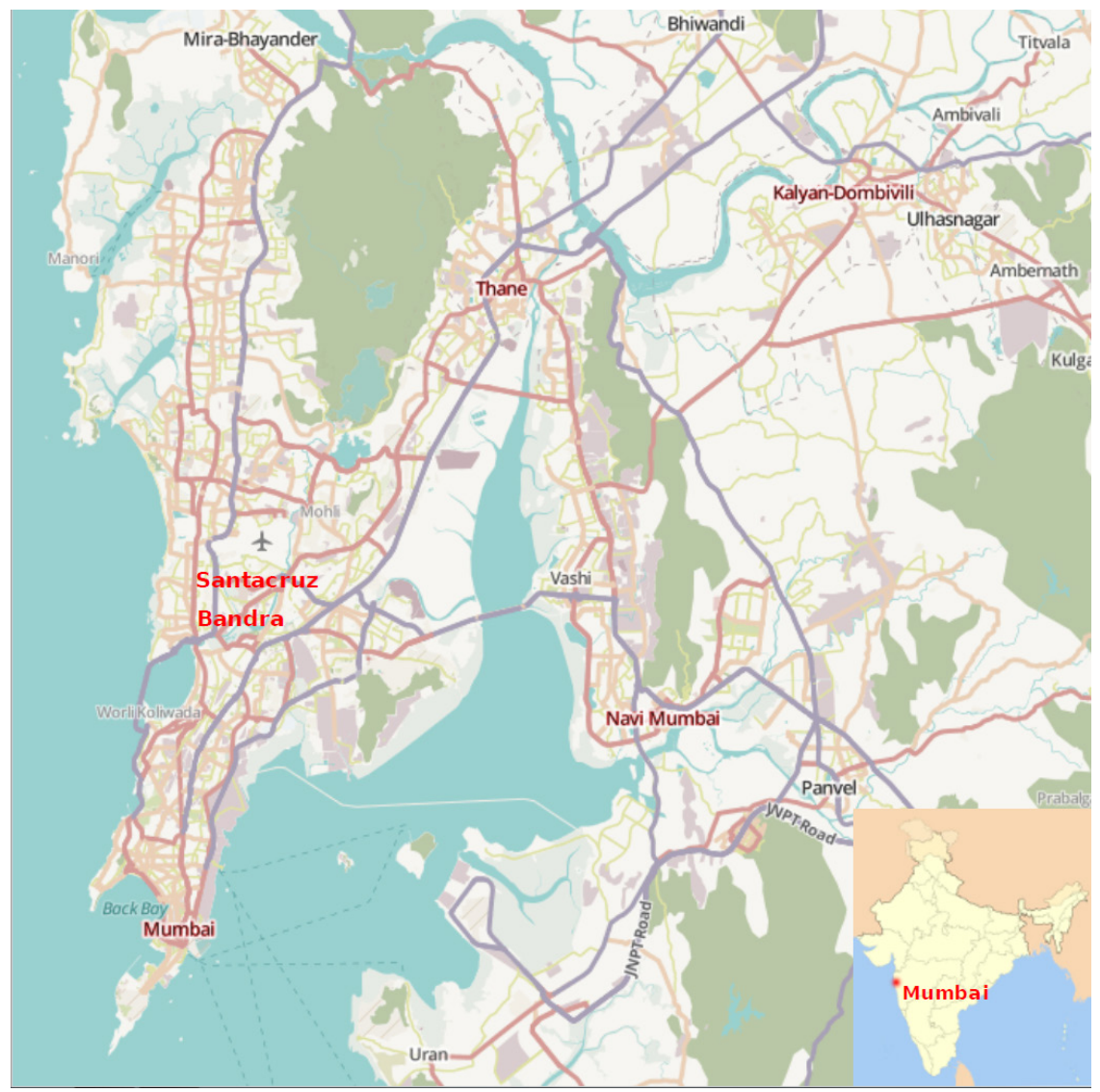

Figure 1.2: carte de la ville et de l'agglomération de Mumbai ${ }^{17}$

17 Carte réalisée grâce à l'aide précieuse de Grégoire Métral, informaticien au Centre interfacultaire de gérontologie et d'études des vulnérabilités (Université de Genève). 
Les mouvements de population depuis les espaces périurbains sont considérables. Les travailleurs empruntent quotidiennement les axes de transport ( 7 millions de personnes par jour en moyenne utilisent les voies de chemin de fer) pour se rendre sur leurs lieux de travail, au sud de la ville, dans un trajet qui peut s'avérer de plusieurs heures dans chaque sens (Appadurai, 2000). Par ailleurs, les constructions d'habitats dans ces zones extérieures sont prévues pour les classes moyennes, non pour les pauvres, d'où une prolifération rapide des bidonvilles, qui plus est sur des terres agricoles, provoquant des désordres écologiques majeurs (Angueletou, 2007). Les migrants, particulièrement défavorisés, sont les premiers susceptibles de s'installer dans ces taudis aux abords de la ville (Saglio-Yatzimirsky, 2013), la montée du prix de la terre dans les années 80 et 90 ayant rendu inaccessible toute parcelle proche du centre (Angueletou, 2007).

Le gouvernement indien a cherché à planifier l'urbanisation au travers de plusieurs plans d'ajustement dès 1969, donnant aux municipalités la charge de gérer leur propre développement. S'il existe initialement un souhait de développement régional équilibré, afin d'éviter les grandes concentrations urbaines, cet objectif se modifie avec l'entrée de l'Inde dans l'économie mondiale (Angueletou, 2007), correspondant à la volonté de favoriser une croissance économique rapide. Ainsi, le plan de 1996-2001 définit comme une priorité l'attrait de capitaux étrangers pour financer les infrastructures urbaines. Les mégapoles comme Mumbai deviennent des pôles d'investissements tout en connaissant en parallèle des problèmes de gouvernance (Dupont, 2008). Rapidement, l'apport financier privé ne suffit pas pour maintenir les infrastructures, qui deviennent inadéquates avec l'augmentation de la population et la persistance de la pauvreté. De son côté, la part de subsides provenant du public est insignifiante, l'Etat perdant son statut de fournisseur de service pour n'être plus qu'un intermédiaire (Angueletou, 2007). En 2005, un programme d'incitation à la relance est entrepris, dans le but de favoriser la planification face au creusement des inégalités, mais il ne suffira pas à endiguer la situation (Mahadevia, 2001).

Mumbai se trouve donc confrontée à une taille de territoire et de population gigantesques, où la pauvreté est grandissante. Ceci, au fond, n'a rien de nouveau : depuis longtemps les bidonvilles y sont abondants. Mais l'échec d'une ville récemment mondialisée à faire profiter des retombées positives de sa croissance à la masse des miséreux qui y vivent est de plus 
en plus cuisant. La libéralisation de son secteur public ainsi qu'un déficit reconnu de gouvernance urbaine entraînent nombre de conséquences dramatiques. Nouvel enjeu phare de la fin du $20^{\text {ème }}$ siècle, la pollution devient un problème considérable dans la métropole, tant à cause de la quantité de véhicules qui y roulent que par les industries qui polluent les abords de la mer et les sols, la mauvaise gestion des déchets, ou encore, au niveau micro, par les effluves de carburant utilisé par les ménages pauvres pour la cuisine. Globalement, l'inégalité entre les niveaux de vie - qui a toujours été de mise à Mumbai - devient véritablement criante avec l'entrée dans le $21^{\text {ème }}$ siècle, alors que la ville se positionne sur la scène internationale comme un pôle éminent de l'économie mondiale. Cette inégalité se constate dans un accès très différencié à une protection sociale et des structures de soins, à un travail ou à un logement décent.

In fine, malgré une volonté tardive de maîtriser et de planifier le développement urbain, l'évolution récente de la métropole et de ses villes périphériques montre une croissance chaotique, définie par les déséquilibres, à l'image de la prolifération des bidonvilles (Narayan, 2014). De nos jours, l'agglomération de Mumbai possède une densité évaluée à 16'000 habitants par $\mathrm{km}^{2}$ (Bhagat \& Jones, 2015), soit deux fois plus élevée que la municipalité de New York. Cette pression immobilière, associée à la montée du prix des locations ${ }^{18}$, contribue à la création de nouvelles dynamiques spatiales ainsi que d'une contrainte exacerbée sur les espaces d'occupation informels (Bautès, Saglio-Yatzimirsky, \& Boissinot, 2011).

\subsubsection{Les dynamiques spatiales en réponse à la croissance}

En plus des changements économiques, de la croissance démographique exponentielle et paradoxalement à la pauvreté urbaine, la mégapole acquiert au début des années 1990 la réputation de ville la plus chère du monde (Saglio-Yatzimirsky, 2013). La cité souffre à la fois d'un manque d'espace, d'une forte spéculation et d'une inadéquation des lois sur l'immobilier. Le coût de la vie et plus spécifiquement du logement a dès lors formidablement augmenté (Saglio-Yatzimirsky, 2013).

18 Sur la seule année 2006, le prix des locations avaient grimpés de $12 \%$ à New York contre $40 \%$ dans le centre de Mumbai (Bautès, Saglio-Yatzimirsky, \& Boissinot, 2011). 
Deux réglementations participent à ce constat. Premièrement, la loi de 1946 a gelé les loyers au niveau de ceux de 1940 (afin de protéger les locataires de l'inflation), créant un marché captif du parc immobilier et figeant le turnover des locations et achats d'appartements. De plus, cela a découragé la rénovation et l'entretien de nombreuses maisons et immeubles (Saglio-Yatzimirsky, 2013). La loi fut abandonnée en 2000, et depuis les propriétaires peuvent progressivement augmenter les loyers, ce qu'ils s'empressent de faire. En second lieu, une loi passée en 1976 imposa un plafond à la propriété foncière (limitant les zones bâtissables afin de laisser des terrains libres pour des infrastructures sociales), créant une augmentation du prix de la terre (Saglio-Yatzimirsky, 2013). Au lieu de garantir des droits aux petites gens, ces règles favorisèrent une spéculation illégale (Bautès et al., 2011). La demande des individus riches pouvant se permettre d'acheter et de construire dans des zones bien situées de la ville, participe finalement à augmenter le prix de l'immobilier dans toute la métropole.

Concrètement, en à peine 5 ans, entre 1991 et 1996, les loyers ont quintuplé dans le centre-ville, s'élevant à la hauteur de ceux que l'on peut trouver à Manhattan (Saglio-Yatzimirsky, 2013). Le logement est résolument inaccessible pour les pauvres et à la part inférieure de la classe moyenne, à moins de se localiser très à l'extérieur de la ville. L'habitat informel s'avère la seule option à la disposition de larges segments de la population. Auparavant, la cité se dessinait essentiellement par quartier selon les langues et les ethnies des résidents. Avec la carence de logements pour les individus pauvres (Chalana, 2010) et la montée de la valeur foncière, ce sont les statuts socioéconomiques qui deviennent les variables principales de cette gestion invisible du marché de l'immobilier (Saglio-Yatzimirsky, 2002). Un déplacement des habitations pauvres du centre-ville vers la périphérie se produit (Bhagat \& Jones, 2015) et, du fait de cette externalisation des masses populaires, une ségrégation apparait (Narayan, 2014). Des dynamiques spatiales se profilent en faveur des plus nantis, accompagnées d'une modification dans la gestion des bidonvilles.

\section{Les «Building Boomers» et la spéculation foncière}

Comme le reste des grandes villes indiennes, Mumbai a dû faire face aux conséquences d'une urbanisation rapide, sans réelle gouvernance de la part de l'état. De prévisibles - quoique difficiles à endiguer - problèmes de planification urbaine sont survenus (Bhagat \& Jones, 2015). Mumbai 
a vu se substituer à l'occupation coloniale une manne de propriétaires immobiliers, d'entrepreneurs, de politiciens et de promoteurs corrompus, protégés par l'administration, qui se lancent dans le jeu de la spéculation foncière et de la construction tous azimuts (les Building Boomers comme les nomme Nainan [2008]). Cette collusion a permis de libéraliser la gestion du marché immobilier, d'alléger les lois foncières par des arrangements de transfert de droit d'exploitation, conduisant à la destruction des usines et à la récupération de leurs terres au centre de la ville, pour y bâtir des immeubles modernes, des appartements coûteux, des centres commerciaux et des bureaux pour les multinationales (Nainan, 2008; Saglio, 2001; Srivastava \& Echanove, 2015).

Ce faisant, le jeu spéculatif participe à constituer des insuffisances dans la demande d'habitations par rapport à l'offre. Ainsi, dans une ville où plus de la moitié des habitants vit dans un taudis, des immeubles flambants neufs demeurent partiellement inoccupés, prévus pour une classe moyenne qui ne peut payer les loyers demandés et demeure dans des immeubles anciens qui se désagrègent. De plus en plus d'appartements très chers se construisent, dédiés aux ultra-riches, où peu de gens consomment une place considérable. Paradoxe s'il en est, le territoire de la cité se trouve en sur-occupation constante, avec une concentration particulièrement élevée dans les lieux d'habitats informels, malgré la sous-occupation des grands immeubles du centre (Echanove \& Srivastava, 2011; Srivastava \& Echanove, 2015).

Les justifications à la construction de gratte-ciels sur des zones territoriales limitées - telles que la péninsule de Mumbai ou l'île de Manhattan vont généralement dans le sens d'une solution au manque de logement et à la densité urbaine. Or, à Mumbai, la spéculation immobilière qui en découle permet au contraire l'explosion du prix des logements dans toute l'agglomération ainsi qu'une gentrification des quartiers, et finalement se trouve à l'origine de la prolifération des bidonvilles (Bhagat \& Jones, 2015; Srivastava \& Echanove, 2015).

L'état de délitement apparaît de manière évidente dans le recensement de 2001: une majorité des ménages $(65 \%)$ ne dispose que d'une pièce pour vivre, plus de la moitié (53\%) n'ayant pas de toilettes intérieures (Dupont, 2008). Autre témoin révélateur, la croissance des slums s'avère plus rapide que la croissance urbaine (Nangia \& Thorat, 2000), se constituant sous l'impulsion de deux éléments: l'établissement des individus qui ne possèdent pas de quoi payer le loyer d'un habitat formel, et l'extension 
urbaine qui englobe les zones villageoises pauvres ou les mangroves à proximité. Près de la moitié de ces bidonvilles s'installent sur des terrains privés, le reste se trouvant sur des terres de l'Etat ou du gouvernement central (Chalana, 2010). En bordure de la ville, les distinctions perçues entre le monde rural et le monde urbain se confondent dans ces taudis, qui possèdent souvent des caractéristiques issues des deux univers (Srivastava \& Echanove, 2015).

L'inflation des loyers, la pollution et la densité de population ont encouragé la réflexion autour de nouvelles politiques d'aménagement, qui visent une reconfiguration de l'espace urbain en direction d'une suburbanisation. Par ailleurs, depuis 1995, un partenariat public-privé appelé «Bombay First», composé de lobbyistes nationaux et internationaux, pousse la métropole à devenir compétitive sur la scène internationale (Bautès et al., 2011). Afin de faire émerger Mumbai comme une ville de «classe mondiale», de vastes programmes sont mis en place pour, d'une part, libérer les terrains du centre-ville qui possèdent une forte valeur et, d'autre part, résoudre la question de la pauvreté urbaine par trop visible (Bautès et al., 2011). A cette occasion, le délogement des pauvres pour une relocalisation en périphérie est la première étape (Saglio, 2001). Le processus d'urbanisation prend une tournure exclusionnaire marquée (Bhagat, 2011) et les zones de taudis sont les premières affectées.

\section{Suburbanisation et relocalisation des slums}

L'expansion horizontale des zones périurbaines de Mumbai se produit à nouveau sans pilotage global (Angueletou, 2007), engendrant des aires métropolitaines de plus en plus vastes, une densification de l'habitat accompagnée d'un manque d'infrastructures (cliniques, écoles, etc.). Dans ce lieu de haute mixité, de nouvelles fractures prennent racine, des fragmentations issues de l'aggravation des inégalités socio-spatiales entre les territoires environnant la métropole (Banerjee-Guha, 2002). Une population composée de migrants ruraux et de citadins pauvres délocalisés du centre ainsi qu'une grande partie de l'industrie polluante cohabitent dans ces territoires de périphérie, témoignant d'un changement de structure démographique et socioéconomique (Angueletou, 2007; Guilmoto, 2005).

Ajouté à ce déploiement, la volonté du lobby «Bombay First» de nettoyer le centre de la cité de ses bidonvilles prend de l'ampleur, bien que résoudre la question des slums ait toujours été dans l'air. Jusque dans les années 70, la seule politique observée (à part le laisser-faire) visait 
l'éradication pure et simple des bidonvilles, pour des raisons d'hygiène (Saglio-Yatzimirsky, 2002, 2013). Dans le corridor de 20 km entre l'aéroport international et l'entrée du centre historique - dans les quartiers de Santa Cruz, Khar, Bandra, Mahim, etc. jusqu'à Dharavi -, la spéculation immobilière a été considérable et de nombreux bidonvilles y ont été démantelés (Bautès et al., 2011; Risbud, 2009). Par la suite, une prise de conscience face à l'urgence de la situation ainsi qu'à l'inutilité de telles pratiques a fait émerger deux options alternatives: la relocalisation et la réhabilitation des slums (Bautès et al., 2011).

Le premier plan d'action, la relocalisation, consiste à simplement déplacer les familles des bidonvilles, de manière souvent forcée, vers des immeubles prévus à cet effet et bien souvent situés loin du centre-ville. Cela concerne essentiellement les slums non-notifiés, c'est-à-dire ceux qui ne sont pas reconnus par le registre foncier ${ }^{19}$. L'autre option, lancée sous la politique de Rajiv Gandhi dans les années 80, puis développée avec l'arrivée en 1995 à la tête de la municipalité du parti de la Shiv Sena, est la réhabilitation in situ des zones de délabrement (Srivastava \& Echanove, 2015). Dans ce deuxième cas, un partenariat public-privé est engagé, où l'état se charge de reconstruire des habitats à l'emplacement même du bidonville au travers d'appels d'offre aux entreprises privées. Ces dernières sont tenues de reloger les habitants sur 30\% du terrain original, après avoir détruit les anciennes structures. Le sol restant peut ensuite être utilisé pour y élever des immeubles ou des espaces commerciaux, vendus au prix du marché (Bautès et al., 2011; Narayan, 2014). Une institution compétente est fondée en 1991, le Slum Rehabilitation Authority (dit SRA), afin de considérer, superviser et ratifier les projets de réhabilitation (Bautès et al., 2011; Saglio-Yatzimirsky, 2013). En outre, une procédure de «Transfert de droits de construction» (TDR) est organisée, permettant l'indemnisation en nature (foncière) des entrepreneurs qui se chargent de bâtir des infrastructures publiques (Bautès et al., 2011).

Loin d'être suffisants pour gérer le problème de la pauvreté urbaine et des slums (à l'heure actuelle, le nombre de familles ayant bénéficié de réhabilitation est bien en dessous des attentes), ces programmes de réhabilitations sont très controversés. En premier lieu, le marché de l'immobilier n'est absolument pas favorable à une telle entreprise. Ensuite, la

19 Selon le National Sample Survey de 2011-12, près de la moitié des bidonvilles de Mumbai ne sont pas notifiés. 
relocalisation gratuite signifie un financement colossal, que l'état n'arrive pas à couvrir. Par ailleurs, les habitants des bidonvilles émettent eux aussi de nombreuses critiques. Le relogement leur est généralement imposé et l'éligibilité à un tel programme est clairement sélectif: il faut pouvoir prouver être sur son emplacement depuis une date buttoir (estimée à 1995 puis révisée à 2000 [McFarlane, 2012]) et seul le rez-de-chaussée d'un habitat est pris en compte (aucune entrée en matière n'est prévue pour les familles des étages supérieurs); enfin, les habitants doivent être regroupés en association (Bautès et al., 2011; Saglio-Yatzimirsky, 2013).

Ensuite, les nouveaux immeubles posent un certain nombre de problèmes. Bien qu'étant prétendument gratuits pour les locataires venus des slums, un loyer est en fait perçu pour l'électricité et l'eau, dont le montant est vite inabordable. Souvent mal finis, les nouveaux immeubles SRA se dégradent rapidement, connaissent des infiltrations d'eau, des moisissures et leur disposition très peu espacée, à quelques mètres les uns des autres afin de gagner en surface, les rend denses et peu lumineux. Autre dimension problématique, le temps intermédiaire entre l'évacuation et le relogement s'effectue dans des camps de transit aux conditions environnementales lamentables, pour une durée minimale de 18 mois mais qui se révèle souvent plus longue.

Finalement, l'idée de déplacer des populations des slums vers des immeubles fait montre d'une incompréhension fondamentale des manières de vivre des personnes concernées, et souligne une vision très occidentale du développement. Alors que la structure communautaire des slums favorise une survie grâce aux réseaux d'entraide et au passage par des moyens de production et d'écoulement des marchandises liés à l'habitat, la vie cloisonnée dans des édifices aux pièces exiguës sans accès direct à la rue est problématique. Bref, la réhabilitation des bidonvilles est une entreprise dont les résultats sont teintés de nuances, du point de vue sociétal comme de celui des individus (Felber \& Schmid 2014).

Dans les megacities du Sud, l'émergence des bidonvilles est ainsi devenue l'icône des fractures sociales, et l'attention portée au problème de la pauvreté urbaine est en augmentation (Narayan, 2014; Risbud, 2009). Néanmoins, pénétrer le monde hétérogène des slums n'est pas évident vu la complexité de ces lieux de (sur)vie, de production, devenus parfois de vrais espaces politiques (Gemenne, 2015; Roy, 2011). 


\subsubsection{Les slums de Mumbai}

La globalisation et la nature du capitalisme ont un impact sur la distribution des richesses (Marx \& Engels, 1999 [1847]; Narayan, 2014); subséquemment, une inadéquation du système immobilier dans une période de forte urbanisation et d'augmentation des inégalités mène logiquement à l'émergence et à la prolifération des taudis. Au niveau mondial, le nombre de personnes résidant dans un bidonville est estimé à un milliard environ, soit un sixième de l'humanité, une très large majorité (90\%) étant localisée dans les pays en développement (Véron, 2006). En 2008, les Nations Unies ont relevé que l'Inde rassemble à elle seule $17 \%$ de cette population (Narayan, 2014). L'expression d'habitat informel désigne aussi bien l'abri fait de bric et de broc que le quartier qui les regroupe (Saglio-Yatzimirsky, 2013).

\section{Le logement urbain pauvre}

La plupart des bidonvilles dans les pays du Sud se caractérisent par une surdensité de population, un habitat pauvre de plain-pied pouvant aller jusqu'à un maximum de deux étages. Les abris sont spartiates, bâtis spontanément avec les matériaux à disposition comme le bambou, le bois, le torchis, voir même uniquement des morceaux de tissus, et les toits sont faits en plaques de plastique ou de tôle ondulée (Saglio-Yatzimirsky, 2013), ce qui implique une faible ventilation, une pollution importante de l'air, et un accès insuffisant à l'eau potable (Narayan, 2014). Usuellement, une distinction est proposée entre les habitats permanents (avec des murs en ciment, un toit en tôle, prévus pour 2 à 20 personnes) et les logements non-permanents (dont les murs sont en bambou, en bois ou en toile, et le toit est en plastique). Dans les slums les plus précaires, les structures fragiles sont pour autant faciles à reconstruire, afin de pouvoir se relever au plus vite après une destruction (d'origine climatique ou humaine).

Les slums de Mumbai sont à la fois larges et très denses (avec des «quartiers» allant jusqu'à un million d'habitants, dont $42 \%$ des logements font moins de $10 \mathrm{~m}^{2}$ et $38 \%$ seulement 15 à $20 \mathrm{~m}^{2}$ ). Très peu voire aucun des foyers n'a l'eau courante ni de toilettes et les équipements sanitaires, s'ils existent, sont à partager avec le voisinage, qui peut être très nombreux. Il arrive régulièrement que l'habitat individuel soit aussi le lieu de travail ou de production de la famille, dans une juxtaposition utilitaire des fonctions (Heuzé, 2009; Saglio-Yatzimirsky, 2013; Srivastava \& Echanove, 2015). 
Pratiquement, certains logements sont divisés en deux parties, avec une pièce de vie ou de travail et une cuisine. Ainsi, la production est effectuée à la maison, qui peut aussi servir de comptoir de vente (Desai, 1994).

De petites ruelles étroites permettent de circuler entre les emplacements, insuffisantes pour laisser passer plus d'un homme à la fois et sans éclairage. Au sol serpentent des égouts à ciel ouvert, régulièrement bouchés par des ordures jetées à terre (Saglio-Yatzimirsky, 2013). De fait, la gestion des déchets ménagers et des eaux usées est un point délicat et, en l'absence de systèmes d'évacuation, les conditions de vie s'en trouvent rapidement affectées. Les maladies se transmettent facilement dans ce milieu insalubre, qui de surcroit est en mal de centres médicaux. Des montagnes de détritus sont situées à proximité, se déversant parfois sur les bords de mer, où les pauvres passent leur journée à faire du recyclage. Durant la saison des moussons, ces quartiers précaires sont particulièrement exposés aux inondations et aux débordements des égouts dans la mesure où ils sont construits au niveau du sol, dans des matériaux peu résistants (Risbud, 2009). De plus, ils se trouvent souvent situés sur des terrains vagues, au bord de rivières ou de zones d'écoulement, sur des terres basses, ou au bord des voies ferrées, qui présentent de forts risques d'accidents.

Il existe trois catégories de logements informels à Mumbai : les chawls, les jhopadpatti (le seul type considéré comme un slum dans les recensements) et le logement à même le trottoir (parfois sous des tentes, sous les ponts, dans les gares, etc.). Ce dernier groupe englobe les personnes les plus pauvres, nouvellement arrivées des zones rurales et contraintes de se satisfaire des moins bonnes conditions de vie. Ils subissent régulièrement les harcèlements policiers et la démolition de leur refuge de fortune, et sont par-là les plus vulnérables (Appadurai, 2000). Cette condition est supportée par une part sans doute conséquente de la population pauvre, mais il est très difficile de l'estimer car elle n'est pas recensée. Les résidents sont soit propriétaires de leur emplacement, auquel cas ils paient une taxe d'habitation, soit locataires (en payant un loyer à la municipalité dans le cas du chawl, ou à un slumlord ${ }^{20}$ dans celui des jhopadpatti ou du logement sur le trottoir) ; les loyers sont évalués entre $40 \$$ (au centre) et $10 \$$ (en périphérie).

20 Personne qui s'est illégalement saisie d'un lopin de terre pour créer un marché, souvent en accointance avec la police et les fonctionnaires. 
Les chawls ont été érigés par les propriétaires d'usine et la municipalité entre 1920 et 1956 pour accueillir les ouvriers des usines de textiles, puis leur famille (sans pour autant que les appartements aient été agrandis, accroissant de ce fait la densification démographique) (Bhowmik \& More, 2001; Chalana, 2010; Mundu \& Bhagat, 2008; Saglio-Yatzimirsky, 2013). Ces petits immeubles devaient être peu chers et efficients (Chalana, 2010): avec le passage des ans et le manque d'entretien, une dégradation de leur structure s'est rapidement produite. Ils sont constitués sur quatre à cinq étages, chaque appartement possédant une seule pièce avec un espace cuisine et des sanitaires partagés sur un couloir commun. Ces maisons persistent encore aujourd'hui et abritent environ $20 \%$ de la population initiale, bien que les vagues de licenciement des années 80 aient chassé une grande partie des anciens locataires. Des entrepreneurs se sont maintes fois proposés pour racheter ces espaces, et ont souvent eu gain de cause (Bhowmik \& More, 2001).

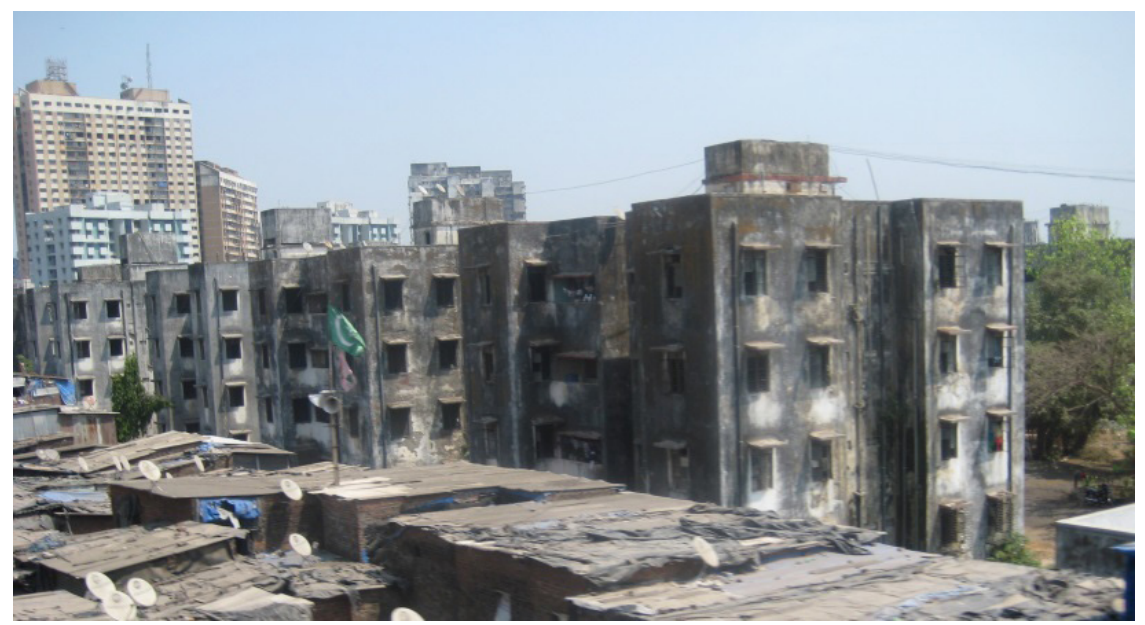

Figure 1.3: un chawl à Mumbai

Source : photo de l'auteure, 2014

De son côté, le jhopadpatti est le terme marathi pour parler d'un bidonville, une zone dégradée, très dense car souvent encastrée dans des espaces limités (entre les rails, les marécages, etc.), généralement située dans les banlieues (Saglio-Yatzimirsky, 2013). Les ménages qui y logent sont en moyenne constitués de 4.5 personnes, $60 \%$ des abris peuvent être 
qualifiés de permanents, plus d'un quart (27\%) de semi-permanents, le reste étant encore plus précaire. Toutefois, près de la moitié des logements ont un ou deux étages (les gens s'arrangeant pour améliorer leur habitat au cours de leur vie en ajoutant une pièce ou un étage), ce qui signifie quand même une certaine solidité de bâti (Saglio-Yatzimirsky, 2002). Si les chawls ont été sciemment érigés dans l'optique de permettre un style de vie communautaire, c'est la promiscuité qui règne dans les jhopadpatti, issue de la forte densité de population et des logements peu isolés, qui a progressivement mené à un mode de vie collectif (Saglio-Yatzimirsky, 2013).

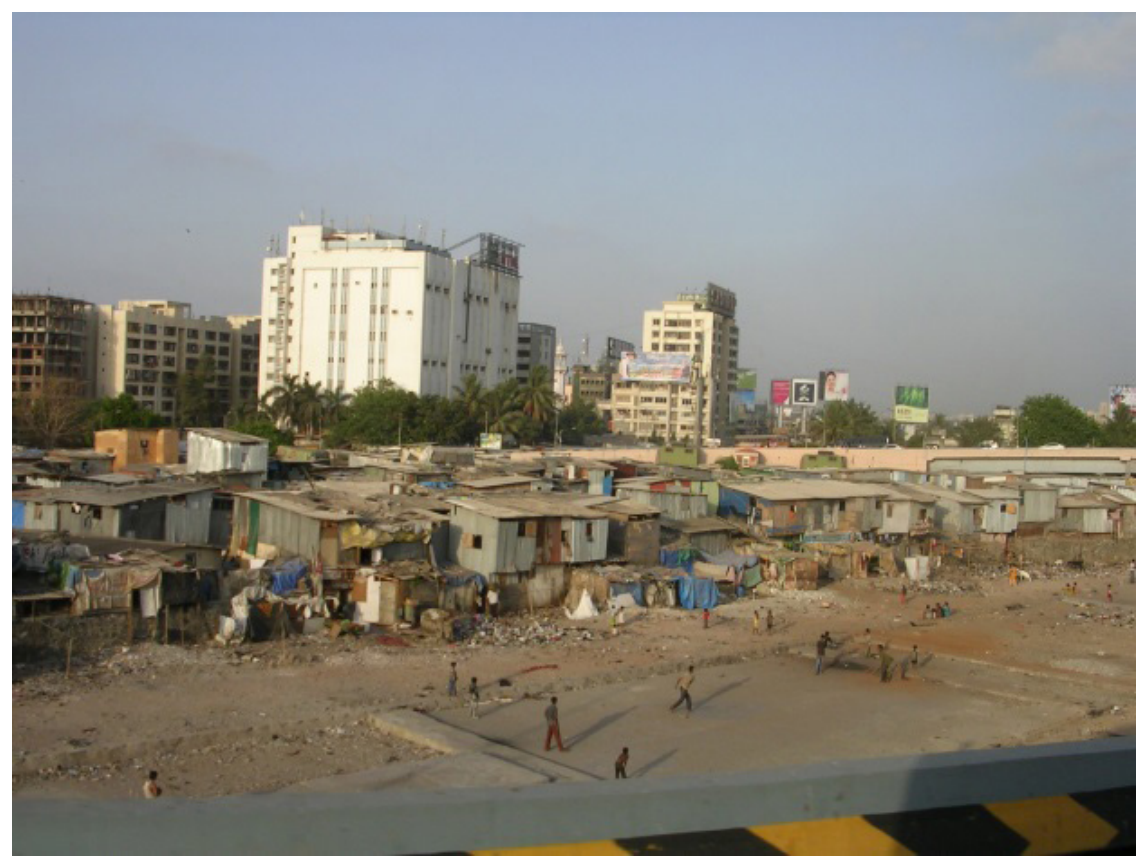




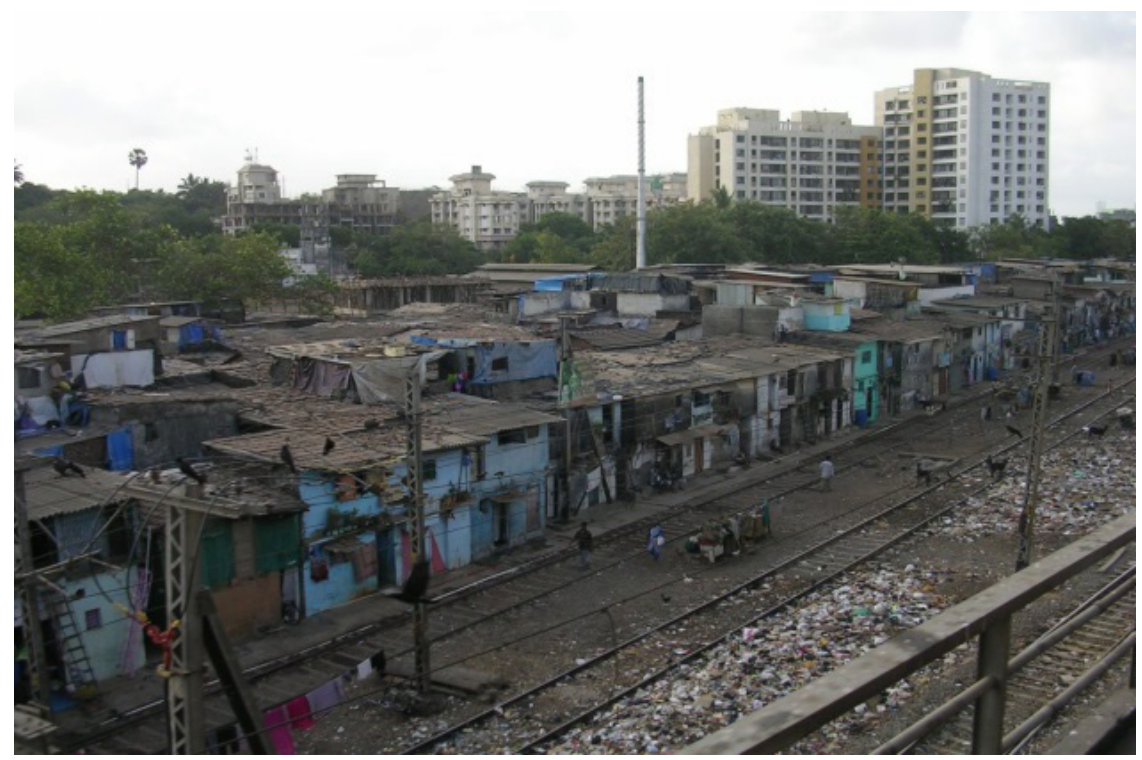

Figure 1.4: deux jhopadpatti à Mumbai

Source : photos de l'auteure, 2010

Le slum le plus peuplé et le plus fameux de Mumbai est Dharavi, situé juste au nord du centre-ville, proche du Bandra Kurla Complex. Cet espace a beaucoup attiré l'attention des chercheurs, permettant d'améliorer notre connaissance du fonctionnement de tels lieux. Dans ce bidonville, en moyenne 40 ménages doivent se partager un robinet d'eau (Saglio-Yatzimirsky, 2013). L'accès à l'électricité dans le quartier est plutôt bon, amélioré depuis 2002. Toutefois, ce n'est pas forcément le cas d'autres slums, où la connexion reste établie de manière illégale et insuffisante (Saglio-Yatzimirsky, 2013). Avec sa médiatisation, Dharavi a été reconnu comme un espace de vie dont la destruction ne pouvait être automatique, malgré l'intérêt de nombreux promoteurs pour son emplacement. Nonobstant, seul $25 \%$ des foyers sont officiellement reconnus comme résidents (car ils peuvent prouver être arrivés avant 2000) et de cette manière ne sont pas expulsables.

\section{Pauvreté, exclusion et organisation des slums}

Sans aucun doute, pauvreté et bidonville présentent des recoupements et sont inter-reliés; cependant, il n'existe pas de relation directe ou simple entre ces deux concepts (D'Costa \& Das, 2002; Narayan, 2014). En effet, 
la plupart des pauvres des villes indiennes sont contraints de se loger dans des slums. Toutefois, dans les bidonvilles ne se rencontrent pas que des pauvres, mais parfois des personnes ayant des revenus moyens, voire plutôt élevés (Risbud, 2009; Saglio-Yatzimirsky, 2002). En moyenne, 40\% de la population vivant dans les bidonvilles indiens sont considérés comme étant en dessous du seuil de pauvreté (Risbud, 2003). Ceci s'explique par les évolutions historiques : une part des générations récentes nées dans les slums a accédé à l'éducation et obtenu des postes plus ou moins confortables, tout en ne souhaitant pas s'éloigner de leur communauté. D'ailleurs, lorsqu'ils sont situés au centre, les slums permettent aux familles d'envoyer les enfants à l'école et de trouver un travail proche, ce qui est particulièrement pratique pour les femmes. Des difficultés de désignation et de recensement entrent aussi en compte. Si dans beaucoup de cas, le niveau économique des habitants des slums est pire que celui des campagnes d'où les migrants proviennent, pour d'autres individus l'habitat dans les slums reste une option de circonstance nécessaire, qui représente une amélioration de la condition initiale, ce qui n'est pas peu dire (Desai, 1994).

Les taudis sont souvent définis comme la marque d'une exclusion sociale, et pour cause. Il y règne un manque important de tous les services de base, la vie quotidienne est marquée par le stress dû à une forte proximité, par la précarité, les risques d'éviction et de harcèlement policier liés à une occupation incertaine (Kundu, 2002). Nonobstant, ces lieux de vie accueillent une population considérable, à l'image de Dharavi, et il est difficile de parler d'exclusion quand plus de la moitié de la population d'une ville est concernée (Perlman, 2010).

Malgré toutes ces nuances, l'hétérogénéité ne doit pas être surestimée; il existe bel et bien une sur-représentation des basses castes, des minorités religieuses et des analphabètes dans les slums. Par ailleurs, s'y côtoient davantage d'hommes que de femmes (Risbud, 2009) ${ }^{21}$. De prime abord d'un aspect totalement anarchique, le bidonville s'avère être en fait une microsociété très organisée, dynamique (Narayan, 2014; Nijman, 2015), établie selon des normes et des hiérarchies parallèles au reste de la société (Risbud, 2009; Saglio-Yatzimirsky, 2013). Les taudis occupant de vastes superficies, ils sont subdivisés en districts, les habitants se regroupant par région d'origine, langue, religion, caste et profession, ce qui facilite l'absorption des nouveaux arrivants. Ce type de lieu est souvent le premier

21 Toutefois, une bonne partie des ménages a un des membres qui est employé dans un poste formel permanent, tel qu'enseignant, banquier, etc. (Desai, 1994). 
rencontré par les migrants, leur offrant une collectivité dans laquelle ils doivent s'insérer et d'où ils apprennent la ville. Ceci explique qu'il n'y ait pas forcément rupture des liens sociaux et des identités, au contraire (Saglio-Yatzimirsky, 2002). Une vie s'organise, au sein d'un voisinage qui se résume au groupe d'habitations entourant le foyer ou sur la même rue (Desai, 1994).

Administrativement, les bidonvilles sont classifiés selon qu'ils sont inscrits au registre foncier (statut de notifiés) ou pas (statut de non-notifiés). Le rejet par l'Etat d'une reconnaissance officielle de ces logements précaires renvoie à la volonté de pouvoir en tout temps en expulser leurs occupants, d'autant plus intéressante lorsque le terrain concerné se trouve au sein un marché compétitif. Les risques qu'un bidonville non-notifié ne possède pas le minimum d'infrastructure (accès à l'eau, toilettes,...) est accru du fait de sa plus grande précarité (Risbud, 2009), justifiant le non-investissement dans leurs infrastructures (Subbaraman et al., 2012). Il en résulte une aggravation des dégradations dans les conditions de vie, tout en discriminant ses ressortissants qui ne possèdent pas le statut de résident officiel (les papiers d'identité étant fortement liés à une adresse de résidence). Les individus dans cette situation sont de fait poussés à la marginalité, à l'informalité et aux revenus illégaux par l'appareil de l'état lui-même (Srivastava \& Echanove, 2015).

Les inondations qui eurent lieu en 2005, le 26 juillet, ont cristallisé un certain nombre de rapports de pouvoir et de profit sur le territoire de Mumbai (McFarlane, 2012). Le centre-ville (les quartiers de Santa Cruz, Khar et Bandra en particulier) a été spécialement touché. A cette date, près d'un tiers de la ville a été recouverte de plusieurs mètres d'eau en quelques heures seulement, provenant d'une mousson particulièrement abondante. Les services de communication et d'électricité ont été coupés pendant plusieurs heures, alors que les transports étaient arrêtés - beaucoup de personnes furent bloquées loin de chez elles pendant plusieurs jours. Plus de 1'000 personnes y ont perdu la vie (noyées, électrocutées ou ensevelies), la majeure partie des victimes étant des habitants des quartiers défavorisés. Le système de drainage s'avéra inefficace, et des quartiers qui sont restés sous l'eau plusieurs jours durant connurent une recrudescence de maladies. La responsabilité de l'état, des entreprises de construction et des régies a été mise en avant temporairement, détournant pour une fois l'attention des slums, souvent les premiers accusés parce qu'ils bloquent les égouts par leur présence. Les débris de chantiers, le manque 
d'investissement public dans le drainage, le développement immobilier incontrôlé sur les espaces verts, les mangroves et les rivières, la montée de la mer et le changement climatique, le manque de prévision étatique concernant les désastres naturels et les crises, et le mépris des règles de construction furent en fait bien plus responsables de la catastrophe que ne l'étaient les bidonvilles. Malheureusement, le retour à la critique habituelle de l'habitat informel ne s'est pas fait attendre (McFarlane, 2012).

\subsection{Structures sociales inégales et discriminations culturelles}

Les sections précédentes l'ont déjà évoqué : l'Inde à l'aube du $21^{\text {ème }}$ siècle est un pays de contrastes, perceptibles tant au niveau macro (entre villes et campagnes, régions privilégiées et contrées oubliées de la modernité) que micro (richesse et pauvreté des individus, du logement, de l'accès à la santé, à l'emploi et à la formation). Les inégalités ont creusé un fossé entre les riches et les laissés pour compte de la mondialisation (Appadurai, 2005 [1996]; Sen, 2007), qui s'illustre aisément en chiffres; ainsi, les 100 personnes les plus riches du pays possèdent l'équivalent d'un quart du PIB national (Roy, 2014; Roy \& Demanuelli, 2015). Les discriminations ainsi provoquées sont notables dans le domaine de l'habitat à Mumbai, ville qui comprend à la fois la résidence individuelle la plus chère jamais construite (de 27 étages) et les taudis les plus délabrés (Roy, 2014). Mais d'autres sources d'inégalités doivent aussi être mises en avant: la culture indienne, qui mêle religions, origines ethniques et langues, contient les ferments d'une classification de la société fortement ségrégée, à commencer par le célèbre système des castes (Drèze $\&$ Sen, 2013). Si le décor a été posé en ce qui concerne l'évolution globale du pays et de Mumbai (au niveau économique et urbain), il reste à se pencher sur des traits culturels mêlant modernité et traditions. Pour ce faire, cette section présente les distinctions culturelles et religieuses, la montée de l'informalité et ses conséquences sociales, ainsi que l'héritage du patriarcat et son impact sur la position des femmes dans la société indienne. Elle offre une ouverture sur les codes qui peuvent régir le quotidien des millions d'habitants d'une mégapole comme Mumbai, davantage discutés dans le chapitre 2. 


\subsubsection{Les transformations récentes de la stratification socioéconomique et sociopolitique}

L'Inde possède une histoire ouvrière forte depuis le début du $20^{\text {ème }}$ siècle, permettant la mise en place lois protectrices du travail, avec l'établissement d'un salaire minimum ${ }^{22}$ et de protections sociales. Toutefois, ces gains sont limités au travail formel, dans le privé comme dans le public (Sakthivel \& Joddar, 2006). De leurs bénéfices sont exclus les masses de travailleurs informels; or, contrairement aux recommandations du BIT sur la promotion du travail décent, le travail informel a particulièrement cru avec la chute des barrières protectrices du marché intérieur (Basu \& Thomas, 2009) et la privatisation d'une partie des usines et du secteur public indien (McFarlane, 2012). A la fin des années 1990, ces travailleurs produisaient déjà $60 \%$ du PIB du pays.

\section{Un secteur informel florissant}

Bien qu'il regroupe énormément de tâches et de métiers différents, le secteur informel (également qualifié d' «inorganisé» ou de «non-enregistré» [Saglio-Yatzimirsky, 2002, 2013]) peut se définir selon trois critères de base. Premièrement, il est aménagé en petites unités entrepreneuriales ${ }^{23}$ ou en affaires familiales. De cette manière, il n'est pas soumis au salaire minimum légal ni à toute autre régulation engageant le droit du travail, ce qui constitue sa seconde caractéristique (Bhowmik \& More, 2001; Desai, 1994). Troisièmement, les activités informelles présentent toutes la spécificité d'être intensives en travail et faibles en capital (Saglio-Yatzimirsky, 2002, 2013). Ainsi, avec le labeur informel, les patrons bénéficient de nombreux avantages tels que l'absence de conflits sociaux et une main d'œuvre flexible, payée à la pièce ou à l'heure (Saglio-Yatzimirsky, 2002), sans avantages sociaux ni adéquation au coût de la vie, ce qui rend ce type de salariat quatre fois plus rentable que l'emploi formel (Heuzé, 2009). L'abondance de main d'œuvre (notamment dans les bidonvilles) permet également de réduire les risques et de produire à flux tendu. En outre, les prolétaires informels se prêtent peu à l'action syndicale classique même si, récemment, des mobilisations de travailleurs informels ont pris quelque

22 Inscrit dans la loi, le salaire minimum indien reste cependant très faible (atteignant à peine $2 \$$ par jour, soit 150 INR).

23 C'est-à-dire de moins de 20 employés, ou de moins de 10 employés s'il y a une utilisation d'énergie. 
ampleur au travers de la revendication envers l'état de l'assurance de leurs droits de citoyens.

L'activité entrepreneuriale de petite envergure, une classe moyenne montante demandeuse de services domestiques et la concurrence mondiale mènent à une fragmentation et une vulnérabilisation de ces travailleurs. En effet, l'informalité représente une forte précarisation d'un côté par l'embauche au besoin, sans contrat, et de l'autre par les salaires qui, déterminés par l'état du marché et la compétition mondiale, sont inévitablement minimes ${ }^{24}$ (Desai, 1994; Marjit \& Kar, 2009). Finalement, les lieux de travail informels se caractérisent par un risque accru d'accidents, d'insalubrité et d'insécurité (chantiers de construction, trottoirs, etc.), alors que les rares bénéficiaires du système de protection social et de santé sont essentiellement des employés du secteur formel (Sakthivel \& Joddar, 2006).

Les années 60 et 70 à Mumbai sont véritablement le théâtre du développement de l'informel, grâce aux transports (portuaires et ferroviaires), aux services et à la finance. Jusque-là, l'économie industrielle avait favorisé les emplois formels, majoritairement qualifiés, synonymes de sécurité et de stabilité. Ils se situaient fréquemment au sein d'entreprises structurées ou d'institutions étatiques, avec des salaires décents (soumis au revenu minimum légal) et une couverture sociale (Desai, 1994). Avec l'effondrement d'une partie de l'industrie et l'augmentation du prix du sol, une fragmentation des usines en petits ateliers a lieu, les tâches devenant peu mécanisées (Saglio-Yatzimirsky, 2002, 2013) et les places qualifiées rares (Heuzé, 2009). Le recours à une externalisation devient courant parmi les usines restantes, qui sous-traitent notamment dans les petits ateliers informels des bidonvilles. En outre, une réorientation des emplois a lieu au moment des grandes grèves de 1982-83 (Bhowmik \& More, 2001). En effet, les ouvriers militants, ainsi que dans bien des cas leurs femmes, furent contraints de chercher des revenus d'appoint durant la période de grève (Bhowmik \& More, 2001). Ils subirent une réduction de leur niveau de vie et une croissance de l'insécurité quotidienne,

24 A Dharavi par exemple, la paie est en dessous du salaire minimum, les travailleurs du cuir - emploi informel relativement bien payé - recevant entre 600 et 900 INR (soit entre 9 et 14 CHF) la semaine de 7 jours en 2010 (Saglio-Yatzimirsky, 2013). Autre chiffre révélateur, à la fin de la première décennie du $21^{\text {ème }}$ siècle, près de $80 \%$ des travailleurs informels (surtout dans l'agriculture) gagnaient moins de 20 INR par jour (soit moins de 0.29 CHF) (Basu \& Thomas, 2009). 
l'informalité leur servant alors de substitut aux assurances sociales, en garantissant leur survie par un cumul de petits emplois.

Ainsi, au moment de l'ouverture économique, le profil du marché de l'emploi local est déjà clairement orienté: selon le recensement de 1991, $65 \%$ des forces de travail à Mumbai se situaient dans l'informel et 35\% dans le formel, soit l'opposé exact de la situation de 1961 (Saglio-Yatzimirsky, 2013). L'emploi informel se décline en deux modalités, d'abord le travail indépendant puis le travail salarié (en 2007, ils représentaient respectivement $64 \%$ et $36 \%$ ) (Basu \& Thomas, 2009). Être indépendant signifie rarement posséder une réelle liberté entrepreneuriale - quoiqu'il arrive que des familles des bidonvilles s'enrichissent grâce à la possession d'un atelier (Saglio-Yatzimirsky, 2002) -, mais plutôt avoir la flexibilité de travailler où et quand on le peut, à des tâches rarement plus aisées que celles qui se rencontrent dans le travail informel employé. A Mumbai, les salariés de l'informel se retrouvent souvent dans la construction - plus de la moitié de ces emplois sont non-enregistrés (Basu \& Thomas, 2009) -, dans l'industrie de petite échelle (Saglio-Yatzimirsky, 2013) ou encore la vente ou l'hôtellerie (Sakthivel \& Joddar, 2006). Les postes sont généralement distribués de manière journalière, et l'information de l'offre de travail passe par le bouche-à-oreille. Pour cette raison, il n'est pas rare de rencontrer le matin devant les gares de Mumbai des dizaines de personnes provenant des campagnes et banlieues alentours, qui attendent tout le jour la possibilité d'une embauche.

\section{Déqualification et marginalisation des travailleurs}

L'informalité est un produit de la modernité urbaine et de la libéralisation économique, servant à qualifier des dimensions aussi diverses que l'habitat ou le travail dans une dichotomie officiel versus illégal. Dans tous les cas, ce processus repose sur la mise au ban d'une partie de la population, et devient ainsi un outil gouvernemental de gestion sociopolitique, permettant de criminaliser sur des bases administratives (McFarlane, 2012). Toutefois, l'informalité en Inde est de moins en moins un statut marginal. Elle est reconnue étatiquement dans un certain nombre de situations, ce qui démontre que les relations formel-informel sont en perpétuelle mutation (McFarlane, 2012).

Une des retombées de l'informalité est l'apparition d'un phénomène de déqualification des artisans (Saglio-Yatzimirsky, 2013). En effet, même dans les petits ateliers, le travail informel connait le principe de la division 
des tâches ; il est standardisé, répétitif, sans possibilité de transfert de connaissances, et les savoirs n'ont plus lieu d'être enseignés. De plus, la paie n'est pas forcément améliorée avec la qualification, n'encourageant pas l'apprentissage. Autre dimension problématique, le recrutement de la main d'œuvre passe parfois par des intermédiaires chargés de l'enrôlement des pauvres dans les zones éloignées du centre-ville, qui se font ensuite rémunérer (Heuzé, 2009). La sujétion des travailleurs à ces intermédiaires est monnaie courante.

Du fait qu'ils possèdent généralement un faible niveau de scolarisation et que, dans le cas des migrants, leur langage ne soit pas toujours celui de l'Etat et leur culture distincte de celle de la ville, les travailleurs informels sont soumis à un isolement important (Heuzé, 2009). A leur arrivée en ville, les migrants ruraux, pauvrement qualifiés, n'ont guère d'autre possibilité que de s'intégrer dans le secteur informel (Bhagat, 2011). Ils entrent alors en compétition avec les locaux (natifs et anciens) (Saglio-Yatzimirsky, 2002). Un sentiment de rejet envers les nouveauxvenus s'est implanté et a été exploité par les politiques en place. Au début $\mathrm{du} 21^{\text {ème }}$ siècle à Mumbai, l'augmentation du chômage - pointe de l'iceberg du sous-emploi - atteignant les 6\% dans les villes (Heuzé, 2009; Yesudian, 2009) et l'arrivée du parti du Shiv Sena ${ }^{25}$ sur le devant de la scène politique ont participé à diffuser cette xénophobie ambiante, accentuant les tensions entre populations migrante et autochtone. Une idéologie culturelle est mise en avant, favorisant l'hindouisme national et le droit du sol pour les Maharastriens d'origine (Saglio-Yatzimirsky, 2002, 2013; Singh, 2015). Montrant du doigt les musulmans et les migrants pour plaire à la classe moyenne montante, le Shiv Sena est, à ses débuts, logiquement antislums où se concentrent les pauvres (Saglio-Yatzimirsky, 1999). Toutefois, la nécessité d'obtenir des voix et le souci du populisme les poussent peu à peu à réviser leur position (Chalana, 2010; Saglio-Yatzimirsky, 2002) ${ }^{26}$.

25 Le Shiv Sena est un parti du Maharastra qui s'est formé en 1966, dont le discours est ouvertement en faveur des natifs de l'Etat et de la langue marathi, prônant un imaginaire retour au sol hindou et dénonçant - le parti détient plusieurs médias - les minorités ethniques présentes à Mumbai. C'est ce parti qui va réclamer le changement du nom de Bombay pour Mumbai en 1995 (Contractor, 2012). Son fondateur, Bal Thackeray, est décédé en 2012. Aujourd'hui, ce parti est majoritaire au Maharastra et fait partie de la coalition nationale de droite Hindouiste (Appadurai, 2000).

26 Toutes ces tensions vont se cristalliser au début des années 1990, à l'occasion de deux événements importants: le second rapport de la Commission Mandal (voir section 1.3.2), qui attise l'animosité entre les castes, et la destruction de la mosquée 
Le genre est une autre dimension largement influencée par la question de l'informalité de l'emploi. Les femmes sont en effet particulièrement concernées par les emplois temporaires et peu rémunérés (Sakthivel \& Joddar, 2006). En offrant moins de risques de syndicalisation que leurs époux, elles ont rapidement suscité l'intérêt des employeurs (Heuzé, 2009). Dans les années 90, la montée du travail féminin sert à pallier le manque de revenu du ménage, à travers des métiers sous-payés comme par exemple employées de maison ou vendeuses ambulantes (Heuzé, 2009). Aujourd'hui, à Mumbai, les vendeuses de rue sont évaluées à 200'000 environ, ce qui est probablement inférieur à la réalité, et les emplois domestiques (majoritairement féminins) à 600'000 (Basu \& Thomas, 2009). Les femmes sont surreprésentées parmi les plus pauvres des travailleurs informels ${ }^{27}$, cumulant les désavantages d'une moindre éducation et d'une moins grande flexibilité puisqu'elles doivent supporter en plus le fardeau du travail domestique dans leur foyer (en moyenne de 35 heures par semaine, contre quatre heures pour les hommes) (Basu \& Thomas, 2009). La construction est une illustration typique de cette inégalité crasse: les femmes y ont les emplois les moins qualifiés, portant de lourdes charges, recevant les plus bas salaires et subissant fréquemment le harcèlement sexuel (Basu \& Thomas, 2009).

L'informel comme secteur économique a pris de l'ampleur dans l'Inde moderne et à Mumbai avec la compétition mondiale croissante qui pousse les gouvernements à baisser les contrôles sur les employeurs pour réduire les coûts de production en diminuant les salaires et les protections sociales (Agarwala, 2008). Dans ce contexte, la proportion de travailleurs informels s'est accrue. Ils souffrent de nombreux facteurs de vulnérabilité. Précaires en termes d'emploi, ils vivent aussi généralement dans des slums

d'Ayodhya qui met le feu aux poudres à de violentes émeutes interreligieuses (voir section 1.1.1) (Appadurai, 2005 [1996]). Dans la ville de Mumbai, cela se traduit concrètement par une exclusion spatiale des musulmans (surtout des pauvres), au travers du départ d'hindous de quartiers mixtes en termes religieux vers d'autres quartiers, laissant derrière eux des espaces qui prennent l'allure de ghettos (Appadurai, 2000; Contractor, 2012). Des bidonvilles où se côtoyaient les croyances deviennent alors à majorité musulmane, réserves de travailleurs informels où de nombreuses ONG tentent de soulager une pauvreté endémique (Contractor, 2012).

27 En effet, le phénomène des working poors est courant en Inde, et les chiffres de $1999 / 2000$ démontrent que cela concerne environ $26 \%$ de la population, dont une large majorité féminine (Unni, 2009). 
et sont communément issus du groupe le plus opprimé dans la structure socioculturelle traditionnelle : le système des castes.

\subsubsection{Religion, castes et classes sociales : une hiérarchisation de la société indienne qui persiste dans la modernité}

\section{Les castes}

La société indienne s'appuie sur une hiérarchisation des individus selon le fameux système des castes (Srinivas, 1974 [1969]). Ce terme, provenant du portugais casta qui signifie non-mélangé, représente une morphologie sociale en place depuis des millénaires (Deliège, 2006). Liée intrinsèquement à la religion hindoue, cette forme organisationnelle régule les relations sociales aussi bien que la vie quotidienne (Deliège, 2011). La religion hindoue possède la caractéristique de ne pas avoir de prophète ou de dogme particulier (c'est également une religion polythéiste). En 1966, la Cour Suprême indienne a défini la foi hindoue comme basée entre autres sur l'acceptation de textes traditionnels (lois des Vedas) et la croyance à la renaissance ou de la préexistence. Les rituels sont multiples (offrandes, purifications, etc.), presque aussi nombreux qu'il y a de castes, et régissent la vie quotidienne et les étapes de la vie. En outre, le culte des ancêtres est une priorité de la religion, qui ne peut être effectuée dans une famille que par le fils.

En principe, tous les Indiens sont catégorisés dans une caste, à l'exception des individus qui ne sont pas de confession hindoue. Toutefois, la présence des castes a impacté les autres religions présentes en Inde (Heuzé, 2012; Srinivas, 1974 [1969]). Les musulmans, par exemple, se divisent en deux sections dont le statut n'est pas aussi clairement défini mais qui n'en opposent pas moins les anciens (la noblesse) et les convertis (Deliège, 2006; Saglio-Yatzimirsky, 2013). Trois facteurs ont permis le maintien des castes à travers les siècles: l'hérédité, l'endogamie et la hiérarchie (Deliège, 2011; Saglio-Yatzimirsky, 2013). La première et la seconde dimension se réalisent par la naissance (les enfants prenant la caste des parents) et le mariage à l'intérieur d'une même caste ${ }^{28}$ (Deliège, 2011). En troisième lieu, la hiérarchie s'explique par la nécessité, pour une caste endogame focalisée sur un domaine d'activité, de pouvoir accéder

28 En 2005, seuls $11 \%$ des femmes étaient mariées à un homme d'une autre caste ou sous-caste (Roy \& Demanuelli, 2015). 
au travail des autres groupes, par le truchement de rapports de pouvoir (Deliège, 2006, 2011).

Il existe environ quatre mille castes et sous-castes associées à des spécialisations professionnelles et aux diverses régions du pays (Deliège, 2006), fondées sur le modèle des quatre castes principales dites varnas: les brahmanes (prêtres qui se situent entre les dieux et les hommes, donc au statut social le plus élevé), les guerriers kshatriya, les artisans vaishya et les serviteurs shudra (Jaffrelot, 1992; Roy \& Demanuelli, 2015). Ensuite, au niveau inférieur de la pyramide se trouvent les hors-castes, les intouchables, censés s'y trouver parce qu'ils purgent leur karma, qu'ils paient le prix d'une vie antérieure mauvaise. Louis Dumont (1966) a proposé une analyse des castes sous la clé d'interprétation de la pureté, alors que d'autres scientifiques l'estiment basé sur la répartition du travail (Saglio-Yatzimirsky, 2002, 2013). Le concept de pureté et son opposé, la pollution, définissent la position hiérarchique des castes entre elles : plus la caste est pure, plus elle possède une situation élevée, ce qui détermine également l'activité qu'elle pratique. Les basses castes sont impures et peuvent donc pratiquer des métiers qui nécessitent d'entrer en contact avec des matières organiques sales (la mort ou les déchets). La manière de se nourrir est aussi influencée par ce critère de pureté, les hautes castes étant végétariennes, ne consommant ni alcool ni tabac.

\section{Les intouchables et les autres castes discriminées}

$\mathrm{Au}$ centre de l'actualité médiatique indienne se situe la vaste question de l'exclusion des basses castes et des hors-castes, dans une discrimination parfois extrêmement violente (Saglio-Yatzimirsky, 2002, 2013). Etre intouchable ${ }^{29}$ - autrement nommé Harijan, Dalit ou caste répertoriée signifie se trouver au plus bas de cette pyramide sociale hindoue, situation qui concernait quelque 185 millions d'individus en 2010 (soit 40\% des hindous) (Deliège, 2006; Guilmoto, 2011; Saglio-Yatzimirsky, 2013). Le terme d'intouchable vient des colons britanniques et est sujet à controverse depuis plusieurs années par sa qualification dénigrante (Saglio-Yatzimirsky, 2002, 2013).

$\mathrm{Au}$ moment de l'Indépendance indienne, l'intouchabilité et la discrimination de caste ont été déclarées anticonstitutionnelles, notamment grâce au travail du Dr. Ambedkar (1891-1956) qui milita pour

29 Ce terme inclut également les tribus répertoriées, ou Adivasis, des groupes aborigènes aussi discriminés que les castes répertoriées. 
l'égalité des droits politiques et rédigea la Constitution indienne de 1950 (Saglio-Yatzimirsky, 2013; Sen, 2007). Lui-même de condition intouchable, il choisit de se convertir au bouddhisme afin de sortir du système des castes inhérent à l'hindouisme (Roy \& Demanuelli, 2015). Leader des mouvements de protestation contre l'oppression des Dalits, il a influencé un grand nombre de partis politiques et d'associations qui sont encore actifs aujourd'hui. Pourtant, les injustices économiques et sociales sont toujours de mise pour ces groupes, encouragées par l'accroissement des inégalités socioéconomiques au cours des dernières décennies. En effet, les individus pauvres des autres castes ont développé un sentiment de frustration face à leur situation, qui les pousse à discriminer encore plus ceux qu'ils perçoivent comme leurs inférieurs. Le terme de Dalit, qui signifie «opprimé», et les mouvements d'opposition à la domination des castes répertoriées, ont progressivement vu le jour et pris de l'ampleur en entrant dans l'arène politique, notamment au Maharastra où la situation s'avère critique depuis la montée au pouvoir du BJP ${ }^{30}$.

Des tendances contradictoires sont à l'œuvre. Dans un sens positif, la migration, l'urbanisation et l'industrialisation ont participé à réduire les pratiques ancestrales et par là les inégalités, particulièrement évidentes dans le monde rural mais qui s'estompent dans le foisonnement de la ville (Saglio-Yatzimirsky, 2013). Effectivement, la ségrégation est rendue difficile dans les espaces publics, alors que la mobilité sociale est favorisée par l'économie capitaliste et l'émergence de nouveaux métiers (Saglio-Yatzimirsky, 2002). En outre, une discrimination positive a été promulguée par le gouvernement. Il s'agit de réservations de quotas, destinés à favoriser la représentation des Dalits dans les hautes écoles et parmi les bénéficiares de bourses, dans les emplois de la fonction publique et dans les parlements (Heuzé, 1991; Ramaiah, 1992; Saglio-Yatzimirsky, 2013). Respectivement $15 \%$ de castes répertoriées et $7.5 \%$ de tribus

30 Le Bharatiya Janata Party (BJP) est le principal parti politique de la droite dure hindouiste. Avec le Congrès national indien, d'obédience centre gauche et associé aux figures de Gandhi ou Nehru, et le Parti communiste indien, il est l'une des principales forces politique présente dans le gouvernement indien. Il est au pouvoir un court temps en 1996 puis à nouveau entre 1998 et 2004. Il revient au pouvoir en 2014 avec l'élection de Narendra Modi, actuel Premier ministre indien. En faveur d'une économie néolibérale, le BJP promeut également le retour à «une Inde pour les hindous» par le développement de sentiments xénophobes lui permettant de rallier le vote des pauvres, qui parfois se traduisent par de violentes émeutes (Roy \& Demanuelli, 2015; Saglio-Yatzimirsky, 1999). 
répertoriées (soit $22.5 \%$ des effectifs) doivent être réservés aux intouchables. Fait intéressant, il est précisé que toutes les femmes doivent être considérées comme discriminées, peu importe la caste, et peuvent donc entrer dans ces quotas.

Toutefois, les Dalits ne sont pas les seuls discriminés du système des castes. Ainsi, lors d'une première tentative en 1953, puis à nouveau en 1979, une Commission a été formée afin de déterminer les Other Backward Classes $(\mathrm{OBC})$ - les autres castes défavorisées ${ }^{31}$ - qui pourraient également bénéficier de quotas de représentation dans les universités, les administrations et les parlements. A la tête de la seconde Commission se trouvait le parlementaire B. P. Mandal, d'où son appellation éponyme. Le rapport issu de cette réflexion, élaboré en 1989 et dont la mise en pratique sera entamée l'année d'après, présente une technique de détermination des individus hindous et non-hindous pouvant être considérés OBC, basée sur des indices économiques, éducationnels et sociaux (Heuzé, 1991). La Commission Mandal a évalué les quotas réservés aux OBC devant atteindre 27\%: soit, additionné aux quotas réservés aux intouchables, une réservation totale de $50.5 \%$ des postes (Heuzé, 1991; Ramaiah, 1992). Ce rapport a suscité de vives réactions anti-réservations de la part des castes non discriminées, qui se sont plaintes d'un favoritisme basé sur l'origine plutôt que le mérite. En 2006, alors que le gouvernement cherche à appliquer ces quotas, de violentes protestations ont lieu, largement encouragées par les médias et certains politiciens, allant jusqu'à l'immolation de plusieurs jeunes étudiants de castes élevées (Ramaiah, 1992).

A l'heure actuelle, les castes n'ont pas disparu mais suscitent de plus en plus de divisions (Saglio-Yatzimirsky, 1999). Les quotas ne fonctionnent que partiellement, relativement peu appliqués, et la discrimination se maintient, par exemple au sein des emplois de fonctionnaires où les Dalits continuent d'être sous-représentés dans les postes importants. Seule l'accès à l'éducation a été facilitée par ces mesures, preuve en est l'augmentation de la représentation des intouchables parmi les diplômés (Dreze \& Sen, 2013; Saglio-Yatzimirsky, 2002). Pour autant, l'importance des castes se maintient au-delà des lois, dans un apartheid politique (une fragmentation ou «castéisation» [Saglio-Yatzimirsky, 1999]) qui ne repose pas

31 Il existe en effet une strate de castes situées entre les intouchables et les trois grandes castes supérieures, qui possèdent historiquement un faible accès à la santé, à l'éducation et aux revenus décents. 
sur la couleur de peau mais dont l'impact est similaire au racisme (Roy \& Demanuelli, 2015).

\section{Les nouvelles imbrications des stratifications socioculturelles et socioéconomiques}

Le rapprochement et la comparaison des castes avec l'analyse marxiste des classes sociales a fait couler beaucoup d'encre parmi les penseurs qui se sont penchés sur le sujet. Toutefois, beaucoup d'entre eux se rejoignent pour constater que l'assimilation des deux concepts est par trop réductrice, bien que les deux dimensions doivent nécessairement être prises en compte et qu'elles se recoupent largement (Roy, 2014; Roy \& Demanuelli, 2015; Sen, 2007). Ce constat se pose avant tout parce que les castes ne sont pas homogènes économiquement (Deliège, 2006). Ensuite, parce que les castes s'appuient sur le statut religieux, de manière explicite, alors que les classes sociales découlent d'une position dans le processus de production, sans nécessairement que les individus en aient conscience (Deliège, 2011). Le système des classes sociales est bien en passe de s'établir progressivement en Inde, mais ne remplace pas les formes de mobilité (ou de rigidité) traditionnelles, cela participe plutôt à en redéfinir les pratiques (Saglio-Yatzimirsky, 2002). Les castes remplissent à présent de nouveaux rôles: elles ne signifient plus (pour les castes dominantes) l'indépendance économique mais demeurent un instrument crucial dans la rivalité entre les groupes (Deliège, 2011; Drèze \& Sen, 2013). Ainsi, les castes supérieures se retrouvent dans les emplois symboliques empreints de pouvoir (dans le public, les tribunaux, les médias, les sports de compétition comme le cricket, etc.), alors que les défavorisés ne parviennent pas à développer leurs libertés (Drèze \& Sen, 2013). Avec la modernité, la mobilité sociale s'est diversifiée pour dépasser la rigueur première des castes (qui figeaient la main d'œuvre et étaient donc des obstacles au progrès), en reposant sur une taxinomie désormais autant socioéconomique que de statut (Dreze \& Sen, 2013).

Nonobstant la tradition des castes qui régente la société indienne, cette dernière supporte en effet simultanément un clivage entre classes supérieures, moyennes et inférieures depuis le $19^{\text {ème }}$ siècle. La langue a notamment joué un rôle considérable, les élites indiennes se définissant par le fait qu'elles parlaient l'anglais (avantage tant sous la colonisation britannique que depuis l'ouverture à l'économie globalisée) (Montaut, 2004). Langue du pouvoir et du commerce, l'anglais fait partie des langues 
officielles nationales ${ }^{32}$ qui sont apprises dans une partie des écoles ${ }^{33}$, mais dont seuls 3\% à $11 \%$ des Indiens ont la maîtrise. La scolarisation en Inde n'est pas universelle et d'une qualité plutôt médiocre, elle est aussi très inégale : si la minorité d'enfants des élites bénéficie de grandes opportunités, la majorité des enfants ont accès à un enseignement de maigre qualité. Ceci s'explique notamment par une forte insuffisance de financement des établissements publics, et par une sélection drastique des bénéficiaires des écoles privées (par des frais inabordables pour la majorité de la population). Par ailleurs, le système éducatif indien est établi en trois langues (l'idiome maternel, régional et national [anglais ou hindi]), qui n'offrent pas les mêmes possibilités d'avenir. Ainsi, l'accès à l'Université n'est possible qu'avec une bonne compréhension de l'anglais et une part très importante des étudiants reste issue des hautes castes (Heuzé, 1991). Autre témoin de la discrimination, malgré des avancées positives de ces dernières décennies, les filles continuent à avoir moins accès à l'éducation que les garçons (Drèze \& Sen, 2013). Une triple hiérarchie de caste, de langue et de genre affecte donc le système scolaire indien.

L'anglais a aussi permis la constitution de la classe moyenne par les colons, qui recrutaient pour les besoins de l'administration parmi les hautes castes (marchands, industriels, propriétaires terriens ou lettrés) (Assayag, 2005). Avec l'Indépendance, cette classe moyenne a soutenu une stabilité de l'ordre social, tout en montrant une capacité d'unification et d'opposition lorsque ses propres intérêts étaient en jeu ${ }^{34}$. A la fin des années 80 , la croissance des services et de la finance a généré une nouvelle classe moyenne dont les préoccupations rejoignent celles des élites, en particulier

32 En plus de l'anglais, il existe 18 langues reconnues en Inde: manipuri, népali, assamais, bengali, oriya, hindi, ourdou, marathi, konkani, goujarati, sindhi, panjabi, kashmiri, sanscrit, telougou, kannada, tamoul, malayam, ainsi que des centaines de dialectes.

33 La quasi-totalité des hautes écoles et des universités sont en langue anglaise, mais seulement $13 \%$ des collèges et $1 \%$ des écoles primaires, dénotant d'une volonté de sélection des élites éduquées.

34 Les mouvements actuels contre la corruption, l'inflation des prix et les violences contre les femmes sont largement portés par une classe moyenne concernée par ces questions et ayant le pouvoir de montrer son opposition, contrairement aux minorités musulmanes, Dalits, kashmiris, etc. qui subissent une répression féroce. Le reproche souvent assené à ces militants est d'oublier, dans leur lutte, de dénoncer la coercition employée contre ces minorités ainsi que leurs conditions de survie, imposées par un système qui simultanément a permis l'ascension de ces mêmes classes moyennes (Roy, 2014). 
dans les grandes villes comme Mumbai (Nainan, 2008). Elle a encouragé la libéralisation économique et adhéré au consumérisme prôné par la globalisation, tout en repoussant au second plan la culture populaire, surtout portée par les basses castes et les intouchables. Et pour cause, l'ouverture à la mondialisation ayant permis l'augmentation des revenus d'une classe moyenne évaluée aux 20\% supérieurs de la population (Drèze \& Sen, 2013). Toutefois, plusieurs niveaux se distinguent au sein de cette classe. La strate supérieure se situe à la limite inférieure du niveau socioéconomique des possédants, riche mais avec peu d'accès au pouvoir (Heuzé, 1991). La classe moyenne inférieure entretient une indépendance économique, la mobilité sociale et navigue entre tradition et modernité, loisir et consommation, visant généralement un conservatisme politique (Assayag, 2005). Elle se reconnait particulièrement parmi les métiers tels qu'employés de bureau, professeurs, ouvriers qualifiés ou boutiquiers, et est dotée d'une instruction et d'un revenu plus élevés que la moyenne indienne mais faibles par rapport aux standards des élites. Ses conditions de vie et notamment de logement peuvent s'avérer médiocres (Heuzé, 1991).

$\mathrm{Au} 21^{\mathrm{ème}}$ siècle, les classes moyennes en Inde représentent en réalité une classe privilégiée intermédiaire plutôt que majoritaire, hétérogène, incontestable pivot de la modernité qui pratique un style de vie occidental et dont l'icône peut être autant les centres d'appels notoires que les cafés internet, les grands centres commerciaux, etc. Cette nouvelle classe intermédiaire participe aussi à l'inégalité démocratique (Deliège, 2011). Les discours xénophobes ainsi que les stigmatisations de l'hindouité se sont répandus au sein de cette classe, en particulier dans le milieu urbain, éduqué (Assayag, 2005). Depuis 1998, cela va même au-delà, parmi les strates moyennes et inférieurs (Assayag, 2005; Heuzé, 1997; Saglio-Yatzimirsky, 2013). Le nationalisme et les mouvements d'extrême-droite ont débordé des couches sociales privilégiées vers les classes moyennes, puis petit à petit vers les groupes vulnérables, menacés (Assayag, 2005). Ces mouvements sont par ailleurs porteurs d'un discours virulent affirmant une conception très traditionnelle de la condition féminine et de la famille.

\subsubsection{Condition féminine et patriarcat}

La famille est une institution capitale dans la régulation de la vie des Indiennes et des Indiens. En effet, le statut d'une personne se définit 
essentiellement par la situation qu'elle occupe au sein de son ménage et de sa famille élargie, position qui évolue en fonction des étapes de son parcours de vie, en particulier en ce qui concerne les femmes. Petite fille, jeune femme prête à se marier, jeune épouse, mère, belle-mère, grandmère, veuve sont autant de positions qu'une femme peut occuper tour à tour (Bourgeois, 2013), et qui impliquent des rôles et des contraintes spécifiques. Toutefois, quel que soit son statut, une norme sociale demeure: son autonomie est réduite et subordonnée aux hommes de la famille, d'abord son père puis son mari, enfin ses fils (Vella, 2003).

Aux origines de cette discrimination se trouvent trois caractéristiques de la famille indienne: patriarcat, patrilinéarité et virilocalité (Joshi, 2016; Vella, 2003). Le patriarcat est fondé sur des rapports de pouvoir hiérarchiques de genre et d'âge - le pouvoir étant exercé par les hommes et les séniors du ménage -, qui ont pour but de régenter notamment le transfert du nom et des biens ainsi que les rapports de parenté (Mullatti, 1995; Vella, 2003). Au sein du foyer, il sert à justifier le contrôle de l'homme sur la femme (Bourgeois, 2013), mais souvent ces règles de subordination sont aussi intériorisées et reproduites par les femmes plus âgées (bellemère vis-à-vis de sa bru par exemple). En outre, plus l'écart d'âge entre les époux est grand, plus cette forme d'ascendance s'accentue. La patrilinéarité est une inégalité qui repose sur l'héritage et qui signifie que les femmes ne peuvent recevoir de biens ou de propriétés de leur lignée, ces derniers se transmettant de pères en fils ${ }^{35}$. Enfin, la virilocalité indique que les épouses partent habiter sous le toit de la belle-famille au moment du mariage et quittent donc le nid originel. Elles sont alors obligées de s'intégrer à cette belle-famille élargie ce qui, en tant que dernière arrivée, représente souvent une nouvelle forme de dépendance (Dimri, 2015).

Les discriminations de genre sont une réalité ancienne (Vella, 2003) qui perdure dans l'Inde actuelle, voire se détériore depuis quelques décennies (Banerjee, 2005), à l'image des viols et des violences à l'encontre des femmes ${ }^{36}$. Effectivement, après l'Indépendance du pays, les quelques

35 Parfois la dot permet de garantir un héritage à la fille, sous formes de biens qu'elle conserve (bijoux, etc.).

36 Les violences contre les femmes ont longtemps été taboues, et le sont encore dans de nombreuses strates défavorisées de la société. L'impression d'augmentation des viols et des violences est donc difficile à vérifier, puisque bien souvent ces crimes ne sont pas dénoncés par les victimes, particulièrement si elles sont d'origine pauvre. Toutefois, la répression croissante à l'égard de l'ascension sociale des Dalits est au cœur de ce phénomène, qui semble pourtant de prime abord très individuel, et le 
zones de l'Inde qui connaissaient des coutumes matrilinéaires ont évolué vers le modèle patriarcal (Mullatti, 1995). De plus, bien que des lois promouvant l'équité de genre aient été votées (à l'image de la loi de 2005 sur les droits de succession pour les enfants des deux sexes), la réalité démontre que l'inégalité reste ancrée dans les mœurs.

Dans la religion hindoue comme dans de nombreux autres systèmes sociétaux, le mariage est utilisé comme un outil pour favoriser l'endogamie, en l'occurrence ici celle des castes, dont elle est une composante cruciale, manifestant leur fermeture et permettant leur reproduction (Joshi, 2016) ${ }^{37}$. Généralement, les unions sont arrangées par les familles, qui choisissent les candidats répondants aux attentes de caste et de souscaste et négocient une dot financée par la famille de l'épouse ${ }^{38}$. En ville, les jeunes bénéficiant d'une éducation obtiennent un peu plus de liberté dans leur choix du conjoint, mais le mariage arrangé reste la norme (Sharangpani, 2010). Associée à la dot, cette forme d'union constitue un risque de perte d'autonomie importante pour les femmes hindoues, au travers de l'accueil dans la belle-famille (Loiselle-Léonard, 2001). Si les termes de l'échange ne conviennent pas aux deux parties, les conséquences peuvent être des pressions, du stress, des conflits à l'encontre de la jeune mariée. Les violences domestiques représentent l'apogée de ces moyens de contrôle de la femme, par ailleurs souvent pratiqués au tout début de l'union (Bourgeois, 2013).

A l'opposé du mariage arrangé endogame, existe le mariage mixte (entre langues, religions ou castes), appelé dans le langage courant un «mariage d'amour» et qui engage - si on considère que tous les mariages exogames appartiennent à cette catégorie - un peu plus d'une union sur dix. Dans ce cas, les conjoints ont fait leur choix seuls, en général sans obtenir l'accord de leurs familles. Mais ce second type d'union est encore peu répandu, et la source de nombreuses tracasseries pour les époux (surtout de la part la belle-famille si celle-ci accueille la mariée). Au travers de ce phénomène, se distingue la tendance collectiviste de la société indienne,

caractère public de celui-ci, lui conférant sa portée mobilisatrice, est bel et bien en augmentation (Dreze \& Sen, 2013; Roy \& Demanuelli, 2015).

37 Les autres religions présentes en Inde se sont adaptées au système des castes et fonctionnent souvent d'une manière similaire en ce qui concerne la famille.

38 En 2000, 95\% des mariages étaient arrangés et accompagné d'une dot (Elkouri, 2000). Bien que cette dernière soit interdite par la Constitution depuis 1961, elle s'est largement répandue et la coutume est plus que jamais de rigueur. 
où les intérêts du groupe priment sur celui de l'individu (Chasles, 2008; Loiselle-Léonard, 2001). Le mariage sert à lier deux familles, en préservant les statuts socioéconomiques, avant d'être le symbole du couple.

Culturellement, il demeure impérieux de marier sa fille ${ }^{39}$. Les frères attendent d'ailleurs communément que toutes leurs sœurs soient mariées avant de suivre à leur tour (Joshi, 2016). On conçoit donc que l'avancée en âge signifie une perte de valeur pour la jeune femme sur le marché matrimonial, mais soit aussi associée à des pressions émanant des membres du foyer, du voisinage, des collègues (Guilmoto, 1996). Il n'est dès lors pas surprenant que l'âge médian au mariage des femmes indiennes ne se soit que très légèrement élevé au fil du $20^{\mathrm{ème}}$ siècle, tout en restant extrêmement précoce : de 16 ans en 1951-1961 (respectivement 22 ans pour les hommes), il est passé à 17 ans en 2005-2006 (respectivement 23 ans ${ }^{40}$. Par ailleurs, le taux de célibat définitif est de moins de 1\% (Guilmoto, 1996).

Une fois le mariage conclu, la reproduction est elle aussi fortement encadrée par la communauté, à travers des pratiques normées telle que l'abstinence, l'allaitement prolongé, le tabou du remariage, etc., qui tendent à réduire la fécondité. Toutefois, l'urbanisation et l'exode rural entraînent une réduction des pouvoirs de contrainte de la famille élargie ; la nucléarisation des foyers contribue de même à une gestion plus étroite de ces dimensions. Cela a probablement favorisé la diffusion de la contraception. A Mumbai, 59\% des femmes mariées la pratiquent, les techniques permanentes (stérilisations) étant davantage employées dans les bidonvilles, où moins de $40 \%$ des femmes utilisent une technique dite moderne, ou réversible, de contraception (Guilmoto, 1996).

La séparation des tâches entre sphère publique et sphère privée est importante entre les genres, ce qui participe au manque d'indépendance féminin (Guérin, 2008). L'épouse est responsable de l'éducation de ses enfants, de la tenue du ménage et de son harmonie. Mais même au sein d'un foyer dont la gestion est reléguée aux membres féminins, l'égalité n'est pas obligatoirement de mise. Des marques de discriminations sont

39 Le mariage reste encore aujourd'hui un passage obligé: selon les chiffres de 20052006, 95\% des femmes de 45 à 49 ans sont mariées et 67\% des hommes de la même tranche d'âge (Véron, 2008).

40 Il a été démontré que la maternité des adolescentes est plus élevée parmi les pauvres et les moins éduqués, en campagne surtout, que parmi les citadins de classe moyenne ou aisée (à Mumbai, 10\% des adolescentes vivant dans les taudis sont concernées, contre 3\% des adolescentes vivant hors des bidonvilles) (Guilmoto, 1996). 
visibles au quotidien, par exemple lorsque les hommes mangent avant les femmes (Dimri, 2015). La femme obtient du respect avant tout lorsqu'elle devient mère, plus précisément si elle met au monde un garçon (LoiselleLéonard, 2001). Avant d'obtenir ce statut de génitrice d'un enfant mâle, de lourdes pressions peuvent s'abattre sur elle de la part de sa belle-famille et de son mari, pouvant aller jusqu'à la répudiation. Dans la sphère publique, les obligations financières reposent sur les épaules du mari, qui doit assurer la subsistance du ménage (Guérin, 2008; Loiselle-Léonard, 2001). Néanmoins, en pratique, ce sont souvent les femmes qui se chargent de l'organisation financière et de la comptabilité (Guérin, 2008).

Un pan conséquent des inégalités de genre provient du fait que les femmes n'ont que peu accès au marché du travail ${ }^{41}$, ce qui est une entrave à l'émancipation féminine (Ghosh, 2015; Guérin, 2008). Par ailleurs, lorsqu'elles participent au revenu du ménage, c'est généralement au travers de travaux infromels très précaires, sans qualifications (vente de rue, travaux domestiques,...) (Mahadevia, 2002). Posséder une source de revenu stable permet de se projeter, d'anticiper et d'être le gestionnaire de sa vie. Sans disposer de ressources (foncière à cause de la patrilinéarité ou salariale du fait qu'elle ne travaille pas), la femme se retrouve dépendante de son mari et démunie en cas de séparation conjugale. En outre, bien qu'il soit de plus en plus fréquent, le divorce demandé par la femme reste un sujet tabou et l'épouse est menacée du rejet de la part de sa communauté ou de sa famille lors d'une séparation, par crainte des retombées sur leur réputation (Bourgeois, 2013).

L'idéologie patriarcale a une autre conséquence bien connue sur le sous-continent: la préférence pour le fils (Guilmoto, 2008; Das Gupta et al., 2003; Vella, 2003; Véron, 2008). Le fils s'occupant des parents dans la vieillesse, des rituels religieux et recevant l'héritage familial alors que la fille part au moment du mariage et coûte en plus de cela le prix de la dot, il est économiquement bien plus sensé d'élever un garçon en Inde. Ainsi, avoir des enfants revête une importance considérable pour tous les Indiens, mais avant tout s'il s'agit de mâles.

Le rapport de masculinité (calculé comme le nombre de femmes pour 100 hommes) est un indicateur important du niveau de discrimination féminin. Sans impact extérieur, il nait environ 105 garçons pour 100 filles, chiffre qui s'équilibre avec l'avancée en âge et la légère surmortalité masculine. Or,

41 Dans l'enquête NFHS-3 de 2005-2006, 43\% des femmes de 15 à 49 ans ont un emploi contre $87 \%$ des hommes de ces âges en Inde. 
en 2011 en Inde, il est né 109.4 garçons pour 100 filles (Véron \& Nanda, 2011). Déséquilibré en faveur des garçons, ce ratio montre qu'il existe avant la naissance des pratiques de sélection des embryons afin de conserver les mâles, ainsi que des facteurs sociaux de discrimination après la naissance ${ }^{42}$. Depuis que la chute de la fécondité s'est répandue dans le sous-continent et que la libéralisation initiée en 1991 a accru les coûts de santé (consultations, médicaments), l'avortement des fœtus féminins se pratique, de même que la discrimination envers les petites filles, creusant l'écart déjà substantiel du ratio de la population féminine par rapport à la masculine.

Pour résumer, la famille en Inde est un lieu où les rôles et les positions sont diverses selon le genre, suivant le schéma classique de la sphère privée réservée aux femmes et de la sphère publique comme l'apanage des hommes. Ce lieu sert aussi de refuge et favorise la coopération de ses membres, d'autant plus lorsqu'aucune assurance sociale ne palie aux difficultés économiques du quotidien. Cette réalité est vraie en particulier parmi les couches les plus pauvres de la population, fortement représentées au sein des habitants des bidonvilles. Cependant, la famille possède également une importance considérable dans les classes moyennes, de par la forte tradition patriarcale et patrilinéaire qui se maintient dans la culture indienne. Se marier reste indispensable, et se marier selon la tradition de l'union endogame et de la dot versée à la famille de l'époux reste une norme largement dominante. En outre, il est crucial d'avoir une progéniture pouvant recevoir un héritage et s'occuper des parents vieillissants, donc d'avoir des garçons. Lors de ces deux moments de construction du ménage (mariage et procréation), la place de la femme subit une discrimination par rapport au sexe masculin.

\subsection{Conclusion}

Ce que cet aperçu du contexte de l'Inde d'aujourd'hui, si succinct soit-il, a permis de saisir est la situation d'un pays émergent, et surtout d'une ville à la croisée des chemins alors que la globalisation s'arrange un passage

42 La surmortalité féminine peut survenir durant les premières années de vie par un accès restreint aux soins, ou à la nourriture équilibrée, liée à la dévalorisation des femmes en Inde (Guilmoto, 1996). 
en son sein. Des ruptures anciennes (hindous contre musulmans, riches et pauvres, castes défavorisées contre castes supérieures, patriarcat) cotoient des ruptures nouvelles (villes contre campagnes, extrême richesse contre extrême pauvreté, capitalisme contre misère). Pour résumer, il s'agit d'une illustration du choc de la modernité contre l'écueil des traditions (LaniBayle \& Mallet, 2006).

Le cœur économique du pays, Mumbai, bat depuis plusieurs décennies au rythme de cette modernisation, largement encouragée par une entrée en force dans l'économie planétaire. Parallèlement, dans un effort de prouver sa valeur urbanistique aux yeux d'un monde bien cruel, l'urbanisme sauvage de la mégacité a ouvert la porte à une répartition spatiale fracturée entre la vertigineuse ascension politique et économique d'une poignée d'individus et le naufrage du plus grand nombre vers les abîmes du dépouillement le plus complet. Ainsi, les disparités d'origine et de religion recoupent les inégalités socioéconomiques et les localisations dans les divers types de quartiers. Ce qui se constate dans le plus misérable des logements : le bidonville. Ce phénomène comprend une montée de l'informalité, tant dans le domaine du logement que de l'emploi, ainsi que la cohabitation compliquée d'une population dans le quartier mais aussi au sein du foyer. 



\title{
Chapitre 2. Parcours de vie et vulnérabilités en Inde
}

\author{
"Social inequality exists, of course, in non-Western \\ as well as in Western societies. Perhaps we can \\ learn more about it from the experiences of the \\ former because it is here that it exists in its more \\ extreme and manifest forms both as a set of facts \\ and as a system of values."
}

(Béteille, 1974, p. 11)

Ce travail de recherche est confronté à un défi: ancré sur un terrain indien que nous avons commencé à présenter dans le chapitre précédent, il s'appuie sur des références théoriques essentiellement occidentales. Plus concrètement, notre enquête interroge la vulnérabilité dans le parcours de vie des individus. Alors que les chercheurs indiens, confrontés à une démographie explosive et à une population de plus d'un milliard d'êtres humains, mettent toujours l'accent sur les masses et les grands chiffres, alors qu'existe une littérature abondante opposant, parfois de manière outrancière, des sociétés individualistes occidentales à des sociétés orientales qui seraient avant tout collectives (Dribe, Manfredini, \& Oris, 2014), notre approche peut apparaître comme une sorte d'hiatus interculturel, en questionnant des individus reconnus dans leur subjectivité, puisque c'est la mémoire de leur vie qui nous documente leurs vulnérabilités. Ces dernières sont, comme nous l'avons vu dans le chapitre 1, largement étudiées, y compris par les chercheurs indiens, dans leurs dimensions concrètes, objectives; mais c'est leur poids mémoriel et leur expression que nous avons enquêtés. Les lignes qui suivent présentent donc un travail de dialogue, de confrontation, de transposition et d'intégration.

Après un rappel succinct des objectifs du paradigme du parcours de vie, les structures sociales qui encadrent l'existence seront analysées plus spécifiquement au regard du contexte indien. Les normes sociales qui imprègnent les vies en Inde dès la naissance sont dictées par différents facteurs (classe sociale, position culturelle de caste et genre). Durant l'existence, des institutions participent à orienter, voire guider, le déroulement 
des trajectoires, en accord avec les normes dont elles sont l'émanation et qu'elles reproduisent. Alors que les chercheurs en sociologie du parcours de vie parlent d'une standardisation des trajectoires liée à une institutionnalisation typiquement occidentale, voire européenne (Kohli, 1986; Mayer, 2004), similarité et homogénéité semblent marquer les vies en Inde, sans que les sources en soient les mêmes. Cela nous amènera à spécifier la force persistante des institutions informelles dans le contexte indien. Les imprégnations culturelles à travers la socialisation construisent également une stratification profonde selon l'âge et le genre des individus (Settersten \& Owens, 2002). De puissantes normes sociales sont imposées aux individus en fonction de leur âge ainsi que de leur sexe, l'une comme l'autre de ces dimensions renvoyant à des rôles attendus.

Les forces qui résultent en une large standardisation des étapes et des transitions de la vie en Inde pourraient donner l'impression d'une société immobile, enfermée dans des traditions immuables. Ce stéréotype, souvent associé aux sociétés asiatiques (Dribe et al., 2014), n'est pas fondé. Rejoignant les constats issus de la théorie du parcours de vie sur les concepts d'âge, de cohorte et de génération, nous démontrerons pourquoi l'impact de l'histoire globale ne doit pas être sous-estimé. Les cohortes nées après l'ouverture économique de l'Inde, particulièrement si elles résident en ville, ont des parcours de vie qui ne peuvent que différer en partie d'avec leur prédécesseurs, dans leur accès à l'éducation surtout, mais aussi aux systèmes d'information, à la contraception, ou à d'autres ressources leur offrant un contrôle (relatif et inégal) sur leur existence.

Notre échantillon d'Indiennes et d'Indiens, résidant dans et hors des bidonvilles, porte les stigmates d'une pauvreté et d'une précarité marquées. Leurs vulnérabilités, celles qu'elles et ils ont bien voulues nous révéler, sont issues de leurs trajectoires personnelles situées dans l'histoire, mais elles s'inscrivent aussi dans une temporalité immédiate et un contexte spatial spécifique, à Mumbai dans les années 2012-2014. La dernière section de ce chapitre s'appuie sur les apports théoriques issus de recherches récentes sur la vulnérabilité. Plus exactement, l'étude des risques liés à la modernité et les stress qui peuvent influer sur l'existence sont des composantes cruciales car elles seront à même de nourrir les interprétations de nos résultats empiriques. Ces réflexions nous offrent d'ailleurs un pont entre la théorie et les analyses car elles permettront de démontrer l'utilité d'une enquête subjective, afin précisément de faire ressortir les multiples formes de vulnérabilité: latente ou patente, partagée ou individuelle, 
immédiate ou de long terme, prévisible ou imprévisible. Questionner les vulnérabilités implique encore nécessairement d'interroger les capacités des individus à faire face, leur autonomie ou capacité d'agir (agency) fondée sur leurs capabilités si l'on suit Amartya Sen (1999), ou leurs capitaux si l'on préfère la perspective de Pierre Bourdieu (2011).

\subsection{L'étude du parcours de vie: un bref état des lieux}

Cette étude s'inscrit dans le paradigme pluri- et interdisciplinaire du parcours de vie. Il s'applique à saisir les influences qui influencent les trajectoires humaines, les étapes et les transitions qui en composent le déroulement, ainsi que les représentations mouvantes des divers âges de la vie (Elder, 1994; Lalive d'Epinay, Bickel, Cavalli, \& Spini, 2005; Levy, 2013). Au fondement de cette approche se trouve l'objectif de comprendre les évolutions des vies et leurs structures afin d'appréhender le changement social au travers des relations entre individus et institutions, entre vies personnelles et histoire collective (Elder, 1995; Heinz \& Krüger, 2001; Heinz \& Marshall, 2003).

D'origine multidisciplinaire', c'est dans les années 1960 que le parcours de vie se cristallise en tant que domaine d'étude à part entière (Lalive d'Epinay et al., 2005; Marshall \& Mueller, 2003; Sapin, Spini, $\&$ Widmer, 2007). Face aux changements économiques, politiques et sociaux qui secouent les sociétés occidentales depuis l'industrialisation, une connaissance approfondie des comportements adaptatifs des individus devient nécessaire, d'autant que l'agencement de l'existence est de plus en plus mis à la charge des individus eux-mêmes (Elder, Shanahan, \& Jennings, 2015; Mills, 1997 [1959]). Les disciplines intéressées à la compréhension des biographies se sont ainsi penchées sur les relations entre vies humaines et sociétés mouvantes (Elder, 1994), en développant un nouvel outil d'analyse focalisant sur «[...] une interprétation des faits centrée sur la dimension temporelle des vies» (Sapin et al., 2007, p. 19).

1 Bien que de toute évidence au centre de préoccupations sociologiques, socio-psychologiques et psychologiques, ce domaine d'études est aussi largement développé en démographie, en histoire sociale, en anthropologie et en neurosciences (Levy, 2013). 
Dans un objectif de reconstruction des trajectoires individuelles à un niveau collectif, la sociologie du parcours de vie se penche sur divers aspects de celles-ci, qu'ils soient spécifiques à l'individu, interindividuels, structurels ou institutionnels (Levy, 2013). Dépassant la simple chronologisation des événements marquants de la vie, les dimensions temporelles et normatives des événements et des âges sont mises en avant, ainsi que les liens et les contraintes liées à l'histoire et aux institutions (Elder, 1994; Elder et al., 2015; Levy, 2013). En plus d'un demi-siècle d'existence, l'étude des parcours de vie a permis de poser un certain nombre de postulats ${ }^{2}$. Glen

2 La base de la réflexion du parcours de vie repose sur un questionnement du déroulement de la vie dans une articulation du biologique, du psychologique et du sociétal (Lalive d'Epinay, Bickel, Cavalli, \& Spini, 2005; Marshall \& Mueller, 2003). Ainsi, diverses disciplines ont conjugué leurs efforts et leurs savoirs pour aboutir à un courant de pensées plurielles, permettant d'interpréter au mieux un phénomène aussi complexe que l'existence humaine.

En psychologie, l'investigation du cycle de vie et des différentes étapes qui le constituent a débuté dans la seconde moitié du $20^{\text {ème }}$ siècle, à l'image des travaux d'Erik Erikson (1959) portant sur l'identité. Mais c'est véritablement la psychologie développementale (dite du lifespan) qui entama la réflexion autour d'un développement présent tout au long de la vie (et non plus uniquement sur une partie de celle-ci ou en apposant des frontières imperméables d'un âge à l'autre), avec les travaux de Baltes et de ses collègues parus dans les années 1980. Ces chercheurs vont pousser la réflexion pour montrer la variabilité des trajectoires, qu'ils qualifient comme multidimensionnelles (en tant que processus biologiques, historiques, culturels et de socialisation) et multidirectionnelles (tous les stades de l'existence comprenant leur lot de gains et de pertes, dans des proportions fluctuantes) (Baltes, Lindenberger, \& Staudinger, 2006; Baltes, Staudinger, \& Lindenberger, 1999). Ils soulignent ainsi que le développement n'est pas linéaire; au contraire, de nombreux facteurs entrent en jeu et expliquent la labilité des trajectoires. Toutefois, la critique principale portée à cette recherche par d'autres auteurs en science sociale concerne une prise en compte parfois insuffisante de l'impact de l'histoire et des normes sociétales sur le déroulement des vies (Lalive d'Epinay et al., 2005; Levy, 2013; Martenot \& Cavalli, 2014).

Dans une contradiction - ou une complémentarité - illustrative des préférences respectives de la psychologie et de la sociologie en termes d'approche analytique, c'est précisément la dimension d'interaction entre l'histoire au sens large et les biographies qui a initié les réflexions de cette seconde perspective sur le parcours de vie. La célèbre enquête de Glen Elder sur les Enfants de la Grande Dépression (1999 [1974]) a été fondatrice, au travers de l'observation des répercussions d'un événement sociohistorique majeur sur les trajectoires de vie ainsi que sur des institutions telles que la famille (Sapin, Spini, \& Widmer, 2007). Analysant une enquête longitudinale entamée en 1920-21, l'auteur met en avant la notion de temporalité en démontrant que, suivant l'étape du parcours dans laquelle la personne se trouve (ou 
Elder (1994; 1999 [1974]) est le premier à avoir formalisé ces fondements théoriques ${ }^{3}$.

\section{Parcours de vie et modernité}

En marche depuis le $19^{\text {ème }}$ siècle, voire le $18^{\text {ème }}$, le processus de modernisation des sociétés est imbriqué avec le mode de production capitaliste et se définit par plusieurs caractéristiques fondamentales: les changements technologiques rapides, l'économie de marché et la globalisation,

rapidement dit: l'âge de cet individu), les conséquences d'un trouble historique sur l'existence n'ont ni la même forme, ni la même portée.

Proche de la sociologie à cet égard, la démographie a contribué au développement des méthodes quantitatives. Partant d'une perspective populationnelle alors assez éloignée des individus, mais s'appuyant sur des concepts favorable à l'intégration des approches du parcours de vie (risque et exposition, en particulier), la discipline a rapidement, dès les années 1980, nourri l'étude des événements qui infléchissent le déroulement des vies et des variables susceptibles d'expliquer ces transitions. Plus récemment s'est fait sentir la nécessité de passer à une analyse à la fois plus holistique et moins englobante, en considérant les trajectoires comme des processus complexes et multidimensionnels, en s'interrogeant sur la manière dont les biographies sont construites et sur les structures résultantes (Courgeau \& Lelièvre, 1989; Guillaume, 2010). La diversité des vies requière le développement d'outils adaptés et de méthodes pour en appréhender la complexité (Antoine, Lelièvre, Collectif, \& Gourgeau, 2006).

3 D'après les résultats de son étude longitudinale sur les Enfants de la Grande Dépression, Elder pose cinq principes de base au parcours de vie. Il reconnaît en premier lieu que le parcours de vie se développe de manière ontogénésique, suivant les constats de la psychologie développementale. En deuxième lieu, il précise que ces vies sont enchâssées et modelées dans un temps et un lieu historique particulier. Comme troisième postulat, il souligne le principe de temporalité des transitions de l'existence. Ainsi, la survenue d'un événement a des conséquences et des significations diverses en fonction de la position de l'individu dans son parcours. Quatrièmement, il relève l'idée que les individus ne se construisent pas en totale indépendance, mais bien de manière inter-reliée à leur entourage. Les transitions du parcours sont vécues collectivement, au travers de rites élaborés (par exemple le mariage, la naissance, le décès...) qui ont un effet structurant sur les vies (Sapin et al., 2007). Enfin, le cinquième axiome s'intéresse à la capacité d'agir des individus (agency en anglais) (Elder, 1994; Shanahan, Elder, \& Miech, 1997). Cette idée édicte que les humains sont acteurs de leur vie, capables de faire des choix et de prendre des décisions qui construisent leur existence, bien qu'influencés par des contraintes structurelles et un contexte sociohistorique défini (Elder, 1994; Elder, 2002). Ces cinq principes présentent l'avantage d'être suffisamment larges pour inclure une palette considérable de domaines de recherche ; nonobstant, cette même largesse n'offre pas un cadre analytique et conceptuel spécifique (Levy \& Bühlmann, 2016). 
l'urbanisation, l'industrialisation, les mutations du système familial (chute de la fécondité et augmentation de l'espérance de vie) et des rapports entre les genres (Beck, 1992; Shanahan, 2000; Sørensen \& Christiansen, 2014). En sociologie, deux courants de pensées, sis de chaque côté de l'Atlantique, ont principalement contribué à l'avancée de la compréhension des parcours de vie dans cette histoire moderne (Heinz \& Krüger, 2001; Marshall \& Mueller, 2003).

Dans le monde anglophone, regroupant l'Amérique du Nord et l'Angleterre essentiellement, le point de départ est un travail de terrain, la reconstruction biographique effectué par Thomas et Znaniecki (1974 [1918]). Issus de cette réflexion et des suivantes, les principaux constats théoriques qui sous-tendent ces connaissances reposent d'une part sur une réflexion autour des normes et des attentes liées à l'âge chronologique, et plus généralement sur la structure par âge comme régulatrice de la société (Cain, 1964; Neugarten, Moore, \& Lowe, 1965; Riley, Johnson, \& Foner, 1972; Settersten \& Gannon, 2005; Settersten \& Hagestad, 1996), et d'autre part sur l'impact d'événements sociohistoriques importants sur les transitions individuelles de différentes cohortes d'individus (Clausen, 1995; Elder, 1999 [1974]; Heinz \& Marshall, 2003).

Le second axe de recherche du parcours de vie a été développé en particulier par des chercheurs en Europe continentale. Ainsi, Martin Kohli (1986) analyse l'impact de la modernité sur les trajectoires humaines, soumises avant tout aux nouvelles exigences du travail salarié. De son côté, Karl Mayer (2004) établit une revue des différents régimes de parcours de vie qui ont existé depuis l'époque préindustrielle jusqu'au post-Fordisme. Ces deux auteurs mettent en exergue la prégnance des institutions renforcées (à l'image de l'école obligatoire pour tous) ou nouvellement créées dans l'âge moderne (comme la retraite). Subordonnées à la gestion étatique, elles modèlent l'existence dans les pays dits développés. Cette présence de plus en plus marquée de l'état s'avère une composante cruciale dans la montée du parcours de vie comme institution en soi, où les vies sont régulées du berceau au tombeau (Kohli, 1986; Shanahan, 2000).

Loin d'affecter uniquement les pays dits riches, la modernisation redessine également le paysage socioéconomique des pays émergents. Les lignes qui suivent ont pour objectif de présenter les structures régissant la société indienne, issues autant des traditions religieuses et culturelles les plus anciennes que de l'impact de la nouvelle économie globalisée. Alors que l'Occident reconnait largement l'importance des institutions 
formelles dans ses structures, dans le cas de l'Inde comme dans celui de pays où l'état est moins présent, il faut sonder les institutions informelles et les normes sociales dont elles sont porteuses pour atteindre les stratifications de la société en termes d'âge et de genre.

\subsection{L'Inde millénaire : puissantes structures d'encadrement des vies et contexte de pauvreté de masse}

Lorsque l'on s'intéresse au déroulement de l'existence humaine comme le fait le paradigme du parcours de vie, la focale est mise avant tout sur les individus et leur environnement immédiat (Levy, 2009). Pourtant, la substance qui cherche à être révélée se situe à un niveau plus large, dans ce qui constitue la société, ses structures et ses normes (Sapin et al., 2007). A ce titre, l'Inde est un laboratoire fascinant où se côtoient anciennes et nouvelles structures, au sein d'une nation où l'Etat-providence n'a pas la même portée qu'en Europe et où les normes sociales sont véhiculées par des vecteurs parfois séculaires (les castes) et parfois liés à la modernité elle-même (les classes sociales) (Appadurai, 2005 [1996]; Kaviraj, 1990; Sakthivel \& Joddar, 2006).

Nonobstant la pléthore de définitions de ce concept (Marshall, 2005), globalement les structures sociales sont des arrangements institutionnels permettant de prédire les agissements et d'identifier des groupes d'individus selon divers critères (Cain, 1964; Hendricks \& Hatch, 2008). Elles existent à des niveaux multiples et sous de nombreuses formes (Dannefer \& Kelley-Moore, 2008). En simplifiant quelque peu, il est possible de parler de forces de type top-down ayant un impact sur les individus et dont il est difficile de se soustraire, permettant d'internaliser ou d'objectiver un certain nombre de comportements qui sont par la suite reproduits par les personnes elles-mêmes (Marshall, 2005; Settersten \& Gannon, 2005). Des dimensions clés composent ces structures, qui permettent d'identifier les individus: les plus classiques sont la classe, l'ethnie, l'âge ou encore le sexe (Macmillan, 2005). Certaines de ces notions sont définies à la naissance et les personnes ont peu, voire pas de possibilités de les modifier, alors que d'autres sont construites au cours de la vie et offrent une relative marge de manœuvre. Toutes ces caractéristiques individuelles servent de 
«proxy» pour saisir les structures (Settersten \& Gannon, 2005), révélant les inégalités sociales par le biais de ces catégorisations.

Les écrits marxistes décrivent la structure sociétale capitaliste comme intrinsèquement constituée afin d'empêcher toute distribution égalitaire des ressources et de favoriser la reproduction des inégalités par une conservation des richesses et des ressources dans les mains d'une minorité (Marx \& Engels, 1999 [1847]). Pour ce faire, le capitalisme, système économique désormais triomphant parmi toutes les sociétés de la planète (Appadurai, 2005 [1996]), a su créer des institutions et des relations de pouvoir afin de contrôler la population et de parvenir à ce que les individus eux-mêmes reproduisent ces schémas (Kaviraj, 1990; Wright, 2005). En outre, l'inégalité économique est répercutée dans diverses sphères de la vie, comme la santé ou l'éducation, qui deviennent également sélec-

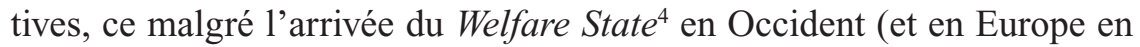
particulier $)^{5}$. Les néo-Marxistes corroborent ce constat, en soulignant que la structure économique et l'ordre social inégalitaire sont conservés par le jeu central des institutions publiques, sociales et juridiques (Mandel, 2007; Oris, Gabriel, Ritschard, \& Kliegel, 2017; Wright, 2000).

\subsubsection{La classe et la caste comme déterminants sociaux des individus}

Karl Marx est parmi les premiers auteurs à avoir observé la société occidentale du $19^{\text {ème }}$ siècle, en pleine industrialisation, et à parvenir au constat que la position des individus à l'intérieur d'une classe sociale signifie un

4 Il s'agit de l'«Etat-providence» ou «Etat-social» en français, une implication de l'Etat dans les lois, l'économie et le social en vue de fournir des prestations sociales pour la population.

5 La notion même d'état diverge entre les chercheurs européens et nord-américains, ce qui explique en partie l'attention différente portée aux institutions étatiques par ces derniers (Mayer \& Schoepflin, 2009). En Amérique du Nord, l'état se réduit plus ou moins à un gouvernement et à son administration, expliquant que les études issues du monde anglo-saxon soulignent les transformations des structures des vies liées à des facteurs individuels de stratification d'âge et aux normes sociales, ainsi qu'à des facteurs collectifs historiques. A l'inverse, en Europe la société civile est davantage gérée par l'état, au travers des institutions sociales (Mayer \& Schoepflin, 2009) et, dès les années 1970, les recherches sur le parcours de vie vont redresser la barre afin de se focaliser sur la dimension d'institutionnalisation des trajectoires au travers des changements culturels et démographiques, ainsi que de structure et des politiques sociales (Hagestad, 1990; Kohli, 1986; Mayer, 2004). 
accès spécifique au pouvoir et aux ressources (Béteille, 1974 [1969]). Ce qualificatif a plus tard été employé par Max Weber, qui a porté un regard sur le découpage social en délimitant les classes comme des groupes d'individus possédant des caractéristiques communes et une dynamique de vie similaire (Chauvel, 2001; Hendricks \& Hatch, 2008; Weber, 2003 [1978] $)^{6}$.

Selon l'analyse marxiste, la société capitaliste se compose de deux classes sociales, qui se définissent par leur accès au système économique. La première (la bourgeoisie) possède les moyens de production et impose son hégémonie à la seconde (le prolétariat), bien plus nombreuse, qui n'a comme ressource que sa propre capacité de travail (Marx, 1993 [1867]). Chaque individu débute sa vie à l'intérieur de l'une ou de l'autre de ces classes sociales, en fonction de la situation économique de ses géniteurs, et incorpore de ce fait toute une palette de comportements particuliers (autrement nommés, des normes sociales). De cette manière, les inégalités sociales, politiques et économiques de la société occidentale capitaliste moderne sont construites et reproduites par le contexte social et appliquées aux individus en fonction de caractéristiques définies à la naissance (Wright, 2005). Ce rapport de force entre dominants et dominés explique également que la dynamique sociale dans son ensemble soit construite autour d'une lutte des classes, le moteur principal de l'évolution historique (Marx \& Engels, 1999 [1847]).

Lorsqu'il vient au monde, l'enfant est donc assigné à une classe qui détermine un grand nombre d'opportunités qu'il aura, ou qu'il n'aura pas, tout au long de sa vie. Cette vision marxiste est aisément transposable à l'Inde, qui après son Indépendance (mais surtout depuis l'ouverture économique des années 1990) a rapidement intégré les modes de fonctionnement de l'économie capitaliste. Le terreau indien était en outre déjà fertile à cet égard, par son organisation sociale et culturelle inégalitaire. Traditionnellement, les structures sociales y sont marquées par le cadre déterministe de la caste, dont les diverses implications ont été dis-

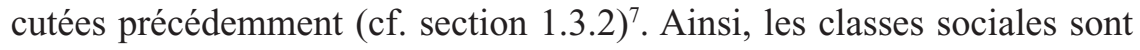

6 Dans la vision wébérienne, la classe est une dimension permettant de définir la structure sociale, réduite ainsi au niveau d'un simple paramètre (Chauvel, 2001; Levy, 2009).

7 Les intouchables sont considérés comme «hors-caste». Toutefois, par soucis de simplification, lorsque nous parlons de la caste comme d'une manière de classifier la société indienne, nous les incluons dans la réflexion. Dans la même idée, les castes ne 
relativement neuves en Inde alors que les castes relèvent d'une pratique ancestrale, consolidée par l'arrivée en force du capitalisme (au travers du colonialisme d'abord, puis par l'ouverture économique du pays ces dernières années).

Comme nous l'avons vu dans le chapitre 1, bien que constituant les fondements du même carcan normatif régissant les vies indiennes, les deux concepts - classe et caste - ne concordent pas entièrement (Roy \& Demanuelli, 2015; Sen, 2007). Le premier repose sur un rapport économique alors que le second représente un statut socio-culturel (avec une assise religieuse importante), généralement relié à une profession il est vrai, mais pas seulement, et de moins en moins. La présence simultanée de ces deux notions a rendu poreux un clivage qui ne l'était pas initialement : les castes supérieures possédaient les richesses et les intouchables en étaient dépourvus, condamnés à une vie laborieuse parfois proche de l'esclavage. Se situer en haut ou en bas de l'échelle socioéconomique n'est aujourd'hui pas - ou plus - l'apanage d'une seule caste, bien que la discrimination envers les Dalits reste indéniable (Heuzé, 1991; Saglio-Yatzimirsky, 1999). Ainsi, malgré une surreprésentation des élites religieuses hindoues parmi les individus avec un niveau d'éducation et de revenu élevé, des Indiens de haute caste se trouvent parfois à un niveau socioéconomique inférieur. De la même façon, grâce à une amorce de démocratisation des études, une proportion réduite d'intouchables a maintenant accès à des métiers très qualifiés qui assurent une rémunération conséquente (Deliège, 2006; Drèze \& Sen, 2013; Heuzé, 1999).

Avec l'entrée dans la globalisation, la société indienne se complexifie et le système traditionnel des castes, reposant sur une transmission héréditaire des tâches, représente un obstacle pour une économie mouvante. En effet, le précédent chapitre nous l'a montré, la division rigide du travail selon le groupe d'appartenance des individus plutôt que sur la base de leurs atouts compétitifs s'oppose au développement de l'économie de marché, ne permettant ni les réallocations de ressources ni les réajustements structurels (Drèze \& Sen, 2013). Pour autant, les castes ont une nouvelle utilité. Comme souligné au sortir de l'Indépendance par le militant de la cause des intouchables Bhimrao Ramji Ambedkar (1990

sont valides que pour les personnes de confession hindoue, largement majoritaires. Néanmoins, nous incluons les affiliés à d'autres confessions dans la considération, car les normes sociales liées aux castes ont influencé la société indienne dans son ensemble. 
[1936]), elles ne se contentent plus de diviser le travail sur le mode indiqué dans les textes sacrés, mais elles permettent désormais d'opposer les travailleurs entre eux (Saglio-Yatzimirsky, 1999). Les castes fractionnent des alliés de classes «naturels» (selon la compréhension marxiste du terme). C'était déjà en 1949 une des raisons pour lesquelles, selon Ambedkar, elles auraient dû être abolies. A cet égard, l'exemple des événements qui ont eu lieu en 1990, notamment dans les universités, lors de la promulgation du rapport de la Commission Mandal (voir section 1.3.2), sont révélateurs. Les hautes castes (non discriminées) ont exprimé avec violence leur opposition à l'établissement de quotas de réservation pour les castes discriminées (OBC) ne faisant pas partie des Dalits (Ramaiah, 1992). Sous l'argumentaire trompeur d'une résistance au castéisme et d'une promotion de la méritocratie, promulgué par les médias et les castes dirigeantes, le refus de l'égalité des chances à toutes les castes inférieures permettait d'entretenir les clivages et les rancœurs entre intouchables et OBC (Saglio-Yatzimirsky, 1999). Parallèlement, il a été constaté que les Dalits ayant bénéficié de ces quotas ont relativement peu aidé à réduire les inégalités sociales, mais plutôt profité des avantages pour eux-mêmes, grossissant les rangs de la classe moyenne montante (Jaoul, 2007).

Ces derniers temps, l'enchevêtrement des castes et des classes a été marqué par la montée d'un nationalisme pro-hindou et d'un sentiment anti-migrants prononcés (Assayag, 2005; Deliège, 2006), qui prennent leurs racines dans les turbulences de l'Indépendance et ont connu plusieurs faux-départs dans les années 70 (Heuzé, 2012). En parallèle du système social traditionnel, les transformations économiques récentes du monde urbain indien, impliquant d'un côté un déplacement des emplois du secondaire vers le tertiaire et de l'autre une montée du secteur informel, ont secoué l'architecture sociale des villes du pays. En réponse, une partie du monde politique a développé une stratégie d'adaptation au travers d'un discours mettant en concurrence les droits des travailleurs selon leur origine, migrante ou native. Ainsi, une ville comme Mumbai a beau être bâtie sur la venue et par le travail des migrants (dans les domaines de la construction, de la manufacture et des services notamment), un climat xénophobe se ressent depuis quelques décennies (Singh, 2015). Ce rejet des immigrés a pour but de diviser la classe des opprimés, empêchant une union qui aurait pu dépasser les castes (Saglio-Yatzimirsky, 1999).

C'est donc dès le berceau que les Indiens subissent un double contrôle, une double imposition sociale issue des structures de castes et de 
classes (Assayag, 1999), qui délimite et imprègne leur bagage social de caractéristiques normatives, auxquelles sont associés des rôles et des statuts particuliers à assumer dans le cours de leur existence. Ce contrôle a également comme objectif de désigner aux individus une place dans la société qu'ils ne quitteront que rarement. La caste, et dans une autre mesure la classe, sont des outils permettant d'inscrire dans l'«ADN social» des individus des déterminants qui conditionnent leur parcours de vie, et ce dès leur plus jeune âge. L'entrée dans la modernité a signifié une modification de ces outils, notamment dans l'Inde urbaine, sur laquelle nous aurons l'occasion de revenir à maintes reprises. Si les normes fondatrices continuent d'agir, tout au long du chemin de la vie, sur les existences, c'est aussi parce que les influences initiales sont renforcées par la présence d'institutions, qu'elles soient formelles ou informelles, dont le fonctionnement pérennise, réduit ou prend appui sur les inégalités sociales (Mayer \& Schoepflin, 1989).

\subsubsection{Puissance des institutions informelles ${ }^{8}$ normatives : religion et famille}

Dans le domaine du parcours de vie, les chercheurs se sont généralement consacrés aux pays développés plutôt qu'en développement, et fort peu à l'Inde. L'exemple des castes et des pratiques familiales indiennes n'a donc pas (à notre connaissance) été utilisé pour étayer les réflexions portant sur les normes et les structures sociales. Néanmoins, de nombreux travaux en Occident ont montré que la religion, la famille et d'autres formes d'institutions informelles ont joué un rôle central dans le déroulement des parcours de vie, notamment lors du passage de l'Ancien régime à l'époque moderne (Heinz \& Krüger, 2001). Sans tomber dans un cliché réducteur prônant une fallacieuse similitude entre l'Inde d'aujourd'hui et l'Occident

8 En sociologie, les institutions représentent des structures sociales dotées d'une certaine stabilité dans le temps (Bourdieu, 1994). Cette définition peut correspondre aux organismes que nous qualifions ici de formels, mis en place par l'état et qui reposent sur des lois, des décisions politiques et sont gérées globalement par le gouvernement (comme l'école, la retraite, l'assurance chômage). Par ailleurs, cela peut également faire référence à des institutions que nous nommons informelles, car elles relèvent avant tout de la sphère privée: la famille, la religion, etc. 
d'avant la révolution industrielle, nous allons tâcher d'appliquer ces réflexions au cas indien.

\section{Religion(s) et castes}

Pour les adeptes de la religion hindoue, appartenir à une caste et respecter ses codes comportementaux est obligatoire. Certains de ces codes peuvent avoir des impacts considérables sur la vie quotidienne, tels que l'obligation de se marier au sein d'une même caste ou le devoir de se rendre en pèlerinage à Varanasi (Bénarès), que l'on soit riche ou pauvre (Ambedkar, 1990 [1936]). Du point de vue du genre, sans développer une question sur laquelle nous reviendrons plus longuement en section 2.2.3, la position de la femme dans l'hindouisme est clairement subordonnée à celle de l'homme, introduisant un rapport très inégalitaire entre les sexes (Banerjee, 2005; Dumont, 1959). Ces règles strictes attestent de la force de la religion majoritaire en tant qu'institution sociale normative qui, de plus, a des restrictions très étroites concernant la définition de ses affiliés. Ainsi, elle ne reconnait pas la conversion : on naît dans une caste ou on n'est pas hindou. Il n'est pas possible de pénétrer une caste en cours d'existence, ni d'en changer à la suite d'un événement personnel (comme le mariage). Cependant, briser les codes imposés par sa caste peut mener à une excommunication. Les musulmans, les sikhs et les chrétiens indiens vivent eux aussi selon un système de caste, mais qui diffère précisément parce qu'il est moins structurant. Dans ces cas, la caste est vue comme une pratique, mais pas un dogme (Ambedkar, 1990 [1936]).

Malgré l'interdiction constitutionnelle de discriminer en fonction de la caste, celle-ci continue à exercer ses effets de manière officieuse; elle a même pris de l'ampleur depuis les années 1990 (Roy \& Demanuelli, 2015). La rigidité durable du système des castes a également participé à ralentir la création d'institutions publiques destinées à compenser cette discrimination (Drèze \& Sen, 2013), confirmant que les castes représentent une entrave vis-à-vis des réformes en faveur de l'égalité économi-

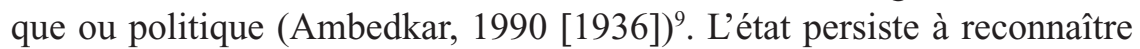
leur statut aux castes de manière officieuse et, en fonction de leur position hiérarchique, ne leur offre pas la même capacité d'intervention en tant que groupes de pression dans l'économie et le marché du travail (Heuzé,

9 " [...] turn in any direction you like, Caste is the monster that crosses your path. You cannot have political reform, you cannot have economic reform, unless you kill this monster." (Ambedkar, 1990 [1936], article 3 alinéa 13). 
1989). Dans les entreprises privées qui fournissent la meilleure protection sociale, comme Siemens, Tata ou Ciba-Geigy à Mumbai, les emplois exigent de tels niveaux de formation (et donc d'origine sociale) que la discrimination de caste passe inaperçue (Heuzé, 1989).

La représentation des Dalits parmi les universités, les administrations, ou encore les parlements (voir section 1.3.2) par le biais de places réservées (quotas) a été une tentative de rétablir une égalité des chances pour cette minorité, afin de dépasser le handicap de l'origine sociale contracté à la naissance. Malheureusement, la mise en pratique de ces quotas en est encore aux balbutiements, et seul un relatif impact sur l'accès aux hautes études a pu être constaté (Dreze \& Sen, 2013; Saglio-Yatzimirsky, 2002). L'Inde est également à la traîne en ce qui concerne la redistribution des revenus par les taxes, entrainant une dégradation des prestations publiques qui avaient peu à peu été mises en place durant la seconde moitié du $20^{\text {ème }}$ siècle (Roy, 2014). Ceci est particulièrement visible dans le domaine de la santé, les hôpitaux publics proposant des services déplorables par manque de moyens, ce qui encourage l'utilisation d'établissements de médecine privée, chers et inaccessibles pour le plus grand nombre (Dreze $\&$ Sen, 2013). Les politiques publiques en Inde ne s'adressent pour l'heure qu'à une infime part de la population, ne répondant pas aux besoins grandissants des laissés-pour-compte de la modernité (Heuzé, 1989; Sakthivel \& Joddar, 2006). Pour ces derniers, l'unique solution de survie face à la maladie, au chômage ou à la vieillesse reste le plus traditionnel des systèmes de sécurité sociale : la famille.

\section{La famille comme structure et comme support, au centre du parcours de vie}

La famille en Inde est donc un élément central dans la vie, en termes de réseau social avant tout, mais aussi comme filet de sécurité. Contrairement à un a priori courant, le foyer indien urbain est majoritairement nucléaire, les périodes de cohabitation avec des membres de la famille élargie s'avérant de court terme (Guilmoto, 2011; Heuzé, 1997). Une de ces périodes se trouve être les quelques années de vieillesse et de retraite (dans le sens concret mais non institutionnel du terme) des parents, lorsqu'ils ne peuvent plus subvenir à leurs besoins et que cette responsabilité repose sur leur fils aîné et leur bru. Un modèle qui offre également l'avantage de la garde des enfants en bas âge et de mettre en commun des finances généralement maigres (Jadhav, Sathyanarayana, Kuma, \& 
James, 2013). Les évolutions démographiques, telles que la montée de l'espérance de vie, la chute de la fécondité et le vieillissement de la population, ont impliqué des modifications à ce schéma familial, néanmoins peu nombreuses. Le nombre moyen d'habitants par foyer va en diminuant alors que la moyenne d'âge de ceux-ci s'élève. Autrement dit, les familles indiennes connaissent simultanément une augmentation du nombre d'années de vie des grands-parents et une réduction du nombre de petitsenfants (Guilmoto, 2011).

Les individus ont ainsi une durée de vie qui s'étend et les personnes âgées ${ }^{10}$ constituent dès lors un poids accru pour les enfants (par leur présence au sein du ménage et leur coût financier d'entretien) durant un nombre croissant d'années. Globalement, les âgés tombent plus facilement dans la pauvreté (Jadhav et al., 2013). En outre, que ce soit en ville ou à la campagne, l'écart d'âge au mariage implique que le nombre de veuves est plus élevé que celui des veufs, et une féminisation de la population âgée et vulnérable a lieu (Jadhav et al., 2013). La solitude des personnes âgées est aussi un problème qui grandit, sans toutefois alléger la charge financière assumée par leurs descendants. Avec la migration vers la ville, les enfants des campagnes ne sont plus là pour s'occuper de leurs parents, contraints de vivre seuls. En ville, le phénomène d'isolement se produit à cause du manque d'espace et de la cherté du logement (en particulier à Mumbai); il devient alors difficile de cohabiter et les âgés doivent loger ailleurs (Jadhav et al., 2013).

Les premières évidences de cette mutation sociale et démographique sont le manque de soin et d'encadrement pour les âgés ainsi que l'absence de soutien pour la garde des enfants, qui doit entièrement être assurée par les parents (plus exactement les mères). Au niveau économique, des stratégies d'adaptation pour la conservation du niveau de vie du foyer (souvent déjà minimal) ont dû être déployées face à l'inflation des prix, modifiant également la structure familiale. La principale stratégie a été la mise au travail de deux individus au sein du ménage, généralement le mari et la femme (Desai, 1994). Néanmoins, les tâches domestiques continuent d'incomber aux épouses et rendent leur accès au marché du travail plus compliqué. Elles reçoivent des salaires bien inférieurs à ceux de leurs époux, sont davantage astreintes au travail informel, au temps partiel et

10 Les 60 ans et plus en Inde représentaient 8.9\% de la population en 2015, en 2050 ils devraient atteindre les 19.4\% (UN 2015 Word Population Prospects. The 2015 Revision). 
aux emplois peu rémunérés, tout en devant gérer entièrement leur propre ménage (voir section 1.3.3). Une autre de ces stratégie consiste en une rentabilisation de l'espace du foyer (même dans les bidonvilles), qui fournit désormais aussi la surface pour développer des activités commerciales (manufacture, vente, etc.) (Desai, 1994).

Si les jeunes adultes en âge de travailler (entre 15 et 65 ans, selon des bornes, il est vrai, très théoriques) représentent la plus grande part de la population indienne (65\% en 2015 selon les estimations de la Banque mondiale), cette majorité doit néanmoins subir les effets de la transformation des structures d'âge, dans un contexte de développement économique qui favorise l'individualisation et la privatisation. C'est d'autant plus le cas que ces adultes ne peuvent s'appuyer sur des institutions publiques pour compenser l'aggravation du poids des individus dépendants (par des crèches, des établissements pour personnes âgées, des pensions de retraites, etc.). Ce qui signifie un nouveau type de pression intergénérationnelle sur les couples, les forçant à dépenser plus pour des âgés plus nombreux (un problème bien connu de la Chine ou du Japon par exemple [Okamoto, 1992; Zimmer \& Kwong, 2003]). De plus, la précarisation d'une grande masse de travailleurs au travers de l'informalité et de la pauvreté (influençant notablement la santé des individus, entre autres choses) rend caduque la vision théorique idéale d'un bonus démographique: seul un tiers des travailleurs urbains en Inde étaient économiquement indépendants en 2004 (Guilmoto, 2011).

Dans cette situation de tensions, le traditionnel care par la famille est de moins en moins la norme en Inde et un besoin urgent de support étatique se fait sentir. Ainsi, le gouvernement indien a lancé un National Social Assistance Programme en 1995 à l'attention des personnes âgées, des orphelins et des femmes enceintes (Justino, 2007). En 1999, le National Policy on Older Persons est conçu, devant permettre un allégement des impôts, du prix des médicaments et une facilitation d'accès au logement pour les enfants en charge de parents âgés. Or, cette dernière loi n'a pas encore été rendue effective, ni même adoptée par les Etats (Jadhav et al., 2013). Par ailleurs, les systèmes de sécurité sociale, qu'ils soient protectifs ou promotionnels ${ }^{11}$, sont à l'heure actuelle uniquement accessibles aux fonctionnaires et aux travailleurs peu nombreux des secteurs

11 Les mesures promotionnelles représentent les subventions à l'éducation, à la santé, etc., alors que les mesures protectives concernent les retraites, les congés maternité, les assurances de santé, etc. (Sakthivel \& Joddar, 2006). 
privés fortement syndiqués (Sakthivel \& Joddar, 2006). Les groupes particulièrement vulnérables, travaillant dans le secteur informel - soit 94\% des Dalits et des OBC -, sont ainsi les plus exclus de ces mesures (Sakthivel \& Joddar, 2006), démontrant le décalage des institutions avec la réalité (Guilmoto, 2011).

Un travail se focalisant sur le passage à l'âge adulte en Afrique subsaharienne a montré qu'un changement important de la prise en charge traditionnelle a lieu lors du passage de la vie en campagne à la vie en ville: l'école moderne prend la place de la famille ou de la communauté, l'emploi urbain éloigne du foyer et ouvre à l'autonomie, la socialisation doit se faire au dehors de la sphère familiale (Calves, Bozon, Diagne, \& Kuépié, 2006). La période de transition en devient plus floue et de nouveaux statuts surgissent (par exemple celui de mère célibataire, prohibé par la famille mais rendu possible avec l'éloignement). Ainsi, la force normative de l'institution informelle familiale pourrait décliner dans l'Inde urbaine du $21^{\text {ème }}$ siècle, qui connaît également des changements démographiques et urbanistiques importants. Pourtant, les institutions formelles manquent ou sont trop faibles pour compenser cette réduction et ce sont encore les informelles, telles que la famille ou la religion, qui portent l'essentiel du fardeau de la protection sociale. En contrepartie, leurs normes restent les repères les plus contraignants au sein de la structure sociale.

\subsubsection{Stratifications d'âge et de genre en Inde}

\section{Homogénéité des parcours de vie}

La constatation d'une organisation des parcours de vie par les institutions formelles, en parallèle d'une individualisation de l'existence, n'est plus à faire dans un contexte européen (Kohli, 2007; Levy \& Bühlmann, 2016; Mayer, 2004) ${ }^{12}$. Inversement, l'agencement des vies est reconnu comme

12 Sous l'Ancien régime (ou période préindustrielle), le ménage était un espace dans lequel s'opéraient la production et la consommation des ressources, la vie reproductive et la socialisation des jeunes. La vie était organisée et régulée par la famille et l'environnement local (Giddens, 2004 [1994]; Hareven, 1986, 1996; Mayer \& Schoepflin, 1989). Avec l'avènement de l'industrie et des villes, cette organisation des trajectoires vole en éclats, sous le coup notamment du déplacement de la sphère du travail et de la formation hors du foyer, vers l'usine ou le bureau (Mayer, 2004; Mayer \& Schoepflin, 2009). L'économie de marché se met à réguler le parcours de vie, au travers de l'état qui édicte des lois portant sur les âges minimaux ou maximaux 
moins impacté par les instances sociétales dans des pays où l'Etat-providence joue un petit rôle, passant avant tout par l'économie, les réseaux sociaux (comme le voisinage, les collègues ou la famille), les choix et décisions individuels (Heinz, 2003). Dans ce deuxième cas, les institutions étatiques sont comprises comme des sources de contraintes subjectives sur les personnes, mais ne sont pas interprétées comme des contraintes structurelles. Ainsi, analysant le travail des enfants dans les pays du sud, Dale Dannefer (2003a, 2003b) constate un fonctionnement institutionnalisé de l'informel, par des phénomènes économiques de production, de marchés globalisés, mais aussi par la famille plutôt que par l'état. Cette description semble correspondre à la société indienne, dont le système ne peut garantir de protection sociale ni d'accès universel aux institutions formelles, où les traditions et les conditions socioéconomiques pèsent encore lourdement sur les destins.

Dans l'espace occidental, l'une des plus importantes conséquences de l'accroissement de la prise en charge et du contrôle des étapes de la vie par les institutions publiques (Marshall \& Mueller, 2003) est une scansion de plus en plus marquée des parcours (van de Velde, 2015). Les séquences se répartissent en trois segments clés, partitionnant ainsi la vie autour du travail et du marché économique: en premier vient la période de formation

(entrée à l'école, sur le marché du travail, au mariage, à la retraite). Au terme de ces évolutions, le Welfare State s'étend progressivement à l'entier des populations occidentales tout en couvrant de plus en plus de besoins, accroissant simultanément les emplois administratifs et du secteur public (Mayer \& Schoepflin, 1989). L'Etat-nation tel qu'il est organisé en Europe continentale insuffle et propose des politiques sociales autour de différents domaines vitaux comme l'éducation et la formation, la santé, la vieillesse, l'assistance sociale, le travail (Weymann, 2009).

Ce processus général d'institutionnalisation des parcours de vie a eu pour conséquences une libération et une déconnection d'avec les traditions, la religion et les communautés anciennes. Les formes d'institutionnalisations qui en découlèrent ont été classifiées selon deux types: structurelle et culturelle (Levy, 2009). La première s'appuie sur le lien entre la société et l'économie de marché et relativement peu sur une implication des individus (par exemple l'école), alors que la seconde décrit les formes de diffusion et de socialisation des représentations et des idéologies (par exemple le mariage). Autre découpage du processus, il peut affecter de manière directe les comportements humains (par exemple au travers de lois) ou de manière indirecte (par exemple au travers du nombre de places en crèches disponible) (Levy, 2009). Tout ceci conduit à un renforcement des normes et de la transmission de celles-ci (Lalive d'Epinay et al., 2005). L'effet fondateur des critères attribués à la naissance dans la construction des inégalités est ainsi renforcé par ces institutions. 
(l'école), suivie des années d'activité, puis de la retraite (Bessin, 1996; Hareven, 1993 [1982]; Kohli, 1986). Ces moments renvoient évidemment aux trois périodes classiques allant de la naissance au décès (ou âges de la vie): jeunesse, âge adulte et vieillesse (van de Velde, 2015).

Ces modifications entraînent divers effets. Premièrement, définir temporellement la survenue des transitions détourne la variable âge de son rôle de statut, pour en faire un simple marqueur temporel (temporalisation). Deuxièmement, un ordre normatif de survenue des transitions s'installe, dans une chronologisation liée à l'âge des événements. Plus largement, le processus provoque un détachement de l'individu face à ses limites de statut, de localité, de famille, pour devenir l'unité de base de la vie sociale (individualisation); dit autrement, ressort une centration des événements de la vie autour de la personne (Kohli, 1986, 2007). Jusqu'à la moitié du $20^{\text {ème }}$ siècle, les populations occidentales connaissent ainsi une standardisation du parcours de vie, largement encouragée par l'institutionnalisation des trajectoires de vie comme la famille, l'éducation et l'emploi (Cavalli, 2007; Heinz \& Krüger, 2001; Kohli, 1989; Lalive d'Epinay et al., 2005). La vie devient prévisible et relève du projet personnel, dont l'individu endosse (quasiment) seul la charge de la construction (Kohli, 2007; Shanahan, 2000) ${ }^{13}$.

En Inde, les carences dans la protection sociale et la montée de l'économie informelle laissent présager que cette tripartition de la vie ne sera pas aussi visible. Pour une grande majorité de la population, le lieu de production n'a pas encore migré du foyer ou de la rue vers l'usine ou le bureau. De plus, les deux extrémités de l'existence montrent des comportements encore peu régulés, comme la sortie brutale de l'enfance par une entrée précoce dans le marché du travail et une trajectoire laborieuse qui peut s'étendre tard dans la vieillesse (en l'absence de retraite), jusqu'à l'invalidité. Toutefois, si une régularisation du parcours de vie par le travail ou les autres institutions semble encore de la musique d'avenir, d'autres critères plus traditionnels sont présents et induisent des normalisations.

13 Par le même phénomène, les structures des parcours de vie deviennent plus flexibles, plus individualisées et davantage autogérées qu'elles ne l'étaient avant l'industrialisation, ce à la fois en Europe et en Amérique du Nord (Heinz \& Krüger, 2001). Malgré ces bouleversements, les étapes de l'existence sont globalement restées les mêmes et continuent de s'identifier aux étapes de la vie laborieuse: l'enfance qui englobe parfois l'adolescence, la jeunesse et l'entrée dans l'âge adulte (formation), puis la maturité (vie active) et enfin la vieillesse (dont le point de départ est la retraite) (Helfter, 2009). 
Les milieux enracinés dans les traditions connaissent une stabilité des mœurs culturelles, familiales et religieuses, toutefois différenciées selon les caractéristiques individuelles de religion, de caste et de sexe (Heuzé, 1997). Les métamorphoses sociales issues de l'urbanisation et des changements qui y sont attachés ont, elles aussi, entraîné des évolutions, comprenant une standardisation des attitudes. Ainsi, la coutume très ancienne de la dot est désormais interdite légalement, mais en réalité elle se trouve étendue à des couches toujours plus nombreuses de la société. La planification des naissances (et la réduction de la fécondité qui s'en est suivie) est également devenue une pratique majoritaire en ville, affectant le parcours de vie (Heuzé, 1997).

Contre intuitivement, et en parallèle de cette homogénéisation de pratiques, le renforcement de l'endogamie de caste et le maintien des structures familiales sont plus ancrés que jamais (Heuzé, 1997). Ce qui n'est pas sans susciter des contradictions. La diffusion de l'idéologie du mariage d'amour, par les films des studios Bollywood notamment, est ainsi en porte-à-faux avec la culture des mariages arrangés. Pour y remédier, les circonstances de rencontre sont hautement surveillées, afin de parer le choix du conjoint du voile illusoire de la rencontre fortuite et de la liberté de décision. Néanmoins, certaines concessions sont inévitables. L'obligation stricte des épousailles au sein de la même sous-caste a été adoucie pour permettre des unions avec des groupes moins proches, demeurant malgré tout «compatibles» (Heuzé, 1997). L'augmentation de l'accès à l'université a été l'une des causes de ces évolutions, puisque de jeunes couples peuvent à présent se former loin des radars familiaux. Cela $\mathrm{a}$-à la fois paradoxalement et logiquement - forcé la mise en place de puissantes stratégies de contrôle sur les individus (Trivedi, 2014).

Ainsi, une forme de standardisation de l'existence se constate dans l'Inde moderne et urbaine qui, sans correspondre à la forme institutionnalisée habituellement reconnue par le paradigme du parcours de vie (Kohli, 1986; Levy, 2013), résulte bel et bien en une unification du déroulement des trajectoires de vie. Un nombre considérable de transitions dans la vie sont toujours effectuées par une très grande majorité de la population indienne, comme le mariage ou le fait d'avoir des enfants, et régulées par des facteurs culturels (les castes et les traditions) et socioéconomiques (les classes sociales), amenant à des parcours de vie normés et homogènes. Si ces moments de transition ne sont pas orchestrés par des organes collectifs, les individus sont malgré tout confrontés aux contraintes et aux 
normes sociales qui les poussent à agir conformément à la règle (Guillaume, 2010; Neugarten et al., 1965).

\section{Une stratification selon les âges de la vie $e^{14}$}

Aux divers âges de la vie, les individus possèdent des capacités et assument des rôles sociaux spécifiques (étudiante, épouse, travailleuse, retraitée par exemple), tout en recevant des droits et des privilèges particuliers (Riley, 1971). Dans les sociétés occidentales, ces âges entrent en concordance avec la tripartition de la vie centrée autour du travail et deviennent ainsi des régulateurs de l'existence, alors que d'autres cultures peuvent définir ces activités sur des temporalités différentes (Riley \& Riley, 2000; van de Velde, 2015). De fait, la plupart des sociétés ont leur propre manière de comprendre les âges, de leur attribuer des rôles et de rythmer les séquences (Bessin, 1996; Elder, 1975; Lelièvre, 2006; Settersten, 1999). Les réalités économiques et les contraintes sociales peuvent toutefois altérer ces régulations et provoquer des sorties de route (Macmillan, 2005). C'est par la socialisation que les gens intériorisent ces normes d'âge, avant de les reproduire eux-mêmes (Neugarten et al., 1965; Settersten, 2002, 2004).

Durant la jeunesse ont lieu deux transitions principales vers le devenir adulte: l'arrivée sur le marché du travail, qui fait suite à la formation, et la constitution d'une famille propre (Bidart, 2005). Avec les mutations de la société post-industrielle, dans les pays riches l'entrée dans le monde professionnel est retardée par une sortie des études plus tardive, en conséquence de quoi la construction de la famille survient plus tard (et moins d'enfants naissent en moyenne). Ensuite, la phase de maturité est caractérisée par une activité professionnelle bien établie, en parallèle de l'éducation des enfants. Par ailleurs, cette génération se trouve «prise en sandwich» entre une progéniture non encore indépendante mais en voie de l'être, et des parents qui avancent vers la vieillesse (Helfter, 2009). A l'autre bout de l'existence, le nombre d'années s'allonge avec l'espérance de vie et une retraite plus précoce offre de belles années, celles d'un troisième âge où les

14 La stratification sociale représente le classement des catégories sociales selon des strates hiérarchiques inégalitaires, généralement basées sur les ressources économiques mais pas uniquement. De fait, la stratification par âge est un marqueur fondamental de la société, aussi déterminant que peut l'être le sexe de l'individu ou son niveau de revenu (Macmillan, 2005; Settersten \& Mayer, 1997). Elle révèle la division de la société en des groupes d'âge significatifs, qui diffèrent en valeurs sociales et en allocation des ressources (George, 1990). 
problèmes de santé sont encore peu nombreux, avant d'atteindre la grande vieillesse (Heinz \& Krüger, 2001; Lalive d'Epinay \& Cavalli, 2013) ${ }^{15}$.

En Inde, les séquences des parcours de vie se découpent aussi en fonction des trajectoires professionnelle et familiale. La tradition classique indienne reconnaît quatre stades (ou ashrama, étapes de la vie) du développement : le brahmacharya (être étudiant - pas forcément dans le cadre d'une structure scolaire), le grhasta (être chef de ménage), le vanaprastha (être retraité) et le sannyasa (la renonciation). L'espérance de vie a augmenté, mais le temps des loisirs et de la retraite n'est pas encore une norme, nous l'avons vu; les deux dernières étapes sont donc atteintes par une faible proportion de la population (Guilmoto, 2011). Aujourd'hui, c'est le niveau social de la personne et son sexe qui sont les principaux déterminants de l'aboutissement ou non de ces stades, notamment du premier et des deux derniers.

La Constitution indienne garantit un accès à la scolarisation à tous les enfants entre l'âge de 6 et 14 ans. Pourtant, jusqu'à ces dernières décennies, les écoles étaient fréquentées surtout par des garçons issus de familles favorisées. Récemment, la formation s'est généralisée parmi les Indiennes et les Indiens, à tout le moins dans le monde urbain. Dans le pays, 74\% des enfants ont suivi l'école primaire (Anand, 2011) et 59\% des garçons, respectivement $49 \%$ des filles, ont atteint le niveau secondaire, déjà en 2007-08 selon le National Family Household Survey. Le niveau secondaire est généralement effectué entre 12 et 18 ans. De fait, parmi les pauvres, il arrive souvent que les enfants soient plus âgés par rapport à la norme, à cause d'autres tâches qui les retardent (travail rémunéré ou

15 Ces nouvelles étapes de la vie, relativement similaires à ce qui pouvait exister dans l'Ancien Régime, cachent une mutation des trajectoires qui subissent davantage de ruptures, de discontinuités, de va-et-vient qu'auparavant. Depuis les années 1960, une nouvelle évolution des parcours de vie prend forme, où le modèle standard de déroulement de l'existence est remplacé au profit d'une pluralisation des transitions. C'est ce qui a parfois été nommé la seconde phase d'individualisation (Shanahan, 2000). Ainsi, les auteurs constatent un amoindrissement des continuités dans les parcours contre une augmentation des ruptures et des responsabilités incombant aux personnes, soit une pluralisation ou déstandardisation des vies (Cavalli, 2007; Kohli, 2007). Tout en restant sous le joug des institutions, les individus connaissent une multiplicité des opportunités dans le domaine éducationnel, professionnel mais aussi familial. Ce constat est illustré avec la montée des changements de carrière, de la reprise des études, des divorces et des configurations familiales multiformes (Sapin et al., 2007). 
domestique essentiellement). En 2007 et 2008, seuls 14\% des collégiens (issus du secondaire) sont ensuite entrés à l'université (Anand, 2011). Si la période de scolarisation a ainsi augmenté, elle reste cependant toujours très dépendante du niveau social.

Garçons et filles sont séparés au moment de l'adolescence, les unes chargées de prendre part au travail domestique et les autres d'apprendre un métier. La plupart des écoles indiennes ont longtemps été unisexes, et c'est encore le cas pour un certain nombre d'entre elles. Dans les quartiers populaires de Mumbai, les hommes sont considérés jeunes jusqu'à 30-35 ans. Ils ont des activités réservées et connaissent une socialisation par les loisirs qui participent de l'émergence d'une culture de pairs. Dès la fin du $19^{\text {ème }}$ siècle, des activités culturelles et religieuses sont proposées pour rassembler la jeunesse (Heuzé, 1997). Des bandes de quartier avaient coutume de se retrouver fréquemment dans la rue, mais cette pratique a diminué avec l'accès à l'école. Ces moments de rencontre ont été utilisés comme lieux de transmission de culture de masse (spécifiquement masculine), reprise plus récemment pour la diffusion d'idéologies de toutes sortes (allant de la valorisation de la réussite scolaire aux haines interreligieuses). L'arrivée des postes de télévision dans chaque foyer, à la fin du $20^{\text {ème }}$ siècle, a eu le même type d'impact, à la fois disséminateur et rassembleur (Heuzé, 1997).

De son côté, le mariage a conservé en Inde son rôle inébranlable de rite de passage. Dans les années 1960, l'âge à la première union s'élevait à 15 ans en moyenne pour les femmes et 18 ans pour les hommes dans les familles populaires de Mumbai. Il est passé à 20 ans ${ }^{16}$ pour les femmes et 24 ans pour les hommes à la fin du $20^{\text {ème }}$ siècle (Heuzé, 1997), ce qui contribue à expliquer la réduction de la fécondité (Mohanty \& Ram, 2011). Plusieurs programmes nationaux ont participé à favoriser une augmentation de l'âge au mariage. Malgré cela, une large proportion de filles est encore mariée durant l'adolescence ( $22 \%$ avant l'âge de 18 ans en ville en 2005-2006, et seulement 2\% d'hommes, avec toutefois quasiment pas de différences selon la religion et la caste [International Institute for Population Sciences \& Population Council, 2008]). Pour les filles, la jeunesse s'achève donc rapidement, au moment du mariage ou plus tôt, lorsqu'elles

16 Somme toute, cette augmentation est faible; de même, l'âge de 20 ans en moyenne pour le premier mariage dans un milieu urbain n'est guère éloigné de l'âge médian au mariage pour les femmes de toute l'Inde (urbaines et rurales) qui se situe à 17 ans (Guilmoto, 1996). 
occupent complètement leur fonction de seconder leur mère dans le foyer. Aujourd'hui encore, elles ont moins que les garçons la possibilité de poursuivre des études, malgré un meilleur taux de réussite. Le mariage précoce des filles est ainsi négativement corrélé avec leur nombre d'années d'étude, mais aussi avec l'apprentissage de comportements sanitaires de base (notamment autour de la santé de la reproduction) et leur capacité d'empowerment ${ }^{17}$ au sein de la famille (International Institute for Population Sciences \& Population Council, 2008).

Une différence notable se constate toutefois entre les foyers pauvres et les autres, ces derniers démontrant (au début du $21^{\text {ème }}$ siècle) près de deux années supplémentaires dans leur moyenne d'âge au mariage (Mohanty \& Ram, 2011). Répondants à une enquête portant sur la préférence subjective des âges au mariage, $66 \%$ des Maharastriens vivant en ville ont indiqué un âge idéal entre 25 et 29 ans, alors que leurs homologues féminines le situaient de manière moins tranchée entre 20 et 29 ans (Ram, Mohanty, Singh, \& Ram, 2010). Pour un homme, se marier nécessite de pouvoir prouver son indépendance financière, ce qui explique le décalage temporel. De plus, les logements peu nombreux et chers à Mumbai ne facilitent pas la sortie du nid.

Les périodes creuses de la vie professionnelle (pour cause de chômage, de problèmes de santé, ou autres) sont rarement accompagnées d'une compensation financière garantie par l'état. Les hommes adultes, en âge d'avoir la charge d'une famille, passent ainsi souvent à l'informalité afin de conserver une rentrée d'argent (Bhowmik \& More, 2001). Ces mauvaises conjonctures poussent aussi à une entrée (ou un retour) sur le marché du travail d'autres membres du foyer (enfants, épouse, parents âgés, etc.). L'informalité a donc un impact direct sur la force normative associée à l'âge, mais aussi au genre, puisque l'incertain et les variations de trajectoires dominent largement, pour l'individu comme pour ses proches directs (Settersten, 2004). Les vulnérabilités sévères liées à la vieillesse, pour des personnes n'ayant pas de retraite, sont aussi nombreuses, la plus évidente étant que si la famille n'est pas là, l'individu âgé est obligé de continuer à travailler jusqu'à ses derniers jours (Singh, 2015).

17 L'empowerment est un concept qui peut être traduit en français par l'«autonomisation», et qui correspond à l'idée de reprise de pouvoir ou de capacité d'agir à un groupe d'individus dominés, sur les conditions sociales, économiques, politiques ou écologiques auxquelles ils sont confrontés (Solomon, 1976; Zappi, 2013). 
La tripartition occidentale, tout comme le modèle traditionnel indien en quatre phases, ne se traduisent donc pas, dans la réalité que nous étudions, par des séquences systématiquement évidentes et des transitions normatives, c'est-à-dire vécues par une grande majorité autour d'un âge donné. C'est toujours le cas du mariage, mais en ce qui concerne le travail s'observe une forte variabilité sociale, notamment selon le genre.

\section{Une variable clé : le genre}

Outre le niveau social, l'une des premières sources de différentiation dans la configuration et le séquencement dans les parcours de vie est d'être un homme ou une femme. En effet, même en Occident, la vision d'une tripartition du parcours de vie (formation/emploi/retraite) s'applique majoritairement aux parcours masculins, moins aux trajectoires féminines (Krüger, 2009; Levy, 2009; Mayer \& Schoepflin, 2009). Dans les recherches, la focale a longtemps été mise sur les trajectoires standard masculines et publiques, en négligeant les parcours plus typiquement féminins (Hughes, 1996; Krüger \& Levy, 2001).

Face à l'hétérogénéité sociale de genre et de classe, il est pourtant réducteur de concevoir un seul et même type de biographie pour tous les individus (Levy, 2009). La stratification par âge pèse différemment sur les destins des hommes et des femmes, en raison de repères, d'attentes et de significations divergents (Hagestad, 1990; Settersten, 1999; Settersten \& Mayer, 1997). Ainsi, en Occident, l'hypothèse du statut-maître a permis d'expliquer les centrations de la trajectoire masculine autour du travail (des sphères économiques et politiques) et des trajectoires féminines autour de la famille et du cycle reproductif (Crompton \& Le Feuvre, 1996; Heinz \& Krüger, 2001; Krüger \& Levy, 2001; Levy, Gauthier, \& Widmer, 2006). Ce jeu entre famille et travail a été institutionnalisé simultanément au reste du parcours de vie dans les pays développés, présentant une segmentation genrée du marché du travail et de la répartition des tâches domestiques (Krüger, 2009). Les institutions (école, travail, retraite) génèrent des différences en fonction des sexes, travaillent avec ces différences (par exemple en organisant un système de garde et d'allocations familiales) et s'appuient sur cette inégalité (qui influence directement la production et la reproduction créées par la société) (Krüger, 2009). En Inde, nous l'avons dit, de telles institutions n'existent pas de manière large, mais les traditions ont imprimé des comportements profondément dissociés entre hommes et femmes. 
Les femmes indiennes connaissent des parcours spécifiques, centrés sur la sphère familiale, le mariage et la constitution de leur famille. Dans ce contexte, l'arrivée dans les bidonvilles de Mumbai a pu signifier des changements importants dans la vie des campagnardes. Dans certains cas, cela a été source d'indépendance, d'opportunités de sorties et de loisirs en comparaison de la vie menée au village ainsi que, plus crument, la simple possibilité de se nourrir au minimum deux fois par jour. De même, la nécessité de participer au revenu du ménage a pu représenter un accroissement de la liberté féminine, autant que l'éloignement d'avec la famille. Dans d'autres cas, l'environnement quelque peu hostile du slums a au contraire conduit à l'enfermement sur la sphère domestique (de manière volontaire, par crainte de l'extérieur, ou involontaire, sous la contrainte du mari ou de la belle-famille) (Saglio-Yatzimirsky, 2002). Toutefois, dans toutes les situations, les femmes continuent d'assumer toutes les tâches ménagères (préparer les repas, amener les enfants à l'école, aller chercher de l'eau, laver..., toutes activités rendues plus ardues par la précarité de l'environnement) ainsi que de petits travaux d'appoint liés aux activités du mari (couture, découpage du cuir,...) (Saglio-Yatzimirsky, 2002). Lorsqu'elles travaillent à côté, dans des métiers instables, difficiles et peu rétribués (par exemple entre 500 et $5^{\prime} 000^{18}$ INR par mois comme femme de ménage), la conciliation entre travail domestique et rémunéré est épuisante et nécessite une forte organisation. Le plus pratique est donc, quand c'est possible, d'effectuer les tâches payées directement à la maison (Basu \& Thomas, 2009).

Le milieu urbain est le cadre d'évolutions paradoxales dans les relations de genre L'atmosphère citadine encourage l'affirmation patriarcale et la prédominance masculine, des comportements genrés qui se standardisent (des hautes castes vers les basses castes). Elle redonne force à ces visions, à une valorisation de la tradition. Pratiquement, la surveillance des jeunes filles par l'entourage dans les milieux populaires est exacerbée. Mais parallèlement, l'instruction des filles domine dans ces mêmes milieux populaires à Mumbai, et l'accès au marché du travail se répand, offrant de nouvelles possibilités d'émancipation féminine (Heuzé, 1997). $* * *$

A l'issue de cette courte description des structures sociales et des institutions qui participent à définir les normes d'âges et de genre en Inde,

18 Soit entre CHF 7.40 et CHF 74.00 par mois (www.oanda.com, consulté le 22 novembre 2016). 
plusieurs constats s'imposent. Les parcours de vie des Indiennes et des Indiens sont soumis à des impératifs liés à l'origine de classe, de caste et de sexe. Parmi ces régulateurs, deux institutions informelles ressortent comme particulièrement marquantes dans un contexte où l'Etat providence est encore réduit et la pauvreté de masse importante : la religion et la famille. Nonobstant, les récentes métamorphoses de la société urbaine, subordonnées à l'économie de marché globalisé, ont entraîné des changements dans les trajectoires de vie. Plus de similitudes s'observent entre les parcours, en parallèle d'un renforcement des inégalités traditionnelles et de genre.

Pierre angulaire de la construction des vies en Inde, la famille est au centre de ce système fortement normé. Tout d'abord, parce que comme nous l'avons déjà noté, se marier et avoir des enfants est une règle sociale quasiment incontournable. Ensuite, par le support qu'elle fournit face aux aléas de la vie, que l'état ne sait prendre en charge (garde des enfants, assurance chômage et vieillesse, ....). Ce principe s'insère parfaitement dans l'un des préceptes du parcours de vie, qui souligne l'interdépendance entre l'acteur et ses proches, soit le phénomène des vies liées (Elder, 1994; Hareven, 1996; Heinz \& Krüger, 2001). Tout au long de son développement, l'individu est influencé par ses amis et parents, et des changements de rôles et de relations surviennent entre eux (Bengtson \& Allen, 2009). Les personnes ne vivent pas en dehors d'un contexte social, mais bien au sein de celui-ci. Elles en subissent les conséquences, qu'elles soient positives (apprentissages, soutiens,...) ou négatives (obligations, dépendances,...) (Thornberry, Freeman-Gallant, Lizotte, Krohn, \& Smith, 2003). Les transitions attendues du parcours sont, au moins en partie, vécues collectivement et possèdent un effet structurant sur les vies (Sapin et al., 2007).

Une fois posé le contexte social sertissant les vies et proposé un aperçu des différents moments de l'existence (âges chronologiques de la vie), y compris de l'impact du genre, il convient de prendre en compte une dimension supplémentaire : les temporalités. En effet, il ne s'agirait pas de laisser croire que le temps n'ait pas d'importance, tant pèseraient les structures sociales et les normes traditionnelles. Au contraire, les transitions dans la vie (le mariage, la sortie de l'école, la retraite ou la dépendance, etc.) surviennent - de manière plus ou moins régulée - à des âges qui évoluent selon le contexte historique (Attias-Donfut, 1991; Bessin, 2009). En plus des strates d'âge et des rôles assignés, le processus de succession des individus (naissances de nouvelles cohortes) et celui de leur vieillissement 
sont deux sources de changement social (Attias-Donfut, 1991). Ages, cohortes et générations sont les trois points d'ancrage des individus dans l'histoire (Elder, 1975; Riley, 1971).

\subsection{Temporalités individuelles et collectives}

D'un point de vue spatial, les mutations sociales provenant de la mondialisation et de l'institutionnalisation des parcours de vie sont en train de modifier le déploiement des existences de manière globale, bien que différemment selon le cadre sociétal (Dannefer, 2004). En termes temporels, il est crucial de tenir compte à la fois des parcours individuels et du contexte sociohistorique (Mills, 1997 [1959]). C'est précisément l'un des objectifs principaux de la sociologie du parcours de vie que d'interpréter les biographies au sein du contexte sociétal et historique dans lequel elles évoluent (Abbott, 1997; Elder, 1994; Lalive d'Epinay et al., 2005; Sapin, Spini, et Widmer, 2007). Les cadres historiques (socioéconomiques, institutionnels et politiques) de chaque société ont en effet une emprise capitale sur l'évolution des vies humaines (Settersten, 2002). C'est ce que Everett Hughes (1996) remarque en soulignant que les étapes successives du vécu «coïncident avec des événements extérieurs» (p.165) (par exemple le fait de débuter sur le marché du travail en période de plein-emploi ou à l'inverse de récession économique).

A l'origine, la signification sociale de l'âge était déjà au centre de l'attention, chez les sociologues (Cain, 1964; Riley, Johnson, \& Foner, 1972) comme chez les démographes (INED, 1982a, 1982b; Lelièvre, 2006). Mais avec le déploiement des recherches sur le parcours de vie, ont été mises en avant trois dimensions qui permettent analytiquement de situer les individus dans l'histoire (Lalive d'Epinay et al., 2005). La première consiste à utiliser la position dans le parcours de vie (ce qui est une manière de reconsidérer l'âge): avoir eu 15, 35 ou 75 ans au moment de vivre un événement comme la grande dépression de 1929 a un impact radicalement différent sur la vie (Elder, 1999 [1974]). En second lieu, le concept de «cohorte» comprend le regroupement des individus d'après un marqueur historique commun, le plus fréquent étant l'année de naissance (Ryder, 1965). Ce moment initial est déterminant et fixe objectivement 
l'insertion du sujet dans le monde et dans l'histoire. Enfin, la «génération» est la troisième notion, élément incontournable pour saisir la période et les mouvements sociaux dans lesquels les individus s'insèrent, pour aller jusqu'à la définition des «générations historiques» selon Karl Mannheim (1990 [1928]), qui est concomitante de celle de «mémoire collective».

\subsubsection{Age et cohortes : un ancrage historique partagé dès la naissance}

Quatre décennies après la publication des Enfants de la Grande Dépression, les principes de base du parcours de vie tels qu'énoncés par Glen Elder (1999 [1974]) sont toujours reconnus (Elder, 1998; Elder \& George, 2016). Deux en particulier se focalisent sur l'articulation des temporalités individuelle et historique, centrale dans de nombreuses études et systématisée par différents auteurs (Lelièvre, 2006; Mayer, 2004; Settersten, 2004). Le premier (timing) évoque la cardinalité d'un indicateur comme l'âge au sein d'une trajectoire, alors que le second (time and place) rappelle que chaque vie est conditionnée par l'époque et l'endroit où elle se déroule. Autrement dit, le timing prend en compte le moment de l'occurrence d'une transition dans la vie d'une personne, au regard d'un événement extérieur collectif; toutefois, s'il permet de désigner les entrées et sorties dans différents rôles que l'individu doit assumer, il n'en dépend pas moins du lieu et du temps, soit de variables comme le contexte social, économique, institutionnel et culturel (Elder, 1994; Hareven, 1996). Ainsi, le paradigme du parcours de vie repose sur l'interdépendance de plusieurs temporalités, individuelle, familiale et historique (Hareven, 1996). De ce constat découlent deux facteurs : l'évolution du moment de survenue des transitions individuelles dans un contexte historique changeant; la synchronisation des transitions individuelles avec celles - collectives - de la famille ou du groupe, et leur impact sur les relations entre générations.

Dans l'interaction entre biographies et histoire, la notion de cohorte permet une agrégation des individus afin d'étudier les temporalités au niveau de la période historique et du groupe (Heinz \& Krüger, 2001; Settersten \& Martin, 2002). L'ancrage historique des personnes est avant tout déterminé par leur date de naissance, à partir de laquelle se construit un ensemble d'individus vivant des expériences à la fois communes et diverses, mais simultanément (Ryder, 1965). En ce sens, la cohorte se distingue du groupe d'âge, qui n'est défini que chronologiquement. Plus 
qualitatif dans sa conception, le concept de cohorte circonscrit un groupe ayant vécu des événements au même moment et au même âge (Elder \& George, 2016; Marshall, 1983). Logiquement, ils ont un point d'entrée dans l'histoire identique, ce qui peut créer une confusion entre les concepts d'âge et de cohorte (Elder, 1975; Elder \& George, 2016). La taille et la composition des cohortes sont particulièrement importantes de par les implications sociales qui en découlent (Ryder, 1965).

\section{La cohorte comme agent du changement social}

Etre né au cours d'une période spécifique implique de partager des caractéristiques communes avec les personnes venues au monde à la même époque (Elder \& George, 2016; Ryder, 1965). Chaque cohorte se distingue au fil du temps de celles qui l'ont précédé et de celles qui la suivent, parce qu'elle traverse une séquence particulière de l'histoire. En termes géographiques, les diverses régions de la planète, avec leurs normes culturelles et leurs règles sociétales propres, offrent également des conditions singulières au déroulement des vies (Hughes, 1996; Sapin et al., 2007). Ainsi, une femme qui a grandi dans la campagne française du début du vingtième siècle puis qui a traversé successivement les Deux Guerres mondiales, aura connu une existence bien différente de celle d'un homme né à Berlin après la chute du Mur, ou d'une fillette née dans les années 1990 à des milliers de kilomètres, dans les bidonvilles de Mumbai.

Le changement social passe par deux vecteurs: le vieillissement (effet d'âge) et le remplacement successif des cohortes (Alwin \& McCammon, 2007, 2004; Chauvel, 1998; Ryder, 1965). Le vieillissement est un processus individuel, une temporalité variable propre à chaque personne, un processus de maturation. Dans ce développement, la jeunesse représente un moment important, susceptible de provoquer une transformation (Alwin \& McCammon, 2004; Chauvel, 2000; Erikson, 1988). Les cohortes, elles, sont les instruments du changement social (Ryder, 1965). Elles portent, inscrits dans leur $\mathrm{ADN}$, les traits caractéristiques de la période historique vécue.

De manière schématique, les différences entre les cohortes se forment au travers de deux phénomènes: le remplacement des cohortes (Riley, 1987) et la diffusion (adoption tôt dans la vie de nouvelles attitudes et comportements) (Elder \& George, 2016). Chaque cohorte se suit mais conserve une cohérence propre définie par une éducation formelle, une socialisation par les aînés, par les institutions mais aussi par les pairs et 
une expérience historique particulière, amenant un développement spécifique de ses membres. L'émergence continuelle de nouveaux participants dans le processus social ainsi que le retrait de leurs prédécesseurs permet de compenser les limites de la flexibilité individuelle face au changement (Draaisma, 2008; Ryder, 1965). La sécularisation, par exemple, a été un élément clé du passage des sociétés prémodernes à celles dites modernes. $\mathrm{Au}$ sein de ces dernières, au travers de l'industrialisation et de l'urbanisation, une organisation sociétale basée sur des valeurs et une autorité religieuses s'est transformée en une société laïque dans ses lois et ses institutions, par la diminution de la participation religieuse institutionnelle, le déclin de l'influence des autorités religieuses sur les vies, l'augmentation de la privatisation de la religion (Elder \& George, 2016).

La cohorte sert d'outil analytique pour considérer l'articulation entre temps historique et temps individuel, entre parcours de vie et changements sociaux. L'observation des cohortes successives permet de relever les schémas des trajectoires et leurs évolutions (en termes de contenu et de timing), tout en informant sur les structures de la société (Elder, 1975). Toutefois, les cohortes ne sont pas des blocs monolithiques; au contraire elles connaissent en leur sein des variations, en fonction notamment du genre, de la classe, du niveau d'éducation et de l'ethnie (Hareven, 1996). Ces différences font que tous les membres d'une cohorte ne sont pas exposés au même risque face à un changement historique (Elder \& George, 2016). En outre, les cohortes successives rencontrent les événements sociohistoriques à différentes étapes de leur parcours, elles impriment donc des expériences différentes (Elder \& Johnson, 2003; Elder et al., 2015; Ryder, 1965). En ce sens, l'adolescence et l'entrée dans l'âge adulte d'une cohorte forment un moment de construction de la mentalité générationnelle largement influencé par les circonstances historiques du moment (Mannheim, 1990 [1928]).

\subsubsection{Entre histoire et biographies : le concept de génération à la croisée des chemins individuels et collectifs}

La génération est une notion à géométrie variable, qui a fait couler beaucoup d'encre (Attias-Donfut, 1988; Devriese, 1989). Elle est entrée dans le langage courant pour désigner des groupes de personnes délimités par un espace de temps qui s'avère élastique, pouvant aller de quelques 
années à une raisonnable trentaine («génération de l'Indépendance» en Inde, «génération des Trente Glorieuses» en Occident, "génération mai 1968» en France, ...). En effet, si la cohorte est davantage un concept méthodologique établi sur la base de bornes chronologiques identifiables, la génération représente au contraire un groupement de cohortes mitoyennes estimées selon des frontières malaisément chiffrables (Marshall, 1983). Trois niveaux d'analyse sont compris sous ce terme, définissant le lien entre individu et contexte historique (Alwin \& McCammon, 2007; Hareven, 1996).

Premièrement, la génération sert à distinguer les différents membres d'une famille, leurs relations et leurs statuts respectifs (Alwin \& McCammon, 2007; Devriese, 1989; Elder \& George, 2016; Settersten, 1999). Elle permet d'approcher les interactions entre relations et transitions familiales, construites dans les conditions historiques spécifiques (Hareven, 1986, 1996). Deuxièmement, la génération décrit des groupes d'individus nés et ayant vécu les mêmes périodes historiques (soit des cohortes démographiques adjacentes), afin de se pencher sur la modification des comportements liée à l'âge (Devriese, 1989; Ryder, 1965). Ici, les générations prennent aussi une fonction de liant afin de cerner une étape de la vie, qui n'est pas obligatoirement vécue par l'entièreté du groupe. C'est ainsi que des individus se reconnaîtront dans une appellation commune du type de la «génération universitaire», qui définit une expérience partagée (Kertzer, 1983).

Une troisième définition place l'intersection entre biographies et histoire véritablement au cœur de l'analyse (Alwin \& McCammon, 2007; Devriese, 1989; Kertzer, 1983; Mannheim, 1990 [1928]). Elle considère les personnes nées approximativement en même temps et partageant des critères communs, mais ne reconnaît la constitution d'une unité générationnelle qu'à la condition de la survenue d'un événement clé, suffisamment en rupture pour marquer les vies. Il faut également que les cohortes «à risque» d'être affectées soient dans leur jeunesse, prêtes à être investies par ce tournant social et aptes à le comprendre en tant que tel (Elder \& George, 2016; Settersten, 1999). Sous cet angle, le concept de génération est basé sur celui de cohorte, mais nécessite en sus la présence marquée de l'histoire, ainsi qu'une réponse des individus (Alwin \& McCammon, 2004) et la reconnaissance par ces acteurs qu'ils ont partagé une même expérience critique dans un intervalle de temps similaire (Alwin \& McCammon, 2004; Mannheim, 1990 [1928]). Il ne s'agit pas seulement de cohortes 
contigües mais de la conscience historique d'une unité autour d'un changement, qu'il soit social, économique, politique ou technologique (Alwin \& McCammon, 2007; Chauvel, 2000; Settersten, 1999).

L'attention porte donc sur les liens entre des individus égaux en âge, qui expérimentent en même temps l'histoire et les courants sociaux. S'ajoute à cela la capacité d'innovation et de changement social propre à la cohorte et le «degré d'exposition à l'événement», particulièrement élevé dans le cas de jeunes adultes (Devriese, 1989). Ainsi, par la notion de génération, les cohortes sont ancrées dans l'histoire et un rapprochement logique peut être fait avec le concept des générations sociohistoriques de Mannheim (Lalive d'Epinay et al., 2005), qui sera développé au chapitre 8.

\subsubsection{Les événements de la vie : croisements, transitions et bifurcations}

\section{Trajectoires, étapes et transitions}

Dans une vie, les trajectoires sont multiples : familiales, professionnelles, cognitives, spatiales, etc. Elles se déroulent sous forme de moments de stabilité apparente, où les structures de l'existence n'évoluent pas ou très lentement, entrecoupés de changements ou de transitions plus ou moins brutaux permettant le passage d'un stade à un autre ou d'un statut à un autre (Sapin et al., 2007).

Dans cette optique, l'analyse mobilise le concept de transition. Dans le but d'insérer davantage les vies humaines dans le contexte qui les entoure et de saisir les interactions entre les diverses trajectoires, les sociologues découpent traditionnellement le parcours de vie en plusieurs étapes. Elles peuvent se révéler plus ou moins longues et sont généralement séparées par des moments charnières permettant de passer d'un stade à l'autre (Sapin et al., 2007). Appelés transitions ou bifurcations (Bessin, Bidart, \& Grossetti, 2010; Cavalli et al., 2006; Oris et al., 2009), ils sont souvent eux-mêmes composés d'événements ou de tournants déclencheurs et impliquent un changement dans la vie des personnes. Ces transitions dans le parcours de vie sont fortement encadrées par des mécanismes institutionnels formels (école, armée, politiques sociales...), au moins en Occident, parfois par la présence de rites de passage (Attias-Donfut, 1991; Lalive d'Epinay et al., 2005), et toujours par des normes sociales ou des institutions informelles (Hareven, 1986; Hareven \& Masaoka, 1988). 
Puisque la réflexion menée dans ce livre se base sur la perception du parcours de vie par les individus eux-mêmes, au travers de leur mémorisation des événements marquants de leur existence, il importe de considérer la manière dont ce façonnement peut s'opérer. Au niveau individuel, les étapes de la vie suivent un ordre plus ou moins préétabli et sont bien souvent associées à des âges auxquels correspondent des attentes. A nouveau, ce cadre varie selon la société dans laquelle s'inscrivent les individus (Hareven \& Masaoka, 1988; Settersten, 1999). Par ailleurs, la position de la personne dans son parcours de vie au moment de la survenue d'un événement influence la perception de ce dernier. Un événement vécu à 20 , 35 ou 60 ans n'aura pas nécessairement le même impact (Settersten, 1999). Dans le même ordre d'idée, les changements qui surviennent alors que la personne s'évalue elle-même trop âgée ou trop jeune («hors-timing») attestant d'un sentiment de décalage par rapport à une norme - marqueront davantage l'individu qu'un même événement qui peut être anticipé et qui survient au bon moment (Elder, 1975; Neugarten et al., 1965).

Ceci nous conduit à interroger la nature des souvenirs. Lorsqu'ils étudient la mémoire autobiographique des individus, Birren et Schroots (2006) soulignent que les moments rappelés sont majoritairement positifs, bien que cela soit moins marqué chez les personnes âgées. Les auteurs remarquent un clivage du contenu des souvenirs selon le sexe, résultat des processus de construction des inégalités de genre. Ainsi, les femmes apparaissent davantage centrées autour des questions familiales (naissances, romances, scolarisation, etc.) alors que les hommes évoquent plus souvent des événements liés à la sphère professionnelle. L'âge participe également à déterminer le choix des souvenirs: en Europe et dans les Amériques, les adolescents entrant dans le monde adulte se concentrent autour de l'amitié, des rencontres, mais aussi de la formation, les jeunes adultes se focalisent plutôt sur la construction de la famille et les adultes plus avancés en âge évoquent la retraite ou les problèmes de santé (Birren \& Schroots, 2006; Cavalli, Lalive d'Epinay, et al., 2013; Lalive d'Epinay \& Cavalli, 2007). Les décès de proches ont une place importante dans les trajectoires personnelles mais sont plus nombreux à avoir marqué la vie des personnes âgées, ce qui explique la couleur plus sombre de leurs souvenirs (Cavalli, Lalive d'Epinay, et al., 2013; Lalive d'Epinay \& Cavalli, 2007). Dans une perspective temporelle en partie indépendante de l'âge, la valeur émotionnelle d'un souvenir change avec le temps qui passe : une démarche 
rétrospective ne peut que capturer la perception, au moment de l'enquête, des événements passés.

\section{Les événements de la vie}

Les moments charnières de l'existence, où se perçoit la (dis)continuité des trajectoires, sont révélateurs des tendances sociales du monde dans lequel ils prennent place (Hareven \& Masaoka, 1988). Propres à chaque contexte historique, ces changements dans la vie ont été largement étudiés en Occident - ou comme le dit Dale Dannefer (2003), dans l'espace atlantique. Dans un cadre socioéconomique perçu comme caractérisé par l'accélération et la multiplication des transitions (Cordazzo et Fichet, 2013), par la déstandardisation et l'individualisation des parcours de vies (Macmillan, 2005; Shanahan, 2000), ainsi que par la montée des risques sociaux (Beck, 2001) correspondant à une insécurisation des trajectoires et identités personnelles, les transitions dans les parcours de vie permettent d'appréhender les évolutions historiques, en particulier les mutations du marché du travail, des formes de vie familiales et des normes de réalisation de soi.

La trajectoire de vie d'un individu est rarement un long fleuve tranquille. Les événements qui surviennent durant l'existence sont parfois sources de tumulte, ou plus drastiquement de ruptures dans la trame de l'existence (George, 1993; Hughes, 1996). Ces moments comme leurs effets sont dépendants tant du contexte sociohistorique dans lequel le vécu se déroule que des caractéristiques de la personne (Elder, 1999 [1974]). En outre, selon la position de celle-ci au sein de son parcours - autrement dit son âge et les rôles sociaux qui y sont associés - la manière dont les événements seront appréhendés, leur signification, mais aussi leur nature et leur nombre vont varier (Cavalli et Lalive d'Epinay, 2008). Ensemble, ces transitions plus ou moins brutales structurent nos vies (Abbott, 2010; Cordazzo et Fichet, 2013) en permettant ou imposant de passer d'une étape à l'autre. Les auteurs qui ont travaillé sur ces moments utilisent un jargon fourni: événements, tournants (Oris et al., 2009) - turning points dans le monde anglophone (Hareven \& Masaoka, 1988) -, crises, bifurcations ou ruptures (Bessin, Bidart, et Grossetti, 2010). Cette diversité de mots exprime la nature multiple de ces passages.

Une transition, ou un changement de statut, peut être déclenchée, marquée ou conclue par un événement de la vie, qu'il soit désiré ou imprévu, individuel ou collectif, brutal ou graduel, et dont l'issue s'échelonnera 
entre le très positif et le très négatif (Bessin, 2009; Bessin et al., 2010; Hughes, 1996; Reese et Smyer, 1983). Pour Bidart et Brochier (2010), un événement déclencheur sera impromptu et ouvrira sur des prises de décisions, alors que l'événement résolutif apparaîtra à l'issue d'une crise, comme une solution émergente. Autre source de diversité, certaines transitions sont constituées de plusieurs événements (Cordazzo et Fichet, 2013; Hogan et Astone, 1986; Shanahan, 2000; Tichit et Lelièvre, 2006), à l'image de l'entrée dans l'âge adulte, alors que d'autres ne sont provoquées que par un seul fait marquant (une migration par exemple).

Il existe aussi des événements qui se caractérisent à l'inverse par une absence de modification des trajectoires. Un tel épisode donne la sensation de ce qui aurait pu avoir lieu grâce à l'événement mais qui ne s'est pas produit, laissant à peine une trace mémorielle (Hélardot, 2010; Leclerc-Olive, 1998, 2010). A l'extrême, certains événements cruciaux deviennent de véritables nœuds dans la biographie d'une personne puisqu'ils induisent une réorientation de la trajectoire: ce sont alors des tournants (Abbott, 1997) ou des bifurcations (Bidart, 2006a; Grossetti, 2010), voire des événement-catastrophes (Leclerc-Olive, 1997, 2010).

Un premier facteur déterminant l'impact sur la trajectoire de vie est la propension de l'événement à être imprévisible et irréversible (Grossetti, 2010; Hélardot, 2010). Une majorité des transitions étudiées en Occident se définissent par leur caractère attendu, à la fois par la société environnante (Cain, 1964; George, 1993) et par l'individu concerné (Hareven \& Masaoka, 1988). Elles sont le produit de décisions dictées par les normes sociales et par le comportement individuel (Cavalli et Lalive d'Epinay, 2008): les membres de la communauté sont encouragés à les vivre et des accompagnements facilitent le périple. La première communion, la soutenance d'une thèse, le départ à la retraite sont autant d'exemples d'événements qui sont régis par une institution formelle (l'église catholique, l'université, l'Etat) et qui marquent le passage à une nouvelle étape de la vie (religieuse, éducationnelle ou professionnelle), avec souvent des rites associés (Attias-Donfut, 1991; Hughes, 1996; Lalive d'Epinay et al., 2005). D'autres changements prennent davantage la forme de «carrefours», où se présentent des orientations obligatoires, limitées et structurées par un organisme extérieur mais déterminées par la personne elle-même. C'est le cas du choix des études par exemple (Bidart, 2006a).

Plusieurs chercheurs soulignent que les règles culturelles ou institutionnelles informelles tendent elles aussi à cadrer les événements vécus 
et à leur imposer un calendrier (Bessin, 1996; George, 1993; Neugarten \& Hagestad, 1976; Neugarten et al., 1965; Settersten \& Hagestad, 1996). Pour illustration, la sortie du nid et l'entrée dans la parentalité sont soumises à des codes sociaux qui réclament leur occurrence. De telles normes incluent aussi, en règle générale, la spécification d'une période de la vie jugée adéquate pour opérer la transition, une survenue trop précoce ou trop tardive signifiant un éloignement de la temporalité «normale», qui peut provoquer des sanctions dans le regard d'autrui (Hareven \& Masaoka, 1988; Settersten \& Hagestad, 1996). En Occident, la plupart des transitions observées sont normatives, surtout en ce qui concerne les trajectoires éducationnelles, professionnelles et familiales (George, 1993; Hareven et Masaoka, 1988).

Dans les cas où les transitions sont contingentes, souvent non normatives, avec des débouchés inattendus, elles possèdent le potentiel de devenir, comme nous l'avons rapidement indiqué ci-dessus, des turning points, des tournants ou des bifurcations qui délimitent un avant et un après dans la vie (Abbott, 2010; Hélardot, 2010; Hughes, 1996; Leclerc-Olive, 1997). Autrement dit, la transition prend de l'ampleur: le parcours de la personne est infléchi et une irréversibilité est admise (Bessin, 2009; Bessin et al., 2010; Grossetti, 2004). L'existence change alors durablement et significativement d'orientation (Bidart et Brochier, 2010; Clausen, 1995; Elder et Johnson, 2003), de nouvelles attitudes ou de nouveaux rôles doivent remplacer les anciens (Bessin et al., 2010) et une discontinuité dans le parcours est observée (Settersten, 1999). L'origine de ces bifurcations peut être idiosyncrasique, institutionnelle ou provenir d'un événement extérieur sociohistorique comme une crise économique ou une guerre (Elder et Johnson, 2003; Shanahan, 2000).

Vivre une transition majeure implique du stress pour l'individu, d'autant plus lorsque celle-ci n'est pas normative (Chiriboga, 1995). Pour cette raison, les sociologues qui s'intéressent au stress et à ses causes se sont dirigés vers l'étude des transitions, spécialement celles qui surviennent durant la vieillesse (George, 1993; Pearlin, 1989). En psychologie, l'observation du stress engendré par les événements de la vie a également été primordiale, notamment pour analyser son impact sur des maladies cognitives survenant à différents âges (la jeunesse et la vieillesse en particulier). La perception que les individus ont de ce stress, les traumas engendrés et les répercussions sur l'identité ont été au centre de ces recherches (Berntsen et Rubin, 2006). 
Cet aperçu de la littérature sur les transitions illustre combien les parcours de vie regorgent de changements dont l'imprévisibilité varie, de même que l'impact sur les trajectoires, en termes de nature ou de temporalité, courte ou longue. La diversité qui caractérise les moments marquants de l'existence ne doit pas cacher qu'in fine, ce qui fait une différence est l'interprétation qui en est faite par la personne qui les vit. C'est dans la valeur donnée à un changement ou un événement que réside la réalité d'une transition de la vie. En posant une telle affirmation, nous nous situons bien sûr dans le débat qui oppose les approches «objective» et subjective (Bessin, 2009). La première option implique que le chercheur pose un regard extérieur sur les transitions et les événements qu'il souhaite approfondir, sélectionne a priori ceux qui lui semblent pertinents, et interroge les individus à ce sujet. Majoritairement utilisée et pourvoyeuse de nombreux avantages, cette approche a comme référent une objectivité complète qui relève de l'illusion (Lelièvre et Robette, 2015; Lelièvre et Vivier, 2001). En effet, ce sont des êtres humains qui observent et sont observés, avec leurs défauts et leurs qualités, et nul ne peut prétendre à une exactitude ou distance absolue. La seconde perspective, qui fut longtemps moins usitée, vise à saisir le point de vue des acteurs, qui sont amenés à définir eux-mêmes les moments décisifs de leur trajectoire (Hélardot, 2010). Loin d'être faillible scientifiquement, ce procédé a déjà révélé tout son potentiel à travers diverses études. Il est de plus en plus souvent utilisé en renfort du mode objectif pour creuser l'interprétation des faits (Bidart \& Gosselin, 2014; Cavalli, Lalive d'Epinay, et al., 2013; Dasoki, 2017; Laborde et al., 2012).

Subjectif ou tangible, un événement dans la vie peut déclencher un changement qui affectera l'équilibre parfois précaire de l'existence, en fonction d'une stratification d'âge. A côté de cela, les inégalités sociales, en particulier dans notre cas les statuts de genre, de caste et de classe, ainsi que les structures formelles et informelles encadrant les vies, prennent une signification particulière dans un monde de pauvreté de masse. Ce double constat de vulnérabilités a été établi dans la littérature portant sur les nouvelles questions sociétales de précarité et d'exclusion, ainsi que sur les risques liés à la globalisation et à la modernité. Au travers du paradigme du parcours de vie, les formes de cette vulnérabilité ont pu être révélées à de nombreuses reprises (Bresson et al., 2013; Oris et al., 2009, 2016; Spini et al., 2013) dans des études de plus en plus nombreuses sur les sociétés occidentales dites riches, ou développées; qu'en sera-t-il pour des Indiennes et des Indiens vivant à Mumbai? 


\subsection{Vulnérabilités et parcours de vie}

Les études portant sur les sciences environnementales et les catastrophes naturelles sont les premières à utiliser le terme de «vulnérabilité», dans les années 1970, pour désigner les vacillements de la condition naturelle et humaine (Thomas, 2008; Martin, 2013). Ce constat s'inscrit dans la montée des guerres, des crises économiques et des accidents industriels (explosion d'une usine chimique à Bhopal en Inde, de la centrale nucléaire de Tchernobyl en URSS, ...) qui caractérise la fin du $20^{\text {ème }}$ siècle (Thomas, 2010). Dans les années 1980, le mot est repris par les sciences sociales, d'abord dans le monde anglo-saxon en même temps que celui de «risque», qui fait également son chemin parmi les domaines du développement et de la lutte contre la pauvreté (Thomas, 2010). Se développe alors une préoccupation liée à la compréhension des risques collectifs, des désastres naturels et des facteurs de stress individuels, prévisibles et imprévisibles (Misztal, 2011; Thomas, 2008, 2010).

Le concept de vulnérabilité a évolué depuis ces premiers temps, souvent en parallèle d'une transformation de paradigme dans les politiques publiques. Suivant le trend de l'individualisation des parcours de vie (voir 2.2.3) et dans un contexte de libéralisation économique et de flexibilisation du marché du travail, une responsabilisation individuelle est mise en avant et remplace ce qui, auparavant, relevait de la gestion collective (Bresson et al., 2013). Dans les pays du Sud et en Inde notamment, l'état est amputé de sa charge protectrice et régulatrice alors qu'il est explicitement attendu des marchés qu'ils comblent miraculeusement les inégalités par le biais de la redistribution des profits liés à la croissance économique. Pourtant, aucune redistribution ne voit le jour (Lautier, 2013; Roy, 2014).

Comprendre les vulnérabilités qui affectent aujourd'hui des Indiennes et des Indiens vivant à Mumbai signifie s'intéresser au concept de pauvreté bien sûr, mais également aux processus de modernisation et de globalisation qui ont transformé le pays en même temps que le reste du monde. Ceci ne veut pas dire pour autant oublier les structures normatives et traditionnelles ni les stratifications sociales qui y exercent leur poids (voir 2.2). Les inégalités d'antan ne sont certes plus les mêmes, mais ont donné lieu à de nouvelles formes non moins exclusives, qu'il est important de souligner. Pour cela, le parcours de vie est un outil utile, essentiellement neuf dans le contexte indien, permettant de mettre en lien 
les vulnérabilités géographique et historique avec celles que ressentent les individus et qu'ils situent dans leurs trajectoires.

\subsubsection{Aux origines de la vulnérabilité : environnement, développement et pauvreté}

Initialement, la vulnérabilité - dont la signification s'apparente à celle de pauvreté, quoique cultivant ses différences ${ }^{19}$ - est un sujet de recherche dans le domaine du développement durable. Dans ce cadre, elle sert à décrire le degré d'exposition des populations vis-à-vis des désastres environnementaux et souligne l'expérience objective et subjective de l'incertain, de la fragilité collective et du manque de contrôle des individus sur leur milieu (Misztal, 2011). Bien vite, la vulnérabilité va dépasser la sphère des risques macrosociaux - sans pour autant la perdre de vue, comme nous le verrons au point 2.4.2 - pour s'intéresser plus particulièrement aux victimes de ceux-ci.

Au début des années 1990, les organisations internationales qui prêchent pour un rattrapage économique du Sud, ainsi que les chercheurs intéressés aux pays moins développés économiquement, se mettent à utiliser largement la vulnérabilité pour décrire les habitants du Sud (ou les «populations vulnérables»), grands perdants du creusement des inégalités à l'échelle mondiale, dont les carences dans la satisfaction des besoins primaires sont profondes (Geronimi, 2013). De nombreux indicateurs sont mis en place par diverses institutions, notamment le FMI et la Banque Mondiale, afin de mesurer l'impact d'une crise économique ou d'une catastrophe naturelle sur un pays et ses habitants, déplaçant ainsi la focale du seul revenu vers davantage de dimensions du dénuement ${ }^{20}$. Selon cette interprétation, la vulnérabilité est associée à la capacité du

19 Pauvreté et vulnérabilité sont des concepts proches mais pas identiques, bien que largement entremêlés (Chambers, 2006). Le premier représente des caractéristiques du groupe ou de l'individu, liées à une exposition aux risques reposant sur une situation socioéconomique: les populations pauvres possèdent peu de défenses (Thomas, 2008). Ainsi, la pauvreté représente un statut, le non-accès à un certain nombre de biens élémentaires. A l'inverse, la vulnérabilité est dynamique, c'est une composante du mécanisme de pauvreté, dont la signification est plurielle.

20 Depuis l'an 2000, l'ONU et le PNUD (Programme des Nations Unies pour le développement) incluent également des indices de vulnérabilité dans leur classement des pays les moins avancés (Geronimi, 2013; Thomas, 2008, 2010). 
pays et des individus à résister à un choc exogène, afin de maintenir un développement et une croissance économique positive (Geronimi, 2013). Dans une perspective micro (personnes et ménages), elle représente une avancée significative dans les travaux portant sur la pauvreté, qui jusqu'alors étaient centrés sur des évaluations monétaires, car l'usage du concept de vulnérabilité permet de prendre en compte la probabilité de perte de bien-être, l'incapacité à prévoir et à anticiper le futur ${ }^{21}$ (Alwang, Siegel, \& Jorgensen, 2001).

L'icône de ce paradigme d'une pauvreté multiforme et multidimensionnelle est Amartya Sen, chercheur indien, détenteur du prix Nobel d'économie en 1998, qui après avoir démontré que la famine n'est pas uniquement la résultante du manque de ressources mais provient surtout d'une mauvaise gestion politique (Sen, 1981), a travaillé sur la notion de développement humain en mettant en avant les concepts de libertés réelles et de capacités de réalisation (ou capabilités) (Sen, 1999). Concrètement, ces initiatives se cristallisent dans la mise en place en l'an 2000 des objectifs du Millénaire pour le développement, plan prévu pour 15 ans, ainsi que sa suite, l'après-2015, qui visent à éliminer la pauvreté au travers de plusieurs axes (alimentation, scolarisation, égalité des sexes, accès à la santé,...) (Bonvin \& Farvaque, 2008; Cartier-Bresson, 2013)22.

Ces deux premiers versants de la notion de vulnérabilité s'intéressent avant tout aux secousses extérieures pouvant affecter l'existence, ainsi qu'aux façons de les prévenir. Au cœur des réflexions se trouve l'idée

21 Toutefois, ces mesures demeurent basées sur un seuil numérique et permettent peu de nuances dans les formes d'indigence (pauvreté et extrême pauvreté sont généralement les uniques échelons de la misère); ce malgré certaines tentatives de compréhension plus nuancée de la pauvreté, qui restent bien limitées, à l'image des 60'000 entretiens de personnes pauvres menés par la Banque Mondiale dans 60 pays au début du millénaire: Voices of the poor [http://web.worldbank.org/] (Cartier-Bresson, 2013).

22 Dans les années 2000, à la suite de ces préoccupations, les pays en développement mettent la lutte contre la vulnérabilité au centre de leurs programmes institutionnels d'action, afin d'agir en faveur d'une catégorie de nouveaux vulnérables issus des récents changements sociaux et économiques. Il s'agit des groupes tombés dans la pauvreté suite à un événement collectif ou individuel, que l'absence de protection sociale a laissé sombrer (working poor, travailleurs déqualifiés ou informels inférieurs, chômeurs,...) (Lautier, 2013). Avec le passage de l'ouragan Katrina, en 2005 aux USA, une réorientation de l'usage de la vulnérabilité vers les populations pauvres du Nord s'effectue. Les travaux permettent de montrer le lien qui existe entre les particularités géographiques, la densité humaine et les caractéristiques socioéconomiques de la population (Thomas, 2010). 
que les garanties étatiques de protection sociale mises en place au Nord permettent d'endiguer ces heurts, tandis que leur partielle (voire totale) inexistence dans les pays du Sud aggrave les conditions de vie dans ces zones de la planète. Pourtant, la vision des politiques publiques comme une étape incontournable dans l'éradication de la pauvreté et de la vulnérabilité des pays en développement avait déjà fait son chemin, voilà plus de 70 ans (Lautier, 2013).

\section{Faillite des réponses institutionnelles face aux vulnérabilités de masse}

Au sortir de la Seconde Guerre mondiale et de la colonisation, beaucoup de pays du Sud (dont l'Inde fait partie) ont lancé un système de protection sociale, afin de protéger toute la population contre tous les risques. Dans les réflexions plutôt occidentalo-centrées menées à ce propos, deux objectifs sous-tendent cette protection universelle: l'augmentation de la productivité d'une part et la paix sociale de l'autre (Lautier, 2013). Dans les faits, ce sont des conséquences alternatives qui émergent: la régulation des bénéficiaires, l'encouragement à suivre les normes institutionnelles ainsi qu'à les reproduire, et la définition de qui est habilité à recevoir un subside, produisent ensemble un effet de «normalisation» sur la population (Misztal, 2011); par ailleurs, la frange à la limite de l'indigence mais qui n'entre pas dans les critères de définition des prestataires constitue de nouveaux défavorisés (Lautier, 2013). Ainsi, le nombre grandissant de personnes vulnérables qui apparaissent à cette période s'explique par des pressions institutionnelles plutôt que par l'arrivée de risques inédits (Lautier, 2013). S'ajoutent à cela des soucis d'application, comme la constitution artificielle d'une hiérarchie des besoins et des risques, la gestion de la corruption, etc. Finalement, la mise en place de ces politiques publiques bute sur une difficulté inhérente aux pays moins développés économiquement: la misère de masse, qui nécessite un encadrement bien plus considérable que dans les pays développés pour sortir les individus de la pauvreté, en parallèle d'un manque abyssal de moyens (Lautier, 2013).

Toutes ces raisons serviraient à elles seules à expliquer l'échec du système de sécurité publique dans le Sud, constaté à partir des années 1980. Toutefois, si ce système laisse les experts sceptiques, c'est d'abord parce que la couverture sociale est arbitrairement répartie, protégeant avant tout ceux qui ont moins besoin de l'être. Effectivement, cette dernière est appliquée le plus souvent par le biais des contrats de travail et garantie par le pouvoir relatif des syndicats, donc plus élevée dans les branches 
dites formelles au sens du BIT, qui sont les plus syndiquées et déjà relativement protégées. Avec les changements économiques de la fin du $20^{\text {ème }}$ siècle (abandon du protectionnisme, compétition mondiale, montée de l'informalité,...) ce lien est invalidé: la plupart des travailleurs ne sont plus employés contractuellement par des firmes et une déconnection de la notion de risque avec celle de revenu du travail et de droits sociaux s'opère (Castel, 2013).

Face à ces déboires, l'idéal de protection généralisée cède le pas à des politiques ciblées, usant de moyens spécifiques et qui ne reposent pas sur des principes de droits universels, la plus évidente de ces batailles étant la lutte contre la pauvreté (Lautier, 2013). Néanmoins, cette aide qui se veut pointue n'affecte pas la vulnérabilité extrême, se contentant de pallier la facette la plus évidente de la pauvreté dans les Suds : la famine. D'autres actions ont pour but de cibler les individus pauvres les moins vulnérables, soit ceux qui se présentent avec certaines ressources et une capacité de réinsertion, afin de leur offrir des chances de développement par des voies microéconomiques (microcrédit, coopératives,...) (Lautier, 2013).

L'Inde, comme la plupart des pays en développement, a acté l'échec de sa politique de protection sociale, malgré une donne quelque peu différente. En effet, sa croissance économique fulgurante au cours des dernières années du $20^{\text {ème }}$ siècle démontre d'une potentielle capacité financière; c'est le choix politique de ne pas redistribuer les bénéfices de cette croissance qui s'est avéré l'un des empêchements au développement de programmes sociaux (Lautier, 2013). Or, dans le contexte d'informalité23 massive et de pauvreté chronique que l'Inde supporte à cette même période (voir chapitre 1), seule une réforme fiscale complète aurait pu assurer aux travailleurs non protégés un encadrement public décent (Lautier, 2013). En son absence, la pauvreté reste massive dans le sous-continent indien et, de manière évidente et officielle, la vulnérabilité des individus y est connue: plus de 350 millions de personnes y ont d'ailleurs été répertoriées comme vivant en dessous du seuil de pauvreté (pourtant peu élevé) en 2011-2012 (LeMonde.fr, 2014; Roy, 1999). Il n'y a aucun doute non plus que l'exposition aux risques soit une réalité objective dans cette nation où les catastrophes naturelles et industrielles frappent régulièrement (cf.

23 La notion d'informalité est utilisée pour la première fois en 1972 par le BIT, qui la définit tout d'abord que par la taille réduite des unités de production; la dimension d'absence de protection ne sera ajoutée que 30 ans plus tard. Si la précarité est définie politiquement, l'informalité l'est institutionnellement (Bouffartigue \& Busso, 2010). 
chapitre 1), où les crises économiques et politiques se multiplient, et dont la population pauvre pourrait aisément illustrer la nécessité du combat pour chacun des huit points des Objectifs du Millénaire.

Nonobstant, ces vulnérabilités ne sont pas de facto reconnues, au sens où aucune réponse institutionnelle n'est clairement apportée. Par ailleurs, le dénuement n'est qu'un aspect de la pauvreté en Inde, le mépris lié au statut de pauvre étant un autre fléau (Saglio-Yatzimirsky, 2002). Autre phénomène trompeur, si les ouvertures économiques de ces dernières décennies ont permis au pays de résorber quelque peu la masse de ses miséreux, tout au moins dans les chiffres, en pratique, la montée des inégalités et les nouvelles fractures sociales marquent toujours profondément l'Inde moderne, particulièrement au sein des zones urbaines. Pour les comprendre, une analyse des travaux sur la pauvreté et l'exclusion, menés essentiellement dans les sciences sociales francophones, s'avère nécessaire.

\section{Pauvreté et nouvelles fractures sociales}

La recherche sur la vulnérabilité dans les pays développés a davantage porté sur la forme latente de cette dernière - une vulnérabilité subjective, multiforme, qui affecte des groupes d'individus à risque et surgit sans crier gare - que sur sa forme évidente et objective issue des privations (Bresson et al., 2013), présente avant tout dans les pays où la pauvreté de masse fait rage. Cette tentative d'identifier les "problèmes sociaux» sous tous leurs angles était déjà apparue avec le courant hygiéniste français des années 1830-1840, où l'utilisation de termes tels que précarité, pauvreté et exclusion servait à pointer la nouvelle question sociale, occultant simultanément la question des inégalités entre prolétariat et sous-prolétariat (Thomas, 2008, 2010). En effet, à la même époque avait débuté la diffusion des écrits de Marx et Engels à propos de la division des travailleurs ${ }^{24}$

24 Selon Marx et Engels, le prolétariat est composé de plusieurs catégories, l'une d'elle étant un «sous-prolétariat» ou main d'œuvre de remplacement, qui désigne une population dont les conditions de vie et surtout de travail sont inférieures au reste du prolétariat urbain (2014 [1845]). Les éléments qui la composent sont particulièrement pauvres et non organisés. La bourgeoisie se sert de cette armée de réserve pour faire pression sur le reste de la classe laborieuse, cherchant par cette division à contenir les germes d'une émancipation populaire. Pour ces auteurs, le capitalisme est fondamentalement instable, avance de crise en crise, vers une société de plus en plus inégalitaire où le désaccord entre les classes est inévitable (Marx \& Engels, 1999 [1848]). Ainsi, dans la pensée marxiste, le moteur de l'histoire réside dans la lutte des 
et la désignation des miséreux autrement que par ces termes controversés servait la classe dominante. L'intérêt pour la question reprend avec le tournant des Deux Guerres mondiales et les améliorations sanitaires et sociales liées à la modernisation, alors que l'espoir est grand de voir la misère disparaître dans ces pays.

Plus récemment, la sociologie francophone s'est à nouveau penchée sur le sujet, en déterminant trois moments de la pauvreté survenant les uns après les autres de manière chronologique, sans toutefois appeler à la disparition des stades précédents. Partant de la pauvreté, la réflexion va de plus en plus laisser de la place au concept de vulnérabilitée ${ }^{25}$. En premier lieu, il s'agit de la grande pauvreté des débuts de la révolution industrielle, à laquelle s'ajoute sur la fin du $20^{\text {ème }}$ siècle une nouvelle forme de dénuement caractérisée par la précarisation d'une partie du salariat, avant que vienne finalement poindre la catégorie des vulnérables (Thomas, 2010). Le premier âge, qui s'étend jusqu'à la période prospère des Trente Glorieuses, définit les pauvres selon leur position dans la société et face au marché du travail (ils sont alors nommés marginaux ou inadaptés). Après cette époque et face à la persistance de la pauvreté, il est envisagé d'étendre la protection sociale à d'autres nécessiteux. Les chocs pétroliers de 1973 et 1979 amorcent la crise de la société salariales et la montée des incertitudes (Castel, 1999). Les institutions mettent alors en place de grandes enquêtes et les notions de «précarité» et de «fragilité» (phase de faiblesse intense qu'un simple événement imprévu peut faire basculer vers l'indigence [Thomas, 2008]) se répandent largement, comme une seconde dimension de la nécessité, coexistant avec la pauvreté extrême (Thomas, 2008, 2010).

A ce moment, l'existence d'une distinction entre pauvreté chronique (profonde et pouvant difficilement être résolue) et pauvreté temporaire (contre laquelle une intervention extérieure de soutien peut porter rapidement ses fruits) est soulignée, et des seuils sont établis afin de les définir (Thomas, 2010). La pauvreté temporaire (aussi appelée relative ou potentielle) distingue la frange de la population en équilibre instable qui, sous l'impulsion d'un événement exogène ou endogène, peut rapidement

classes et la classe bourgeoise s'oppose au prolétariat, en procédant par la division de la conscience de classe du peuple.

25 Jusqu'à la fin des années 1990, les experts européens dans le domaine de la pauvreté et des inégalités ne font guère usage du mot vulnérabilité ni de lien avec les concepts de risques ou de populations vulnérables, les sciences sociales utilisent toutefois des idées proches pour identifier une pauvreté multiforme (Thomas, 2010). 
chuter sous le seuil de pauvreté. Cette précarité sociale désigne les victimes de l'inégalité de répartition des ressources (Thomas, 2010), nouvellement issues de l'évolution économique mondiale, alors que la pauvreté extrême semble une ancienne catégorie inextinguible - le fameux sous-prolétariat de Marx et Engels (2014 [1845]).

Dans les années 1990 naît le troisième âge de la pauvreté, recentré autour de l'«exclusion», où la distinction entre précaires et exclus s'affine. Les marges les moins qualifiées du prolétariat (essentiellement les femmes et les travailleurs pauvres ou working poor) sont qualifiés comme une forme inédite de pauvreté (Thomas, 2010). Ces vulnérables représentent les individus figés dans une situation salariale instable, non protégés, avec une insuffisance de ressources économiques, trop peu d'accès aux droits et aux protections sociales, ainsi qu'un manque de représentation parmi les groupes de pression (Bourdelais, 2005; Martin, 2013). Il ne s'agit pas d'une pauvreté crasse ni d'une précarité de l'existence, mais plus largement d'une dérive d'individus abandonnés socialement et institutionnellement. Apparaissent alors des mots plus spécifiques, plus travaillés, comme les «désaffiliés» de Robert Castel (1999). Une forme de renouveau du contrôle social se constitue avec la responsabilisation du citoyen, la disqualification des vulnérables au travers de l'étiquette de l'indignité (Thomas, 2010). On cesse de parler d'égalité des droits pour évoquer une équité des chances.

Robert Castel (1994) est bien connu pour ses travaux sur le sujet de la vulnérabilisation (qu'il nomme plutôt marginalisation), dont il comprend l'émergence comme l'addition des phénomènes de précarisation du travail et d'individualisation. Selon lui, la post-modernité introduit une double dégradation, celle du statut dans le marché de l'emploi (qui se précarise fortement avec une montée de l'instabilité et du chômage) et celle des liens sociaux (à travers les décompositions et recompositions familiales) (Castel, 1994). Dans sa perspective, la vulnérabilité est le résultat d'un décrochage au niveau professionnel et relationnel, d'un processus dynamique qui affecte les personnes déstabilisées, qu'une exclusion peut mener progressivement à la grande marginalité. Castel (2013) rappelle que des travailleurs sont pauvres parce que leur travail ne leur permet pas de se nourrir et que leur vulnérabilité reflète en fait cette crise de la société salariale qu'il a mis en exergue dans ses travaux. Il considère que le risque ne serait pas tant de se retrouver hors du marché de l'emploi que de subir les divisions induites par ses contraintes et son insécurité (Cingolani, 2013). En effet, l'individualisation et les mutations socioéconomiques de 
la modernité ont entraîné un clivage entre les travailleurs, que ce soit selon leur type de profession (qualifiée ou non) ou selon des caractéristiques intrinsèques (hommes et femmes, étrangers et locaux). La mise au ban professionnelle, et dès lors sociale, dont la responsabilité est directement attribuée à l'individu, est l'ultime stade de la précarité telle qu'elle se rencontre dans nos sociétés occidentales et modernes.

\section{Les vulnérabilités en Inde}

Ce savoir occidental appliqué à l'Inde permet de souligner les diverses formes de pauvreté et de vulnérabilité qui touchent la population. La pauvreté extrême affectait a minima 350 millions d'individus en 2011-2012, se traduisant par un manque d'accès aux besoins élémentaires ainsi qu'à un revenu décent, et ce de manière chronique. Par ailleurs, une précarité de l'existence est aussi présente, rendant la rencontre d'événements prévus (comme payer la dot de sa fille) ou imprévus (rembourser des frais médicaux par exemple) difficile à surmonter pour davantage de monde encore. Cette seconde modalité prend un caractère plus temporaire, lié aux aléas de l'existence. Typiquement, au sein des ménages, la vulnérabilité des femmes et enfants ressort de la sensibilité au risque. Ils sont en effet encore largement dépendants de l'homme pourvoyeur de revenus, la perte de l'emploi, la maladie ou la mort du mari (respectivement du père) induisant de nombreuses conséquences pour la famille entière (malnutrition, maladies etc.) (Pryer, 1989).

En ce qui concerne la vulnérabilité au sens de la marginalisation, la réponse est plus nuancée. Dans un contexte urbain comme celui de Mumbai, ville où plus de la moitié des habitants survit dans un dénuement certain, il semblerait logique de se raccrocher à ce que Castel (1994) nomme la pauvreté intégrée, dénuée d'exclusion. Cette idée repose sur le constat que, puisque le gouvernement ne reconnait pas ces individus - il ne les rejette pas non plus, comme c'est le cas en Europe, au travers de lois discriminatoires et criminalisantes telle l'interdiction de la mendicité - et que l'Etat-providence est inexistant pour l'essentiel de la population, la marginalisation n'existerait pas (Castel, 1994).

Toutefois, dans un contexte où les disparités sont palpables (entre les castes bien sûr, mais également au travers du phénomène de ghettoïsation des bidonvilles, eux-mêmes stratifiés selon le revenu, la caste et la religion, par la distanciation flagrante entre classes moyennes et pauvreté populaire, et plus récemment par la marginalisation de sous-populations 
spécifiquement vulnérables: les chômeurs, les femmes, les enfants), l'exclusion est malgré tout une réalité (Saglio-Yatzimirsky, 2002). Diverses institutions politiques encouragent ces divisions en se montrant ouvertement anti-pauvres, notamment en ordonnant la destruction de bidonvilles ou leur déplacement aux extrémités de la ville, etc. ${ }^{26}$. Des fractures sociales se distinguent, bien que d'une forme différente de celles que l'on connait en Occident, par un jeu d'échelle évident : une majorité bien plus grande des individus en est la cible. Ainsi, il n'est pas comparable de se trouver sans toit à Paris ou à Mumbai, sans même considérer les critères météorologiques. Dans le premier cas, il ne s'agit que d'une faible minorité aisément circonscrite, alors que dans le second cas le pourcentage de personnes sans domicile fixe est si élevé que le groupe peut même être subdivisé. Une rupture se dessine, excluant une partie des habitants de Mumbai du marché de l'emploi et du logement, de l'accès à l'éducation et aux soins, notamment une partie des Dalits, femmes, musulmans et/ou étrangers. Les clivages naissent de critères de langue, de religion, de caste, de genre (voir chapitre 1) et prennent des apparences qui ne nous sont pas si étrangères : xénophobie, sexisme, islamophobie.

Dans le monde anglo-saxon, la notion de vulnérabilité est directement mise en lien avec le processus de modernisation (Beck, 1992; Giddens, 2004 [1994]), d'une manière quelque peu différente des travaux de Robert Castel. Par ce processus, les individus sont soumis à des risques extérieurs nouveaux, globaux, qui bien qu'affectant la société dans son ensemble (remettant en question les anciennes stratifications sociales) sont contrés par des protections individuelles.

\subsection{2 "A risk society": vulnérabilités sociétales et protections individuelles}

Dans toutes les sociétés, les structures génératrices d'inégalités sociales

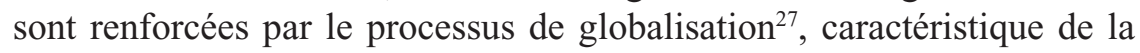

26 Il y a bien eu une certaine combativité institutionnelle à l'égard des inégalités, par exemple au travers des quotas de réservation, mais ces derniers ont davantage participé à fragmenter les pauvres, suivant le jeu des politiques (Saglio-Yatzimirsky, 2002).

27 Quatre tendances macroéconomiques définissent cette globalisation: l'internationalisation des marchés et l'augmentation de la compétition entre les pays; ce qui enjoint les états à diminuer leurs taxes sur le commerce, à déréguler et privatiser, en un mot: à libéraliser; à cela s'ajoute une interdépendance des acteurs via la croissance des réseaux d'individus (migrations) et des technologies de communication; enfin, 
nouvelle étape historique dite de la «seconde modernité» ou «modernité avancée» (Bergeron, 2013; Giddens, 2004; Lalive d'Epinay, 2012). L'affaiblissement de l'Etat-nation, l'accroissement des imaginaires transnationaux et la dérégulation des parcours de vie en sont des conséquences directes (Appadurai, 2005 [1996]; Lalive d'Epinay, 2012; Mills \& Blossfeld, 2003; Spini, Hanappi, Bernardi, Oris, \& Bickel, 2013) ${ }^{28}$. En outre, l'élévation du niveau économique de la société et l'augmentation numérique des classes moyennes durant les 30 Glorieuses, puis la progression des inégalités depuis, ont été largement reconnues comme conduisant à une diminution de la conscience de classe, tout au moins en Occident (Beck, 1992; Chauvel, 2001). La globalisation fait donc plus que remettre en question le régional et le national, elle ébranle les structures politiques et culturelles. Elle n'est pas l'apanage du Nord: une réappropriation identitaire par l'adaptation à la modernité voit également le jour dans les pays du Sud (Appadurai, 2005 [1996]).

Des réponses culturellement et historiquement orientées permettent de faire face aux pressions, provoquant des variations locales au sein de la globalité (Mayer, 2001). L'incertain et les inégalités induits par les formes de mondialisation sont filtrés par un certain nombre de cadres nationaux spécifiques : systèmes éducatif, marchés du travail, politiques et institutions sociales plus ou moins développées, normes de soutien dans les réseaux familiaux, etc., qui peuvent signifier autant des atouts que des handicaps. Pays émergent au milieu de cette transformation planétaire, particulièrement soumis aux aléas climatiques (inondations, sécheresses,...) et tractant un passé belliqueux encore douloureux (guerres civiles, conflits indo-pakistanais, ...), l'Inde subit de plein fouet ces dynamiques d'intégration, tant nationalement, par un positionnement laborieux dans l'échiquier

l'importance des marchés mondiaux en parallèle d'une forte volatilité des marchés locaux, de plus en plus vulnérables face aux chocs économiques, sociaux et politiques (Buchholz et al., 2009; Mills \& Blossfeld, 2003).

28 Deux écoles analysent cette situation. La première voit la désinstitutionalisation et la pluralisation des parcours de vie comme étant ralentie par des structures plus profondes, qui empêchent les institutions sociales de répondre trop vite aux changements (Riley \& Riley, 2000; Schaie \& Abeles, 2008). D'autres pensent que les forces comme la globalisation et l'économie de marché (qui provoquent la diminution de la protection sociale et des mutations dans le marché du travail et les structures familiales) agissent avec les changements démographiques pour éroder les institutions traditionnelles des sociétés industrielles et désinstitutionnaliser les parcours de vie (Esping-Andersen, 1999; O'Rand, 2004), ce qui mène à l'individualisation. 
des superpuissances mondiales, qu'au niveau des ménages et des individus, encore trop majoritairement submergés par la pauvreté et des vulnérabilités multiples. L'Etat social peu développé peine à combler une diminution de la présence des institutions informelles telles que la famille, nous l'avons vu précédemment (voir 2.4.1). Des stratégies individuelles doivent alors être déployées pour affronter les événements extérieurs.

\section{Enjeux de la globalisation et différentiels dans les facteurs d'exposition aux risques}

La modernité a également été étudiée comme une transformation de la société, certes liée à la globalisation mais aussi définie par une exposition à des risques nouveaux au regard des dangers préétablis de naguère ${ }^{29}$ (Beck, 1992). Une partie d'entre eux est prévisible mais une autre est imprévisible; Ulrich Beck (2003) regroupe au sein de cette dernière trois types principaux: les menaces terroristes, les crises financières et les catastrophes environnementales. Ces risques sont en effet essentiellement issus de décisions humaines et moins de causes naturelles, comme c'était le cas jadis avec des dangers tels que maladies, sécheresses, etc. (Beck, 2003). Par ailleurs, selon Beck (2001 [1986]), les origines et le niveau de vie des individus ne déterminent plus l'incertain : le risque est présent pour tous les individus, qui n'ont pas l'opportunité de s'assurer contre (à l'image de la catastrophe de Tchernobyl). De ce fait, les différences de classe s'estompent, n'étant plus des critères déterminants de vulnérabilité face aux épreuves de la vie, et l'existence même des classes sociales est soumise à critique (Beck \& Beck-Gernsheim, 2002). Parallèlement, les sécurités traditionnelles (réseaux d'entraide) sont menacées par le processus d'individualisation. Ainsi, la protection diminue (délitement de la

29 Les anciens risques (analphabétisme, maladies, chômage, pauvreté dans la vieillesse) ont été fortement présents jusqu'à la première moitié du $20^{\text {ème }}$ siècle dans les sociétés occidentales, basés sur une absence d'information et de contrôle sur les hasards de la vie (O'Rand, 2004). Une réponse institutionnelle a pu être apportée à ces risques, qui a limité énormément leurs impacts négatifs (en particulier au niveau de la santé, comme le prouve la hausse de l'espérance de vie). Toutefois, de nouveaux risques sont apparus, basés sur une présence accrue de l'information (O'Rand, 2004). Les anciens risques sont considérés comme essentiellement exogènes, alors que les nouveaux risques sont davantage endogènes et rendent l'individu, plus que le groupe, vulnérable. Trois facteurs sont étroitement liés à cette individualisation des risques: le vieillissement, la privatisation des systèmes de protection sociale, la nouvelle économie (la flexibilisation du marché de l'emploi) (O'Rand, 2004). 
prévention collective) alors que les risques grandissent. Les individus sont responsabilisés dans ce processus et chargés de dépasser les vulnérabilités par eux-mêmes, de faire des choix et même de savoir saisir des «opportunités risquées» (Misztal, 2011).

Cette analyse des risques qui caractérisent la société moderne a été largement discutée, d'abord pour son amalgame rapide entre crises socioéconomiques et environnementales, jugées pareillement imprévisibles. Par ailleurs, l'apologie du risque que cette théorie sous-entend a été plusieurs fois qualifiée d'instrumentalisation de la peur, dont l'archétype est le terrorisme (Boucher, 2015; Martin, 2013). Autre point crucial, la théorie de la société du risque tire ses fondements d'études presque exclusivement centrées sur l'Occident et relativement peu empiriques. De ce fait, elle accorde une attention considérable aux enjeux globaux mais oublie les anciens risques, tels que l'exclusion et la pauvreté, qui affectent le niveau des individus toujours profondément au sein des structures socioéconomiques (Misztal, 2011).

Centrale dans le discours de Beck (2007), la remise en question des classes sociales a soulevé une critique pertinente (Atkinson, 2007; Chauvel, 2001) ${ }^{30}$. Nombres d'études ont prouvé le renforcement des inégalités sociales durant la période récente (Atkinson, 2007; Buchholz et al., 2009; Chauvel, 2001; Oris et al., 2009, 2017). Avec la globalisation et la modernisation, les travailleurs déjà désavantagés le sont encore plus : les personnes à basse qualification, les femmes, les jeunes adultes en passe de construire une famille, les employés en fin de carrière... le nombre des perdants de la mondialisation est grand. Inversement, les hommes qualifiés en milieu de carrière sont les plus protégés de ses retombées négatives (Buchholz et al., 2009). Le sexe, l'éducation et la classe professionnelle

30 Selon Beck et Beck-Gernsheim (2002), l'individualisation et la modernité auraient entraîné la fin des catégories de classe, d'emploi, de famille, ainsi que des rôles de genre, comme cadres aux identités individuelles et aux biographies. Les individus seraient devenus les maîtres de leur propre destin et les risques sociétaux se répartiraient de manière démocratique. Ainsi, Beck (1992) et Giddens (2004 [1994]) voient la société comme individuellement atomisée, où les sociétés du risque ne sont plus des sociétés de classe au sens marxiste du terme (Atkinson, 2007). Par ailleurs, les institutions et l'Etat-providence, ne viseraient plus les intérêts de groupe mais présumeraient que l'individu est acteur et responsable de sa propre biographie. Pourtant, la persistance des inégalités structurelles liées aux positions dans le système économique et la distribution hiérarchique des richesses amènent des auteurs à critiquer cette position (Atkinson, 2007; Chauvel, 2001; Oris, Gabriel, Ritschard, \& Kliegel, 2017). 
continuent bel et bien à déterminer les risques sur le marché du travail, accrus avec la globalisation.

Mais il n'est pas que dans la trajectoire professionnelle que les inégalités persistent. L'égalité présumée face au risque a été admise comme étant à deux vitesses en ce qui concerne tant les désastres naturels que les vulnérabilités individuelles. Beck (2007) lui-même souligne que les pays en développement sont les plus affectés par la modernisation globale. Il précise qu'il existe une conjonction cruciale entre pauvreté, vulnérabilité, corruption et accumulation des dangers: "The poorest of the poor live in the blind spots which are the most dangerous death zones of world risk society» (Beck, 2007, p.693). Progrès et inégalités croissent en parallèle (Sauvain-Dugerdil, 2014); ce phénomène est dramatiquement visible dans le cas de l'Inde (Drèze \& Sen, 2013), qui plus est dans un environnement aussi insalubre que les bidonvilles de Mumbai. Un tel milieu de vie présente des risques quotidiens en termes d'alimentation, de santé et d'accident. Les dangers globaux n'en sont pas non plus absents : émeutes interreligieuses, attentats, inondations (voir chapitre 1.3). En outre, les inégalités inter-individuelles se trouvent amplifiées par les risques (Sørensen \& Christiansen, 2014). «L'individualisation ne signifie pas la dissolution, mais pour l'instant le renforcement continuel des inégalités sociales comme en attestent plusieurs indicateurs.» (Beck, 1998, p.22). Effectivement, un accroissement des différentiels de revenu est constaté globalement et surtout dans les Suds (Atkinson, 2007; Piketty, 2013).

Il existe donc des risques sociétaux objectifs du fait de vivre dans un pays comme l'Inde, accablée par des impératifs environnementaux, économiques et sociaux. Si ces risques rendent toute la population vulnérable par essence, les individus ne sont pas uniformément affectés. Vivre dans un bidonville accroit nettement cette vulnérabilité, par exemple. De plus, les inégalités de classe et de caste, liées à la structure sociale indienne, persistent (voir 2.2); elles correspondent à une répartition inéquitable des ressources utilisables pour faire face aux vulnérabilités (Thomas, 2008).

\section{Les capitaux et la reproduction des inégalités sociales}

Les ressources mises à l'épreuve par les vulnérabilités rencontrées tout au long de la vie, ne sont pas immuables; elles se transforment au gré des changements socioéconomiques et personnels (Martin, 2013). La première de ces ressources est certainement le «capital économique». Il s'agit des 
avoirs monétaires et plus généralement de la richesse (immobilière, etc.) possédée par les personnes. Etre riche offre bien évidemment des atouts que les pauvres n'ont pas pour se battre contre des fléaux comme la maladie, les désastres naturels, la famine. Figure emblématique, Karl Marx a démontré comment l'emprise sur ce capital n'est pas aléatoire mais bien favorisée par une situation de classe (Marx, 2009 [1867]). Travaillant sur les stratégies de reproduction et les dominations sociales, Pierre Bourdieu (1994) a aussi dévoilé le dynamisme des structures de la société permettant aux élites de mettre en place des règles (ou stratégies) pour préserver leur situation. Cette reproduction sociale signifie la perpétuation des hiérarchies et des stratifications, par des mécanismes comme l'école ou la famille qui assurent les continuités intergénérationnelles de classe (Dannefer, 2003a).

L'innovation de Pierre Bourdieu a été de repenser le capital pour parler de «capitaux», afin de sortir de l'enclave des moyens financiers pour englober les diverses formes de ressources. Selon leur nature, les capitaux $^{31}$ divisent le socle des ressources sur lequel tout individu prend appui (Bourdieu, 1997, 2011). Chacun de ces capitaux bénéficie des autres pour s'accroître et en engendrer de nouveaux, participant ainsi à la perduration des inégalités entre les êtres humains. Certains individus peuvent manquer de l'un ou de l'autre et beaucoup manquent de tout, en opposition à une minorité qui jouit de la totalité, ou presque, des avantages.

En somme, les capitaux sont des réservoirs de ressources possédant des qualificatifs variables. Le premier capital défini par Bourdieu (1997) correspond à celui de Marx, comprenant les revenus et la fortune ainsi que tous les biens économiques (et moyens de production) appartenant à une personne. Le second est nommé «capital culturel» et Bourdieu (1979) lui octroie trois états complémentaires. Le premier correspond à une incorporation ou un «habitus» que l'acteur porte dans son enveloppe corporelle, sous condition d'un temps d'acquisition. Ce capital culturel incorporé fait partie intégrante du sujet et ne survit qu'avec lui. Second état, sa forme objectivée, entretient une relation directe avec l'état incorporé, bien que possédant des propriétés matérielles qui lui permettent une transmission au même titre que le capital économique (par exemple un tableau, qui

31 Les capitaux sont de caractères divers, dépassant les moyens financiers qui ne constituent pas l'unique forme de ressource à disposition des êtres humains (bien qu'au final, tout puisse se réduire au capital économique ou à l'accumulation du travail humain [Portes, 1998, 2000]). 
peut être légué mais que seul l'apprentissage de sa lecture permet d'apprécier). L'état institutionnalisé constitue la troisième facette du capital culturel. Cette institutionnalisation du culturel (une école pouvant délivrer des diplômes par exemple) permet de dépasser le lien intrinsèque entre le corps biologique du sujet et le capital culturel incorporé en lui offrant une autonomie relative. Elle permet également de faire reconnaître une acquisition de compétences à la communauté.

Troisième pilier, le «capital social» est décrit par Bourdieu (1980, p. 2) comme «[...] l'ensemble des ressources actuelles ou potentielles qui sont liées à la possession d'un réseau durable de relations [...], à l'appartenance à un groupe [...].». Autrement dit, le nombre de personnes membres du réseau social d'un individu, ainsi que le volume des autres formes de capitaux que ceux-ci possèdent, définissent le capital social. Il s'agit d'un capital non monétaire, mais qui offre des profits de par l'appartenance à un groupe, la reconnaissance mutuelle de profils similaires et l'exclusion des autres; en bref, il souligne les conséquences positives de la socialisation (Portes, 1998). Un travail de construction du groupe y est à l'œuvre, impliquant droits et devoirs, avec comme objectif plus ou moins admis la reproduction sociale. Ce travail est constant, et même une institution telle que la famille ne peut se targuer de proposer un ordre donné et immuable, mais doit se bâtir constamment. Les effets du capital social sont pluriels, allant du négatif (comme l'exclusion, la restriction des libertés individuelles, etc.) au positif (le soutien familial et les réseaux extra-familiaux d'entraide, etc.). Bien évidemment, le capital social est tributaire des institutions (famille, nation, association, etc.) pour rassembler, mais il l'est tout autant de la sociabilité de la personne. Divers autres capitaux ont été énoncés par Bourdieu et d'autres sociologues après lui, qu'il n'est pas utile d'énumérer ici, mais qui montrent que le concept est extensible et adaptable aux moyens variés dont dispose l'être humain.

Les différents capitaux constituent ainsi un système de ressources dans lequel les individus peuvent puiser du support. Dans un contexte donné, lorsqu'un ou plusieurs de ces capitaux sont déficitaires, une vulnérabilité latente de l'existence s'instaure ${ }^{32}$ (Spini et al., 2013). Un événement critique ou un stress supplémentaire peuvent alors avoir des conséquences négatives sur le parcours de vie (exclusion, stigmatisation, dépression,

32 A ce moment, l'adjectif «latent» représente un état de manque de ressources, dont les conséquences négatives ne sont pas encore visibles (Spini, Hanappi, Bernardi, Oris, \& Bickel, 2013). 
fragilité ou pauvreté), cette fois de manière manifeste (Spini et al., 2013). L'identification et la reconnaissance de la vulnérabilité changent suivant la position historique et le lieu, mais elle est toujours présente, notamment subjectivement. Ainsi, les pauvres ont partout une propension plus élevée à être vulnérables à cause de leur accès limité aux capitaux, subissant de ce fait une perte de bien-être ou la crainte de celle-ci. Le degré de vulnérabilité dépend des caractéristiques de l'événement ou du risque, ainsi que de la capacité de l'individu (ou du ménage) à y répondre (Alwang et al., 2001).

Malgré ses apports indéniables, l'approche par les capitaux présente une limite : elle assume que les individus utilisent les ressources dont ils disposent, pour prévenir ou faire face. En se fondant sur des analyses qui portent en grande partie sur les pays moins développés économiquement, Amartya Sen a remis en cause ce postulat. Il questionne la mise en pratique et offre une compréhension plus fine de l'utilité des ressources, biens et services, marchands ou non, dont l'individu peut user pour compenser les risques et les sources de stress (stresseurs) dans son existence. Il constate ainsi que la possession des capitaux (qu'il nomme ressources), en plus d'être répartie de manière fortement inégalitaire sur la planète, ne garantit pas d'avoir la liberté réelle d'en profiter (Bonvin \& Farvaque, 2008). Aux ressources doivent donc être ajoutés la capacité d'en jouir et le choix individuel d'y avoir recours.

\section{Absorption des risques : l'approche par les «capabilités» et le concept d'agency}

Dans ses écrits, Amartya Sen (Sen, 1981; Sen \& Hawthorn, 1987) appelle à intégrer dans le calcul des ressources, puis dans leur comparaison, les inégalités culturelles, naturelles et socioéconomiques qui séparent les personnes (Bonvin \& Farvaque, 2008; Sen, 1999). Ce faisant, Sen part littéralement du constat de l'inégalité qui existe entre les individus et s'interroge sur la manière dont les institutions, quelle que soit leur forme, peuvent y répondre. Sa théorie postule que les personnes ont à disposition des ressources, ainsi qu'un certain nombre de facteurs de conversion spécifiques à la société dans laquelle ils vivent (d'ordre institutionnel, normatif, culturel,...) et individuels (âge, sexe, niveau de formation,...), qui aident ou entravent l'utilisation de ces ressources (Sen \& Hawthorn, 1987). Autrement dit, détenir des capitaux est une chose, mais la liberté réelle de choix, la «capabilité» individuelle, se mesure lorsque chaque 
personne est capable de faire avec ceux-ci ce qu'il estime être le mieux pour sa vie (Bonvin \& Farvaque, 2008; Sen, 1999).

Dans cette approche par les capabilités, les personnes vulnérables ne sont pas seulement dépourvues de ressources, elles manquent également de facteurs de conversion leur permettant de les transformer en compétences ou niveau de vie (Bengtsson, 2004; Sen, 1988). Un bas standard de vie se constate lorsque la capacité à endiguer les stress économiques de court terme (par exemple un changement du niveau des prix alimentaires ou du revenu) est faible. A l'inverse, avoir des plans à long terme (se marier, avoir des enfants, ...) et les tenir - en dépassant les obstacles du quotidien - démontre une sécurisation de l'existence (Bengtsson, 2004). Sen (1981, 1999) révise la définition de la pauvreté et du bien-être en affirmant que la collectivité est en charge de fournir les facteurs de conversion. Il offre un nouvel angle d'attaque pour s'opposer à la vulnérabilité, reposant sur le développement des personnes tout en leur laissant liberté et donc choix. Dans un monde de pauvreté de masse, il est courant d'observer une focalisation institutionnelle sur la santé ou l'épargne pour promouvoir le développement ; or, la réussite scolaire des enfants et le fait d'avoir un travail sont par exemple également des préoccupations majeures dans les foyers. Décider en amont de programmes de lutte contre la pauvreté n'offre pas aux pauvres l'opportunité de choisir ce qui leur fait défaut.

Laisser à chaque personne la latitude d'évaluer pour elle-même ce qui est important pour son bien-être coïncide avec le principe d'agency, abondamment utilisé dans le domaine du parcours de vie (Elder, 1994; Marshall, 2005; Shanahan, 2000). L'agency est la capacité des individus d'être acteurs de leur propre destinée, de choisir et d'orienter leur vie. Le basculement dans la modernité a accru la nécessité d'autogestion et de planification, soit un phénomène de «biographisation» (Kohli, 2007; Levy \& Bühlmann, 2016). Ainsi, les individus sont moins guidés par les transitions traditionnelles et les rites de passage (Chiriboga, 1995). Ils participent au contraire activement à écrire leur biographie, vivent les changements individuellement et sont seuls garants des réussites et des échecs de leur existence. Les chercheurs mettent en exergue l'attitude d'être «un agent» qui se généralise, alors que les aléas de l'existence étaient précédemment imputés aux forces spirituelles, puis socioéconomiques, et que l'individu avait peu d'espace de décision (Fijalkow, 2013; Settersten, 2003) ${ }^{33}$.

33 Avec la libération de la famille et du local qui caractérisent la modernité (Beck, 1992; Giddens, 1991) le parcours de vie devient un projet délibéré, alors qu'avant 
Ayant constaté la multitude d'utilisations et de sens attribués à l'agency, Marshall et Clarke (2010) préconisent de l'employer dans son interprétation large: la capacité humaine, intentionnelle, à choisir ${ }^{34}$. Elle se définit également comme une résistance face à l'ordre social établi, au travers de comportements «hors normes», bien que suivre ces normes ne signifie pas obligatoirement une absence de prise de décision. Dans cette configuration, l'agency représente la manière dont les personnes interprètent le monde, soumises aux contraintes normatives et institutionnelles et y façonnent leur voie (Giddens, 1991; Hendricks \& Hatch, 2008).

Mais le versant sombre de la biographisation des parcours de vie est qu'elle entraîne une vulnérabilité subjective, parallèlement à la vulnérabilité objective issue du manque de capitaux et de facteurs de conversion. Les insuffisances ne sont plus imputées à la société inégalitaire ou à l'absence de protection sociale mais aux individus eux-mêmes, responsables de leurs déboires, provoquant un stress important (Levy \& Bühlmann, 2016; Spini et al., 2013). Ainsi, les solutions proposées pour surmonter ces vulnérabilités ne sont pas collectives, comme le recommande pourtant Amartya Sen, mais individuelles (tels l'empowerment et la résilience) (Fijalkow, 2013). La focale est à nouveau détournée des facteurs de causalité structurels pour ne concerner que l'individu isolé, désencastré du social (Dannefer, 2006), de la même façon que les risques liés à la globalisation et aux mutations

il résultait de décisions prises par la famille (Hareven, 1986, 1993). La modernité représente ainsi une première phase d'individualisation, laissant de l'espace à de nouveaux schémas de vie: chute de la mortalité infantile, augmentation de la longévité, accès accru à l'éducation et subséquemment réduction de l'étape laborieuse de la vie, régularisation du marché du travail et généralisation des systèmes de sécurité publique (Shanahan, 2000). Une seconde phase d'individualisation a ensuite lieu à la fin des années 1960, où la déstandardisation des trajectoires augmente encore drastiquement cette prise en main par l'individu de sa destinée (Kohli, 1986). Le parcours de vie passe entièrement sous l'autorité et la responsabilité de l'individu.

34 Le concept d'agency a été essentiellement envisagé sous deux angles: celui de la psychologie développementale (comme capacité de créer et de s'adapter à son environnement ou trait de personnalité) et celui du parcours de vie (capacité de choix, d'action sur les transitions). Selon Marshall et Clarke (2010), quatre types d'agency coexistent au sein de la vision du parcours de vie. Premièrement la capacité humaine à choisir intentionnellement. Deuxièmement les ressources de l'individu pour soutenir ce comportement intentionnel. Troisièmement le comportement des individus qui reflète leur intention. Quatrièmement la structure sociale et physique des choix, soit la structure des opportunités à disposition pour l'acteur. La première de ces quatre dimensions semble la définition idoine à utiliser ici. 
sociétales ont laissé de nombreux auteurs évoquer une généralisation de ces vulnérabilités au-delà des anciens clivages socioéconomiques (Beck, 2007). Pourtant, de plus en plus de travaux soulignent également la persistance des effets de classe et des forces sociales (Crystal, 2006; Dannefer, 2006; Ferraro, Shippee, \& Schafer, 2009; Oris et al., 2017). En Inde, les inégalités ne sauraient être négligées pour comprendre la situation de pauvreté et de précarité qui assaille la population (cf. 2.2), bien qu'une croissance des nouveaux risques soit indéniable (cf. 2.4.1).

Outre sa position parmi les pays du Sud, qui sont menacés par des risques globaux plus nombreux qu'au Nord (cf. 2.4.2), la population de l'Inde présente incontestablement une vulnérabilité structurelle au niveau des institutions formelles (cf. 2.2.2) et des normes culturelles (cf. 2.2.1). Ces deux facteurs se traduisent par une pauvreté de masse, qui frappe selon une stratification d'âge et de sexe marquée (cf. 2.2.3). S'y ajoute une vulnérabilité expérimentée au niveau personnel (des risques partagés et du stress individuel) issue de l'individualisation et des changements de la modernité, deux phénomènes qui ne font que débuter en Inde mais dont l'influence se perçoit déjà. Ces deux formes de vulnérabilisation sont des processus qui se renforcent mutuellement (Marshall, 2011).

\subsubsection{Processus de vulnérabilisation et construction des inégalités à travers le parcours de vie}

La vulnérabilité dans ses multiples aspects se présente comme une dynamique entre les ressources, les risques et le stress qui surviennent à tous les âges, entre les différentes sphères de l'existence; elle se prête donc aisément à l'étude selon une perspective du parcours de vie (Oris et al., 2016; Spini et al., 2013). Au sein de ce paradigme, deux ensembles théoriques ont approfondi les processus de vulnérabilisation des individus. Le premier, que nous venons d'évoquer, fait référence à la biographisation, soit aux phénomènes de transformations aux niveaux individuel et familial liés à la modernité, avec une augmentation des événements majeurs de la vie, ainsi que de la diversité dans les parcours, marqueurs d'une croissance à la fois de la vulnérabilité et de l'agency (Spini, Bernardi, \& Oris, 2017).

La seconde alternative s'en remet à de plus anciens constats et ramène la stratification sociale au centre du débat (Dannefer, 2006; Spini et al., 2017). Sans nier l'existence d'une transformation des parcours et des liens 
sociaux, ni la montée de risques dits modernes, ce courant attribue toujours une importance cruciale au milieu d'origine. Il enrichit des études du parcours de vie en attribuant la diversité des trajectoires et les inégalités au concept de «destinée», c'est-à-dire à une accentuation au fil du temps des caractéristiques personnelles assignées avant la naissance, dans un processus inégalitaire et prévisible (Dannefer, 2006; Moffitt \& Caspi, 2001). Bâtissant dans les années 1980 sur une idée ancienne de Merton (1968), Dale Dannefer (2003a) a ainsi rendu populaire la théorie des (dés)avantages cumulatifs. Une fois encore élaborée dans un cadre occidental, elle trouve à s'appliquer en Inde.

\section{Les (dés)avantages cumulatifs ou la construction des inégalités à travers les parcours de vie}

Dans toutes les sociétés, une inégalité fondamentale se constitue entre les individus sous l'impulsion de l'avancée historique (comprenant le développement économique, technologique et médical), favorisant certains et desservant d'autres d'après des critères contextuels (Elder, 1994; Mayer, 2015). Les cohortes de naissance successives (cf. 2.3.1) ainsi que les stratifications d'âge (cf. 2.2.3) révèlent ainsi des formes de vulnérabilité différentes (Dannefer, 2006; Oris et al., 2016; Riley et al., 1972). Déterminants structurels de l'inégalité, les processus démographiques (de cohorte) et développementaux (d'âge) sont au cœur du renouvellement social mais aussi des discriminations générationnelles (Ferraro et al., 2009).

Néanmoins, aucune cohorte n'est socialement et économiquement homogène, comme nous l'avons déjà souligné (cf. 2.3.1). Au contraire, l'hétérogénéité s'est affirmée avec l'arrivée de la modernité, soulignant le renforcement des inégalités au sein des cohortes (Dannefer, 1987; O'Rand, 1996) comme la conséquence de conditions imposées avant la naissance puis consolidées durant l'enfance, à l'école, à travers la famille, l'épargne et l'accumulation du capital, qui se répercutent finalement jusque dans la vieillesse (Burton \& Whitfield, 2006; Ferraro et al., 2009; Mayer, 2015; Oris et al., 2017). Les structures et les institutions sont les agents qui allouent les opportunités ou les désavantages aux membres d'un groupe d'âge selon des critères précis, stratifiant les individus sur le long terme et participant à la reproduction des inégalités (O'Rand, 1996). Cette stratification, dans le cas de l'Inde, est, comme nous l'avons vu, d'ordre traditionnel, de classe et de genre (cf. 2.2). Elle se concrétise par un différentiel de répartition des ressources, des opportunités et des influences. En outre, 
une transmission intergénérationnelle des capitaux, basée sur l'origine familiale, encourage le maintien des richesses et des opportunités dans les lignées favorisées (Dannefer, 2003a; Ferraro et al., 2009; Spini et al., 2017).

La construction des inégalités ne s'arrête toutefois pas à ces dimensions. Un processus d'accumulation des avantages et des désavantages est à l'œuvre, nommé effet Matthew, qui menace inéquitablement et partialement les individus (Dannefer, 1987; Merton, 1968, 1988; Price, 1965). Entre les différentes trajectoires de l'existence d'abord, mais aussi au fil des âges, les inégalités interagissent entre elles et se cumulent: les avantages objectifs de formation, de statut social et de ressources creusent les écarts entre ceux qui les possèdent et ceux qui en sont dépourvus (Dannefer, 1987, 2003a). Cet effet a été observé par plusieurs chercheurs en parcours de vie, qui parlent alors du principe des «dés/avantages cumulatifs» (CAD) et mettent en exergue le lien existant avec les notions de cohortes, de structure d'âge et de processus institutionnels (Burton \& Whitfield, 2006; Dannefer, 2003a, 2006; Ferraro et al., 2009).

Ils ont ainsi constaté que des expériences ou des événements majeurs survenus tôt dans la vie ont le pouvoir d'influencer le reste de l'existence, ces conséquences pouvant se circonscrire à un domaine de la vie (économie, santé,...) ou au contraire interagir avec les autres (Alter \& Oris, 2008; Burton \& Whitfield, 2006; Crystal, 2006). Les avantages gagnés précocement croissent avec l'avancée en âge, ou dit rapidement: les riches deviennent toujours plus riches, les pauvres toujours plus pauvres (Dannefer, 2003a; Ferraro et al., 2009). Pour cela, l'enfance, l'adolescence et l'entrée dans l'âge adulte sont des moments cruciaux de distribution des déterminants futurs, basés sur des opportunités scolaires et de socialisation qui conditionnent la structure du parcours de vie ultérieur, également en termes de bien-être (Dannefer, 2003a; Marshall \& Mueller, 2003). La perception subjective des CAD a également une importance non négligeable (Ferraro et al., 2009).

Durant l'existence, la variabilité impliquée par les CAD au sein des cohortes devient plus importante; autrement dit, les écarts de niveau de vie entre les individus proches de la mort sont plus grands qu'à la naissance (Burton \& Whitfield, 2006; Dannefer, 2003a, 2006; Marshall \& Mueller, 2003). Cette situation se révèle drastiquement au moment de la retraite, même dans des sociétés usant de systèmes de pension, expliquant le taux plus élevé de pauvreté parmi les personnes âgées, aggravé encore par les 
coûts de la santé (Dannefer, 2006; Oris et al., 2017; Tholomier, 2017). Dans le contexte indien, où l'essentiel de la population n'a pas accès à une assurance vieillesse, ce constat est encore plus criant. Il convient également de ne pas oublier que les personnes encore en vie à des âges avancés font partie des survivants, et que les plus vulnérables ont déjà succombés (effet de sélection) (Dannefer, 2006).

Les CAD et la reproduction sociale des inégalités permettent de prédire dans une certaine mesure les risques encourus, notamment dans le domaine de la santé (Burton \& Whitfield, 2006; Ferraro \& Kelley-Moore, 2003). Naître dans une famille de classe moyenne ou supérieure résidant dans les quartiers formels de Mumbai et ayant un accès régulier à des soins renforce les avantages de santé, d'éducation et de revenu (Marshall \& Mueller, 2003). Inversement, posséder un faible niveau socioéconomique représente un risque plus élevé de rencontrer des maladies, des problèmes mentaux, voire de les cumuler (co-morbidités) (Burton \& Whitfield, 2006; Ihle, Oris, Fagot, \& Kliegel, 2017); à cela s'ajoute la vulnérabilité due au fait d'avoir grandi dans un environnement insalubre comme les bidonvilles de la mégapole indienne. Les pauvres connaissent donc une redondance d'inégalités qui transcende les différentes trajectoires de vie: chômage, basse qualité du logement, manque d'infrastructures sanitaires, santé fragile, éducation faible, conduisant à un désavantage renforcé par le stress et les difficultés du quotidien (Burton \& Whitfield, 2006).

\section{Stress, temporalité et prégnance des transitions: les multiples dimen- sions de la vulnérabilité}

Qu'elle provienne d'inégalités sociales ou individuelles (manque de ressources), la vulnérabilité se traduit par la probabilité qu'une pression déstabilise les capitaux des individus (Alwang et al., 2001; Spini et al., 2017). Ainsi, le sociologue Leonard Pearlin (1989) et ses collègues ont observé la manière dont le bien être (qui à bien des égards pourrait être interprété comme l'inverse de la vulnérabilité) est affecté par les structures encadrantes et les expériences de la vie, arrivant à la conclusion qu'il existe un processus de «stress» affectant l'existence (Pearlin, Menaghan, Lieberman, \& Mullan, 1981).

Le stress passe par des «stresseurs», des circonstances stressantes dont la forme se subdivise entre événements ponctuels et contraintes chroniques, dont les effets peuvent être altérés par des «médiateurs» (institutions formelles et informelles, capitaux, etc.) avant de se traduire en 
résultat. Ces trois composantes sont bien sûr influencées par les inégalités et la stratification sociale (Pearlin, 1989; Pearlin et al., 1981). Les individus dont les ressources socioéconomiques sont faibles ont des risques plus élevés de connaître un stress perturbateur au cours de leur vie (Oris et al., 2016), et le fait de posséder peu de ressources et d'opportunités signifie se situer à un statut inférieur qui est lui-même source de stress (Pearlin, 1989). Par ailleurs, les institutions et les normes sociales impliquant des rôles ne sont pas systématiquement des soutiens pour les personnes; elles peuvent aussi imposer des pressions chroniques (Oris et al., 2016). Ces contraintes se caractérisent par des problèmes qui durent, des situations de conflits, ou d'autres formes de menaces courantes pesant sur la vie quotidienne. La «résilience» (la capacité à s'adapter) et le coping (la capacité à faire face) contribuent à réduire ce stress ou à le gérer. Le capital social est déterminant dans ces processus, mais comme les institutions formelles, les proches et l'entourage peuvent aussi être des sources de stress (Oris et al., 2016).

Divers travaux ont bâti sur les fondations posées par Pearlin, spécifiant un certain nombre de processus. Les sociologues ont ainsi établi de manière plus précise le lien avec la position personnelle dans les structures sociales, montrant que les événements indésirables de la vie (le chômage ou une maladie par exemple) ont plus d'impact sur la vie des personnes qui sont déjà dans une situation de vulnérabilité (McLeod, \& Kessler, 1990). Particulièrement illustratif de ce constat, les inégalités de santé physique et mentale s'expliquent par des déterminants liés à la position des individus, les plus bas de l'échelle sociale étant aussi les plus à risque de contracter un ennui de santé (Thoits, 2006) - ce qui ne doit néanmoins pas conduire à négliger la part d'influence de l'agency. Parmi les groupes vulnérables, les femmes cumulent les pénalités en étant plus sujettes aux conséquences de ces épisodes, ce qui s'expliquerait par un moindre accès au support social ainsi que par une implication émotionnelle accrue envers leur proches (Kessler, \& McLeod, 1984). Le genre est donc bien l'un des principaux critères influençant le stress, comme le pressentait Pearlin (1989).

Dans le champ d'étude du parcours de vie, plusieurs chercheurs ont réfléchi les relations entre trajectoires individuelles et stress, ainsi que la manière dont elles affectent la vulnérabilité des individus (Spini et al., 2013). Le stress lui-même peut provenir de diverses origines: un événement critique entraînant un changement dans la vie de la personne (une transition); une contrainte chronique; une difficulté quotidienne mineure; 
l'absence d'un événement attendu ou encore sa survenue hors-timing (Spini et al., 2013). Les contraintes chroniques sont généralement des problèmes liés à des rôles sociaux fixes (mariage, travail,...), à des privations ou à des obstacles de longue durée (comme la maladie) (Avison \& Turner, 1988; Folkman, Lazarus, Pimley, \& Novacek, 1987). A l'inverse, certains événements sont plus courts et circonscrits. Les deux cas de figures peuvent également interagir, se renforcer ou se cumuler (Glickman, Tanaka, \& Chan, 1991).

Dans tous les schémas, les transitions dans la vie apparaissent bel et bien comme des moments particuliers d'exposition aux risques car ils impliquent une modification de la situation, du statut, voire de l'identité personnelle. Tous les changements sont potentiellement stressants, comme nous l'avons vu ci-dessus, car ils nécessitent un réajustement, mais ils peuvent provoquer plus ou moins de stress selon leur importance et leur configuration $^{35}$. La nécessité de prendre en compte la temporalité s'avère toujours cruciale. C'est pour cela que les stresseurs et le bien-être doivent être évalués en fonction de la position de l'individu dans son parcours de vie, et pas uniquement dans leur contenu.

\subsection{Conclusion}

Les inégalités proviennent tant de conditions inscrites avant même la naissance que de bénéfices ou de désavantages qui tendent à cumuler leurs effets durant le développement des individus, en raison des institutions gérant les trajectoires, du contexte socioéconomique et des événements historiques rencontrés. A ces dynamiques de différenciation s'oppose le travail d'unification réalisé au sein de cohortes de naissance qui traversent l'histoire par les normes, les valeurs, la culture partagées. C'est dans ces cadres que se planifient les existences, que s'opèrent les choix de vie, de celles et ceux qui ont assez de liberté pour pouvoir se permettre de choisir (Clausen, 1995; Dannefer, 2003a; Dannefer \& Kelley-Moore, 2008; Lee,

35 Les événements de rupture peuvent être normatifs ou non-normatifs, attendus ou inattendus pour des raisons de timing (survenant trop tôt ou trop tard, au «mauvais» âge), de deséquencialisation (inversion dans l'ordre des transitions espérées) (Neugarten \& Hagestad, 1976; Neugarten et al., 1965; Settersten, 2004). 
Campbell, \& Bengtsson, 2004; Shanahan, 2000). En règle générale, dans les societies occidentales, les structures sociales et l'agency entretiennent donc une relation dialectique, l'individu construisant sa trajectoire de vie parmi plusieurs options disponibles (Giddens, 1979; Marshall \& Clarke, 2010; Thoits, 2006) dans un temps historique et un lieu donnés (Hitlin \& Elder, 2007).

En Inde, nombreux sont les individus aux capitaux limités par leur niveau socioéconomique, celles et ceux que l'ont peut qualifier de pauvres et qui constitutent encore la masse de la population. Malnutrition, exposition exacerbée aux maladies et à leur diffusion, manque d'hygiène dû à des installations sanitaires déficientes, accès limité aux soins: autant d'exemple de vulnérabilités qui se cumulent du fait de ne pas avoir de revenu suffisant et/ou d'appartenir à une minorité discriminée, ces traits de structures semblant ne laisser que bien peu de place à l'agency (Bengtsson, 2004). Ceci rencontre l'analyse de sociologues, qui considèrent que les inégalités sont perpétuées par les structures sociales et non pas la résultante de décisions individuelles (Bourdieu, 1994; Ferraro et al., 2009). En effet, Bourdieu (1998b) a su illustrer comment des individus, situés à des positions similaires dans la structure sociale, partagent des situations de vie analogues.

Certaines nuances doivent être apportées à la théorie des CAD, qui mêle avantages et désavantages. Les deux phénomènes surviennent effectivement en parallèle; malgré tout, les nuisances des uns ne constituent pas les privilèges des autres comme le laisserait illusoirement croire l'expression (Ferraro et al., 2009). Certains auteurs préfèrent utiliser le terme d'inégalités cumulatives (Ferraro et al., 2009), qui suppose une vision où les désavantages représentent des positions ou statuts défavorables à cause de déterminants structurels ou d'effets liés au passé, alors que les avantages résulteraient d'une exposition accrue aux opportunités (soit la probabilité d'une réussite future). Cette définition laisse opinément place à l'agency, à la mobilisation de capitaux et au contexte sociohistorique dans le processus cumulatif (Ferraro et al., 2009). D'autres études ont montré que la perception subjective de la vulnérabilité est cruciale, la notion de bienêtre étant d'ailleurs souvent utilisée pour la mesurer (Oris et al., 2016). Des départs difficiles peuvent révéler une vie où la vulnérabilité ne se ressent pas, tout comme un niveau social prometteur ne conduit pas nécessairement à un sentiment de sécurité d'existence, à un ressenti de bonheur (Mayer, 2015). 
Par rapport à d'autres pays, nonobstant la corruption et tant d'autres imperfections, n'oublions pas que l'Inde est et reste la plus grande démocratie du monde : l'individu n'est pas là le plus vidé de sa substance. Mais qu'en est-il entre le public et le privé, lorsque l'on descend jusqu'au sein de son propre foyer où tous, et surtout toutes, n'ont toujours pas le même droit à la parole? 



\title{
Chapitre 3. Enquêter la subjectivité des parcours de vie à Mumbai
}

\author{
«Essayer de savoir ce que l'on fait, lorsqu'on instaure \\ une relation d'entretien, c'est d'abord tenter de \\ connaître les effets que l'on peut produire sans le \\ savoir par cette sorte d'intrusion toujours un peu \\ arbitraire qui est au principe de l'échange [...]»
}

(Bourdieu, 1998a, p. 905)

Sortant des chantiers les plus fréquentés en sciences sociales, qui explorent les liens de causalité entre divers phénomènes et analysent des structures et des processus, l'intérêt porte ici sur la perception des événements forts s'imposant dans la mémoire des personnes. Qui plus est, au lieu de se saisir du souvenir d'un événement prédéfini comme marquant et de procéder à l'étude de sa construction et de ses implications, la recherche repose sur l'évaluation par les individus eux-mêmes des différents points d'inflexion de leur biographie prise dans sa totalité.

L'appréciation subjective de son propre parcours est une thématique qui a attiré les chercheurs en Occident depuis quelques décennies déjà. Dans les pays du Sud cependant, elle reste encore largement minorisée, surtout lorsqu'on la compare aux études qui mettent en avant les informations démographiques, socioéconomiques ou sanitaires dites «objectives». Etudier la perception des changements survenus dans la vie et dans l'environnement sociétal depuis la naissance est innovant dans le contexte d'une des plus grandes mégapoles du monde, en l'occurrence Mumbai. Cela nous impose une réflexion sur les riches acquis de la recherche, sur les concepts théoriques, mais aussi, une fois encore, sur les difficultés inhérentes à l'application d'un savoir occidental dans un espace culturel et socioéconomique pour le moins différent. Ce sera l'objet de la première section de ce chapitre.

Dans ce travail, un questionnaire $a d$ hoc a été utilisé pour explorer la perception des moments importants de la vie des individus, ainsi que la mémoire qu'ils ont du positionnement de leur existence dans l'histoire. 
Dans ses deuxième et troisième parties, ce chapitre décrira cet outil et la méthode à travers laquelle les données nécessaires à ce livre ont été récoltées. Derrière le terme très généraliste de méthode, ce sont des retours (auto-)critiques sur l'enquête, le recrutement, la formation et l'encadrement des enquêteurs qui sont visés. Assurément, le travail de terrain à Mumbai, a fortiori dans les bidonvilles, comporte des spécificités qu'il est important de mettre en évidence, avant de passer à une description et à une spécification de l'échantillon ainsi obtenu.

Une quatrième section sera consacrée à l'opérationnalisation des données, c'est-à-dire au travail qui a été effectué sur les questionnaires une fois ceux-ci saisis, dans le cadre de leur nettoyage, de leur codification pour en arriver à la forme finale que les bases de données revêtent. Enfin, la cinquième et dernière partie de ce chapitre méthodologique apportera un regard critique sur la procédure développée. Avant d'entrer dans l'analyse des données, il convient en effet que le lecteur se fasse une idée concrète de la réalisation d'un terrain d'enquête en sciences sociales à Mumbai. Si une récolte de données en Suisse (ou ailleurs en Occident) est toujours source de surprises, malgré une préparation intensive, et se heurte de plus en plus à des taux de non-réponses élevés (Oris et al., 2016), le sous-continent indien n'en est pas moins surprenant, plus inattendu encore, offrant des obstacles d'origines diverses. Ce bilan nous montrera en quoi certains de ces cahots s'expliquent par les conditions du terrain. La discussion des biais sera cependant balancée par celles des bénéfices des enquêtes réalisées.

\subsection{La perception des changements marquants de la vie}

Le travail d'élaboration de la mémoire de son parcours de vie dépend à la fois des personnes et de la société qui les entourent. Nous l'avons vu à la section 3 du chapitre 2 , des générations peuvent se constituer au gré de l'histoire, devenant ainsi les garantes d'une mémoire collective. Toutefois, le phénomène mémoriel démarre avant tout au sein de l'individu, comme un processus cognitif lié aux caractéristiques de chacun. C'est cet aspect-ci, théorique, de la mémoire qui nous interpelle dans cette première section, avant d'en venir aux représentations sociales qui en découlent et 
à la subjectivité qu'elle représente, à la fois en tant qu'atout et que biais pour la recherche (Bourdieu, 1982; Jodelet, 1989a; Moscovici \& Buschini, 2003). Puis, nous verrons comment cette mémoire peut être pratiquement étudiée, au travers de l'exemple d'une enquête internationale portant sur les événements et changements au cours de la vie.

\subsubsection{Se rappeler sa vie: le processus de mémoire}

Traditionnellement, la mémoire individuelle a été le sujet de recherches menées en psychologie et dans les neurosciences, ainsi que dans divers domaines de sciences sociales (Berntsen \& Rubin, 2002; Buckner \& Wheeler, 2001; Cavalli, et al., 2013; Conway, Wang, Hanyu, \& Haque, 2005; Lalive d'Epinay \& Cavalli, 2007; Rubin, 1982). Les neurosciences se cantonnent au fonctionnement mécanique, s'affairant à expliquer le processus de rappel et de sélection qui est à l'œuvre dans le cerveau des individus. En psychologie développementale, l'accent a été mis davantage sur les étapes de l'existence où cette mémoire devient particulièrement active et intense, par le truchement de la distribution des souvenirs au cours du temps (Baltes, Staudinger, \& Lindenberger, 1999; Conway \& Pleydell-Pearce, 2000; Rubin, 1982; Rubin, Wetzler, \& Nebes, 1986).

Ainsi, les psychologues distinguent trois composantes de la mémoire autobiographique: la plupart des individus ne conservent pas de souvenirs précis de ce qui s'est passé dans les premières années de leur vie (childhood amnesia); on retient davantage les informations encodées entre 10 et 30 ans environ, c'est-à-dire pendant l'adolescence et le début de la vie adulte (reminiscence bump); les souvenirs deviennent moins accessibles avec le passage du temps (forgetting) ou, pour le dire simplement, on se souvient davantage des événements récents (Conway \& Pleydell-Pearce, 2000; Rubin, 1982; Rubin, Wetzler, \& Nebes, 1986). Par ailleurs, un souvenir peut être perçu par l'individu qui le vit selon une tonalité, pouvant aller du très négatif au très positif. Lorsqu'ils étudient la mémoire autobiographique des individus, Birren et Schroots (2006) soulignent que les moments rappelés sont majoritairement positifs, bien que cela soit moins marqué chez les personnes âgées.

L'effet de réminiscence est un phénomène robuste qui a été supporté par des travaux portant tant sur les souvenirs d'événements publics que personnels (Glück \& Bluck, 2007; Grob, Krings, \& Bangerter, 
2001; Holmes \& Conway, 1999; Janssen, Murre, \& Meeter, 2008). Il a été observé dans des études réalisées dans plusieurs pays d'Europe, d'Amérique du Nord et d'Asie (Conway, Wang, Hanyu, \& Haque, 2005; Demiray, Gülgoz, \& Bluck, 2009), quelle que soit la technique de récolte des informations: d'une simple remémoration de faits aux associations de mots visant à réveiller des souvenirs, jusqu'à l'étude des préférences littéraires ou musicales (Janssen, Chessa, \& Murre, 2007).

Plusieurs explications ont été avancées pour rendre compte de cette augmentation de la densité mémorielle (Draaisma, 2008 [2001]; Jansari \& Parkin, 1996). Selon certains auteurs, la propension à citer des événements survenus au moment de l'entrée dans la vie adulte serait due à une plus grande aptitude sur le plan neurophysiologique à emmagasiner des souvenirs vers l'âge de vingt ans, lorsque le développement du cerveau atteint son optimum (Janssen et al., 2008; Rubin, Schulkind, \& Rahhal, 1999). Une autre explication met en avant la primauté de nombre de changements vécus pendant l'âge critique, les individus étant davantage marqués par les événements rencontrés pour la première fois: le premier partenaire, le premier travail, un voyage initiatique, mais aussi les premières confrontations à l'histoire, tout comme les engagements civiques et politiques (Berntsen \& Rubin, 2002; Conway \& Haque, 1999; Schrauf \& Rubin, 1998). Dans une interprétation ultérieure, l'accent est mis sur la rétention préférentielle des événements dans la période de formation et de consolidation de l'identité (Conway et al., 2005). Le début de la vie adulte représente une période critique pour la formation de la personnalité: un individu d'âge mur se souviendra alors surtout des événements de cette époque, ceux qu'il perçoit comme ayant fait de lui ce qu'il est devenu (Draaisma, 2008 [2001]).

\section{La mémoire dans les sciences sociales : un bref aperçu}

Largement traitée également en sciences sociales, la mémoire intéresse les sociologues depuis de nombreuses années. Maurice Halbwachs (1997 [1950], 2004 [1925]) s'est penché sur la question et a développé le concept de "mémoire collective», en faisant référence aux souvenirs partagés par les individus composant une société. Selon cet auteur, la mémoire prend différentes formes: elle peut être autobiographique (concernant les événements directement expérimentés par les personnes) ou historique (désignant les événements qui participent à élaborer une identité collective, à définir la situation du groupe dans le temps) (Guichard, 2015; Halbwachs, 1997 [1950]). Ces différents niveaux se retrouvent chez 
plusieurs chercheurs suivant des définitions parfois changeantes: mémoire transmise (familiale), apprise (collective) et vécue (individuelle) selon Devriese (1989); expérience vécue (connaissances sociales et historiques acquises durant l'existence) versus expérience perçue (savoir diffusé par les discours et les représentations publiques et retenu par le biais de la socialisation) selon Thompson (2012 [1963]).

Bien évidemment - comme l'évocation du dernier auteur l'a rappelé -, les historiens sont aussi particulièrement concernés par l'étude de la formation de la mémoire (Nora, 1989; Thompson, 2012 [1963]). Sous cette perspective, la mémoire représente une conception collective de la réalité sociale, structurant les relations et permettant d'expliquer le présent (Ricoeur, 2003). Dans ce processus, la mémoire historique est un aspect seulement de la mémoire collective, soutenue par les représentations publiques et les lieux de mémoire (Nora, 1989). Les définitions théoriques et les constats empiriques à propos de la mémoire collective seront davantage explicités dans le chapitre 8 .

Du point de vue de l'approche du parcours de vie, l'étude de la construction de la mémoire se focalise davantage sur le lien entre société et biographies. Il s'agit de tenir compte de l'écoulement du temps dans son aspect collectif et historique, mais aussi personnel. Ainsi, c'est à la fois le poids subjectif du souvenir qui est pris en compte (mis en lien avec le contexte au sein duquel il s'est réalisé), et l'âge que possède la personne lorsqu'elle le vit. En fonction de cet âge, l'impact et l'interprétation du souvenir ne sera pas le même (Devriese, 1989; Settersten, 1999). Néanmoins, la position occupée par les individus dans leur parcours de vie, à elle seule, ne suffit pas à expliquer la sélection des changements qu'ils jugent marquants et la prise en compte des représentations sociales portées sur l'événement rappelé est indispensable, ce que par ailleurs d'autres sociologues avaient déjà souligné (Durkheim, 1898; Moscovici, 2000).

\subsubsection{Penser sa vie : entre normes collectives et réflexion individuelle}

Tout processus réflexif à propos d'un objet social - et en particulier celui visant à analyser et parler de sa propre vie - repose sur des représentations partagées (ou collectives) de celui-ci (Jodelet, 1989b). Ces représentations sociales se constituent en partie à l'intérieur des sujets (phénomène cognitif et psychologique) et en partie à l'extérieur, par le biais des normes 
et des statuts socialement construits (Haas \& Jodelet, 2007). Précisément parce qu'elles sont au croisement entre la société et l'individu, il n'est pas étonnant que ce soit dans le domaine de la psychologie sociale que l'étude des représentations sociales ait pris son envol (Moscovici, 1961, 2001).

\section{Des règles du jeu imposées par le contexte...}

Pour les chercheurs, les représentations sociales sont une opportunité de connaître le contexte sociétal (Doise, 1989; Thomas \& Znaniecki, 1974 [1918]): liens entre les individus et ce contexte, elles révèlent les structures sociales et le processus de changement qui les affecte (Campos \& Lima, 2017).

De façon générale, les représentations sociales constituent «une forme de connaissance, socialement élaborée et partagée, ayant une visée pratique et concourant à la construction d'une réalité commune à un ensemble social» (Jodelet, 1989b, p. 36). Plusieurs éléments composent ces représentations et permettent de parler de la réalité sociale: informations, idéologies, normes, croyances, valeurs, attitudes, opinions, etc. (Durkheim, 1898). Ils sont produits au travers d'un travail de compréhension mené par les personnes et guidé par les règles normatives et comportementales présentes au niveau collectif (Moscovici, 1961, 2001). Les représentations évoquent donc un savoir commun plutôt que scientifique, qui se base sur les processus d'objectivation (la concrétisation d'objets sociaux abstraits) et d'ancrage (la catégorisation d'objets mal connus) (Doise, 1989). Ce sont des symboles mais aussi des moyens par lesquels la société devient consciente d'elle-même (Moscovici, 1989). Ce savoir offre la possibilité de saisir les nouvelles données sociales et de les intégrer, fortifiant au passage les normes et les comportements (Haas \& Jodelet, 2007).

Puisque les représentations sont collectives (c'est-à-dire partagées par une collectivité), elles poussent les membres du groupe à penser et agir uniformément (Moscovici, 1989). En effet, les croyances, normes et savoirs communs agissent comme des institutions sociales, élaborant l'identité du groupe au travers de l'édiction de règles et de lignes de conduites (Haas \& Jodelet, 2007; Moscovici, 1961, 2001). En cela, les représentations sociales font partie du contexte social qui influence les parcours de vie (cf. chapitre 2), différant selon la société considérée, dont les produits culturels (langage, mythes, religions, ...) suivent les codes. Mais elles sont aussi autonomes, dépassant les individus pour devenir une 
réalité (Moscovici, 2001). Ainsi, elles sont issues de la structure sociale pour devenir à leurs tours structurantes.

Pourtant, si les membres d'un groupe partagent un savoir commun à propos d'un objet social, leur évaluation de ce dernier peut varier. Ainsi, le fait d'appartenir à un groupe ne signifie pas directement ou obligatoirement partager des attitudes et des opinions avec les autres membres (Doise, 1989). Ceci, non pas en fonction d'éléments personnels mais bien à cause des effets des positions sociales (Doise, 1989; Doise \& LorenziCioldi, 1989). Pour discerner les représentations, il est donc nécessaire de tenir compte aussi de la subjectivité au sens de la place de l'individu en tant que sujet.

\section{...au retour à la subjectivité}

Toute représentation sociale comprend un sujet qui se rapporte à un objet (Jodelet, 1989b, 2008). Ce faisant, l'individu (sujet) démontre une conscience du monde où il vit, définit son identité et son existence en tant qu'acteur (Jodelet, 2006). Au cours du vingtième siècle, le statut du sujet a été questionné, considéré parfois comme un jouet social incapable de penser ou trop imbriqué dans les structures pour être pris en compte. La recherche a ainsi été jusqu'à sortir l'individu de son centre d'observation, en éliminant le sujet comme entité (Jodelet, 2008; Touraine, 2007). Depuis plusieurs décennies, un renversement s'opère et une réintégration du sujet dans la réflexion survient, comme une (ré)émergence de la subjectivité.

Ce retour de la subjectivité (au sens de l'individu comme sujet) concorde avec la constatation d'une montée de l'injonction normative d'être l'agent de son existence (cf. 2.4.2). L'époque où les chercheurs se penchaient sur les structures sociales comme uniques déterminants des parcours de vie est passée; à présent, le sujet doit être «actif et pensant», il doit être acteur (avec une autonomie réduite) ou agent (effectuant des choix) (Giddens, 1982, 1986): autant de qualificatifs répondant aux mutations sociales modernes telles que l'individualisation ou la construction de l'identité dans la globalisation (Touraine, 2007). Ceci ne doit pas nous mener d'un extrême à l'autre et conduire à négliger de considérer aussi le sujet comme un acteur social soumis aux contraintes de son milieu; mais un espace se constitue bel et bien pour prendre en compte la subjectivité (Jodelet, 2006).

Dans la recherche, s'intéresser à la subjectivité signifie se pencher sur ce que pensent les individus, leurs perceptions du monde et la compréhension qu'ils ont de leurs comportements (Bertaux, 2014). La subjectivité prend un 
sens qui va au-delà du simple reflet inverse de l'objectivité (vue comme la transcription fidèle de la réalité), s'accordant avec le principe de singularisation ou d'affirmation du sujet (Ertul, Melchior, \& Lalive d'Epinay, 2014). Partant de l'idée que toute personne «est le produit d'une histoire dont il cherche à devenir le sujet» (Ertul et al., 2014, p. 17), se raconter revient à produire un récit, bien évidemment empreint de subjectivité, mais dont le résultat n'en reste pas moins cohérent et dont les faits relatés révèlent la réalité (Bertaux, 2014). Pour ces raisons, tenir compte de la subjectivité a à nouveau de plus en plus marqué les enquêtes en sciences sociales, qui sont ainsi revenues à une pratique qui avait déjà fait son chemin.

\subsubsection{Questionner au travers d'une approche dite «subjective»}

L'étude des vies humaines selon le point de vue de l'acteur lui-même est en effet à la fois récente et ancienne. Elle s'est développée en anthropologie puis en sociologie dès les années 1920, au travers de l'approche biographique puis, plus tard et dans une moindre mesure, de celle du parcours de vie (Bessin, 2009). Aux origines se situe la recherche restée fameuse de Thomas et Znaniecki (1918), dont les résultats ont ouvert la porte à tout un pan de recherches au sein de l'Ecole de Chicago. Analysant les attitudes de personnes dans leur pays d'origine (la Pologne) puis celles d'un groupe de migrants polonais dans leur pays d'arrivée (en Europe ou aux EtatsUnis), ce texte fondateur illustre combien l'examen des existences se doit de mobiliser à la fois objectivité des phénomènes sociaux et subjectivité de leur interprétation par les individus qui les ont vécus.

Dans l'approche des parcours de vie, la subjectivité a pris de plus en plus de place depuis une vingtaine d'années seulement. Dans l'étude des changements marquants des parcours de vie, les enquêtes lui donnant une place prépondérante se sont alors multipliées (Cavalli, et al., 2013; Fiske \& Chiriboga, 1990; Laub \& Sampson, 1993; Rönkä, Oravala, \& Pulkkinen, 2003). Toutefois, ce procédé reste marginal par rapport à l'observation dite objective. A l'inverse, analyser la perception des événements vécus importants ou des moments de stress est courant dans le monde de la psychologie, que cela concerne la mémoire autobiographique (Schrauf et Rubin, 1998), la mémoire historique (Brown et Kulik, 1977) ou encore les traumas de l'existence, signes manifestes d'une souffrance subjective (Loftus et Ketcham, 1994; Ofshe et Watters, 1994; Schacter, 1996). 
En démographie, les années 1970 voient apparaître un changement de paradigme dans l'étude des événements vécus. Les méthodes d'analyses biographiques viennent compléter et parfois remplacer l'analyse classique, que la multiplicité et l'individualisation des trajectoires de vie ont rendue plus difficile à appliquer. Les outils permettant d'étudier les biographies dans leur totalité, comme un processus complexe qui dépasse l'unité d'analyse de l'événement, se développent fortement (Courgeau \& Lelièvre, 1989; Lelièvre \& Vivier, 2001). A cette occasion, des dessins d'enquête quantitatifs ou qualitatifs ont parfois laissé une place à la subjectivité (Laborde et al., 2012; Lelièvre \& Robette, 2015). Néanmoins, dans la plupart des enquêtes démographiques, la subjectivité est utilisée comme un complément aux questions objectives permettant de creuser des aspects soulevés par ailleurs. Elle ne devient pas l'objet principal de la recherche (Crenner et al., 2002).

Pourtant, les événements perçus comme marquants dans la vie sont des révélateurs, des symboles du vécu, qui ont autant de validité scientifique que les registres administratifs par exemple, et dépassent leur cadre formel. Ils proposent une richesse d'information considérable qui prévient les présupposés du chercheur, tout en permettant de saisir l'articulation des trajectoires. La signification de ces événements ne saurait d'ailleurs pas être dissociée de l'existence dans laquelle ils sont survenus (Leclerc-Olive, 1997). Toutefois, si aujourd'hui la perception subjective des changements est admise comme un aspect capital de la réalité, la manière de récolter ces informations a suscité de nombreux débats.

Pour être reconnu comme marquant, un événement doit avoir été approprié et validé par son acteur (Leclerc-Olive, 1998). Le tournant ou l'évènement ne peut donc être défini comme tel par la personne qui le vit qu'après sa survenue, en effectuant une rétrospection sur soi-même. Or, cela signifie passer par une reconstruction a posteriori du vécu et l'effort demandé à l'individu au cours du travail de rappel l'amène à réinterpréter sa biographie, à la façon d'un bilan de son existence (Hélardot, 2010). Outre les différents biais que cela peut impliquer, comme celui de l'oubli, la rétrospection pose la question de savoir si une réalité du passé réécrite au présent peut toujours être fidèle (Settersten, 1999).

En sociologie, Pierre Bourdieu (1986) soulignait déjà ce paradoxe: lors d'une remémoration, l'individu cherche à expliquer le présent en structurant les séquences de son passé en un récit qui a du sens. Le sujet souhaite livrer un message cohérent au chercheur et transforme l'enchaînement, le choix 
et les conséquences des événements vécus dans ce but, en posant un regard global sur sa vie (Bidart, 2006a). A sa manière, Daniel Bertaux (1980, 1997) reprend ce constat en parlant de «l'idéologie» résidant dans l'approche biographique et de l'organisation du récit en fonction d'une logique significative pour la personne elle-même et l'entourage.

D'autres chercheurs vont plus loin, démontrant qu'en quelque sorte les défauts de la collecte rétrospective biographique en sont les qualités et qu'il faut en retirer ce qui peut l'être (Courgeau et Lelièvre, 1989). L'exercice mémoriel reste un moyen fondamental pour comprendre l'existence, puisqu'il permet en effet d'associer et d'organiser entre eux les moments du parcours de vie. Par ailleurs, le procédé a été homologué par divers travaux méthodologiques récents, qui ont confronté des données objectivement établies avec des rétrospections subjectives afin d'en établir la fiabilité (Lelièvre \& Robette, 2015; Mazuy \& Lelièvre, 2005). L'idée est d'étudier des individus aujourd'hui, qui ils sont ou en d'autres mots leur identité, telle qu'elle s'exprime à travers leurs sélections mémorielles. Il s'agit assurément d'une forme de choix, mais qui ne remet pas en cause la fiabilité, la véracité, du travail, puisque ce n'est pas l'exhaustivité qui nous importe mais ce que ces mémoires nous disent des personnes. Il faut sortir de l'objectif comme référent et plutôt prendre comme repère l'identité qui est bien sûr un mélange de personnel et de social - et dont le capital mémoriel est une composante.

Les méthodes pour récolter des données subjectives sont multiples : des récits de vie et entretiens qualitatifs approfondis jusqu'aux vastes enquêtes quantitatives. Historiquement cependant, c'est davantage la perspective qualitative qui a su exploiter le subjectif. En effet, elle apparait toute indiquée pour se pencher sur la perception des individus de leur vie, afin de faire ressortir les mécanismes de causalité et les relations entre les événements (Verd et López, 2011). Un avantage du qualitatif réside également dans le fait que la rigueur méthodologique et le durcissement des consignes sont moins cruciaux que pour la récolte de données objectives (Berthier, 2010), ce qui permet de s'ouvrir aux informations imprévues et innovantes.

Toutefois, dépasser l'opposition quelque peu stéréotypée entre la méthode quantitative qui serait apte à étudier l'objectivité des trajectoires versus la méthode qualitative utilisée pour analyser la subjectivité, a récemment fini par s'imposer comme une évidence en sciences sociales (Bidart et Gosselin, 2014; Heinz, 2003). Les études du parcours de vie y ont contribué car, d'emblée, elles ont accepté les deux approches. Leur 
combinaison est plus récente. Walter Heinz (2003), en particulier, a mis en évidence les avantages de l'utilisation conjointe du quantitatif et du qualitatif pour comprendre les trajectoires.

Le défi à relever dans ce livre est d'adhérer à cette posture et dépasser des dichotomies réductrices (Small, 2011) pour placer l'individu au centre du questionnement et s'appuyer sur une méthodologie correspondant à l'objet de recherche, tout en sachant nous adapter aux contraintes de l'environnement dans lequel la passation s'est effectuée. L'approche proposée ici consiste donc à employer une méthode mixte pour questionner la subjectivité des moments marquants de la vie.

\subsubsection{L'enquête Changements et événements au cours de la vie}

Dans le cadre de ce travail, deux enquêtes ont été effectuées dont la méthodologie et le questionnaire sont issus d'un programme de recherche plus vaste, l'étude Changements et événements au cours de la vie (CEVI). Créé en 2003 par Christian Lalive d'Epinay et Stefano Cavalli, ce programme a vu le jour à l'Université de Genève (il est aujourd'hui hébergé par la Scuola universitaria professionale de la Suisse italienne), puis s'est successivement déroulé dans une dizaine de pays. Utilisant la notion de changement (ou événement) comme levier d'analyse, il étudie l'articulation entre les trajectoires de vie des individus et les changements sociétaux. L'étude CEVI se focalise uniquement sur la perception subjective qu' ont des adultes de différents âges de leur propre vie et de l'histoire.

Avec l'ambition de lire le lien entre vies humaines et structures sociohistoriques, le programme CEVI use de la représentation que les personnes se font des changements les plus importants qui déterminent leur biographie. Ce n'est donc pas un repérage factuel et exhaustif des événements survenus dans la vie des interviewés ou dans leur environnement sociétal qui est proposé, mais plutôt la sélection de ceux qui, à leurs yeux, ont été les plus frappants. Par ailleurs, l'étude ne permet aux individus que de sélectionner des changements survenus au cours de leur vie, soit depuis leur naissance. Cette borne temporelle a pour principales conséquences de différencier les répondants suivant leur âge ainsi que de privilégier l'histoire vécue par rapport à l'histoire apprise.

La recherche CEVI est conçue sur la base d'un questionnaire simple, établi de façon que le répondant puisse le remplir lui-même (format 
auto-administré), avec des conditions méthodologiques réduites pour permettre une reproductibilité aisée en tout temps et en tout lieu. Elle n'a pas pour ambition d'être représentative d'une population et son échantillon est construit de manière arbitraire, c'est-à-dire non-aléatoire. Cet échantillon doit, selon la convention de CEVI, atteindre un minimum de 500 individus, groupés le plus égalitairement possible entre 5 classes d'âge (20-24, 35-39, 50-54, 65-69 et 80-84 ans) et entre les sexes, soit des sous-groupes comprenant au minimum 50 personnes. Ainsi, seuls les critères d'âge et de sexe sont imposés dans le choix des répondants, ce qui soutient plusieurs types de comparaisons : entre les individus interrogés selon leur cohorte de naissance et selon leur sexe, ainsi que des comparaisons internationales grâce à la standardisation du questionnaire.

Lorsque le choix d'utiliser le questionnaire CEVI pour récolter des données sur la perception des parcours de vie et de l'histoire à Mumbai s'est posé, il a été évident que la spécificité culturelle et les conditions de vie hétérogènes dans cette ville, allant de l'extrême pauvreté à une richesse considérable, ne permettraient pas de comparer de manière simple et stricte les résultats obtenus à ceux issus des enquêtes passées à Genève, Buenos Aires, Padoue, Nancy... En revanche, et précisément dans l'idée de creuser l'influence culturelle versus l'influence socioéconomique présente dans les réponses des Mumbaikars, un premier terrain a été réalisé dans un quartier de bidonvilles, puis un second s'est déroulé deux ans plus tard dans un quartier mixte. La particularité des données qui fournissent matière à ce livre est donc de permettre de confronter les réponses obtenues dans deux quartiers d'une même ville, possédant des niveaux socioéconomiques distincts. La section qui suit se charge de dévoiler le contenu exact du questionnaire et des hypothèses de recherche ainsi que de décrire les critères de constitution de l'échantillon. La section 3.3 présentera les deux récoltes de données plus en détail, ainsi que les répondants indiens.

\subsection{Design d'enquête et questions de recherche}

L'Inde n'est pas un pays qui manque de ressources démographiques et socioéconomiques. Réservoir de population gigantesque, des chercheurs indiens et internationaux se penchent sur son cas depuis longtemps, 
travaillant sur des données provenant de vastes études quantitatives. Le gouvernement, britannique puis indien, effectue depuis 1872 des recensements réguliers, mobilisant des moyens humains et économiques considérables afin de récolter des informations socioculturelles (éducation, langue, religion, ...), économiques (activité, équipement du ménage, ...) et démographiques (fécondité, mortalité, ...) permettant de brosser un tableau détaillé de la structure et des dynamiques de la population. Par ailleurs, des institutions privées ou publiques, comme l'International Institute for Population Sciences (IIPS), effectuent parallèlement leurs propres recherches.

Toutefois, peu d'enquêtes se centrent autour des parcours de vie des individus et encore moins de leur perception des événements de la vie. Rompant avec cette dominante, ce livre est issue d'une opportunité qui s'est présentée il y a cinq années de cela, celle de faire passer le questionnaire CEVI dans, successivement, un quartier de bidonvilles puis une zone mixte en termes socioéconomiques. Les terrains organisés pour la récolte des données utiles à ce livre reprennent donc les principaux critères méthodologiques de l'étude CEVI, tout en étant soumis à un certain nombre de contraintes liées au contexte.

Par sa méthodologie, le format de son questionnaire ainsi que la taille de son échantillon, CEVI se conforme à une enquête quantitative. Toutefois, les réponses à ses principales questions permettent, après codage (cf. section 3.4), outre des analyses statistiques, de laisser la place à une appréciation qualitative du contenu, puisque comme nous le verrons plus en détail ci-dessous, il a aussi été demandé aux répondants de justifier en quelques mots le choix des événements qu'ils ont mentionné. Des travaux d'analyse de contenu ont été effectués à diverses occasions sur des données de différents pays. (Aeby, 2006 ; Guichard, 2015).

\subsubsection{Trois questions sur la perception du parcours de vie}

Le questionnaire standardisé de l'étude CEVI (cf. annexe 1 et 2 pour les versions anglaise et en marathi) est simple et court afin que, entre autres, sa traduction et sa passation soient réalisables à faible coût et que l'enquêté puisse y répondre sans l'aide de l'enquêteur. Trois volets principaux ${ }^{1}$

1 Il est arrivé que dans l'un ou l'autre des pays, le questionnaire ait compté un ou deux volets supplémentaires, mais ce ne fut pas le cas en Inde. 
composent l'essentiel du support, complétés par une dizaine de questions fermées ${ }^{2}$ portant sur des informations sociodémographiques décrivant le répondant. Les questions centrales sont ouvertes mais la plage de réponse est définie, ce qui permet de garantir la taille réduite du questionnaire. Cette particularité le rend propice à une distribution dans un pays tel que l'Inde, a fortiori une ville comme Mumbai, où il est facile de trouver un nombre suffisant de participants mais dont les habitants n'ont que peu l'habitude de répondre à des questions ouvertes. Ainsi, avoir un nombre restreint de questions à soumettre aux répondants a permis aux enquêteurs, à la fin de l'interview, de prendre le temps de revenir en arrière si l'une ou l'autre d'entre elles n'avait pas été immédiatement saisie.

Chacun des volets centraux de cette enquête concerne les changements ou les événements les plus importants ayant marqué la mémoire des répondants, sur la base d'une variation temporelle (en tenant compte uniquement de la dernière année vécue ou de l'entier de la vie) et de contenu (événements personnels ou sociohistoriques). La disposition des questions est organisée de manière logique : l'interviewé est amené à se souvenir des événements personnels qui lui sont arrivés au cours de la dernière année, puis des changements les plus importants de son existence, avant d'élargir sa réflexion pour considérer les événements survenus au cours de sa vie ayant eu, selon lui, le plus d'impact dans son pays ou dans le monde.

Les éléments fournis peuvent être au nombre de quatre au maximum pour chaque question, impliquant une sélection de la part de l'individu. Ainsi, les réponses représentent une vision doublement subjective des moments marquants d'une vie : évalués par l'acteur principal et choisis par ce dernier parmi tous les changements qui ont eu lieu au cours de son existence. Si le nombre des réponses est limité, le choix de celles-ci par contre est délibérément ouvert. L'objectif de cette enquête étant de laisser l'individu libre dans l'interprétation de ce qui constitue un changement dans sa vie ou dans l'histoire, il est bien évident qu'aucune consigne ni indication de la part de l'enquêteur n'est tolérée, afin de ne pas influencer les réponses. Néanmoins, pour chacun des éléments répondus, le répondant est sollicité de donner la raison de son choix en quelques mots ou breuses, et des données économiques les ont complétées (c'était le cas lors du second terrain indien). 
phrases. Enfin, l'interviewé est chargé de décrire les changements en les situant dans le temps et dans l'espace.

\section{Volet I : Les changements récents}

Dans le courant de la dernière année, y a-t-il eu des changements importants dans votre vie?

Cette première interrogation met à l'épreuve les changements perçus par des individus se situant à différentes positions de la vie. En effet, l'échantillon interrogé comprend des adultes depuis la fin de l'adolescence jusqu'aux personnes âgées, appréhendant ainsi diverses étapes du parcours de vie. Parmi les individus qui vivent ces différents phases de l'existence, voit-on apparaître des changements dans le contenu des réponses, sous-entendant des rôles et des enjeux caractéristiques des divers âges de la vie adulte (Neugarten et al., 1965)?

Par ailleurs, un constat réunit aujourd'hui les chercheurs se penchant sur la construction des parcours de vie dans le monde occidental, indiquant que certaines phases de l'existence sont davantage propices au changement que d'autres (Bidart, 2010). En particulier, de précédentes études ont attesté que l'entrée dans l'âge adulte est associée à la perception d'une plus grande quantité de changements (Fiske et Chiriboga, 1990; Folkman, Lazarus, Pimley, et Novacek, 1987). Ce premier volet du questionnaire soumet à examen cette affirmation, en observant la fréquence des changements récents mentionnés, selon les divers âges.

Une spécificité supplémentaire de cette première question est de demander au répondant de donner une évaluation subjective du changement évoqué. Une échelle lui permet de cocher la case correspondante : était-ce «un gain», «une perte», «les deux», «ni l'un ni l'autre», «ne sait pas»? Cette information a pour objectif de tester l'hypothèse de la distribution des gains et des pertes au cours de la vie (Heckhausen, Dixon, et Baltes, 1989), issue de la psychologie développementale. Ces gains et pertes dépendent-ils de l'âge ou du domaine de la vie affecté ?

Aussi bien en ce qui concerne le contenu des événements rappelés, leur fréquence ou leur évaluation en termes de gains ou de pertes, deux phénomènes peuvent $a$ priori être à l'origine d'une variation entre les groupes d'âge: la position des individus dans leur parcours de vie ou le fait qu'ils aient vécu au sein d'un contexte historique, institutionnel et social influant de manière caractéristique sur leur vie (Lalive d'Epinay et al., 
2005). Ces deux dimensions doivent être prises en compte dans l'analyse des résultats. En outre, l'identité de la personne qui vit le changement peut fournir des éléments d'information et de compréhension. En effet, le répondant n'ayant pas de consigne de réponse, il arrive que le changement évoqué ne le concerne qu'indirectement (par exemple en faisant allusion à une maladie d'un parent). La perception des événements qui marquent une étape de la vie peut ainsi révéler des imbrications majeures entre la vie des proches et celle d'une personne, les «vies liées» (linked lives) qui sont un des principes de base dans l'analyse des parcours de vie (Elder, 1998; Elder \& George, 2016).

\section{Volet II : Les tournants dans la vie}

Si vous considérez l'ensemble de votre vie, quels en ont été les principaux tournants, ces moments qui ont marqué un changement important dans votre vie?

Ce deuxième volet du questionnaire amène l'individu à s'interroger sur sa vie entière et à en sélectionner les moments les plus marquants ${ }^{3}$. Dans l'optique d'analyser la vie dans son ensemble, une première dimension concerne la manière dont la biographie est retranscrite : se représente-t-on sa vie de façon continue ou l'accent est-il plutôt mis sur les discontinuités et les ruptures ? Une seconde dimension a trait à la répartition des souvenirs au cours de la vie, puisqu'une sous-question demande l'âge de l'individu au moment du tournant. L'hypothèse d'une concentration de ceux-ci durant la période qui va de l'adolescence à l'entrée dans la vie adulte se confirme-t-elle en-dehors de l'Occident (Martenot et Cavalli, 2014), dans une toute autre culture et face à des modalités de transition à l'âge adulte si différentes (cf. chapitre 2)?

A l'évidence, au cœur de cette seconde question se trouve le contenu des tournants évoqués. La dimension familiale a été observée dans plusieurs études comme prévalant dans les souvenirs personnels, et ce dans différents contextes culturels (Cavalli et al., 2013; Inglehart, 2003; LaniBayle et Mallet, 2006). Cette valorisation maximale de la famille dans la structure du parcours de vie individuel devrait, selon cette hypothèse,

3 Les événements important survenus lors de la dernière année de vie, que le répondant a donc eu l'opportunité d'évoquer dans le précédent volet, pouvaient à choix être répétés ou être considérés comme déjà cités, ce qui offrait une place à un autre souvenir. 
se traduire dans les réponses par une surreprésentation des événements démographiques typiques tels que mariages, naissances, divorces, décès de proches, etc. Revers de la médaille, quelle place est-elle accordée aux autres trajectoires, comme l'éducation, la profession ou la santé ? Il y a fort à parier que ces dernières, fortement régulées par le marché et les institutions étatiques dans les pays nord-atlantiques mais bien souvent dépendantes de la condition sociale et sans guère de contrôle de la part des autorités dans les pays du Sud, aient une présence variable en fonction des contextes.

Comme le premier, ce second volet offre des opportunités de comparaison entre les groupes d'âge et les sexes à propos de la perception des grandes articulations de la vie, ainsi que de l'âge auquel ces changements sont apparus. Existe-t-il un modèle dominant, une représentation collective et stable du parcours de vie ? Si la représentation n'est pas unique mais se modifie entre les groupes, ces fluctuations opèrent-elles sous l'influence de l'âge (de la position dans le parcours de vie), de la période (du contexte dans lequel l'on a grandi et qui définit un certain nombre de conditions du déroulement des trajectoires) ou encore de la cohorte de naissance à laquelle les individus appartiennent ?

\section{Volet III : Les événements et changements sociohistoriques}

Considérons maintenant les grands événements et
changements qui se sont produits dans votre pays et
dans le monde au cours de votre vie. Quels sont ceux
qui vous ont le plus frappés?

Dans ce troisième volet, la mémoire collective historique est mise en avant, tout en restant dans le cadre temporel du vécu, excluant l'apprentissage par transmission (scolaire, familiale, ...) du passé. Le lien entre les biographies et l'histoire est au centre de cette dernière question, puisque les groupes d'âge interrogés ne peuvent puiser dans les mêmes souvenirs selon leur âge (les jeunes n'auront vécu qu'un quart environ de la durée de vie des aînés). Quels sont les changements dans l'histoire qui marquent les mémoires d'individus de sexes et de classes d'âge différents ? Les événements récents, vécus par l'ensemble des répondants, auront-ils le même impact dans leurs mémoires ? Schuman et Scott (1989), sociologues étudiant le souvenir d'un événement frappant, ont revisité l'idée que l'adolescence et l'entrée dans la vie adulte constituent une «période critique» de 
formation du socle de la mémoire historique (voir section 3.1). Est-ce donc l'âge qui importe, davantage que l'événement lui-même ? A l'inverse, des événements particuliers (comme une guerre ou une crise économique) s'inscrivent-ils dans la mémoire des personnes déjà installées dans la vie adulte au moment de leur survenue, donnant ainsi origine à des mémoires collectives trans-générationnelles (Guichard, 2015)?

Le référent géographique principal de la mémoire historique est $a$ priori l'Etat-nation et son histoire récente (Anderson, 2002 [1983]). Toutefois, le processus de globalisation culturelle en marche depuis quelques décennies tendrait à donner une dimension planétaire et partagée aux mémoires historiques des cohortes récentes (Appadurai, 2005 [1996]). Les références à l'histoire nationale seront-elles différentes en fonction des groupes d'âge ? L'appartenance à un pays fortement tourné vers l'international (comme la Suisse) ou à un autre qui ne s'est ouvert au commerce mondial que depuis peu (comme l'Inde) aurait-elle un impact sur la perception des événements sociohistoriques marquants?

\section{Questions complémentaires}

Une série de questions fermées complémentaires, portant sur les caractéristiques sociodémographiques du répondant, ont été ajoutées à la fin du questionnaire. Il s'agit : du sexe; de l'année de naissance; de la nationalité ; de l'état civil ; du fait d'avoir des enfants (ou non); des co-résidents (s'il y en a); du niveau d'éducation; de la situation professionnelle; de la santé autoévaluée.

Tant les questions centrales que les questions complémentaires ont été établies d'abord en français, puis traduites pour les besoins des différents pays. Pour chaque passation, il était possible d'ajouter une ou deux questions principales en fonction des intérêts des chercheurs, et toutes les questions supplémentaires nécessaires à la compréhension de l'échantillon.

\subsubsection{Un échantillon non-aléatoire, structuré selon les «âges de la vie» adulte}

L'objectif de l'enquête CEVI est d'offrir une comparaison de la perception des changements personnels et historiques marquants selon les choix effectués par des adultes situés à des moments différents de leur parcours 
de vie. Ainsi, cinq classes d'âge quinquennales ont été retenues, séparées entre elles par un intervalle de 10 ans afin de les typifier par jeu de contraste.

Ces groupes d'âge recouvrent l'entièreté de la vie adulte. En outre, ce découpage est construit pour que chaque classe soit installée dans une étape identifiable de la vie. Le premier groupe est établi à 20-24 ans, soit l'entrée dans l'âge adulte. Le second représente l'installation dans la vie professionnelle et familiale et se trouve à 35-39 ans. Le troisième se situe à 50-54 ans, lorsque la vie professionnelle et familiale est avancée. L'avant-dernier groupe correspond au début de la retraite, à 65-69 ans. Enfin, le dernier groupe, à 80-84 ans, équivaut à la notion nouvelle de «quatrième âge», c'est-à-dire la grande vieillesse, l'âge de la fragilité (Lalive d'Epinay et Cavalli, 2013). Ces différents âges chronologiques sont des étapes classiques de la vie, largement étudiées sous le paradigme du parcours de vie (Cain, 1964; Fiske et Chiriboga, 1990). Un tel schéma de découpe de la vie adulte est aussi utilisé dans la psychologie du lifespan (Heckhausen et al., 1989). Toutefois, ces âges restent approximatifs, puisqu'ils subissent des variations temporelles et spatiales (Conway, Wang, Hanyu, et Haque, 2005; Hareven et Masaoka, 1988; Settersten, 2004).

Comme nous l'avons montré dans le chapitre 2, en Occident des institutions (école, etc.) sont en charge de certaines de ces étapes et des lois qui les régissent (scolarité, retraite, etc.), ce qui garantit une relative homogénéité dans l'âge des individus qui les traversent. Cela a également comme effet de resserrer les liens entre les personnes appartenant à une même cohorte de naissance, puisque la majorité d'entre elles effectueront le passage d'un stade à l'autre en même temps. Néanmoins, depuis le dernier tiers du vingtième siècle, un flou règne autour des âges propres aux diverses étapes de la vie (Hughes, 1996) en partie dû à une déstandardisation ou désinstitutionnalisation (Macmillan, 2005) des trajectoires, qui rendrait obsolètes ces âges chronologiques. En outre, dans des pays où une large partie de la population ne bénéficie pas de la protection universelle et de la régulation de telles institutions, à l'instar de l'Inde, la définition d'un âge typique aura vraisemblablement des frontières poreuses, souvent contrebalancées par une plus grande incidence de la famille et des traditions sur les modes de vie (cf. chapitre 1 et 2).

Aucune exactitude stricte ne peut donc être attendue de l'assimilation d'un ou de plusieurs âges donnés aux paliers du parcours de vie. Pour cette raison, les cinq classes d'âges retenues dans la constitution de 
l'échantillon CEVI ne doivent être considérées autrement que comme des repères. Ce d'autant plus que le concept de l'enquête repose sur la subjectivité des participants et leur choix personnel des changements rappelés. Partir d'événements survenus (avoir eu des enfants, être actif, être à la retraite, etc.) pour constituer des groupes et définir des limites d'âge serait prendre le problème à l'envers. En même temps, le découpage par âges circonscrit des cohortes de naissance distinctes et permet donc d'ancrer les individus dans l'histoire (Lalive d'Epinay et al., 2005; Ryder, 1965). Par conséquent, l'âge ou la position dans le parcours de vie n'est pas le seul angle d'analyse qui distingue ces groupes, mais également leur appartenance à des générations historiques selon la définition de Karl Mannheim (1990 [1928]).

Lors de la constitution de l'étude, il a été décidé de ne pas élaborer un échantillon strictement aléatoire et représentatif ; c'eut à vrai dire été hautement compliqué dans les conditions de terrain qui seront décrites plus avant ${ }^{4}$. La volonté de préserver la facilité de passation du questionnaire a poussé à favoriser un dessin d'échantillon selon de simples critères d'âge et de sexe. La stratification est donc double : avoir autant de personnes dans chaque groupe et au sein de ces groupes autant de femmes que d'hommes. Ainsi, bien que suivant des critères précis, le panel obtenu est non-aléatoire, ce qui signifie que toute la population n'est pas soumise à une probabilité identique et calculable d'être interrogée. Pour cette raison notamment, le déroulement de l'enquête - la phase de terrain - nécessite d'être comprise et explicitée en profondeur.

4 Outre le fait que le programme CEVI n'envisage pas un tel échantillon, la grande mobilité de la population des bidonvilles (rendant difficile la connaissance de sa structure), ainsi que les complexités d'accès aux personnes et de passation du questionnaire nous ont découragé d'entreprendre la réalisation d'un échantillon représentatif. Bien que certaines techniques aient sans doute permis d'envisager la représentativité, nous avons opté pour nous en tenir aux critères d'échantillonnage préconisés par l'étude CEVI. 


\subsection{Pratique de la recherche : deux terrains à Mumbai}

Deux récoltes de données utilisant le questionnaire CEVI ont été effectuées à Mumbai, à deux ans d'intervalle. Une première passation s'est déroulée en 2012 (entre le 5 avril et le 5 juin), dans le quartier de Bandra East. Le second terrain eut lieu en 2014 et n'a duré qu'un mois (du 16 mars au 17 avril), à Santa Cruz East. Lors de ces deux enquêtes, il fut tenté de respecter les normes de l'étude CEVI au plus près, mais la réalité urbaine et les particularités sociales de la mégapole indienne imposèrent des adaptations.

Bandra East et Santa Cruz East se situent au centre de la zone suburbaine de Mumbai, proches d'un riche quartier d'affaire : le Bandra Kurla Complex. Tous deux sont des quartiers mixtes en termes socio-économiques, allant de l'extrême pauvreté (visible au travers des personnes dormant à même le trottoir ou dans des taudis) à une classe moyenne parfois aisée (résidant en immeubles dont le standing peut à l'occasion s'avérer très élevé). Toutefois, la récolte de données effectuée à Bandra East s'est déroulée exclusivement au sein de bidonvilles, alors que celle passée à Santa Cruz East a été réalisée dans des espaces globalement de classe moyenne inférieure. Ce sont donc deux environnements très différents qui ont été le cadre contextuel de nos terrains, présentant des situations d'enquêtes disparates et justifiant d'autant l'intérêt d'une comparaison entre les deux (Dupont, 2008).
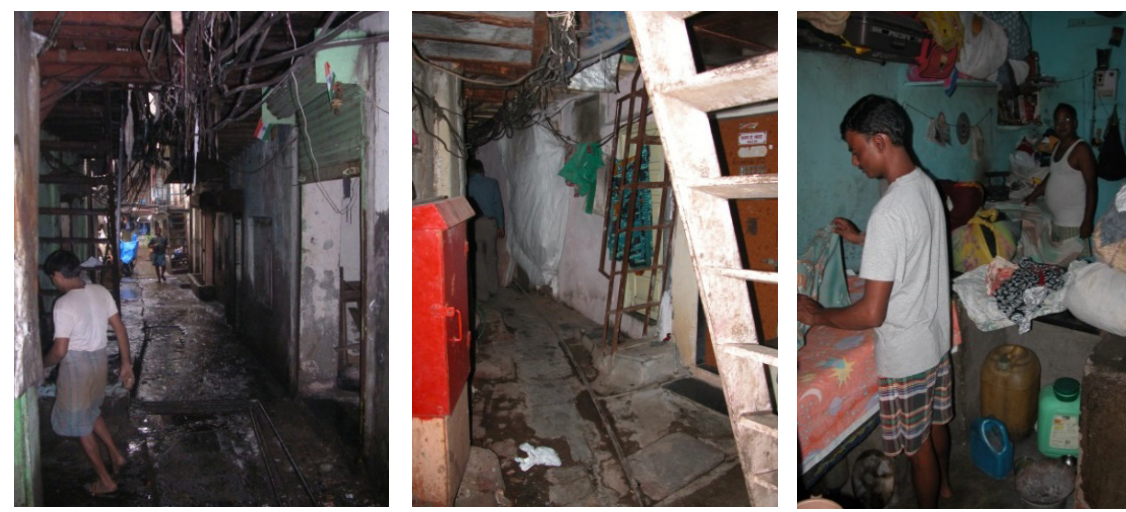

Figure 3.1: l'intérieur d'un slum de Bandra East

Source : photos de l'auteure, 2010 
La zone de bidonvilles de Bandra East s'étend sur plusieurs kilomètres, et les différents slums qui la composent sont autant de villes dans la ville, avec leur identité linguistique et religieuse (cf. figure 3.1 et 3.3). Pénétrer dans cet univers n'est pas aisé sans une clé d'entrée et la capacité à interagir avec les habitants. En effet, une grande partie des slums $d w e l l e r s^{5}$ provient de la campagne du Maharastra où ne se parle pas le marathi (langue officielle de l'Etat) mais divers dialectes, et une minorité est issue d'Etats plus lointains et parle l'idiome du lieu (gujarati, rajastani, ...). Rares sont les résidents des bidonvilles qui parlent anglais, privilège de l'élite indienne (Montaut, 2004) ou d'enfants ayant reçu une éducation supérieure ${ }^{6}$, et relativement peu de gens s'expriment dans une autre langue indienne commune comme le hindi.

Lors de la seconde passation en 2014, c'est dans l'ensemble d'un quartier mixte de la ville que les questionnaires ont été distribués. Santa Cruz se trouve près de Bandra East, deux secteurs plus au nord. Les rues sont bordées d'immeubles de classes moyennes, et de classes moyennes inférieures, parfois quelques maisons individuelles existent encore. Certaines barres d'immeubles abritent des logements pour habitants de bidonvilles ayant passé par le programme du Slum Rehabilitation Authority (SRA) afin de sortir des zones précaires pour être relogés (cf. chapitre 1). Toutefois, ces nouvelles habitations (de longues séries d'immeubles décrépis, collés les uns aux autres) n'offrent guère d'améliorations dans l'environnement de vie des personnes ${ }^{7}$ (voir figure 3.2).

Une fois posées ces quelques informations générales et avant de passer à la présentation proprement dite de chacun des terrains, un retour succinct sur les circonstances - plus ou moins fortuites - nous ayant conduit à effectuer une récolte de données dans un lieu inaccoutumé tel que celui des slums de Mumbai s'avère utile.

5 Habitants des bidonvilles.

6 Supérieur ne signifie pas seulement plus avancée. En Inde, les classes primaires publiques sont divisées entre les apprentissages dans la langue de l'Etat et le hindi et les apprentissages en anglais, ces dernières étant plus difficilement accessibles aux familles avec peu de moyens ou pas de moyens du tout.

7 Pour plus d'informations sur les relocations, voir Felber et Schmid, 2014. 

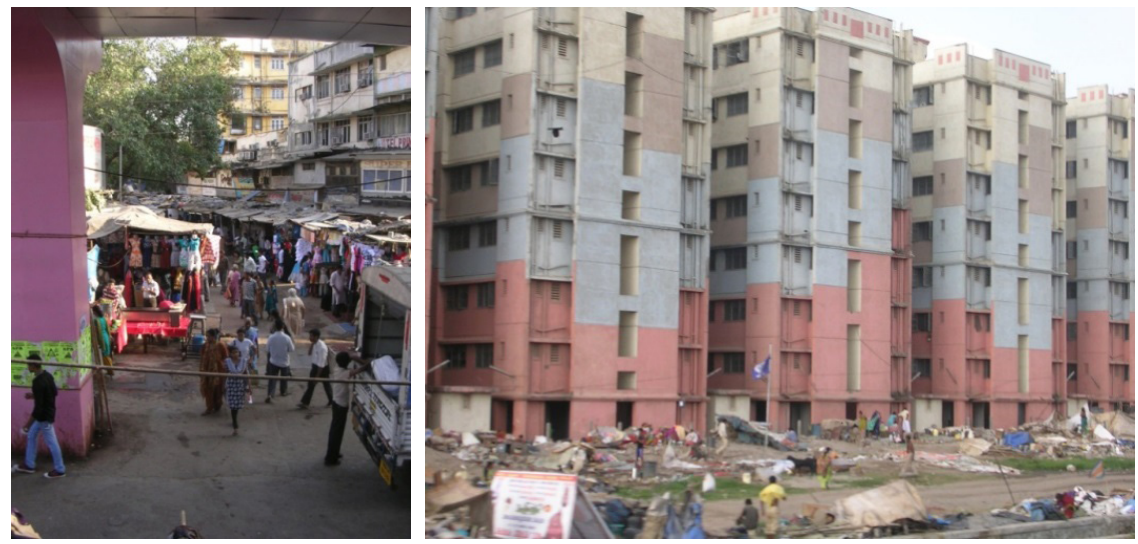

Figure 3.2: le quartier de Santa Cruz East et l'exemple d'immeubles SRA

Source : photos de l'auteure, 2014

\subsubsection{Genèse d'une recherche sise dans un environnement atypique}

Il n'est pas évident - malgré l'intérêt patent que présente ce milieu - d'entrer en tant que chercheuse (qui plus est, issue d'un pays occidental) dans le monde bigarré des bidonvilles de Mumbai. Au travers d'une succession de collaborations entre des étudiantes et des étudiants de l'Université de Genève et une ONG locale (le Centre for the Study of Social Change, CSSC), un certain réseau s'est toutefois mis sur pied. Ce dernier forme la pierre angulaire qui rendit possible la réalisation du premier terrain à Bandra East, en nous mettant en contact avec des personnes proches ou résidantes des slums, et simultanément en nous permettant de découvrir la configuration et la population de ces derniers.

\section{Rencontre avec le CSSC, autour de données sur la grande pauvreté}

La collaboration entre l'Université de Genève (en la personne du Professeur Oris) et le CSSC débuta en 2008, par le biais de la Fondation Internationale pour la Population et le Développement (IFPD) sise à Lausanne. Elle se concrétisa avec le départ de deux étudiantes du master de Socioéconomie à destination de Mumbai. 
Le CSSC est une organisation non gouvernementale avec une vocation médicale. Il favorise la santé reproductive et l'accès aux soins de première nécessité des habitants (surtout des habitantes) des zones défavorisées du quartier de Bandra East (et d'une partie des quartiers voisins de Khar East et Santa Cruz East) à Mumbai. Une vingtaine de doctoresses généralistes et quelques spécialistes (gynécologues et pédiatres) donnent bénévolement de leur temps pour tenir une vingtaine de cliniques de consultation dans les bidonvilles (voir figures 3.3 et 3.4). Ces dispensaires sont en fait de petites salles dépourvues de tout ce que la médecine occidentale qualifierait de basique, afin de prodiguer des soins aux femmes et enfants (parfois les hommes sont admis), offrir des consultations, suivre des grossesses, etc.

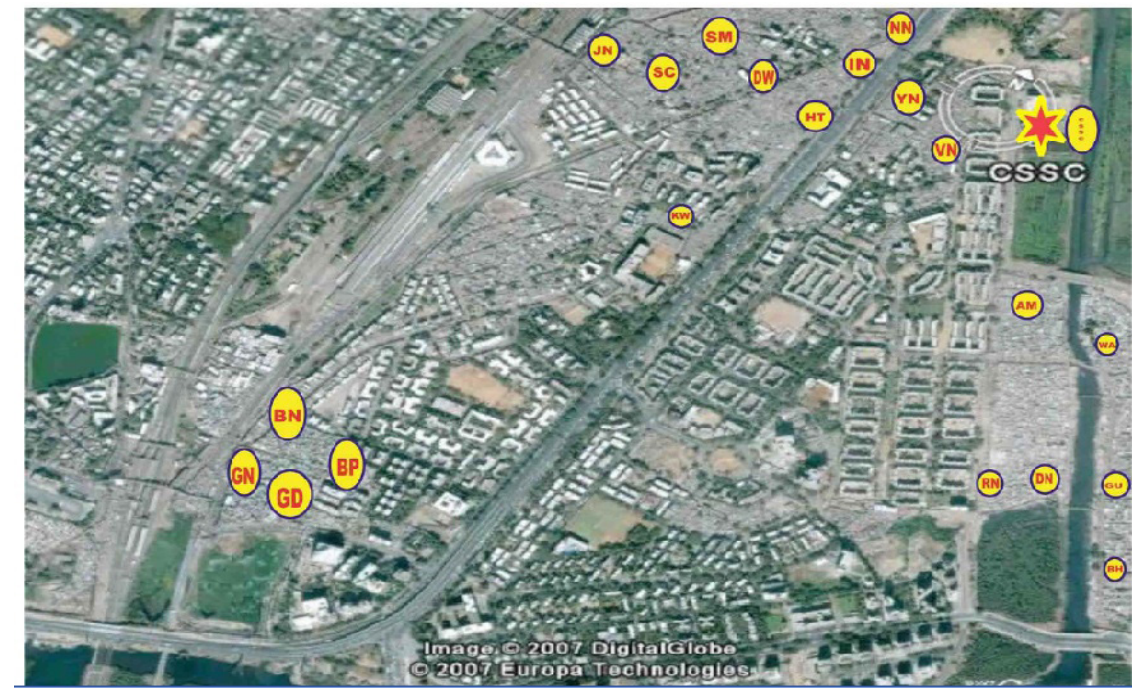

Figure 3.3: la zone de bidonvilles de Bandra East et les 20 cliniques de l'ONG CSSC

Source: Henke \& Schlaefli, 2009, p. 146 

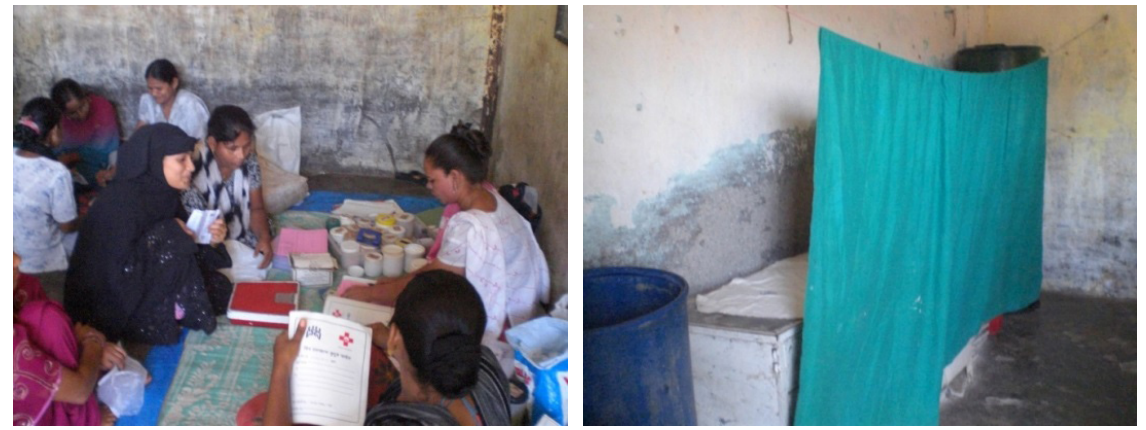

Figure 3.4: l'exemple d'une clinique du CSSC

Source: photos de l'auteure, 2010

Principalement, le CSSC gère un projet d'envergure nommé WIN (Women of India Network), qui a pour objectif d'amorcer un changement social, dont les femmes sont les actrices privilégiées (Henke et Schlaefli, 2009). Le concept d'empowerment féminin est très présent, c'est-à-dire la capacité à s'autonomiser, à ne plus être en posture de dépendance ou d'infériorité, à prendre sa vie en main. Les conditions de vie des pauvres doivent être améliorées au travers de différents moyens médicaux ou sociaux comme la santé générale et la santé reproductive pour les femmes, la santé et la survie infantiles, mais aussi l'accès à la contraception ou l'éducation informelle.

Lors du premier contact avec le CSSC, Julia Henke et Katja Schlaefli (2009) ont eu pour tâche d'éplucher le recensement de la population de Bandra East effectué par l'ONG, qui possède de nombreuses données sur les familles de ses patientes et patients. ${ }^{8}$. Un autre enjeu crucial fut de comprendre la structure de l'organisation ainsi que du projet WIN.

8 En 2006, le CSSC a effectué un vaste recensement de la population de Bandra East (près de 100'000 individus furent concernés). Des données sur les structures familiales, le revenu du ménage, le niveau d'éducation et le statut socioprofessionel des habitants ont ainsi été collectées. Parallèlement, chaque mois les assistantes sociales sont chargées de noter les pratiques contraceptives des femmes. Chacun des répondants à ces enquêtes possède un code unique, permettant de lier les informations socioéconomiques et les pratiques de planning familial. Un second recensement fut réalisé en 2009, toujours à Bandra East parmi les patients de WIN, portant sur près de 80 '000 personnes. A cette occasion, des sur-échantillons furent sélectionnés pour répondre à quelques questions supplémentaires portant sur des domaines spécifiques (migration, travail,...). 
Fortuitement, c'est aussi au cours de ce voyage qu'eu lieu la rencontre des étudiantes avec Dinesh Shenai, sur le campus du Tata Insitute of Social Sciences où elles suivirent des cours. Depuis, Dinesh fut régulièrement sollicité pour servir de traducteur. Il remplit le rôle de collaborateur lors du second terrain réalisé à Santa Cruz East.

Dans les années qui suivirent, plusieurs autres travaux de master virent le jour, bénéficiant toujours de l'appui et de l'accès aux données du CSSC, afin d'étudier les facettes de la population des slums de Bandra East (sur les sujets de la fécondité [Bertrand, El Rassi, \& Weibel, 2011], des trajectoires de vie [Eggimann \& Yannick, 2012] ou encore de la migration [Rossignon \& Gaillard, 2013]). C'est en amont de ces travaux, mais après le passage de Julia et Katja, que j'ai moi-même eu mon premier contact avec le CSSC, mais aussi avec le milieu des slums de Mumbai et plus généralement avec l'Inde, en 2010.

\section{Six mois pour connaître la population de WIN}

Durant six mois, en compagnie de Camille Guignet (également étudiante en master de Socioéconomie), j'ai eu l'opportunité de travailler pour le CSSC afin de traiter les nombreuses données que le centre possède sur la santé reproductive de ses patientes. Au cours de ce stage, nous avons découvert la réalité des bidonvilles, au travers des conditions de vie bien sûr, mais aussi dans leur configuration spécifique à Bandra East. A la différence de Dharavi par exemple, les quartiers n'y sont généralement pas organisés par secteurs de métier, mais par religion (quartier hindou, musulman, bouddhiste) et par lieu d'origine (donc, plus ou moins selon les dialectes et les langues). Précisons que parmi la population patiente de WIN, la grande majorité des Hindous sont des Dalits.

Notre travail consista à analyser les bases de données afin d'en extraire des observations permettant d'évaluer l'impact du projet WIN sur l'empowerment féminin. Après avoir établi un certain nombre d'informations générales sur la population de 16 cliniques du projet (en présentant les pyramides des âges et les sex ratio par âge, les niveaux d'éducation, la distribution des revenus par ménage, les taux de vaccination des enfants, et le statut matrimonial féminin), nous avons réalisé des comparaisons entre les cliniques, pour déterminer le niveau de pauvreté et d'éducation en fonction, notamment, du statut matrimonial féminin et, le cas échéant, de l'âge moyen au premier mariage. 
Bien que fort intéressant et formateur, ce stage n'a pas constitué mon travail de mémoire de fin de master, effectué auparavant à Genève et portant sur une toute autre perspective. Néanmoins, ce dernier joua un rôle prépondérant dans la suite d'éléments qui menèrent aux données constitutives du présent livre. En effet, ce fut à cette occasion que je m'insérais dans le programme CEVI.

\section{Au départ du projet, une enquête à Genève}

Parallèlement à l'établissement de nos premiers liens avec le CSSC et Mumbai, j'ai réalisé mon travail de mémoire en accomplissant - sous la direction de Stefano Cavalli et de Michel Oris et avec l'aide de deux autres étudiantes - une récolte de données à Genève dans le cadre d'une étude particulière : le programme international CEVI.

Durant 6 mois, entre septembre 2009 et février 2010, je me suis familiarisée avec l'outil CEVI, participant activement à la collecte des données, à leur opérationnalisation en base de données puis à son étude. Cet apprentissage a été complété, à mon retour de Mumbai en septembre 2010, par l'obtention d'une place d'attachée de recherche au sein d'un projet financé par le Fond national suisse de la recherche scientifique (FNS), dédié à CEVI et conçu sur deux ans ${ }^{9}$. J'ai alors travaillé à harmoniser les différentes bases de données des pays ayant rejoint le projet, ainsi qu'à en présenter et publier les résultats.

$\mathrm{Au}$ printemps 2012, nous avons saisi l'opportunité de lancer une enquête CEVI à Mumbai, dans les bidonvilles de Bandra East, grâce à notre attache avec le CSSC et à la présence sur place d'une étudiante de Socioéconomie, Anouk Piraud. En amont de cette récolte et surtout quelques mois après, alors munis des premiers résultats, nous avons pris contact avec l'International Institute for Population Science (l'IIPS est un centre de formation, de recherche et d'enseignement autour des questions de population, situé à Mumbai) afin de présenter notre étude et de proposer des collaborations. L'intérêt envers nos constats fut certain ; cependant, l'enquête en tant que telle, portant sur un aspect subjectif des vies, ne souleva pas de velléités de participation de la part des professeurs de l'IIPS. Ceci peut s'expliquer par l'intérêt encore prégnant, à l'heure actuelle, des démographes indiens envers les vastes enquêtes et la recherche sur des

9 Il s'agit du projet intitulé «CEVI. Changements et événements dans le cours de la vie», subside n 100017_132047 / 1 dont le requérant responsable est Stefano Cavalli. 
populations au sens large (Spoorenberg, 2005) plutôt que sur des sujets comme la perception des parcours de vie individuels (voir par exemple la publication We, the Billion de Rajini Sen [2003]). A noter qu'une approche du côté de la sociologie au Tata Institute of Social Sciences aurait peut-être conduit à un autre résultat.

De notre côté, nous avons donc continué notre collaboration avec le CSSC et, lorsque le subside du FNS pour l'étude CEVI s'est achevé, alors que je débutais mon assistanat à l'Université de Genève et l'élaboration d'une thèse, l'idée d'un second terrain pour valoriser les premières données a vu le jour. Après une courte hésitation sur le lieu de concrétisation de cette deuxième enquête (une première idée avait été de répliquer l'enquête dans les quartiers informels de Ouagadougou au Burkina Faso, où nous connaissions des chercheurs), nous avons finalement opté pour Santa Cruz East, à Mumbai mais avec une population socioéconomiquement différente de celle des bidonvilles de Bandra. Revenons sur le déroulement de chacun de ces terrains.

\subsubsection{Collecter des données dans une zone défavorisée}

C'est avec le soutien de l'ONG CSSC et l'aide d'Anouk Piraud ${ }^{10}$, que 633 questionnaires ${ }^{11}$ ont été remplis par des habitants des bidonvilles de Bandra East, entre mars et juin 2012. Le souhait de faire passer les questionnaires sur la perception des changements et des événements marquants de la vie dans des slums de Mumbai était irréalisable sans un encadrement qui aménage un accès aux résidents, ainsi que des connaissances suffisantes des divers dialectes parlés par ceux-ci, pour effectuer ensuite des traductions vers l'anglais. Le CSSC fut pour nous cet intermédiaire clé.

$\mathrm{Au}$ sein du projet WIN, des femmes résidant dans les slums ont été engagées pour aider les médecins dans leurs tâches. Plus précisément, ces quelques 40 personnes sont chargées soit d'assister le médecin en tant que pharmaciennes, soit de suivre les patientes en tant qu'assistantes sociales. Sans aucune illusion, la gestion du CSSC reste très hiérarchisée : somme

10 Alors stagiaire au Centre interfacultaire de gérontologie et d'études des vulnérabilités de l'Université de Genève, Anouk a été encadrée par Aude Martenot et Michel Oris. Pour plus d'informations sur ce terrain voir: Piraud, 2012.

11646 personnes ont en fait été interrogées, mais 13 d'entre elles ne rentraient pas dans les classes d'âge prédéfinies ou avaient à peine remplis le questionnaire. 
toute, nous sommes toujours en Inde. Ainsi, chaque équipe d'assistantes dans les cliniques est gérée par une assistante superviseure ayant plus d'expérience. Mais l'embauche de femmes vivant dans les bidonvilles permet astucieusement de faciliter l'approche vers la population locale (qui au départ ne fut pas toujours aisée à convaincre, une méfiance initiale vis-àvis de la médecine régnant) et de fournir un revenu à ces dernières. Si ce salaire est plus symbolique qu'autre chose, joint à la formation qu'elles reçoivent et au statut conféré, l'ensemble leur assure une certaine reconnaissance et fait d'elles des figures reconnues dans leur communauté. D'un point de vue concret, leur fonction de suivi des patientes les amène à remplir des fiches d'informations sur celles-ci, sur leur ménage, à divers niveaux démographiques et médicaux. Ce sont donc des femmes ayant une scolarisation de base qui leur permet d'écrire et de saisir parfaitement les modes de remplissage d'un questionnaire.

Le prof. Michel Oris a pris contact avec le CSSC afin de pouvoir engager un certain nombre de ces assistantes sociales pour faire le premier travail d'enquête sous la direction d'Anouk Piraud. Considérées par les responsables de l'ONG comme ayant plus d'expérience et un statut en conséquence de la tâche, ce sont des assistantes supérieures qui ont eu pour mission de faire remplir les questionnaires à l'occasion de leurs visites mensuelles. Les assistantes sociales sont chacune en charge d'une clinique de l'ONG et de ses patientes, se répartissant sur toute l'aire de Bandra East ${ }^{12}$, parmi lesquelles les personnes interrogées ont été recrutées.

Ces bidonvilles regroupent des communautés qui partagent une même religion ; ils peuvent donc être distingués entre les slums hindous, musulmans ou bouddhistes. Au moment de démarrer notre collecte, il a été décidé que les répondants seraient, outre les variables d'âge et de sexe, également répartis entre les deux religions majoritaires (hindoue et musulmane), ce qui équivaut à un sur-échantillonnage des musulmans. En conséquence, 22 enquêtrices de l'ONG ont été recrutées pour réaliser ces entretiens, qui se sont déroulés chez les interviewés, durant l'après-midi. Une formation des enquêtrices a été organisée sur deux après-midis, par un responsable du CSSC et Anouk Piraud. Chacune d'elle a rempli un questionnaire en auto-administré, après que les questions principales aient été explicitées. Une discussion autour des incompréhensions et des difficultés perçues a permis d'ajuster quelque peu le questionnaire (par exemple, en

12 La zone s'étend de fait à deux quartiers voisins, mais nous avons préféré nous concentrer sur des cliniques de Bandra East pour trouver des participants. 
commençant par les questions complémentaires sociodémographiques plutôt que les questions ouvertes, afin de sécuriser le répondant).

Plusieurs autres adaptations ont dû être opérées dans ce contexte si spécifique de grande pauvreté, d'habitat précaire et de mobilité continue. Premièrement, vis-à-vis du mode de passation usuel de l'enquête, le remplissage du questionnaire en auto-administré (par le répondant lui-même) était inenvisageable. En effet, le taux élevé d'illettrisme, en particulier chez les adultes âgés (D'Costa \& Das, 2002), rendait impossible la lecture des questions et la rédaction des réponses pour une grande partie de l'échantillon. Or, un élément important dans les choix méthodologiques est de garantir au maximum des conditions similaires de réponses. L'option choisie a donc été de renoncer à l'auto-administré et de procéder à l'enquête en mode face-à-face, c'est-à-dire en faisant rédiger les réponses des participants par les enquêtrices. Elles posaient les questions une à une aux individus, puis transcrivaient leurs réponses directement sur le questionnaire papier. Cette manière de faire implique bien sûr des limites, puisqu'elle nécessite un passage de l'oral à l'écrit par une tierce personne (cf. figure 3.5). Nonobstant, cela restait le moyen le plus équitable d'interroger tout le monde de manière similaire.

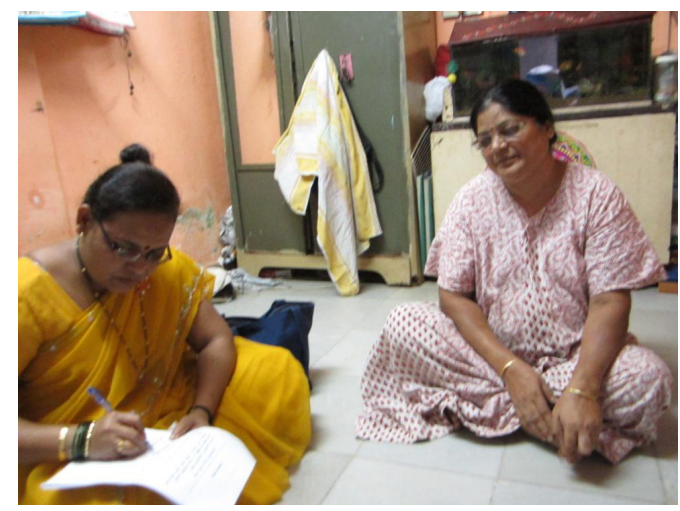

Figure 3.5: l'une des assistantes sociales du CSSC faisant passer le questionnaire à Bandra

Source: Piraud, 2012 p. 37.

$\mathrm{Au}$ départ, une liste d'une quarantaine de noms de personnes entrant dans les critères d'âge, de sexe et de religion avait été fournie à chaque enquêtrice à partir du recensement réalisé par l'ONG en 2006 (Henke \& 
Schlaefli, 2009), afin d'obtenir une répartition équitable entre les slums. Toutefois, cette liste n'a pas été suffisante pour compléter l'échantillon, la population des bidonvilles ayant une grande propension à fluctuer (suite aux migrations ou aux décès). Par ailleurs, WIN ne travaille qu'avec des femmes et, bien que dans leurs fichiers de recensement les hommes des ménages soient inscrits, un manque de participants masculins s'est vite ressenti, pour des raisons que nous discuterons plus en avant. Outre cela, certaines enquêtrices se sont révélées plus efficaces à trouver des participants et d'autres moins. Ainsi, la répartition géographique n'a pas pu être complétement contrôlée et certaines zones sont donc surreprésentées. Très vite, il est apparu que l'option d'un 50/50 sur la religion ne pourrait pas non plus être maintenue.

Quelles qu'aient été ces difficultés, l'aide du CSSC nous a offert une opportunité d'étude inespérée. Dans les bidonvilles, mêmes les recensements sont ardus à faire passer : d'une part parce que comme nous l'avons déjà évoqué, les habitants connaissent une très grande mobilité, pour des raisons sociales ou économiques, et d'autre part car ils ne font que rarement partie des registres de population, de la population de jure. Ceci explique également pourquoi, dans une telle situation, construire un échantillon aléatoire et représentatif relève de la pure fiction. Ce fut en fait notre ambition initiale, avec des tirages aléatoires à partir du recensement de WIN en 2009, mais la réalité nous a rapidement rattrapés... Par ailleurs, pénétrer un milieu si précaire peut s'avérer impossible pour un chercheur qui ne fait pas partie de la communauté. Un certain nombre d'autres d'obstacles concrets s'érigeaient sur notre chemin. La langue, le sexe, l'âge entre autres, qui pouvaient constituer autant de restrictions dans la communication avec la population interrogée. Bénéficier de l'aide de travailleuses issues de ces quartiers défavorisés a été la garantie du succès d'une telle opération.

\subsubsection{Diriger une enquête à Mumbai}

De mars à avril $2014^{13}$, une nouvelle récolte de données a été dirigée, cette fois dans le quartier de Santa Cruz East. L'objectif de ce second terrain était d'étayer les premiers résultats issus des questionnaires passés

13 Sont exclus de la durée des terrains: l'embauche et la formation des enquêteurs, la saisie et la traduction des réponses, le travail de codification des données. 
à Bandra East, en les confrontant au point de vue de Mumbaikars qui ne résident pas dans des bidonvilles. Ainsi, la variable du lieu de résidence devrait permettre de définir les impacts sur les réponses liés à l'environnement socioéconomique différent, quoique dans un cadre spatial et culturel partagé.

Durant ces deux mois et demi, j'ai été assistée par un collaborateur, Dinesh Shenai, recruté sur place pour encadrer 15 enquêteurs. Ils ont interrogé 622 personnes ${ }^{14}$, dans la rue mais également dans divers immeubles de la zone choisie. Deux personnes ont servi de superviseurs aux enquêteurs et de lien avec le collaborateur et moi. Le recrutement des intervieweurs a eu lieu sur plusieurs campus universitaires, mais la période des examens a empêché de nombreux étudiants de participer à notre enquête. Ensuite, des agences spécialisées dans la gestion de récolte de données (avec négociations des prix) ont été contactées. Elles ont surtout permis de rencontrer des enquêteurs intéressés à nous rejoindre, mais elles-mêmes n'ont pas été employées car leur coût dépassait le budget prévu. Finalement, le bouche-à-oreille a été des plus efficaces.

Se retrouver à la tête d'une équipe relativement nombreuse d'enquêteurs (qui ne comprenait que deux femmes), dont la majorité ne parlait que hindi ou marathi, a fait de la présence du collaborateur une nécessité. En effet, il a rendu possible la communication entre les enquêteurs, les superviseurs et moi, mais aussi entre les enquêteurs et les répondants lors des premiers tests sur le terrain. Lors des formations, il a servi d'interprète, et dans l'adaptation des questionnaires de traducteur ${ }^{15}$. Ses connaissances préalables dans la passation d'enquêtes ont fait également de lui une source d'échanges intéressants, sur le contenu même de la recherche. Au sein d'un monde où tout se marchande, il m'a aidée à déjouer les difficultés d'interaction avec l'équipe et les participants afin de gérer au mieux cette étude.

La présence des superviseurs a, par exemple, été une surprise qui n'avait pas été envisagée. A la fin de la première formation organisée pour les enquêteurs, où une quinzaine de personnes étaient présentes, trois d'entre elles se sont approchées pour me signifier qu'elles ne voulaient pas être enquêtrices mais superviseurs. Autrement dit, elles avaient fait

14 De fait, 661 personnes ont répondu; 14 questionnaires ont été ôtés car hors classes d'âge; 25 mal remplis ont été écartés.

15 En effet, quelques questions ont été ajoutées au questionnaire de 2012; toutefois, les questions principales ont été dûment conservées identiques. 
venir à cette formation un petit groupe d'enquêteurs (qu'elles connaissaient auparavant) et souhaitaient en avoir la gestion plutôt que d'aller sur le terrain elles-mêmes. Evidemment, un paiement particulier devait leur être réservé, qui n'entrait ni dans le coût d'un questionnaire rempli, ni dans celui du rôle du collaborateur. Ils ont indiqué de manière plus ou moins explicite que si leur poste était refusé, les enquêteurs venus avec eux repartiraient de même. Finalement, le collaborateur a choisi les tâches qu'il souhaitait attribuer aux superviseurs (nous en avons gardé deux) et pour lesquels il leur réservait un revenu sur son propre salaire.

Un montant forfaitaire par questionnaire (de $\left.100 \mathrm{INR}^{16}\right)$ a été décidé dès le départ, afin de n'avoir pas à négocier de prix à chaque paiement. Ce prix a été évalué en fonction du temps moyen de remplissage. Les enquêteurs parvenaient à environ une dizaine de questionnaires par journée de travail, soit une paie de 1'000 INR (18.00 CHF) par jour. Sachant que le revenu moyen mensuel par habitant (avec toutes les limites qu'un tel chiffre, dans pareil contexte d'informalité, puisse contenir) est de 10'417 $\mathrm{INR}^{17}$ à Mumbai (Times of India, 23.03.2011), nous dépassions les normes en vigueur. Toutefois, un jeu de rétention a été mené durant toute la récolte, qui consistait, pour ma part, à ne pas payer tous les questionnaires faits en une seule fois (afin que les enquêteurs reviennent chercher leur paie ainsi que d'autres questionnaires) et du côté des enquêteurs à ne jamais me remettre l'entièreté des questionnaires remplis pour être sûr de conserver leur place et de se faire payer.

Plusieurs demi-journées de formation ont été organisées. Nous y avons présenté le questionnaire et la méthodologie d'enquête. En fonction des langues que les enquêteurs pratiquaient le plus aisément à l'écrit (anglais ou hindi), la décision de faire imprimer le matériel dans les deux langues (avec davantage d'exemplaires en hindi) a été prise. Le questionnaire n'a pas semblé revêtir de difficulté, grâce notamment au fait qu'une partie des enquêteurs professionnels avait l'habitude de poser des questions dans le domaine du marketing, des sciences économiques. Un essai a été pratiqué en présence de tous les participants, afin de les confronter au contenu de l'enquête. A cette occasion, il est ressorti que le volet II (les grands tournants de la vie) représentait plus de difficultés de compréhension que les

16 Soit environ $1.80 \mathrm{CHF}$.

17 Soit environ 156.00 CHF. Le taux de change de la roupie indienne étant fluctuant, ces chiffres ne correspondent plus exactement à l'heure de rédiger ce livre. 
deux autres, essentiellement parce que les Indiennes et les Indiens ne sont pas habitués à repenser leur existence de manière subjective et sélective ${ }^{18}$.

Toutes les informations méthodologiques n'ont pas été divulguées lors de ces journées de formation. Notamment, la consigne de respect des classes d'âge n'a pas été mentionnée au départ, afin de conserver la motivation des enquêteurs. Par ailleurs, la difficulté de trouver des individus correspondant aux critères de l'échantillon aurait pu ouvrir des négociations sur le paiement, qui n'étaient pas souhaitées. Lors des tests en situation réelle, passés avec chacun des interviewers les jours suivants la formation, ces compléments de sélection étaient révélés. Une fois sur le terrain et ayant connaissance de la tâche attendue, les enquêteurs ont facilement intégré ces nouvelles instructions.

Ainsi, des tests en situation réelle ont été prévus à la suite de la formation pour chacun des enquêteurs, sous la supervision du collaborateur. Cela permettait d'expliquer plus précisément le mode de remplissage du questionnaire. Des fiches techniques pour faciliter la récolte des informations sociodémographiques et économiques ont été construites. Dans ces renseignements, nous demandions l'âge de l'individu. Or, les Indiens indiquent leur âge six mois avant et six mois après leur anniversaire ; pour cela, il a été préconisé de préciser que nous souhaitions connaître leur âge révolu ${ }^{19}$. Il est arrivé que cette consigne ne soit pas appliquée, ce qui explique la quantité non négligeable d'années de naissance et d'âges déclarés qui ne coïncident pas, à une année près. Dans ces cas, l'année de naissance a été privilégiée.

Afin de respecter le mode de passation appliqué lors de la précédente récolte de données, les enquêteurs ont été formés à passer le questionnaire en face-à-face. Une fois encore, nous reconnaissons que le passage de l'oral à l'écrit par une tierce personne représente une source potentielle de biais, et ce alors qu'à Santa Cruz les répondants auraient - dans une grande majorité - pu écrire eux-mêmes leurs réponses. Mais cela a permis de limiter les divergences méthodologiques entre les deux enquêtes, dont la comparabilité est de la sorte assurée. Comme à Bandra, la taille réduite du questionnaire a laissé aux enquêteurs davantage de temps pour retranscrire

\footnotetext{
18 En effet, l'Inde pratique régulièrement des recensements de sa population, mais il s'agit de questions fermées et précises sur les modes de vie (âge au mariage, nombre d'enfants, années d'études, etc.).

Complied en anglais
} 
les réponses et les interviewés, n'ayant pas à subir la fastidiosité d'une passation interminable, ont pris leur temps pour réfléchir.

Les enquêteurs se sont vu attribuer des immeubles et des rues par les superviseurs et le collaborateur, afin de couvrir largement la zone de Santa Cruz East (cf. figure 3.2). Ils sonnaient aux portes des appartements, se présentaient et demandaient si la personne était d'accord de répondre. Une carte de légitimation avec le logo de l'Université de Genève, expliquant qu'ils étaient des enquêteurs engagés par l'institution pour faire passer ces questionnaires, a permis de faciliter l'approche. En cas d'acceptation, l'âge de la personne était vérifié ${ }^{20}$. Généralement, les individus sollicités se prêtaient facilement à l'exercice ; le seul défi était donc de trouver les personnes à la maison et qu'elles correspondent aux critères d'âge. Certains immeubles du quartier n'étaient accessibles qu'aux habitants de ceux-ci, et nos enquêteurs ont parfois été refoulés par le gardien à l'entrée. A plusieurs reprises, demander la permission a suffi pour accéder aux logements. Il est aussi arrivé que nous nous retrouvions le dimanche matin dans un parc du quartier, pour y interroger les passants (souvent des personnes âgées).

Des immeubles SRA ont été pris en compte, avant que je ne m'en rende compte et demande de préférer ceux de classes moyennes. Pour rappel, les immeubles SRA représentent des édifices voués à la relocalisation des habitants des bidonvilles (cf. figure 3.2). Le processus de relogement est multiple et complexe, mais le résultat explique ces longues barres d'immeubles massifs, rapprochés, peu avenants, qui abritent des centaines de personnes dont la situation socioéconomique est similaire aux slums $d$ wellers. Une minorité $(31 \%, n=185)$ des membres de l'échantillon interrogé en 2014 appartient donc à cette catégorie, inférieure en termes de niveau de vie au reste des participants de Santa Cruz.

La passation s'est très bien déroulée dans l'ensemble et ce sur une courte période. Une seule consigne a été modifiée en cours de route dans la méthodologie. Après environ 200 questionnaires récoltés, je me suis rendu compte que souvent le nombre de réponses était faible (un ou deux événements mentionnés, pas plus). J'ai alors demandé explicitement à avoir plusieurs réponses par question, ce qui a représenté plus de temps de travail pour les enquêteurs.

20 D'autres personnes du ménages pouvaient être présents, et même être interrogés s'ils entraient dans les classes d'âge, mais il était préconisé d'éviter les passations en présence d'un tiers. 


\subsubsection{Particularités et difficultés des terrains}

Les conditions de réalisation des enquêtes ont été à la fois imposées et décidées avec le souci de conserver une similitude aussi grande que possible, de même que lors de la constitution des bases de données. Malgré tout, des différences structurelles existent. Les deux terrains ont été réalisés avec deux années d'écart, ce qui est suffisamment faible pour qu'il n'y ait pas de grande modification des groupes d'âges et que ces derniers soient comparables d'une enquête à l'autre. Toutefois, des changements - historiques surtout - ont eu lieu entre ces deux dates, qui impliquent la présence de quelques réponses spécifiques dans l'enquête la plus récente.

L'enveloppe financière limitée pour les deux enquêtes a imposé des choix nécessaires. Il fallut trouver comment répartir les diverses dépenses et salaires, bien que ces derniers soient bien inférieurs au coût escompté dans un pays autre, comme la Suisse. Pour la première enquête, ce fut relativement simple, alors que dans le cas du second terrain cela s'avéra l'une des principales difficultés. En 2012, nous avons hésité à rémunérer les enquêtrices et les répondants ; nous y avons renoncé car cela aurait introduit une inégalité de traitement entre les individus (d'une assistante sociale à l'autre et d'un répondant à un non-répondant) et surtout présentait le risque de discréditer les autres récoltes d'informations du CSSC, qui ne peut guère retirer la moindre somme du budget de fonctionnement de ses cliniques et du salaire de ses employés non-bénévoles. Par contre, un don substantiel a été versé au CSSC. En 2014, le jeu typiquement indien des négociations salariales a impliqué de nombreuses restrictions sur le déroulement du terrain, en particulier à propos de l'explication des critères d'échantillonnage et de la réception des questionnaires remplis.

Concernant les questionnaires eux-mêmes, peu de modifications ont été apportées à leur forme originelle issue du programme CEVI. Des questions complémentaires ont été ajoutées, avec quelques différences entre 2012 et 2014. En plus des renseignements habituels (voir p.15), nous avons demandé que soient indiqués : la «date de l'interview»; le «nom (de l'enquêteur)»; le «nom (de l'enquêté)»; l'《âge» ${ }^{21} ; l^{\prime}$ '《état d'origine»; la «confession religieuse». En 2012, dans les slums de Bandra, nous avons demandé en plus : un «numéro d'identifiant» si la personne figurait sur les listes de l'ONG ; le «quartier» (zone précise de Bandra) dans lequel elle/

21 Pour les cas où les gens ne connaîtraient pas leur année de naissance. Si les résultats ne correspondaient pas, nous avons privilégié cette dernière. 
il résidait ; le «nom de la clinique» si elle/il appartenait au programme WIN. En 2014, dans le quartier de Santa Cruz, les indicateurs suivants ont été récoltés : la «rue»; le «nom de l'immeuble»; une «évaluation de la situation économique» par l'enquêteur' ${ }^{22}$; le «type du lieu d'origine» ${ }^{23}$; le «nombre d'enfants» (si l'individu en avait) ; le «revenu moyen du ménage ${ }^{\prime 2}$; en complément de l'information relative au revenu, nous demandions si le participant «possédait» une série de biens ${ }^{25}$. Finalement, une dernière et courte question ouverte a été ajoutée au questionnaire de 2014. Il s'agissait de savoir si le répondant percevait sa vie comme étant plus semblable à celle de ses parents, et/ou à celle de ses enfants (s'il en avait), ou encore à aucun des deux (voir chapitre 6).

Lors de la passation à Santa Cruz, la supervision des enquêteurs exigeait de pouvoir contacter les répondants afin de procéder à des contrôles aléatoires. Pour cette raison, un certain nombre d'informations personnelles (adresse et numéro de téléphone essentiellement) ont été demandées aux enquêtés et indiquées sur les questionnaires. Une fois la base de données construite, ces informations ont été supprimées afin de garantir l'anonymat.

Comme cela a déjà été précisé, le déroulement des questions - au sein du questionnaire - a été inversé pour les deux enquêtes passées à Mumbai. En effet, en Inde il est plus courant de répondre à des questions fermées du type des questions complémentaires. Les placer en premier lieu permettait dès lors au répondant de prendre confiance avant d'arriver aux trois volets principaux (qui eux étaient dans l'ordre habituel). Le questionnaire ainsi constitué a d'abord été traduit vers l'anglais puis vers le hindi et le marathi (langue de l'état du Maharastra), en prenant garde à ne pas modifier le sens des questions principales. Les différents langages et la traduction de concepts qui demandent une certaine finesse d'interprétation n'ont pas

22 De manière ternaire: pauvre / non pauvre / ne sait pas.

23 Était-ce une ville: grande / moyenne / petite / un village?

24 Selon une répartition en six niveaux, basée sur des situations allant de l'extrême pauvreté à une classe moyenne supérieure: <3'700 INR ; 3'700 - 7'999 INR ; 8'000 - 14'999 INR ; 15'000 - 29'999 INR ; 30’000 - 49'999 INR ; >49'999 INR.

25 Les items choisis étaient: posséder un climatiseur; un ordinateur; avoir l'accès à internet; posséder des toilettes dites «indiennes»; des toilettes dites «anglaises»; un frigidaire; avoir la télévision ou la radio; posséder un téléphone portable; une voiture; une imprimante; un chauffage de type liquid petroleum gas (LPG); ou de type compress natural gas (CNG). Les deux types de toilettes étaient demandés comme un moyen pour savoir si la personne en possédait vraiment, peu importe leur forme, le risque étant qu'une question trop vague entraîne une réponse erronée. 
rendu cette tâche facile. Par la suite, une traduction des réponses a été effectuée vers l'anglais. A nouveau, le passage d'une langue à l'autre a impliqué le risque de perdre un certain nombre de nuances dans la réponse.

La communication avec les équipes de recherche (que ce soit les assistantes sociales en 2012 ou les enquêteurs en 2014) s'est également révélée difficile à cause de la langue. En ce qui concerne les choix des intervieweurs, il a fallu s'en remettre à un intermédiaire, soit le CSSC pour l'enquête de 2012 et le collaborateur pour l'étude de 2014. Le recrutement occasionna aussi un certain nombre de complications. Le choix est apparu plutôt simple à Bandra East où les assistantes sociales, de par leur propre origine sociale, étaient les meilleures enquêtrices souhaitables pour un tel environnement. Seule la quantité d'assistantes que nous voulions engager fut soumise à réflexion, et a dépendu de la rapidité avec laquelle nous souhaitions faire avancer la récolte des questionnaires. A Santa Cruz, la décision sur le nombre d'enquêteurs et l'évaluation de ceux-ci s'est révélée plus ardue. Trouver des enquêteurs et les former ne fut pas aisé et un encadrement plus strict a été nécessaire. Néanmoins, au final un seul d'entre eux dut être exclu de la récolte après avoir rendu des questionnaires mal remplis.

Des contacts réguliers avec l'équipe de recherche ont eu lieu, afin de permettre le suivi du nombre de questionnaires rentrés et de vérifier les comptes. Pour l'équipe de 2014, cela permettait des paiements réduits mais réguliers. Les difficultés majeures ont concerné l'échantillon. Dans les bidonvilles, il fut plus compliqué de trouver des hommes car les entretiens avaient lieu durant la journée, alors que la plupart d'entre eux étaient au travail. De plus, toutes nos enquêtrices étaient des femmes, pour qui parler à des hommes hors la présence de leur propre mari n'est pas une situation sociale acceptable. Par ailleurs, trouver des personnes âgées - en particulier des femmes - s'est révélé difficile en raison de la structure démographique de la population et d'une crainte plus grande d'ouvrir sa porte à des inconnus. Ceci explique la faiblesse du nombre de femmes âgées interrogées en 2014. Pour résoudre ce problème et maintenir une répartition égale des individus entre les groupes d'âge, il a été finalement décidé d'élargir la dernière classe d'âge de plus et moins deux années. Les plus âgés interrogés en 2014 avaient donc entre 78 et 86 ans $^{26}$. Cette difficulté fut moins lourde en 2012, précisément grâce au fait que notre

26 Bien évidemment, ces âges élevés peuvent être soumis à questionnement, car connaître sa date de naissance exacte n'était pas une évidence dans l'Inde des années 1928-1934. Toutefois, les contrôles menés par le biais des réponses aux différents 
équipe d'enquêteurs était exclusivement composée de femmes et que la confiance était déjà établie avec les interviewés par le biais de l'ONG.

\subsubsection{Les répondantes et les répondants mumbaikars}

Au cours de ces deux terrains d'enquête, 1255 individus entrant dans les critères d'âge et de sexe ont été interrogés (cf. tableau 3.1). Le minimum de 50 femmes et hommes par classe d'âge et par quartier a été atteint, à l'exception des femmes de 80-84 ans à Santa Cruz comme cela a déjà été indiqué. Ces groupes d'âge circonscrivent des cohortes, dont la plus ancienne (personnes de 80-84 ans interrogées dans la première enquête) est venue au monde entre 1928 et 1932, et la plus récente (personnes de 20-24 ans interrogées dans la seconde enquête) dans la première moitié des années nonante.

Les caractéristiques de l'échantillon final se trouvent exposées dans le tableau 3.2. L'état civil des répondants révèle ce que les chiffres à l'échelle de la population laissaient prévoir (Véron, 2006): passé l'âge de 25 ans, une large majorité des gens sont mariés. Néanmoins, dans le quartier pauvre, le veuvage est une réalité dès la trentaine. La perte du conjoint n'affecte que plus tard, vers la soixantaine, les individus vivant à Santa Cruz. De la même manière, les répondants ont presque tous des enfants dès la trentaine, voire plus tôt, en particulier parmi les habitants des slums.

Tableau 3.1: effectifs de 2012 et 2014, selon la classe d'âge et le sexe ${ }^{27}$

\begin{tabular}{|c|c|c|c|c|c|c|}
\hline \multirow[b]{3}{*}{$20-24$ ans } & \multicolumn{3}{|c|}{ Bandra 2012} & \multicolumn{3}{|c|}{ Santa Cruz 2014} \\
\hline & Hommes & Femmes & Total & Hommes & Femmes & Total \\
\hline & 63 & 77 & 140 & 63 & 63 & 126 \\
\hline $35-39$ ans & 56 & 89 & 145 & 65 & 72 & 137 \\
\hline $50-54$ ans & 59 & 59 & 118 & 66 & 72 & 138 \\
\hline $65-69$ ans & 58 & 63 & 121 & 67 & 69 & 136 \\
\hline $80-84$ ans & 50 & 59 & 109 & 52 & 33 & 85 \\
\hline Total & 286 & 347 & 633 & 313 & 309 & 622 \\
\hline
\end{tabular}

volets de l'enquête ont été concluants et ont semblé attesté de l'âge des individus (par exemple, en regardant quels changements sociohistoriques la personne avait vécu).

27 Une marge de plus ou moins deux ans a été acceptée dans les groupes d'âge (de quatre ans dans le cas des plus âgés) au moment de la récolte de données; toutefois, par souci de clarté, nous conservons dans ce texte l'étiquette des classes quinquennales. 
Les répondants sont en majorité de confession hindoue (entre 43 et $87 \%$ en fonction des âges et des lieux de résidence), avec un pourcentage élevé de musulmans parmi les résidents de Bandra. Ceci corrobore le phénomène de discrimination socioéconomique subi par les musulmans en Inde. Une autre forme de discrimination apparaît nettement dans notre échantillon, il s'agit de l'accès aux études. De manière évidente, les individus âgés ont peu eu de moyens pour poursuivre leur scolarité, voire la débuter. Cette situation est sans surprise particulièrement évidente en ce qui concerne les individus vivant dans les bidonvilles. Inversement, suivre une formation, parfois de niveau tertiaire, est bien plus accessible aux jeunes générations, avant tout parmi les classes moyennes.

La variable «activité» dévoile un clivage de genre peu surprenant : une large part (95\% dans chacun des terrains d'enquête) des individus se déclarant «au foyer» sont des femmes. A la différence des pays occidentaux, ceci ne signifie pas qu'elles se cantonnent au travail domestique, il arrive qu'elles aient un petit revenu d'appoint pour aider le ménage (cf. chapitre 1). Notons la progression avec l'avancée en âge du pourcentage de personnes se disant dans une situation professionnelle autre qu'étudiant, actif ou sans emploi. Parmi les sexagénaires et les octogénaires, il s'agit des répondants trop âgés pour conserver leur ancien emploi : soit ils se déclarent «à la retraite», soit ils précisent leur nouveau moyen de gagner un salaire (mendicité, emplois au jour le jour, soutien dans la boutique des enfants, etc.). La santé auto-évaluée permet de constater une perception plutôt positive de sa propre situation, qui se dégrade pour les derniers groupes d'âge, avec toutefois peu d'individus estimant leur santé comme mauvaise ou très mauvaise. 
Tableau 3.2: caractéristiques de l'échantillon selon les groupes d'âge et le lieu de résidence

\begin{tabular}{|c|c|c|c|c|c|c|c|c|c|c|}
\hline & \multicolumn{5}{|c|}{ Bandra } & \multicolumn{5}{|c|}{ Santa Cruz } \\
\hline classes d'âge & \multicolumn{5}{|c|}{$20-2435-3950-5465-6980-84$} & \multicolumn{5}{|c|}{$20-2435-3950-5465-6980-84$} \\
\hline$n$ & 140 & 145 & 118 & 121 & 109 & 126 & 137 & 138 & 136 & 85 \\
\hline Age moyen & 22.9 & 37.2 & 51.9 & 67 & 80.8 & 22.1 & 36.9 & 51.9 & 66.4 & 79.7 \\
\hline (écart-type) & $(1.7)$ & (2) & $(2.1)$ & $(1.9)$ & $(2.4)$ & |(1.9) & $(1.7)$ & $(1.7)$ & $(2.2)$ & $(2.9)$ \\
\hline \multicolumn{11}{|c|}{ Sexe (\% de femmes) } \\
\hline & 55 & 61 & 50 & 52 & 54 & $\mid 50$ & 53 & 52 & 51 & 39 \\
\hline \multicolumn{11}{|l|}{ Etat civil (\%) } \\
\hline Célibataire & 53 & 2 & - & - & - & 75 & 4 & 5 & 2 & 4 \\
\hline Marié(e) & 46 & 92 & 86 & 61 & 42 & 24 & 94 & 88 & 80 & 75 \\
\hline Divorcé(e) & 1 & 1 & - & - & - & 1 & - & - & - & - \\
\hline Veuf, veuve & - & 5 & 14 & 39 & 58 & - & 2 & 7 & 18 & 21 \\
\hline \multicolumn{11}{|l|}{ Religion (\%) } \\
\hline Hindou & 52 & 61 & 54 & 68 & 43 & 66 & 78 & 78 & 73 & 87 \\
\hline Musulman & 37 & 35 & 38 & 25 & 54 & 26 & 20 & 16 & 15 & 8 \\
\hline Autre $^{28}$ & 11 & 4 & 8 & 7 & 3 & 8 & 2 & 6 & 12 & 5 \\
\hline \multicolumn{11}{|c|}{ A au moins un enfant (\%) } \\
\hline & 35 & 94 & 99 & 99 & 99 & $\mid 12$ & 91 & 96 & 96 & 94 \\
\hline \multicolumn{11}{|l|}{ Habite seul (\%) } \\
\hline & - & 2 & 3 & 6 & 2 & $\mid 5$ & 2 & 7 & 6 & 4 \\
\hline \multicolumn{11}{|c|}{ Niveau d'éducation atteint ou en cours (\%) } \\
\hline Sans & 2 & 12 & 20 & 30 & 54 & 0 & 2 & 4 & 6 & 12 \\
\hline Primaire & 16 & 30 & 44 & 46 & 41 & 1 & 10 & 22 & 37 & 33 \\
\hline Secondaire & 32 & 36 & 31 & 14 & 4 & 17 & 35 & 40 & 35 & 33 \\
\hline Tertiaire & 50 & 22 & 5 & 10 & 1 & 82 & 53 & 34 & 22 & 22 \\
\hline \multicolumn{11}{|l|}{ Activité (\%) } \\
\hline En études & 23 & 1 & - & - & - & 50 & - & 1 & - & - \\
\hline Actif/ve & 29 & 48 & 41 & 12 & 6 & 27 & 53 & 41 & 17 & 3 \\
\hline Au foyer ${ }^{29}$ & 37 & 43 & 38 & 41 & 36 & 18 & 41 & 49 & 46 & 28 \\
\hline Sans emploi & 8 & 5 & 10 & 10 & 33 & 5 & 4 & 3 & 5 & 5 \\
\hline Autre $^{30}$ & 3 & 3 & 11 & 37 & 25 & - & 2 & 6 & 32 & 64 \\
\hline \multicolumn{11}{|c|}{ Santé auto-évaluée (\%) } \\
\hline Très bonne & 25 & 6 & 6 & 2 & 1 & 45 & 53 & 10 & 8 & 2 \\
\hline Bonne & 63 & 69 & 62 & 22 & 22 & 39 & 23 & 53 & 51 & 52 \\
\hline Moyenne & 10 & 24 & 30 & 60 & 59 & 16 & 21 & 34 & 36 & 38 \\
\hline (Très) Mauvaise & 2 & 1 & 2 & 16 & 18 & 0 & 3 & 3 & 5 & 8 \\
\hline
\end{tabular}

28 Il s'agit surtout de chrétiens, de bouddhistes, de jaïns et de sikhs.

29 Dans chacune des enquêtes, 95\% des individus s'étant déclaré "au foyer" sont des femmes.

30 Y compris les individus s'étant déclarés "à la retraite". 

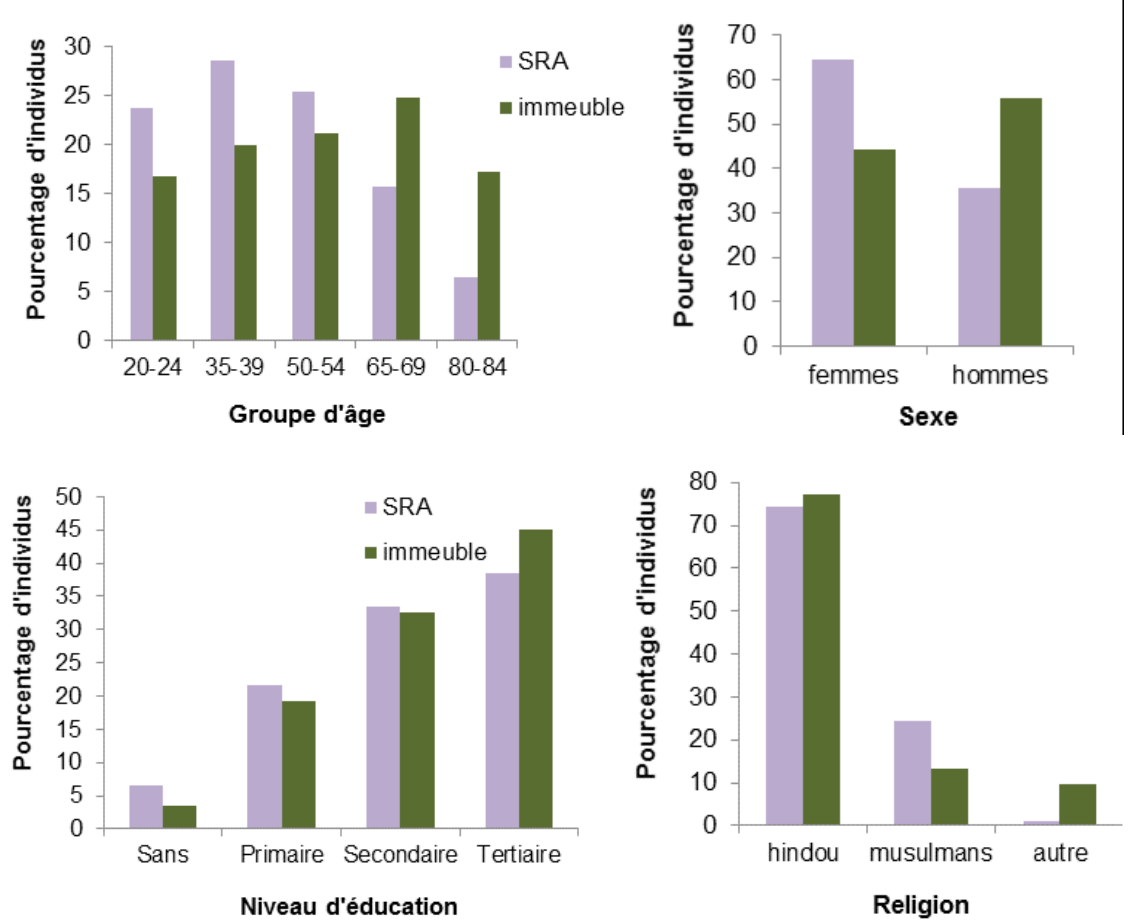

Figure 3.6: la répartition (\%) par âge, sexe, niveau d'éducation et religion des répondants interrogés à Santa Cruz

\subsection{Transformer les données pour les analyser}

La récolte des questionnaires (et leur traduction) n'a été que la première étape de la préparation des données en vue de leur analyse. En second lieu, les réponses aux différents volets, traduites en anglais, ainsi que les données complémentaires, ont été retranscrites sur des fichiers informatiques Excel. Puis, nous avons procédé à l'élaboration des bases de données et à la construction des variables descriptives sociodémographiques nécessaires aux analyses. Finalement, un long processus de codification des trois volets de l'enquête, sur le modèle des typologies utilisées dans 
le programme CEVI, a permis l'opérationnalisation des réponses et leur traitement de manière quantitative.

\subsubsection{Bases de données et variables sociodémographiques}

Lors du terrain passé à Bandra, la saisie et la traduction ont été entièrement exécutées par des personnes recrutées par le CSSC et rémunérées à cet effet. Pour le terrain de Santa Cruz, je me suis moi-même chargée de l'essentiel de la saisie (les questionnaires ayant été auparavant traduits en anglais à même le papier). Par ailleurs, je suis repassée sur l'ensemble des questionnaires pour vérifier l'exactitude des retranscriptions. Il m'était impossible de reprendre la traduction; en revanche, un certain nombre de détails ont pu être contrôlés, complétés voire corrigés. Les numéros (dates et âges), certains mots récurrents, les lieux ainsi que les oublis de traduction étaient à ma portée.

Une fois les fichiers Excel complétés, ils ont été transférés sur SPSS pour leur nettoyage et l'opérationnalisation des variables. Une base spécifique aux questions complémentaires, sociodémographiques, a été constituée, ainsi qu'une base par volet, pour chacune des enquêtes. Ensuite, il a été aisé de les fusionner au fur et à mesure des besoins analytiques. A ce stade, seules des bases «individu» ont été constituées : il s'agit de fichiers où chaque ligne représente un répondant.

Le nettoyage des données (c'est-à-dire le contrôle et l'homogénéisation des variables sociodémographiques et des questions ouvertes entre les deux enquêtes) a représenté l'étape suivante. Une fois les bases portant sur les différents volets du questionnaire rigoureusement similaires, il a été possible de constituer de nouveaux fichiers SPSS, cette fois restructurés selon la logique d'un événement par ligne (soit des bases «changement», comprenant quatre fois plus de lignes que les bases individus). Ce second format offre l'opportunité de se focaliser sur les réponses données par les personnes, il a notamment permis d'effectuer des analyses de régression.

\section{Les variables sociodémographiques}

Cinq variables sociodémographiques et socioéconomiques ont été utilisées pour spécifier les caractéristiques individuelles des répondants. La première tient compte du lieu de résidence, soit du quartier où s'est déroulé le terrain (Bandra ou Santa Cruz). Les deux variables suivantes 
sont directement liées aux critères d'échantillonnage : le groupe d'âge et le sexe. Pour ces trois premières variables, indispensables à la construction de nos échantillons, aucune valeur manquante n'est à déplorer.

Un quatrième indicateur a été constitué d'après le niveau d'éducation, séparé en quatre degrés : illettré, école primaire, secondaire et supérieure (ce dernier groupe comprenant université et secondaire supérieur), afin d'obtenir des catégories plus ou moins égales en quantité d'individus (cf. tableau 3.2) ${ }^{31}$. Une seule valeur manquante apparait, à Bandra. Finalement, la santé auto-évaluée a aussi été retenue comme variable explicative et ne contient que 11 valeurs manquantes (à Bandra également). Elle a été subdivisée en quatre niveaux : très bonne, bonne, moyenne et mauvaise (cette dernière catégorie incluant les personnes se déclarant en très mauvaise santé) (cf. tableau 3.2).

Les autres variables descriptives (religion, cohabitation, avoir des enfants, état civil, activité) présentent trop de déséquilibres (entre l'un et l'autre des items, ou entre l'une et l'autre des classes d'âge); elles n'ont pas été conservées dans les analyses (à une exception près, la variable religion, que nous avons utilisé une fois, voir chapitre 8). De plus, la variable d'activité est soumise à interprétation: au vu du contexte de forte informalité du marché du travail, le fait d'être en étude, sans emploi, au foyer ou à la retraite ne signifie pas pour autant que la personne ne travaille pas; il aurait donc été délicat de séparer ces catégories de personnes.

\section{La caste: une variable impossible}

En outre, au regard de la présentation du contexte indien faite dans le chapitre premier, l'absence de l'utilisation de la caste comme indicateur de situation sociale ne peut que surprendre. Il est clair que les castes jouent toujours un rôle majeur dans la société indienne : en tenir compte dans nos analyses semble de prime abord évident. Néanmoins, plusieurs explications justifient que ce ne soit pas le cas dans ce travail.

Tout d'abord, bien que chaque Indienne et chaque Indien de confession hindoue naisse dans une caste (ou comme Dalit) et soit capable de reconnaître d'un regard la position d'autrui, l'interdiction de discrimination sur la base des castes - inscrite dans la Constitution indienne depuis

31 L'école primaire en Inde s'étend de l'âge de 6 à 12 ans; l'école secondaire de 13 à 16 ans et le supérieur de 16 ans jusqu'à la fin des études universitaires. Le 12th standard est l'année de l'examen final décisif pour l'entrée à l'université, qui se trouve inclus dans cette dernière catégorie. 
1949 - crée un environnement pesant lorsqu'il s'agit de demander à quelqu'un sa caste dans le cadre d'une enquête. Ce qui explique que nous ayons choisi de ne pas réclamer cette information aux répondants de Santa Cruz, afin de ne pas susciter de tension, ou d'inconfort, pour les enquêtés et les enquêteurs ; qui plus est, le rapport de force inhérent à la relation interviewé-intervieweur s'en serait trouvé affecté, probablement maximisé (voir ci-dessous pour une discussion plus approfondie de ce point).

Sans doute, une certaine dose de prudence (possiblement exagérée) s'est-elle aussi imposée à nous, chercheurs occidentaux s'immisçant dans un monde dont nous étions en partie ignorants, pour ne pas y transgresser des règles que nous ne saurions évaluer ou que nous n'aurions pas prévues, provoquant des conséquences que nous ne saurions maîtriser.

Par ailleurs, d'après les informations fournies par le CSSC, nous savions que les habitants de Bandra sont très généralement Dalits. Si l'on ajoute à cette catégorie les musulmans résidant dans les bidonvilles, dont les conditions de vie et d'exclusion sont proches, une très large majorité de notre échantillon interrogé dans les slums est Intouchable. L'information se recoupe donc avec le lieu de résidence. Face à ces arguments, nous avons préféré nous contenter de cette dernière variable comme indicateur socioéconomique, en reconnaissant toutes les limites de ce choix.

\subsubsection{Interpréter le subjectif pour le rendre quantifiable : typologies et codifications des trois questions ouvertes}

Le travail le plus long fut celui du codage des trois volets de l'enquête selon deux critères de classification. La première de ces typologies concerne les changements personnels (volet I et II) et la seconde les changements sociohistoriques (volet III). Elles sont présentées ci-dessous.

Tout au long de ce livre, nous utilisons en outre plusieurs autres typologies, reposant sur des concepts différents : les capitaux ou ressources à disposition (chapitre 4), l'agency et l'individualisation présentes dans les souvenirs (chapitre 9), le degré de bifurcation compris dans ces mentions (chapitre 9), la connotation positive ou négative des réponses (chapitre 9). Ces grilles de lecture sont explicitées dans les chapitres concernés. Finalement, lorsque cela s'avère nécessaire, nous élaborons aussi une codification des raisons avancées par les répondants pour expliquer les choix 
des événements cités (volet II et III). Les divers critères utilisés seront expliqués dans les sections y relatives.

\section{Les changements personnels}

La classification des événements personnels, divisée en ensembles généraux et en catégories plus fines, est commune aux enquêtes CEVI (Cavalli et al., 2006; Lalive d'Epinay \& Cavalli, 2007) et s'inspire de celle développée par Reese et Smyer (1983).

Partant d'une division des changements selon leur origine (biologique, socioculturelle, physique-environnementale ou psychologique), Reese et Smyer (1983) croisent ces quatre catégories avec les principales trajectoires de la vie concernées par les événements (famille, éducation, profession,...) afin d'obtenir une grille de codification précise. Dans notre cas, la typologie est basée sur 12 «domaines»: famille, amitié, décès, santé, développement personnel (self), éducation, profession, mobilité, voyages, activités (participation sociale, religieuse, etc.), économie, environnement (événement extérieur affectant l'existence, comme une inondation).

A un second niveau (les «types»), une subdivision de ces domaines est faite en fonction d'événements plus précis. Par exemple, le domaine famille comprend les types : naissance des enfants, mariage, mise en couple, séparation, autre. Ces sous-catégorisations représentent au total une cinquantaine de changements différents, plus ou moins nombreux suivant les domaines.

Dernière opération, les dates des événements (que les enquêteurs avaient dû demander aux répondants et inscrire sur le questionnaire) ont été vérifiées grâce à l'âge du souvenir (également indiqué par l'interviewé). Nous avons en plus procédé à une codification de l'identité de la personne concernée par ce changement : le répondant, son conjoint, un parent (beau), un enfant (beau), un frère ou une sœur (beau), un grand-parent, un petit-enfant, un autre proche, la famille sans précision, un ami ou une connaissance. Le lieu de survenue de l'événement a aussi été retranscrit selon ce que l'enquêteur avait écrit sur le questionnaire.

\section{Les changements sociohistoriques}

Sur le même modèle que les événements personnels (soit sur deux niveaux, domaines et types), les changements sociohistoriques ont été codifiés selon une typologie commune à l'enquête CEVI. Une première partie de cette grille d'analyse considère les changements internationaux (Seconde 
Guerre mondiale, 11 Septembre 2001, crises économiques, ...) mentionnés dans tous les pays, alors qu'une seconde partie est spécifique à chaque lieu d'enquête.

En ce qui concerne l'Inde, nous avons constitué 6 domaines principaux pour répartir les changements sociohistoriques nationaux: conflits et guerres impliquant l'Inde (comprenant des types tels que la Partition de 1947, les guerres indo-pakistanaises, la question du Cachemire); la politique indienne (les diverses élection, l'assassinat d'Indira Gandhi,...); les affrontements religieux; les attentats; les événements relatifs à Mumbai (effondrement d'un immeuble, conflits dans les bidonvilles, etc.); les événements relatifs à l'Inde (les violences contre les femmes par exemple).

Dernier élément, nous avons procédé à l'élaboration d'une variable indiquant la date de survenue des changements sociohistorique. Lorsque l'événement concerné n'a eu lieu qu'en un moment ponctuel de l'histoire, la codification fut aisée. Lorsqu'au contraire il s'est étendu dans le temps (comme une guerre), nous avons opté pour conserver la date moyenne (par exemple, la Seconde Guerre mondiale a pour date de survenue 1942). L'utilité de cette date fixe est de permettre le lien avec l'âge de l'individu au moment où ce dernier vivait le changement. Enfin, le lieu d'occurrence de l'événement a également été codé, selon son continent d'appartenance.

Bien que ces diverses codifications reposent sur des typologies existant au préalable, elles ont malgré tout impliqué une démarche inductive, en fonction surtout de la quantité de mentions faites. Ainsi, un domaine largement évoqué a justifié une subdivision plus fine en nombre de types. $* * *$

La section qui suit se propose d'offrir un questionnement critique sur la capacité de l'étude à identifier les événements et changements vécus les plus importants, selon les individus. Le nombre moyen de réponses aux trois questions ouvertes rejoint-il ce qui a pu être établit à d'autres occasion (Laborde et al., 2012; Lelièvre \& Robette, 2015)? Outre ce constat statistique, peut-on distinguer un effet du quartier, du genre, de l'âge ou du niveau d'éducation sur le nombre de réponses ? Quelques analyses permettront de soutenir la réflexion.

Par ailleurs, dans une seconde partie, une considération plus théorique offrira le loisir de soulever divers questionnements à propos de la méthode employée. Pour part, des réponses à ces observations seront amenées à la fin de ce livre, dans les conclusions générales, en se basant sur les résultats empiriques. 


\subsection{Regards critiques sur l'enquête}

\subsubsection{Evaluation des données}

A l'issue de ces deux enquêtes, l'exercice apparu avoir fonctionné. En effet, entre 87 et $98 \%$ des individus ont répondu aux deux questions portant sur l'entièreté de la vie (volet II et III). Le premier volet, qui concernait uniquement la dernière année de vie, n'a pas été rempli par tous les individus (mais tout de même 56\% de l'échantillon de 2012 et $61 \%$ de l'échantillon de 2014), sans surprise puisque le laps de temps durant lequel un événement pouvait survenir était réduit. Concernant le nombre moyen d'événements cités (parmi les personnes en ayant mentionné au moins un), il se situe au-dessous de deux [volet I = 1.64 (écart-type : 0.825 ) ; volet II $=1.94$ (écart-type : 0.932 ) ; volet III $=1.80$ (écart-type : $0.815)]$.

Des variations sont observables principalement entre les deux enquêtes et selon l'âge (ou le niveau d'éducation, ces deux variables étant fortement corrélées dans nos échantillons), mais relativement peu selon le sexe de l'enquêté (cf. tableau 3.3). Les réponses fournies lors de la première passation dans les bidonvilles étant plus nombreuses, cela laisse supposer qu'avoir demandé à une organisation déjà implantée dans la zone, le CSSC, de se charger des entretiens a encouragé les participants dans la mention des événements.

La fluctuation qui se remarque entre les groupes d'âge est un peu plus délicate à interpréter. En effet, la première question sur les changements récents a stimulé davantage de réponses parmi les jeunes habitants des bidonvilles, mais pas de la part de leurs homologues à Santa Cruz. Or, nous l'avons évoqué, de nombreuses études ont démontré que l'entrée dans l'âge adulte était un moment où beaucoup de transitions avaient lieu (Cavalli et al., 2013). Une explication à ce phénomène peut être liée à la position dans le parcours de vie, distincte de l'âge chronologique : comme nous le verrons dans plusieurs chapitres, les jeunes de niveau socioéconomique inférieur (vivant dans les bidonvilles) connaissent une entrée dans l'âge adulte légèrement plus précoce que leurs homologues issus des classes moyennes, et ont déjà vécu certaines transitions que les seconds n'ont pas encore connues. Dans les questions où les interviewés pouvaient se fonder sur toute leur existence (volets II et III), les plus jeunes 
ont généralement moins répondu que les autres. Ceci donne à penser que le nombre d'événements que l'on cite est tout de même dépendant du nombre d'années que l'on a vécu (une longue vie offrant plus d'opportunités de moments marquants).

Dans l'enquête Biographies et entourage (Bonvalet et Lelièvre, 2012), une question subjective a été posée, ressemblant fortement à celles utilisées dans notre propre questionnaire: "Y a-t-il des événements personnels ou historiques qui ont marqué votre vie?». Les réponses données montrent qu'en moyenne trois événements marquants ont été cités par personne, davantage donc que ce que nous constatons dans notre enquête indienne (Laborde et al., 2012; Lelièvre \& Robette, 2015). Toutefois, la question évoquée dans Biographies et entourage ne différencie pas la mémoire personnelle et la mémoire historique, ce qui peut largement expliquer l'écart observé. En effet, nos trois questions additionnées montrent une moyenne de 4.5 événements cités par personne. Cette valeur, supérieure à celle de l'enquête française, soutient l'intérêt de dissocier les questions en les ciblant de manière plus spécifique.

Tableau 3.3: nombre moyen de changements cités parmi les individus ayant cité au moins un événement, selon les trois questions de l'enquête

\begin{tabular}{l|c|c|c}
\hline & $\begin{array}{c}\text { Volet I } \\
\text { Changements } \\
\text { récents }\end{array}$ & $\begin{array}{c}\text { Volet II } \\
\text { Grands tour- } \\
\text { nants de la vie }\end{array}$ & $\begin{array}{c}\text { Volet III } \\
\text { Changements } \\
\text { historiques }\end{array}$ \\
\hline Nb moyen de changements & 1.64 & 1.94 & 1.80 \\
$\begin{array}{l}\text { Selon l'enquête } \\
\text { Bandra East }\end{array}$ & 1.90 & 2.34 & 2.01 \\
Santa Cruz & 1.45 & 1.58 & 1.60 \\
$\begin{array}{l}\text { Selon le sexe et l'enquête } \\
\text { Bandra East }\end{array}$ & 1.99 & 2.36 & 1.94 \\
femmes & 1.80 & 2.32 & 2.10 \\
hommes & 1.35 & 1.56 & 1.59 \\
$\begin{array}{l}\text { Santa Cruz East } \\
\text { femmes } \\
\text { hommes }\end{array}$ & 1.53 & 1.61 & 1.61 \\
\hline
\end{tabular}




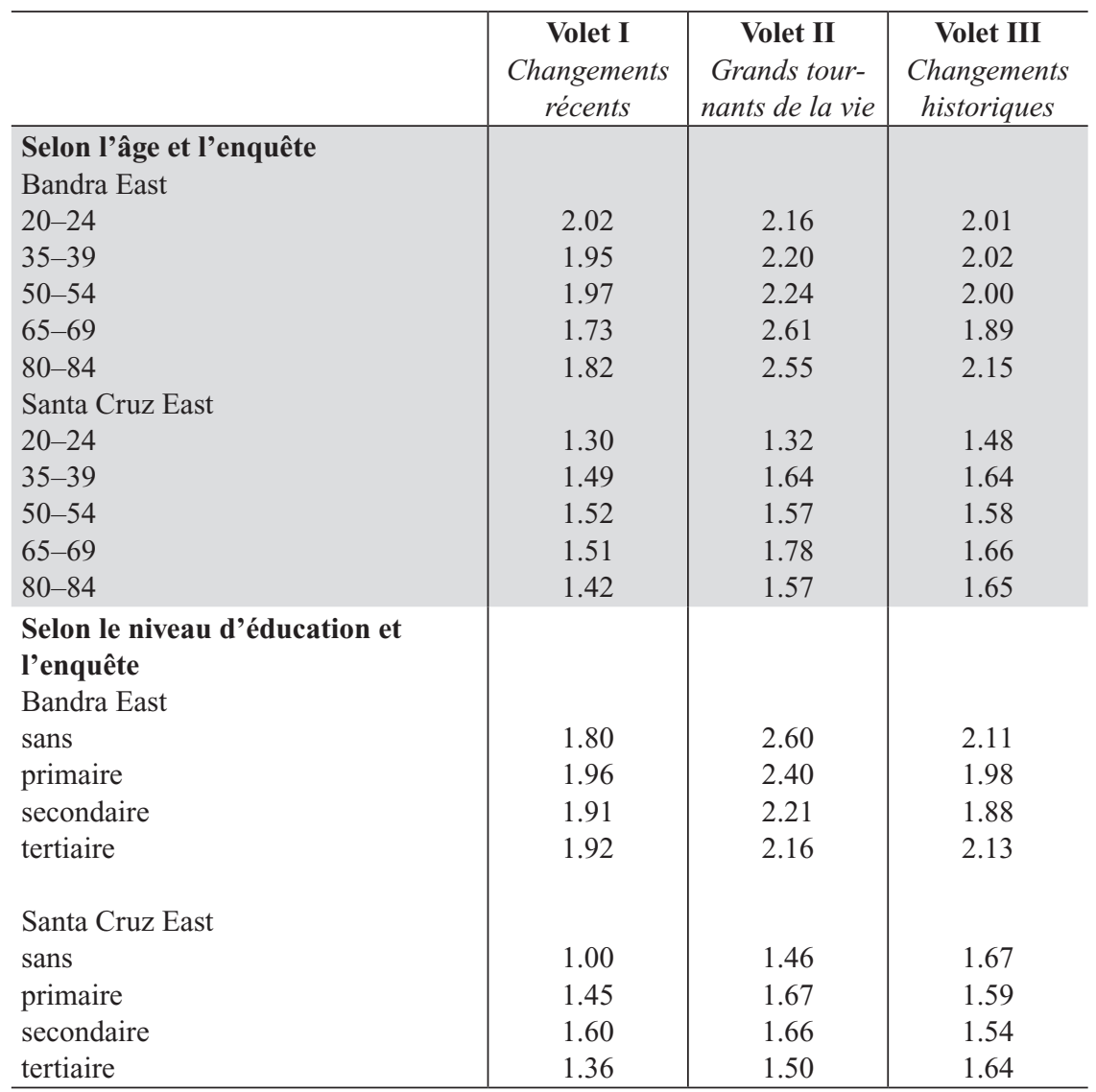

Par ailleurs, l'articulation entre les questions portant sur le domaine du micro (histoire personnelle, volet II) et du macro (histoire globale, volet III) permet, dans notre étude, de creuser à la fois les événements marquants des trajectoires personnelles, en lien avec la toile historique de fond qui a marqué les années de vie des répondants (Elder, 1999 [1974]; Mills, 1997 [1959]). Cela donne également accès à une clé d'interprétation du fonctionnement mémoriel de groupes d'individus définis par leur âge (donc leur cohorte de naissance), mais aussi leur genre et leur niveau de vie. Enfin, la perspective subjective qui sous-tend chacun des volets du questionnaire donne lieu à une vaste liberté d'interprétation par les individus de ce qui constitue un événement important, offrant un complément utile à 
l'usuel aspect objectif majoritairement présent dans les recherches en Inde à l'heure actuelle.

\subsubsection{Parler de sa vie : l'enquête comme outil et comme filtre du discours}

Au cours de ce chapitre, les contraintes liées à la réalisation des enquêtes, aux particularités de la population étudiée et du contexte culturel dans lequel elle évolue, à notre dessin d'étude et à la composition de nos équipes d'enquêteurs ont été soulignées. Accepter cette situation et en tirer parti (dit autrement, reconnaître et jouer avec la subjectivité du chercheur, de l'enquêteur et du répondant) est fondamental (de Sardan, 2000). Demander à un individu de parler de sa vie ou de tout objet social est un moyen fantastique d'obtenir une connaissance de la réalité, mais signifie conjointement apposer un filtre sur les informations perçues. La réflexion qui suit a pour objectif de mettre en évidence quelques dimensions importantes pouvant influencer les réponses des enquêtés.

\section{L'utilité d'une méthode mixte : l'apport du qualitatif au savoir quanti- tatif...et vice-versa}

Nous l'avons dit, enquêter la subjectivité des parcours de vie est habituellement envisagé au travers d'une méthode d'étude qualitative, et plus rarement quantitative. Le format de notre questionnaire nous a toutefois permis de ne pas opter pour l'une ou l'autre de ces options méthodologiques.

Ce choix s'explique par deux considérations fondamentales : la prépondérance de recherches portant sur les parcours de vie occidentaux et la nécessité de dépasser les interprétations préconçues des événements de la vie tels qu'ils sont étudiés dans l'espace atlantique. Le premier argument souligne la méconnaissance du déroulement des trajectoires hors du monde dit le plus développé économiquement (Dannefer, 2004). Pour les atteindre, le quantitatif s'est révélé adéquat en fondant une première analyse générale des événements ayant un impact sur les vies. Relevons que les questions posées dans le cadre du projet CEVI sont ouvertes, afin de laisser autant que possible la liberté aux répondants d'évoquer les changements qu'ils considèrent importants. Ainsi, au lieu de se saisir du souvenir d'un événement prédéfini comme marquant et de procéder à l'étude de sa construction et de ses implications, la recherche repose sur l'évaluation 
par les individus eux-mêmes des différents points d'inflexion de leur biographie prise dans sa totalité.

Malgré cette liberté d'élection des changements évoqués, tenir compte de la subjectivité appelle à dépasser les résultats chiffrés pour aller creuser les éléments qualitatifs des réponses. Le second argument repose donc sur le besoin de révéler les dimensions supplémentaires présentes dans les événements mentionnés par des individus se situant dans une culture et une société encore peu étudiée sous l'angle du parcours de vie, pour mieux en approcher la portée. En cela, le questionnaire CEVI permet, au travers de quelques phrases, de mettre en avant la parole individuelle.

Néanmoins, CEVI demeure un outil simple, dont les possibilités analytiques qualitatives (et quantitatives) sont limitées. Qui plus est, dans notre cas, la double traduction effectuée, d'une part dans la retranscription écrite de l'entretien en face-à-face par les enquêteurs; d'autre part dans la traduction effective du marathi ou du hindi vers l'anglais, rend ce matériau impropre à l'analyse textuelle. De même, des analyses statistiques poussées des données quantitatives sont parfois impossibles au vu de la taille de l'échantillon et de sa non-représentativité. Enfin, s'ajoutent à ces limites celles imposées par le contexte de passation de l'enquête.

\section{De la position intervieweur-interviewé dans la relation d'enquête}

Les méthodes qualitatives, tout comme celles d'obédience quantitative, parce qu'elles sont le produit d'une rencontre entre deux ou plusieurs individus, se fondent sur des relations sociales situées au sein d'une structure plus large, dont on ne peut ignorer les effets au moment de l'échange (Bourdieu, 1998a). Quelle que soit sa forme (du questionnaire dit fermé à l'entretien ouvert), un entretien est une interaction entre l'enquêteur, le répondant et l'objet de l'étude (Kaufmann, 2011; Masson \& Haas, 2010; Moscovici \& Buschini, 2003). Il est important de s'intéresser à la dynamique entre ces trois pôles, notamment entre l'intervieweur et l'interviewé puisque chacun d'eux possède des caractéristiques, définissant des positions et des rôles particuliers (Bourdieu, 1998a; Masson \& Haas, 2010). La subjectivité (soit la capacité de s'exprimer comme sujet) est alors soumise aux rapports de force sociaux ambients (Amossy, 2010).

Par ailleurs, il est crucial de tenir compte de la représentation que se fait l'enquêté de la situation d'enquête et de la relation particulière qu'elle introduit, afin de percevoir les contraintes en jeu au moment de la réponse (Amossy, 2010; Bourdieu, 1998a). L'entretien n'est pas une 
simple discussion et n'a rien de neutre, même si ce constat ne doit pas condamner le processus. En effet, des apports bénéfiques comme négatifs se remarquent. D'un côté, il permet un échange différent entre l'enquêteur et l'enquêté, plus égalitaire (par exemple lorsque le répondant perçoit qu'il dispose d'un espace d'écoute et de la prise en compte de son avis), et de l'autre il modifie leur relation et par-là la nature de l'information produite (Blanchet \& Gotman, 2010).

$\mathrm{Au}$ sein de cette relation, peuvent émerger de la part des répondants des divergences d'interprétation des questions posées, ou des résistances face aux consignes imposées. L'enquêteur a pour objectif de mettre en valeur la parole du répondant (Amossy, 2010); de son côté, ce dernier communique sa façon de percevoir la réalité, selon ce qui lui semble avoir compris de la demande et ce qui lui paraît pertinent comme message à donner. Dans d'autres circonstances, face à un autre enquêteur, il est possible que ce message varie, sans pour autant que cela implique machiavélisme ou transformation de la vérité. C'est le lien social avec l'enquêteur qui tend à mettre en avant tel ou tel aspect du problème, encourage à employer telle ou telle expression, tel ou tel mots (Moscovici, 1996 [1969]). Ainsi, les réponses à notre enquête se doivent d'être comprises en tenant compte de l'interaction dans laquelle elles ont été formulées, que ce soit dans leur contenu ou dans leur forme.

\section{La parole et la langue dans le discours}

Issus des rapports de force entre les individus, outre leur fonction de communication, les échanges linguistiques sont aussi des rapports de pouvoir symboliques $^{32}$ (Bourdieu, 1982). «[D]es pressions multiples s'exercent sur les catégories dominées, leur imposant un vocabulaire qui ne correspond pas à leur expérience, ne leur donnant pas les clés de l'accès au pouvoir des décisions centrales, même multipliant sous une forme apparemment neutre ou même favorable les signes de dépendance.» (Touraine, 2003, p. 132). Lintervention sociologique doit tenir compte de ces contraintes,

32 Des discours stylistiquement caractérisés, propres aux classes, entre des agents dotés des clés de compréhension: il s'agit en fait d'une facette du capital symbolique (ou culturel). La langue est alors à la fois une production de l'habitus et l'habitus linguistique (lorsqu'elle est uniquement pratique, avant la codification rigide) (Bourdieu, 1982). La langue standard apparaît avec l'Etat nation, propagé par le système éducatif. De son côté, le capital linguistique se transmet comme le capital culturel et dépend également du niveau d'éducation. 
ne pas donner à l'acteur une liberté totale de choix et d'action qui serait factice, mais connaître les filtres imposés par la langue (Touraine, 2003).

Manier plus ou moins la parole incite à parler d'un sujet et à avoir la capacité à le faire, ou à choisir de ne pas en parler (Bourdieu, 1982). Il s'agit de tenir compte de la dénomination des faits sociaux, de l'impact des classes sur la compréhension et l'expression de ceux-ci, afin de saisir comment la pensée et la perception du monde se structurent. La langue est aussi une représentation, faite de présupposés, qui fonctionne comme un pouvoir. Le seul fait de montrer ou dire une chose est déjà une prise de position (critique ou complice) (Bourdieu, 1982).

Nous avons déjà souligné les implications des doubles traductions de notre étude, de l'oral enquêté à l'écrit enquêteur, du marathi ou du hindi à l'anglais. A quoi s'ajoute le passage au français dans ce livre, qui met en avant des citations apparemment «polies» et «lissées», alors qu'elles ont probablement été dites à l'origine dans une langue populaire. Comme cela a été indiqué ci-dessus, l'analyse textuelle n'est pas envisageable ; il s'agit tout de même de garder à l'esprit ces multiples travaux effectués sur nos données, qui pourraient conduire à penser que le discours de nos interviewés est plus artificiel et académique qu'il ne l'a sans doute été au moment de son énonciation. Il demeure que réaliser la présence et - peutêtre même davantage - l'absence de certaines paroles, de certains mots, comporte un intérêt notoire.

***

De ces quelques réflexions théoriques, le terrain de Mumbai et ses spécificités ne ressortent guère ; nous pourrions comprendre que le lecteur s'interroge sur la raison d'être de la section qui précède. Ce sont en fait les chapitres empiriques qui feront ressortir les différentes dimensions évoquées ci-dessus. Leur confrontation à la théorie sera reprise dans les conclusions générales du livre.

Au-delà, nous avons d'emblée noté, au début de ce chapitre, que l'appréciation subjective par les individus de leur propre parcours de vie est une thématique qui a attiré les chercheurs en Occident depuis quelques décennies déjà. Dans les pays du Sud, elle reste peu courue, surtout lorsqu'on la compare aux études qui mettent en avant les informations démographiques, socioéconomiques ou sanitaires «factuelles». Pourtant, questionner la perception des trajectoires de vie et des changements sociétaux qui ont pu les affecter (les changements au niveau micro étant interdépendants de ceux du niveau macro) a fait sens lors des deux 
enquêtes réalisées à Mumbai, l'une dans un quartier de bidonvilles et l'autre dans un quartier de classe moyenne inférieure, pour les chercheurs comme pour les enquêtés.

Cette démarche, qui vise avant tout à placer les individus au centre de la réflexion, a certes dû être adaptée au contexte (parfois très vulnérable), un processus facilité par l'utilisation d'un questionnaire simple et court. Par ailleurs, avoir demandé aux répondants de sélectionner les événements marquants de l'année écoulée, de leur vie entière, puis de l'histoire vécue, ajoute à la subjectivité des réponses celle du choix électif de quelques changements parmi un large potentiel. Afin de comprendre ces choix, la méthode utilisée est mixte : l'analyse des éléments de réponse, aisément traitables quantitativement, se double de celle des raisons avancées par les répondants eux-mêmes, qui donnent une certaine profondeur qualitative aux résultats. Cette imbrication des matériaux d'enquête offre donc des possibilités de mettre en œuvre des méthodes mixtes d'analyses de données, selon la définition proposée par Mario Small (2011). En cela, elle correspond à la nécessité cruciale en sciences sociales de repenser les méthodologies, en cessant d'opposer quantitatif et qualitatif, afin de reconnaître que les fondements de l'interprétation sont multiples (Small, 2011). 



\section{Chapitre 4. Temporalités et contenus des souvenirs personnels}

"Greater attention must be paid to the "subjective"
sides of socialization experiences: how "actors"
understand the experiences they have. How they
make and remake, and assign and reassign,
meanings to experiences as they move through
life."

(Settersten, 2002, p. 23)

Les trajectoires de vie ne sont pas de longs fleuves tranquilles, comme la théorie nous l'a montré (voir la section 2.3.3). Des phases de continuité sont interrompues par des changements, plus ou moins abrupts (Cavalli \& Lalive d'Epinay, 2008; Levy, Ghisletta, Le Goff, Spini, \& Widmer, 2005). Parallèlement, aux différents âges sont attribués par les normes sociales des rôles, qui participent à stratifier la société (Levy \& Bühlmann, 2016), et des transitions spécifiques assurant le passage des uns aux autres. Le nombre et le contenu des événements qui composent ces transitions ne sont donc nécessairement pas les mêmes en fonction de la position de l'individu dans son existence (life stage) et, bien évidemment, de son sexe, certaines étapes connaissant une fréquence de changements plus élevée (Bidart, 2005; Cavalli \& Lalive d'Epinay, 2008; Fiske \& Chiriboga, 1990). La première partie de ce chapitre se penche sur cet aspect. Combien et quels changements marquent subjectivement les vies d'Indiennes et d'Indiens de différentes classes d'âge habitant à Mumbai ?

Bien sûr, la façon dont les groupes d'âge appréhendent les événements est également impactée par l'évolution historique influençant le développement des cohortes de naissance correspondantes (Settersten, 1999). Suivant ce principe, la seconde partie de ce chapitre se penche sur l'observation des grands tournants de la vie, qui offre une compréhension plus fine de l'articulation des trajectoires. Dans cette section, nous abandonnons le point de vue des diverses positions au sein du parcours de vie pour considérer l'entièreté de l'existence. Les âges auxquels les tournants ont lieu, ainsi que leur contenu, sont des sources précieuses d'informations sur la manière 
dont les vies se sont déroulées pour des femmes et des hommes vivant en cette aube du $21^{\text {ème }}$ siècle dans une mégapole, en plein cœur de l'Inde.

Les transitions de la vie sont des moments où la vulnérabilité individuelle prend par essence appui (Levy et al., 2005; Oris et al., 2009). Ce sont des périodes où les capitaux à disposition des individus se modifient, et avec eux leur niveau de vie. Historiquement, trois moments de l'existence où la vulnérabilité des individus est particulièrement forte ont été répertoriés: lors de la prime enfance, au moment de la reproduction $\mathrm{du}$ couple (lorsque les enfants sont encore très jeunes) et dans les vieux jours (Rowntree, 1971 [1901]) ${ }^{1}$. D'un point de vue subjectif, nous postulons que les changements évoqués par les individus sont des révélateurs des incertitudes et des obstacles qui ont marqué leur existence (Cavalli \& Lalive d'Epinay, 2008). En termes de position d'âge ou d'un point de vue rétrospectif embrassant l'entier de la vie, nous nous penchons dans cette troisième partie de chapitre sur les moments (âges) et les formes (contenus) que peuvent prendre ces vulnérabilités.

\subsection{Le nombre et la distribution des événements perçus comme marquants à différentes étapes du parcours de vie}

Lorsqu'il a été demandé aux individus de lister les changements qu'ils jugent importants et qui sont survenus durant leur dernière année de vie, l'objectif était autant de connaître le contenu de ces événements que de

1 L'exposition au stress s'accroit inévitablement au cours de la vie (Lowenthal \& Chiriboga, 1975), chaque étape apportant son lot de difficultés, d'épreuves. Toutefois, certaines en sont plus remplies que d'autres (Holmes \& Rahe, 1967). En 1901, Rowntree explique la pauvreté dans un lien entre parcours de vie et tensions économiques familiales (Rowntree, 1971 [1901]). Prenant appui sur ses observations de familles miséreuses en Angleterre, il démontre l'existence de trois moments de l'existence où la vulnérabilité des individus est particulièrement forte: lors de la prime enfance, au moment de la reproduction du couple (lorsque les enfants sont encore très jeunes) et dans les vieux jours. A ces moments, le salaire d'un seul individu suffit difficilement à nourrir toute la famille car les enfants sont encore dépendants et les âgés ne gagnent plus assez. Au travers de ce cycle reproductif des ménages, Rowntree explique la persistance de la pauvreté, que l'arrivée des politiques publiques occidentales a permis de briser (Bengtson \& Allen, 1991). 
comparer leur fréquence, pour des femmes et des hommes se situant à diverses positions de leur parcours de vie. Les recherches menées en Occident ont étayé le fait que les adultes et surtout les personnes âgées vivent moins de changements marquants que les jeunes entrant dans l'âge adulte (ayant entre 15 et 30 ans), essentiellement parce que ces années de jeunesse sont associées à des transitions sociodémographiques importantes, des bifurcations telles que la sortie des études, le début de la phase active de la vie, la mise en couple et la constitution de la famille (Bidart, 2010; Calves et al., 2006; Tichit \& Lelièvre, 2006).

Ceci a aussi été constaté dans des recherches focalisant sur la perception subjective des changements vécus (Fiske \& Chiriboga, 1990; Folkman et al., 1987), avec quelques nuances toutefois en ce qui concerne les retraités. Il arrive en effet que le nombre d'événements stagne après la sortie de la période active, alors que dans d'autres études il continue de chuter (Chiriboga, 1996; McLeod, 1996). Dans l'Inde urbaine moderne, la question de la retraite ne se pose pas pour grand monde. Par ailleurs, en fonction du sexe et du niveau socioéconomique, l'accès aux études n'est pas toujours envisageable. En ce sens, dans ce contexte si différent, la partie qui suit a pour objectif de questionner la densité de changements à des positions d'âge diverses, en tenant compte de l'environnement de résidence (soit de l'enquête) et du sexe des enquêtés.

Les questionnaires ont permis aux répondants d'évoquer librement ce qu'ils considéraient comme un changement au cours de l'année écoulée : ce point de vue subjectif laisse toute latitude à l'évocation d'événements imprévus par les chercheurs, permettant également de mentionner des faits qui ne concernent pas immédiatement la personne (ex.: «My eldest son got job in office» [femme de 40 ans, Bandra]). Néanmoins, nous partons du principe que si l'interviewé l'a évoqué, c'est que le changement affectant autrui a aussi une importante conséquence sur sa propre vie. C'est donc l'ensemble des changements récents mentionnés par nos enquêtés qui sont considérés, afin de déduire la densité événementielle dans les vies d'adulte en Inde.

\subsubsection{Fréquence des changements selon la position dans le parcours de vie}

Au total, 1231 changements récents ont été signalés (670 en 2012 et 553 en 2014). A Bandra comme à Santa Cruz, les interviewés sont près de deux 
tiers à évoquer un souvenir au moins au cours de la dernière année de vie (respectivement $57 \%$ et $61 \%$ ) (voir le tableau 4.1). Dans les deux échantillons, les écarts entre les sexes et entre les classes d'âge sont modestes, évoluant entre $50 \%$ et $65 \%$ d'individus ayant cité au moins un événement.

Le nombre moyen de changements récents parmi les individus ayant rapporté au moins un événement se situe autour de 1.8 en 2012 et de 1.5 en 2014. L'explication de cet écart réside sans doute dans le mode de passation de chacune des enquêtes. Pour rappel, à Bandra les enquêtrices n'étaient pas directement rémunérées au questionnaire, alors que c'était le cas à Santa Cruz. Dans ce second terrain, la perspective de se voir refuser un questionnaire trop peu rempli a dû encourager les enquêteurs à trouver des individus ayant au moins un événement récent à raconter; à l'inverse, les enquêtrices en 2012 ont davantage laissé les répondants s'exprimer quand ils avaient un changement à évoquer, voire plusieurs.

Tableau 4.1: le pourcentage d'individus n'ayant pas ou ayant cité au moins un changement récent, selon l'enquête, le sexe et l'âge

\begin{tabular}{lcccc}
\hline & \multicolumn{2}{c}{ Bandra } & \multicolumn{2}{c}{ Santa Cruz } \\
\cline { 2 - 5 } & $\begin{array}{c}\text { Au moins un } \\
\text { changement } \\
\%(n)\end{array}$ & $\begin{array}{c}\text { Pas de } \\
\text { changement } \\
\%(n)\end{array}$ & $\begin{array}{c}\text { Au moins un } \\
\text { changement } \\
\%(n)\end{array}$ & $\begin{array}{c}\text { Pas de } \\
\text { changement } \\
\%(n)\end{array}$ \\
\hline $20-24$ ans & 58 & 42 & 65 & 35 \\
$35-39$ ans & 58 & 42 & 57 & 43 \\
$50-54$ ans & 50 & 50 & 56 & 44 \\
$65-69$ ans & 65 & 35 & 65 & 35 \\
$80-84$ ans & 51 & 49 & 65 & 35 \\
\hline Femmes & 58 & 42 & 58 & 42 \\
Hommes & 55 & 45 & 65 & 35 \\
\hline Général & $57(357)$ & $43(276)$ & $61(381)$ & $39(241)$ \\
\hline Moyenne (écart-type) & $1.83(0.80)$ & \multicolumn{3}{c}{$1.45(0.62)$} \\
\hline
\end{tabular}

Des analyses complémentaires ont confirmé le peu de divergences dans le nombre de changements récents répondus entre les deux terrains d'enquêtes. Une régression logistique (cf. tableau 4.2) a établi dans quelle mesure le fait de mentionner ou non au moins un événement au cours de la dernière année peut être mis en relation avec les caractéristiques sociodémographiques des répondants. Le lieu de vie (bidonville ou quartier de classe moyenne inférieure), le sexe, l'âge et le niveau d'éducation ont été un à un contrôlés, une fois l'effet des autres facteurs neutralisé, 
et aucune différence significative n'a été constatée (à l'exception des sexagénaires par rapport aux plus jeunes et des individus avec un niveau d'étude secondaire par rapport aux universitaires ; toutefois, ces résultats sont peu significatifs).

Contrairement aux observations faites en Occident, à Mumbai le nombre moyen d'événements rapportés ne varie guère entre les groupes d'âge. La différence majeure surgit lorsque les jeunes cohortes de chaque enquête sont comparées l'une avec l'autre. En effet, le groupe des 20-24 ans vivant en bidonvilles et ayant cité au moins un changement mentionne en moyenne 2 événements, contre 1.3 pour leurs homologues habitant à Santa Cruz. Au niveau des sexes, les femmes des bidonvilles ayant cité au moins un changement évoquent en moyenne davantage d'événements que les hommes ( 2 contre 1.8). Somme toute, les différences restent faibles : dans nos enquêtes, être à l'orée de la vie adulte ne semble pas signifier une période de vie plus turbulente, plus riche en moments marquants.

Cette absence de diversité dans la densité événementielle de nos échantillons ne reflète pas forcément une absence de stratification selon l'âge, car elle peut s'expliquer de diverses façons. En premier lieu, il est possible que les jeunes Indiennes et Indiens vivent moins d'événements entre 19 et 24 ans que ceux mentionnés par les jeunes Occidentaux, en raison de leur moindre accès à l'éducation et de la nécessité associée d'entrer plus précocement, avant 19 ans, sur le marché du travail. En deuxième lieu, les personnes d'âge mûr à Mumbai peuvent subir davantage de changements probants que dans des contextes plus favorisés, en lien avec les conditions de vie plus difficiles en termes sanitaires et de protection sociale.

Dans une étude méthodologiquement similaire à la nôtre mais passée à Genève en 2003, Cavalli et Lalive d'Epinay (2008) observent bel et bien une densité de changements plus élevée durant la jeunesse, mais pas de diminution du nombre d'événements rapportés entre 50 et 84 ans, laissant entrevoir une maturité et une vieillesse qui connaissent également leur lot d'agitation. Dans le contexte indien, nous l'avons dit, la retraite ne représente pas une étape universelle, loin s'en faut. Toutefois, ne pas avoir de changement important en référence à la sortie de la vie active (qui marquerait en particulier les individus positionnés entre 65 et 69 ans) ne semble pas un obstacle au fait de mentionner des événements dans la vieillesse, dénotant d'une certaine similarité avec les résultats suisses. 
Tableau 4.2: rapports de cotes issus de modèles de régression logistique sur la variable «avoir mentionné au moins un changement récent»²

\begin{tabular}{|c|c|c|c|c|c|}
\hline & modèle 1 & modèle 2 & modèle 3 & modèle 4 & modèle 5 \\
\hline \multicolumn{6}{|c|}{ Lieu de vie (référence $=$ Santa Cruz East) } \\
\hline Bandra East & $0.806^{\circ}$ & $0.810^{\circ}$ & $0.810^{\circ}$ & 0.865 & 0.891 \\
\hline \multicolumn{6}{|c|}{ Sexe (référence $=$ Hommes) } \\
\hline Femmes & & 0.928 & 0.928 & 0.970 & 1.025 \\
\hline \multicolumn{6}{|c|}{ Classe d'âge (référence $=20-24$ ans) } \\
\hline $35-39$ ans & & & 0.863 & 0.859 & 0.937 \\
\hline $50-54$ ans & & & $0.724^{\circ}$ & 0.737 & 0.843 \\
\hline $65-69$ ans & & & 1.207 & 1.296 & $1.494^{\circ}$ \\
\hline $80-84$ ans & & & 0.846 & 0.953 & 1.100 \\
\hline \multicolumn{6}{|c|}{ Education (référence $=$ Supérieure) } \\
\hline Sans & & & & 0.821 & 0.792 \\
\hline Primaire & & & & 0.898 & 0.925 \\
\hline Secondaire & & & & $1.306^{\circ}$ & $1.326^{\circ}$ \\
\hline \multicolumn{6}{|c|}{ Santé auto-évaluée (référence $=$ Très bonne) } \\
\hline Bonne & & & & & $0.700^{\circ}$ \\
\hline Moyenne & & & & & $0.532 * *$ \\
\hline Mauvaise & & & & & 1.375 \\
\hline
\end{tabular}

Seuils de significativité ${ }^{\circ} \leq 0.1 ; * \leq 0.05 ; * * 0.01 ; * * * 0.001$.

Fondamentalement, c'est moins la fréquence que le contenu des événements qui peut faire ressortir une stratification selon l'âge. A cet égard, un aspect intéressant est révélé par les tests de régression. Il concerne la perception de sa propre santé par l'interviewé, et plus précisément la propension à évoquer autant de changements lorsque l'on se considère en mauvaise santé qu'en très bonne. Etre mal en point provoque une perception discontinue de l'existence, hachée par des événements. Ceci suggère bien que la nature des changements varie entre les groupes plus que leur nombre. Avant de se pencher sur le contenu, la section suivante sort des positions dans le cours de la vie pour explorer une vision rétrospective longitudinale à travers laquelle les individus portent un regard global sur leur existence et y «disposent» les événements cruciaux de leur vie.

2 Lors de chaque régression présentée dans ce travail, une vérification de la corrélation des variables a été faite. Il n'est jamais apparu de corrélation trop élevée pouvant conduire à un biais dans les résultats des modèles. 
4.1.2 La temporalité des grands tournants de l'existence selon les habitants de Mumbai

Partant du principe qu'une transition dans la vie représente une source potentielle de vulnérabilité (Levy et al., 2005; Oris et al., 2009), déterminer à quels âges se situent les grands tournants de l'existence permet de situer des moments «typiquement» vulnérables, d'après la perception des acteurs eux-mêmes. La question de leur fréquence durant certaines phases de l'existence - elles-mêmes davantage propices au changement - se repose à nouveau, mais documentée différemment par le second volet de l'enquête.

\subsubsection{La répartition des tournants selon les groupes d'âge}

La figure 4.1 présente la répartition des grands tournants de l'existence selon l'âge des répondants au moment de leur survenue. Les classes d'âge déterminées au moment des enquêtes (respectivement en 2012 et 2014) ont été considérées séparément, afin d'observer les profils de chacune. En effet, nos données comportent un biais méthodologique important : la durée de vie des interviewés n'est pas la même (plus courte pour les jeunes, plus longue pour les aînés); ainsi, la concentration des tournants devra forcément être plus grande pour les 20-24 ans que pour les autres cohortes.

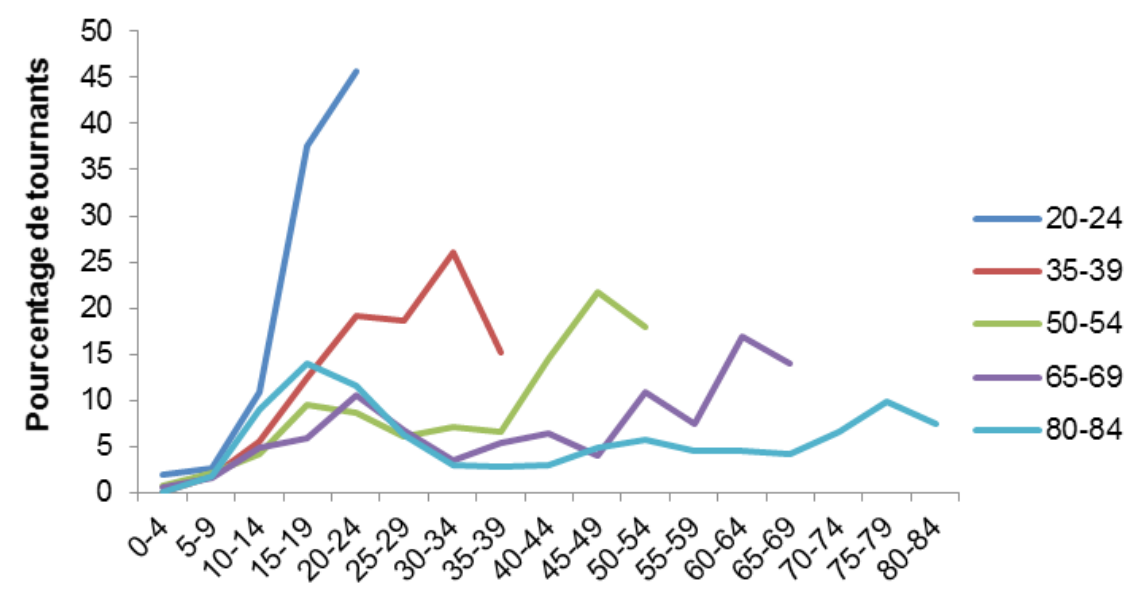

Age au moment du tournant

Figure 4.1: la répartition des changements selon l'âge des répondants au moment de leur survenue $(\%)$ 
La répartition des tournants ne présente pas de tendance très nette, même s'il semble qu'entre 15 et 30 ans une légère croissance du nombre de changements s'observe dans la plupart des groupes. Ce phénomène est particulièrement visible chez les plus jeunes et les plus âgés. Dans les autres groupes, un second pic survient plus tard et prend le pas sur cette première bosse. Il se situe autour de 30-34 ans pour les trentenaires, de 45-49 ans pour les quinquagénaires, de 60-64 ans pour les sexagénaires et - bien que faible - entre 75 et 79 ans pour les octogénaires. Autrement dit, quelques années seulement (entre 1 et 5 ans) avant la passation de l'enquête.

Nous avons évoqué dans l'introduction de ce chapitre les moments typiquement vulnérables de la vie qui ont été constatés empiriquement dans des familles pauvres des pays industrialisés au $19^{\text {ème }}$ siècle par Rowntree (1971 [1901]). S'ils devaient se retrouver dans l'Inde urbaine, à Mumbai, ils auraient émergé et sur la figure 4.1 les courbes de nos groupes d'âge se seraient superposées. Au contraire, après un premier rebond (sauf pour les jeunes où il est plus marqué) chaque classe montre une remontée sur les années les plus récentes, les plus proches de la collecte de données (donc, chacune à un âge différent). Davantage que des moments de fragilité ou de turbulence inscrits dans la structure de l'existence, il semble que les souvenirs de nos répondants se répartissent selon la courbe classique de distribution des souvenirs au cours du développement (voir chapitre 3.1) (Conway, 1997; Holmes \& Conway, 1999; Rubin, Wetzler, \& Nebes, 1986). Ainsi, dans nos données se constatent une légère tendance au reminiscence bump et surtout un effet de récence plus net (cf. section 3.1.1).

\subsubsection{Un faible pic de souvenirs durant l'adolescence et l'entrée à l'âge adulte}

Pour mieux comprendre le reminiscence bump, plusieurs modèles de régression logistique ont été estimés sur la propension à «avoir cité au moins un tournant entre 15 et 30 ans», selon les caractéristiques sociodémographiques : lieu de vie, sexe, classe d'âge, niveau d'éducation et santé autoévaluée (cf. tableau 4.3). La variable dépendante a été construite de manière binaire : 0 ne pas avoir cité de changement et 1 en avoir cité au moins un.

Les rapports de cotes présentés dans le tableau 4.3 montrent globalement un effet lié au lieu de vie, à l'âge et au niveau d'éducation. Etre âgé de 20 à 24 ans augmente très nettement le risque de citer un événement entre l'adolescence et le début de l'âge adulte, ce qui n'a rien de surprenant, eut égard au biais méthodologique déjà évoqué (voir 4.1.2.1). Alors que 
le sexe n'apparaît pas comme déterminant, être éduqué et résider dans un bidonville accroissent également significativement la propension à mentionner un tournant entre 15 et 30 ans.

Un effet intéressant se dégage en scrutant de manière plus approfondie ce lien entre l'éducation et le lieu de vie. A éducation égale, les résultats montrent que les résidents des bidonvilles ont 1.5 fois plus de risque de citer un tournant situé durant la phase d'entrée dans l'âge adulte. Les habitants de Bandra possédant un niveau socioéconomique et une capacité d'accès à l'enseignement moindres, la prise en compte de l'effet de l'éducation fait ressortir un risque notable de reminiscence bump, peu apparent jusque-là.

Tableau 4.3: rapports de cotes issus de modèles de régression logistique sur la variable «avoir mentionné au moins un tournant situé au cours de l'entrée dans l'âge adulte»

\begin{tabular}{|c|c|c|c|c|}
\hline modèle 1 & modèle 2 & modèle 3 & modèle 4 & modèle 5 \\
\hline \multicolumn{5}{|c|}{ Lieu de vie (référence $=$ Santa Cruz East) } \\
\hline Bandra East & $1.239^{\circ}$ & 1.215 & $1.469 * *$ & $1.478 * *$ \\
\hline \multicolumn{5}{|l|}{ Sexe (référence = Hommes) } \\
\hline Femmes & 1.027 & 0.973 & 1.072 & 1.082 \\
\hline \multicolumn{5}{|c|}{ Classe d'âge (référence $=20-24$ ans) } \\
\hline $35-39$ ans & & $0.328 * * *$ & $0.366^{* * *}$ & $0.377 * * *$ \\
\hline $50-54$ ans & & $0.104 * * *$ & $0.127 * * *$ & $0.132 * * *$ \\
\hline $65-69$ ans & & $0.114^{* * *}$ & $0.149 * * *$ & $0.150 * * *$ \\
\hline $80-84$ ans & & $0.130 * * *$ & $0.183 * * *$ & $0.184 * * *$ \\
\hline \multicolumn{5}{|c|}{ Education (référence $=$ Supérieure) } \\
\hline Sans & & & $0.535^{*}$ & $0.508 * *$ \\
\hline Primaire & & & $0.576^{* *}$ & $0.570 * *$ \\
\hline Secondaire & & & 0.858 & 0.859 \\
\hline \multicolumn{5}{|c|}{ Santé auto-évaluée (référence $=$ Très bonne) } \\
\hline Bonne & & & & 0.854 \\
\hline Moyenne & & & & 0.882 \\
\hline Mauvaise & & & & 1.308 \\
\hline
\end{tabular}

Seuils de significativité : ${ }^{\circ} \leq 0.1 ; * \leq 0.05 ; * * 0.01 ; * * \leq 0.001$.

Les figures 4.2 et 4.3 exposent à nouveau la répartition des tournants selon l'âge des individus au moment de leur survenue, mais cette fois en séparant les deux lieux d'enquête, afin de mettre en lumière ces divergences liées au niveau socioéconomique des répondants. De manière générale, il ne ressort pas de grande divergence entre les deux figures. Toutefois, le 
premier pic de rappel (pouvant correspondre au reminiscence bump) se situe entre 10 et 24 ans à Bandra et entre 15 et 29 ans à Santa Cruz, et ce pour toutes les cohortes sauf la plus jeune. Emerge ainsi un décalage des souvenirs personnels en fonction du lieu de résidence, qui suggère la fin précoce de l'enfance, l'obligation de la quitter trop tôt, là où les conditions de vie sont les plus dures.

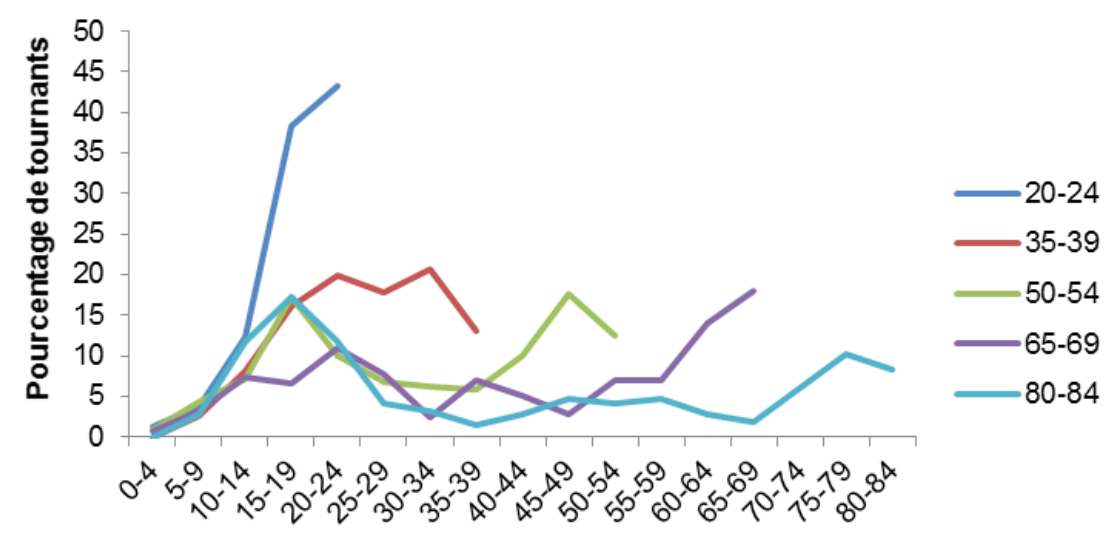

Age au moment du tournant

Figure 4.2: les tournants de la vie (\%) selon l'âge des répondants de Bandra au moment de leur survenue

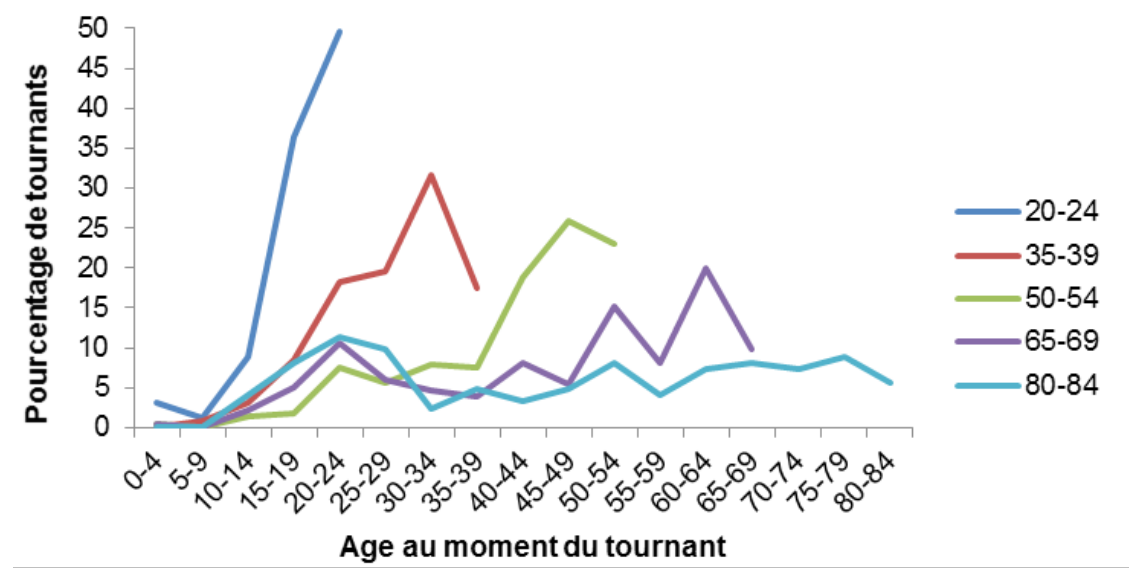

Figure 4.3: les tournants de la vie (\%) selon l'âge des répondants de Santa Cruz au moment de leur survenue 
Plus généralement, la présence d'un reminiscence bump se doit d'être nuancée. Comparativement aux constats d'autres études empiriques, conduites dans l'espace atlantique, les données indiennes ne montrent pas un pic de rappel très important (Conway, 1997; Holmes \& Conway, 1999). En outre, il est envisageable que ce soit le contenu des tournants qui explique cette propension plus élevée de mentions. Par exemple, nous avons constaté qu'être éduqué est associé à une probabilité plus grande d'évoquer un événement majeur durant cette période. A rebours, il est aussi possible que d'avoir été à l'école, voire à l'université, signifie posséder des souvenirs liés à la formation, ce que les personnes peu scolarisées ne peuvent bien sûr pas avoir. En somme, c'est l'idée que les parcours de vie des individus avec les statuts socioéconomiques les plus bas posséderaient une densité mémorielle plus faible que les trajectoires ascendantes, jalonnées d'étapes importantes. La partie qui suit a précisément pour objectif d'observer quels types d'événements sont cités, afin de brosser un premier tableau - qui sera approfondi dans les chapitres 5, 6 et 7- des divergences ou des ressemblances dans les contenus selon le lieu de vie, les groupes d'âge et le sexe.

\subsection{La perception des événements de la vie}

D'après la typologie par catégories de changement présentée en section 3.5.2, nous avons pu établir la distribution des événements les plus mentionnés par les individus. Tout d'abord, nous revenons sur les événements survenus au cours de la dernière année (volet I), afin d'avoir un regard transversal sur ce qui affecte l'existence d'habitants et d'habitantes de Mumbai adultes, en 2012 et en 2014, sur une temporalité courte. Ensuite, les tournants de la vie (volet II) sont à leur tour classifiés, afin de se rendre compte, d'un point de vue rétrospectif et longitudinal, de la façon dont les vies se déploient et s'articulent.

$\mathrm{Au}$ centre de la réflexion sur le contenu des changements dans la vie, l'hypothèse testée ici est qu'à chaque âge correspondent des événements particuliers (Cavalli et al., 2013; Cavalli \& Lalive d'Epinay, 2008). En Inde comme ailleurs, les parcours de vie sont soumis à des attentes différentes selon les positions (Elder, 1998; Elder \& George, 
2016). Il est donc intéressant de se pencher sur les événements typiques de l'existence pour des adultes situés à divers moments de leur trajectoire de vie. Mais plus en particulier dans un contexte de pauvreté de masse, des événements révélateurs de vulnérabilité peuvent trancher avec les résultats observés en Occident; ils seront mis en avant dans nos analyses.

\subsubsection{Des changements spécifiques aux diverses positions d'âge}

Le tableau 4.4 offre une vision liminaire du pourcentage d'événements apparus l'année précédant l'enquête et mentionnés par les individus, soit les réponses au volet I. L'addition des domaines en colonne (identifiés en gras) est égale à la totalité des mentions $(100 \%)$.

Tableau 4.4 les domaines et les types de changements récents les plus mentionnés (\%), selon l'âge et le lieu de résidence ${ }^{3}$

\begin{tabular}{l|cc|cc|cc|cc|cc|cc}
\hline Domaine & \multicolumn{2}{|c|}{ Total } & \multicolumn{2}{|c|}{$\mathbf{2 0}-\mathbf{2 4}$} & \multicolumn{2}{|c|}{$\mathbf{3 5}-\mathbf{3 9}$} & \multicolumn{2}{|c|}{$\mathbf{5 0}$} & \multicolumn{2}{|c|}{$\mathbf{6 5}-$} & \multicolumn{2}{|c}{$\mathbf{8 0}$} \\
Type & BA $^{4}$ & SC & BA & SC & BA & SC & BA & SC & BA & SC & BA & SC \\
\hline Famille & $\mathbf{1 7}$ & $\mathbf{1 3}$ & $\mathbf{2 3}$ & $\mathbf{2 0}$ & $\mathbf{1 4}$ & $\mathbf{1 6}$ & $\mathbf{2 3}$ & $\mathbf{1 2}$ & $\mathbf{1 4}$ & $\mathbf{7}$ & $\mathbf{9}$ & $\mathbf{8}$ \\
Mariage & 7 & 4 & 6 & 5 & 5 & 6 & 10 & 5 & 8 & 3 & 6 & - \\
Naissance & 7 & 5 & 10 & 5 & 7 & 7 & 8 & 3 & 4 & 3 & 2 & 5 \\
Décès & $\mathbf{4}$ & $\mathbf{4}$ & $\mathbf{2}$ & $\mathbf{2}$ & $\mathbf{4}$ & $\mathbf{2}$ & $\mathbf{2}$ & $\mathbf{6}$ & $\mathbf{7}$ & $\mathbf{5}$ & $\mathbf{4}$ & $\mathbf{4}$ \\
Santé & $\mathbf{4 1}$ & $\mathbf{3 9}$ & $\mathbf{1 2}$ & $\mathbf{1 6}$ & $\mathbf{3 3}$ & $\mathbf{2 4}$ & $\mathbf{4 1}$ & $\mathbf{3 2}$ & $\mathbf{5 8}$ & $\mathbf{5 6}$ & $\mathbf{8 0}$ & $\mathbf{7 4}$ \\
Maladie & 18 & 11 & 4 & 3 & 17 & 4 & 16 & 11 & 29 & 17 & 29 & 18 \\
Accident & 2 & 3 & - & 2 & 1 & 5 & 3 & 1 & 3 & 6 & 2 & 3 \\
Hospitalisation & 7 & 4 & 2 & 2 & 4 & 2 & 4 & 2 & 11 & 8 & 15 & 9 \\
Déclin graduel & 6 & 11 & 1 & 3 & 3 & 2 & 6 & 6 & 7 & 17 & 18 & 35 \\
Amélioration & 4 & 4 & 3 & 6 & 2 & 4 & 8 & 5 & 3 & 3 & 6 & 3 \\
Travail & $\mathbf{1 3}$ & $\mathbf{1 1}$ & $\mathbf{2 3}$ & $\mathbf{1 7}$ & $\mathbf{1 5}$ & $\mathbf{2 2}$ & $\mathbf{1 5}$ & $\mathbf{4}$ & $\mathbf{7}$ & $\mathbf{8}$ & - & $\mathbf{3}$ \\
Reprise/nouveau & 7 & 4 & 12 & 10 & 9 & 7 & 5 & 2 & 4 & 1 & - & 3 \\
Chômage & 3 & 1 & 6 & 1 & 2 & 2 & 2 & 1 & 2 & 2 & - & - \\
Promotion & 2 & 3 & 1 & 3 & 3 & 8 & 5 & - & - & 4 & - & - \\
Education & $\mathbf{6}$ & $\mathbf{6}$ & $\mathbf{1 7}$ & $\mathbf{2 0}$ & $\mathbf{4}$ & $\mathbf{7}$ & $\mathbf{2}$ & $\mathbf{3}$ & $\mathbf{1}$ & $\mathbf{1}$ & - & - \\
Début & 1 & 1 & 5 & 2 & 1 & 3 & 1 & - & - & 1 & - & - \\
Réussite & 3 & 3 & 7 & 11 & 3 & 3 & 1 & 3 & - & - & - & - \\
Economie & $\mathbf{6}$ & $\mathbf{8}$ & $\mathbf{4}$ & $\mathbf{9}$ & $\mathbf{1 2}$ & $\mathbf{1 0}$ & $\mathbf{7}$ & $\mathbf{1 1}$ & $\mathbf{7}$ & $\mathbf{6}$ & $\mathbf{1}$ & - \\
\hline
\end{tabular}

3 En gris les pourcentages où moins de cinq individus sont représentés.

$4 \quad \mathrm{BA}=$ Bandra

$5 \quad \mathrm{SC}=$ Santa Cruz 


\begin{tabular}{l|cc|cc|cc|cc|cc|cc}
\hline Domaine & \multicolumn{2}{|c|}{ Total } & \multicolumn{2}{|c|}{$\mathbf{2 0 - 2 4}$} & \multicolumn{2}{|c|}{$\mathbf{3 5}-\mathbf{3 9}$} & \multicolumn{2}{|c|}{$\mathbf{5 0}-\mathbf{5 4}$} & \multicolumn{2}{|c|}{$\mathbf{6 5}-$} & \multicolumn{2}{|c}{$\mathbf{8 0}-\mathbf{8 4}$} \\
Type & BA $^{4}$ & SC & BA & SC & BA & SC & BA & SC & BA & SC & BA & SC \\
\hline Mobilité & $\mathbf{3}$ & $\mathbf{3}$ & $\mathbf{2}$ & $\mathbf{2}$ & $\mathbf{1}$ & $\mathbf{4}$ & $\mathbf{4}$ & $\mathbf{1}$ & $\mathbf{2}$ & $\mathbf{3}$ & $\mathbf{4}$ & $\mathbf{4}$ \\
$\begin{array}{l}\text { Développement } \\
\text { personnel }\end{array}$ & $\mathbf{2}$ & $\mathbf{2}$ & $\mathbf{7}$ & $\mathbf{4}$ & $\mathbf{1}$ & - & $\mathbf{2}$ & $\mathbf{4}$ & - & $\mathbf{2}$ & - & $\mathbf{1}$ \\
Activités & & & & & & & & & & & & \\
Autres & $\mathbf{2}$ & $\mathbf{3}$ & $\mathbf{5}$ & $\mathbf{1}$ & $\mathbf{2}$ & $\mathbf{3}$ & - & $\mathbf{9}$ & - & $\mathbf{2}$ & - & $\mathbf{3}$ \\
\hline
\end{tabular}

La plupart des titres du tableau 4.4 sont explicites. Toutefois, nous avons opté pour ajouter quelques sous-domaines (des «types») afin de détailler les réponses contenues dans ces catégories. Parmi les domaines qui ne sont pas précisés, celui concernant l'«économie» regroupe des citations en lien avec l'inflation des prix, l'augmentation des dépenses du ménage ou des achats de biens. De son côté, la catégorie «mobilité» englobe les déplacements d'un lieu d'habitation à l'autre, généralement sur une distance plutôt courte : au sein du même Etat, voire d'un quartier à l'autre de la ville. Sous le terme générique de «développement personnel» se retrouvent les réponses ayant trait à une évolution comportementale, émotionnelle ou de la personnalité des individus (ex. : «My confidence decreased» [femme de 79 ans, Santa Cruz]) ou simplement à l'avancée en âge (ex. : «My son become 19 years old», [homme de 52 ans, Santa Cruz]). Enfin, les «activités» rassemblent les réponses évoquant le sport, la musique, la participation sociale, les activités extra-scolaires, etc.

Globalement, les deux terrains d'enquêtes présentent peu de divergences, les mêmes domaines de la vie affectent les individus. Seules les mentions «famille» et «santé» ressortent légèrement moindres à Santa Cruz qu'à Bandra. Nonobstant, dans un cas comme dans l'autre, c'est clairement la trajectoire de santé qui apparaît comme la source d'événements la plus citée (41\% des mentions en 2012 et 39\% en 2014). Qui plus est, le nombre de personnes évoquant la santé (qui n'est pas affiché dans le tableau 4.4, centré sur les changements) est de près d'un tiers pour Bandra (30\%) et de plus d'un quart à Santa Cruz (26\%), dénotant bien d'une vision des événements récents de la vie assez nettement centrée autour du domaine sanitaire. Au sein de ces mentions, ce sont les maladies que les personnes ont le plus souligné.

Cette présence de la santé est notable et distingue nos résultats de ceux observés dans des enquêtes similaires passées dans d'autres pays. En effet, c'est la famille, puis la profession et les déménagements qui ont été 
constatés comme les domaines les plus mentionnés par des Genevoises et Genevois interrogés en 2003 (Cavalli \& Lalive d'Epinay, 2008). De manière similaire, une enquête effectuée en 2004 à Buenos Aires a montré que les trajectoires familiales et professionnelles étaient bien davantage citées que la santé (Gastrón \& Lacasa, 2009). La santé constitue donc une dimension particulièrement prenante de la vie en milieu urbain en Inde. Ce résultat sera approfondi dans le chapitre 7.

Après la santé, la famille est le domaine le plus mentionné par les individus. Essentiellement, il s'agit de mariages et de naissances. L'évocation des décès a été placée dans une catégorie à part, mais de fait, ces disparitions concernent toutes des membres la famille et pourraient donc être ajoutées au domaine familial, portant de la sorte la fréquence des événements familiaux à $21 \%$ dans les slums et $17 \%$ à Santa Cruz. Entre les deux quartiers, ce sont toujours les habitants des bidonvilles qui évoquent davantage de changements familiaux.

Jusqu'ici, le contenu des changements les plus rapportés a trait à la sphère privée : famille et santé. Inversement, les domaines qui viennent ensuite font référence à la sphère publique. Il s'agit de la profession, de l'économie et de l'éducation. Ces trois dimensions peuvent être considérées comme appartenant à une même équation, qui décrit la situation socioéconomique des répondants. En effet, l'éducation mène aux opportunités professionnelles, et en cela revête une importance cruciale pour le parcours de vie. Bien sûr, il est vraisemblable que les jeunes soient tout particulièrement affectés par la formation, puisqu'une partie de ceux qui ont été interrogés sont toujours en étude aux âges considérés. Mais les autres cohortes peuvent également avoir des raisons majeures de mentionner ce domaine. Par exemple, la réussite des enfants, ou leur échec, auront des implications significatives pour leurs parents qui investissent en eux et sont ou seront dépendants de la solidarité intergénérationnelle dans leurs vieux jours. Cela étant, la quantité d'individus indiquant des événements éducatifs est similaire entre les deux quartiers.

Un certain nombre d'autres événements ont été cités par peu de personnes car plus inhabituels, plus rares et très liés au parcours de vie individuel : avoir vécu une agression, obtenir un permis de conduire, faire du sport par exemple. Selon la typologie plus fine des événements cités (par types et non par domaines), dans les deux quartiers les répondants offrent 
une palette de réponses autour d'une quarantaine de types ${ }^{6}$. Toutefois, les réponses données par les habitants des slums montrent une concentration plus forte autour de l'événement le plus cité : les maladies. En effet, 19\% de l'échantillon de Bandra a évoqué ce changement, le suivant (la reprise du travail) ne l'étant que par 7\% des individus. A Santa Cruz, les événements de santé comme la maladie ou le déclin graduel, bien que les plus cités, ne le sont que par un maximum de $8 \%$ de la population interrogée.

Par ailleurs, une large majorité des changements récents mentionnés concerne l'individu lui-même, et ce dans les deux terrains. Le classement a été organisé en trois catégories, selon que la description du changement désigne comme principal auteur le répondant, un membre de sa famille (même élargie) ou de manière imprécise la population. Dans cette répartition, la naissance d'un fils ou d'une fille sera placée dans le groupe se référant à la famille, bien que de toute évidence la personne ait également vécu directement l'événement. Si le répondant évoque un changement qui l'inclut aussi bien que sa famille, ledit changement est classé comme appartenant à la famille. A Bandra, ce sont $69 \%$ des événements qui sont imputables au répondant et $29 \%$ à son entourage familial (les 2\% restants se rapportant à la société dans son ensemble). A Santa Cruz, la répartition s'opère entre $70 \%$ de mentions liées à l'individu, $20 \%$ à ses apparentés et $10 \%$ à la société (comme l'inflation des prix).

\subsubsection{A chaque âge ses tracas}

L'éventail des événements cités se réduit avec l'avancée en âge, dans les deux quartiers. Bien que le nombre moyen d'événements récents cités par les différentes classes d'âge soit proche, la diversité des contenus de ces changements diminue et passe d'une trentaine chez les jeunes à une quinzaine chez les aînés. Autrement dit, une plus grande concentration des réponses s'observe chez les personnes âgées, qui sans surprise vivent plutôt des turbulences autour de leur santé, alors que les jeunes évoquent des changements récents de natures plus diverses.

\section{La santé : un désagrément qui n'est pas réservé aux personnes âgées}

La santé se confirme comme le domaine le plus cité, dans toutes les classes d'âge sauf chez les plus jeunes (cf. tableau 4.4). Les problèmes

6 Parmi cette quarantaine d'événements distincts, seuls 6 dans l'enquête de Bandra et 2 à Santa Cruz ont été mentionnés par plus de $5 \%$ des répondants. 
de santé sont ainsi évoqués précocement, de nombreux individus y faisant référence même parmi les adultes d'une trentaine ou d'une cinquantaine d'année, particulièrement dans les bidonvilles. Outre le pourcentage élevé de réponses concernant la trajectoire de santé, l'éventail des âges de la vie auxquels surviennent des événements sanitaires est bien plus large que ce qui a pu être constaté ailleurs. Dans l'enquête effectuée à Genève par exemple, seuls les 65-69 ans et les 80-84 ans évoquent massivement des événements de santé (Cavalli \& Lalive d'Epinay, 2008).

Lorsque l'on regarde les types d'événements que les individus citent dans ce domaine, il ressort que la maladie est la première source de souci évoquée à Bandra, et ce à partir de la trentaine. Par ailleurs, il s'agit du changement récent le plus mentionné par ces tranches d'âge s'étalant de 35 à 84 ans. Dans une large majorité, ces pathologies concernent les interviewés eux-mêmes $(86 \%)$ et plus rarement un proche. En revanche, si les maladies représentent $17 \%$ des changements cités par les sexagénaires et $18 \%$ de ceux mentionnés par les octogénaires interrogés à Santa Cruz, dans ce quartier de classe moyenne inférieure ils ne constituent que 4\% et $11 \%$ des réponses des 35-39 et des 50-54 ans. Inversement, dans tous les groupes d'âge sauf parmi les quinquagénaires, les habitants de Santa Cruz sont plus nombreux que dans les slums à citer des accidents, ce qui compense en partie la moindre mention de maladies.

Le déclin de la santé, les symptômes ou les douleurs ${ }^{7}$ sont fréquemment évoqués par les 80-84 ans de Santa Cruz (35\% des changements rapportés par les personnes âgées sont concernés, contre $18 \%$ dans les bidonvilles), alors que les maladies le sont moins que chez les pauvres (18\% à Santa Cruz contre $29 \%$ à Bandra).

Dans les deux terrains d'enquêtes, les 20-24 ans parlent moins des événements de santé que les autres, sans doute très simplement parce qu'ils sont eux-mêmes en meilleure santé et parce qu'ils n'ont généralement pas encore de parent ou d'enfant à charge. En effet, les jeunes qui mentionnent leur état évoquent avant tout des améliorations (sortir d'une maladie, ou avoir un problème médical «sous contrôle»). Par ailleurs, les problèmes de santé d'un membre proche de la famille signifient souvent des dépenses supplémentaires, des tracas, etc., et ne pas avoir encore de responsabilité vis-à-vis d'une autre personne peut expliquer

7 "I always have bodyache" (femme de 54 ans, Bandra); "There is pain in knees" (homme de 50 ans, Santa Cruz,); "As I am getting old, I am getting a little unwell" (femme de 80 ans, Santa Cruz). 
que les jeunes soient moins concernés. Dans la même logique, parmi les événements de santé, ils évoquent nettement moins de maladies que leurs aînés.

\section{Famille, travail et formation pour les jeunes}

A Bandra comme à Santa Cruz, une diminution du pourcentage d'individus évoquant des événements familiaux récents se remarque avec l'avancée en âge. Les problèmes de santé prennent peu à peu le pas sur des événements comme les mariages, les naissances, les rencontres.

D'un quartier à l'autre, ce sont les mêmes moments de la vie familiale qui sont mentionnés aux différents âges de la vie, sauf parmi les jeunes adultes de 20-24 ans. Pour ces derniers, la famille est évoquée comme source de changement plus souvent à Bandra, mais le contenu diffère également. Il semble que les jeunes des bidonvilles aient de l'avance sur leurs pairs pour ce qui est des événements de la vie (mariage, naissance, ...), les premiers mentionnant davantage de ces éléments démographiques (et plus de jeunes de l'échantillon de Bandra sont mariés et déjà parents (ce constat sera creusé davantage au chapitre 6).

Quant aux mariages et aux naissances, ils sont mentionnés dans toutes les classes d'âge. Toutefois, la question posée exigeait de parler des événements récents ; ainsi, selon l'âge de l'enquêté, ce n'est pas le même acteur qui est au centre du changement. Suivant une logique chronologique, les jeunes parlent majoritairement de leurs propres fiançailles, alors que lorsque ces mentions apparaissent dans les autres groupes, c'est plutôt pour rappeler celles de leurs enfants. Son propre mariage et celui de ses frères/ sœurs (membres de la même génération) se retrouvent aussi essentiellement chez les jeunes. Par contre, les unions des enfants sont plutôt citées par les groupes d'âge 35-39, 50-54 et 65-69 ans. Dans le même ordre d'idée, lorsque les 20-24 et 35-39 ans parlent de naissance, il s'agit essentiellement d'eux-mêmes entrant dans la parentalité, alors que parmi les 50 ans et plus, cela concerne leurs enfants, au travers de la naissance des petits-enfants.

Seuls les individus appartenant aux trois plus jeunes cohortes à Bandra et aux deux plus jeunes à Santa Cruz sont nombreux à évoquer la vie professionnelle. En leur sein, une variabilité s'observe lorsque l'on dépasse le niveau d'agrégation du domaine pour explorer les contenus plus spécifiques des mentions. Ces considérations seront approfondies au chapitre suivant. 
Le côté positif du travail (obtenir de la reconnaissance, avoir une promotion - en termes de poste ou de salaire, avoir son business qui va bien ou grandit) apparaît peu, aussi bien à Santa Cruz qu'à Bandra $(3 \% \text { et } 2 \%)^{8}$. Cette situation est cependant plus apparente chez les 35-39 ans, 8\% de leurs mentions à Santa Cruz évoquant une reconnaissance professionnelle (contre 3\% à Bandra). Nonobstant la prudence requise, puisque ces valeurs sont faibles. A l'inverse et sans surprise, le côté négatif du travail (licenciement, chômage) ressort plutôt parmi les réponses données dans les bidonvilles ( $3 \%$ contre $1 \%)$. A nouveau, si la fréquence globale est faible, des disparités ressortent selon l'âge. Les licenciements et le chômage sont quand même évoqués par $6 \%$ des jeunes pauvres et presque pas par leurs homologues de classe moyenne inférieure (1\%). Il n'est guère possible de dissocier ici ce qui relève de l'impact réel de la condition socioéconomique et de l'économie informelle, ou d'une perception plus négative de sa trajectoire professionnelle.

Comme attendu, l'éducation est présente essentiellement parmi les réponses de la jeune cohorte, dans les deux terrains d'enquêtes. Les changements rapportés par les 20-24 ans et concernant une réussite scolaire (un examen ou une année scolaire, voire un diplôme) ou la fin des études sont moindres à Bandra (7\%) qu'à Santa Cruz (11\%), où c'est de plus l'événement le plus fréquemment évoqué par ces jeunes gens. Y a-t-il un effet du moindre d'accès à l'éducation et en conséquence d'un nombre de réussites scolaires inférieur pour la population des bidonvilles ? Parmi les 20-24 ans qui en parlent, tous font référence à eux-mêmes. A l'inverse, les cohortes plus âgées qui citent une réussite scolaire évoquent leur(s) enfant(s).

Etre en formation, reprendre des études, ou avoir été admis dans un cursus de formation provoque plus de mentions des habitants de Bandra (7\%) que de Santa Cruz (4\%). Une satisfaction ou une fierté, sans doute issue de l'importance significative pour l'avenir qui en découle, se ressent : "I got admission in college. I was very happy" (femme de 23 ans, Bandra) "My sister got admission in Diploma. She passed first semester with 68\%") (femme de 23 ans, Bandra). A l'opposé, l'absence presque complète de mentions d'échec scolaire vient probablement de ce que, si l'on a échoué à l'école cela remonte sans doute à avant cette dernière année.

8 Plus de $75 \%$ de ces mentions concernent le répondant. 


\subsubsection{Les événements de l'année écoulée selon le sexe : des stéréotypes nuancés}

Dans chaque quartier, les femmes ont montré une centration vers des événements familiaux et de santé, alors que les hommes répartissent davantage les changements récents entre santé, profession et famille. D'un point de vue global, les clichés sont respectés : les femmes tournées vers la sphère familiale et les hommes vers le public. Néanmoins, les hommes se montrent aussi concernés par leur trajectoire familiale et les femmes ne sont pas désintéressées des questions professionnelles, ce qui tend à atténuer ce clivage.

Plus spécifiquement, les femmes interrogées à Bandra parlent plus de maladies, d'opérations ou d'hospitalisations, ainsi que d'une amélioration de la santé, que leurs homologues masculins. A Santa Cruz, il n'y a pas de différence selon le sexe dans le pourcentage de mention des maladies, qui est néanmoins l'événement le plus cité par les hommes alors que les femmes mettent plus l'accent sur le déclin de la santé, les symptômes ou les douleurs. Par contre, les hommes à Santa Cruz sont nettement plus impactés par des accidents : $14 \%$ des mentions y font allusion, contre $2 \%$ de celles des femmes de ce même quartier et $1 \%$ des citations des hommes de Bandra. Dans les deux enquêtes, très peu de problèmes psychologiques ou de dépression ressortent ${ }^{9}$.

Les répondantes des bidonvilles apparaissent donc plus concernées par les questions de santé. Toutefois, dans ce domaine de la vie, il ne semble pas y avoir parmi les changements récents de tendance au $c a r e^{10}$ ou à une préoccupation plus grande du bien-être de la famille que chez les hommes. En effet, c'est dans une même proportion que leurs homologues masculins qu'elles mettent elles-mêmes - plutôt que leurs proches - au centre de ces événements. Les questions de santé sont moins invoquées par les individus interrogés dans un quartier de classe moyenne comme Santa Cruz, sauf en ce qui concerne les accidents. Ces derniers sont particulièrement présents

9 Anecdotique: toutes les mentions de consommation de boisson alcoolisée (que ce soit une diminution ou une augmentation) sont faites par des hommes, et une femme parlant de son mari. Les problèmes de poids ou de taille (maigrir ou grossir) sont évoqués exclusivement par des hommes. Ils sont aussi les seuls à parler de problèmes oculaires ou de devoir porter des lunettes.

10 Le care signifie à la fois les soins, l'attention, les sollicitudes (rémunérés ou non) apportés aux proches pour améliorer leur bien-être, leur situation de santé (Razavi, 2007). 
dans la mémoire des hommes qui, inversement, mentionnent moins de maladies. Ce constat laisse songeur lorsque l'on sait que les individus pauvres en Inde sont plus souvent obligés de pratiquer des métiers à risques ou de vivre dans des lieux peu protégés (Narayan, Patel, Schafft, Rademacher, $\&$ Koch-Schulte, 2000).

Tableau 4.5: les domaines et les types de changements récents les plus mentionnés $(\%)$, selon le sexe et le lieu de résidence ${ }^{11}$

\begin{tabular}{l|cc|cc}
\hline \multirow{2}{*}{$\begin{array}{l}\text { Domaine } \\
\text { Type }\end{array}$} & \multicolumn{2}{|c|}{ Femmes } & \multicolumn{2}{c}{ Hommes } \\
\hline Famille & $\mathbf{1 8}$ & $\mathbf{1 7}$ & $\mathbf{1 5}$ & $\mathbf{9}$ \\
Mariage & 7 & 7 & 7 & 2 \\
Naissance & 8 & 7 & 5 & 3 \\
Décès & $\mathbf{4}$ & $\mathbf{4}$ & $\mathbf{4}$ & $\mathbf{1}$ \\
Santé & $\mathbf{4 6}$ & $\mathbf{4 1}$ & $\mathbf{3 5}$ & $\mathbf{3 8}$ \\
Maladie & 20 & 12 & 16 & 10 \\
Accident & 3 & 2 & 1 & 5 \\
Hospitalisation & 8 & 4 & 5 & 5 \\
Déclingraduel & 6 & 16 & 6 & 8 \\
Amélioration & 6 & 5 & 1 & 4 \\
Travail & $\mathbf{8}$ & $\mathbf{7}$ & $\mathbf{2 0}$ & $\mathbf{1 4}$ \\
Reprise/nouveau travail & 5 & 2 & 9 & 6 \\
Chômage & 2 & 1 & 4 & 1 \\
Promotion & 1 & 1 & 4 & 4 \\
Education & $\mathbf{6}$ & $\mathbf{8}$ & $\mathbf{5}$ & $\mathbf{4}$ \\
Début & 1 & 2 & 2 & - \\
Réussite & 3 & 4 & 2 & 3 \\
Economie & $\mathbf{6}$ & $\mathbf{6}$ & $\mathbf{7}$ & $\mathbf{9}$ \\
Mobilité & $\mathbf{3}$ & $\mathbf{3}$ & $\mathbf{2}$ & $\mathbf{2}$ \\
Développement personnel & $\mathbf{1}$ & $\mathbf{3}$ & $\mathbf{3}$ & $\mathbf{2}$ \\
Activités & $\mathbf{1}$ & $\mathbf{2}$ & $\mathbf{3}$ & $\mathbf{5}$ \\
Autres & $\mathbf{7}$ & $\mathbf{9}$ & $\mathbf{6}$ & $\mathbf{1 5}$ \\
\hline
\end{tabular}

Les femmes évoquent un peu plus la famille que les hommes à Bandra (18\% contre $15 \%)$ et nettement plus à Santa Cruz (17\% contre $9 \%)$. Parmi ces mentions, ce sont les naissances et les mariages qui font la différence. Des différences relativement faibles au final, mais qui laissent apparaître une focalisation plus grande des femmes sur leur couple et leur(s) enfant(s). Nonobstant, si les unions sont plus mentionnées à Bandra, c'est

11 En gris les pourcentages où moins de cinq individus sont représentés. 
essentiellement à travers l'évocation du mariage des enfants ou de la fratrie, avant le sien. A Santa Cruz par contre, où les divergences de mentions sont plus creusées entre les sexes, c'est son propre mariage qui est évoqué, autant ou davantage que celui des autres membres de la famille.

La sphère professionnelle reste plus mentionnée par les hommes. Ce qui est surtout vrai à Bandra, où ils travaillent le plus souvent dans le secteur informel et où assurer un revenu est une pression constante. Avoir un travail, changer de poste ou reprendre le travail est davantage cité par les hommes à Bandra (9\%) et à Santa Cruz (6\%) que par les femmes de ces quartiers (respectivement $5 \%$ et $2 \%$ ). De la même manière, la promotion ou la reconnaissance professionnelle est plus évoquée côté masculin que féminin. Enfin, les mentions de licenciements et de chômage sont plus fréquentes de la part des habitants de Bandra (4\%) que des répondants de Santa Cruz (1\%) et des femmes des deux quartiers. Quel que soit le type de mention professionnelle, les femmes ont moins tendance à en parler que les hommes, ce qui exprime la situation dominante des Indiennes qui, surtout une fois mariées, accèdent beaucoup moins au marché du travail que les hommes et ont pour tâche de s'occuper du foyer.

Le double standard masculin et féminin s'impose encore plus clairement lorsqu'est analysée l'identité de la personne concernée par le changement récent. Dans près de la moitié des citations concernant le travail et faites par des femmes (49\% à Bandra et $41 \%$ à Santa Cruz), c'est un autre membre (masculin) de la famille (conjoint, enfant ou fratrie) qui est concerné. Alors qu'entre 80\% (à Santa Cruz) et 95\% (à Bandra) des mentions faites par des hommes les concernent eux-mêmes. Cela souligne à quel point la responsabilité du revenu du foyer incombe aux Indiens, sur qui est dès lors exercée une pression conséquente par les normes sociales. Ce n'est toutefois pas une situation unidirectionnelle, puisque plus de la moitié des mentions professionnelles des femmes les concernent elles-mêmes : dans une vaste ville comme Mumbai, les femmes de classes inférieures et moyennes commencent à prendre place dans l'espace public, par les études mais aussi par l'accès à la carrière.

L'éducation et l'économie, faisant traditionnellement partie de la sphère publique, sont d'ailleurs aussi largement mentionnées par les femmes. C'est le cas de la réussite scolaire à Bandra, par exemple. Ces événements concernent majoritairement la personne elle-même, mais aussi ses enfants : les aider à réussir leurs études ou simplement pouvoir les nourrir. Les contraintes financières sont mentionnées par les femmes 
et les hommes relativement égalitairement dans les slums. A Santa Cruz, près de deux fois plus d'hommes (5\%) que de femmes (3\%) évoquent les changements de situation économique, mais les préoccupations sont identiques. De plus, femmes et hommes semblent pareillement impactés par les évolutions géographiques de leur vie (des déménagements essentiellement). Par contre, à Bandra les déménagements, les sorties du nid ou encore le fait de vivre seule sont uniquement des mentions de femmes (seul un homme parle de la migration de son fils).

\subsubsection{Les grands tournants de la vie}

Considérer les tournants majeurs de la vie offre l'opportunité de vérifier ces résultats en prenant en compte toutes les étapes de l'existence. Si les interviewés les plus jeunes n'ont pas un parcours aussi étendu que les plus âgés, ces derniers, eux, peuvent porter un regard rétrospectif sur leur longue trajectoire, nous offrant un point de comparaison intéressant. Cependant, les biais méthodologiques inhérents à notre enquête ne peuvent être oubliés : la limitation du nombre d'événements à évoquer rend la tâche plus ardue pour les aînés que pour les jeunes, tout comme le temps qui passe peut affecter la mémoire et l'influence de l'histoire modifier les regards portés sur les expériences antérieures.

Le tableau 4.6 présente les domaines des tournants, selon la même logique typologique que pour les changements récents. Cette fois, c'est la famille qui prévaut (27\% à Bandra, 24\% à Santa Cruz) parmi les mentions, à nouveau avec une focale sur le mariage et la naissance des enfants. La famille est aussi citée par le plus d'individus, à Bandra (38\%) et à Santa Cruz (32\%). Apparaissent ensuite dans une même proportion les décès et les problèmes de santé, particulièrement évoqués par nos interviewés. Toutefois, le poids de la santé pèse moins que ce qui est ressortit dans la question sur les événements récents, ceux de l'année écoulée. De ce point de vue, la profession et l'éducation, eux, gagnent en importance lorsque l'entier de la vie est considéré, avec une fréquence proche de celle de la santé, voire supérieure à Santa Cruz.

Une fois les décès ajoutés au domaine familial, la profession prend la seconde place, talonnée par la santé, et ce quel que soit le lieu de résidence. Notons que l'apparition d'une citation surprenante dans sa forme et nouvelle par rapport aux mentions de changements de l'année écoulée : évoquer 
des années de travail ou le simple fait de travailler (assemblés dans le tableau 4.6 sous le type «travail»). Certainement une marque de la précarité au quotidien et du soulagement de ne plus la connaître.

Tableau 4.6: les domaines et les types de tournants les plus mentionnés (\%), selon l'âge et le lieu de résidence ${ }^{12}$

\begin{tabular}{|c|c|c|c|c|c|c|c|c|c|c|c|c|}
\hline Domaine & To & tal & & -24 & & & & & & & & \\
\hline Type & $\mathrm{BA}^{13}$ & $\mathrm{SC}^{14}$ & $\mathrm{BA}$ & $\mathrm{SC}$ & $\mathrm{BA}$ & $\mathrm{SC}$ & $\mathrm{BA}$ & $\mathrm{SC}$ & $\mathrm{BA}$ & $\mathrm{SC}$ & $\mathrm{BA}$ & $\mathrm{SC}$ \\
\hline Famille & 27 & 24 & 19 & 22 & 31 & 33 & 23 & 23 & 28 & 20 & 31 & 20 \\
\hline Mariage & 14 & 13 & 7 & 12 & 15 & 16 & 13 & 13 & 14 & 11 & 19 & 13 \\
\hline Naissance & 7 & 7 & 6 & 4 & 11 & 13 & 5 & 5 & 7 & 5 & 8 & 2 \\
\hline Décès & 15 & 12 & 7 & 7 & 12 & 10 & 13 & 9 & 23 & 17 & 19 & 18 \\
\hline Santé & 14 & 14 & 13 & 12 & 12 & 13 & 15 & 16 & 19 & 16 & 11 & 15 \\
\hline Maladie & 6 & 5 & 4 & 4 & 4 & 3 & 6 & 3 & 9 & 8 & 6 & 10 \\
\hline Accident & 4 & 5 & 5 & 6 & 4 & 4 & 5 & 6 & 3 & 5 & 1 & 2 \\
\hline Hospitalisation & 2 & 2 & 1 & - & 3 & 2 & 2 & 2 & 4 & 2 & 2 & 1 \\
\hline Travail & 14 & 16 & 13 & 11 & 14 & 10 & 17 & 17 & 13 & 21 & 16 & 19 \\
\hline Premier emploi & 4 & 3 & 4 & 4 & 5 & 2 & 3 & 5 & 1 & 3 & 6 & 4 \\
\hline Travail & 4 & 4 & 4 & 4 & 5 & 2 & 5 & 5 & 2 & 5 & 5 & 5 \\
\hline Reprise/nouveau & 2 & 4 & 1 & 1 & 2 & 3 & 4 & 5 & 3 & 6 & 1 & 6 \\
\hline Chômage & 3 & 1 & 2 & - & 1 & - & 4 & 1 & 4 & - & 2 & 2 \\
\hline Promotion & - & 2 & - & 2 & - & 3 & - & 1 & 1 & 2 & - & 1 \\
\hline Education & 11 & 8 & 28 & 23 & 12 & 8 & 9 & 4 & 2 & 3 & 3 & 3 \\
\hline Début & 1 & 1 & 4 & 3 & 1 & - & - & 1 & - & - & - & - \\
\hline Réussite & 3 & 5 & 10 & 11 & 3 & 7 & 1 & 2 & - & 1 & - & 3 \\
\hline Pas d'étude & 2 & - & 4 & 1 & 4 & 1 & 3 & - & 1 & - & 1 & - \\
\hline Economie & 3 & 5 & 2 & 4 & 4 & 5 & 5 & 10 & 3 & 4 & 2 & 3 \\
\hline Mobilité & 6 & 6 & 5 & 2 & 5 & 8 & 8 & 8 & 3 & 8 & 8 & 2 \\
\hline $\begin{array}{l}\text { Développement } \\
\text { personnel }\end{array}$ & 2 & 3 & 3 & 3 & 3 & 2 & 1 & 3 & 1 & 2 & 1 & 5 \\
\hline Activités & 1 & 2 & 3 & 4 & 1 & - & - & 1 & 1 & - & - & 2 \\
\hline Autres & 7 & 10 & 7 & 12 & 6 & 11 & 9 & 9 & 7 & 9 & 9 & 13 \\
\hline
\end{tabular}

Les types d'événements santé mentionnés sont similaires entre les deux quartiers. La maladie demeure le type cité par le plus d'individus. Arrivent ensuite les accidents et les hospitalisations (qui comprennent les opérations, et tout passage en milieu hospitalier). L'amélioration de la santé,

12 En gris les pourcentages où moins de cinq individus sont représentés.

$13 \quad \mathrm{BA}=$ Bandra

$14 \quad \mathrm{SC}=$ Santa Cruz 
elle, disparaît presque complètement (une seule mention à Bandra et cinq à Santa Cruz).

L'éducation est plus évoquée à Bandra qu'à Santa Cruz. Dans les slums, la réussite scolaire (ou la fin des études) est mentionnée à hauteur de $3 \%$, alors qu'à Santa Cruz elle constitue $5 \%$ des mentions et se trouve à la place du $6^{\text {ème }}$ type d'événement le plus cité. Une nouveauté apparaît également dans ce domaine : la mention du fait de ne pas avoir pu faire ou continuer ses études. Si elle est apparue dans l'échantillon des bidonvilles $(2 \%)$, elle reste quasi absente (quatre mentions) dans celui de Santa Cruz.

\subsubsection{Impacts croisés du lieu de résidence et de la cohorte}

La fourchette de changements évoqués est relativement similaire d'une classe d'âge à l'autre (variant entre 31 et 40 types d'événements différents). Exception faite du groupe le plus jeune, qui n'a pas encore pu vivre un certain nombre d'événements démographiques, la famille est le premier domaine cité par toutes les cohortes à Bandra East. A part un pourcentage légèrement plus faible parmi les mentions des cinquantenaires $(23 \%)$, les classes d'âge réservent toutes près d'un tiers des souvenirs de leur vie aux questions de famille. Le constat est moins clair à Santa Cruz. Le taux de citations familiales tourne autour de $20 \%$ dans la plupart des groupes, sauf pour les 35-39 ans qui se démarquent avec un pic de 33\% de changements liés à la famille.

Les souvenirs des jeunes (28\%), des trentenaires (12\%) et des cinquantenaires $(9 \%)$ de Bandra sont davantage orientés vers l'éducation que leurs homologues de Santa Cruz $(23 \%, 8 \%$ et $4 \%)$, malgré un accès plus difficile aux études des premiers. La différence s'explique justement par la fréquence du type d'événement «pas d'étude», majoritairement évoqué à Bandra, qui manifeste un manque douloureux au point que les personnes pensent à l'évoquer lorsqu'on leur demande de se remémorer les tournants de l'existence. En termes d'individus concernés, ce sont les trois premières classes d'âge interrogées à Bandra qui comptent le plus d'individus mentionnant l'absence de suivi ou l'abandon contraint des études (respectivement $6 \%, 8 \%$ et $5 \%$ du sous-échantillon). Néanmoins, parmi les jeunes des slums, c'est avant tout la réussite scolaire (avoir passé un degré, réussi un examen décisif ou fini ses études) qui est mentionnée, soulignant la progression de la démocratisation de la scolarité à Mumbai. 
Lorsqu'ils mentionnent des souvenirs d'ordre formatif, les jeunes parlent essentiellement d'une expérience personnelle. A Bandra, 96\% des événements cités les concernent, et $97 \%$ à Santa Cruz. Les autres groupes parlent également de leurs enfants mais toujours avec une large majorité d'événements personnels. La totalité des mentions «pas d'étude» concerne directement l'interviewé, ainsi que la quasi-totalité des mentions d' 'échec scolaire». Cependant, la réussite scolaire engage parfois les enfants, dans les deux terrains d'enquêtes.

Les tournants professionnels sont évoqués dans une proportion similaire par les habitants des slums quel que soit leur âge (entre 13\% et 17\%), cette variabilité étant plus distendue à Santa Cruz (entre 10\% et 21\%). En majorité, ils concernent directement le répondant. A Bandra, le conjoint est le second membre de la famille qui est évoqué, sauf dans le cas du premier emploi qui concerne toujours ego et des quelques mentions de satisfaction professionnelle, alors qu'à Santa Cruz ce sont les enfants qui suscitent l'attention.

Une première stratification par âge ressort de nos résultats. Les jeunes sont très centrés sur l'éducation, et dans une moindre mesure également les trentenaires. La famille influence davantage les souvenirs des cohortes plus âgées, avec une concentration particulière autour des naissances et des mariages pour les 35-39 ans. Dans les classes d'âge suivantes, ce sont les décès qui viennent accroître les mentions de la famille. En second lieu, une stratification d'ordre socioéconomique, liée au lieu de résidence des répondants, voit le jour. Les décès concernent davantage d'individus dans l'échantillon de Bandra que dans celui de Santa Cruz, même si le nombre final de pertes mentionnées est comparable. L'éducation, bien que beaucoup évoquée par les 20-24 et 35-39 ans des deux quartiers, l'est différemment. Dans les slums, les réussites scolaires prennent de l'importance, et la frustration de ne pouvoir continuer ou débuter ses études est aussi palpable, ce qui n'est pas le cas à Santa Cruz. Enfin, concernant le monde professionnel, les deux cohortes les plus âgées de Santa Cruz évoquent plus fréquemment le fait d'avoir dû reprendre ou changer de travail, ce qui sera développé au chapitre 5 et mis en lien avec l'évolution du tissu industriel de Mumbai.

\subsubsection{Les tournants de la vie au prisme du genre}

Sans surprise, une différence de genre conséquente se remarque dans les tournants de la vie évoqués par les répondants. Le tableau 4.7 souligne une 
forte prévalence familiale dans les tournants féminins, et une centration autour de l'éducation et de la profession pour les hommes.

Tableau 4.7: les domaines et les types de tournants les plus mentionnés $(\%)$, selon le sexe et le lieu de résidence $(\%)^{15}$

\begin{tabular}{l|cc|cc}
\hline \multirow{2}{*}{ Domaine } & \multicolumn{2}{|c|}{ Femmes } & \multicolumn{2}{c}{ Hommes } \\
Type & Bandra & Santa Cruz & Bandra & Santa Cruz \\
\hline Famille & $\mathbf{3 5}$ & $\mathbf{3 1}$ & $\mathbf{1 7}$ & $\mathbf{1 8}$ \\
Mariage & 17 & 18 & 10 & 9 \\
Naissance & 11 & 8 & 4 & 5 \\
Décès & $\mathbf{1 8}$ & $\mathbf{1 8}$ & $\mathbf{1 1}$ & $\mathbf{6}$ \\
Santé & $\mathbf{1 3}$ & $\mathbf{1 3}$ & $\mathbf{1 6}$ & $\mathbf{1 5}$ \\
Maladie & 6 & 5 & 6 & 6 \\
Accident & 3 & 4 & 5 & 6 \\
Hospitalisation & 2 & 2 & 3 & 1 \\
Travail & $\mathbf{9}$ & $\mathbf{9}$ & $\mathbf{2 0}$ & $\mathbf{2 2}$ \\
Premier emploi & 3 & 2 & 5 & 4 \\
Travail & 3 & 3 & 5 & 5 \\
Reprise/nouveau travail & 1 & 2 & 3 & 6 \\
Chômage & 2 & - & 4 & 1 \\
Promotion & - & - & 1 & 3 \\
Education & $\mathbf{9}$ & $\mathbf{7}$ & $\mathbf{1 2}$ & $\mathbf{8}$ \\
Début & 1 & 1 & 1 & 1 \\
Réussite & 3 & 5 & 3 & 5 \\
Pas d'étude & 3 & - & 2 & 1 \\
Economie & $\mathbf{2}$ & $\mathbf{5}$ & $\mathbf{4}$ & $\mathbf{6}$ \\
Mobilité & $\mathbf{5}$ & $\mathbf{6}$ & $\mathbf{7}$ & $\mathbf{6}$ \\
Développement personnel & $\mathbf{1}$ & $\mathbf{2}$ & $\mathbf{2}$ & $\mathbf{3}$ \\
Activités & $\mathbf{1}$ & - & $\mathbf{1}$ & $\mathbf{2}$ \\
Autres & $\mathbf{7}$ & $\mathbf{9}$ & $\mathbf{1 0}$ & $\mathbf{1 4}$ \\
\hline
\end{tabular}

Les souvenirs des femmes contiennent plus de mariages (17\% à Bandra et $18 \%$ à Santa Cruz), de naissances $(11 \%$ et $8 \%)$ et de décès $(18 \%$ dans les deux enquêtes) que ceux des hommes (10\% et $9 \%$ pour les mariages, $4 \%$ et $5 \%$ pour les naissances, $11 \%$ et $6 \%$ pour les décès). Ces événements familiaux concernent, quel que soit le sexe de l'interviewé, autant soimême que la famille proche. Par contre, les hommes évoquent moins les unions de leurs enfants (15\% à Bandra et $17 \%$ à Santa Cruz des changements familiaux) que les femmes (26\%).

15 En gris les pourcentages où moins de cinq individus sont représentés. 
Au niveau de la santé, les mentions sont très proches en général mais aussi lorsque l'on se penche sur le détail des réponses. Toutefois, les hommes sont plus nombreux à mentionner des accidents que les femmes, que ce soit à Bandra $(11 \%$ et $4 \%)$ ou à Santa Cruz $(9 \%$ et $6 \%)$, ce qui ne ressort pas dans le tableau 4.7, laissant entrevoir que les hommes concernés mentionnent plusieurs fois la santé. A Bandra, ce sont exclusivement des femmes qui évoquent les déclins graduels.

Les hommes à Bandra sont plus nombreux à évoquer des événements professionnels, l'éducation par contre est citée pratiquement au même niveau. Néanmoins, des divergences apparaissent lorsque l'on creuse ces réponses ; elles seront développées dans le chapitre 5. Les différences de mentions économiques et des déménagements sont aussi minimes entre hommes et femmes.

\subsection{Evénements récents et tournants : les capitaux à disposition des habitants de Mumbai pour surmonter la vulnérabilité}

La typologie utilisée dans le projet international CEVI renvoie à des domaines de vie, à des trajectoires situées dans les espaces publics ou privés. Nous avons opté pour confronter cette classification assez neutre à une grille de lecture plus engagée, relevant d'une pensée néo-marxiste. Cette dernière s'appuie sur une élaboration proposée par Pierre Bourdieu $(1979,1980)$, puis reprise par de nombreux autres auteurs. Il s'agit du concept de capital, décliné en plusieurs modalités : capital social, capital culturel, capital économique et capital symbolique.

L'intérêt majeur de cette typologie est de rassembler des événements divers qui suggèrent l'exploitation d'une même ressource. En sus, cette catégorisation permet de conserver un regard centré autour de l'individu, qui est pour autant situé au sein des stratifications socioéconomiques, en fonction de la position qu'il y occupe. Etant donné que nous observons des changements, ce n'est pas le stock des capitaux à disposition de chaque individu qui est analysé ici, mais l'évolution de ce stock. Autrement dit, ce sont les capitaux concernés par les transitions rapportées par les répondants qui sont étudiés. 
Pour catégoriser les changements récents évoqués par les répondants, il s'agit de cibler les mentions se référant aux divers capitaux mentionnés ci-dessus, en prenant en compte la signification de ceux-ci pour la personne. Le capital social est donc composé des changements définis par le domaine de la famille, les décès, mais aussi de la santé, du développement personnel, de l'éducation et des déménagements pour autant qu'ils soient survenus à un membre de la famille de l'interviewé. Ce sont ainsi les événements ayant trait aux membres du réseau et aux différentes ressources que ceux-ci possèdent qui sont réunis. Dans le capital santé se trouvent bien sûr toutes les citations qui se rapportent au domaine de la santé, mais en se limitant à celles qui concernent la personne elle-même. Ce ne sont donc que les événements évoquant la capacité physique (ou psychique) de l'individu qui sont retenus. Le capital culturel est constitué en grande partie par l'éducation (uniquement concernant le répondant), le développement personnel (surtout les quelques mentions ayant trait au religieux), les voyages et les activités (sport, participation sociale, etc.).

Quant au capital économique, il rassemble les citations du domaine économique et professionnel, ainsi que les déménagements vécus par l'interviewé. Considérant qu'en Inde la protection sociale est loin d'être universelle et la plupart du temps compensée par du soutien familial (voir section 2.2.2), les mentions entrant dans le domaine de la profession sont interprétées comme du capital économique même si elles concernent un membre de la famille. Lorsque les individus citent une mobilité qu'ils ont vécue, ce peut être pour venir vivre en ville depuis la campagne, passer de résidant d'un slum à locataire d'un immeuble ou encore devenir propriétaire. Ces diverses configurations se recoupent en raison de leur impact socioéconomique : les emplois disponibles en ville, vivre loin de la communauté et sans les emplois informels qui s'y développent (par le bouche-à-oreille, les petits commerces de rue, etc.), détenir la sécurité $\mathrm{du}$ logement et ne plus supporter de loyer. Elles ont donc leur place toute trouvée au sein du capital économique.

\section{Changements de la dernière année : des capitaux stratifiés par âge}

La figure 4.4 présente la répartition des changements récents cités à Bandra selon les différents capitaux et la classe d'âge, la figure 4.5 concernant Santa Cruz. Au premier regard, on remarque que les jeunes évoquent des événements plus diversifiés : les quatre capitaux sont mentionnés, bien que la santé soit moitié moins présente. A l'inverse, les groupes d'âge du milieu 
de la vie évoquent essentiellement trois capitaux (santé, économie et social) et les octogénaires sont surtout cantonnés aux capitaux santé et social. Ainsi, le premier constat d'un éventail plus large de types de changements mentionnés par les jeunes se confirme et même s'accentue : ce ne sont pas seulement les types d'événements mais surtout les ressources que ceux-ci engagent qui sont plus variés chez les jeunes à l'orée de la vie adulte.

Une grande similitude s'observe entre les cohortes d'un terrain d'enquête à l'autre, surtout, justement, pour les jeunes adultes. Les mutations dans la réserve de capitaux sont résolument plus nombreuses dans la jeunesse, puis dans la trentaine et la cinquantaine les variations s'observent majoritairement au niveau des capitaux économiques et sociaux (confirmant qu'il s'agit d'un moment délicat de la vie, où l'on construit son foyer et où les responsabilités croissent). Enfin, chez les sexagénaires et les plus âgés, les modifications ont lieu avant tout dans le capital santé.

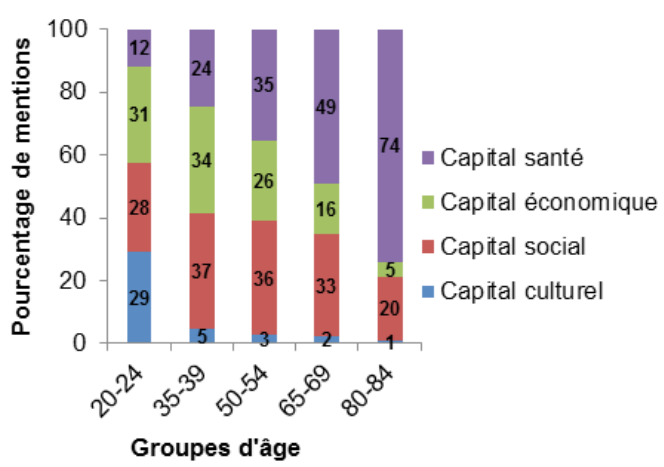

Figure 4.4: les changements récents (\%) à Bandra, selon la classe d'âge

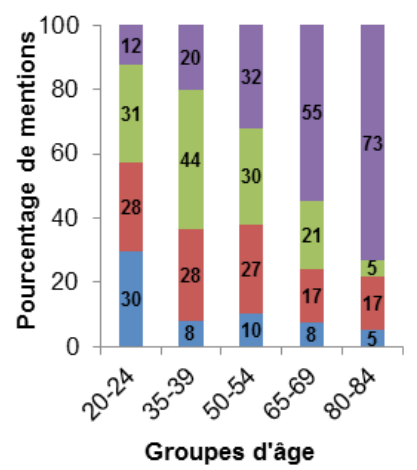

Figure 4.5: les changements récents (\%) à Santa Cruz, selon la classe d'âge 


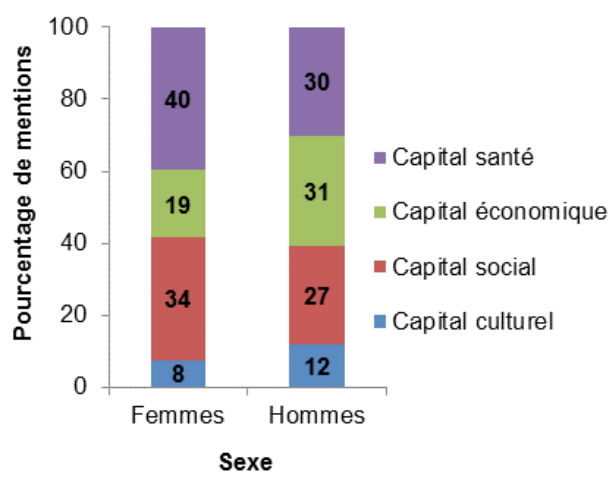

Figure 4.6: les changements récents (\%) à Bandra, selon le sexe

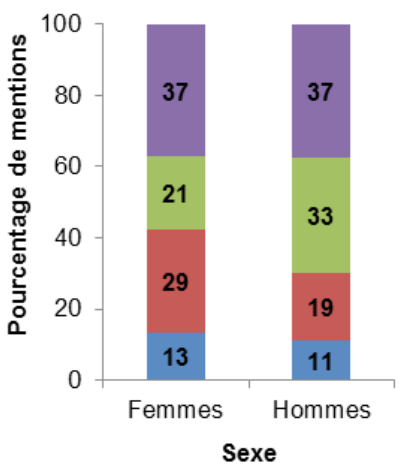

Figure 4.7: les changements récents $(\%)$ à Santa Cruz, selon le sexe

La répartition des événements cités selon les quatre capitaux est relativement proche entre les hommes et les femmes (voir figure 4.6 et 4.7). Néanmoins, le capital économique est davantage mentionné côté masculin (environ $30 \%$, pour environ $20 \%$ côté féminin). Les habitantes de Bandra se réfèrent plus au capital santé (40\%) que leurs compagnons (30\%) alors qu'il y a égalité entre les sexes à Santa Cruz (37\%). Le capital social est moins évoqué par les hommes, résidant dans un quartier de classe moyenne inférieure.

Tournants de la vie : une perception commune de l'existence, où le socioéconomique et le genre prennent l'avantage

Concernant les tournants de l'existence, les différentes classes d'âge montrent bien plus de similarités que lorsque l'on se penche sur les souvenirs liés aux positions d'âge (voir les figures 4.8 et 4.9). Seule l'évolution du capital culturel demeure franchement marquée chez les plus jeunes, les autres groupes d'âge évoquant des tournants principalement situés parmi les capitaux social, économique et de santé.

Toutefois, à Santa Cruz, certaines fluctuations apparaissent entre l'économique et le social. Les trentenaires montrent plus de mentions de type social, tout comme les plus âgés, alors que les cinquantenaires et les sexagénaires se focalisent presque autant sur le capital économique. Les soucis de dépenses et de revenus marquent davantage les trajectoires de 
ces groupes, que ce soit liés à leur âge ou à leur histoire. Nous creuserons ce constat dans le chapitre 5 .

Par rapport aux figures 4.6 et 4.7 , les figures 4.10 et 4.11 montrent des résultats plus similaires entre les sexes qu'entre les lieux de résidence. Les femmes sont nettement orientées vers le social, alors que les hommes se répartissent presque équitablement entre les différents capitaux, avec une prévalence pour l'économique et le social. Les stéréotypes de genre se trouvent soulignés dans les souvenirs des grands tournants de la vie.

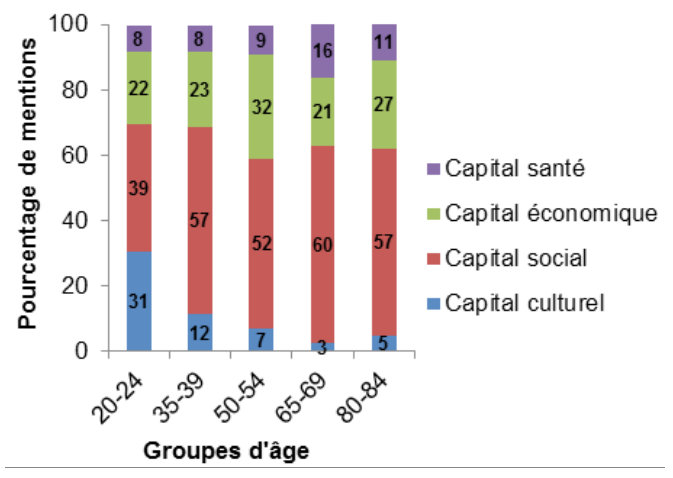

Figure 4.8: les tournants (\%) à Bandra, selon la classe d'âge

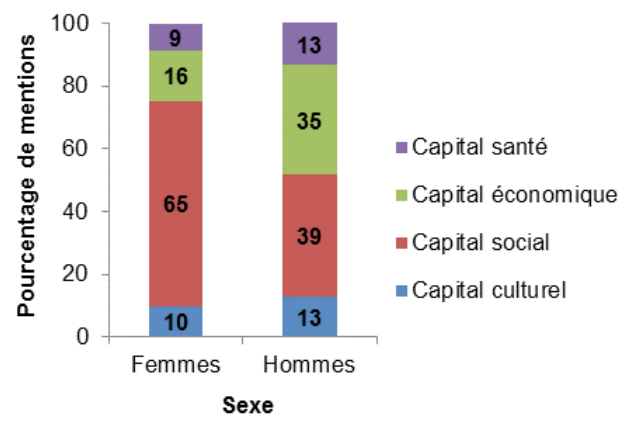

Figure 4.10: les tournants (\%) à Bandra, selon le sexe

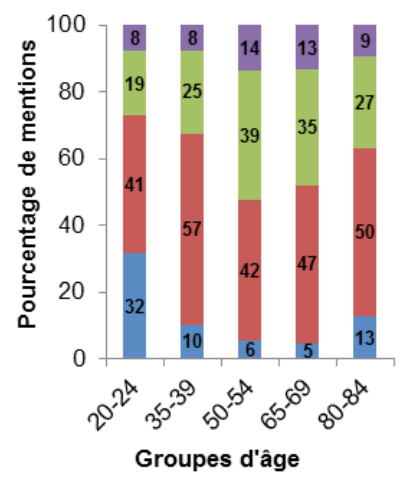

Figure 4.9: les tournants (\%) à Santa Cruz, selon la classe d'âge

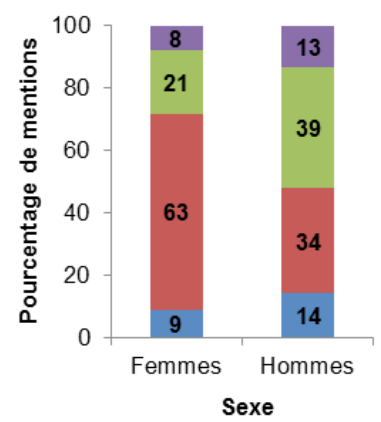

Figure 4.11: les tournants (\%) à Santa Cruz, selon le sexe 


\subsection{Conclusion}

Un des objectifs de cette recherche est d'interroger la perception subjective du parcours de vie dans un espace culturel qui déborde du cadre atlantique, le seul à avoir été étudié tant qu'à présent. En l'occurrence, globalement, les domaines concernés par les grands tournants de la vie ne paraissent pas différer des résultats obtenus dans d'autres enquêtes passées en Occident.

La prévalence et l'ordre d'apparition de ces domaines ne dérogent pas à ce qui a déjà été souligné dans d'autres recherches portant sur les événements de la vie (Cavalli et al., 2013; Laborde et al., 2012), avec cependant la différence notable de la santé plus fréquemment citée en Inde. Cette surreprésentation de mentions concernant ce domaine reflète la conscience individuelle, subjective, des conditions de vie difficiles au niveau de l'habitat, de la nourriture, du travail, de l'accès aux soins et de leur qualité fluctuante. Les Indiennes et les Indiens ne sont pas aveugles sur ce qui rend les individus vulnérables face aux maladies et aux accidents, les chapitres suivants le confirmeront. A l'inverse, il est surprenant que les mentions explicites de difficultés économiques soient peu présentes pour des gens qui en subissent la réalité et les conséquences quotidiennement. Dans le même ordre d'idée, les mobilités (déménagements en ville ou de la campagne à la ville) sont très peu mentionnées par un échantillon majoritairement composé de migrants.

Dans un pays où le mariage et la parentalité sont quasiment incontournables, moins de la moitié des personnes interrogées y font allusion comme à un moment fort de leur vie. Il est vrai, toutefois, que les décès mentionnés renvoient presque exclusivement aux membres de la famille. Ainsi, sur ces deux domaines conjugués, un total de 53\% des individus évoquent un tournant familial au moins une fois dans nos enquêtes. Ceci mérite d'être interprété en termes de normalité et d'anormalité, d'événements normatifs ou attendus versus crises ou ruptures (voir section 2.3.3). Notons encore l'absence de «nouveauté» dans les domaines évoqués par des personnes ancrées dans une autre culture, d'autres conditions et modes de vie que ceux déjà étudiés. Par exemple, il n'aurait pas été étonnant de trouver des mentions de type religieux (dans un pays où toute la population possède une confession religieuse, déterminante pour le statut 
social) ; or n'ont guère été mentionnés que quelques fêtes religieuses et un pèlerinage.

Santé, famille, profession, éducation... les événements récents mentionnés par les individus interrogés à Mumbai se rassemblent ainsi en différents sous-groupes selon un découpage plutôt classique des facettes de l'existence. Ces domaines font écho aux trajectoires qui sont habituellement étudiées dans le paradigme du parcours de vie: familiale, professionnelle, de santé... (Sapin et al., 2007). Par ailleurs, ces domaines se divisent selon qu'ils concernent davantage la vie privée (relations, foyer, etc.) ou la vie publique (formation, emploi, etc.). Pour creuser ces résultats encore grossiers, la section 4.1 .3 a proposé une grille de lecture plus fine que la simple dichotomie opposant les sphères publique et privée mais moins restrictive que les trajectoires (qui empêchent un certain nombre de regroupements logiques transversaux comme de placer les événements professionnels avec l'économie) au travers de l'utilisation du concept de capital (Bourdieu, 1997).

S'appuyant sur les changements récents qui marquent la vie à différents âges, une vision individuelle se dessine, où l'acteur se place au centre des mouvements de son existence. La famille a certes de l'importance, et ce davantage pour les résidents d'un milieu urbain précaire que pour des habitants d'un quartier de classe moyenne, mais l'individu reste la référence principale.

En séparant les réponses données par classe d'âge, peu de divergences ressortent. Une première exception est l'éducation qui se dégage comme le domaine le plus important parmi les jeunes. Ayant entre 20 et 24 ans, ces derniers sont à l'aube de la construction de leurs propres trajectoires : de nombreux tournants évoqués par les plus âgés n'ont simplement pas encore eu lieu. En outre, une pression très forte est mise sur les épaules des jeunes générations indiennes pour la réussite scolaire et l'échec est parfois socialement difficile à surmonter. Cela étant, que l'éducation soit le premier souci de ces jeunes adultes indique que de plus en plus de jeunes prolongent leurs études jusqu'au niveau supérieur, acquérant un haut capital humain (voir les données de base du questionnaire). Ensuite, la présence des décès est, comme la théorie le prédit, plus marquée au sein des cohortes 65-69 et 80-84 ans (qui ont vécu davantage de départs de proches) que chez les plus jeunes. Enfin, la santé est mentionnée en priorité par les quatre cohortes plus âgées, particulièrement par les sexagénaires. 
Il est cependant frappant que les groupes d'âge intermédiaires (35-39 ans et 50-54 ans) évoquent autant la santé que les 80-84 ans.

En ce qui concerne les mentions des tournants de l'existence par les hommes et les femmes, les divergences sont peu marquées mais révélatrices d'une société où les rôles sont profondément genrés. Les domaines de la famille et celui joint des décès sont mentionnés par $63 \%$ des répondantes mais à peine plus de $40 \%$ des hommes. Inversement, ces derniers concentrent plus de souvenirs dans les domaines de la profession, de la santé et de l'éducation, qui sont en fait tous illustratifs d'un intérêt lié à la capacité de travail affectant directement le revenu (également les dépenses) du ménage. Cela nous donne indirectement témoignage de leur vulnérabilité. Il est aussi probable que la prévalence de l'éducation chez les hommes découle du contexte indien : en effet, proportionnellement moins de filles ont (ou avaient) accès à l'école, ce qui explique que ce domaine soit plus souvent mentionné côté masculin.

Les femmes citent un peu plus la famille (le capital social) mais de peu, la différence étant plus marquée à Santa Cruz. Ces femmes ne sont pas si cantonnées à la sphère privée, au moins pour ce que leur perception subjective des changements récents de la vie laisse apercevoir. Le chapitre qui suit a pour objectif de développer les réponses liées exclusivement aux capitaux économiques et culturels, afin de creuser les représentations concernant la sphère publique. 


\title{
Chapitre 5. Profession, économie, formation: expressions et déterminants des inégalités
}

\author{
"[...] the official estimates of poverty, based on a \\ normative level of consumption expenditure, \\ would be totally unsuited to capture the concept. \\ In fact, no measure based on a univariate \\ criterion can be adequate."
}

(Kundu \& Mahadevia, 2002, p. 6)

Inégalité flagrante dans une ville comme Mumbai, l'accès aux ressources financières (capital économique) et formatives (capital culturel) détermine sinon la survie des individus, à tout le moins leur qualité de vie actuelle et future. La teneur de ces capitaux explique une part considérable des vulnérabilités qui marquent les existences de femmes et d'hommes, pauvres et moins pauvres, logeant dans des bidonvilles ou des quartiers de classe moyenne inférieure. Concrètement, les degrés d'inégalités varient de manière importante au cours de la vie: habitat salubre ou insalubre, accès aux études ou non, travail stable ou informel, pauvreté ou niveau de vie décent...

Dans le chapitre précédent, les divers domaines de l'existence concernés par les réponses aux volets I et II ont été mis en exergue. Nous savons donc qu'en leur sein, les mentions liées à la sphère publique (majoritairement l'économie du ménage, la mobilité, l'éducation et la profession) représentent une part minoritaire; elles sont néanmoins dignes d'intérêt. Ce chapitre se propose de revenir sur ce qui compose les deux capitaux que recouvre cette sphère: culturel et économique.

Pour aller au-delà du chapitre précédent, une focale est mise dans la première section sur le capital culturel, plus précisément les mentions d'éducation (concernant le répondant) et d'autres types d'activités non scolaires. Par la suite, nous nous concentrons sur les ressources économiques, notamment la place des changements liés à la situation du foyer (rentrées d'argent ou dépenses, achat ou vente de biens,...), à celle de l'emploi et son insuffisance, enfin à la mobilité résidentielle comme événement majeur de l'existence...ou son absence, sous forme d'immobilité de contrainte. Pour certains habitants, ces événements soulignent-ils la 
présence d'avantages cumulatifs (réussite scolaire, travail stable, épanouissement personnel,...) et pour d'autres de désavantages qui s'enchaînent (non accès aux études, chômage, inflation des prix,...)?

\subsection{Quitter l'école, quitter l'enfance: l'éducation en tant que palier de la vie et clé de sortie de la pauvreté}

L'utilisation d'une codification basée sur les capitaux (cf. section 4.3) a permis de souligner que les changements de l'année écoulée se rapportent plus souvent aux capitaux économiques et culturels parmi les jeunes groupes d'âge. Les tournants de l'existence soutiennent le même constat regardant le capital culturel, alors qu'une présence égalitaire du capital économique apparaît entre les cohortes, avec une prévalence légèrement plus élevée parmi les cinquantenaires. En outre, la formation demeure majoritairement citée par les habitants des bidonvilles, alors que l'économie est surtout évoquée à Santa Cruz.

Il ressort du premier aperçu de ces capitaux que les jeunes groupes d'âge considèrent les deux alors que les plus âgés ne focalisent leurs souvenirs que sur un, le capital économique (plus particulièrement le travail). Cette diversification marque la modernité et les changements survenus à Mumbai, toutefois non sans rappeler que l'impossibilité pour les plus pauvres de bénéficier des études pour des raisons socioéconomiques est toujours la première source d'inégalité.

Le capital culture ${ }^{1}$ plus marqué chez les jeunes est avant tout dû à une focalisation plus grande autour des études. Les divergences sont plutôt faibles selon le lieu de résidence, sauf à se pencher sur les types d'événements mentionnés: les réussites scolaires sont plus citées par les jeunes de Santa Cruz et la mention «pas d'étude» par les habitants de Bandra, reflétant les inégalités entre les deux quartiers.

1 Le tableau en annexe 3 présente les capitaux culturels et économiques en détail, pour les changements récents et les grands tournants de l'existence, à Bandra et à Santa Cruz. Les chiffres différent quelque peu des tableaux 4.4 et 4.6 , puisque le codage des capitaux a été établi selon une typologie légèrement différente (pour rappel de la typologie, voir section 4.3). 


\section{L'éducation comme marqueur de réussite ou d'échec}

L'état institutionnalisé du capital culturel (voir section 2.4.2) ou, dit autrement, la détention de diplômes ou toute autre forme de reconnaissance d'une formation, apparaît explicitement dans les réponses fournies aux deux premiers volets de l'enquête, quoique dans un pourcentage relativement faible. C'est avant tout le fait de débuter des études ou de les réussir qui est mentionné. Néanmoins, un second aspect de ce capital est également présent: il s'agit de son manque, de la constatation de l'absence d'opportunité ou de l'échec scolaire. Cette seconde dimension n'est logiquement présente que dans les tournants de l'existence.

L'éducation est donc mentionnée selon deux angles évidents: la réussite et l'échec. Sous l'aspect positif, il faut inclure les débuts de scolarité ou la reprise d'études, qui sont explicitement un signal d'espérance de succès futur (voir chapitre 4). Dans le volet I, le positif prend nettement le pas. Douze débuts de formation et 24 réussites scolaires, contre un seul échec et 19 citations factuelles (descriptif de situation, inquiétudes, cours spécifiques, etc.):

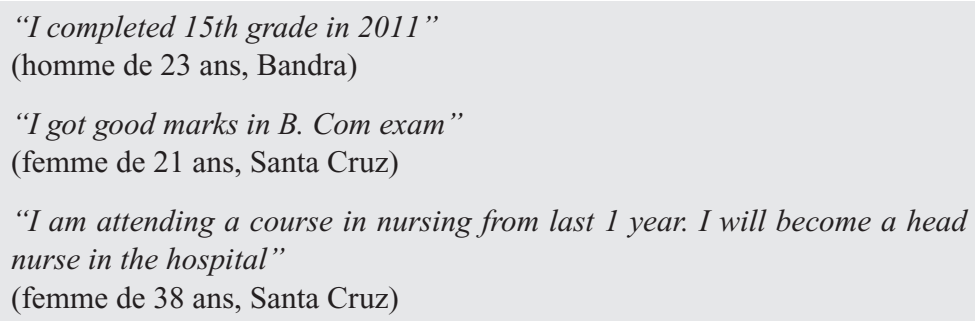

Concernant les quelques tournant (TOU) évoquant une réussite scolaire ( 2 et $3 \%$ des mentions, voir annexe 3 ), et les raisons (RS) du choix, une espérance, mais aussi une pression de réussite apparaissent, aussi prégnantes chez les jeunes cohortes de Bandra que de Santa Cruz: 
TOU: "I passed 10th grade with good percentage. I got good Diploma"

RS: "My dream came true. I got admission in engineering"

(homme de 25 ans $^{2}$, Bandra)

TOU: "Without classes, I passed 10th grade. I was not sure that I would pass"

RS: "I passed with 42\%. I was happy. I thought I have done something big" (femme de 39 ans, Bandra)

TOU: "I completed $10^{\text {th }}$ exam nicely"

RS: "I and my family were happy"

(femme de 20 ans, Santa Cruz)

TOU: "I got good mark in 2nd year of engineering"

RS: "I fulfilled the hopes of people"

(homme de 21 ans, Santa Cruz)

La dimension positive de l'éducation apparaît donc à égalité dans les deux enquêtes, alors que l'angle négatif est surtout évoqué par les répondants de Bandra (8 sur les 10 citations) au travers de l'échec scolaire ou du regret de ne pas avoir suivi de formation.

Le peu d'échecs scolaires évoqués, ainsi que leurs raisons, montrent un regret très personnel, avec également l'importance que ceux-ci revêtent pour la famille :

TOU: "I was unable to get more education"

RS: "I would have get good job if I could study"

(homme de 67 ans, Bandra).

TOU: "I got failed in D.Ed for me D.Ed education is very important. I lost my confidence because I got failed"

RS: "It is important education for me and my family"

(femme de 22 ans, Bandra)

Les mentions «pas d'études» allèguent explicitement la contrainte structurelle imposant l'impossibilité de faire ou de suivre des études. Pauvreté, obligation de s'occuper de la fratrie, école trop éloignée du domicile ou mariage précoce sont quelques-unes des explications données à cette carence d'apprentissage :

2 A moins d'une précision, les âges indiqués correspondent à ceux des répondants au moment des enquêtes et non au moment de l'événement rapporté. 
TOU: "I didn't go to school"

RS: "Because we had poor condition"

(femme de 50 ans, Bandra)

TOU: "I wanted to study after 10th grade but I couldn't study because of poverty"

RS: "I left education and started doing job"

(homme de 36 ans, Bandra)

TOU: "I want to do something after study. But due to poverty not able to complete study"

RS: "As per today's generation due to more inflation expenses are increasing, I want to help my family financially"

(femme de 39 ans, Santa Cruz)

TOU: "I had small brothers and sisters, family condition was bad so I couldn't study"

RS: "Because it would have been good if I would study"

(femme de 66 ans, Bandra)

Globalement, les individus de chaque quartier montrent une tendance similaire à citer des moments forts de leur parcours éducationnel comme des tournants de leur existence ; toutefois, le désavantage de la pauvreté a une influence qui s'exprime par l'accroissement des mentions des plus jeunes cohortes vivant dans les bidonvilles. De manière surprenante, ce ne sont pas les plus âgés qui évoquent le plus le regret de ne pas avoir été du tout (ou pas assez longtemps) à l'école bien que, de toute évidence, leur accès à la scolarité ait été moindre que celui des classes d'âge plus jeunes (de 20-24, 35-39 et 50-54 ans). Comme ce fut la destinée d'une majorité d'entre eux, et surtout d'entre elles, nous pouvons assumer qu'ils en ressentent moins la frustration.

\section{Une entrée dans l'âge adulte plus précoce sans les études}

Il est évident, à Bandra comme à Santa Cruz, que ce sont les jeunes de 20-24 ans qui ont le plus mentionné l'éducation comme un tournant de leur vie. Cependant, l'inscription de ces événements dans le parcours de vie n'est pas exactement similaire. La figure 5.1 présente la répartition de ces événements selon l'âge et le lieu d'habitation du répondant, toutes cohortes confondues. Nous n'avons ici considéré que les 26 premières années de la vie, autrement dit les années les plus concernées par l'éducation et les seules ayant été vécues par tous les groupes d'âge. 
La courbe issue des tournants rapportés par les habitants des bidonvilles est visiblement décalée sur la gauche, démontrant une mention d'événements survenus plus tôt dans le parcours de vie des individus (voir figure 5.1). Ce qui se constate ici est l'effet des mentions d'abandon forcé ou contraint des études, survenu à des âges jeunes. Ainsi, plus que les résidents d'un quartier de classe moyenne inférieure, les individus vivant dans les slums et soumis aux impératifs de la pauvreté entrent plus rapidement dans la vie active. Entre les sexes par contre, il n'y a pas de divergence marquante dans les âges qui ressorte (voir figure 5.2).

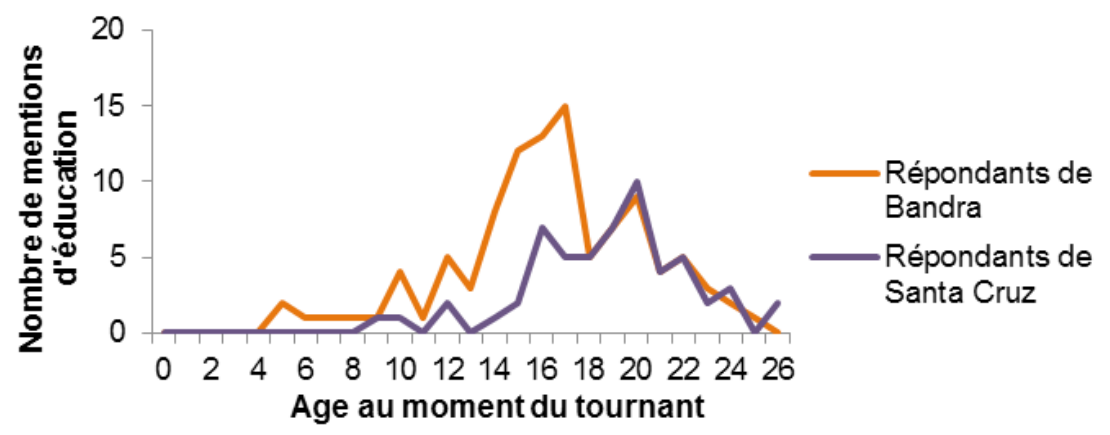

Figure 5.1 les mentions d'éducation $(n)$ selon l'âge du répondant au moment du tournant et le lieu de résidence

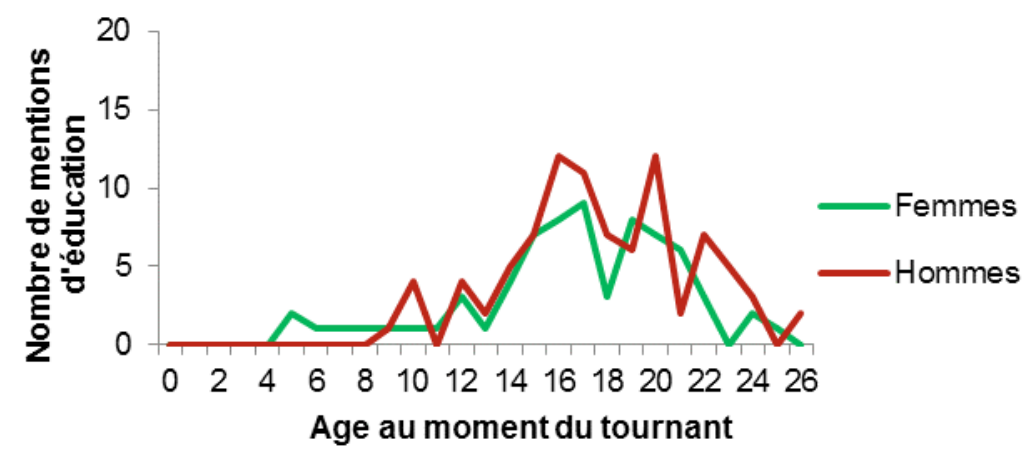

Figure 5.2: les mentions d'éducation $(n)$ selon le sexe et l'âge du répondant au moment du tournant 
Si l'éducation représente un socle déterminant de capital culturel, ce dernier n'est pas très étoffé. Il est même surprenant de constater le peu de mentions faites, en comparaison du poids décisif pour l'existence que représente la formation dans des conditions de précarité aussi extrêmes que celles que supportent la plupart des individus interrogés. La figure 5.3 expose la répartition des individus ayant évoqué au moins un tournant marquant de leur formation, selon le niveau d'éducation atteint au moment de l'enquête et leur lieu de résidence.

Le faible pourcentage d'individus ayant mentionné au moins un tournant éducationnel alors qu'ils n'ont pas été scolarisés (sans éducation) ou ont atteint seulement l'école primaire, désigne essentiellement les répondants ayant cité l'absence d'études. Par ailleurs, une différence ressort entre les lieux d'habitation : davantage d'habitants des slums de niveau secondaire ou tertiaire mentionnent un tournant de formation. C'est une marque de l'importance de celle-ci dans le parcours de vie de personnes pauvres, qui y voient leur seule chance de changer leur destinée.

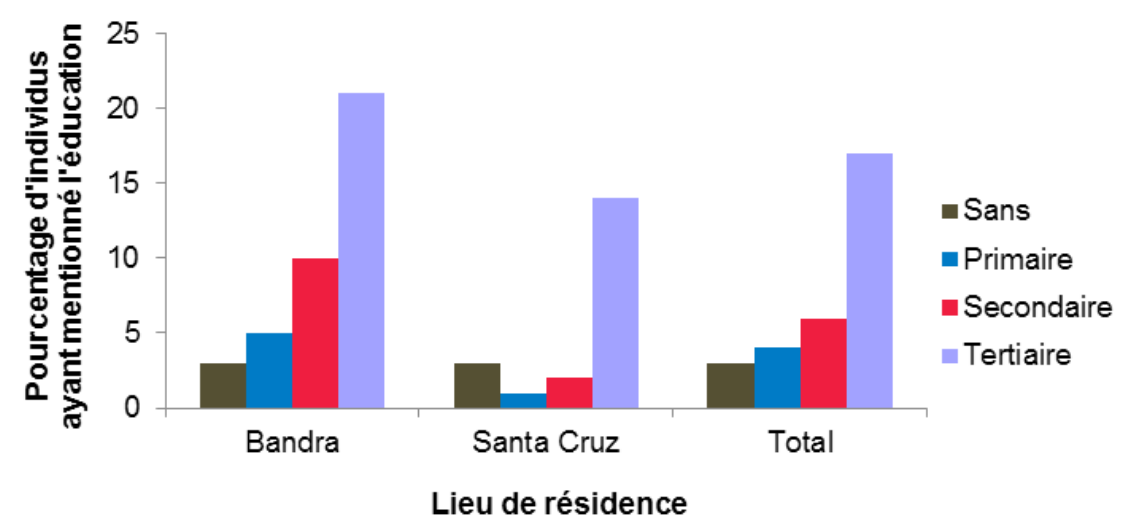

Figure 5.3: les individus (\%) ayant mentionné au moins un tournant du domaine éducation, en fonction du lieu de résidence et du niveau d'éducation

Quelques dimensions supplémentaires du capital culturel : développement personnel, voyages, activités

Autre aspect du capital culturel, le développement personnel a été faiblement évoqué (de 1 à $3 \%$ des mentions). Parmi les changements de l'année écoulée, ce dernier est exprimé dans une même mesure à Bandra et à Santa Cruz, et majoritairement par les plus jeunes. Il s'agit surtout de sa propre maturité : 
"My nature is changing. Atmosphere is also changing because of which life is also changing"

(homme de 22ans, Santa Cruz)

"Now I don't fear to go anywhere"

(homme de 21 ans, Bandra)

"My behaviour changed since $1^{\text {st }}$ January 2011"

(femme de 22 ans, Bandra)

Au sein des grands tournants de l'existence, le développement personnel se trouve surtout cité à Santa Cruz, et parmi les cohortes les plus âgées. Cette fois, les citations tendent plutôt à poser un constat de son propre vieillissement ou font référence à des pratiques religieuses :

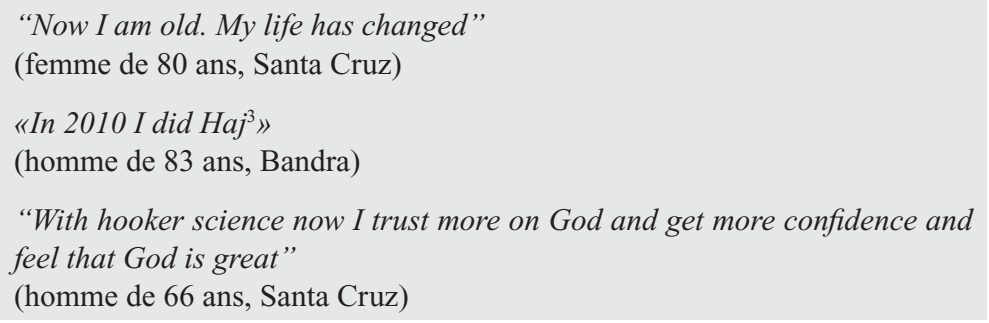

Sans surprise pour des individus possédant des moyens limités, voire très réduits, peu de voyages ont été évoqués. Les mentions d'activités autres que professionnelles ou scolaires sont aussi peu nombreuses, mais démontrent que certains répondants possèdent du temps libre, dédié à de la musique, de la danse, de la peinture ou d'autres loisirs $(n=26)$, à de la participation sociale politique, associative ou religieuse $(n=8)$, ou du sport $(n=24)$. Toutefois, pour le volet I comme pour le II, ces activités sont surtout citées par les jeunes, à l'exception des cinquantenaires de Santa Cruz, qui dans leurs souvenirs récents ont laissé une place relativement large au sport. Dans presque tous les cas ( 28 des 29 changements récents et 24 des 27 tournants), les individus à l'origine de ces mentions possèdent un niveau de formation secondaire ou tertiaire : ce n'est que lorsque les répondants détiennent un certain niveau de vie que des activités «triviales» sont mentionnées.

3 Haj (ou hajj) représente le pèlerinage des musulmans vers les lieux saints de la ville de La Mecque. 


\subsection{Pauvreté matérielle, financière et humaine}

Le capital économique repose sur plusieurs dimensions (les changements d'ordre professionnel, économique et de logement) alors que le capital culturel, on l'a vu, se compose presque exclusivement d'un seul aspect (la formation). Il est davantage mentionné parmi les changements de l'année passée des quatre plus jeunes groupes d'âge, et moins parmi ceux des octogénaires. Dans les tournants par contre, ses évocations restent stables avec l'avancée en âge (voir annexe 3). Cela tient beaucoup au domaine professionnel, largement cité par les plus jeunes, les trentenaires et les cinquantenaires de Bandra comme changement de l'année écoulée, et par les quinquagénaires, sexagénaires et octogénaires de Santa Cruz pour les tournants.

\subsubsection{Le travail, au centre de la survie}

Au sein des tournants professionnels, le premier emploi et avoir un travail sont généralement la transition et la situation les plus citées, même chez les personnes âgées.

\section{Débuter sa vie active : contrainte ou espoir?}

Débuter sa vie active a fait l'objet de 48 tournants à Bandra, 32 à Santa Cruz. En observant les raisons apportées à ces mentions, un premier ensemble laisse entendre une contrainte, un regret, une charge familiale. En bref, il s'agit des conséquences de la pauvreté, bien sûr davantage présentes parmi les réponses des habitants des bidonvilles (34 raisons à Bandra, 4 à Santa Cruz):

TOU: "Since 9-10 years of age I am working"

RS: "Because house economical condition was bad"

(femme de 38 ans, Bandra)

TOU: "Since 12 years at the age. I started going to father's mutton shop"

RS: "I could not concentrate my studies"

(homme de 80 ans, Santa Cruz)

TOU: "I started doing farming since I was 13 years old"

RS: "We are 5 sisters and brother"

(femme de 20 ans, Bandra) 
Le second type de raisons est explicitement positif, où se ressentent la satisfaction de travailler et d'avoir un revenu (y compris par le biais des enfants) avec cette fois-ci 14 raisons à Bandra, 28 à Santa Cruz :

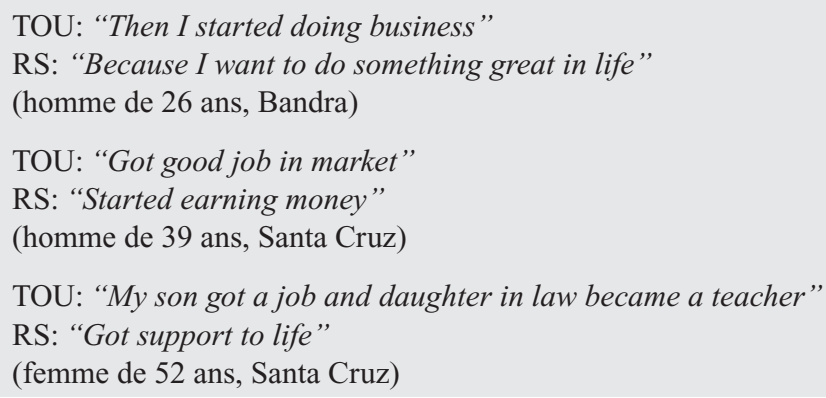

L'entrée dans le monde professionnel a été peu évoquée comme un changement crucial de l'existence. Toutefois, parmi les quelques mentions à ce sujet, un clivage semble avoir lieu en fonction du niveau socioéconomique des individus. Les personnes de classe moyenne inférieure apparaissent plus en mesure d'apprécier l'indépendance et l'avancée vers l'âge adulte que cette transition représente, alors que les individus résidant dans les bidonvilles subissent davantage les revers de celle-ci (quitter l'école, devoir de participer au revenu du ménage, etc.), à des âges parfois très jeunes.

\section{Réorienter sa vie professionnelle ou seulement la stabiliser : une protec- tion contre la vulnérabilité du quotidien}

Parmi les changements récents, les jeunes cohortes citent facilement le fait de recommencer un travail, de réorienter sa carrière ou d'avoir modifié son emploi. Ce type de transition professionnelle est remémoré avant tout par les 20-24 et les 35-39 ans vivant en bidonvilles (respectivement 12\% contre $10 \%$ des mentions des jeunes de Santa Cruz et 9\% contre 7\% des trentenaires) $)^{4}$.

En arrière-fond de ces évocations, s'exprime généralement un sentiment de soulagement, lié à la fin de l'incertain et de l'informalité avec l'obtention d'un emploi formel, ainsi que la sortie d'une période de chômage :

4 De plus, les 20-24 ans de Bandra sont 14\% à en parler (contre 10\% à Santa Cruz), et les trentenaires de Bandra 7\% (contre 6\% à Santa Cruz). 
"I got new job in 2011. I was at home for 2 years after losing job" (homme de 34 ans, Bandra)

"I got a good job. That is of importance"

(homme de 26 ans, Santa Cruz)

"My husband got BMC job"

(femme de 24 ans, Bandra)

"One son is working in HSBC bank and other is in shipping company" (homme de 83 ans, Santa Cruz)

Dans le même ordre d'idée, un nouveau type de mention apparaît parmi les tournants, d'autant plus significatif qu'il ne s'agit par d'un événement (alors que la question posée appelait explicitement une réponse comprenant une dimension de changement): avoir un travail, un constat généralement assorti d'une notion de durée (51 citations à Bandra, 37 à Santa Cruz). Se ressentent derrière la conscience d'avoir un emploi relativement stable, un revenu assuré, mais aussi pour certains d'être soumis à la condition laborieuse par la nécessité de survivre :

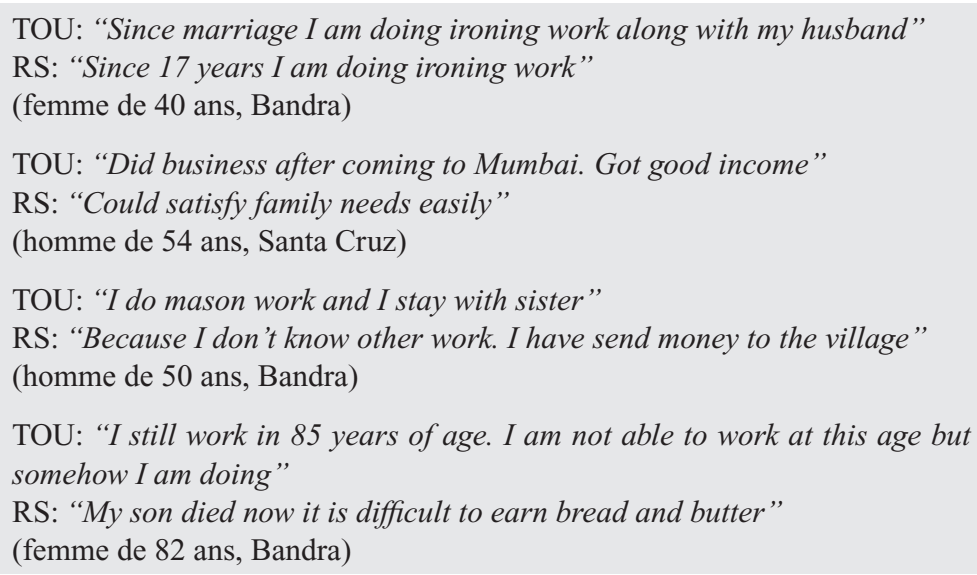

A Santa-Cruz, des tournants font aussi référence à la reprise d'un travail pour les 50-54 ans (5\%), les 65-69 ans (6\%) et les 80-84 ans (6\%), et ce bien plus fréquemment que dans les réponses des résidents de Bandra.

5 Brihanmumbai Municipal Corporation: il s'agit de la municipalité du Grand Mumbai, donc le budget annuel dépasse celui de beaucoup de petits états. 
Pour ces derniers, le chômage et les licenciements sont une préoccupation, légèrement plus marquée pour les cinquantenaires et les sexagénaires à cause des fermetures des usines de textile dans les années 1980 (voir chapitre 1). Douze mentions sur les 39 font explicitement rapport à cette désindustrialisation de la ville. Dans les raisons, se perçoivent également les impacts directs du chômage pour la famille :

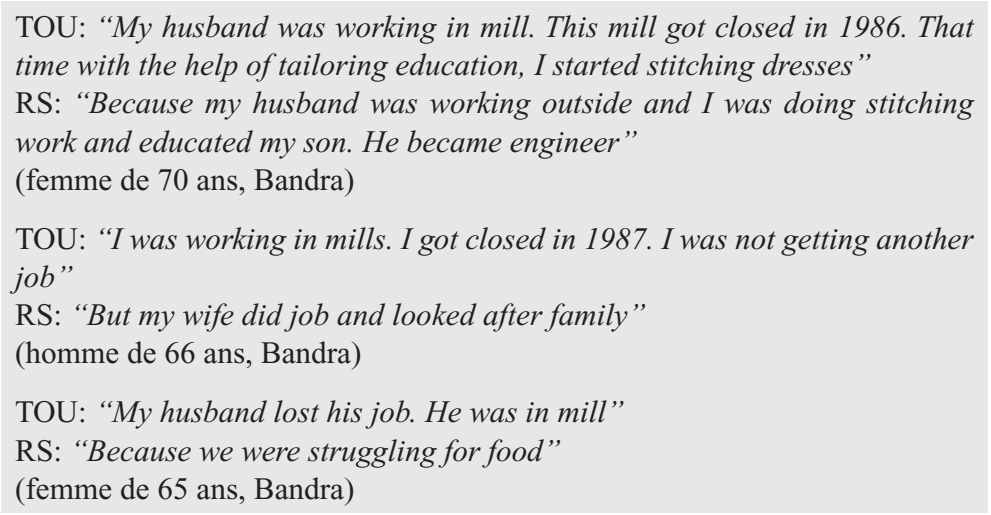

\section{Les perceptions positives et négatives du travail}

Une mémoire professionnelle ouvertement positive (obtenir de la reconnaissance, avoir une promotion - en termes de poste ou de salaire -, avoir son business qui va bien ou grandit) apparaît peu, aussi bien à Bandra qu'à Santa Cruz (seulement $2 \%$ et $3 \%$ des changements récents). Cette situation est cependant plus apparente chez les 35-39 ans, avec quand même $8 \%$ des mentions du volet I faites à Santa Cruz (contre 3\% à Bandra).

Du côté des tournants professionnels, les chiffres sont encore plus dérisoires. Seules trois personnes à Bandra et 15 à Santa Cruz en font mention :

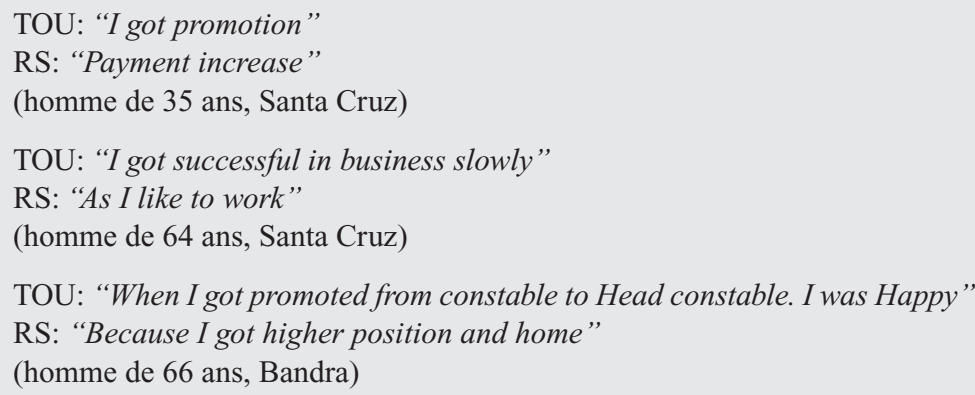


Nonobstant la prudence requise, puisque ces valeurs sont faibles, se situer dans une classe moyenne inférieure offre plus d'opportunités de réussite que les bidonvilles.

A l'inverse et sans surprise, le côté négatif du travail (licenciement, chômage) ressort plutôt parmi les réponses données à Bandra, bien que les chiffres demeurent petits ( $3 \%$ contre $1 \%$ des changements récents). A nouveau, si la fréquence globale est faible, des disparités sont présentent selon l'âge. Les licenciements et le chômage sont ainsi évoqués par $6 \%$ des jeunes des zones défavorisées (soit 10 individus) et presque pas par leurs homologues de classe moyenne inférieure ( 1 seul individu). Il n'est guère possible de dissocier ici ce qui relève de l'impact réel de la condition socioéconomique et de l'économie informelle, ou d'une perception plus négative de sa trajectoire professionnelle.

$* * *$

A l'inverse du domaine de l'éducation, les tournants professionnels ont été cités par toutes les classes d'âge dans une proportion semblable. Pourtant, à nouveau se dessine une entrée plus précoce dans le monde du labeur pour les individus résidant en bidonvilles (voir figure 5.46).

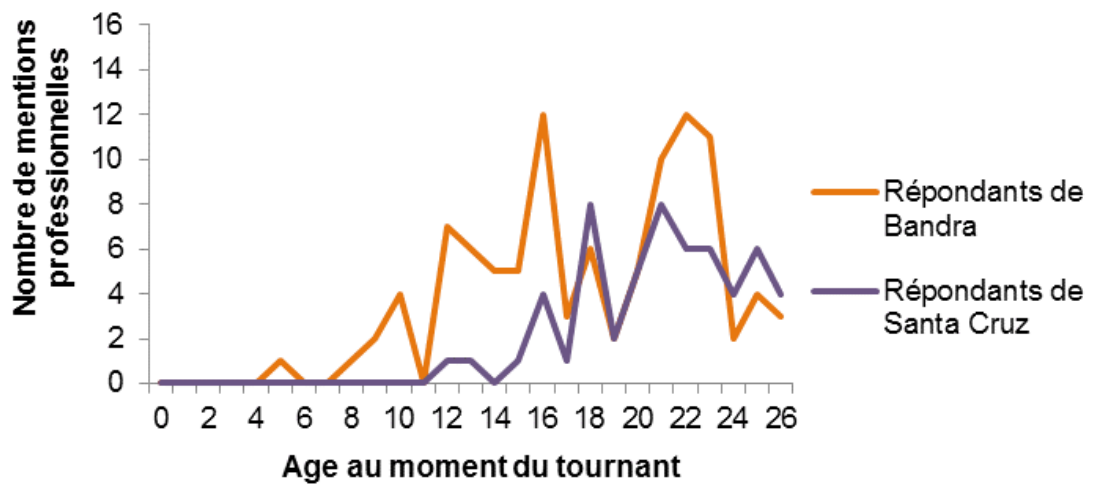

Figure 5.4: les mentions professionnelles ( $n$ ) selon l'âge du répondant au moment du tournant et le lieu de résidence

Concernant les différences de genre (figure 5.5), les débuts des courbes sont à peu près semblables, mais un clivage ressort nettement dès l'âge

6 A nouveau, seules les 26 premières années de l'existence ont été considérées, afin de permettre à toutes les classes d'âge d'avoir une même possibilité de mention. 
de 20 ans, lorsque les hommes évoquent beaucoup plus fréquemment un tournant dans cette sphère de leur vie.

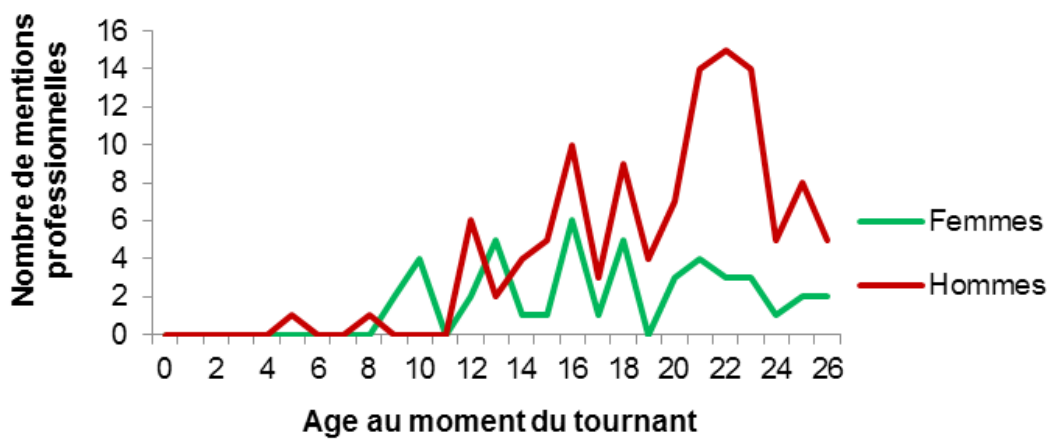

Figure 5.5: les mentions d'éducation $(n)$ selon le sexe et l'âge du répondant au moment du tournant

\subsubsection{Au gré de l'économie: responsabilité globale, vulnérabilités individuelles}

Dans les changements récents et les tournants, les individus évoquant des évolutions de situation économique, en mentionnant des augmentations de revenus ou de dépenses ainsi que l'achat ou la vente de biens, sont très peu nombreux. Plus spécifiquement à Santa Cruz, apparaît également la constatation négative d'une inflation générale des prix, affectant les ressources financières du ménage. Au final, malgré sa faiblesse, ce domaine nous informe de ce qui peut influencer le quotidien en termes de dépenses ou de rentrées d'argent et, à ce titre, il n'est pas surprenant qu'il soit surtout cité par les 35-39 et les 50-54 ans, âges pivots où les responsabilités familiales sont les plus lourdes.

La quinzaine de tournants citant l'achat ou la vente d'un bien $(n=17)$ est passablement hétéroclite, pouvant aller de l'acquisition d'un vélo à la perte d'une maison : 
TOU: "My son purchased a house"

RS: "We used to live in a rented house I was very happy when he purchased a house"

(femme de 70 ans, Bandra)

TOU: "Son had bought new flat and settled"

RS: "Seen my son get settled and grown up with my eyes"

(homme de 80 ans, Santa Cruz)

TOU: "My grandfather sold his house"

RS: "Because we were houseless. Now we stay in rented house. It is difficult to run house"

(homme de 25 ans, Bandra)

Parmi les tournants évoquant une amélioration économique $(n=23)$, les raisons laissent la plupart du temps entendre les conséquences positives influant sur la famille de manière immédiate (davantage de nourriture, etc.) ou sur le long terme (possibilité d'envoyer les enfants à l'école, de régler la dot pour le mariage d'une fille, etc.) :

TOU: "Income increased so financial condition improved"

RS: "Due to the income of children now there is better support for running the household"

(homme de 51 ans, Santa Cruz)

TOU: "Since 2007 I am working as a watchman at Shivalik builder's office. Before that I was ironing cloths and earning money. With that earning only I married my daughter in 2004"

RS: "Now I have fixed payment. So I can manage house. I did marriage of my 2nd daughter"

(homme de 53 ans, Bandra)

TOU: "I have profit from flour mill shop"

RS: "The income from my flour mill allowed me to educate my children well" (homme de 66 ans, Bandra)

TOU: "My elder son got job. Now I have this support. Now our condition is improving"

RS: "Once upon a time we had very bad condition. They was nothing to eat. Now condition is good. We shouldn't forget bad days"

(femme de 52 ans, Bandra)

Du côté des détériorations économiques, les raisons pour avoir mentionné ces tournants mettent en avant, pour les habitants de Santa Cruz, surtout les causes (dans 7 cas sur 18, elles sont explicitement liées à la récente 
crise économique ayant entraîné une inflation considérable en Inde) et, pour les résidents des slums, plutôt les impacts (imminence de la pauvreté et de ses retombées pour la famille) :

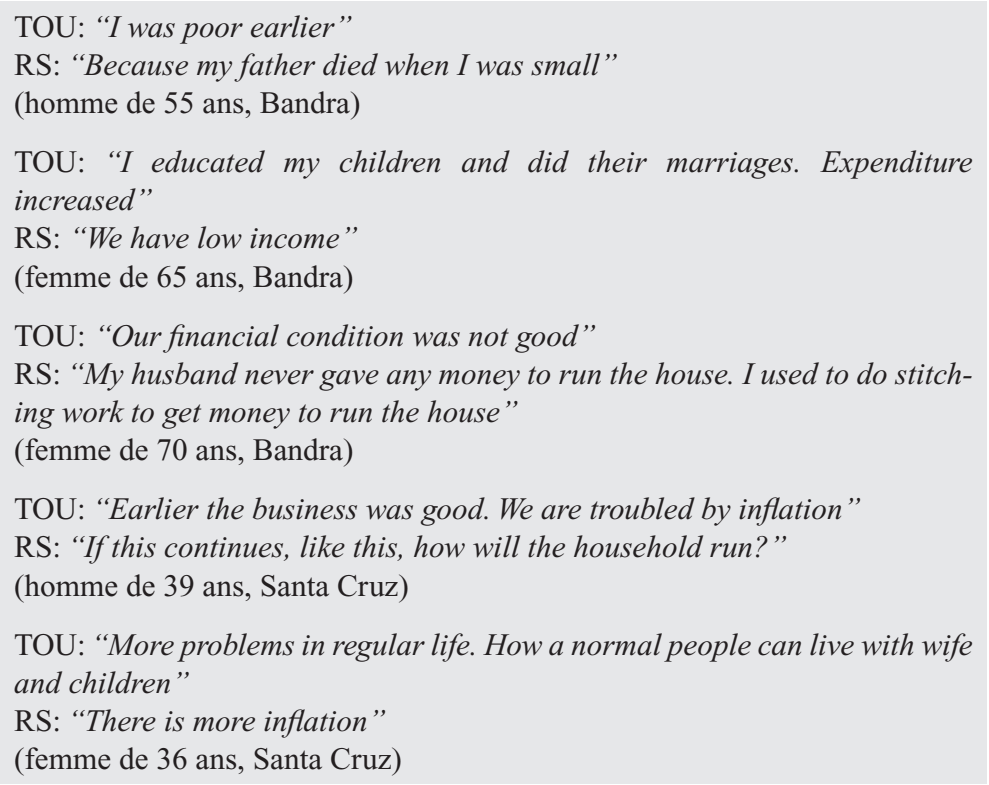

Nonobstant, le peu de mentions dans ce domaine économique laisse perplexe lorsque l'on connait la pauvreté et le dénuement dans lesquels vivent la plupart des individus interrogés. Les dimensions concrètes de la pauvreté (impossibilité d'étudier, nécessité de travailler, etc.) sont davantage formulées que la misère elle-même.

\subsubsection{Le lieu de vie comme déterminant social}

La catégorie mobilité, autre facette du capital économique, regroupe, en ce qui concerne les changements récents, les transhumances d'une autre ville ou de la campagne vers Mumbai, ainsi que certains déménagements à l'intérieur même de la ville. Les migrations de longue portée n'apparaissent pas du tout dans les changements récents (probablement car, si la personne avait vécu une migration au cours de l'année passée, elle ne serait pas présente au moment de l'enquête pour l'évoquer) et relativement 
peu dans les tournants, où seuls quelques départs vers le Moyen-Orient sont cités.

Dans le volet II, ce sont avant tout des transports d'un autre Etat (ou d'un autre lieu dans le Maharastra) vers Mumbai qui sont mentionnés, ou encore d'un quartier à l'autre dans la mégapole, généralement d'un slum vers un immeuble en dur. Ces déménagements (qui ne représentent que 5 à $6 \%$ des tournants, voir annexe 3) soulignent tant des obligations familiales (comme de rejoindre son époux ou épouse) que des opportunités professionnelles (venir à Mumbai depuis un village de campagne par exemple) ou des changements de statut socioéconomique (devenir propriétaire d'une maison) :

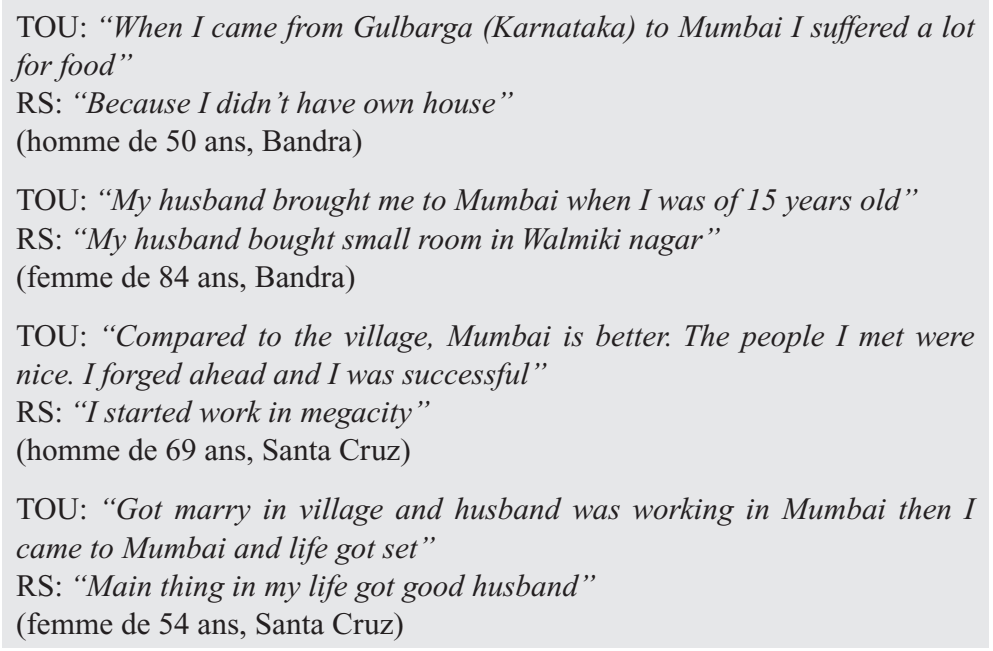

Les habitants de slums ont bien souvent vécu plusieurs de ces mobilités, à l'occasion de leur arrivée en ville puis d'allers et retours vers leur village d'origine. Néanmoins, les quelques citations qui sont faites par les habitants des slums ne concernent que des déménagements survenus dans la ville de Mumbai. De leur côté, les répondants de Santa Cruz évoquent quelques événements comme devenir propriétaire, passer des slums aux immeubles SRA (Slum Rehabilitation Authority, immeuble prévu pour reloger les habitants des bidonvilles dans le cadre de campagnes municipales, voir 
chapitre 1), mais cela reste des chiffres très faibles : 30 tournants évoqués par des habitants de Santa $\mathrm{Cruz}^{7}$ et 4 par ceux de Bandra.

Les actions de délocalisations des bidonvilles en Inde, et en particulier à Mumbai, sont très controversées. Visant officiellement à sortir les individus de ces zones insalubres et officieusement à récupérer de l'espace au centre-ville pour des opérations de spéculation immobilière, les conséquences pour les familles ne se révèlent généralement pas positives (Felber \& Schmid, 2014). En se penchant sur les raisons de ces tournants, il apparaît néanmoins que la majorité relève les aspects bénéfiques de ces déplacements (24 à Santa Cruz et 2 à Bandra). Sans doute car l'une des premières critiques de ces programmes est leur sélectivité ; en profiter relève déjà du positif :

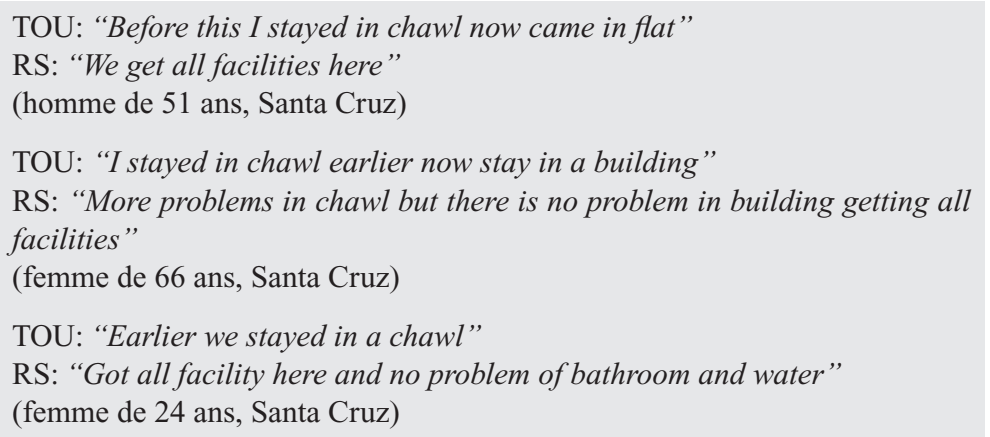

Ces citations raisonnent de manière très concrète, notamment pour les femmes, en lien avec l'accès à l'eau courante par exemple. A nouveau, il est étonnant de constater si peu de présence de mentions portant sur les lieux de vie insalubres, en particulier à Bandra. C'est a priori la marque d'une accommodation générale.

\section{L'environnement, une dimension supplémentaire du capital économique} Finalement, une catégorie supplémentaire «autre» du capital économique (voir annexe 3) regroupe en majorité des évocations d'événements environnementaux (crise économique, événements politiques, catastrophes naturelles, émeutes interreligieuses, etc.) que l'on aurait arbitrairement

7 Vingt-six personnes sur les 30 de Santa Cruz ayant cité un déménagement proche vivent en SRA, soit 14\% des individus interrogés dans le quartier de classe moyenne et résidant en building SRA. 
attribués au volet III de l'enquête, mais qui ont eu tant d'impact sur le quotidien des individus que ces derniers les ont cités comme des événements personnels. La portée de ces changements sociohistoriques étant négative, en général supposant une perte de biens, l'impossibilité de se déplacer ou l'arrêt des activités économiques de la ville durant plusieurs jours, ils ont été considérés comme affectant directement le capital économique des individus :

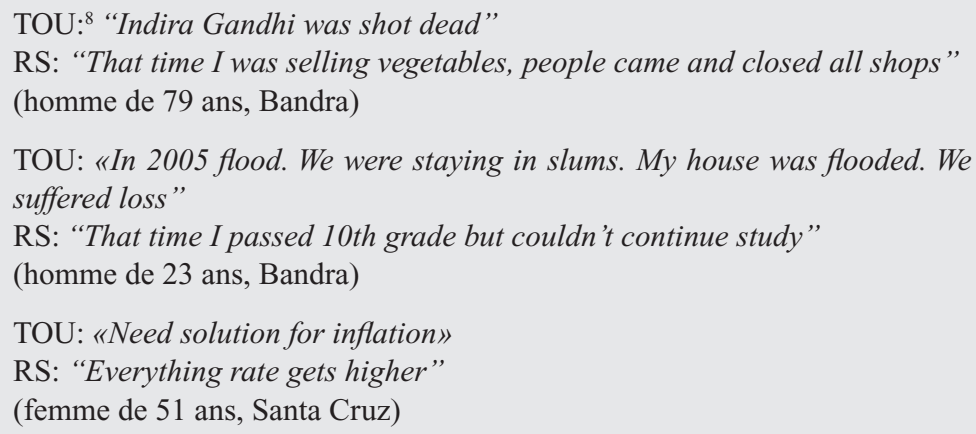

\subsection{Conclusion}

Au sein d'un monde occidental où la seconde modernité a augmenté la variabilité entre les parcours de vie, où les institutions régissent toujours les trajectoires tout en définissant moindrement l'ordre et l'âge de survenue des événements marquants de l'existence, le passage à l'âge adulte a subi des modifications. La plus marquante de ces mutations a été l'étalement des transitions, leur complexification et diversification, en bref, une montée des incertitudes et un report de ce passage de quelques années (Bidart, 2005; Mauger, 1995).

Dans une étude portant sur la perception du «devenir adulte», Claire Bidart a souligné l'existence de seuils biographiques de plus en plus différenciés selon que l'on soit d'une origine sociale modeste ou plutôt aisée, et d'une culture à l'autre (Bidart, 2005, 2006b). L'inégalité d'accès à l'âge

8 Les tournants (TOU) et les raisons (RS). 
adulte (Battagliola et al., 1997; Bourdieu, 1984) se traduit concrètement par une entrée plus précoce dans les transitions menant à la vie adulte: fin des études et entrée sur le marché du travail; sortie du nid et constitution d'une famille propre (Bidart, 2005). De manière inattendue, ce constat transparaît également dans les réponses fournies par les habitants de Mumbai, alors même que les institutions formelles sont quasiment inexistantes dans la gestion des trajectoires. Leur mémoire confirme que l'âge de la fin de l'école et du début du travail survient plus tôt chez les défavorisés, qui ont toujours (eu) moins accès à l'éducation. L'accès à la formation montre ici son importance décisive sur la normalisation des événements liés à l'entrée dans l'âge adulte.

Mais in fine, le résultat le plus marquant de ce chapitre est l'absence. La misère, la faim, les conditions de logement pitoyables, toute une série d'expressions de la pauvreté pourtant toujours omniprésente, ne ressortent que de manière ponctuelle, sur des fréquences basses. Ce large silence est singulier et interpelle. Il peut illustrer ce que Galbraith, économiste qui fut ambassadeur en Inde, appelait «l'accommodation à la pauvreté», qui n'est pas à proprement parler de la résignation, mais la conscience diffuse de la difficulté, voire de l'impossibilité de sortir de sa condition, impliquant de ne pas ajouter à sa souffrance en nourrissant des espérances qui seront déçues (Bi, 1981; Galbraith, 1980).

Dans l'Inde contemporaine en transition, les classes moyennes sont bel et bien en émergence et avides de saisir de nouvelles opportunités, mais encore assez fragiles pour se sentir menacées par l'inflation. Dans les bidonvilles, l'espoir n'est pas absent, mais il ne s'appuie guère que sur de jeunes générations marquées par une forte ambivalence, dans la mesure où elles héritent de la «culture de la pauvreté» de leurs parents, parents qui souhaitent pour autant les outiller de capitaux éducatifs afin qu'ils puissent avoir une autre vie que la leur, mais dont les espérances se confrontent parallèlement aux contraintes structurelles qui subsistent dans l'accès à l'éducation. 


\title{
Chapitre 6. La place de la famille dans une société en transition
}

\author{
"[...] l'analyse de «l'économie pratique» de la famille \\ nous invite plutôt à l'envisager comme un espace de \\ coopération et de conflit permanent, tant d'un point de \\ vue matériel que symbolique.»
}

(Guérin, 2008, p. 62)

Le parcours de vie d'un individu est jalonné de moments forts parmi lesquels, nous l'avons vu, le cadre familial ressort comme central, en tant que lieu de vie et de développement durant l'enfance, puis dans la construction de son propre foyer à partir de l'âge adulte (Bonvalet \& Lelièvre, 2012; Cavalli et al., 2006; Gastrón \& Lacasa, 2009). Ne dérogeant pas à ce constat, les événements familiaux sont nombreux dans les souvenirs rapportés par nos répondants. Nous les analysons ici, et ce selon une perspective genrée.

Dans les changements récents comme dans les grands tournants de la vie, les Indiennes et les Indiens interrogés ont mis en avant des moments importants de leur trajectoire familiale : unions, naissances et décès. Une première section se penche sur ces principaux aspects des relations familiales. A partir de ces résultats, et grâce aux informations qualitatives recueillies, nous mettrons en avant plusieurs types de vulnérabilités. La place accordée aux proches dans ces souvenirs sera également mise en exergue, cette dernière étant révélatrice des évolutions récentes, tant socioéconomiques que démographiques et sociétales.

Ces transformations ont affecté les structures familiales, mais aussi les normes matrimoniales, les rapports de genre et la distribution du pouvoir au sein du foyer (Chadda \& Deb, 2013), conduisant soit à des ruptures entre les générations, soit servant de support à la continuité des traditions face à la modernisation croissante du pays. Ainsi, la seconde partie de ce chapitre se focalisera sur la cohabitation entre plusieurs générations, situation typiquement indienne et néanmoins en pleine mutation, en particulier à Mumbai où se conjuguent changement social et manque d'espace (en 
termes immobiliers) (cf. chapitre 1). La perception par les individus des différences de comportements au sein de leur famille, capturée par le biais d'une question subsidiaire posée aux répondants logeant à Santa Cruz, permettra de creuser cette question.

Dans un troisième temps, les questions de genre implicites dans les événements familiaux mentionnés par les répondants seront soulignées. Nous questionnerons la répartition classique de la sphère privée pour les femmes et de la sphère publique pour les hommes en observant la répartition des événements de chacun des domaines à travers les âges. Par la suite, en observant les mentions de naissances, l'une des dimensions cruciales des inégalités entre les sexes en Inde sera illustrée : la préférence pour un fils.

\subsection{La famille indienne dans une modernité ambivalente}

Dans les questions portant sur les événements de l'existence (volet I et II), le domaine de la famille représente respectivement $15 \%$ et $26 \%$ du total des mentions. Pour affiner ces chiffres, des subdivisions plus fines (les types) ont été prévues (voir annexe 4, qui présente ces résultats selon le lieu de résidence et la classe d'âge) : entrées en couple, mariages, naissances, divorces (ou séparations). Comme indiqué précédemment, à ces catégories peut être ajoutée celle des décès, qui avait été codée à part mais qui s'avère concerner essentiellement des membres du ménage ou de proches parents. Au final, ces mentions ne varient guère, en ce qui concerne leur contenu, des résultats observés dans des enquêtes similaires provenant d'autres pays (Cavalli \& Lalive d'Epinay, 2008; Gastrón \& Lacasa, 2009). Malgré tout, un certain nombre de particularités propres à la société indienne résident dans ces réponses, que ce chapitre entend souligner.

\subsubsection{Les dits et les non-dits des trajectoires familiales indiennes}

Les mariages et les naissances sont les types les plus mentionnés dans toutes les classes d'âge (sauf pour les jeunes de Santa Cruz dans le volet I, qui montrent un intérêt plus important envers leur mise en couple). 
Suivant une logique chronologique, lorsque les jeunes parlent de changements récents familiaux de type mariage, il s'agit majoritairement de leur union et de celle de leurs frères/sœurs (membres de la même génération). A l'inverse, les mariages de sa progéniture sont plutôt mentionnés par les groupes d'âge 35-39, 50-54 et 65-69 ans ; que cela soit possible dès 35-39 ans illustre 1'âge toujours très faible au premier mariage en Inde. Dans le même ordre d'idée, lorsque les 20-24 et 35-39 ans parlent de naissances survenues cette dernière année, il s'agit essentiellement d'euxmêmes entrant dans la parentalité, alors que parmi les 50 ans et plus, cela concerne leurs enfants, au travers de la naissance des petits-enfants. Finalement, les décès rassemblent une faible proportion des changements récents $(4 \%)$, et ce de manière semblable entre les groupes d'âge.

Les différenciations sociales sont peu apparentes. D'un quartier à l'autre, ce sont les mêmes moments de la vie familiale qui sont cités aux divers âges de la vie, sauf en ce qui concerne les jeunes adultes de 20-24 ans. Pour ces derniers, les événements familiaux sont évoqués comme sources de changement plus souvent par les résidents des slums, mais le contenu varie également : dans la zone de bidonvilles de Bandra. Là, ce sont les naissances et les mariages qui prévalent alors qu'à Santa Cruz les rencontres sont également mentionnées. Traduction indienne oblige, lorsque l'on parle de «rencontres», il s'agit en fait des fiançailles : nous reviendrons sur cette particularité ultérieurement. Comme cela a déjà été remarqué aux chapitres 4 et 5 , le contenu des changements récents laisse entrevoir une transition à l'âge adulte plus précoce pour les habitants des secteurs les plus pauvres. En revanche, un écart se creuse entre les tournants concernant la famille des personnes âgées de 65-69 et 80-84 ans résidant à Bandra (28\% et $31 \%)$ et celles vivant à Santa Cruz (20\%).

Alors que la part du domaine famille se réduit avec l'avancée dans les classes d'âge pour le volet I de l'enquête, elle prend au contraire de l'ampleur au sein des tournants, mis à part une petite réduction dans la cinquantaine. Le reflet de la méthodologie de notre enquête s'observe ici : le volet I, en ne demandant de trouver d'événements qu'au cours de la seule dernière année, laisse davantage d'espace - notamment aux jeunes pour évoquer des éléments que l'on peut qualifier de «moins cruciaux» (comme des fiançailles) que ne le fait le volet II, portant sur l'entièreté de l'existence.

A noter que très peu de divorces sont mentionnés dans toutes les classes d'âge quel que soit le lieu de vie, soulignant qu'en Inde, même 
dans un monde urbain qui vit une modification socioéconomique rapide, la rupture explicite des couples n'est pas encore une pratique courue.

Singulièrement, la dot n'est évoquée qu'une fois dans les changements récents et trois fois parmi les tournants (dont deux fois par la même personne), ce qui est bien peu si l'on considère que la plupart des mariages impliquent une telle transaction, qui pèse profondément sur les familles et qui, à la fois, exprime et affecte lourdement les rapports de genre (LoiselleLéonard, 2001). Néanmoins, il est envisageable que le caractère illégal et très privé de cette négociation encourage une certaine forme d'autocensure à son encontre. Par ailleurs, aucun aspect de la dimension rituelle n'apparaît en lien avec ces changements qui, la plupart du temps, sont pourtant marqués par des codes et des pratiques religieuses (fête, cérémonie, voyage, initiation, ...).

\subsubsection{Fiançailles et mariages : effilochement des traditions ou transformation liée à la modernité?}

La modernisation a transfiguré les structures familiales, même si des normes anciennes persistent. Très ancré dans la tradition indienne, le devoir de se marier est source de multiples codes et cérémonies durant la jeunesse. Les parents ne sont pas en reste, puisqu'ils sont en charge de l'arrangement des fiançailles (dans la majorité des cas) et des termes de l'union. C'est une réalité qui se retrouve dans les réponses aux volets I et II, où mises en couple (fiançailles) et mariages représentent une part non négligeable des souvenirs rapportés.

\section{Les fiançailles : devoir parental pour les uns, expression de joie pour les autres}

Les changements récents ont à cet égard suscité légèrement plus de mentions de rencontre ou de mise en couple $(n=13)$ que les tournants $(n=8)$, des chiffres qui demeurent très faibles. Dans les deux cas, la répartition entre les sexes est presque équitable. Sur ces 21 mentions, 6 proviennent de parents parlant de leurs enfants (4 mères et 2 pères), exprimant le devoir qui pèse sur eux : 
"My daughter's marriage got fixed"

(femme de 37 ans, Bandra)

«In 2012 my son got engaged»

(homme de 52 ans, Bandra)

Ce sont les jeunes plus que les autres groupes qui ont cité de tels événements, plus proches d'eux ( $n=14)$, parlant de leur mise en couple, de leurs fiançailles ou de leur proche mariage :

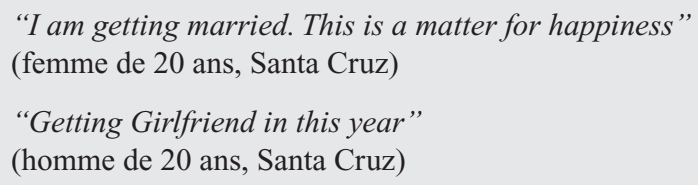

Cinq raisons sur 8 expriment un sentiment de joie, tant de la part des parents que des principaux concernés :

"We all were very happy"

(femme de 20 ans, Bandra)

"We will make love marriage as arrange marriage»

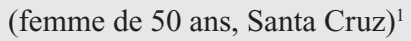

A deux reprises, la tension de ne pas parvenir à réaliser ce mariage et le soulagement d'y être finalement arrivé sont palpables :

"My mother and father were very concerned \& worried about my marriage" (femme de 23 ans, Santa Cruz,).

"My mother's dream of daughter getting married is going to be fulfilled" (femme de 21 ans, Bandra).

Il ne s'agit certes que de quelques réponses, souvent laconiques, mais elles soulignent une dualité de la vie dans l'Inde contemporaine, une modification profonde de la signification du mariage. Ce dernier conserve son statut d'objectif absolu, tant pour les hommes que les femmes; toutefois, la planification (très rigide traditionnellement) faite par les parents (le

1 Ce qui révèle l'opportunité de pouvoir joindre l'utile à l'agréable: un mariage arrangé (selon les codes sociaux) sera organisé à partir d'une union choisie et consentie par les conjoints. 
fameux mariage arrangé) est en train de s'émousser. Les parents continuent malgré tout à porter la charge du bien-être de leur famille en ayant le devoir de s'assurer qu'un mariage ait finalement lieu. Les répondants évoquant leur mariage comme un grand tournant de leur vie éclairent encore davantage cette concomitance du changement social et des traditions qui ont la vie dure.

\section{Bonheur et regrets, amour et arrangement : les mariages indiens révèlent une complexité des rôles et des choix}

Parmi les tournants majeurs de l'existence, les citations de mariage sont plus nombreuses que celles concernant les naissances $(13 \% \text { contre } 7 \%)^{2}$. Ce constat souligne bien l'importance des unions dans un pays comme l'Inde, tout en restant étonnamment faible lorsque l'on sait que $66 \%$ des personnes interrogées à Bandra et $73 \%$ de celles vivant à Santa Cruz sont mariées.

Pour mieux atteindre les marqueurs culturels et ceux spécifiques aux mutations socioéconomiques de Mumbai, il faut se pencher sur la manière dont les réponses sont expliquées. Cinq catégories ont été utilisées pour coder ces raisons ${ }^{3}$ : la responsabilité de marier ses enfants, la reconfiguration familiale issue du mariage, le mariage d'amour, les regrets, les joies ${ }^{4}$.

Dans la tradition indienne, les parents ont le devoir de marier leur fille et les frères attendent communément que toutes leurs sœurs soient mariées (Joshi, 2016). Cette pression qui repose sur les épaules des parents a déjà transparu dans les raisons des fiançailles (voir ci-dessus) et apparaît également dans les tournants concernant les mariages, quoique relativement peu: 11 mentions, faites tant par des femmes que des hommes, sur la totalité des 201 citations de mariage.

2 Pour plus de détails, voir le chapitre 4, section 4.2.2.

3205 tournants sur les 299 au total ont pu être codés. Les tournants qui n'ont pas été catégorisés soit étaient trop peu indicatifs pour décider du contenu, soit ne possédaient pas de raison, soit décrivaient une situation objective (le déroulement de la cérémonie, de la vie quotidienne,...), ou encore évoquaient les conséquences de l'union (avoir eu des enfants, acheté une maison,...).

4 Les changements de l'année précédente contiennent également une part importante de souvenirs de mariages; néanmoins, nous nous pencherons uniquement sur les grands événements de la vie, qui offrent l'opportunité de creuser les réponses à l'aide des raisons et qui permettent aux individus des classes d'âge supérieures d'avoir mentionné leur union. 
Un autre aspect intéressant est celui du mariage d'amour, évoqué comme extraordinaire dans une société qui repose sur l'arrangement des unions. Seules 14 mentions en ont été faites, également réparties entre les sexes, un peu plus souvent à Santa Cruz $(n=9)$ qu'à Bandra $(n=5)$. Avant tout, ce sont les plus jeunes cohortes (20-24 et 35-39 ans) qui mentionnent une union choisie par amour ; toutefois, quelques individus de 50-54 ans l'ont aussi évoquée, ces derniers ayant cependant fait cette expérience «depuis l'autre côté du miroir», en tant que parents. En effet, en un paradoxe qui nous instruit, l'enjeu des sentiments pour les jeunes rencontre la responsabilité lourde de marier ses enfants pour les pères et les mères, le tout se traduisant ici en une situation complexe : préférer la tradition ou encourager le changement?

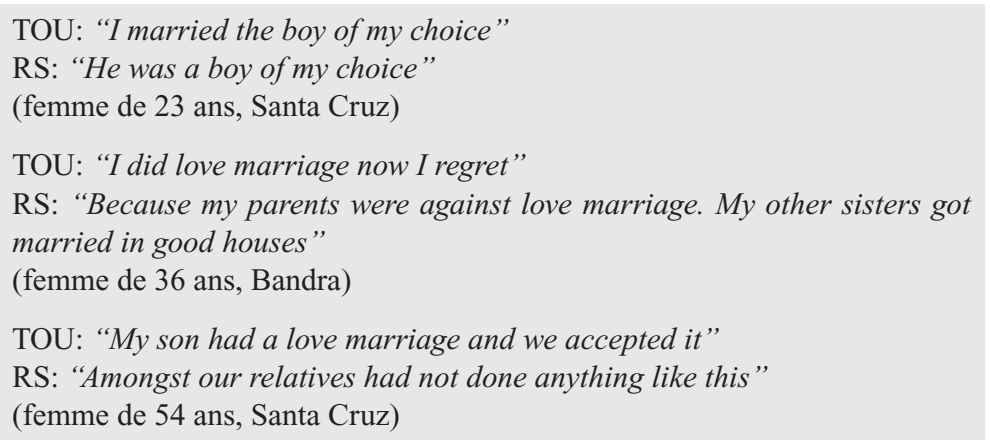

Se discernent aussi, avec un peu plus de force, des évocations de mariage comme un moment de transformations des configurations familiales $(n=43)$, notamment suite à l'arrivée de la bru dans le foyer de l'époux. La charge des parents lors de l'arrangement de l'union est donc aussi de jauger le futur lieu d'accueil de sa fille, ou la compatibilité de sa future bru avec sa famille. Cette responsabilité est indéniable, et la reconfiguration familiale touche évidemment la belle-famille autant que l'épouse, de même que la famille d'origine, qui perd parfois un salaire, en tout cas une aide et un membre de la famille. Mais en réalité cela affecte en premier lieu la mariée, esseulée dans un nouveau ménage (parfois loin de son lieu d'origine), ensuite la belle-mère, qui doit accueillir et faire une place à la nouvelle. Ce qui explique la large majorité féminine de ces mentions $(n=33$ sur 43) : 
TOU: "I got married"

RS: "I had to move to a new house and I had responsibilities"

(femme de 70 ans, Bandra)

TOU: «My son's wedding»

RS: "Because we were getting Daughter in law"

(homme de 51 ans, Bandra).

TOU: "Two son's got married"

RS: "I have 2 daughters in laws. They do household work. Now I take rest"

(femme de 65 ans, Bandra)

TOU: «After marriage the life change»

RS: "I was a girl then. Now I am a daughter in law"

(femme de 22 ans, Santa Cruz).

Les groupes d'âge ayant le plus cité ce changement de configuration sont les trentenaires $(n=11)$ et les cinquantenaires $(n=11)$, mais aucun groupe n'en a pas fait mention du tout. Comme nous l'évoquions plus haut, il ne faut pas surestimer la nucléarisation des ménages urbains indiens et sous-estimer la persistance de la coutume du mariage patrilocal, lequel implique une transition profonde qui affecte tout particulièrement la vie des femmes. D'ailleurs, les habitants du quartier de classe moyenne modeste évoquent plus cette raison $(n=29)$ que ceux des bidonvilles $(n=14)$.

Une quatrième catégorie a servi à regrouper les raisons des tournants où transparaissent des regrets liés au mariage. Cette catégorie rassemble 73 mentions, dont 50 faites par des femmes. En majorité, il s'agissait de personnes des groupes d'âge 35-39 $(n=16), 65-69(n=11)$ et 80-84 ans $(n=30)$, surtout vivant à Bandra $(n=54)$. L'émotion derrière ce regret s'inscrit dans un éventail allant de très marquée (le répondant exprime sa tristesse, parfois vivement) à ambivalente (les conséquences semblent négatives mais ce n'est pas explicite). Le regret le plus marquant est celui d'avoir été marié trop jeune, ce qui est bien plus souvent évoqué par des femmes. Par deux fois, le fait que le mariage soit survenu tard a été mentionné, mais par des hommes : 
TOU: "I got married when I was 14"

RS: "I was too young to get married"

(femme de 81 ans, Bandra)

TOU: "Many changes came after marriage»

RS: "My marriage was late"

(homme de 54 ans, Santa Cruz)

TOU: "I was happy before marriage"

RS: "Now I feel my life is wasted"

(femme de 34 ans, Bandra)

TOU: "When got married, after that there was tension"

RS: "It is fights always"

(homme de 35 ans, Santa Cruz)

TOU: "My life changed after marriage"

RS: "As new responsibilities had to shouldered"

(femme de 19 ans, Santa Cruz)

A l'inverse de la catégorie précédente, un certain nombre de citations de mariage contiennent des expressions heureuses (64 mentions, dont 40 provenant de femmes). Ce sont surtout des individus âgés de 35-39 $(n=16), 50-54(n=10)$ et 65-69 ans $(n=22)$, et la répartition entre les quartiers est relativement équilibrée ( $n=38$ à Bandra, $n=26$ à Santa Cruz) :

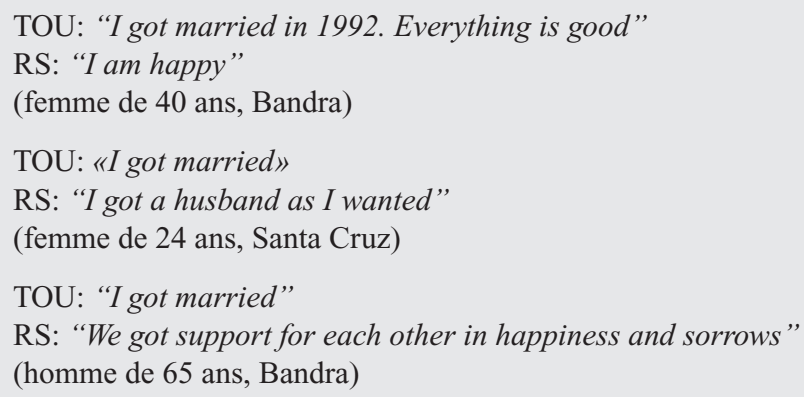

$* * *$

Ces mentions de mariage permettent de percevoir des sources de stress ou de vulnérabilité propres au mariage à l'indienne : parvenir à marier ses enfants, être bien accueillie dans son nouveau foyer en tant que dernière arrivée, vivre en harmonie ou en conflit, etc. A cela s'intègrent des éléments issus de la modernisation du pays et des mentalités depuis quelques décennies: le mariage d'amour, tellement prôné dans les films de 
Bollywood, qui entre en contradiction avec les arrangements traditionnels (Trivedi, 2014). Mais également la prise de conscience, dans les générations anciennes, que l'âge au mariage a évolué et que sa propre union est survenue trop jeune. Parfois, ces contradictions sont librement exprimées par des regrets, d'autres fois, elles transparaissent au travers de l'évocation de responsabilités supplémentaires, de tout le carcan de rôles nouveaux que les individus doivent endosser. Finalement, il apparait que les mariages, même arrangés, ne sont pas uniquement faits de tensions quotidiennes ou de regrets, mais que nombre d'entre eux ont été souhaités et sont associés au bonheur.

Ce dernier point coïncide avec une nouveauté dans les manières de concevoir le mariage. Tout comme la famille élargie issue de l'union patrilocale, la pratique du mariage arrangé n'a pas disparu au contact de la modernité, au contraire. L'arrivée de l'économie de marché et du néolibéralisme a plutôt dopé la consommation liée à son organisation et à sa célébration (agences matrimoniales, cadeaux, fêtes, etc.). Ainsi, alors que la réussite personnelle est de plus en plus mise en avant, les couples s'accrochent à une pratique qui prône l'inverse : l'ingérence de la famille dans un choix intime de vie. Surpris que se conjuguent ces deux courants de prime abord antagonistes, plusieurs auteurs ont étudié les comportements des jeunes de classes moyennes dans l'Inde urbaine et tenté d'expliquer ce paradoxe (Sharangpani, 2010; Trivedi, 2014). Il en ressort une forme d'adaptation - à nouveau similaire à la restructuration familiale - des jeunes filles notamment (alors que leurs aînées d'une génération apparaissent comme davantage encline au mariage d'amour [Sharangpani, 2010]) qui, bien que de plus en plus émancipées, acceptent et même revendiquent une tradition où leurs choix personnels sont peu pris en compte, en échange de davantage d'exigences face au conjoint. La relation de pouvoir évolue, même si aucune révolution n'a encore eu lieu, face à une modernité alternative (Sharangpani, 2010).

\subsubsection{L'impact du décès sur les proches}

On l'a vu précédemment, les trajectoires familiales comprennent plusieurs types d'événements, dont la connotation varie entre tragédie et bonheur (mariages, séparations, naissances, ...). Sans conteste, les décès représentent le versant le plus sombre de ces souvenirs. 
Sans surprise, les décès sont moins nombreux dans la mémoire des individus les plus jeunes et atteignent leur maximum dans les citations des deux dernières classes d'âge. Entre les terrains d'enquête, les taux de pertes mentionnées sont relativement proches (voir annexe 4). Cependant, le nombre d'individus citant au moins un décès n'est pas identique dans les groupes d'adultes mûrs et âgés : 20\% des 50-54 ans résidant à Bandra évoquent au moins une perte contre $12 \%$ à Santa Cruz; respectivement $41 \%$ contre $27 \%$ des $65-69$ ans ; et $34 \%$ contre $24 \%$ des $80-84$ ans. En fait, les répondants de Bandra ont régulièrement mentionné plusieurs décès.

Toutefois, perdre un proche n'est pas forcément une transition inattendue ou traumatisante. C'est le cas, par exemple, de petits-enfants mentionnant la mort de leur grand-père ou grand-mère (Cavalli et al., 2006). Dans les parcours de vie d'Indiennes et d'Indiens soumis à une pauvreté plus ou moins importante, quelles pertes sont-elles mentionnées et à quels âges surviennent-elles? Pour répondre à ces questions, nous analysons les décès évoqués comme des tournants par les répondants (soit le volet II de l'enquête). Par la suite, nous nous penchons sur les raisons invoquées pour les avoir cités, afin d'extraire l'impact de la mort d'un «autre significatif» sur la vie de la personne.

Quatre catégories ont été retenues : la perte du conjoint (40\%), celle d'un parent (28\%), d'un enfant (ou d'un beau-fils, d'une belle-fille) (21\%) ou encore d'autres proches $(11 \%)^{5}$. Les figures 6.1 et 6.2 exposent le nombre de décès mentionnés par les individus comme ayant été des turning points de leur existence, selon le lien familial de la personne décédée et la classe d'âge du répondant. Comme dans les figures qui suivent, les chiffres présentés ici sont faibles; nous avons donc choisi de ne pas donner de pourcentage, seulement les effectifs. Ils permettent toutefois de dégager des tendances.

5 Comprenant: les grands-parents, la fratrie (ou les beaux-frères, belles-sœurs), d'autres parents et des amis. Ces derniers étaient très peu nombreux: une seule mention à Bandra et 8 à Santa Cruz. 


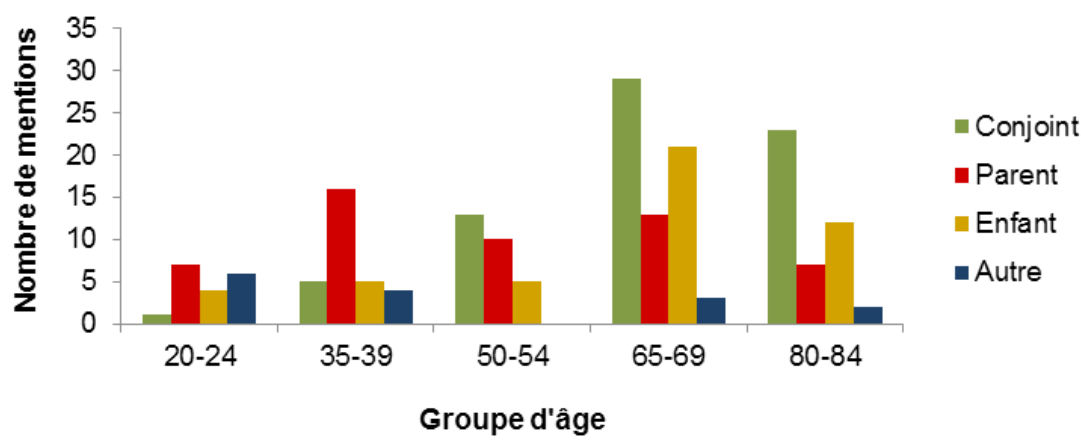

Figure 6.1: lien de parenté des personnes décédées (n) à Bandra, selon le groupe d'âge du répondant au moment de l'enquête

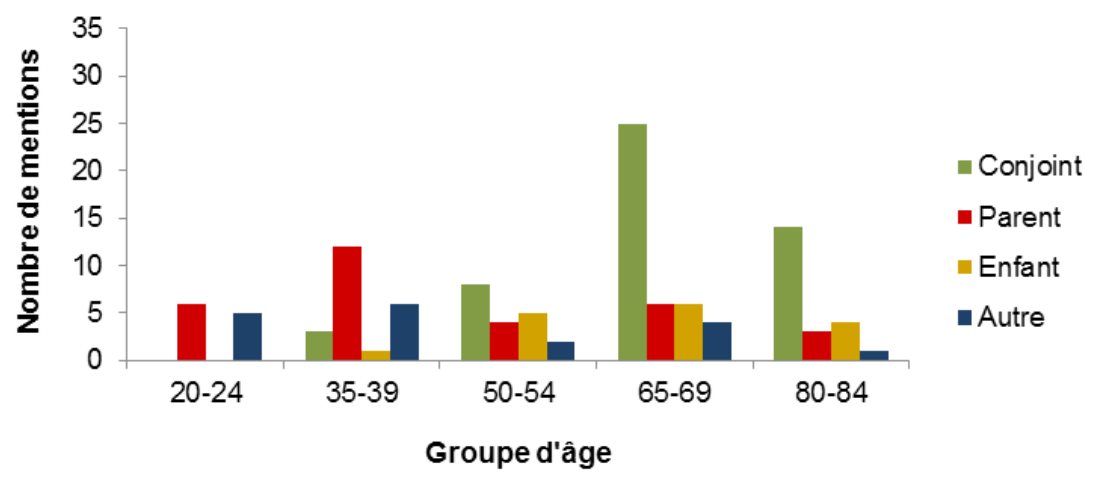

Figure 6.2: lien de parenté des personnes décédées $(n)$ à Santa Cruz, selon le groupe d'âge du répondant au moment de l'enquête

Les jeunes de Santa Cruz mentionnent autant de décès de parents que leurs pairs de Bandra ce qui peut, de prime abord, sembler contre intuitif, puisque les difficultés sanitaires de la résidence dans un quartier fortement défavorisé ont tendance à raccourcir l'espérance de vie, et perdre un parent a sans doute des impacts concrets sur la vie (quitter l'école pour partir travailler). En outre, parmi les répondants de Santa Cruz, le groupe d'âge 20-24 ans se focalise avant tout sur les parents et les autres membres de la famille, les décès de conjoint et d'enfant ne surviennent que plus tard. Or, les jeunes de Bandra ont aussi cité la perte d'enfant, un décès qui relativise et prend sans doute le pas sur la mort d'un parent. Ainsi, côtoyer la mort serait une expérience plus courante. 
Ces mentions de décès d'enfants plus élevé non seulement parmi les 20-24 ans, mais aussi les 35-39, 65-69 et 80-84 ans à Bandra, comparativement à Santa Cruz, sont un signal fort. Au vu de la dureté des conditions de vie parmi les pauvres des bidonvilles, il n'est pas étonnant que les personnes âgées (65-69 ans) voire très âgées (80-84 ans) aient vécu, à un moment de leur existence, le décès de leur progéniture. Malgré les incertitudes inhérentes à la démarche rétrospective appliquée à plusieurs cohortes d'âges, il reste que les morts d'enfants mentionnées par les trentenaires relèvent indéniablement de plus hauts taux de mortalité infantile et enfantine.

\section{Il n'y a pas d'âge idéal pour perdre ses parents}

Les figures 6.3 et 6.4 présentent la répartition des pertes de géniteurs selon la période de l'existence du répondant lorsqu'ils sont survenus : entre 0 et 9 ans, 10 et 29 ans, 30 et 69 ans. Chaque classe d'âge a été considérée séparément, puisque toutes n'ont pas vécu le même nombre d'années.

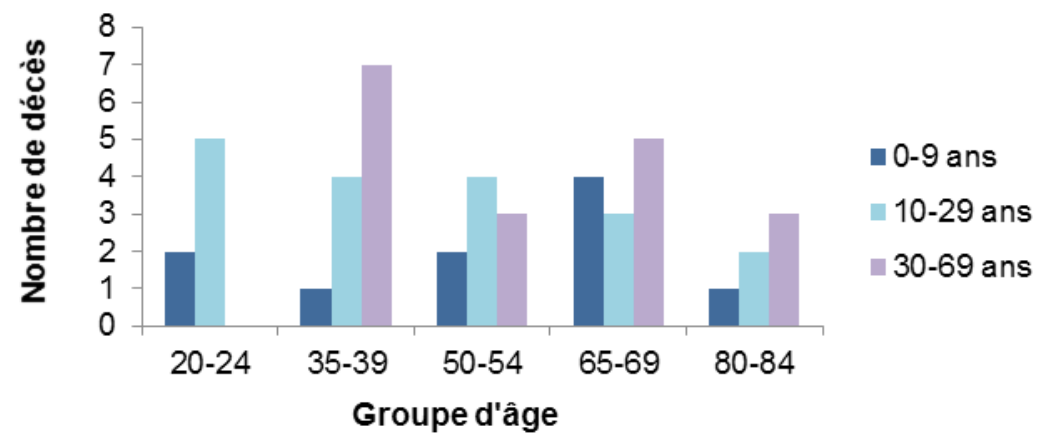

Figure 6.3: âge au moment de la perte du géniteur à Bandra, selon le groupe d'âge 


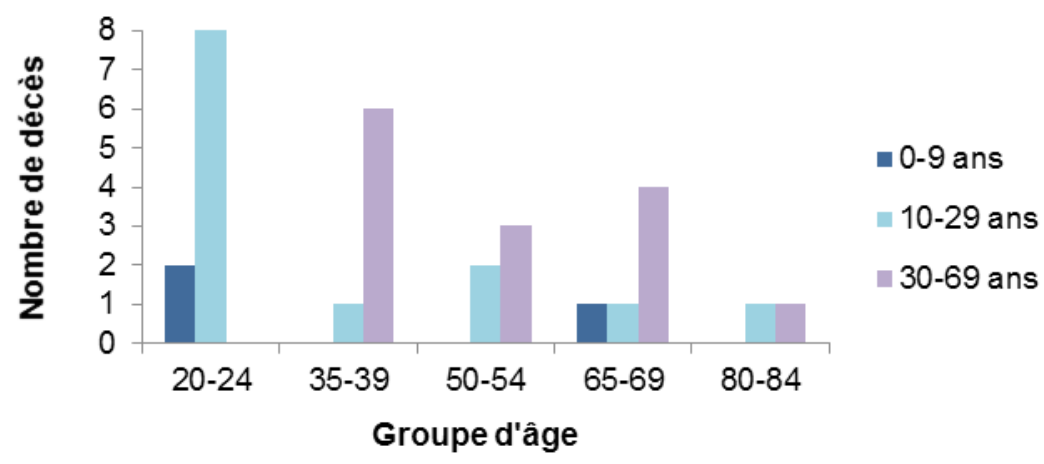

Figure 6.4: âge au moment de la perte du géniteur à Santa Cruz, selon le groupe d'âge

Les résultats soulignent qu'une large partie des décès de parents mentionnés sont situés plus tôt dans la vie à Bandra qu'à Santa Cruz. Dans toutes les classes d'âge, il est fait davantage de référence, lorsque les individus vivent en bidonvilles, à la perte d'un père ou d'une mère alors que le répondant avait entre 0 et 9 ans. Les autres variations sont plus délicates à interpréter.

La différence entre les deux enquêtes souligne le plus fort impact du décès d'un parent sur le parcours de vie d'un enfant ou d'un jeune adulte pauvre. La vulnérabilité face à la mort, ainsi que les impacts sur la vie des enfants sont ainsi soulignés. Les raisons de parler de la mort d'un autre comme tournant de sa vie éclairent ces conséquences.

\section{Emotion et responsabilités croissantes en arrière-plan des mentions de décès}

Alors que la mention d'un décès a parfois pu être soulignée comme porteuse d'une forme de soulagement (à la fin d'une maladie longue et douloureuse par exemple) (Cavalli et al., 2006), ce n'est pas le cas dans les deux enquêtes passées à Mumbai. La plupart des justifications avancées pour expliquer la citation d'une perte laissent transparaître une émotion négative ; dans un certain nombre de cas, survient également une forme nouvelle de stress. Deux axes ont donc guidé la codification des raisons attribuées à ce type d'événement : la tristesse (ou une forme d'émotion de regret) et les responsabilités croissantes (ou le stress).

Sur les 305 pertes considérées, 237 ont été codées selon que les raisons portaient explicitement sur une dimension émotionnelle (la tristesse) 
ou plutôt sur les conséquences pratiques, à caractère stressant, de la perte (responsabilité du ménage, des enfants, pauvreté, etc.). La première catégorie a rassemblé $48 \%$ des raisons $(n=146)$ contre $30 \%$ pour la seconde $(n=91)^{6}$. Les douleurs ou regrets sont plus évoqués par les hommes $(55 \%)$ que par les femmes $(45 \%)$, presque de manière identique par les habitants de Santa Cruz (50\%) et ceux de Bandra (47\%). Toutes les classes d'âge ont donné des raisons empreintes de tristesse, dans des proportions variant entre $41 \%$ (les 65-69 ans) et 63\% (les 35-39 ans):

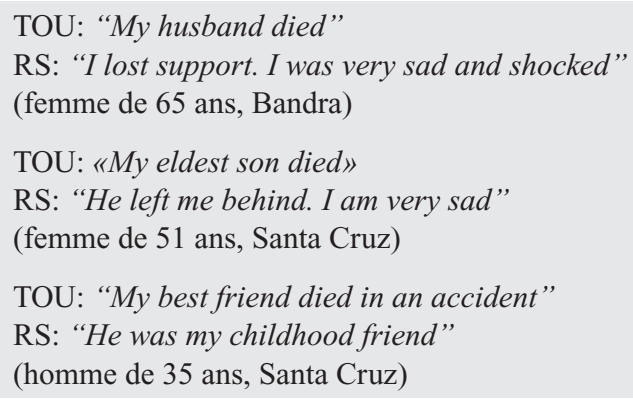

Le second type de raison renvoie aux implications pratiques issues de la perte d'un proche (généralement un mari ou un père, parfois une mère). Il est autant cité dans les deux quartiers (30\%), mais ressort comme plus mentionné par les trois cohortes les plus âgées (respectivement 38, 35 et 29\%).

Qu'elles concernent la gestion du ménage, la nécessité de trouver un emploi pour remplacer le revenu perdu ou la responsabilité des enfants, les femmes sont plus concernées par ce stress (34\% des raisons) que les hommes $(20 \%)$. Ceci reflète la configuration traditionnelle des rôles genrés au sein des ménages indiens, où les femmes assument le fardeau domestique pendant que les hommes ont la charge de rapporter un revenu. Dès lors, à la suite de la perte de leur mari, les épouses sont contraintes de trouver un emploi, en cumul de leurs tâches usuelles, pour permettre au foyer de tourner. C'est une contrainte que certains répondants évoquent également en référence à ce qu'ils ont vécu lorsqu'ils étaient enfants, au moment de la perte du père ou de la mère :

6 Les $22 \%$ restant représentent les raisons non codées $(n=68)$. Le codage n'a pas été possible lorsqu'aucune raison n'a été donnée, ou lorsqu'une description très factuelle du décès a été fournie, ne permettant pas de déduire une émotion ou une responsabilité supplémentaire. 
TOU: "My father died when I was 12 years old"

RS: "I got responsability of house"

(homme de 69 ans, Bandra)

TOU: "My mother died when I was 10 years old"

RS: "I was the eldest so responsibility of cooking came on my shoulder"

(femme de 81 ans, Bandra)

TOU: "I did hard work when my husband died"

RS: "Because I brought up my children. I educated them and I did their marriages"

(femme de 54 ans, Bandra)

TOU: "My life changed from the time of my husband death"

RS: "He was the only one who earned. The children were very small"

(femme de 65 ans, Santa Cruz)

L'analyse des décès révèle des inégalités entre les répondants de Bandra et Santa Cruz. Outre les divergences dans les conditions de vie, bien sûr plus difficiles dans les bidonvilles, s'y marier et y faire souche plus tôt inclut, comme nous l'avons vu plus haut, le risque d'être confronté dès l'âge de 20-24 ans au décès d'un enfant, ce dont sont relativement protégés les répondants de classe moyenne inférieure.

En revanche, pour une population comme pour l'autre, la perte d'un parent représente une vulnérabilisation importante. La perte d'un proche est un événement particulièrement marquant émotionnellement dans la vie d'une personne. C'est d'autant plus le cas lorsqu'il s'agit d'un parent (mère ou père) et que le décès survient «trop tôt», c'est-à-dire alors que les enfants sont encore dépendants économiquement de leurs géniteurs. Dans un pays marqué par la pauvreté et l'absence d'un état capable d'encadrer tous les nécessiteux, une telle disparition peut prendre une signification encore plus brute. En effet, la dimension émotive se double d'une composante pratique : la mort d'un pourvoyeur de revenu a des répercussions considérables sur les conditions de (sur)vie des survivants. Les témoignages de nos répondants se font ainsi l'écho de vies qui furent fragilisées.

En plus d'une tristesse évidente, une nouvelle forme de stress lié à la dépendance envers le breadwinner intervient bien parfois. Elle marque davantage les femmes que les hommes, étant donné que le pourvoyeur de ressource était généralement le père ou le mari. Afin de pallier le manque de revenu, la mère ou les enfants ont dû assurer la survie du ménage, avec notamment des garçons en bas âge, qui se rappellent avoir été contraints 
de quitter l'école pour se mettre à travailler, et des femmes confrontées à un marché du travail qui, traditionnellement n'était pas accessible aux Indiennes. Mais le poids des normes persiste : les veuves ne bénéficient d'aucun soulagement pour le ménage, la gestion du foyer et des enfants.

Un marqueur des modifications démographiques qui traversent l'Inde urbaine de ces dernières décennies se doit d'être souligné. Malgré le creusement de la distance intergénérationnelle issu du recul de l'âge aux transitions (comme le mariage et les naissances), le nombre d'années où plusieurs générations ${ }^{7}$ se croisent est en expansion, essentiellement grâce au gain en termes d'espérance de vie. Ce constat apparaît déjà parmi les changements récents rapportés : dès la cinquantaine, une partie des naissances marquantes sont, nous l'avons vu, celles des petits-enfants. Plus fréquemment et plus durablement qu'auparavant, la coexistence de trois générations a lieu. Elle constitue sans conteste un défi.

\subsection{Continuité et rupture entre les générations}

\subsubsection{Cohabitation et linked lives : de la difficile coexistence de plusieurs générations dans un environnement urbain saturé}

Dans les deux terrains d'enquêtes, plus de la moitié des tournants familiaux mentionnés par les 20-24, 35-39 et 80-84 ans concernent le répondant lui-même (entre 55 et $68 \%$ ). Le reste regarde les enfants, la fratrie, les petits-enfants pour les plus âgés, ou encore d'autres membres du ménage. Les quinquagénaires et sexagénaires évoquent de $44 \%$ à $51 \%$ de tournants familiaux propres, et dédient les autres citations aux enfants ou petits-enfants (soit les naissances des descendants ou leurs mariages). Les événements qui engagent l'individu directement sont surtout les unions. Le conjoint est relativement peu évoqué (évidemment, il faut deux personnes pour s'unir, mais le fait de citer son mariage comme une transition majeure de sa vie renvoie davantage à une étape dans sa trajectoire

7 Le terme de génération permet ici de distinguer les membres d'une famille selon leur statut et leurs relations les uns aux autres (Alwin \& McCammon, 2007; Elder \& George, 2016; Hareven, 1996; Settersten, 1999). Les dimensions historiques ne sont pas prises en compte à ce stade (Alwin \& McCammon, 2007; Devriese, 1989). 
personnelle qu'à la prise en compte de la compagne ou du compagnon). Les parents ou les beaux-parents sont également les grands absents de ces mentions familiales, mais interviennent massivement - nous le verrons plus tard - dans les mentions de décès.

Reconnaître l'identité de la personne au centre des tournants de mariage et de naissance (les événements familiaux les plus évoqués) a pour but de situer le répondant et son entourage ${ }^{8}$ dans les souvenirs de famille, afin de déterminer quels «autres significatifs ${ }^{9} »$, ceux avec lesquels l'interaction sociale est suffisamment décisive pour influer la trajectoire du répondant, au point que ce dernier l'a mentionné au cœur d'un tournant de sa propre vie.

La figure 6.5 expose la répartition des mentions de mariage, en fonction de la personne concernée par l'union, ainsi que du sexe et du lieu de résidence du répondant. Les résultats démontrent que c'est avant tout la propre union de l'interviewé qui est évoquée, davantage parmi les réponses féminines, en particulier à Bandra. Les mentions de mariage d'enfants sont faites relativement uniformément entre les sexes et les quartiers, et renvoient - outre à la joie de voir sa famille s'agrandir - au devoir parental de marier sa descendance. Les autres membres de la famille ne sont cités que de manière anecdotique (fratrie et petits-enfants).

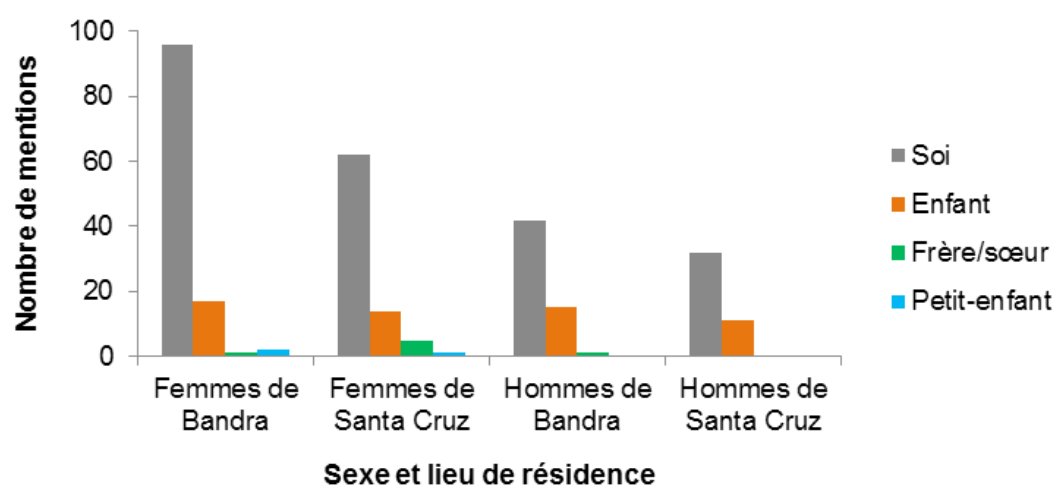

Figure 6.5: qui se marie $(n)$, selon le sexe et le lieu de résidence du répondant

8 Il s'agit de l'idée des linked lives, c'est-à-dire que les individus ne se construisent pas en totale indépendance, mais bien de manière inter-reliée à leur entourage (Elder, 1998; Sapin et al., 2007)

9 Le concept d' «autres significatifs» désigne les membres du réseau d'une personne, qu'ils se situent géographiquement dans le ménage ou non. Il s'agit des individus jouant un rôle important dans la vie de cette personne, que ce soit en termes positifs ou négatifs (Girardin, 2017; Kuhn, 1964; Woelfel \& Haller, 1971). 
La figure 6.6 représente la répartition des unions selon l'identité de la personne concernée et le groupe d'âge du répondant (sans distinction de lieu de résidence). Bien qu'une majorité des unions fasse référence au mariage du répondant dans toutes les classes d'âge, quelques groupes se distinguent par une certaine mixité. Les plus jeunes notamment évoquent quelques mariages de leur frère ou sœur. Par ailleurs, les 50-54, 65-69 et 80-84 ans mentionnent également les mariages de leurs enfants. Ce qui souligne à la fois la coexistence des générations, et à nouveau la responsabilité qui incombe aux parents de marier leur progéniture (que nous avons déjà explicité au-début de ce chapitre).

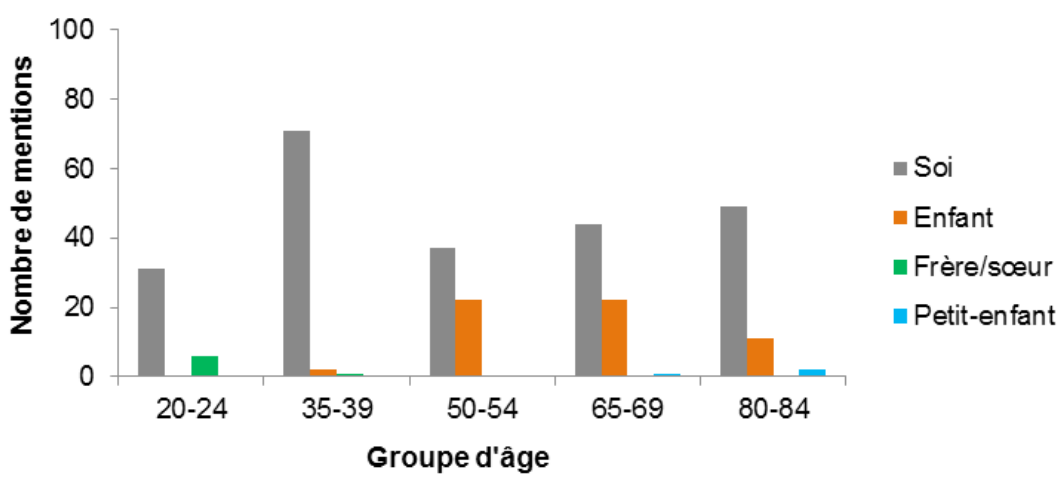

Figure 6.6: qui se marie (n), selon le groupe d'âge

Quant à l'identité de celles et ceux dont la venue au monde a été citée comme un tournant de leur vie par les femmes et les hommes de Bandra et Santa Cruz (voir figure 6.7), un consensus se fait autour de la naissance de ses propres enfants, ce qui s'avère peu étonnant dans un pays dont la culture repose sur la transmission filiale. Une minorité rapporte l'arrivée de leurs petits-enfants, ainsi que d'autres membres de la famille (neveu ou nièce principalement). 


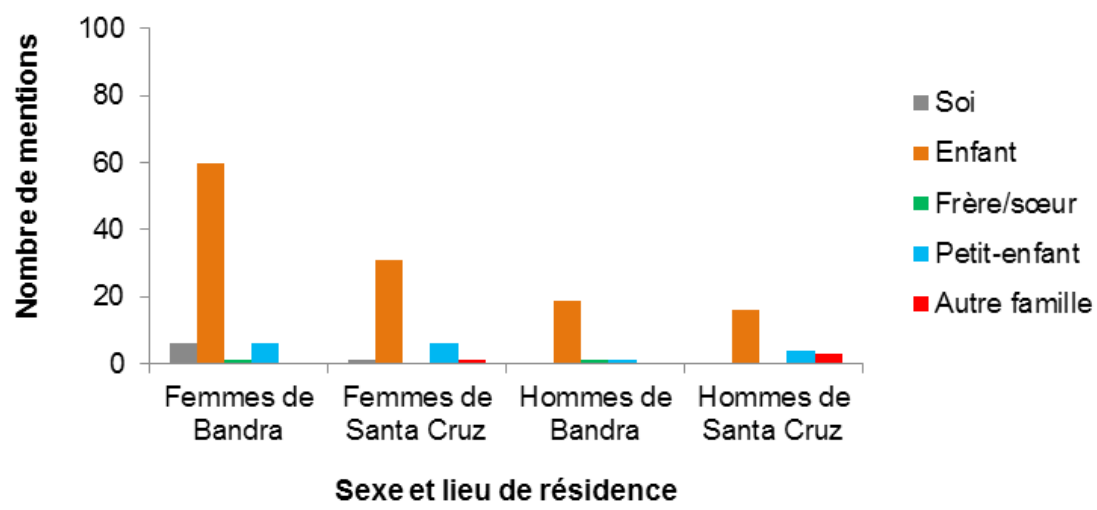

Figure 6.7: qui vient au monde $(n)$, selon le sexe et le lieu de résidence du répondant

Contrairement aux mariages, il y a peu d'effet de la classe d'âge du répondant sur les contenus de la mémoire des naissances (figure 6.8). La focalisation sur la venue au monde de son propre enfant reste constante. Malgré tout, quelques rares autres naissances (la sienne, celle de son frère ou de sa sœur, ou d'autres membres de la famille) sont également citées par les jeunes dans la vingtaine. Avec l'avancée en âge, ce sont les mentions de l'arrivée des petits-enfants qui surgissent. Comme pour les changements récents, on voit ici la coexistence des générations.

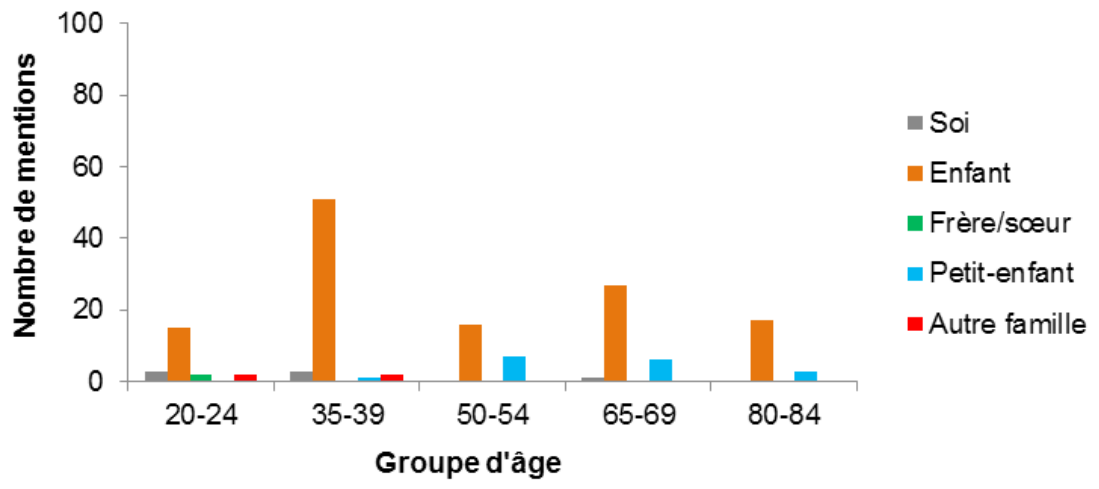

Figure 6.8: qui vient au monde (n), selon le groupe d'âge

$* * *$ 
Dans les populations occidentales, la sociologie de la famille a mis en évidence une transition, au cours des dernières décennies, d'un modèle familial dominant structuré par la parentalité, la reproduction, la transmission, bref organisé autour des enfants, à une centration sur la relation conjugale de l'individu et ses aléas (Hareven, 1993; Kellerhals \& Widmer, 2007; Mayer, 2004). Même si la pluralité des familles est reconnue, cette évolution générale n'est pas remise en cause (Kellerhals \& Widmer, 2007). En Inde, à Mumbai, nos analyses attestent d'une claire centration autour des enfants, plus exactement leur venue au monde et leur mariage qui, dans les classes d'âge les plus âgées, s'étend aux petits-enfants. C'est la chaîne de la vie, l'expression de la continuité de la famille et du groupe.

Certes, les plus jeunes, qui souvent ne sont pas encore engagés dans cette dynamique intergénérationnelle, montrent quand même un intérêt envers les membres de leur propre génération, au premier chef leur fratrie, ainsi qu'envers leur famille élargie. Mais ces dernières mentions sont faibles proportionnellement et quasiment absentes dans les autres classes d'âge, faisant écho aux modifications des structures familiales de l'Inde urbaine d'aujourd'hui. Depuis quelques décennies, se constate en effet une mutation du système de cohabitation familiale élargie vers une nucléarisation des ménages (Allendorf, 2013; Chadda \& Deb, 2013; Gupta, 1994; Heuzé, 1997) ${ }^{10}$.

Cette évolution fait l'objet de controverses interprétatives qui portent d'abord sur l'état initial. Dans un schéma caricatural, les grandes familles élargies collectivistes (typiques de l'Inde et plus en général de l'Asie) ont longtemps été vues comme formant le point central de la structure sociale,

10 En démographie, la famille élargie a été définie selon ces termes: un groupe composé de parents, d'enfants ainsi que d'ascendants et/ou de collatéraux, avec possibilité de polygamie (Tabutin \& Bartiaux, 1986). En Inde, elle est décrite comme des personnes résidant dans le même lieu, cuisinant ensemble et mettant leurs biens en commun, reliées les unes aux autres par des liens familiaux. En outre, les membres sont sous l'autorité des plus âgés (Gupta, 1994). Caractéristique des pays du Sud, cette structure a été longtemps vue comme inconciliable avec une avancée économique (Gupta, 1994) et a servi d'alibi pour expliquer la persistance des inégalités entre Nord et Sud. Généralement, ce précepte est issu d'une comparaison trop rapide avec la situation européenne. Pourtant, il apparaît de plus en plus qu'en Inde notamment, les structures familiales élargies n'entravent ni les initiatives commerciales (Tabutin \& Bartiaux, 1986), ni la modernité (Gupta, 1994). Au contraire, elles peuvent favoriser la protection des plus vulnérables et ne s'avèrent pas un système plus aliénant que les familles nucléaires (Allendorf, 2013; Kadir, Fikree, Khan, \& Sajan, 2003). 
promouvant l'interdépendance et la coopération, ainsi que la prise en charge des membres [le care]) (Chadda \& Deb, 2013; Dribe et al., 2014; Gupta, 1994; Heuzé, 1997). Divers chercheurs ont contesté ce portait unilatéralement positif, notamment en démontrant que les périodes de réelle cohabitation sont en fait minoritaires (en particulier lorsque l'espérance de vie est basse) (Allendorf, 2013; D’Cruz \& Bharat, 2001; Shah, 1996). Le concept même de famille élargie doit être compris avec des nuances, regroupant sans doute moins d'individus qu'imaginé (deux à trois générations qui cohabitent, avec parfois la présence d'un autre membre de la lignée). Il reste que dans l'espace urbain saturé de Mumbai, ces configurations familiales ne peuvent tout simplement perdurer (Chadda \& Deb, 2013; Gupta, 1994; Heuzé, 1997). Outre le manque de place, le travail salarié (par opposition à la paysannerie) ne permet pas la survie d'une famille élargie par le biais d'un seul pourvoyeur de revenu (Heuzé, 1997).

Pour autant, il s'agit de se prémunir contre la généralisation : la situation n'est pas uniforme, loin de là. Les transformations socioéconomiques du pays ont influé sur le modèle familial traditionnel, en particulier dans les mégapoles; néanmoins, les traits culturels dont l'ancien modèle de famille élargie était le conservateur et reproducteur n'ont pas disparu (Allendorf, 2013; Bongaarts, 2001; Gupta, 1994). Selon certains chercheurs indiens, ce n'est pas tant une famille «à l'occidentale» qui a émergé dans les milieux urbains qu'un «entre-deux» (Nijman, 2015). Les normes qui fixent les reproductions de pouvoir, d'échanges et de responsabilités, adaptées aux forces de la modernité (ou en réaction à elle) maintiennent les rôles sociaux et à travers la socialisation, le façonnage de l'identité individuelle, l'apprentissage des devoirs et de la place de chacun au sein de cette modernité menaçant de briser la continuité culturelle. Le rôle traditionnel de la famille conserve tout son intérêt et perdure même dans les grandes villes, malgré une montée des ménages nucléaires, et pousse notamment à un fort maintien des liens avec le reste de la famille à la campagne (Gupta, 1994) ${ }^{11}$.

11 Cinq facteurs ont été énoncés comme pouvant expliquer la persistance des familles élargies en Inde malgré les changements socioéconomiques. Premièrement, le fait que la majorité des Indiennes et des Indiens résident à la campagne plutôt qu'en ville doit être pris en compte. Deuxièmement, les fonctions et les tâches sont clairement définies au sein d'une famille élargie, permettant l'entre-aide mais aussi le maintien des rôles attribués selon le genre et la génération. Troisièmement, le système des castes, qui impose les mariages endogames, rigidifie la situation, empêchant la mobilité sociale et géographique. Quatrièmement, ce même système a sans doute 
Ces restructurations impliquent des rapports de force nouveaux entre les générations, qui peuvent mener à des clivages importants entre injonctions nouvelles et anciennes traditions.

\subsubsection{La perception d'un clivage générationnel}

Une question portant spécifiquement sur la perception d'un clivage générationnel a été posée lors du terrain passé à Santa Cruz en 2014. Elle permet de se pencher sur la compréhension de l'environnement sociétal dans lequel les individus évoluent. Plus exactement, son objectif est de saisir si une fracture se dessine entre les manières de vivre dites traditionnelles (ou à l'ancienne mode) et une «modernité» des comportements surgie ces dernières décennies. Même si elle existe, la frontière entre des attitudes anciennes et nouvelles ne peut bien évidemment pas être objectivement datée et, si impression de changement il y a, elle est d'abord individuelle et il ne saurait être attendu qu'émerge une unanimité. Non seulement la diversité des répondants ne pourrait le permettre, mais aux raisons personnelles (en fonction de chaque parcours de vie) s'ajoutent les aspects sociohistoriques, ici ancrés dans les générations (les comportements des autres groupes d'âge n'apparaissent pas sous le même jour selon le point de vue d'une personne âgée de 20,50 ou 80 ans). Nonobstant, l'éclairage du ressenti des individus face à une brisure entre les générations, l'explication subjective qu'ils en donnent documentent des mutations présentes dans une mégapole indienne en pleine recomposition socioéconomique. Qui plus est, ces témoignages sont des voix from the bottom, d'individus plus ou moins vulnérables - plutôt plus que moins - et différenciés selon le sexe et l'âge.

Seuls trois individus n'ont pas répondu à cette question supplémentaire, dont la formulation exacte était : «Selon vous, votre style d'existence [lifestyle] est-il plus proche de celui : de vos parents / de vos enfants / aucun des deux». Plusieurs choix étaient possibles, une catégorie «les

aussi freiné l'encouragement populaire à une modification des traditions vers plus d'individualisme, sur le modèle occidental. Cinquièmement, les lois indiennes concernant l'héritage ne jouent pas en faveur de la nucléarisation. Finalement, il ne s'agit pas de négliger l'impact de facteurs idéologiques culturels tels que la valorisation d'un individu par le biais de sa famille (plutôt que par son statut sur le marché du travail par exemple) (Gupta, 1994). 
deux» a donc été ajoutée. Par ailleurs, une modalité «autre» a été construite, afin de rassembler les réponses minoritaires (le mari et la religion ont parfois été mentionnés comme alternative).

La figure 6.9 présente la répartition (en pourcentage) de cette perception de similarité ou de rupture entre les générations. Les catégories «les deux» $(1 \%)$ et «autre» $(2 \%)$ rassemblant extrêmement peu de réponses, elles ont été ôtées des analyses. Une majorité nette des répondants a opté pour un style de vie proche des parents (62\%), une moyenne qui est tenue par la plupart des classes d'âge avec toutefois une légère baisse parmi les plus âgés $(51 \%)$. Un comportement plus proche de celui des enfants a rassemblé au total $14 \%$ des choix. Les plus jeunes n'en ont pas fait mention, puis la proportion augmente avec l'avancée en âge (de $8 \%$ chez les trentenaires jusqu'à $28 \%$ pour les plus âgés). Quant à la catégorie «aucun des deux», elle est plutôt bien répartie entre les cohortes et globalise $21 \%$ des réponses de tout l'échantillon. Notons d'emblée que les différences de perception des écarts générationnels sont très faibles entre hommes et femmes.

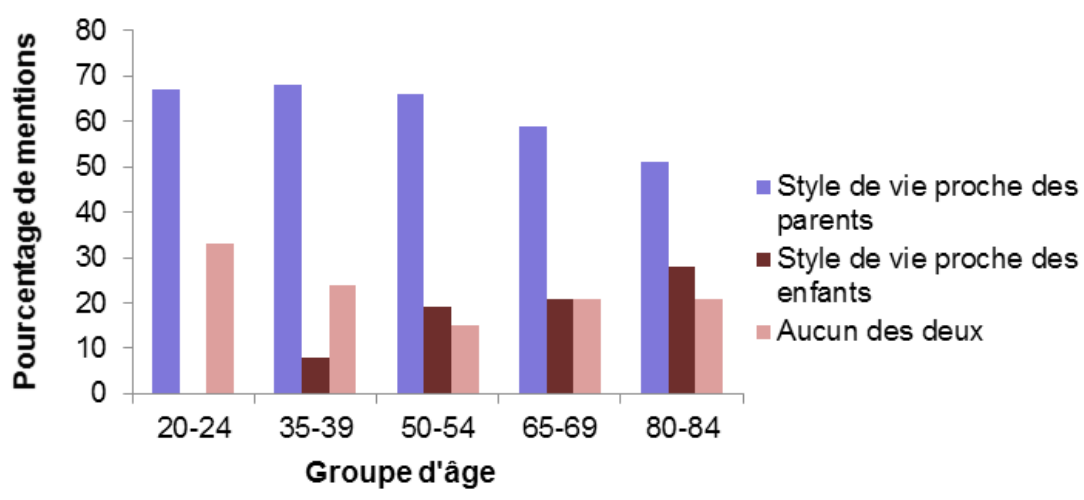

Figure 6.9: les écarts générationnels (\%) selon la classe d’âge

Il n'en va pas de même pour les cohortes d'âges. Que les individus de 80-84 ans soient ceux qui affichent la plus forte ambivalence entre style de vie proche de l'ancienne génération et de la nouvelle n'est pas sans surprendre. Evidemment, les jeunes ne connaissent pas encore de génération plus neuve que la leur, et pouvaient difficilement s'identifier à «plus moderne» qu'eux. Ce qui explique sans doute qu'ils soient un tiers à se qualifier de différents dans leur manière de vivre (33\%), une façon de se 
distancer des traditions sans savoir encore de quoi leurs propres enfants seront porteurs. Néanmoins, que les trentenaires et les quinquagénaires aient respectivement 17 et 15 points de différence avec les octogénaires quant à leur perception d'avoir un style de vie proche de leurs parents souligne deux effets. D'un côté, les anciens ont sans doute une conscience (et une vision) meilleure des évolutions récentes de la société car ils ont été témoins de plus de changements et ont plus de recul. D'un autre côté, la génération les précédant n'est pas la même que celle préexistant les 35-39 et 50-54 ans (qui, de fait, correspond précisément aux 65-69 et 80-84 ans de notre échantillon). Leurs parents avaient des vies encore radicalement différentes ; ils ont grandi dans ce qui était un monde colonial, probablement rural, fort éloigné de leur réalité actuelle.

Les signes d'une émancipation des traditions (ou d'une modernisation des comportements) sont relativement faibles dans la perception d'un clivage générationnel. Une majorité des individus se sent accrochée à des comportements inculqués par la génération précédente. Néanmoins, un cinquième des répondants de Bandra se perçoit en dissonance avec tant les nouvelles que les anciennes cohortes, en particulier les jeunes dans la vingtaine, signal peut-être d'un éloignement des normes en lien avec le changement socioéconomique ambient. Les raisons de ces choix subjectifs sont à bien des égards plus éclairantes que les chiffres. Elles illustrent notamment le sentiment de plus en plus marqué avec l'avancée en âge d'avoir un style de vie proche de ses enfants.

\section{Un style de vie proche des parents}

Lorsque les individus ont estimé vivre d'une manière similaire à leurs parents, plusieurs raisons ont été avancées pour expliquer ce choix. La plus courue renvoie à une enfance durant laquelle le répondant a été élevé selon les normes, les traditions, les valeurs de ses parents, avant de les reproduire $(n=267)$ : 
"My mother is the one who taught me everything"

(femme de 52 ans)

"Parents are of humble nature so my nature is like that"

(homme de 35 ans)

"Because we follow the discipline as maintained by my parents"

(homme de 22 ans)

"Parents were good. My lifestyle is like them only"

(femme de 80 ans)

Un autre raisonnement met en avant, sans autre, la ressemblance de ses propres comportements avec ceux de ses parents $(n=70)$ :

"My parents were uneducated and I am also uneducated"

(homme de 50 ans)

"I am like my mother and doing my all work like her"

(femme de 50 ans)

"As per his expressions, way of working, etc. is like my father"

(homme de 22 ans)

Une part plus réduite des raisons avancées fait un lien évident avec les anciennes traditions, qui servent de référence comme d'une meilleure façon de vivre $(n=26)$ :

\footnotetext{
"I like the old style"

(homme de 23 ans)

"I live with old lifestyle"

(femme de 66 ans)

"We are following old culture"

(homme de 51 ans)
}

Enfin, quelques raisons se réfèrent à la religion inculquée par les parents $(n=11)$. Il s'agit d'un des rares endroits du questionnaire où la religion apparaît comme marquant les trajectoires (et ceci n'engage qu'une minorité d'individus) : 
"They follow Islam which is a true religion. We follow them"

(homme de 23 ans)

"They follow life as per religion. I also do that only"

(femme de 51 ans)

"I like the way parents do prayers and worship. I like that only"

(homme de 69 ans)

\section{Un style de vie qui s'adapte aux attentes des enfants}

Du côté de la perception d'une manière de vivre proche de ses enfants, les raisons les plus fréquentes montrent une prise de conscience de la modernité, des changements sociaux en place depuis quelques années, et plus qu'une acceptation, une adhésion $(n=41)$ :

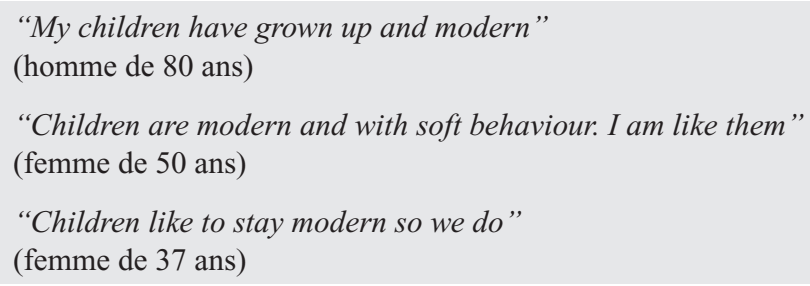

Mais à l'inverse, certaines explications soulignent sans fard la position de dépendance des parents vis-à-vis de leurs enfants, passé un certain âge $(n=35)$. Pour ces témoins, la solidarité intergénérationnelle ne survivrait pas à un conflit de valeur, et ce sont alors les plus faibles, ceux qui reçoivent, qui s'ajustent, de plus ou moins bon gré, aux plus forts, ceux qui aident :

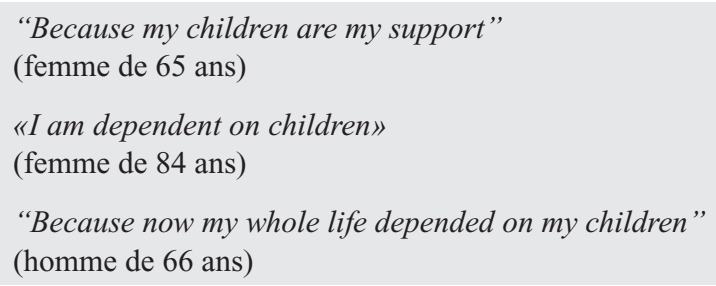

Enfin, quelques raisons à peine $(n=6)$ se réfèrent à nouveau à l'enseignement ou à la transmission de normes, mais cette fois-ci en partant du point de vue des parents et non des enfants : 
"I have to raise them. I am the father"

(homme de 50 ans)

"My children are nice and their values \& ideals are nice"

(femme de 81 ans)

La transmission des normes apparaît comme le principal critère poussant les individus à s'identifier au mode de vie de leurs parents. Il se retrouve, quoique dans une très faible mesure, parmi les raisons de s'identifier à ses enfants. Avoir un parcours de vie «objectivement» similaire à ses parents (en termes de niveau d'éducation, de travail ou encore de pratique religieuse) ressort également comme déterminant, mais ne s'est pas retrouvé dans le sens de s'identifier à l'existence de ses enfants. Sans ambages, la socialisation des parents vers les enfants est un phénomène qui perdure dans l'Inde urbaine, encore peu détachée des anciennes normes; elle explique le sentiment net d'une majorité des individus d'avoir un mode ou style de vie similaire à leurs parents.

L'augmentation de la perception d'évoluer vers ses enfants avec l'avancée dans la vieillesse semble s'expliquer par la situation de dépendance dans laquelle les personnes âgées se retrouvent lorsqu'elles ne peuvent plus subvenir à leurs besoins et vivent grâce au soutien de leur progéniture. Nonobstant, il est difficile de déduire si cette disposition pèse sur les personnes âgées, d'autant plus au vu de l'absence d'alternatives fournies par l'état (pension de retraite, établissement pour personnes âgées, etc.).

In fine, la conscience d'une manière de vivre «ancienne» (celle de la génération précédente) ou moderne (celle des descendants) est explicitement évoquée par une large part des individus. Dans la section qui suit, nous revenons sur les réponses concernant la famille dans le questionnaire, afin d'appréhender cette modernité, présente malgré tout, sous une forme ou sous une autre, dans les évocations des décès, des fiançailles et des mariages.

$$
* * *
$$

Au travers de ce livre, y compris de ce chapitre, nous n'avons eu de cesse de souligner les différents traitements accordés aux femmes et aux hommes dans la sphère familiale. Pourtant, dans nos analyses, le sexe ressort comme une variable relativement peu déterminante. Loin de sous-entendre que les inégalités de genre n'existeraient plus, il semble plutôt qu'elles ne se dévoilent pas si aisément. La section qui suit se focalise sur la perception 
globale des trajectoires issues de la sphère domestique par opposition à celle concernant la sphère publique, précisément sous l'optique du genre. Puis, les naissances évoquées par les répondants seront étudiées afin de creuser un phénomène tristement célèbre en Inde, celui de la préférence pour un fils.

\subsection{Des inégalités de genre contre vents et marées}

Les complexifications des relations et positions de genre sont issues d'une société en transformation dont les traits culturels, souvent d'une extrême ancienneté, n'évoluent pas sans douleur. Marqués par le système des castes, ces traits sont manifestés et reproduits à travers des normes familiales qui affectent profondément la condition féminine, dans un pays où la préférence pour les garçons est manifeste, mais où les hommes sont aussi mis sous pression, comme nous le verrons ci-dessous plus en détail (Guilmoto, 2008; Joshi, 2016; Vella, 2003).

Nous considérons la vie familiale dans une double perspective, comme une ressource vitale pour les pauvres gens, ainsi que comme le lieu par excellence de (re)production des normes de genre. Comment ces normes transparaissent-elles dans les réponses?

Les femmes sont légèrement plus nombreuses à mentionner la famille que les hommes dans les deux quartiers (voir tableau 6.1). Cependant, parmi les individus de classe moyenne inférieure interrogés, les divergences de mentions sont plus creusées entre les sexes que parmi les ressortissants des bidonvilles. Ce sont principalement les naissances et les mariages qui font la différence. Huit pourcents des femmes des slums évoquent des venues au monde contre $5 \%$ des hommes, et $6 \%$ des épouses le font à Santa Cruz contre 3\% des maris. Les mariages sont mentionnés par $7 \%$ des femmes comme des hommes de Bandra, mais seulement par 5\% des femmes et $2 \%$ des hommes de Santa Cruz. Des différences relativement faibles au final, mais qui laissent apparaître une focalisation un peu plus grande des femmes autour de leur couple, de leur(s) enfant(s). 
Tableau 6.1: les changements récents et les tournants concernant la famille $(\%)$, selon le sexe et le lieu de résidence ${ }^{12}$

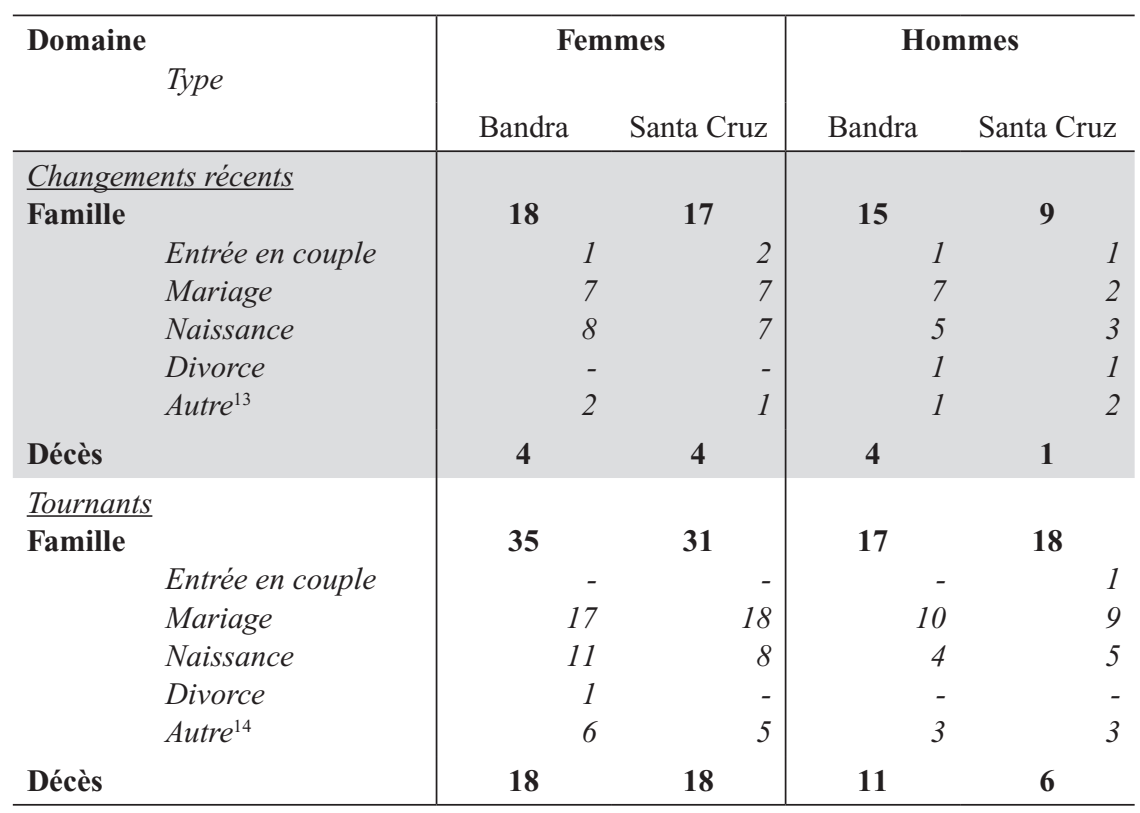

Dans le système patriarcal, patrilinéaire et virilocal qui caractérise la plupart des sociétés, y compris indienne (voir section 1.3), les jeunes épouses sont censées rejoindre leur belle-famille et résider sous leur toit avec leur mari (d'où l'existence de nombreuses familles élargies). Comme nous l'avons entrevu dans la section précédente, ce faisant, elles vivent une transition profonde, se retrouvant au bas de la hiérarchie domestique, en tant que femme et dernière arrivée (Allendorf, 2013; Kadir, Fikree, Khan, \& Sajan, 2003). Pour accroître leur statut, elles doivent réaliser l'injonction d'avoir un fils, qui un jour se mariera et amènera sa propre femme, dont

12 En gris les pourcentages où moins de cinq individus sont représentés.

13 La catégorie "autre" regroupe avant tout: divers problèmes familiaux conflictuels, les mentions d'anniversaire de proches, être heureux/satisfait de sa vie de famille, la responsabilité du ménage, le fait de n'avoir pas pu se marier ou pas pu avoir d'enfant.

14 Dans cette catégorie "autre" se retrouvent essentiellement: les citations de plusieurs types d'événements familiaux (naissances et mariage des enfants par exemple), les conflits ou à l'inverse le fait d'être heureux/de se soutenir en famille (ou dans la bellefamille), le devoir de se charger de l'éducation des enfants ou de la responsabilité (sans précision) du ménage, le fait de n'avoir pas pu se marier ou pas pu avoir d'enfant. 
elle deviendra la belle-mère à son tour. La position de bru peut donc être vue comme celle d'une main d'œuvre supplémentaire corvéable à merci, ou celle d'une entrée dans une famille qui prend soin de tous ses membres (sans que cela ne soit forcément opposé).

\subsubsection{Sphère privée versus sphère publique}

Les figures 6.10 et 6.11 représentent les changements récents (volet I) faisant partie de la sphère privée (majoritairement ceux ayant trait à la famille et à la santé) et de la sphère publique (éducation, profession, économie du ménage, etc.), selon le sexe et la classe d'âge des répondants. Seules $3 \%$ des réponses n'ont pas été codées car leur contenu pouvait entrer dans l'une comme dans l'autre dimension (l'amitié ou les déménagements par exemple). Globalement, la sphère privée rassemble $66 \%$ des changements récents, et la sphère publique 34\%.

A Bandra comme à Santa Cruz, les différences entre les sexes apparaissent fortement dans les trois premières cohortes (de 20-24 à 50-54 ans), où les hommes ont davantage évoqué la sphère publique. Chez les personnes âgées et très âgées, l'écart de genre est faible puisque l'essentiel des mentions (voir chapitre 4) se rapporte aux décès ou à la santé (ce qui explique la prévalence de la sphère privée). Le lieu de résidence est aussi un facteur de différenciation. A Santa Cruz, les femmes dans la vingtaine et la trentaine mentionnent autant de souvenirs publics que privés, montrant ainsi moins de divergences avec leur compagnon du même âge que dans les bidonvilles où le clivage est très marqué.

Cela étant, la sphère publique n'est jamais absente des réponses féminines, au contraire. Parmi les plus jeunes et les classes moyennes inférieures, la perception subjective des changements récents de la vie montrent une focalisation au moins aussi grande sur l'éducation, la profession et l'économie que sur la famille. En résumé, les femmes ne sont pas si cantonnées que cela à la sphère privée, tout au moins pour ce que leur perception subjective des changements récents de la vie laisse apercevoir.

La même codification sphère publique/sphère privée a été appliquée aux tournants de l'existence (volet II), dont $6 \%$ n'ont pu être classés. Les proportions sont similaires aux événements récents, puisque la totalité des tournants du type privée atteignent $62 \%$ contre $34 \%$ pour ceux renvoyant à la sphère publique. En observant la répartition des tournants par sexe et 
par classe d'âge (figures 6.12 et 6.13), le clivage de genre apparaît plus marqué que pour les changements récents. A tous les âges, les femmes se situent nettement plus dans le privé et les hommes davantage dans le public. Ce contraste se perçoit dans les deux quartiers.

Si parmi les changements récents la sphère privée est d'autant plus citée que l'on est âgé, tant chez les pauvres que chez les résidents d'un quartier de classe moyenne inférieure, les tournants de la vie évalués comme les plus marquants par les répondants montrent une différence de genre persistante dans toutes les classes d'âge et dans les deux terrains d'enquête. Ceci nous conduit à penser que les normes sociales, celles qui indiquent aux individus les événements qu'il leur faut valoriser, sont prégnantes et imposent leur loi comme filtre mémoriel. Par contre, la réalité quotidienne, celle qui a un impact sur la mémoire courte, ne suit pas forcément ce précepte et - en particulier dans le grand âge - elle est bien souvent faite d'événements privés tant pour les femmes que les hommes.

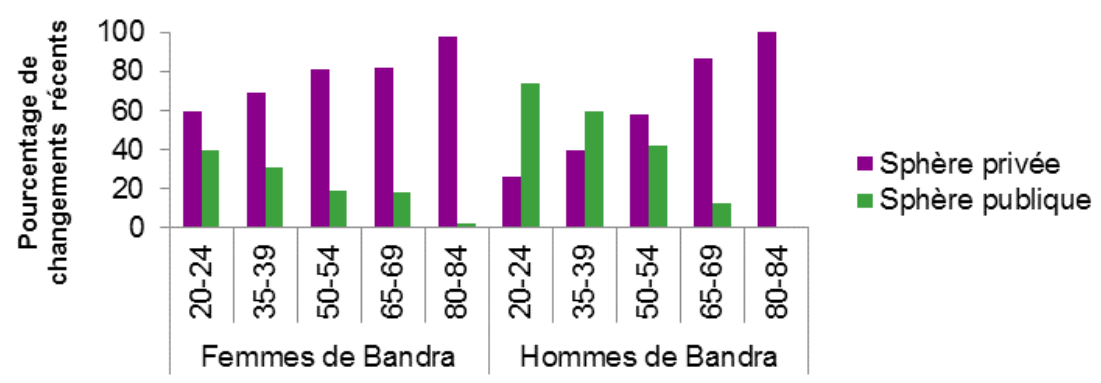

Figure 6.10: les changements récents répartis entre sphères privée et publique (\%), selon le sexe et la classe d'âge à Bandra

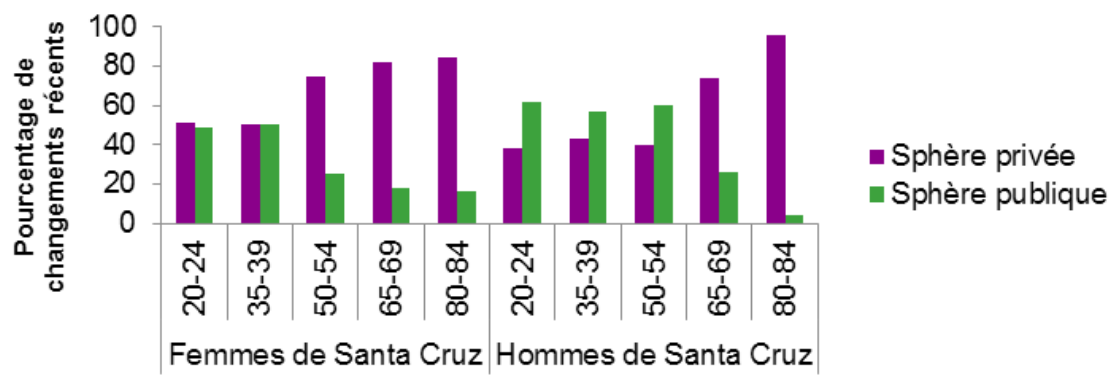

Figure 6.11: les changements récents répartis entre sphères privée et publique $(\%)$, selon le sexe et la classe d'âge à Santa Cruz 


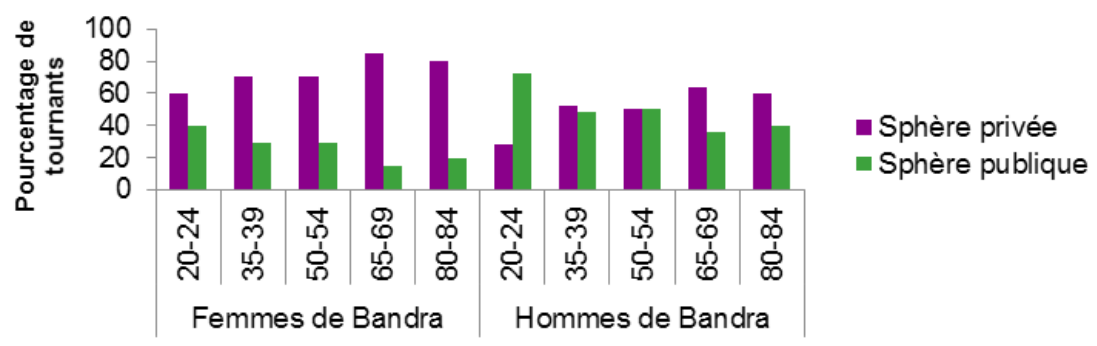

Figure 6.12: les tournants répartis entre sphère privée et sphère publique (\%), selon le sexe et la classe d'âge à Bandra

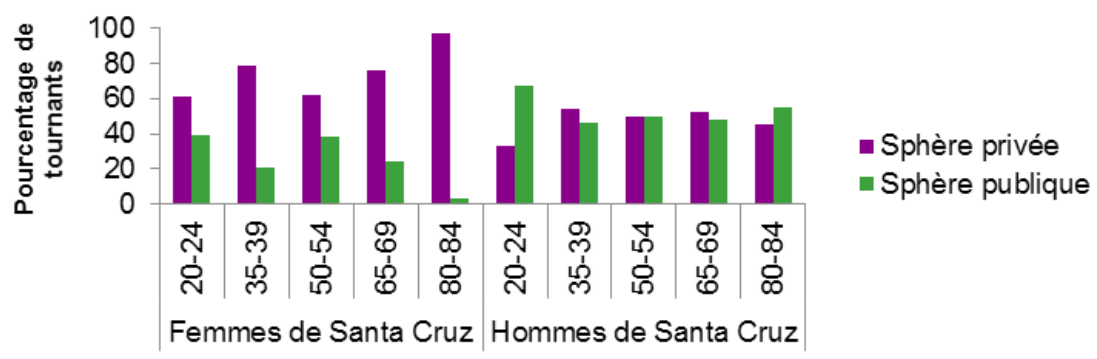

Figure 6.13: les tournants répartis entre sphère privée et sphère publique $(\%)$, selon le sexe et la classe d'âge à Santa Cruz

Un événement normatif par excellence de la société indienne est d'avoir des enfants, en particulier un fils. Tant dans l'injonction de donner la vie que dans celui de faire naître un garçon, les inégalités de genre sont criantes. Elles ont d'ailleurs engendré la disparition de dizaines de millions de petites filles ou de fœus féminins depuis près d'un demi-siècle (Guilmoto, 2005, 2008).

\subsubsection{La préférence pour un fils}

L'une des inégalités de genre les plus médiatisées en Inde concerne le phénomène des femmes manquantes, qui d'ailleurs s'étend - pour des raisons parfois différentes - à d'autres pays d'Asie (Attané, 2005; Gilgenkrantz, 2007; Guilmoto, 2008; Das Gupta et al., 2003; Vella, 2003; Véron, 2008). Ainsi, en 2011, 109.4 naissances de garçons ont été recensés pour 100 naissances de filles (Véron \& Nanda, 2011), chiffre significatif (le sex ratio normal étant évalué à 105 fils pour 100 filles) qui souligne le 
déséquilibre en faveur des garçons, lui-même issu de pratiques de sélection prénatals. Plus avant dans la vie, surtout durant les jeunes années, s'y ajoutent l'impact de facteurs sociaux de discrimination qui affectent les taux de mortalité infantile, enfantine et juvénile (cf. chapitre 1).

La source en est in fine un phénomène culturel, celui de la préférence pour un fils. En Inde, avoir une fille peut être considéré comme une malédiction $^{15}$. Les raisons de cette préférence s'ancrent dans des traditions ancestrales, notamment la coutume selon laquelle, dans la religion hindoue, seul le fils est en mesure d'apporter le repos éternel aux défunts de sa famille en allumant le bûcher funéraire. Mais elles prennent aussi racine dans des explications socioéconomiques plus récentes: la pratique ancienne de la dot a toujours fait des filles à marier une charge pour leurs parents (Das Gupta et al., 2003). Désormais constituée non seulement de bijoux, tissus, mais aussi d'appareils ménagers, etc., transmise des parents de la mariée vers la famille de l'époux, elle conduit généralement à un endettement lourd, voire parfois à la ruine (Gilgenkrantz, 2007) ${ }^{16}$. En outre, une fois passé le mariage, la dot demeure souvent l'objet d'âpres conflits, les victimes par excellence étant les mariées, esseulées dans leur belle-famille, corvéables à merci et parfois victimes d' «accidents» terribles pour inciter leur famille à payer (Banerjee, 2016; Banerjee et al., 2004). La mariée s'en trouve réduite à être l'objet d'un échange commercial, et dès lors le reste de sa vie est d'autant plus tourné vers le seul espoir de reconnaissance: porter un fils (Gilgenkrantz, 2007).

A la question sur les tournants de la vie, 157 réponses ont été faites concernant une naissance. Parmi elles, 34\% n'ont pas évoqué explicitement le sexe de l'enfant (ou du petit-enfant), ou ont mentionné en une réponse plusieurs naissances d'enfants des deux sexes. Mais 35\% des explications données formulent l'arrivée d'une fille et $65 \%$ celle d'un fils. Ce souci spécifique pour une descendance mâle s'exprime sans différences entre les quartiers de résidence ${ }^{17}$ ou selon le sexe ${ }^{18}$ des répondants.

15 D'où un célèbre proverbe du pays: Avoir une fille, c'est arroser une plante dans le jardin de son voisin (Gilgenkrantz, 2007).

16 Néanmoins, il n'y a pas de confirmation de l'idée que les pauvres discrimineraient davantage que les riches (Das Gupta et al., 2003).

17 A Bandra, 34\% des tournants de naissance évoquent l'arrivée d'une fille contre $66 \%$ un fils; à Santa Cruz il s'agit de $36 \%$ pour une fille et $64 \%$ pour un fils.

18 Alors que 34\% des tournants mentionnés par des femmes concernent des filles, $66 \%$ se rapportent à des fils. Les naissances citées par des hommes désignent $36 \%$ de filles et $64 \%$ de garçons. 


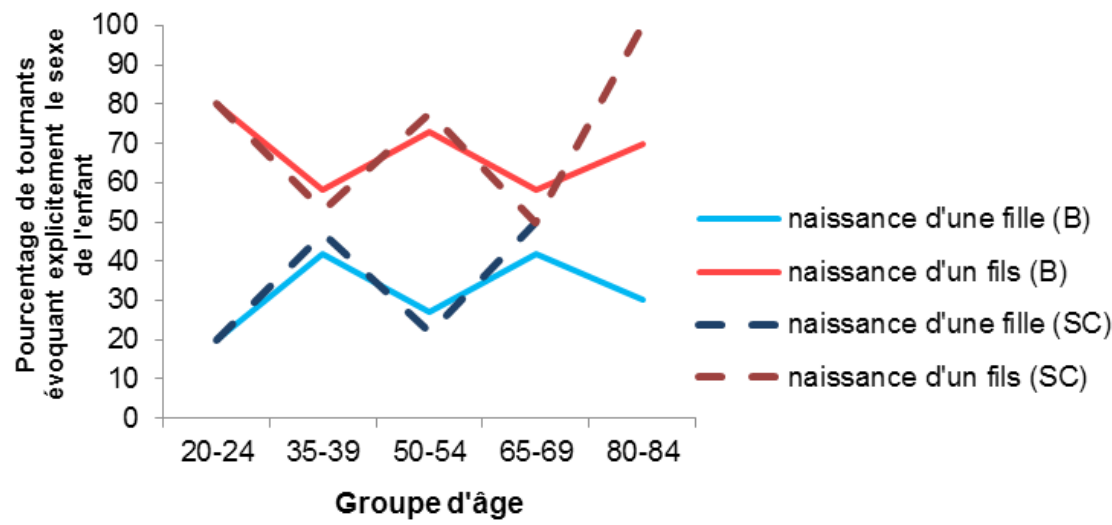

Figure 6.14: les naissances de filles et de fils (\%), selon le groupe d'âge et le lieu de résidence du répondant

La figure 6.14 présente la répartition par groupe d'âge et par lieu de résidence des mentions de naissances de filles et de fils. Les chiffres effectifs utilisés sont petits, les résultats doivent donc être pris avec précaution. Par ailleurs, il s'agit de la perception des individus concernant les tournants les plus marquants de leur existence et certainement pas d'un rapport de masculinité objectif et représentatif. Néanmoins, il ressort que les enfants mâles sont toujours plus cités que les filles, en particulier chez les jeunes répondants (sans doute encore sous la pression de parvenir à avoir une filiation), les cinquantenaires (qui voient arriver leur premiers petits-enfants) et les octogénaires.

Cette illustration de la préférence du fils dans nos questionnaires doit être nuancée. Une large part des raisons montre une joie ressentie, quel que soit le sexe de l'enfant (38\%) :

TOU: "I got a baby girl. It was the happiest moment"

RS: "I wanted a girl child"

(femme de 35 ans, Bandra)

TOU: "I got a daughter"

RS: "We treat the child as "Laxmi 19". Because of her arrival the days are better" (homme de 38 ans, Santa Cruz)

TOU: "In 1982 I got son"

RS: "I was happy»

(homme de 54 ans, Bandra)

19 Déesse de la fortune, de l'abondance, qui apporte la prospérité selon la religion hindoue. 
En outre, nonobstant que $20 \%$ de raisons n'ont pu être classées ${ }^{20}, 42 \%$ ont clairement une tonalité négative. Ces dernières se répartissent entre : naissances trop précoces (3\%), trop tardives $(12 \%)$, liées à un stress imposé par la famille (13\%) ou à l'arrivée de responsabilités ou d'une dépendance $(14 \%)$.

La temporalité est cruciale. Ainsi, des naissances alors que la mère s'estimait encore trop jeune pour cela ont suscité des regrets, que ce soit d'actualité ou concernant un souvenir ancien :

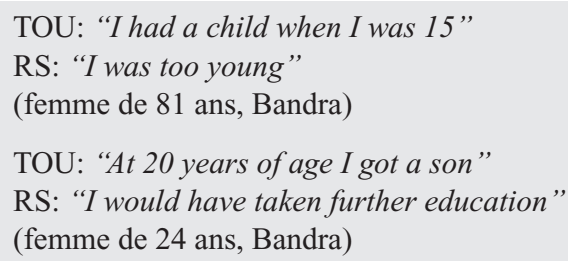

Dans d'autres cas, la temporalité surgit à nouveau, mais dans une relation inverse. C'est davantage l'attente trop longue qui est soulignée, ou l'urgence qui s'est faite sentir (pour la famille davantage que pour le répondant) :

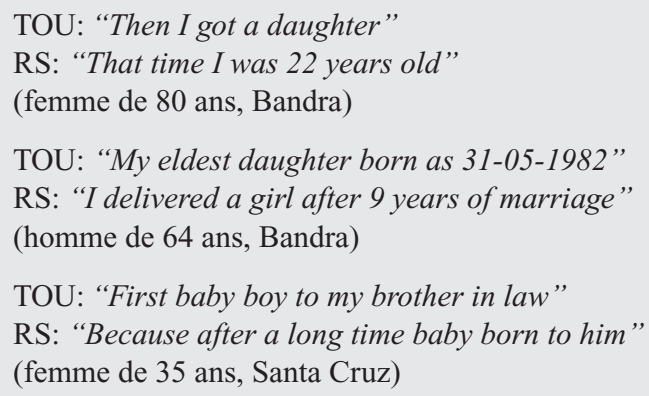

Le stress subit par le couple de la part de la famille est également palpable en soi :

20 La codification n'a pas été possible soit parce qu'aucune raison n'est donnée, soit qu'elle n'est pas assez précise, soit enfin que la raison fait appel à un souvenir précis qui n'entre pas dans nos catégories (l'accouchement, une césarienne, l'arrêt ou le début d'un travail, etc.). 
TOU: "I delivered a girl in 2011"

RS: "Because my parents restarted talking with me after my girl borned"

(femme de 24 ans, Bandra)

TOU: "I delivered a boy after 2 daughter's"

RS: "Because my mother in law was torched me for 2 girls"

(femme de 50 ans, Bandra)

TOU: "After the birth of daughter husband started living without trouble and with peace"

RS: "Less fights and peace at home"

(femme de 50 ans, Santa Cruz)

Enfin, les responsabilités engendrées par la naissance ont également été rapportées, ainsi que l'idée, ambivalente, que lorsque la dépendance viendra cet enfant subviendra aux besoins du répondant en ses vieux jours :

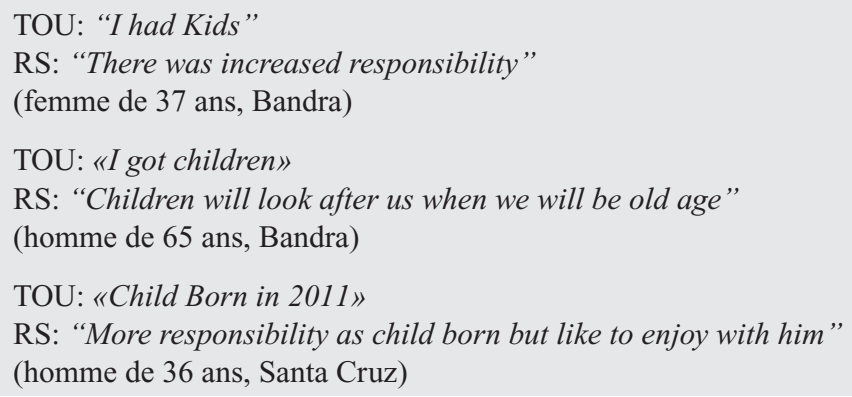

Il ressort sans équivoque des tournants et de leurs raisons que la mémoire de la parentalité est extrêmement normée. La famille participe bien souvent au maintien des règles en émettant une pression - explicite ou implicite - sur le nouveau couple, jusqu'à la première naissance. Toutefois, l'arrivée d'une fille, bien que moins souvent mentionnée que celle d'un fils, n'est pas mal vue par nos répondants. En revanche, les naissances ont pu être sources de drames si elles survenaient au mauvais moment du parcours de vie des individus (trop tôt ou trop tard), représentant souvent des responsabilités supplémentaires dans la vie d'individus déjà soumis à une forte précarité. Pour cela, l'assurance vieillesse que signifient les enfants dans un pays comme l'Inde est tout à fait évidente pour les répondants. 


\subsection{Conclusion}

Si globalement, et non sans nuances, les influences socioculturelles l'emportent sur le socioéconomique, le bilan sur les inégalités de genre, sur les discriminations envers les femmes, prend lui-aussi les teintes de l'ambivalence. Alors que la littérature met l'accent sur le maintien du contrôle exercé par l'homme sur la femme (Bourgeois, 2013), dans nos échantillons, la perception des événements récents et des tournants diffère peu selon le sexe, tant du point de vue quantitatif que dans les expressions qualitatives.

Mariages et naissances enchaînent les générations selon une logique patrilinéaire. Même si elle est mise en cause par des lois comme celle de 2005 sur le partage des héritages entre enfants des deux sexes, ces changements législatifs affectent peu la population, et la préférence pour les fils ressort tant explicitement qu'implicitement. En ce sens, nos résultats confortent la littérature existante selon laquelle la femme indienne obtient du respect avant tout lorsqu'elle devient mère, plus précisément si elle met au monde un garçon (Loiselle-Léonard, 2001). Pour autant, les témoignages des interviewés de Bandra et Santa Cruz montrent que la naissance de filles peut être une source de joie. Ceci nous rappelle qu'avec un sex-ratio à la naissance de l'ordre de 109.4 garçons pour 100 filles à l'échelle de l'Inde en 2011 (Véron \& Nanda, 2011), la discrimination existe mais aussi qu'une majorité de couples indiens n'en pratique pas. Finalement, les répondants ajoutent une dimension supplémentaire à ce tableau, en mettant en avant le caractère crucial de la temporalité des naissances dans le parcours de vie.

De même, les données récoltées à Mumbai confirment la continuité d'un autre trait de la famille indienne présenté comme fondamental : la virilocalité. Selon cette pratique, les épouses partent habiter sous le toit de la belle-famille au moment du mariage et quittent donc le nid originel. Elles sont alors obligées de s'intégrer à cette belle-famille élargie ce qui, en tant que dernière arrivée, représente souvent une nouvelle forme de dépendance (Loiselle-Léonard, 2001). Nos analyses amènent à nouveau la nuance. D'abord, le ménage nucléaire est le modèle familial dominant dans les slums, simplement, en raison des conditions de logement. Ensuite, les témoignages récoltés portent une évaluation ambivalente, neutre, voire favorable à ce changement de situation («[...] j’ai eu une nouvelle amie à 
la maison»), tout en évoquant la famille élargie, lorsqu'elle existe, comme un espace parfois conflictuel.

Autre considérations surprenantes : les non-dits autour de dimensions capitales telles que la religion, qui rythme pourtant toutes les célébrations des moments marquants de la vie (naissance, mariage, etc.), ou les castes, qui sont pourtant des marqueurs indélébiles de la vie en Inde (à tout le moins pour les hindous, mais pas seulement, voir chapitre 1). La première absence interroge, à propos de la description mêmes des événements vécus. Les circonstances religieuses seraient-elles trop évidentes pour être contées à des enquêteurs provenant du même milieu ? Ou est-ce que, finalement, c'est le rite de passage plus que la cérémonie qui importe lors d'un mariage ou d'une naissance ? La seconde absence, celle de la mention des castes, renvoie sans doute à une certaine forme de tabou. Comme cela a déjà été indiqué (voir section 3.4.1), les discriminations sur la base des castes sont sanctionnées par la loi, il n'est donc pas évident de les exprimer (par exemple, s'agissant de l'impossibilité d'avoir épousé la personne de son choix car n'appartenant pas à sa caste). Les mentions de mariage d'amour - qui demeurent peu nombreuses - seraient-elles un moyen d'y faire allusion de manière détournée ?

Somme toute, interroger directement la subjectivité des personnes, y accorder une pleine valeur, ne nous fait pas entrer en contradiction avec les nombreuses recherches de qualité qui ont été consacrées à la population et aux familles de l'Inde, en particulier de ses grandes métropoles. Mais cette approche, qui donne la parole aux acteurs et actrices eux/elles-mêmes, met en évidence les ambivalences d'une société qui, malgré le poids des traditions, trouve son équilibre et change, sous l'effet des grandes logiques de la globalisation et d'une économie émergente, mais aussi sous l'impact lent et obstiné de ses membres. 



\title{
Chapitre 7. La santé : un bien précieux dans un contexte de pauvreté urbaine
}

\author{
"Social class differences in health are explained by a \\ process of health-related social mobility in which the \\ healthy are more likely to move up the social hierarchy \\ and those in poor health to move down."
}

(Blane, Smith, \& Bartley, 1993, p. 1)

Les premières analyses des réponses aux questions portant sur les souvenirs personnels des répondants (volets I et II) ont montré une singulière prévalence de la santé (voir chapitre 4). Tant parmi les changements survenus au cours de l'année précédente (où, dès l'âge de 35 ans, tous les autres domaines - y compris celui de la famille - sont surpassés par les questions de santé) que dans les grands tournants de l'existence, la santé est au cœur des mémoires des habitants de Mumbai, résidant en bidonville ou dans un quartier de classe moyenne inférieure.

Ulrich Beck (1992) a souligné que, dans le contexte actuel de mondialisation, les pays du Sud sont particulièrement affectés par les nouvelles formes de vulnérabilités globales, aggravées par le creusement des inégalités. Selon la perception individuelle des changements caractérisant les différentes étapes de la vie adulte en Inde, il ressort que l'incertain est fortement lié au capital santé des individus. Effectivement, dans un pays où les ménages ne sont pas (ou guère) assurés contre les aléas de l'existence (chômage, accident, maladie, ...), pour des populations pauvres qui dépendent de maigres revenus pour assurer leur survie, la santé est sans aucun doute un bien précieux, déterminant pour l'avenir de la personne et de sa famille.

En Inde comme ailleurs, le statut et le niveau socioéconomique des individus sont à cet égard des facteurs cruciaux (Alwin \& Wray, 2005; Bengtsson, 2004; Pearlin, Schieman, Fazio, \& Meersman, 2005); ils participent fortement à créer des disparités de survie en fonction de critères tels que la classe sociale, le sexe ou la caste, et cela de façon cumulative dans le temps, de la naissance au décès (Alwin \& Wray, 2005; Dannefer, 
2003a; O'Rand, 1996). En outre, dans le contexte spécifique de Mumbai, le lieu de vie insalubre des bidonvilles accroit l'exposition aux maladies contagieuses et la pauvreté de masse précarise la population par le manque de ressources et d'accès aux soins (Bengtsson, 2004). C'est clairement ce que met en évidence la littérature discutée dans la première partie de ce chapitre, mais les analyses fondées sur nos enquêtes, exploitées dans la deuxième section, offriront des résultats nuancés, documentant la place qu'occupent les événements de santé dans les mémoires des individus, selon des critères socioéconomiques et démographiques.

La transition sanitaire du Sud a débuté dans les villes, centres du progrès et du développement économique du monde en développement, entrainant un recul du risque de décès dans l'enfance (Garenne, 2016; Kuate Defo, 2016). Mais la santé reproductive reste mauvaise en comparaison des valeurs observées dans les pays du Nord, des maladies infectieuses anciennes ne sont pas éradiquées et de plus récentes (VIH notamment) menacent. En parallèle, de nouveaux fardeaux se sont déployés dans les milieux urbains, les maladies chroniques et les accidents qui sont liés aux comportements et aux modes de vie, affectant davantage les adultes (Rossier, Soura, Lankoande, \& Roch, 2016). La seconde partie du chapitre interroge la présence de ce que les épidémiologues appellent le multiple burden of disease (Boutayeb, 2006).

Ce chapitre s'achève en proposant de dépasser les analyses chiffrées pour creuser les raisons invoquées pour mentionner des tournants de santé. En effet, les ennuis dans ce domaine possèdent des implications considérables pour l'individu concerné comme pour le reste de sa famille, en termes économiques et émotionnels. Les points de vue des individus concernés, la manière dont ils les expriment, les éléments qu'ils mettent en avant, justifient pleinement la collecte du matériau qualitatif.

\subsection{Le fardeau de la santé, de l'enfance à la vieillesse, à travers la stratification socioéconomique}

Le niveau de santé d'une population est fréquemment évalué grâce à l'indicateur démographique de l'espérance de vie à la naissance, soit le nombre moyen d'années qu'un nouveau-né peut s'attendre à vivre si les conditions 
de vie au moment où il voit le jour se maintiennent. Dans les pays du Nord comme du Sud, cette espérance de vie est passée de 20-40 ans au $18^{\text {ème }}$ siècle, à 60-80 ans au 20 ème siècle (Johansson \& Kasakoff, 2000; Meslé $\&$ Vallin, 2002). En ce début de $21^{\text {ème }}$ siècle, le nombre d'années à vivre reste généralement en-dessous de 65 ans dans les pays en développement (Horiuchi, 2000; Véron, 2008). A l'inverse, les pays les plus développés économiquement atteignent des niveaux de survie inégalés, le Japon et la Suisse culminant avec plus de 83 ans en 2015 (chiffres de l'OMS, publiés en 2016). Cet écart fondamental dans la survie exprime de profondes inégalités de santé.

Ainsi, a contrario de ce que l'opulence alimentaire et les avancées médicales de la seconde modernité pourraient laisser croire, les gradients socioéconomiques de santé se distendent depuis des décennies (Bartley, 2012; Bengtsson \& van Poppel, 2011; Burton-Jeangros, 2009; OMS, 2008). Cette augmentation des inégalités entre les pays mais surtout au sein de ceux-ci, résulte de l'amélioration de l'espérance de vie des groupes avantagés et de sa stagnation ou faible progression parmi les plus vulnérables (les plus pauvres, les moins éduqués, les sans emploi, les femmes et les minorités discriminées). Une deuxième explication s'appuie sur le travail des épidémiologues Link et Phelan (Link \& Phelan, 1995; Phelan, Link, Diez-Roux, Kawachi, \& Levin, 2004; Phelan, Link, \& Tehranifar, 2010), soulignant que les groupes avantagés préservent mieux leur santé, quel que soit le contexte épidémiologique. En résumé, au cours du temps les disparités de santé vont en s'aggravant, ce qui a amené les chercheurs à parler d'un «gradient socioéconomique de santé» (Marmot, 2005).

Le gradient socioéconomique de santé est confirmé par les principaux indicateurs (santé auto-évaluée, taux de mortalité, morbidité, etc.) et se retrouve dans la plupart des cultures et des contextes. Il découle de plusieurs facteurs issus des normes sociales, des comportements et des modes de vie, dont les conséquences sur la santé sont majeurs (Marmot, 2005). Ils changent en fonction des couches sociales et s'ajoutent généralement à la liste des pénalités qui affectent les plus pauvres ${ }^{1}$. Les principes du parcours de vie contribuent eux-aussi à expliquer les différenciations entre les individus en faisant référence à la théorie des (dés)avantages cumulatifs (CAD) (voir section 2.4.3). Cette dernière est ancrée dans le champ théorique très large de la stratification sociale que nous venons d'évoquer

1 Quoique dans des situations très spécifiques - comme les questions de surpoids -, ce constat est parfois inversé dans les pays du Sud, nous y reviendrons. 
ci-dessus; elle y a apporté une perspective dynamique. Les recherches ont montré que, tant les impacts des événements de santé que la capabilité de les surmonter varient en fonction des ressources que les individus ont à disposition (Burton-Jeangros, 2009; Cullati, 2013; Sen, 1992).

Comprendre la construction des inégalités de santé nécessite donc de partir d'un constat de stratification sociale de la santé, ce dernier conditionnant les chances ou les handicaps de survie depuis la naissance et qui s'accumulent tout au long de la vie (Bartley, Blane, \& Montgomery, 1997).

\subsubsection{Des inégalités de santé assignées à la naissance, liées à la classe sociale et au sexe}

Tenir compte de l'ensemble du parcours de vie pour expliquer l'aggravation des inégalités de santé est devenu une évidence pour de nombreux chercheurs (Bartley, 2016; Burton-Jeangros, Cullati, Sacker, \& Blane, 2015; Ferraro \& Kelley-Moore, 2003). En effet, cette approche permet de saisir, sans les opposer, les implications des facteurs biologiques, économiques et sociaux, et ce au cours des diverses parties de l'existence (période intra-utérine jusqu'à l'extrême vieillesse), en tenant compte des effets cumulatifs à travers le temps (Kuh, Ben-Shlomo, Lynch, Hallqvist, $\&$ Power, 2003). Le dialogue entre épidémiologie et parcours de vie a également permis de déterminer que les inégalités de santé issues des différences socioéconomiques ou de genre débutent dès les premiers jours d'existence et que leurs répercussions se font sentir durant toute la vie (Balarajan, Ramakrishnan, Özaltin, Shankar, \& Subramanian, 2011; Bartley et al., 1997; Kuh et al., 2003; Kulkarni, Kulkarni, \& Gaiha, 2017).

La caractéristique du sexe représente l'une des premières sources d'inégalité de santé, dont la responsabilité est d'ordre social bien plus que biologique. Dans les pays occidentaux, les études sur ce thème se sont souvent focalisées sur les difficultés de conciliation entre activités rémunérées et domestiques pour les femmes (Burton-Jeangros, 2009; Moss, 2002), alors que dans les pays d'Asie du sud tels que l'Inde, ce sont les discriminations et la santé de la reproduction qui ont été beaucoup mises en avant (Iyer, Sen, \& George, 2007; Osmani \& Sen, 2003). Influencées par les rôles sociaux de sexe, les implications sanitaires de ces disparités sont complexes. Généralement, il est constaté que les femmes 
vivent plus longtemps que les hommes (Véron, 2008), à de rares exceptions près dont l'Inde a fait partie, mais elles sont en moins bonne santé (Burton-Jeangros, 2009; Tholomier, 2017). Néanmoins, c'est une donne extérieure à l'individu (par exemple l'accès aux établissements de soins et le fait de résider dans un environnement à risque) qui accentue les divergences au cours de l'existence en termes de santé physique, mais aussi mentale, comportementale ou auto-évaluée (Cullati, 2013).

Les trajectoires familiales et professionnelles sont profondément différenciées selon le sexe, notamment en Inde (cf. chapitre 1). Cependant, si elles sont apparues comme ayant un impact considérable sur la santé, c'est de manière similaire pour les deux sexes (Burton-Jeangros, 2009). Le fait de posséder un emploi stable et protégé préserve de la survenue de maladies et d'accidents; alors que des périodes d'insécurité professionnelle et de chômage sont associées à plus de mortalité et de morbidité (Bartley, 1994). La stabilité familiale est aussi un atout pour posséder une qualité de vie élevée (Uhlenberg \& Mueller, 2003). A l'inverse, le conflit et l'accumulation des rôles peuvent détériorer cet équilibre, en ajoutant des responsabilités et du stress. Il est donc important de tenir compte de toutes les sphères de la vie pour comprendre les inégalités de santé (Bartley et al., 1997; Cullati, 2013).

Plus que la variable de genre ou les trajectoires professionnelles et familiales, c'est le niveau socioéconomique des individus qui est considéré dans la littérature comme la variable la plus cruciale pour expliquer les écarts de santé (Alwin \& Wray, 2005; Bartley, 2016; George, 2005). La montée des maladies dégénératives ou chroniques, qui a transformé le paysage sanitaire de ces dernières décennies (Meslé, 2004), a eu un effet complexe sur ces disparités. Les personnes dont le statut économique et social offrait une certaine protection face aux maladies infectieuses sont aujourd'hui soumises au risque de contracter des maladies chroniques ou de souffrir d'un accident (de la route par exemple) (Rossier et al., 2016).

Toutefois, en particulier dans les pays moins développés économiquement, les riches ne sont pas autant exposés que les pauvres à ces facteurs de mortalité, soulignant que l'idée d'Ulrich Beck (1992) à propos de la diffusion globale des risques aurait atteint ses limites (voir section 2.4.2). Incontestablement, les pauvres ont toujours une espérance de vie plus faible (entre les pays mais aussi au sein de ceux-ci). Aucune réelle démocratisation des risques ne se constate, quand bien même se propage une perception trompeuse que les maladies chroniques frapperaient aujourd'hui 
indistinctement, au-delà des barrières bienfaitrices de la richesse. Dans le contexte des pays du Sud, la colonisation suivie par la globalisation, centrales dans la diffusion des inégalités sanitaires, se sont ajoutées à cet état de fait complexe, expliquant en grande partie la situation de santé déplorable dans laquelle de nombreux individus vivent encore.

\subsubsection{Du Nord vers le Sud: diffusion de la médecine et des inégalités de santé}

Dans les pays les plus développés économiquement, l'augmentation de l'espérance de vie s'est effectuée au cours d'une «transition sanitaire», ou «transition épidémiologique», qui a permis le recul radical de l'âge moyen au décès (Omran, 2005) ${ }^{2}$. Celui-ci s'est avéré plus rapide pour les femmes que pour les hommes, creusant l'écart de l'espérance de vie entre ces deux populations (Meslé, 2004). Les causes d'une telle évolution sont multifactorielles (économiques, sociales mais aussi politiques et urbanistiques) et bien que de nombreux auteurs de différentes disciplines se soient penchés sur le sujet, un consensus se dégage difficilement (Johansson \& Kasakoff, 2000; Meslé \& Vallin, 2002). Le débat se situe entre la part de bénéfices provenant de l'amélioration globale du niveau de vie (économique, nutritionnel, éducationnel...) et celle issue du progrès des techniques

2 La transition épidémiologique représente le passage d'un modèle épidémiologique à un autre (vers une nouvelle structure pathologique), et plus généralement une augmentation considérable de l'âge au décès (Omran, 2005). Par la suite, des auteurs lui ont préféré le terme de transition sanitaire, qui reprend la même signification et y ajoute l'évolution des réponses sociétales fournies à cet état sanitaire (Meslé \& Vallin, 2002).

$\mathrm{Au} 19^{\mathrm{ème}}$ siècle en Occident, cette transition a connu une première phase avec la chute drastique des décès causés par les maladies infectieuses, particulièrement dévastatrices durant les premières années de vie. Une deuxième chute de mortalité est observée au cours de la seconde moitié du $20^{\text {ème }}$ siècle, grâce au recul des maladies chroniques et dégénératives (cardiovasculaires, cérébrovasculaires et cancers) qui concernent cette fois les individus les plus âgés (Horiuchi, 2000; Meslé, 2004; Rossier, Soura, Lankoande, \& Roch, 2016). Depuis quelques années, a été observé un nouveau recul de la mortalité grâce aux soins apportés aux maladies chroniques (Meslé, 2004). Ainsi, l'espérance de vie continue d'augmenter, les décès surviennent plus tard et les individus se maintiennent en meilleure forme (Horiuchi, 2000; Meslé \& Vallin, 2002). 
médicales et de santé publique (Johansson, 2000; Johansson \& Kasakoff, $2000)^{3}$.

Les progrès et le savoir médicaux se sont ensuite diffusés dans le reste du monde au cours du $20^{\text {ème }}$ siècle, et la plupart des pays ont à l'heure actuelle entamé leur transition épidémiologique. Néanmoins, l'arrivée de la médecine occidentale (dite «moderne») dans les pays du Sud est loin d'avoir été neutre politiquement. De tous temps, l'accès aux services de santé a été utilisé comme une arme pour asseoir la suprématie d'une classe sur une autre ${ }^{4}$. En Inde, la médecine occidentale a représenté un outil politique pour les colonisateurs anglais contre le peuple indien, afin de renforcer leur position de domination, détruisant au passage les

3 En effet, les facteurs de la transition sanitaire sont sujets à débat. Pour certains scientifiques, ce sont avant tout les progrès médicaux (comme les découvertes de Pasteur ou les antibiotiques) qui ont permis des avancées en matière de lutte contre les maladies (Preston, 2013 [1976]). Pour d'autres, si une première amélioration est indubitablement obtenue par le biais des connaissances scientifiques et des pratiques médicales, c'est l'augmentation du niveau de vie économique, urbanistique (par exemple le fait de parvenir à fournir l'eau courante à tous les ménages) et social (y compris l'éducation) qui a essentiellement permis à la santé humaine de progresser (McKeown, 1980 [1976]). En fait, le phénomène englobe ces deux explications, qui se conjuguent et s'additionnent, offrant ensemble un cadre propice à la protection des populations face à la propagation: accroissement des denrées, amélioration des transports et des stockages, démocratisation de l'éducation (Johansson \& Mosk, 1987). Ainsi, certaines zones de l'Inde (comme le Kerala) ont rapidement atteint des niveaux d'espérance de vie proches de ceux qui existent en Europe, malgré leur retard économique (Meslé \& Vallin, 2002). Toutefois, cette conjugaison n'est pas toujours identique suivant les contextes. Une comparaison des variations de l'espérance de vie au Japon, en Italie et en Angleterre de la fin du $19^{\text {ème }}$ siècle aux années 1960, a permis de mettre à jour des divergences en fonction des situations culturelles et institutionnelles (Johansson \& Mosk, 1987). C'est également le caractère politique de la gestion de la santé, de l'accès aux soins et de la recherche médicamenteuse, qui explique les difficultés de conciliation entre les divers points de vue (Banerji, 1979).

4 Il n'est d'ailleurs pas étonnant que le développement de la médecine en Europe ait eu lieu parallèlement à la révolution industrielle, alors que les ouvriers souffraient de plus en plus de maux liés à leurs conditions de vie et de travail. La médecine a alors permis de soulager ces maux, générés par le système et entravant le travail. Le progrès médical a servi les travailleurs en les soulageant, mais a surtout bénéficié aux capitalistes en permettant la reprise de la productivité, tout en devenant une nouvelle industrie lucrative. Paradoxalement, c'est aussi la transformation des modes de vie liés à l'industrialisation, ainsi que la «conquête» des problèmes de santé, qui a induit la survenue des problèmes sanitaires modernes: accidents, maladies chroniques, etc. (Banerji, 1979). 
connaissances en médecine «traditionnelle» indienne comme l'Ayurvédique ou l'Unani, et les pratiques médicales populaires (Banerji, 1979).

Ce contact de l'Inde avec la médecine moderne a débuté au $17^{\text {ème }}$ siècle par le biais de la British India Company. Le premier hôpital est fondé à Madras (Chennai) en 1679 (Mushtaq, 2009). Dès ses débuts, la médecine occidentale est conservée dans les mains d'une petite élite anglaise, alors même que l'exploitation du peuple colonisé conduit à de nouveaux problèmes sanitaires, menant à un appauvrissement et à une fragilisation de la population - qui, outre se voir refuser l'accès à la médecine, subit simultanément les répercussions de la disparition de son système traditionnel de santé (Banerji, 1979). L'inégalité se remarque également entre les Etats, bien que la santé publique ait été gérée par le gouvernement central jusqu'en 1919 avant d'être déléguée aux provinces (Mushtaq, 2009). Après l'indépendance, une égalité d'accès est prononcée mais elle n'est toujours pas effective ; les anciens écarts restent importants.

Actuellement en Inde, les maladies infectieuses sont les secondes causes de décès, à hauteur d'un taux de mortalité de 253/100'000 personnes en 2012 (WHO, 2015) . Bien qu'elles aient largement reculé avec l'arrivée des médicaments modernes, elles restent importantes, particulièrement dans les milieux pauvres urbains (Dyson et al., 2004). Outre cette réduction des affections contagieuses anciennes, de nouvelles épidémies ont pointé leur nez, comme le VIH depuis la moitié des années 1980 (qui conserve toutefois une prévalence faible) (Dyson et al., 2004; Véron, 2008).

Pour les pays qui se confrontent à une pauvreté de masse comme l'Inde, la diffusion des bénéfices sanitaires au plus grand nombre reste difficile. Elle est aussi rendue plus compliquée avec la transition sanitaire et la montée des maladies chroniques liées aux risques comportementaux, qui nécessitent des soins sur la durée et une prise en charge plus complexe, que le primary health care ne peut pas offrir (Meslé \& Vallin, 2002). L'état ne parvient pas à l'heure actuelle à proposer des soins à toute la population ; en outre, le niveau de vie et l'accès à l'éducation sont insuffisants pour améliorer les techniques de prévention et de protection face aux maladies. Par ailleurs, le pays cumule les aspects négatifs du progrès et de la globalisation (mode de vie sédentaire, malbouffe et fast food, pollution, etc.) avec des difficultés liées à sa situation de pays économiquement en

5 Comparativement, les maladies chroniques représentaient en 2012 un taux de mortalité de 682/100'000 personnes et les accidents de 116/100'000 personnes (WHO, 2015). 
développement (contrôle économique par l'Occident, urbanisation chaotique et bidonvilles, pauvreté et mauvais accès aux soins, etc.) (Mushtaq, 2009). A ce titre, Mumbai - et en particulier ses quartiers les plus insalubres - se révèle un environnement potentiellement risqué en termes sanitaires; conjointement, la ville est cependant aussi synonyme de progrès médical, de développement scientifique et médicamenteux, malheureusement pas à la portée de tous (Oris \& Ramiro Fariñas, 2016).

\subsubsection{Mégapole du Sud et bidonvilles : des facteurs défavorables pour la santé}

Il existe deux facteurs principaux empêchant la propagation des maladies à travers une population à un moment donné: la protection contre l'exposition et la capacité de récupération (Johansson \& Mosk, 1987). La seconde dépend avant tout de l'inscription des individus dans les stratifications socioéconomiques et socioculturelles, ainsi que du progrès et du développement de l'Etat social. De manière similaire, la protection peut être obtenue au travers d'un niveau de vie suffisant, de l'éducation ainsi que de la possibilité de résider dans un environnement isolé et sain (Johansson \& Mosk, 1987). En ce sens, l'urbanisation dans les pays du Sud représente une ambiguïté: elle a su amener à la fois le progrès à un grand nombre de personnes, tout en étant constitutive d'un lieu de résidence malsain pour une grand part de la population la plus démunie (Johansson \& Mosk, 1987). Cette dualité a également été observée durant la transition épidémiologique dans les pays du Nord, la mortalité ayant évolué de manière non linéaire ${ }^{6}$.

6 Au $19^{\text {ème }}$ siècle en Occident, les taux urbains de mortalité ont été parfois plus élevés que leurs équivalents ruraux, malgré des revenus plus conséquents au sein de la population des villes. Des moments de stagnation voire de rabaissement de l'âge au décès ont été constatés, en lien avec le développement du capitalisme industriel et la dégradation des conditions de vie dans les zones insalubres des cités, les travaux industriels mettant en danger la vie des travailleurs - notamment des enfants, qu'aucune législation ne protégeait encore du labeur -, ainsi que le manque d'alimentation et d'hygiène (Meslé \& Vallin, 2002). Des études ont ainsi démontré qu'en Angleterre, malgré des revenus plus élevés en ville, l'espérance de vie urbaine était un temps inférieure à celle des ruraux (Alter, Neven, \& Oris, 2004; Johansson, 2000). Les Londoniens étaient plus riches en moyenne, mais la cité offrait un environnement plus malsain que les campagnes arriérées et pauvres, où l'espérance de vie moyenne était 
Depuis leurs débuts, les villes du Sud ont globalement connu un niveau de mortalité plus faible que dans le reste de leur pays. Néanmoins, de grosses disparités se sont constituées à l'intérieur du tissu urbain, surtout en raison de l'augmentation de l'habitat informel et des bidonvilles (Fink, Günther, \& Hill, 2016). Ainsi, si la mortalité juvénile est moindre en ville que dans les campagnes, elle demeure similaire entre taudis urbains et zones rurales (Vlahov et al., 2007). Certains slums possèdent même des conditions de santé pires que les campagnes, à cause de la densité et du manque de services de santé (Rossier et al., 2016). Les maladies sont fortement corrélées au niveau de vie, et le manque de système institutionnel de prise en charge ne réduit pas ces inégalités. Les impacts de l'urbanisation sont ainsi apparus multidirectionnels, soulignant l'existence de paradoxes au cours du développement. Toutefois, un constat demeure: en ville comme à la campagne, les élites se sont toujours trouvées nettement avantagées par rapport aux pauvres en ce qui concerne leur santé (Bengtsson \& van Poppel, 2011; Johansson, 2000; Link \& Phelan, 1995).

\subsubsection{La perception de la santé à travers les lieux et les étapes de la vie adulte}

Dans nos enquêtes passées à Bandra et Santa Cruz, les questions de santé sont apparues comme marquant fortement les parcours de vie ${ }^{7}$. En comparant aux nôtres les réponses issues de questionnaires semblables

plus élevée. Des conditions de santé plus dégradées en ville que dans les campagnes désindustrialisantes de la Belgique du $19^{\text {ème }}$ siècle ont aussi été soulignées, malgré une augmentation globale des salaires des citadins (Alter et al., 2004). Avec l'introduction de la santé publique au $20^{\text {ème }}$ siècle, cette pénalité urbaine a définitivement été compensée (Fink, Günther, \& Hill, 2016).

7 Dans les deux quartiers, les changements récents les plus cités concernent avant tout la santé (respectivement $41 \%$ et $39 \%$ des mentions). A travers les classes d'âge, le pourcentage de ces événements prend de l'importance dans les deux échantillons, évoluant entre $12 \%$ des mentions faites par les 20-24 ans jusqu'à $80 \%$ pour les $80-84$ ans de Bandra, respectivement $16 \%$ et $74 \%$ à Santa Cruz (cf. tableau 4.4). Parmi les grands changements de l'existence perçus par les enquêtés, la santé apparaît également comme un domaine fréquemment cité, juste après ceux de la famille et de la profession (cf. tableau 4.6). En outre, 10\% des enquêtés à Bandra ont cité non pas un mais deux tournants de santé, et c'est le cas de $7 \%$ des gens interrogés à Santa Cruz. Parmi eux, 16 personnes citent trois événements de santé (10 à Bandra et 6 à Santa Cruz) et trois individus citent quatre événements de santé (à Bandra). 
passés dans différents pays (avec toute la prudence qu'un tel rapprochement requiert), le pourcentage de changements récents et de tournants ayant trait à la santé se révèle clairement plus considérable dans les mémoires indiennes que dans les autres pays (voir figure 7.1 et tableau 7.1).

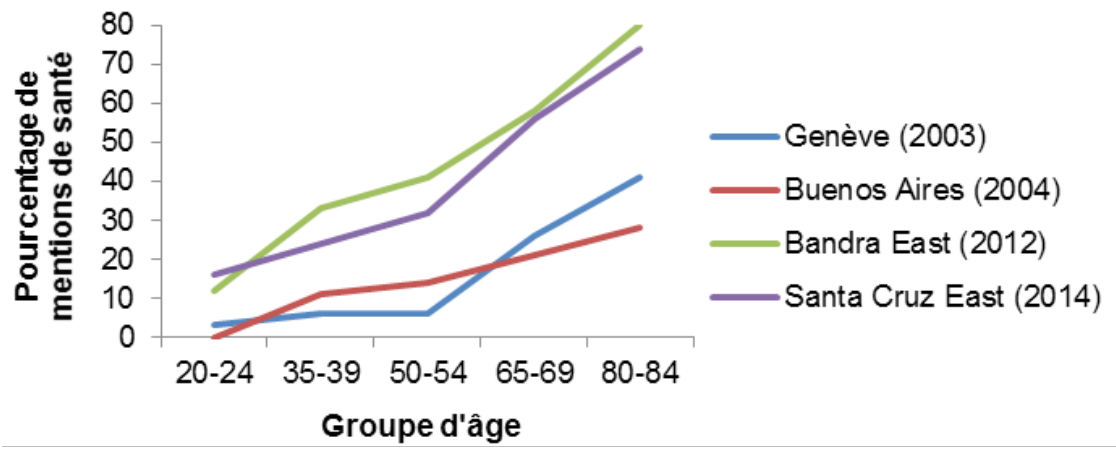

Figure 7.1: les changements récents de santé (\%), selon les groupes d'âge et le pays de résidence

Source: Cavalli \& Lalive d'Epinay, 2008; Gastrón \& Lacasa, 2009

L'avancée en âge se traduit par une présence accrue de la santé dans les réponses des individus (cf. figure 7.1). Toutefois, les enquêtes passées à Mumbai soulignent des mentions plus nombreuses et plus précoces de changements récents de santé, déjà importantes chez les jeunes qui n'en font presque pas mention à Genève $(3 \%)$ et pas du tout à Buenos Aires (Cavalli \& Lalive d'Epinay, 2008; Gastrón \& Lacasa, 2009).

Il n'est pas surprenant que ce soient les résidents d'une mégapole dans un pays où l'écart des inégalités de santé est abyssal, en particulier ceux habitant dans les slums, qui évoquent davantage la santé. Néanmoins, la simple explication du vécu en milieu insalubre ou de la pauvreté pour éclaircir la prévalence de la santé parmi les réponses ne suffit pas, puisque cette dernière se remarque dans les deux quartiers étudiés. En première instance, cela suggérerait que les niveaux socioéconomiques auraient peu d'impact. Dit autrement, ce serait le fait de vivre dans le milieu urbain chaotique d'un pays en développement (plutôt que son degré de pauvreté) qui impliquerait la forte prévalence des événements liés à la santé. 


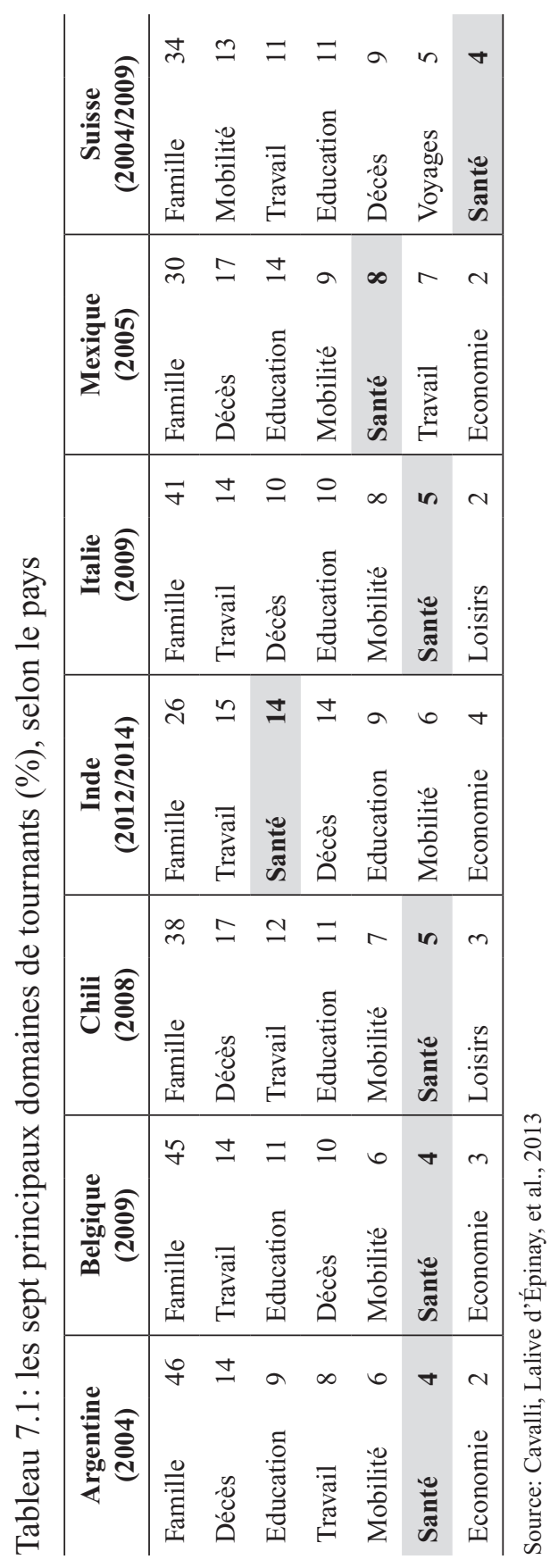




\section{Mentionner sa santé comme un changement important}

Pour saisir le profil des individus mentionnant des changements récents et des tournants de vie engageant la santé, plusieurs modèles de régression logistique ont été estimés sur la propension à "avoir cité au moins un événement de santé», selon les caractéristiques sociodémographiques suivantes : lieu de résidence, sexe, classe d'âge, niveau d'éducation et santé auto-évaluée ${ }^{8}$. La variable dépendante a été construite de manière binaire : 0 ne pas avoir cité de changement récent de santé et 1 en avoir cité au moins un. Afin de se focaliser sur les personnes ayant cité des événements négatifs de santé, les individus qui ont mentionné une amélioration ont été ôtés dans cette analyse ${ }^{9}$.

Les rapports de cotes présentés dans le tableau 7.2 vérifient l'absence de divergences significatives entre les deux quartiers. Il semble que le trait spécifique de la santé, lié à la vie en Inde et à Mumbai dans un milieu urbain largement pollué, se confirme. Ressort par ailleurs un effet lié à l'âge en ce qui concerne l'évocation de changements récents de santé. Toutes choses égales par ailleurs, être âgé de 20 à 24 ans diminue très nettement le risque de citer un événement récent de santé, par rapport aux trentenaires et aux cinquantenaires, mais surtout aux sexagénaires et aux octogénaires. Rien d'étonnant à cela, les personnes âgées rencontrant davantage d'ennuis de santé que les plus jeunes.

Une situation complexe apparaît par contre lorsque l'éducation est considérée. Si les individus illettrés ne présentent pas de différence significative avec ceux au capital humain élevé, les personnes ayant une éducation secondaire (et primaire pour les changements récents) ont un risque plus grand que celles avec un niveau tertiaire d'évoquer leur santé. Que les universitaires mentionnent peu leur santé laisse à penser qu'ils sont tout simplement moins affectés que les autres catégories d'individus. Il est en effet reconnu que l'éducation est protectrice à cet égard (Alwin \& Wray, 2005; Bartley, 2016; Marmot, 2005), surtout pour les élites (Link \& Phelan, 1995; Phelan et al., 2010). Reste que la propension plus élevée de parler de sa santé lorsque l'on a connu quelques années d'école (par

8 Voir tableau 7.2, où seuls les modèles avec toutes les variables indépendantes sont présentés et annexe 5 pour tous les modèles.

9 Au total, 349 individus ont mentionné au moins une fois le domaine de la santé parmi les changements récents, 320 une fois les réponses du type «amélioration de la santé» ôtées. Du côté des tournants, 277 individus ont mentionné au moins une fois le domaine de la santé, 271 sans les réponses du type «amélioration de la santé». 
rapport à n'avoir jamais été à l'école) entre au moins partiellement en contradiction avec ce constat.

Tableau 7.2: rapports de cotes issus de modèles de régression logistique sur la variable «avoir mentionné au moins un événement de santé»

\begin{tabular}{lcc}
\hline & Changements récents & Tournants \\
\hline Lieu de résidence (référence $=$ Santa & Cruz East) & \\
Bandra East & 1.270 & 1.250 \\
Sexe (référence $=$ hommes) & 1.160 & $0.709^{*}$ \\
Femmes & & \\
Classe d'âge (référence $=\mathbf{2 0 - 2 4}$ ans) & $2.257^{* *}$ & 0.886 \\
$35-39$ ans & $2.113^{*}$ & 1.054 \\
$50-54$ ans & $5.653^{* * *}$ & $1.562^{\circ}$ \\
$65-69$ ans & $6.262^{* * *}$ & 0.847 \\
$80-84$ ans & 1.082 & 1.430 \\
Education (référence $=$ Supérieure) & $1.732^{*}$ & 1.439 \\
Sans & $2.248^{* * *}$ & $2.322^{* * *}$ \\
Primaire & 1.459 & $1.686^{*}$ \\
Secondaire & 1.474 & $1.622^{\circ}$ \\
Santé auto-évaluée (référence $=$ Très bonne) & $2.995^{* *}$ \\
Bonne & $3.959^{* *}$ & \\
Moyenne & $50.01 * * 4001$ & \\
Mauvaise & & \\
\hline
\end{tabular}

Seuils de significativité $:^{\circ} \leq 0.1 ; * \leq 0.05 ; * * \leq 0.01 ; * * * 0.001$.

Cette situation est difficile à interpréter. Le concept d'illusion objective, utilisé originellement par Karl Marx (2009 [1867]) et repris par Amartya Sen (Sen, 1993, 2009), peut apporter quelques éclaircissements. Ce concept mobilise à la fois l'idée d'une croyance objective du point de vue de l'individu et le diagnostic que cette croyance est, en fait, erronée (Sen, 2009). Ainsi, dans le cadre d'une comparaison interétatique d'auto-évaluations de la santé en Inde, Sen $(1993,2009)$ a remarqué que des répondants résidant au Kerala (un Etat relativement bien développé en matière de santé publique, avec en moyenne de bons niveaux d'éducation et une espérance de vie élevée) mentionnaient des taux de morbidité auto-évalués bien plus hauts que les habitants de l'Uttar Pradesh ou du Bihar (des 
Etats où la pauvreté est élevée, l'accès aux soins difficile et l'illettrisme important). Il explique ce paradoxe par le fait que les Kéralais ont une conscience accrue des maladies les affectant, précisément parce que leur niveau d'éducation est plus élevé et que les moyens mis en œuvre par l'état pour les prévenir sont plus nombreux. Inversement, les individus vivant en Uttar Pradesh ou au Bihar, ont une compréhension plus floue des maux qui les affectent, car moins habitués à en parler et à les prévenir.

Dans une analyse plus récente, portant sur la santé perçue parmi les personnes âgées en Inde, un résultat similaire s'est dégagé: les femmes, pourtant en moins bonne condition, se déclarent en meilleurs santé que les hommes (Husain \& Ghosh, 2017). Ainsi, les variations contre intuitives dans les réponses collectées par nos enquêtes pourraient s'expliquer par une perception accrue des problèmes médicaux parmi les individus ayant accédé à l'école, même si, de fait, les illettrés sont, selon toute vraisemblance, plus à risque de rencontrer des ennuis de santé. Néanmoins, cette explication d'une illusion objective est soumise à controverses.

Le sexe apparaît significativement décisif dans le fait de mentionner un tournant de santé. Les femmes ont un risque inférieur d'évoquer un tournant de santé (une fois contrôlé l'impact du lieu de résidence, de l'âge, du niveau d'éducation et de la santé auto-évaluée). Ce résultat est cohérent avec ceux des études mentionnées plus haut, suggérant que les femmes déclarent moins de problèmes de santé alors même que, selon toute vraisemblance, elles ont objectivement été plus affectées. Cela peut s'expliquer par le mécanisme d'illusion objective, mais aussi par une spécificité de notre dispositif d'enquête : la concurrence avec d'autres domaines de la vie, familiaux notamment, que les femmes ont pu prioriser lorsqu'elles ont été invitées à mentionner les tournants les plus significatifs de leur existence.

Finalement, la variable de la santé auto-évaluée souligne que les personnes émettant une réserve faible ou importante quant à leur niveau de santé, montrent toutes un risque plus grand de mentionner un événement de santé négatif que les très bien portants. Corroborant ce que nous avons vu dans la section 4.1.1, ce résultat confirme qu'être en mauvaise santé amène le risque de considérer sa trajectoire de santé plus sombrement (l'essentiel des réponses du domaine santé étant des maladies ou des accidents), ce tout au long de son parcours. Ces problèmes ont eu un impact sur l'existence et de ce fait restent dans une mémoire autoalimentée par l'état présent. 
Voyons à présent si le fait que l'enquête effectuée à Bandra l'a été dans les bidonvilles a pu avoir une influence sur ces réponses. En effet, le choix méthodologique de recourir à des assistantes sociales de l'ONG CSSC (cf. chapitre 3) aurait pu avoir une influence sur les réponses des individus.

\subsubsection{Un effet WIN?}

Ce que les réponses apportées aux volets I et II ont démontré est une prévalence certaine de la santé dans les changements de la vie et dans les tournants des Indiennes et des Indiens interrogés à Mumbai. Dans le cas de l'enquête passée en 2012 dans les bidonvilles, ce constat peut avoir été biaisé par la méthodologie même de l'étude. En effet, les enquêtrices étaient des assistantes sociales du projet WIN, qui se penche sur les premiers soins et en particulier la santé de la reproduction dans les bidonvilles de Bandra (voir chapitre 3). Un certain nombre de répondants faisaient également partie du projet WIN en tant que patients, ayant l'habitude de rencontrer mensuellement ces assistantes sociales et de discuter avec elles de problèmes médicaux, ce qui peut avoir eu un impact sur les réponses données par ces répondants.

Toutefois, les critères d'échantillon ont imposé d'interroger également des personnes qui n'étaient pas des patients dans le projet WIN dans le terrain à Bandra. Au final, 59\% de l'échantillon de Bandra faisait partie du projet, à peu près égalitairement entre les classes d'âge ${ }^{10}$. Il est difficile de supposer a priori que le fait d'être patient dans le projet WIN soit un marqueur de fragilité, ou au contraire le signal d'un meilleur accès aux soins. Néanmoins, puisqu'une proportion également importante de l'échantillon de Santa Cruz mentionne des changements récents dans le domaine de la santé, le biais ne semble pas exister ou être minime. Ceci se confirme du fait que, parmi les habitants de Bandra interrogés dans notre enquête et patients du projet WIN, 32\% évoquent des événements de santé, ce qui est à peine plus que les répondants des bidonvilles ne faisant pas partie du réseau WIN (27\%).

10 Les répondantes de Bandra patientes du projet WIN selon la classe d'âge: $46 \%$ (20-24 ans); 45\% (35-39 ans); 42\% (50-54 ans); 21\% (65-69 ans); 48\% (80-84 ans). Les répondants de Bandra recensés dans le projet WIN selon la classe d'âge: 46\% (20-24 ans); 30\% (35-39 ans); 58\% (50-54 ans); 31\% (65-69 ans); 46\% (80-84 ans). 
Le tableau 7.3 présente à nouveau les modèles de régression logistique sur la variable dépendante «avoir cité au moins un événement récent/un tournant de santé», toujours en fonction des caractéristiques sociodémographiques et cette fois se basant sur l'entièreté des mentions de santé (y compris l'«amélioration de la santé»). Toutefois, la variable indépendante concernant le lieu de résidence a été remplacée par le fait d'appartenir, ou non, au projet WIN. Ainsi, la population étudiée ne comprend que les résidents des bidonvilles. Par ailleurs, nous avons choisi de ne retenir que des individus ayant fait mention au moins une fois d'un événement de santé qui les concernait (les problèmes de santé survenus à un membre de la famille ne sont donc pas pris en compte). En effet, percevoir un possible «effet WIN» ne peut être faisable qu'en considérant la santé du répondant directement.

Tableau 7.3: rapports de cotes issus de modèles de régression logistique sur la variable «avoir mentionné au moins un événement de santé (à soi)», à Bandra

\begin{tabular}{|c|c|c|c|c|}
\hline & \multicolumn{2}{|c|}{ Changements récents } & \multicolumn{2}{|c|}{ Tournants } \\
\hline & modèle 1 & modèle 2 & modèle 1 & modèle 2 \\
\hline \multicolumn{5}{|c|}{ Etre patient dans le projet WIN (référence $=$ ne pas être patient) } \\
\hline Patient & - & 1.296 & - & 1.018 \\
\hline \multicolumn{5}{|c|}{ Sexe (référence $=$ Hommes) } \\
\hline Femmes & $1.796^{* *}$ & $1.753^{* *}$ & $0.599^{*}$ & $0.598^{*}$ \\
\hline \multicolumn{5}{|c|}{ Classe d'âge (référence $=20-24$ ans) } \\
\hline $35-39$ ans & $1.879^{\circ}$ & 1.823 & 0.814 & 0.813 \\
\hline $50-54$ ans & $2.168^{\circ}$ & $2.134^{\circ}$ & 1.197 & 1.198 \\
\hline $65-69$ ans & $4.592 * * *$ & $4.218^{* * *}$ & 1.714 & 1.706 \\
\hline $80-84$ ans & $4.625^{* * *}$ & $4.451 * * *$ & 1.019 & 1.017 \\
\hline \multicolumn{5}{|c|}{ Education (référence = Supérieure) } \\
\hline Sans & 1.421 & 1.534 & 1.083 & 1.088 \\
\hline Primaire & 1.597 & 1.690 & 0.767 & 0.769 \\
\hline Secondaire & $2.650 *$ & $2.701 * *$ & 1.635 & 1.637 \\
\hline \multicolumn{5}{|c|}{ Santé auto-évaluée (référence = Très bonne) } \\
\hline Bonne & 0.745 & 0.726 & 1.580 & 1.575 \\
\hline Moyenne & 1.128 & 1.084 & 2.352 & 2.343 \\
\hline Mauvaise & 2.200 & 2.219 & $3.498^{\circ}$ & $3.498^{\circ}$ \\
\hline
\end{tabular}

Seuils de significativité : ${ }^{\circ} \leq 0.1 ; * \leq 0.05 ; * * \leq 0.01 ; * * * 0.001$. 
Le risque de biais constitué par l'engagement à Bandra d'assistantes sociales ayant l'habitude de poser des questions médicales aux individus se révèle bien être infondé (voir tableau 7.3). Il n'y a pas de différence significative entre les deux modèles, une fois l'impact des autres variables indépendantes contrôlé, ce tant pour les changements récents que pour les tournants ${ }^{11}$.

$* * *$

$\mathrm{Au}$ sortir de ces analyses concernant les individus susceptibles de mentionner des événements de santé, une certaine distance se ressent par rapport aux inégalités sociales de santé attendues. Ainsi, le sexe n'apparaît pas comme une variable significative du fait d'évoquer un souvenir sanitaire récent, mais il ressort à l'échelle de l'ensemble de la trajectoire de vie (soit sur les tournants) et de manière paradoxale (les hommes ayant une propension de mentions supérieure aux femmes). Le lieu de résidence n'a pas non plus d'incidence, et le niveau d'éducation souligne plutôt à nouveau un paradoxe, lié au fait d'être habitué à parler de sa santé ou pas, à en être conscient ou non. Seules les inégalités de santé par rapport à la position dans son parcours de vie apparaissent claires, en particulier pour les changements récents. Les adultes les plus âgés ont, comme attendu, une plus grande probabilité d'évoquer un événement de santé que les jeunes, que ce soit pour les changements récents ou les tournants.

Pourtant, la réalité objective des inégalités de santé en Inde ne saurait être remise en question. Serait-ce que la subjectivité et la méthodologie de notre enquête ont effacé ces divergences ? La partie qui suit a pour but de creuser cette question en usant d'une typologie nouvelle, dans l'objectif de mettre à jour la possible existence d'autres formes d'inégalités, jusque-là effacées par l'utilisation «en bloc» des réponses de santé.

11 Seuls les modèles avec toutes les variables indépendantes sont présentés dans le tableau 7.4, afin d'en faciliter la lecture. Toutefois, les modèles intermédiaires (où chacune des variables a été ajoutée tour-à-tour) ne montrent pas davantage de significativité au niveau de la variable explicative «être patient de WIN». 


\subsection{Le phénomène du multiple burden of disease}

Les pauvres ont une santé moins bonne que les riches en général, surtout dans les zones urbaines insalubres des mégapoles des pays du Sud (Bartley, 2016; Marmot, 2005). Par ailleurs, le développement économique est associé à une amélioration de l'espérance de vie, une diminution des décès liés aux maladies infectieuses, et un rythme de vie sédentaire conduisant à des comportements à risques et aux maladies chroniques (Singh et al., 2007). Ainsi, les changements démographiques et socioéconomiques que le continent indien a connus ces dernières décennies ont fortement accéléré sa transition sanitaire.

\subsubsection{Le contexte de vie comme facteur de différenciation sociale dans les pathologies}

L'urbanisation modifie durablement les comportements sociaux et physiques des humains, et sa rapidité dans les pays moins développés économiquement représente l'un des principaux enjeux pour la santé publique (Kroll, Bharucha, \& Kraas, 2014). Dans ce contexte marqué par les disparités socioéconomiques et les stratifications sociales, les interventions dans le domaine de la santé mènent généralement à une croissance des inégalités. Les bidonvilles en sont les meilleures illustrations, tout spécialement à Mumbai où ils côtoient sans complexe les plus modernes des buildings.

Les quartiers informels concentrent des situations sanitaires calamiteuses, en particulier néfastes pour les enfants, et une population qui subit déjà des désavantages de santé liés à une situation économique défavorable (Kroll et al., 2014; Vlahov et al., 2007). L'UN-Habitat (2006) a d'ailleurs démontré que plus une cité abrite de bidonvilles, plus grande est la prévalence d'infections diarrhéiques parmi la population urbaine. Ainsi, les enfants vivant dans les taudis subissent des taux de diarrhées plus élevés que les enfants pauvres ruraux, car plus exposés aux mauvaises eaux et à la nourriture contaminée (Mundu \& Bhagat, 2008).

De surcroît, les disparités ne se contentent pas d'apparaître entre les quartiers décatis et ceux bâtis en dur. Dans la ville de Mumbai, les niveaux de santé diffèrent considérablement entre les bidonvilles. Les slums de 
la ville se divisent en effet entre ceux qui sont notifiés et ceux qui ne le sont pas (voir chapitre 1) ${ }^{12}$. La gestion de l'eau et des sanitaires par la municipalité est soumise au fait d'être un bidonville notifié, soit de se trouver sur une parcelle non gouvernementale (appartenant à l'Etat ou à la municipalité), et de s'être établi là avant la deadline du $1^{\text {er }}$ janvier 1995 (la fameuse 1995 cut-off rule) ${ }^{13}$. Autrement dit, être un slum non notifié pose des barrières à l'accès aux services basiques comme l'eau et les sanitaires, mais aussi aux centres de premiers soins, influençant directement le niveau de santé des populations concernées (Subbaraman et al., 2012; Vlahov et al., 2007).

Les slums (notifiés et surtout non notifiés) sont très souvent localisés dans des espaces malsains, comme le bord de mer, sur des terrains bas susceptibles d'être inondés ou des mangroves, sur des montagnes de détritus, sur des cimetières, sous des lignes à haute tension, en bordure de voie ferrée (Murthy, 2012). Ces lieux présentent des risques élevés d'accidents, de contraction de problèmes respiratoires (issus de la pollution, notamment à proximité des grands axes routiers) et de propagation de maladies (dans des zones humides et marécageuses) (Vlahov et al., 2007). Ces terrains sont également vulnérables face aux désastres naturels, mettant en danger les individus qui y résident (Vlahov et al., 2007).

L'absence d'accès à l'eau potable, d'égouts, de ramassage des ordures et des latrines partagées par des dizaines de personnes favorisent les maladies d'origine hydrique et les voies de contagion des maladies infectieuses (diarrhées, choléra, etc.) (Murthy, 2012; Vlahov et al., 2007). Dans la pratique, le manque de sanitaires condamne les résidents des slums à utiliser des toilettes publiques non hygiéniques (c'est le cas de $63 \%$ des habitants des slums de Mumbai, pour un partage par sanitaire évalué entre 80 et 270 personnes), ce qui accroit fortement les risques de contagion (Murthy, 2012). Sans écoulement des eaux usées, ces toilettes fonctionnent par un système de fosses septiques, dont le manque d'entretien et de vidange provoque régulièrement des débordements. Par ailleurs, ce sont

12 Pour rappel: selon le National Sample Survey de 2011-12, près de la moitié des bidonvilles de Mumbai ne sont pas notifiés.

13 Malgré cette cut-off rule, les autorités étatiques et surtout le gouvernement central auraient l'opportunité de s'occuper de l'accès en eaux et des égouts pour tous les slums, mais les moyens et les décisions politiques manquent pour le faire (Murthy, 2012). En l'absence de politiques publiques, ces zones sont off the map des autorités, qui leur dénient l'accès à la santé et aux services basiques (Subbaraman et al., 2012). 
des toilettes qui ne possèdent pas d'électricité, ne sont donc pas éclairées la nuit, ce qui résulte en de longues files d'attente au matin ou encourage à se soulager dehors $(5 \%$ des habitants des slums effectuent leurs besoins à ciel ouvert) (Murthy, 2012).

Les femmes et les enfants sont les plus victimes physiquement, mentalement et émotionnellement de ces conditions de vie déplorables (Mundu \& Bhagat, 2008). Les femmes, qui doivent souvent attendre la fin de leurs tâches domestiques pour aller aux toilettes, préfèrent parfois l'extérieur au vu de l'état calamiteux des sanitaires publics. Ce qui les contraint à patienter jusqu'à la nuit, en évitant de boire et de manger trop, et les expose à des risques d'agression (Murthy, 2012).

La pollution est également un facteur cardinal du niveau de santé de la population dans les villes du Sud. Les déchets abandonnés, l'eau sale déversée sans traitement dans l'océan ou les réseaux fluviaux proches des bidonvilles sont de graves sources de danger pour les individus et pour l'environnement (Murthy, 2012). De plus, la pollution de l'air, que ce soit à l'extérieur par les émissions de gaz ou à l'intérieur par l'utilisation de combustibles solides (biomasse et charbon) pour la cuisine et le chauffage, dans des endroits fermés et mal ventilés, participe hautement à développer des maladies comme l'asthme ou les problèmes cardiovasculaires (Smith, 2000).

Caractéristique des slums, la densité est souvent accusée de la propagation des maladies, dans une surprenante similarité avec la théorie des miasmes dans les villes européennes à l'époque de la révolution industrielle, par ailleurs tout aussi trompeuse qu'en ce temps-là (Corbin, 1982) ${ }^{14}$. La réalité est plus nuancée. Certes, la densité humaine entraîne un risque plus élevé de transmission des maladies et accroît les problèmes de

14 L'Ancien Régime occidental finissant voit apparaître les villes et le cortège d'insalubrités qui les accompagnent en l'absence d'une gestion efficace des déchets: ordures, excréments, charognes et carcasses, eaux contaminées et air putride. En pleine période de propagation des maladies infectieuses, cette situation provoque une anxiété généralisée qui pousse à la croyance de «miasmes», mauvais airs responsables des infections et des fièvres. Le résultat est une transformation des cités vers davantage d'espace (selon le modèle haussmannien), mais aussi la montée des courants hygiénistes démontrant que, si la crainte des maladies a permis la diffusion de ces idées au sein des masses - par la suite largement infirmées -, c'est avant tout la peur de la colère du prolétariat et de la révolte du peuple qui poussa les élites à dompter la rue et les comportements populaires, au travers du développement de la médecine et de pratiques urbanistiques nouvelles (Corbin, 1982). 
pollution. Néanmoins, un nombre élevé de gens sur un périmètre réduit facilite aussi l'accès aux centres de soins, particulièrement ardu dans les vastes campagnes indiennes, tout en offrant l'opportunité aux individus d'augmenter leur capital social, grâce à la vie en communauté et au soutien des proches (Vlahov et al., 2007).

Beaucoup reste à faire pour que l'espérance de vie s'égalise entre les habitants de la mégapole. Le niveau de progrès médical que certains hôpitaux privés de Mumbai atteignent n'est accessible que pour une petite élite. Nonobstant, ce sont avant tout des déterminants sociaux qui font les inégalités de santé, comme les conditions d'habitation, l'éducation, la qualité et l'accès aux infrastructures de soins. La santé des habitants de la mégapole est ainsi illustrative des inégalités économiques et sociales qui divisent les quartiers de la ville.

\subsubsection{En quelques chiffres, la santé des habitants de Mumbai à l'aube du $21^{\text {ème }}$ siècle}

En 2007 à Mumbai, l'espérance de vie à la naissance était de 57 ans (53 ans pour les hommes et 58 ans pour les femmes). Ce chiffre, inférieur à l'espérance de vie globale indienne (64 ans) et à celle du Maharastra (69 ans) (Mumbai Human Development Report, 2009), souligne une plus grande mortalité en ville, à l'image de la situation européenne du $19^{\text {ème }}$ siècle. Les premières causes de décès dans la mégapole sont les attaques cardiaques et plus largement les déficiences cardiovasculaires, négligées dans l'agenda du développement indien (Ramaraj \& Alpert, 2008), suivies par la tuberculose et le cancer, les problèmes rénaux, le diabète et le VIH. Ensuite viennent les autres maladies infectieuses (le choléra, les hépatites, la malaria, la fièvre dengue, les diarrhées), transmises par la mauvaise qualité de l'eau, le manque d'hygiène et les inondations dues aux moussons (Murthy, 2012).

La tuberculose est une maladie particulièrement présente en Inde car typiquement liée à la pauvreté et à la vie en milieu urbain insalubre et pollué, qui accroit également le risque de contracter des affections respiratoires, des méningites, de l'asthme (Radkar, Kanitkar, \& Talwalkar, 2010). Depuis la fin du $20^{\text {ème }}$ siècle, la maladie a réémergé comme problème majeur de santé, souvent liée au diabète et à la propagation du VIH (Dye, Trunz, Lönnroth, Roglic, \& Williams, 2011; IIPS, 2007). 
Par ailleurs, les habitants de Mumbai sont fortement sujets aux désordres de la tyroïde et au diabète (IIPS, 2007). Ce dernier affecte à l'heure actuelle davantage les habitants de classe moyenne ou riche, à cause du style de vie sédentaire impliquant peu d'activité physique et du surpoids, voire de l'obésité (Srinath Reddy, Shah, Varghese, \& Ramadoss, 2005). Ainsi, si un niveau de vie plus élevé protège généralement des maladies, dans les pays économiquement en développement l'acquisition du mode de vie occidental (contenant des pratiques à risque) par les plus riches leur vaut également d'être plus vulnérables face à certaines maladies, comme le VIH, les ennuis cardiovasculaires et les accidents (Rossier et al., 2016).

Ces derniers ont en effet cru depuis les années 1990, la mortalité résultante variant entre les groupes d'âge ( $8 \%$ avant 14 ans, $62 \%$ entre 15 et 44 ans, $20 \%$ entre 45 et 59 ans) (Upadhyay, 2012). Le ratio est de trois pour un entre homme et femme ( $73 \%$ des décès dû aux accidents arrivent aux hommes) (Upadhyay, 2012). Néanmoins, une étude à Ouagadougou au Burkina Faso a démontré que les femmes subissent plus d'accidents, possiblement car elles sont exposées à la fois aux accidents domestiques et aux accidents extérieurs au foyer (Rossier et al., 2016). Cette étude souligne aussi la survenue plus nombreuse d'accidents dans les quartiers informels, peut-être à cause du manque d'infrastructure du quartier (les routes ne sont pas finies, ont des trous, etc.). Mais les plus éduqués rencontrent aussi plus d'accidents, probablement car ils bougent davantage autour de la ville et sont donc plus exposés aux risques de la route (Rossier et al., 2016), ce qui va dans le sens d'une pénalité affectant davantage les plus favorisés.

A l'autre bout du spectre social, un déterminant crucial de l'état de santé et de la résistance face aux maladies infectieuses, la malnutrition dépend largement du fait de vivre en milieu urbain ou pas. A Mumbai, elle court littéralement les rues, puisque 33\% des enfants de moins de 5 ans étaient en sous poids et $10 \%$ en sous poids sévère (malnutrition aiguë ou chronique) en 2006 (IIPS, 2007). Néanmoins, la situation en ville est meilleure que dans le reste de l'Etat mais reste inférieure à d'autres centres urbains mieux organisés pour combattre la faim. Au sein même de Mumbai, des divergences apparaissent entre les quartiers, les zones de taudis étant les plus affectées (IIPS, 2007). 


\subsubsection{La montée des maladies chroniques}

Bien que moins répandues qu'au Nord, les maladies non transmissibles sont en augmentation dans les pays du Sud (40\% des décès leur sont attribuables, contre 75\% dans le reste du monde) (Shetty, 2002). En Inde, elles contribuent à $53 \%$ des décès et à $44 \%$ des DALY s ${ }^{15}$ perdus (Ramaraj \& Alpert, 2008; Srinath Reddy et al., 2005; Upadhyay, 2012). Comme cela a déjà été précisé, la pauvreté urbaine induit des risques sanitaires avant tout parce que l'accès à la santé est très inégal et la prévention contre les maladies chroniques - mal (re)connues en comparaison des maladies infectieuses - n'est pas une priorité gouvernementale (Mallath et al., 2014). Les écarts sanitaires au sein de la population, poussés par les inégalités socioéconomiques, soulignent également une diffusion des maladies chroniques parmi les individus qui subissaient auparavant seulement l'impact des infections. Les maladies non transmissibles, comme les maladies cardio-vasculaires par exemple, ne sont plus réservées aux riches (Ramaraj et Alpert, 2008). Les cancers sont aussi en augmentation parmi la population démunie, frappant à des âges précoces, principalement à cause de la mauvaise capacité de détection et de l'incapacité de traitement.

L'augmentation rapide des maladies non transmissibles est due aux inégalités et à la pauvreté ainsi qu'aux changements de mode de vie (sédentarisation, pollution, télévision,...) allant avec la mondialisation et l'urbanisation (Shetty, 2002; Shukla, 2007). Les principaux comportements à risque favorisant les maladies chroniques sont d'ordre alimentaires (déficits et excès, malnutrition), associés au manque d'activité physique et à la consommation de tabac et d'alcool (Nugent, 2008; Upadhyay, 2012). Posséder un faible statut socioéconomique est généralement un facteur aggravant, mais pas systématiquement (Ramaraj \& Alpert, 2008).

Alors que les pauvres des pays développés sont globalement plus vulnérables face aux maladies chroniques, en Inde la prévalence de ces maladies varie entre les groupes socioéconomiques en fonction des facteurs de risques comportementaux (Nugent, 2008). Le tabagisme (passif et actif) et la surconsommation d'alcool sont des pratiques en augmentation, caractéristiques des couches défavorisées de la population (pauvres, sans emploi, peu éduqués, basses castes) qui ont un lien direct avec la montée des maladies chroniques (Sorensen, Gupta, \& Pednekar, 2005). A Mumbai,

15 Disability-Adjusted Life Year (DALY): un DALY représente une année en bonne santé perdue. 
la prévalence du tabagisme parmi les individus âgés de 15 à 49 ans est de $57 \%$ chez les hommes et $11 \%$ chez les femmes (IIPS, 2007; Upadhyay, 2012). Par ailleurs, la consommation d'alcool est en hausse depuis les années 1970, en particulier dans les slums et son impact prévaut, à travers la prise de risque, dans le développement de maladies telles que le VIH (Sharma, Tripathi, \& Pelto, 2010).

Inversement, le manque d'activité physique et le diabète de type 2 frappent davantage les riches (Upadhyay, 2012), bien que le dernier soit en train de se répandre dans les bidonvilles, essentiellement à cause du manque d'accès aux soins et de conscience des risques (Srinath Reddy et al., 2005). Cela étant, c'est avant tout parmi les individus aisés que de plus en plus de personnes sont en surpoids ou obèses (Nugent, 2008; Shetty, 2002; Singh et al., 2007). Ce problème est issu de changement dans la quantité et la qualité de la nourriture ingérée (s'orientant vers la viande, les sucres et les graisses, alors que les céréales se réduisent), mais aussi de phénomènes collatéraux comme la réduction du travail physique (et donc la réduction de l'énergie dépensée), une augmentation des loisirs sédentaires et des déplacements motorisés ${ }^{16}$. De son côté, la malnutrition

16 Le modèle de la transition nutritionnelle souligne que l'augmentation des revenus entraîne le passage d'une consommation constituée avant tout de céréales, vers une consommation faite de produits diversifiés (Kulkarni, Kulkarni, \& Gaiha, 2017; Landy, 2009). La première étape de ce schéma survient lorsque la population commence à avoir un revenu moyen suffisant pour manger plus de céréales en quantités absolues (faisant de ce fait disparaître la famine). La seconde étape apparaît lorsque la population change son régime alimentaire pour aller vers une nourriture plus calorifique et plus chère (viande, laitages, fruits et légumes). Cette transition ne s'est pas déroulée linéairement en Inde. En effet, un déclin du nombre moyen de calories ingéré se constate ces dernières décennies, surtout chez les personnes au revenu confortable, mais peu ou pas chez les très pauvres signifiant que la première étape n'est pas achevée (Deaton \& Drèze, 2009). Toutefois, la seconde partie de la transition a parallèlement débuté (Kulkarni et al., 2017; Landy, 2009).

La diminution du nombre de calories ingérées par les Indiens ne s'explique ni par une chute des revenus ni par une augmentation des prix, mais plutôt par la baisse du taux d'activité physique (notamment pour le travail) et l'amélioration de l'environnement sanitaire (permettant une meilleure assimilation de la nourriture) (Deaton \& Drèze, 2009). Un impact culturel, avec le strict végétarianisme des hautes castes et des individus très religieux, peut également expliquer une part de ce déclin (Landy, 2009). Ainsi, en Inde se côtoient la sous-nutrition (pour la frange pauvre et souvent rurale de la population) et la croissance de la quantité de calories (où les nutriments diminuent pour laisser la part belle aux graisses) ingérées en moyenne par personne. Toutefois, il n'existe pas de lien évident entre la consommation de calories et le 
frappe chez les pauvres, ce qui a des répercussions majeures pour les maladies chroniques. En effet, connaître la sous-nutrition durant l'enfance accroit les risques de surpoids et de maladies comme le diabète durant l'âge adulte (Kulkarni et al., 2017).

La pauvreté n'est pas que source de maux ; elle en est aussi une des conséquences les plus évidentes parmi ceux qui sont déjà fragilisés socioéconomiquement. A cet égard, l'âge auquel les maladies chroniques surviennent est crucial. Bien plus précoces dans les pays en développement, elles s'observent souvent au cours des années d'activité économique (entre 35 et 64 ans), alors que les personnes ont une famille à charge (Nugent, 2008; Quigley, 2006; Upadhyay, 2012). Les implications collatérales sont énormes pour les proches, tant dans le cas d'un décès que d'une maladie longue qui empêche l'individu de travailler. Ces impacts sont accentués par les défaillances du système de santé publique. Le coût des médicaments et des traitements dissuade les pauvres de se faire soigner ou ne leur permet pas de le faire correctement (augmentant le nombre de rechutes et la mise en danger par des consultations auprès de médecins/ pharmaciens non qualifiés), provoquant un appauvrissement sur le long terme (Ramaraj \& Alpert, 2008).

\subsubsection{Le cumul des risques ou multiple burden of disease}

Dans les pays les plus développés économiquement, la transition sanitaire a modifié la situation épidémiologique jusqu'à ce qu'un seul fardeau persiste, celui des maladies dégénératives ou chroniques, affectant surtout les individus âgés (Omran, 2005; Rossier et al., 2016). Or, du fait de l'accélération des changements sanitaires en Inde comme dans beaucoup de pays du Sud, l'arrivée des maladies chroniques, telle que nous l'avons analysée dans la section précédente, est survenue alors que les maladies infectieuses - ayant particulièrement d'impact durant les premières années de vie - n'avaient pas disparu. C'est ce phénomène que les chercheurs ont appelé le multiple burden of disease (Boutayeb, 2006) ${ }^{17}$. Ce «fardeau

niveau de santé, car d'autres facteurs entrent en compte (manger équilibré, les activités physiques, l'accès à l'eau potable,...).

17 Cette situation a amené les chercheurs à parler de «polarisation épidémiologique», pour signifier la coexistence de deux schémas de mortalité: le premier (infectieux) lié aux pauvres conditions de vie et le second (chronique, dégénératif combiné avec les 
multiple des maladies», représente pour une population vulnérable le risque élevé de contagion au début de l'existence, additionné de la menace de contracter une maladie non transmissible durant la vie adulte (comme le cancer, le diabète et autres maladies chroniques) ou de décéder d'une mort accidentelle violente (Shukla, 2007). Il est souvent issu du fait de résider dans un environnement urbain malsain, insalubre et pollué, ainsi que de facteurs économiques, sociaux, comportementaux et démographiques, comme l'élévation de l'espérance de vie (Quigley, 2006).

La diffusion du multiple burden of disease n'est pas homogène dans la population indienne. Ainsi, si une partie de la société indienne suit le schéma classique de la transition sanitaire (les individus riches et de classe moyenne aisée), deux autres groupes se distinguent. Dans les villes, les pauvres résidant dans les slums et travaillant dans le secteur précaire informel, ayant généralement effectué une ou plusieurs migrations depuis le monde rural, sont les premières victimes des «nouvelles» maladies (chroniques, mais aussi le VIH) et souffrent particulièrement de la polarisation épidémiologique. A l'inverse, dans les campagnes, les plus pauvres n'ont pas encore débuté la transition et souffrent encore majoritairement de mortalité infantile et de maladies infectieuses (Shukla, 2007).

Les divergences épidémiologiques entre les classes sociales sont aussi susceptibles de varier en fonction du sexe et de la position dans le parcours de vie. A Ouagadougou, il a été constaté que les enfants qui vivent dans des bidonvilles meurent avant tout d'infections alors que les principales causes de décès des 15-59 ans sont le sida, les maladies cardiovasculaires et les accidents (Rossier et al., 2016). A Mumbai, l'obésité est particulièrement importante pour les individus entre 35 et 44 ans, en particulier les femmes plus aisées (Kulkarni et al., 2017). Dans les pays occidentaux, la relation est inverse: ce sont les femmes pauvres qui montrent le plus de prévalence pour l'obésité (Kulkarni et al., 2017). Pour une part, la question de la culture entre en jeu, l'obésité étant parfois synonyme d'opulence dans des pays délaissés par le développement. Ainsi, l'Inde cumule à l'heure actuelle un haut taux de malnutrition (risque de maladie et de mortalité élevée) et des signes d'une montée de l'obésité (accroissant les risques de diabètes et de problèmes cardiaques). Cette coexistence est symptomatique du multiple burden of disease (Kulkarni et al., 2017).

décès accidentels) caractéristique des pays économiquement développés. Ce terme permet de souligner l'écart de santé entre plusieurs groupes sociaux (Shukla, 2007). 
Prises globalement, les réponses concernant la santé que les répondants ont évoquées ne laissaient pas transparaître d'importantes inégalités en termes économiques ou de genre. Au regard du multiple burden of disease, est-il possible d'en détecter de nouvelles?

\subsubsection{Les événements de santé rapportés par les habitants de Mumbai, selon le multiple burden of disease}

Afin d'explorer les réponses classées sous l'étiquette générique de "santé», nous avons opté pour une classification plus fine, basée sur le contenu des événements et reprenant les différents problèmes de santé. Ainsi, quatre sous-groupes ont été constitués : les «maladies contagieuses» (telles que la malaria, la fièvre typhoïde, la tuberculose, etc.), les «maladies non-transmissibles» (comme le diabète, les problèmes cardiaques ou rénaux, les hémorroïdes, etc.), les «accidents» et finalement une catégorie «autre» (qui regroupe essentiellement les maladies mentales, l'alcoolisme et les problèmes oculaires $)^{18}$. Précisons que seules les réponses concernant l'individu ont été conservées, dans le but de cerner l'impact sur le parcours de vie du répondant ${ }^{19}$. Cette subdivision des réponses de santé réduit malheureusement considérablement le nombre de changements évoqués dans chaque catégorie : des analyses statistiques poussées ne sont donc pas envisageables et les résultats sont présentés sous forme d'effectifs.

Les figures 7.2 à 7.5 révèlent la prégnance des mentions de maladies non transmissibles parmi les changements récents et les tournants de santé, quel que soit le lieu de résidence. La répartition par classe d'âge du nombre de changements de santé évoqués par les individus de Bandra (cf. figure 7.2) montre avec netteté l'impact de la transition sanitaire à Mumbai, où le fardeau des maladies chroniques pèse dès l'âge de 35-39 ans. En outre, la présence encore marquée des maladies infectieuses parmi les réponses des plus jeunes souligne le poids du multiple burden of disease. A Santa Cruz, les répondants ont mentionné des changements récents de type maladies chroniques dans toutes les classes d'âge, même bien que de manière réduite - parmi les plus jeunes (voir figure 7.3). Autre

18 En suivant la catégorisation proposée par Rossier et al. (2016).

19 Les changements récents de santé sont au nombre de 496, dont 433 concernent l'individu; les tournants de santé sont au nombre de 318, dont 114 concernent l'individu. 
différence conséquente avec Bandra, les maladies infectieuses sont quasiment absentes des réponses.

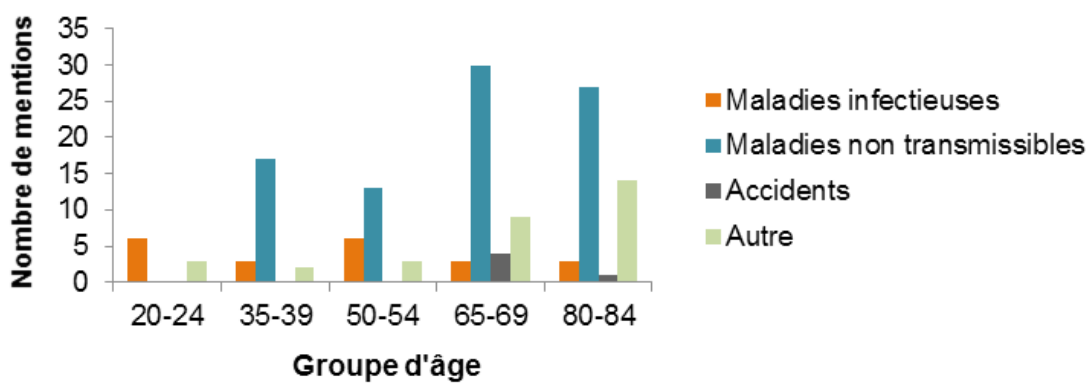

Figure 7.2: les changements récents de santé $(n)$ concernant l'interviewé, selon le type de maladie et le groupe d'âge à Bandra

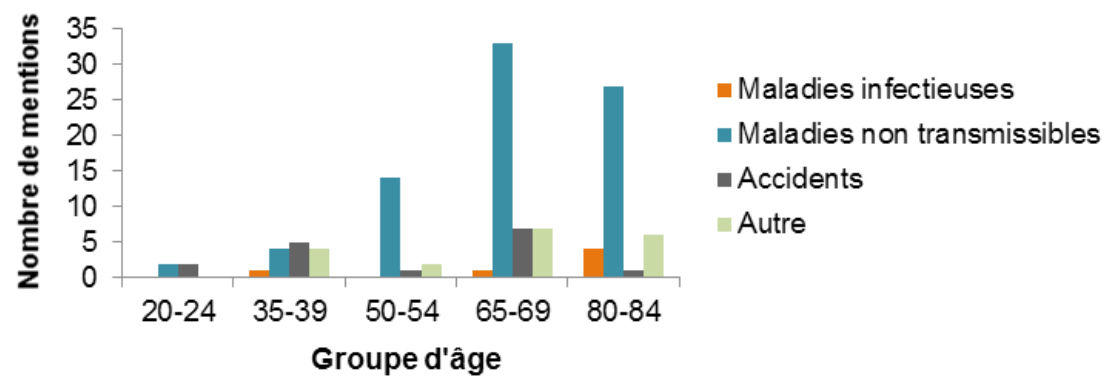

Figure 7.3: les changements récents de santé $(n)$ concernant l'interviewé, selon le type de maladie et le groupe d'âge à Santa Cruz

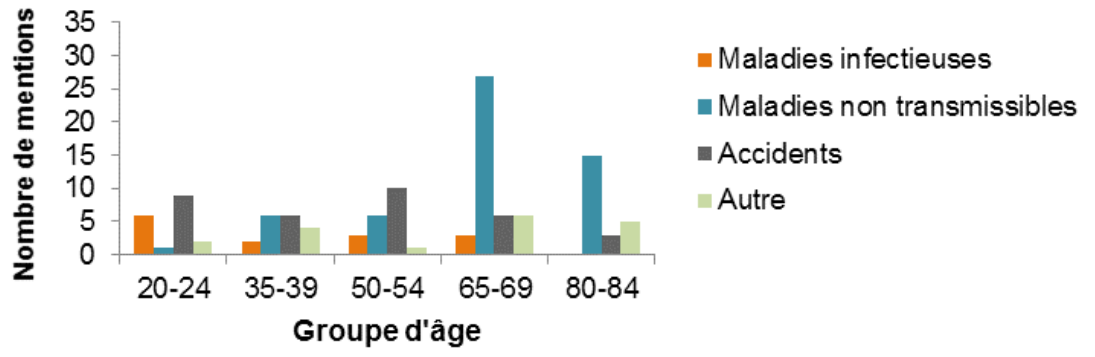

Figure 7.4: les tournants de santé $(n)$ concernant l'interviewé, selon le type de maladie et le groupe d'âge à Bandra 


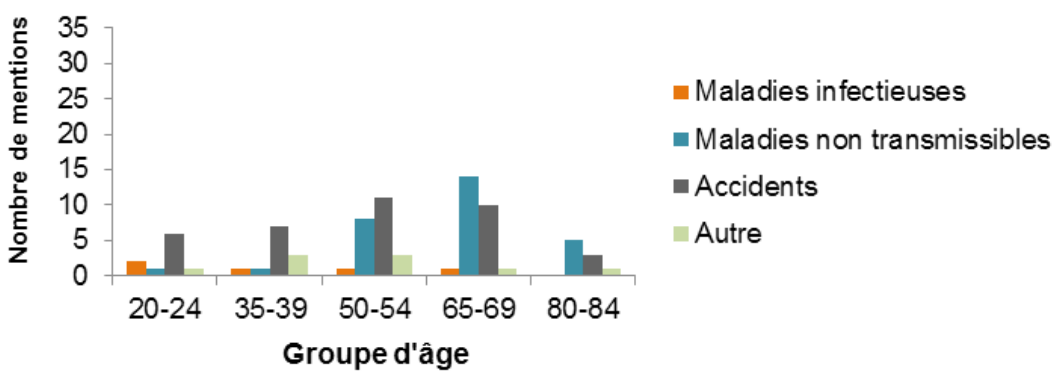

Figure 7.5: les tournants de santé $(n)$ concernant l'interviewé, selon le type de maladie et le groupe d'âge à Santa Cruz

A propos des tournants de santé évoqués à Bandra, la répartition selon les types de maladies souligne à nouveau un multiple burden of disease plus prononcé qu'à Santa Cruz (voir figures 7.4 et 7.5). Les jeunes évoquent ainsi plus de maladies infectieuses que les autres groupes d'âges, qui eux se concentrent sur les accidents et les maladies chroniques. De leur côté, les personnes âgées ne mentionnent pas de maladie infectieuse comme tournant, alors que de nombreux problèmes chroniques de santé (demandant davantage de soins et dont l'impact sur la vie est davantage prolongé) sont cités. Il est aussi possible qu'un effet de sélection soit à l'œuvre : les octogénaires ayant rencontré des maladies infectieuses graves n'ont malheureusement pas survécu ou n'étaient pas aptes à répondre à notre questionnaire.

A Santa Cruz, les citations d'accidents - surtout chez les jeunes et de maladies chroniques (par les 50-54, 65-69 et 80-84 ans) sont les plus présentes parmi les tournants de santé mentionnés. Il est vrai qu'un accident est, plus que nombre de maladies guérissables, susceptible de «casser» un être humain et son parcours, de le mettre significativement en danger et de laisser une marque traumatique en sa mémoire.

Les figures 7.6 à 7.9 exposent les changements récents et les tournants de santé relatifs à l'interviewé, selon le sexe et l'enquête. Concernant les maladies, les divergences sont minimes ; néanmoins, une présence un peu plus marquée des maladies chroniques se constate dans les changements récents évoqués par les femmes des bidonvilles. Par contre, les accidents sont indéniablement plus cités par les répondants de Santa Cruz, par les plus jeunes groupes d'âge et par les hommes dans les deux terrains d'enquête ${ }^{20}$.

20 Anecdotique, quoique: les évocations d'alcoolisme sont essentiellement le fait d'habitants des slums et d'hommes (ou de femmes parlant de leur époux). La santé 


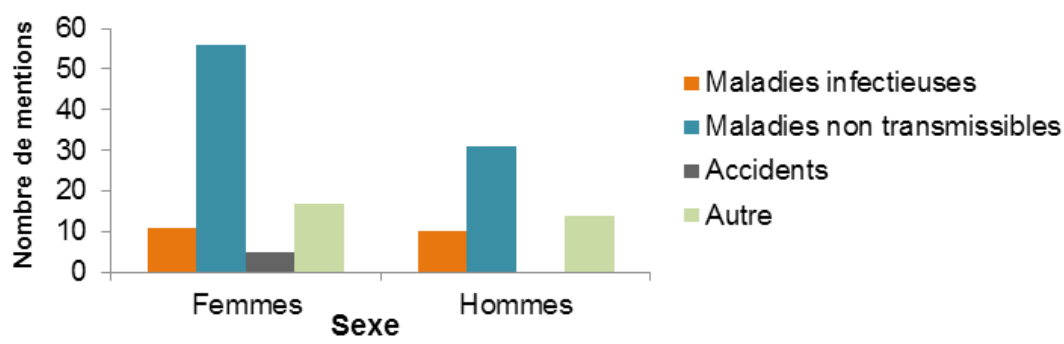

Figure 7.6: les changements récents de santé $(n)$ concernant l'interviewé, selon le type de maladie et le sexe à Bandra

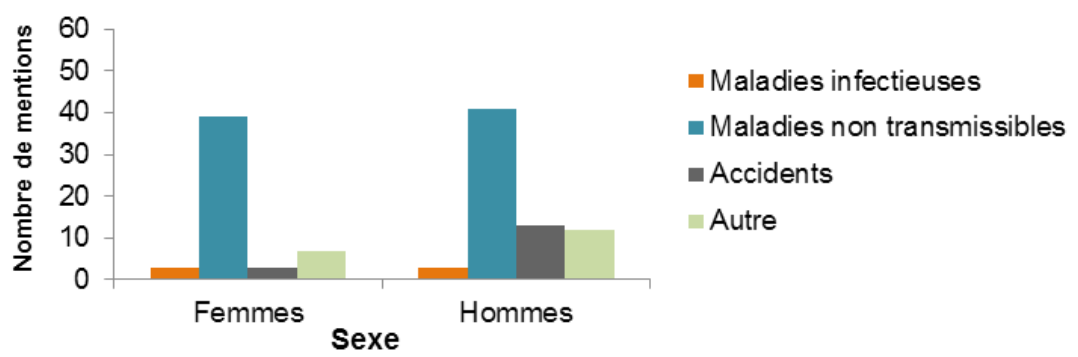

Figure 7.7: les changements récents de santé $(n)$ concernant l'interviewé, selon le type de maladie et le sexe à Santa Cruz

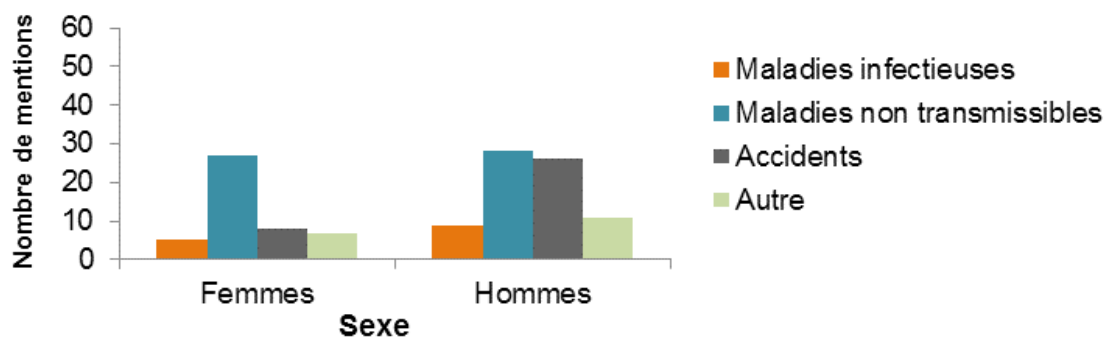

Figure 7.8: les tournants de santé $(n)$ concernant l'interviewé, selon le type de maladie et le sexe à Bandra

reproductive (santé des enfants de moins de 10 ans et des grossesses, pas les accidents mais maladie, malnutrition, diarrhées; la contraception) est pratiquement absente des réponses (5 mentions à Bandra, 3 mentions à Santa Cruz). 


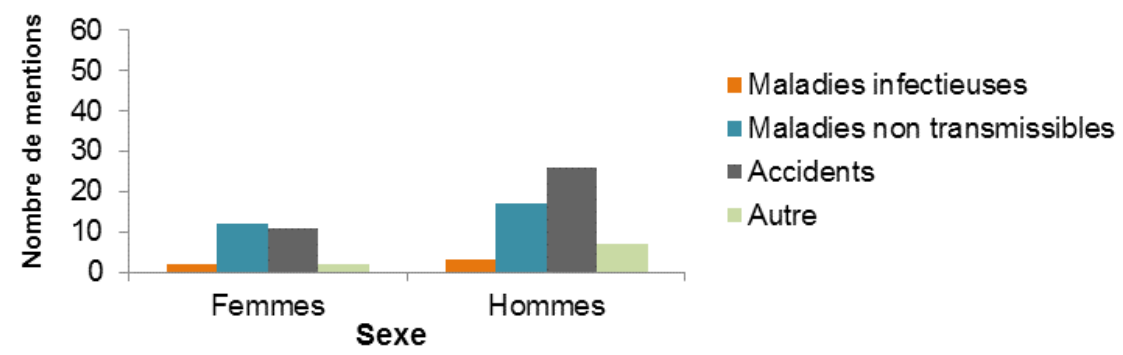

Figure 7.9: les tournants de santé $(n)$ concernant l'interviewé, selon le type de maladie et le sexe à Santa Cruz

\section{$* * *$}

La division selon leur nature des ennuis de santé ayant frappé le répondant (et qu'il a jugé digne de mention) révèle plusieurs constats qui soutiennent l'existence d'un désavantage envers les plus pauvres. En premier lieu, les changements récents montrent l'impact du multiple burden of disease en termes de temporalité et de niveau de vie : les maladies infectieuses sont peu présentes à Santa Cruz, alors qu'à Bandra elles sont évoquées par les plus jeunes. Inversement, les maladies chroniques sont fortement citées dès la trentaine par les répondants tant de Bandra que de Santa Cruz, quoique dans ce dernier cas les 35-39 ans les citent dans une moindre mesure. Ainsi, les maladies infectieuses existent encore pour les plus pauvres alors qu'elles ont disparu pour les classes moyennes inférieures; de plus, les habitants des slums y ajoutent un risque plus précoce de connaître une maladie chronique.

L'inscription historique - générationnelle - de la transition épidémiologique, d'un multiple burden of disease qui survient au niveau agrégé plutôt qu'au niveau individuel (surtout dans les bidonvilles), se constate également dans les tournants de santé, sachant que personne, quasiment, ne cumule de mentions de maladies transmissibles avec des problèmes chroniques ou des accidents ${ }^{21}$. Par contre, le schéma de l'addition des maladies infectieuses et chroniques est à nouveau perceptible dans l'échantillon de Bandra à travers les groupes d'âge, moins parmi les répondants vivant à Santa Cruz.

21 Seuls 35 individus citent deux tournants de santé (23 habitants de Bandra et 12 habitants de Santa Cruz), et trois d'entre eux (résidant à Bandra) citent trois événements santé. 
Par ailleurs, les évocations d'accidents sont plus nombreuses à Santa Cruz, en particulier chez les deux plus jeunes groupes d'âge et les hommes. Les individus résidant dans le quartier de classe moyenne apparaissent protégés des maladies infectieuses mais avec un parcours de vie davantage entravé par la survenue d'une maladie chronique ou d'un accident. Une analyse des raisons données par les individus pour rapporter ces tournants de santé permet de compléter et nuancer ces résultats.

\subsection{Les raisons pour mentionner un tournant de santé : vulnérabilité économique, émotions et description factuelle}

Les analyses des événements de santé ont montré que la vulnérabilité liée aux questions sanitaires n'apparaît pas forcément au premier regard, entre autres parce que leur nature peut être variée (nous l'avons vu concernant les types de maladies). Ainsi, cette dernière partie a pour objectif de détailler les raisons ayant poussé à évoquer des tournants de santé, afin de saisir les implications de ces derniers dans la vie des individus et de leurs proches.

\subsubsection{Trois catégories de raisons au fait de mentionner la santé}

Les motivations à citer des tournants de santé ont été codées selon leur appartenance à trois catégories : (1) le stress économique et lié aux responsabilités familiales ( $n=86)$; (2) le ressenti en termes subjectifs et émotionnels $(n=143)$; (3) les réponses descriptives et factuelles des changements, des symptômes ou des traitements du problème évoqué $(n=47)$.

\section{Economie et famille}

La première catégorie de raisons fait directement ou indirectement référence aux conséquences économiques que crée l'événement de santé ou aux responsabilités familiales qui ne peuvent être remplies. Certaines $(n=28)$ évoquent explicitement l'empêchement de travailler ou le fait de devoir arrêter l'école (à cause de l'ennui de santé ou pour compléter les rentrées d'argent du ménage, amputées par la maladie ou l'accident d'un proche) : 
"My husband was the only earner then"

(femme de 40 ans, Santa Cruz)

"Because I am unable to do job"

(homme de 66 ans, Bandra)

«My studies were interrupted»

(homme de 21 ans, Santa Cruz)

D'autres $(n=44)$ soulignent l'impossibilité d'assumer ses responsabilités familiales ou au contraire les implications morales qui découlent du problème de santé. Ces raisons sont inter-reliées avec les questions économiques, mais soulignent davantage le stress et les difficultés de gestion du ménage :

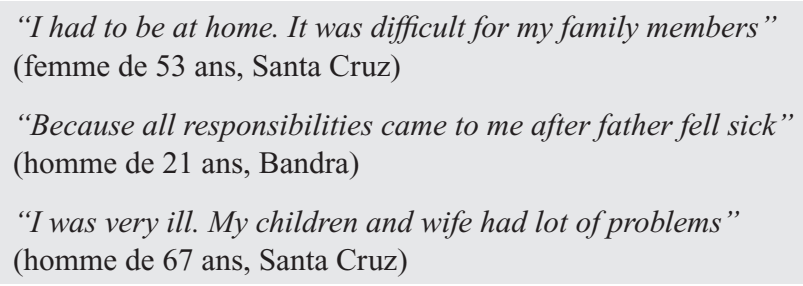

Finalement, toujours au sein de la catégorie économique, quelques raisons $(n=14)$ cernent les frais médicaux et le coût élevé des médicaments qui résultent du traitement du problème de santé :

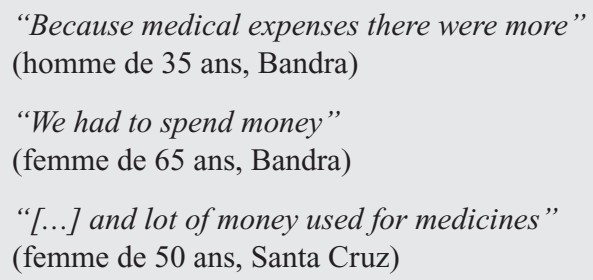

\section{Emotions liées au tournant de santé}

Une partie des explications se rapporte aux émotions perçues par l'individu face à sa maladie ou son accident, constituant la deuxième catégorie. Par définition, les répondants sont des survivants, et ce d'autant plus qu'ils sont âgés. Dès lors, ces émotions sont positives et expriment le soulagement $(n=64)$ : 
"I was saved from death and paralysis"

(homme de 65 ans, Santa Cruz)

"My husband did on time treatment and survived me"

(femme de 37 ans, Bandra)

"She [my daughter] survived from death"

(homme de 70 ans, Bandra)

D'autres $(n=55)$ soulignent des craintes ou la douleur ressentie :

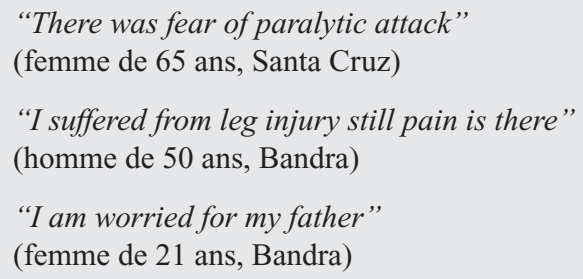

Enfin, dans ce groupe de raisons ont aussi été ajoutées les évocations de la pénibilité qui s'étend dans le temps $(n=24)$ :

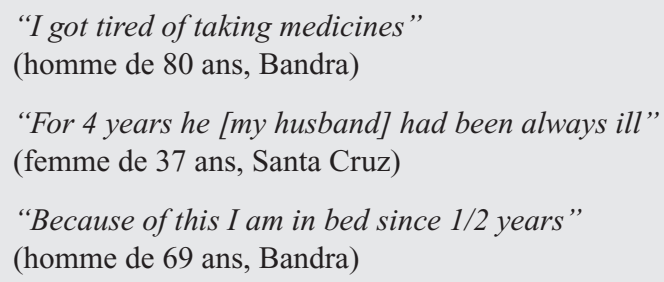

Cette catégorie laisse une large place à la subjectivité, au ressenti des personnes et s'éloigne des conséquences pratiques telles que le premier groupe de raisons le laissait entendre. Ainsi, même face à la maladie ou aux accidents de santé, il est possible de percevoir un aspect positif à la situation (lorsqu'il y a rémission, évidemment). Quittant l'émotionnel et le subjectif, la dernière dimension se focalise sur les aspects objectifs des ennuis médicaux.

\section{Description factuelle du tournant de santé}

La troisième catégorie de raisons correspond aux descriptions précises et factuelles des symptômes, des circonstances, des conséquences ou du traitement des ennuis de santé cités : 
"Right side part of body is not working"

(homme de 65 ans, Santa Cruz)

"Because while walking on road Rickshaw ran over my leg"

(femme de 24 ans, Bandra)

«My leg was burnt»

(femme de 65 ans, Santa Cruz)

\subsubsection{Des raisons communes pour parler de sa santé}

Entre les deux terrains d'enquêtes, peu de divergences apparaissent entre les trois catégories de raisons, à l'exception des événements factuels (22\% à Bandra contre $11 \%$ à Santa Cruz), ce qui traduit sans doute un certain stoïcisme des plus démunis. En ce qui concerne le groupe économie et famille, il représente $28 \%$ des raisons évoquées à Bandra et $35 \%$ de celles apparues à Santa Cruz. Quant aux émotions, elles dominent et correspondent à $50 \%$ des raisons des tournants de santé mentionnés par les répondants des bidonvilles, pour respectivement $54 \%$ de celles du quartier formel.

Entre les classes d'âge, les divergences sont également minimes à Bandra comme à Santa Cruz (voir figures 7.10 et 7.11, qui présentent les effectifs des raisons). Seules différences, par rapport aux autres groupes d'âge, les plus âgés interrogés dans les bidonvilles fournissent plus d'explications émotionnelles que liées à l'économie et aux responsabilités familiales, tout comme les sexagénaires de Santa Cruz. Cela peut s'expliquer parce que ces charges sont passées pour eux à la génération suivante.

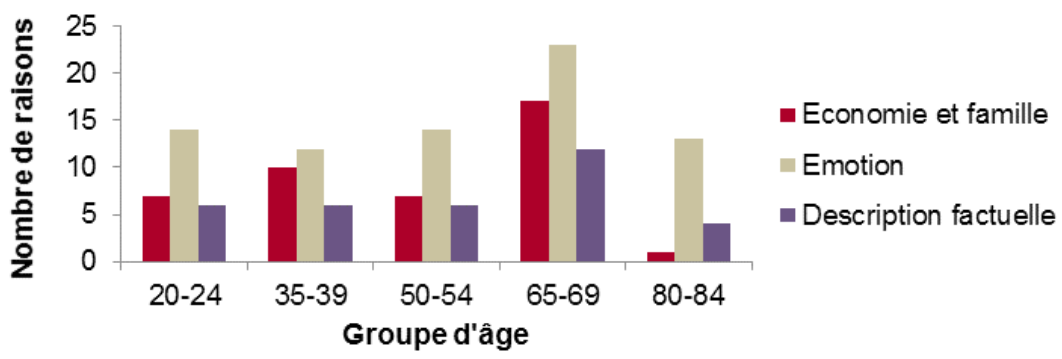

Figure 7.10: les raisons des tournants de santé (n), selon le groupe d'âge, à Bandra 


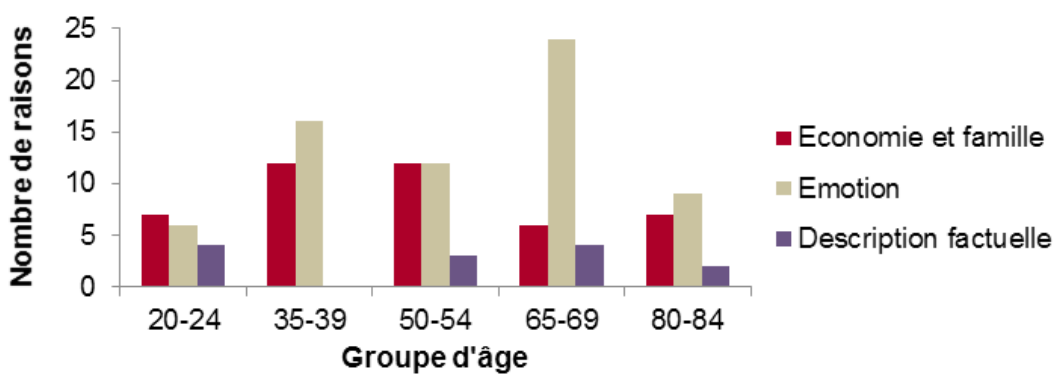

Figure 7.11: les raisons des tournants de santé (n), selon le groupe d'âge, à Santa Cruz

Quelques divergences s'affirment lorsque le sexe du répondant est pris en compte (cf. figures 7.12 et 7.13). En effet, les émotions sont un peu plus présentes parmi les raisons masculines alors que les femmes évoquent quelques explications supplémentaires liées au stress économique et familial. Ce constat est présent dans les deux quartiers. Il peut renvoyer à des attributions genrées de rôles, mais les écarts restent faibles et il convient donc de rester prudent dans l'interprétation.

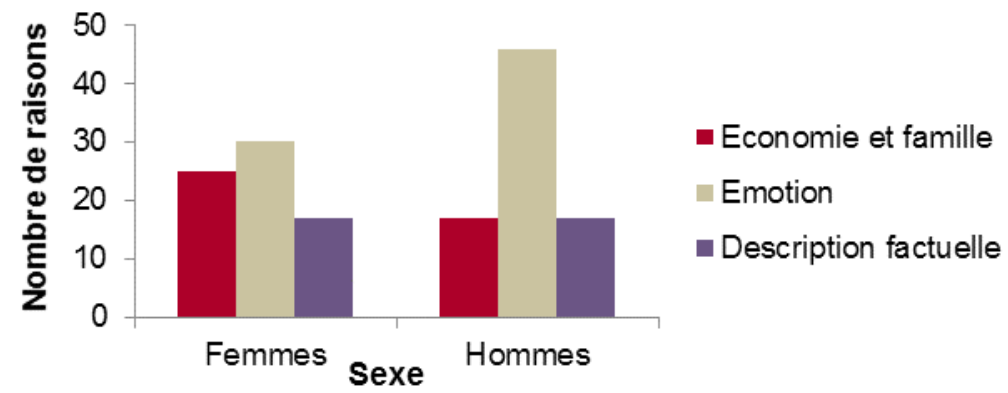

Figure 7.12: les raisons des tournants de santé $(n)$ à Bandra, selon le sexe 


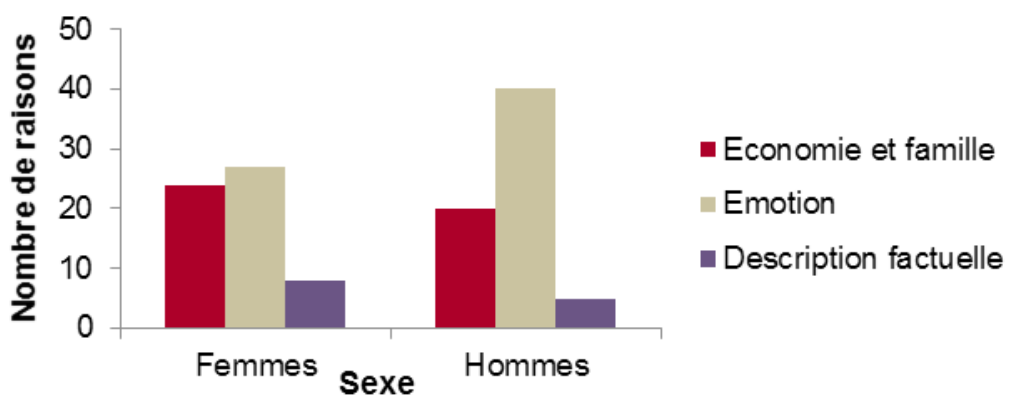

Figure 7.13: les raisons des tournants de santé (n) à Santa Cruz, selon le sexe

Finalement, la répartition des raisons selon les trois catégories et le niveau d'éducation des enquêtés confirme le peu de clivage entre les réponses (cf. figure 7.14). Une légère montée des préoccupations économiques et familiales apparaît avec l'augmentation du niveau économique, alors que les raisons émotionnelles sont plus présentes parmi les raisons des personnes d'éducation primaire et secondaire. Les descriptions factuelles, elles, ne varient guère.

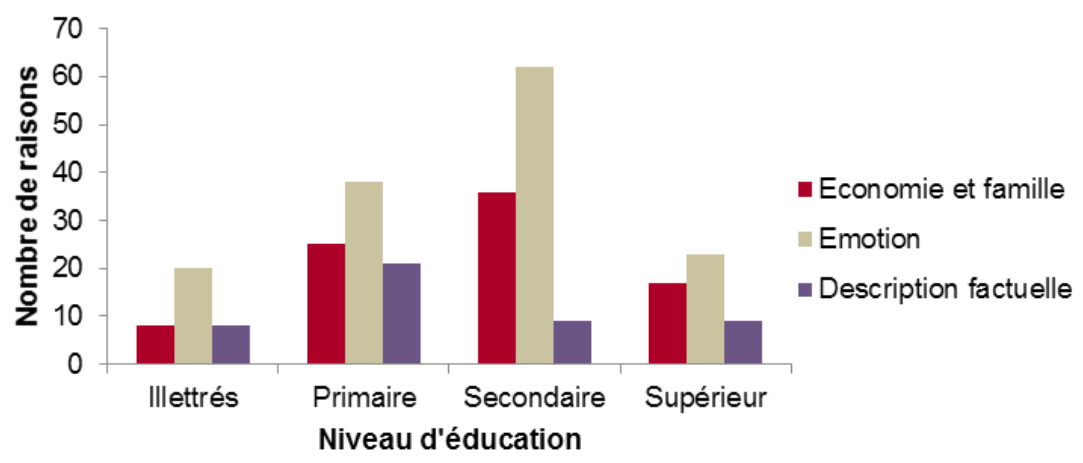

Figure 7.14: les raisons des tournants de santé $(n)$, selon le niveau d'éducation 


\subsection{Conclusion}

Bien que la présence de la santé soit plus grande parmi les événements personnels rapportés par les habitants des bidonvilles, ce résultat est à nuancer : s'il est aisé d'expliquer pourquoi la santé est une composante importante de la mémoire d'une population dans un pays émergent pauvre, il n'en reste pas moins surprenant que les habitants d'un contexte particulier de bidonvilles n'apparaissent pas bien davantage affligés par ce fléau que les résidents de Santa Cruz. Par ailleurs, l'analyse des raisons a montré la présence d'une vulnérabilité issue des pressions que ressentent les individus dans presque tous les groupes d'âge quel que soit le lieu de résidence, par le biais des mentions de difficultés économiques et de responsabilités familiales. Notons malgré tout que les femmes sont davantage soumises à ces pressions, indifféremment de leur niveau socioéconomique.

Des divergences de vulnérabilités se précisent lorsque l'on se penche sur le multiple burden of disease, plus prononcé en ce qui concerne les habitants des slums, même si la population urbaine de classe moyenne affronte également sa part de risques sanitaires. Notamment, c'est le cas des accidents, nettement plus mentionnés par les groupes d'âge jeunes ainsi que par les hommes, sans grande surprise au vu de la littérature récente consacrée à cette thématique. A l'inverse, les maladies non contagieuses apparaissent plus marquantes pour les personnes âgées de 35 ans et plus, et ce particulièrement parmi les femmes et les personnes résidant à Bandra. Chez les trentenaires, ces mentions sont très illustratives du multiple burden of disease, puisqu'elles concernent des individus encore jeunes comparativement aux pays occidentaux.

Nonobstant l'effet de sélection, les sexagénaires et les octogénaires sont plus nombreux à parler de leurs ennuis de santé que les autres groupes, ce qui appuie leur situation de fragilité sans susciter d'étonnement. En outre, les deux classes d'âge les plus âgées citent nettement plus de maladies chroniques qu'infectieuses, ce qui souligne autant la montée de ce type de problème sanitaire dans les pays moins développés économiquement que les difficultés quotidiennes que cela engendre pour les individus.

En Inde, les maladies ont souvent été attribuées à la forte densité de population, aux espaces limités et à la proximité, qui créent des sources de stress importants. Dans les centres urbains, les masures grouillantes de 
saleté et d'ordures, le trafic et la foule sont les stresseurs les plus critiques, suivis par le bruit et la pollution de l'air (Siddiqui \& Pandey, 2003). Le bruit est également un aggravant de morbidité non négligeable, affectant l'écoute, la tension, etc. (Siddiqui \& Pandey, 2003; Vlahov et al., 2007). L'environnement dans lequel les vies se déroulent est donc un facteur crucial dans la compréhension des événements qui marquent l'existence, ce que le chapitre qui vient s'attelle à présenter. 


\title{
Chapitre 8. Histoire et biographies : souvenirs des vulnérabilités environnementales et sociohistoriques
}

\author{
«De façon large, la mémoire collective peut être définie \\ comme la transmission à travers les générations des \\ événements qui ont modifié de façon importante une \\ société»
}

(Deschamps, Paez, \& Pennebaker, 2002, p. 245)

«L'existence de l'individu et l'histoire de la société ne se comprennent qu'ensemble», souligne C. Wright Mills (1997 [1959], p.5), qui précise que l'imagination sociologique doit permettre aux chercheurs de "saisir histoire et biographie, et les rapports qu'elles entretiennent à l'intérieur de la société» (p.8). Dans ce travail réflexif, la notion de cohorte de naissance est capitale: c'est elle qui permet d'ancrer les vies dans l'histoire (cf. section 2.3.1) (Ryder, 1965; Settersten, 1999). Dans les études du parcours de vie, Glen Elder a été l'un des tout premiers à mettre en évidence les effets du contexte économique et social sur la destinée des membres de deux cohortes américaines, au travers d'une étude longitudinale dont les résultats ont été présentés dans son livre fameux, Children of the Great Depression (1999 [1974]). Il a démontré qu'un changement économique important (la dépression économique de 1929 aux Etats-Unis) a eu des influences diverses sur les trajectoires des individus en fonction de leur âge au moment de l'événement, façonnant ainsi différentes affiliations générationnelles. Au cours des dernières décennies, d'autres recherches ont pris le relais et creusé ce type d'interrelations (voir Guichard 2015, pour un bilan). Ce chapitre a pour objectif de questionner ce savoir occidentalo-centré, en interrogeant la perception historique d'habitantes et d'habitants de Mumbai.

Nous commencerons en posant un cadre théorique qui considère notamment comment une mémoire historique partagée est susceptible de transformer des cohortes de naissances en générations sociohistoriques. Nous soulignerons l'importance de l'expérience vécue, de près ou de loin, dans la formation des capitaux mémoriels, et présenterons le concept 
d'âge critique ou de période critique. L'apport de la psychologie sociale ne sera pas négligé. Les chercheurs issus de ce domaine ont également travaillé sur la représentation des événements historiques chez les individus. Ils montrent que cette représentation est globale puisque présente dans tous les pays observés, avec une vision essentiellement eurocentrique, nuancée par une importante mémoire nationale. De plus, ils soulignent que les événements rappelés sont principalement violents, politiques, avec une signification négative. Sous ces conditions et dans un contexte culturel précis (une mégapole indienne à l'orée de la mondialisation) et de vulnérabilités multiples (environnementale, sociale, économique), quels seront les aspects historiques qui ressortent dans les souvenirs des répondants?

L'analyse empirique, appuyée sur nos deux enquêtes, apporte des réponses d'abord en termes de contenus et de localisation temporelle des souvenirs qui composent la mémoire sociohistorique d'habitants des bidonvilles et d'un quartier de classes moyennes inférieures. Les analyses tant quantitatives que qualitatives éclairent la terrible vulnérabilité des premiers aux désastres naturels, en raisons même de leurs conditions de vie dans un espace urbain chaotique. La vulnérabilité exprimée est aussi, de plus en plus, celle des femmes, qui ressort cette fois surtout au sein des classes moyennes. Une autre trame de lecture se dégage dans l'héritage long, sanglant et renouvelé, qui lie la colonisation à la globalisation, de manière faussement paradoxale, à travers un nationalisme qui rejette les minorités, en particulier musulmanes.

\subsection{Mémoire collective : construction générationnelle et représentation partagée de l'histoire}

L'appartenance à une cohorte de naissance (soit l'année de naissance d'une personne) représente le premier point d'ancrage dans l'histoire d'un individu (cf. chapitre 2), initiant la période historique qu'il parcourra au cours de son existence (Heinz \& Krüger, 2001; Ryder, 1965; Settersten \& Martin, 2002). Le groupe ainsi constitué rencontrera ensemble et au même âge des expériences historiques (guerres, crises économiques, désastres naturels, etc.) majeures et mineures, locales et mondiales, qui marqueront 
son époque (Elder, 1994). Toutefois, ces cohortes ne sont pas homogènes; au contraire, les facteurs sociaux de genre, de classe, de religion, etc. impliquent une exposition différente des individus aux divers changements et événements historiques (Elder \& George, 2016; Hareven, 1996).

Un second ancrage provient de l'appartenance générationnelle. Ici, le terme générationnel perd sa signification familiale (cf. chapitre 6) pour correspondre à des individus nés plus ou moins dans la même période et possédant des caractéristiques communes (cf. chapitre 2), avant tout celle d'avoir rencontré, au cours de leur vie, un événement historique clé ayant marqué leur trajectoire (Alwin \& McCammon, 2007, 2004; Devriese, 1989; Kertzer, 1983; Mannheim, 1990). Ce concept de génération a été réfléchi depuis de nombreuses décennies et porte en lui l'idée de l'existence - lorsque les circonstances s'y prêtent - d'une conscience historique de groupe, d'une unité autour d'un changement, qu'il soit social, économique, politique ou technologique (Alwin \& McCammon, 2007; Chauvel, 2000; Settersten, 1999).

\section{L'apport de Karl Mannheim, ou la question de l'existence de généra- tions sociohistoriques}

A partir de quand une cohorte de naissance devient-elle une génération ? Mannheim (1990 [1928]) est le premier sociologue à s'être penché sur la question voilà près d'un siècle, soulevant l'idée d'une prise de conscience générationnelle en opposition à une simple contemporanéité (Eyerman \& Turner, 1998). Une période contient plusieurs générations à la fois, voire des générations plurielles (Devriese, 1989), et lorsqu'un événement sociohistorique survient (que ce soit une guerre, une crise économique, un désastre naturel,...), il frappe tous les groupes d'âge à la fois; toutefois, chacun de ces groupes se trouve à une position différente de son parcours (Mauger, 2015). Une stratification de l'expérience se produit alors, la différence subjective du poids de l'événement en fonction de l'âge participant à révéler une identité générationnelle chez les jeunes (Alwin \& McCammon, 2007). En particulier, Mannheim rappelle que les jeunes adultes vivent généralement cet événement pour la première fois de leur existence: l'âge ne définit pas la génération, c'est l'expérience qui s'en charge (Mannheim, 1990 [1928]).

Ainsi, la génération se construit sous le coup d'un événement précoce, dont les conséquences se répercutent sur les autres âges de la vie. La sortie de l'adolescence et l'entrée dans l'âge adulte, situées par Mannheim 
(1990 [1928]) entre 17 et 25 ans mais que l'on peut sans doute allonger quelque peu aujourd'hui (Martenot \& Cavalli, 2014), sont des moments cruciaux dans la construction des générations, malléables ou vulnérables face au changement (Settersten, 1999). Le point central de la réflexion porte sur le fait d'être jeune, en phase de socialisation et d'adaptation aux rôles de l'âge adulte lorsque survient l'événement (Chauvel, 2000).

L'effet de cette socialisation - unificateur pour la classe d'âge concernée, influençant même les individus n'ayant pas été affectés personnellement par le changement - est durable mais différent pour chaque groupe (Mauger, 2015). Ainsi, les membres d'une génération sont cimentés par une culture, un habitus et des comportements communs, en excluant par ce processus les autres cohortes (Bourdieu, 1994; Eyerman \& Turner, 1998). Les fractures intergénérationnelles et les inégalités sociales ressortent alors, à la manière des classes sociales marxistes qui se révèlent avec les tensions et la lutte interclasses.

Par ailleurs, la génération réalise une jonction entre mémoire collective et histoire (Attias-Donfut, 1988; Eyerman \& Turner, 1998). En plus d'être le témoin d'une époque, la mémoire collective ne vit que par le groupe qui s'y raccroche, y puisant son identité (Halbwachs, 1997 [1950], 2004 [1925]). Elle transcende parfois les générations, mais chacune d'elles possède des souvenirs propres qui participent à la définir (Attias-Donfut, 1988).

Ce qui est implicite dans ce qui précède, est que mémoire et histoire ne sont pas redondantes mais entretiennent une relation dialectique (Nora, 1989). Si l'histoire repose sur des faits écrits et reconnus du passé, la mémoire évoque l'aspect social, ou vivant, de celui-ci (Halbwachs, 1997 [1950]). De plus, la mémoire ne cherche pas à s'affirmer comme une source impartiale et exhaustive de savoir, ce que l'histoire tend à être. Suivant ces considérations, la mémoire collective (ou mémoire sociale) représente une cohésion populaire autour de la compréhension du passé, de souvenirs partagés au sein de groupes plus ou moins larges (comme la nation ou la famille), transmis oralement, mais qui peuvent aussi être affichés officiellement au travers de commémorations, de musées,... participant à construire une identité commune (Assmann \& Czaplicka, 1995; Moscovici, 2000; Ricoeur, 2003). Cette reconstruction du passé s'effectue à la demande du présent, afin de permettre la stabilisation sociale (Lalive d'Epinay et al., 2008). 
Cette mémoire collective a été principalement étudiée selon deux axes, celui de l'âge au moment des souvenirs (en sociologie et en psychologie notamment) et celui de la représentation de l'histoire (autrement dit le contenu des souvenirs, ayant intéressé avant tout les psychologues sociaux).

\section{Mémoire collective (1): l'impact de l'âge sur les souvenirs historiques}

Les apports théoriques sur la mémoire collective proviennent de différentes disciplines. Un premier ensemble de recherches d'ordre sociologique est dû à Howard Schuman et ses collègues, qui ont effectué des études systématiques sur les mémoires collectives contemporaines (Schuman, Akiyama, \& Knaüper, 1998; Schuman \& Corning, 2012; Schuman \& Rodgers, 2004; Schuman \& Scott, 1989; Scott \& Zac, 1993), articulant la perception des événements et des changements aux notions de parcours de vie et de génération ${ }^{1}$. Ils mettent ainsi en évidence des différences dans la perception de l'histoire en définissant un «âge critique» (ou «période critique»), situé pendant l'adolescence et au cours de l'entrée dans la vie adulte, durant lequel les événements ont plus d'impact sur la mémoire des individus. Coïncidant avec le moment de construction des générations selon Mannheim (1990 [1928]), les souvenirs de cette époque inspirent la vision ultérieure du monde et sont déterminants pour la formation d'une conscience historique.

Plusieurs courants de chercheurs parviennent indépendamment à cette conclusion. Outre les sociologues précités, des travaux portant sur la formation des générations politiques (Jennings \& Zhang, 2005; Jennings, 1996; Sears \& Valentino, 1997) ainsi que la littérature psychologique se focalisant sur la distribution des souvenirs au cours du développement (Berntsen \& Rubin, 2004; Birren \& Schroots, 2006; Rubin, 1995) le soulignent ${ }^{2}$.

1 La question posée lors de ces études a été formulée de manière explicitement subjective: il est demandé à des personnes âgées de 18 ans et plus de considérer le dernier demi-siècle et de mentionner les événements ou changements, nationaux ou mondiaux, qui ont été particulièrement importants à leurs yeux. Une première enquête a été menée en 1985 aux Etats-Unis (Schuman \& Scott, 1989) ; elle a ensuite connu plusieurs prolongements, permettant des comparaisons internationales (Schuman, Akiyama, \& Knaüper, 1998; Scott \& Zac, 1993) et dans le temps (Schuman \& Corning, 2012; Schuman \& Rodgers, 2004).

2 En psychologie et psychologie sociale, plusieurs explications ont été avancées pour rendre compte de ce phénomène (Draaisma, 2008 [2001]; Jansari \& Parkin, 1996). 
La position occupée par les individus dans leur parcours vie, à elle seule, ne suffit cependant pas à expliquer le choix des changements marquants. Ainsi, la survenue d'un nouveau fait historique peut condamner à l'oubli (au moins temporairement) des événements plus anciens ou au contraire en renforcer la remémoration (Schuman \& Corning, 2012; Schuman \& Rodgers, 2004) 3 .

Du point de vue de la sociologie du parcours de vie, un certain nombre de travaux sur la perception des changements sociohistoriques a montré que l'impact de l'âge sur les souvenirs rappelés n'est pas automatique, notamment lorsqu'un événement tragique - telle une dictature ou une grave catastrophe naturelle - affecte la mémoire de tous les individus l'ayant vécu, quel que soit leur âge à ce moment-là (Guichard \& Henríquez, 2011; Lalive d'Epinay \& Cavalli, 2009; Lalive d'Epinay et al., 2008; Martenot \& Cavalli, 2014; Oddone \& Lynch, 2008). En outre, se focalisant sur la Chine, une étude a démontré l'absence d'effet d'âge ou de génération dans le cas d'événements historiques s'étant étendus dans le temps (dans ce cas, les programmes de réforme économique chinois instaurés en 1978) (Jennings \& Zhang, 2005). Des événements à teneur traumatique ou de longue durée peuvent donc dépasser les schémas classiques de rappel ainsi

Selon certains auteurs, la propension à citer des événements survenus au moment de l'entrée dans la vie adulte serait due à une plus grande aptitude sur le plan neurophysiologique à emmagasiner des souvenirs vers l'âge de vingt ans, lorsque le développement du cerveau atteint son optimum (Janssen, Murre, \& Meeter, 2008; Rubin, Schulkind, \& Rahhal, 1999). Une autre explication met en avant la primauté de nombre de changements vécus pendant l'âge critique, les individus étant davantage marqués par les événements rencontrés pour la première fois: le premier partenaire, le premier travail, un voyage initiatique, mais aussi les premières confrontations à l'histoire, tout comme les engagements civiques et politiques (Berntsen \& Rubin, 2002; Conway \& Haque, 1999; Schrauf \& Rubin, 1998). Dans une interprétation ultérieure, l'accent a été mis sur la rétention préférentielle des événements dans la période de formation et de consolidation d'une identité adulte (Conway et al., 2005). Et le début de la vie adulte représente la période critique pour la formation de la personnalité et de l'identité. Un individu d'âge mur se souviendra alors surtout de ces événements, qui ont fait de lui ce qu'il est devenu (Draaisma, 2008 [2001]).

3 Ayant interviewé près de 600 personnes à deux reprises - d'abord à la fin de l'an 2000, puis début 2002 - Howard Schuman et Willard Rodgers (2004) ont pu constater que des conflits tels que la Seconde Guerre mondiale ou la guerre du Vietnam sont plus cités après les attentats du 11 Septembre 2011, tandis que la fin du communisme ou les progrès liés à l'informatique le sont beaucoup moins. 
que les générations, pour rassembler plus largement les individus autour du souvenir, indépendamment de leur âge.

Finalement, une étude comparative entre l'Allemagne et le Japon a établi une divergence de rappel entre les événements anciens (remontant jusqu'à 50 ans avant la passation de l'enquête) et les événements récents (Schuman et al., 1998). Majoritairement, les événements anciens ont été expérimentés personnellement, d'où leur ancrage de longue haleine dans la mémoire des répondants. Inversement, les événements récents n'ont pas tous été vécus et, dans ces cas, le rappel a surtout été stimulé par les médias. Cette dernière situation montre également une diminution de l'effet d'âge ou de génération, puisque tout l'échantillon a été exposé similairement aux médias. La présence médiatique est démontrée comme ayant un impact large sur la mémoire collective portée par les individus (Deschamps, Paez, \& Pennebaker, 2002; Schuman et al., 1998).

\section{Mémoire collective (2): des représentations partagées de l'histoire}

Un second ensemble d'études issues de la psychologie sociale s'est davantage focalisé sur le contenu historique de la mémoire collective, notion saisie dans la lignée de Maurice Halbwachs (1997 [1950]). Les résultats provenant de divers pays font état de l'existence d'une remémoration essentiellement eurocentrique (Deschamps, Paez, \& Pennebaker, 2001; Liu et al., 2009; Liu et al., 2012; Paez \& Liu, 2012; Pennebaker, Paez, \& Deschamps, 2006; Pennebaker, Paez, \& Rimé, 1997; Techio et al., 2010); cela étant, l'importance des événements nationaux ou régionaux tend à être surestimée par les individus de tous les pays (Liu et al., 2012; Liu et al., 2005; Techio et al., 2010). Ils soulignent également la prévalence des événements violents et politiques, dont la connotation est majoritairement négative (Techio et al., 2010). Ce constat est appuyé par des sociologues s'intéressant à la mémoire historique collective et nationale dans divers pays (Bergeron, 2015; Guichard \& Henríquez, 2011; Lalive d'Epinay et al., 2008; Martenot \& Cavalli, 2014; Oddone \& Lynch, 2008).

Toutefois, l'évolution globale de l'histoire est généralement perçue comme positive, en opposition à l'image fréquente d'un regret du passé (illustré par la maxime populaire «c'était mieux avant») (Techio et al., 2010). Par ailleurs, il ressort de plusieurs enquêtes un effet de récence, indiquant que les événements surgis durant les dernières années sont interprétés comme plus importants (Liu et al., 2009; Pennebaker et al., 2006; Techio et al., 2010). Corollaire de cet effet de récence, les turbulences plus 
anciennes de l'histoire qui perdurent dans les mémoires représentent donc de profonds marqueurs historiques.

Une part considérable de la mémoire sociale est laissée aux conflits (violents), aux guerres d'indépendance et aux événements fondateurs de l'identité nationale (qui permettent de se définir soi-même versus les autres), généralement ravivés par les institutions à travers les programmes d'enseignement, par les médias et les images choc qu'ils diffusent, en particulier lors de divers éléments commémoratifs (Deschamps et al., 2002). Si leur souvenirs ressort dans la mémoire sociale, cela peut aussi s'expliquer par un effet de rappel, activé par les conflits plus récents qui, de manière dialectique, reposent sur les idéologies dérivant des anciens affrontements (Liu \& László, 2007; Paez \& Liu, 2009). Enfin, une vision glorifiée de la nation est généralement portée par ces souvenirs belliqueux (Liu \& László, 2007; Paez \& Liu, 2009) ${ }^{4}$. Certains conflits sont ainsi sciemment maintenus dans la mémoire collective. La réactivation est ensuite facilement obtenue par de simples piqûres de rappel.

Plus récemment, la possibilité d'une extension de la mémoire nationale vers une mémoire «transnationale» ou transfrontalière a aussi été abordée en sociologie (Lalive d'Epinay, 2012). Ce phénomène est une conséquence culturelle des transformations liées à la globalisation, parmi lesquelles les migrations internationales et les nouvelles formes de communication, auxquelles l'imaginaire national s'adapte (Anderson, 2002; Appadurai, 2005). Selon ces chercheurs, dans une perspective de temps long, dès les débuts du capitalisme, la conscience de classe a incorporé l'économie dans sa mémoire collective; cette conscience a progressivement été couverte par une mémoire de portée nationale (avec la montée des Etats Nations et la fin du colonialisme), puis la mondialisation des échanges (financiers, de biens et de personnes) de la fin du $20^{\text {ème }}$ siècle a provoqué une convergence des imaginaires parallèlement à une conservation des particularités culturelles (Appadurai, 2005; Lukacs, 1974 [1960]). $* * *$

4 Ce faisant, un aspect «moral» de la mémoire ressort, participant à développer l'identité de groupe (Halbwachs, 1997 [1950]) et introduisant un biais de positivité: le rappel des martyrs, des héros sera plus vivace, les guerres «justes» davantage que les guerres controversées (par exemple la Deuxième Guerre mondiale plutôt que la guerre du Vietnam aux Etats-Unis) (Deschamps et al., 2002). Une forme de légitimation de ces violences donc même si, malgré tout, elles conservent une signification négative (Paez \& Liu, 2012). 
La mémoire collective peut être présentée comme une courroie de transmission entre les générations, servant à transmettre le souvenir des changements et des événements sociohistoriques qui ont modifié la société (Deschamps et al., 2002). Elle forge l'identité sociale des individus en construisant un référent commun, qui s'appuie cependant sur les rôles sociaux de genre, d'ethnie, d'âge, etc. (Assmann \& Czaplicka, 1995; Deschamps et al., 2002; Pennebaker et al., 1997). C'est pourquoi, des divergences mémorielles ont été observées selon le sexe: les hommes se mettent au centre des changements rappelés et évoquent fréquemment des événements publics (économie, progrès, etc.), alors que les femmes élargissent les souvenirs sociohistoriques à la collectivité (par exemple, elles emploient plus souvent le pronom «nous») et mentionnent davantage de mouvements sociaux, de maladies, de désastres naturels, etc. (Deschamps et al., 2002).

Qu'elles portent sur les effets d'âge et de génération ou sur les représentations partagées de l'histoire, les observations empiriques de la mémoire collective sont majoritairement concentrées dans des pays issus de la culture occidentale. Dans l'une des études qui sort de cet espace, se focalisant sur le Japon, des dissemblances sont apparues au regard des résultats occidentaux ou anglo-saxons (Deschamps et al., 2002). Alors qu'une tonalité positive des changements historiques de long terme est constatée dans l'espace atlantique, la situation est plus nuancée au Japon, où cette vision peut aller jusqu'à être résolument négative. En Inde, une étude passée en 2005 dans trois grandes villes du pays (Mumbai, Chennai et Hyderabad), interrogeant des adultes de différents âges, montre également des variations entre âge et sexe, les femmes et les âgés ayant une mémoire aux tonalités plus sombres (Lani-Bayle \& Mallet, 2006). Néanmoins, cette enquête conforte certains résultats observés ailleurs: les événements sociohistoriques rappelés sont avant tout nationaux (victoire de l'Inde au cricket, élection du Premier Ministre, conflits avec les pays voisins, catastrophes naturelles,...) et, dans une seconde mesure, internationaux (11 Septembre, guerre en Bosnie, etc.); les événements violents sont surreprésentés (tsunami, tremblement de terre et inondations, mais aussi les guerres, les attaques terroristes sur le sol indien et les conflits entre les religions) (Lani-Bayle \& Mallet, 2006).

Les castes indiennes forment historiquement les fondements de la culture populaire, de l'identité collective et d'une mémoire partagée, dépassant en cela les différences entre cohortes, générations et classes sociales (Heuzé, 1991). Avec la modernisation et les changements sociaux induits 
par l'ouverture économique, il y a fort à parier que ces processus se modifient et laissent apparaitre une ou des ruptures générationnelles (Settersten $\&$ Martin, 2002). La rapidité des mutations socioéconomiques et le développement de l'accès à la formation dans l'Inde urbaine, elles aussi créatrices de fossés générationnels, ne font que renforcer ce pronostic (Settersten \& Martin, 2002). Pour saisir cette complexité, il convient de se tourner vers l'empirie pour identifier les événements qui ont scandé l'existence des participantes et participants à nos deux enquêtes. Leur nature s'avère variable.

\subsection{La perception des moments sociohistoriques marquants à Mumbai}

Le troisième volet du questionnaire passé à Bandra et Santa Cruz interroge les individus sur leur perception des événements majeurs de l'histoire survenus au cours de leur vie. Après un premier aperçu des réponses données, de leur répartition selon l'âge et le lieu de résidence du répondant, nous nous focalisons sur les années auxquelles ces moments correspondent. Les données corroborent-elles le constat d'une mémoire collective différenciée selon les groupes d'âge, estompant les facteurs supplémentaires comme le sexe ou le niveau de vie des répondants? Au contraire, certains moments de l'histoire indienne contiennent-ils davantage de souvenirs et sont-ils susceptibles d'affecter durablement tous ceux qui les ont connus de leur vivant?

\subsubsection{Des événements proches localement et temporellement}

Le tableau 8.1 présente le pourcentage d'individus ayant mentionné au moins une fois les différents domaines de changements sociohistoriques. Etant donné que chaque personne pouvait donner jusqu'à quatre éléments de réponse, l'addition de ces pourcentages excède 100\% (à la différence des tableaux 8.2 et 8.3 qui, eux, exposent les pourcentages de changements et dont le total atteint $100 \%$ ).

Bien que les mêmes domaines principaux aient été mentionnés dans les deux enquêtes, quelques écarts se remarquent. Les individus interrogés à Bandra sont plus nombreux à s'être souvenus de catastrophes, des attentats 
et des pogroms entre hindous et musulmans, ainsi que d'événements «mineurs» survenus dans les quartiers proches de leur lieu de résidence. A Santa Cruz, les répondants ont été davantage à se rappeler d'événements internationaux, concernant l'économie indienne, des guerres et des questions politiques (élections, etc.), ainsi que d'autres événements affectant toute l'Inde (avant tout les violences contre les femmes).

Sans ambages, les catastrophes constituent le principal souvenir commun quel que soit leur lieu de résidence : $60 \%$ des habitants des bidonvilles et $42 \%$ des résidents de Santa Cruz mentionnent au moins une fois un tel événement. Ces chiffres impressionnants illustrent crument à la fois le vécu et le ressenti d'une vulnérabilité environnementale lourde. En particulier, ce taux de rappel concerne l'inondation qui eut lieu en 2005 à Mumbai $(57 \%$ des individus de Bandra et 19\% des habitants de Santa Cruz). Bien que les répondants de Santa Cruz se soient moins focalisés autour de cet événement de 2005, les catastrophes demeurent importantes dans leur mémoire ; ainsi évoquent-ils plus que leurs pairs des bidonvilles d'autres événements de ce type survenus ailleurs en Inde ou dans le monde, qu'ils n'ont pas tous vécu directement mais qui n'en font pas moins partie de leur capital mémoriel.

Ce raz-de-marée mémoriel à propos de l'inondation qui eut lieu dans le Maharastra le 26 juillet 2005 s'explique aisément par le caractère tragique de la catastrophe. Durant plusieurs jours, des inondations ont paralysé diverses zones de l'Etat du Maharastra, y compris sa capitale. A l'origine, une mousson très abondante s'est abattue durant une journée et n'a pu être jugulée, faute de moyens adaptés de drainage. A Mumbai, certaines parties de la ville ont été recouvertes de 5 mètres d'eau. Certains quartiers, et avant tout les espaces vulnérables et les environnements précaires, furent détruits, des cahutes emportées et la vie des habitants pauvres logeant dans ces bidonvilles ou à même le trottoir dangereusement menacée. Très concrètement, plus d'un millier de victimes ont été recensées. Par ailleurs, le réseau de transport fut bloqué, l'électricité et les réseaux téléphoniques coupés et des milliers de personnes restèrent bloquées loin de leur foyer pendant plusieurs heures, voire des jours entiers, sans nouvelles de leurs proches, alors que des résurgences de maladies pourtant disparues se déclaraient dans les espaces dont l'insalubrité structurelle avait été encore aggravée par l'inondation (Bhagat, Guha, \& Chattopadhyay, 2006; McFarlane, 2012). Les données quantitatives attestent d'ores-et-déjà que les survivants en ont été profondément traumatisés. 
Tableau 8.1: les individus (\%) mentionnant au moins une fois les principaux domaines de changements sociohistoriques, selon le lieu de résidence

\begin{tabular}{lcc}
\hline Domaine & Bandra & Santa Cruz \\
\hline Catastrophes & $\mathbf{6 0}$ & $\mathbf{4 2}$ \\
Inondation de Mumbai (2005) & 57 & 19 \\
\hline Evénements mondiaux & $\mathbf{4}$ & $\mathbf{1 1}$ \\
Changements économiques & $\mathbf{5}$ & $\mathbf{7}$ \\
\hline Guerres et politique indiennes & $\mathbf{1 1}$ & $\mathbf{1 6}$ \\
\hline Attentats et émeutes en Inde & $\mathbf{5 1}$ & $\mathbf{2 4}$ \\
$\quad$ Emeutes à Mumbai (1992-93) & 22 & 4 \\
$\quad$ Attentats à Mumbai (2006) & 8 & 6 \\
$\quad$ Attentats à Mumbai (2008) & 13 & 9 \\
\hline Evénements du quartier (Mumbai) & $\mathbf{1 6}$ & $\mathbf{1 0}$ \\
Autres événements en Inde & $\mathbf{1 1}$ & $\mathbf{2 5}$ \\
$\quad$ Viols et violences contre les femmes & 1 & 19 \\
\hline
\end{tabular}

A un autre extrême du spectre mémoriel, les individus ayant mentionné au moins une fois des événements mondiaux (tels que le 11 Septembre, la Seconde Guerre mondiale, la conquête de l'espace, des mobilisations sociales comme mai 68 , l'écologie et le climat, la misère et les épidémies, ou encore le progrès dans les technologies d'information) atteignent 11\% parmi les habitants de Santa Cruz (contre seulement 4\% des répondants de Bandra). Somme toute, ce chiffre reste faible comparativement aux résultats observés dans d'autres enquêtes couvrant l'Europe et les Amériques (Bergeron, 2015; Lalive d'Epinay \& Cavalli, 2009; Lalive d'Epinay et al., 2008; Oddone \& Lynch, 2008; Schuman et al., 1998). Les changements économiques - cités par une même proportion de personnes dans les deux terrains d'enquête à Mumbai - ne peuvent être ajoutés à ces éléments internationaux, puisque la quasi-totalité de ceux-ci se rapportent à l'inflation et la crise économique que l'Inde connait depuis 2008.

Les différentes guerres menées par l'Inde (comprenant l'Indépendance et la Partition de 1947, la question du Cachemire et les guerres successives avec le Pakistan) et la politique indienne (plusieurs élections de Premiers Ministres, l'assassinat d'Indira Gandhi, ...) sont un peu plus mentionnées par les habitants de Santa Cruz. Néanmoins, les répondants de Bandra ne sont pas en reste, d'autant plus que le second terrain à Santa Cruz - qui s'est déroulé juste avant l'élection de 2014 qui a amené Narendra Modi et le parti ultra nationaliste hindouiste au pouvoir - est sans doute spécifique à cet égard, compte tenu d'un effet de rappel sur les anciens événements politiques. 
Sous l'appellation «politique indienne» se trouvent plusieurs éléments comme le mouvement d'Anna Hazare (un leader politique connu notamment pour son combat contre la corruption, apparu en 2011), la dénonciation de la corruption ou les récentes élections à la chambre basse du Parlement. Ces résultats confortent une observation faite par d'autres auteurs: les personnes qui sont exclues de la politique (pour des raisons de pauvreté ou d'exclusion sociale) démontrent une indifférence envers ce sujet qui révèle en fait une dépossession de pouvoir (Bourdieu, 1979; Bourdon, 1992). A ce sujet, il serait intéressant de reposer la question à l'heure actuelle, quelques mois après que la démonétisation des billets de 500 INR ait impacté fortement les plus pauvres des Indiennes et des Indiens, sous couvert de combattre la corruption. Il est probable que les mentions de politique soient bien différentes.

Eléments historiques largement dispersés sur l'échelle du temps, mais liés par une source commune, la Partition de 1947 (cf. chapitre 1), les attentats et émeutes interreligieuses qui ont été mentionnées révèlent la conscience d'une forme de guerre civile qui ravage le pays depuis 70 ans. Toutefois, deux fois plus d'individus résidant à Bandra en ont fait mention, ce qui n'est pas sans rappeler un résultat observé dans d'autres mégapoles où la pauvreté et les inégalités sont légion (comme à Rio de Janeiro par exemple) (Vincent-Geslin et al., 2015). C'est dans les quartiers défavorisés de ces cités, entre des personnes soumises à une précarité exacerbée de survie, que se cristallisent généralement des conflits qui prennent pourtant leurs origines dans des événements de portée nationale (une guerre, l'Indépendance, etc.). Ceci se confirme avec la présence plus marquée des émeutes de 1992 et 1993, particulièrement sanglantes, qui se sont avant tout déroulées dans les bidonvilles de la mégapole indienne.

Les événements survenus dans une zone proche des lieux d'habitation des répondants (deux incendies dans les bidonvilles de Bandra, l'effondrement d'un building dans le quartier de Santa Cruz, divers conflits et des violences à Mumbai ou encore le projet de réhabilitation des bidonvilles) ont été cités par plus d'habitants du quartier d'habitat précaire. Finalement, un domaine a rassemblé les individus mentionnant des événements divers survenus en Inde. En particulier, les violences envers les femmes, incluant les viols, constituent dans le cas de Santa Cruz une majorité des citations dans cette catégorie. 
Tableau 8.2: les changements sociohistoriques (\%), selon l'âge et le lieu de résidence ${ }^{5}$

\begin{tabular}{|c|c|c|c|c|c|c|c|c|c|c|c|c|}
\hline Domaine & To & tal & 20 & & & -39 & 50 & -54 & 65 & -69 & 80 & -84 \\
\hline Type & BA & $\mathrm{SC}$ & BA & $\mathrm{SC}$ & BA & $\mathrm{SC}$ & $\mathrm{BA}$ & $\mathrm{SC}$ & BA & $\mathrm{SC}$ & BA & $\mathrm{SC}$ \\
\hline Catastrophes & 36 & 31 & 31 & 26 & 39 & 27 & 41 & 35 & 30 & 36 & 36 & 28 \\
\hline Inondation à $M$ & 32 & 13 & 26 & 10 & 35 & 7 & 36 & 17 & 27 & 18 & 33 & 12 \\
\hline (Inde) (2013) & - & & - & 6 & - & 5 & - & 5 & - & & - & \\
\hline $\begin{array}{l}\text { Tremblement de terre Bhuj } \\
\text { (Inde) (2001) } \\
\text { Chute du Malaysia Airlines }\end{array}$ & - & & - & 1 & - & 2 & - & 2 & - & & - & 6 \\
\hline (2014) & - & 6 & - & 7 & - & 8 & - & 6 & - & 4 & - & 2 \\
\hline Autre & 4 & 4 & 5 & 2 & 4 & 5 & 5 & 5 & 3 & 4 & 3 & 6 \\
\hline $\begin{array}{l}\text { Evéner } \\
\text { Atter }\end{array}$ & 2 & 10 & 4 & 10 & 1 & 12 & 3 & 10 & 1 & 7 & 2 & 9 \\
\hline 2001 & - & 3 & 1 & 2 & - & 5 & - & 1 & - & 3 & - & 2 \\
\hline Autre & 2 & 7 & 3 & 2 & 1 & 7 & 3 & 9 & 1 & 4 & 2 & 7 \\
\hline Changements économiques & 3 & 5 & 2 & 3 & 3 & 4 & 3 & 5 & 8 & 5 & 1 & 7 \\
\hline Inflation et a & & & & & & & & 3 & 8 & 4 & 1 & 6 \\
\hline des prix & 3 & 4 & 2 & 2 & 3 & 4 & 3 & 2 & - & 1 & - & 1 \\
\hline Autre & 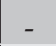 & 1 & 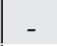 & 1 & - & - & - & & & & & \\
\hline $\begin{array}{l}\text { Guerres et politique indi- } \\
\text { ennes }\end{array}$ & 7 & 12 & 7 & 5 & 3 & 11 & 5 & 12 & 12 & 13 & 9 & 20 \\
\hline $\begin{array}{l}\text { Indépendance/Cachemire } \\
\text { (1947-48) } \\
\text { Assassinat d'Indira Gandh }\end{array}$ & 1 & 1 & - & - & - & - & - & - & 1 & 1 & 3 & 8 \\
\hline $\begin{array}{l}\text { (1984) } \\
\text { Anna Hazare et anticorr }\end{array}$ & 1 & - & - & - & - & - & 1 & 1 & 2 & 1 & 3 & 1 \\
\hline $\begin{array}{l}\text { tion (2011) } \\
\text { Election à la Lok Sabha }\end{array}$ & 2 & 4 & 3 & 3 & 1 & 5 & 2 & 4 & 3 & 2 & - & 5 \\
\hline (2014) & - & 2 & - & 2 & - & 2 & - & 1 & - & 2 & - & - \\
\hline Autre $^{9}$ & 3 & 5 & 4 & - & 2 & 4 & 2 & 6 & 6 & 7 & 3 & 6 \\
\hline
\end{tabular}

5 En gris les pourcentages où moins de cinq individus sont représentés.

6 Comprenant les ouragans, inondations et tremblements de terre mentionnés sans précision.

7 Comprenant diverses mentions: conquête de l'espace, Deuxième Guerre mondiale, progrès et NTIC, mouvements sociaux, climat et écologie, misère et épidémies,, etc.

8 Il s'agit de la chambre basse du Parlement indien, élu au suffrage majoritaire tous les 5 ans. En 2014, l'élection a eu lieu en avril-mai.

9 Une part de ces mentions concerne l'Alliance démocratique nationale (NDA), une coalition indienne de partis politiques de droite et du centre-droit. Des mentions d'autres guerres menées par l'Inde sont également rassemblées sous cette étiquette. 


\begin{tabular}{|c|c|c|c|c|c|c|c|c|c|c|c|c|}
\hline \multirow{2}{*}{$\begin{array}{c}\text { Domaine } \\
\text { Type }\end{array}$} & \multicolumn{2}{|c|}{ Total } & \multicolumn{2}{|c|}{$20-24$} & \multicolumn{2}{|c|}{ 35-39 } & \multicolumn{2}{|c|}{$50-54$} & \multicolumn{2}{|c|}{$65-69$} & \multicolumn{2}{|c|}{$80-84$} \\
\hline & BA & $\mathrm{SC}$ & BA & $\mathrm{SC}$ & BA & SC & BA & $\mathrm{SC}$ & BA & $\mathrm{SC}$ & BA & $\mathrm{SC}$ \\
\hline Attentats et émeutes en Inde & 36 & 18 & 36 & 19 & 37 & 21 & 31 & 19 & 30 & 17 & 43 & 12 \\
\hline Pogroms interreligieux (1984) & 1 & - & - & - & - & - & 1 & 1 & 1 & - & 2 & - \\
\hline Attentats à Ayodhya (1992) & - & 3 & - & 1 & - & 3 & - & 2 & 1 & 3 & - & 4 \\
\hline Pogroms interreligieux & & & & & & & & & & & & \\
\hline$(1992-93)$ & 12 & 2 & 2 & - & 13 & 3 & 12 & 4 & 16 & 2 & 20 & 2 \\
\hline Pogroms interreligieux (2011) & 4 & - & 5 & - & 3 & - & 1 & - & 3 & - & 9 & - \\
\hline Attentats en Inde en général & 4 & 1 & 6 & 1 & 5 & 2 & 4 & 1 & 2 & 1 & 1 & 1 \\
\hline Attentats à Mumbai (2006) & 4 & 4 & 6 & 5 & 5 & 5 & 3 & 3 & 2 & 3 & 4 & 2 \\
\hline Attentats à Mumbai (2008) & 7 & 6 & 13 & 9 & 7 & 5 & 6 & 6 & 3 & 6 & 4 & 2 \\
\hline Attentats à Mumbai (2011) & 2 & 1 & 2 & 2 & 3 & - & 4 & - & 1 & 1 & 1 & 1 \\
\hline Autre & 2 & 1 & 2 & 1 & 1 & 3 & - & 2 & 1 & 1 & 2 & - \\
\hline $\begin{array}{l}\text { Evénements du quartier } \\
\text { (Mumbai) }\end{array}$ & 10 & 7 & 10 & 9 & 9 & 3 & 12 & 8 & 13 & 9 & 7 & 8 \\
\hline Incendies & 4 & - & 4 & - & 4 & - & 6 & - & 2 & - & 2 & - \\
\hline Projet SRA $A^{10}$ & 2 & - & 1 & - & 3 & - & 2 & - & 3 & - & 1 & - \\
\hline Effondrement d'un building & & & & & & & & & & & & \\
\hline $\begin{array}{l}\text { (2014) } \\
\text { Autre (o }\end{array}$ & - & 4 & - & 5 & - & 2 & - & 5 & - & 4 & - & 2 \\
\hline divers) & 4 & 3 & 5 & 4 & 2 & 1 & 4 & 3 & 8 & 5 & 4 & 6 \\
\hline $\begin{array}{l}\text { Autres événements en Inde } \\
\text { Viols et violences contre les }\end{array}$ & 6 & 17 & 10 & 28 & 8 & 22 & 5 & 11 & 6 & 13 & 2 & 16 \\
\hline femmes & 1 & 13 & 1 & 20 & 1 & 16 & - & 9 & - & 9 & 1 & 12 \\
\hline Culture/sport & 2 & 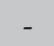 & 6 & 1 & 1 & 1 & 1 & - & 2 & - & - & - \\
\hline Autre & 3 & 4 & 3 & 6 & 6 & 5 & 4 & 2 & 4 & 4 & 1 & 4 \\
\hline
\end{tabular}

Le tableau 8.2 détaille les pourcentages de mention de chacun des domaines et des principaux types de changements, par lieu de résidence et par classe d'âge (le total par colonne atteignant cette fois-ci 100\%). Outre les constations déjà évoquées, plusieurs résultats complémentaires méritent d'être soulignés.

Tout d'abord, se confirme de manière explicite que les répondants résidant à Santa Cruz ont diversifié les mentions de catastrophes (l'inondation dans le Kedarnath au nord du pays, le tremblement de terre dans le district de Bhuj au Gujarat en 2001, la chute et la disparition du Malaysia Airlines dans l'océan Indien le 8 mars 2014 et divers autres événements),

10 Slum Rehabilitation Authority, politiques gouvernementales controversées visant à déplacer les habitants des bidonvilles vers des immeubles en dur (voir chapitre 1). 
alors que les habitants des slums se sont fortement concentrés autour du rappel de l'inondation de 2005 à Mumbai ${ }^{11}$.

Chez les jeunes, contrairement aux hypothèses sur les effets générationnellement différenciés de la globalisation, il n'y a pas de citation particulière de l'international. L'inflation économique est plus marquante pour les sexagénaires et octogénaires. Les 20-24 et 35-39 ans interviewés à Santa Cruz sont très affectés par les violences et viols contre les femmes. La corruption est également un sujet mentionné par les jeunes.

Les guerres datent historiquement, et sont donc forcément plus citées par les cohortes les plus âgées. Néanmoins, comme nous l'avons déjà évoqué, les attentats et les émeutes interreligieuses sont évidemment des émanations des conflits liés à la Partition, réutilisés et parfois exacerbés tout au long de l'histoire de l'Inde indépendante. Cela ressort à travers les mentions considérables d'attentats parmi les jeunes. Les 50-54, 65-69 et 80-84 ans interrogés à Bandra se focalisent davantage sur les pogroms, notamment celui de 1992-93 survenu dans les bidonvilles de Mumbai, qui fit près de 2000 morts. Certains étaient déjà âgés à l'époque, et on sait qu'ils sont les victimes préférentielles de ce type d'émeutes meurtrières parce qu'ils ne peuvent fuir (Bergouignan, 2011; Bergouignan \& Eljim, 2013); ou ils ont connu la peur, pour eux-mêmes et leur famille.

Quant aux effets de genre, contrairement aux hypothèses classiques, ils sont faibles (voir tableau 8.3). Seules les guerres et la politique indiennes sont plus évoquées par les hommes de Santa Cruz, alors que les violences faites aux femmes ont été nettement plus mentionnées du côté féminin, dans le même quartier.

11 Il n'était pas possible pour les répondants de Bandra d'évoquer la disparition du Malaysia Airlines ni l'inondation au Kedarnath, puisque ces deux événements sont survenus après la passation de l'enquête de 2012. 
Tableau 8.3: les changements sociohistoriques (\%), selon le sexe et le lieu de résidence ${ }^{12}$

\begin{tabular}{|c|c|c|c|c|}
\hline \multirow{2}{*}{$\begin{array}{l}\text { Domaine } \\
\text { Type }\end{array}$} & \multicolumn{2}{|c|}{ Femmes } & \multicolumn{2}{|c|}{ Hommes } \\
\hline & Bandra & Santa Cruz & Bandra & Santa Cruz \\
\hline $\begin{array}{l}\text { Catastrophes } \\
\text { Inondation à Mumbai (2005) } \\
\text { Inondation au Kedarnath (Inde) (2013) } \\
\text { Tremblement de terre à Bhuj (Inde) } \\
\text { (2001) } \\
\text { Chute du Malaysia Airlines (2014) } \\
\text { Autre }\end{array}$ & $\begin{array}{c}38 \\
33 \\
-\end{array}$ & $\begin{array}{r}27 \\
13 \\
3\end{array}$ & $\begin{array}{c}32 \\
30 \\
-\end{array}$ & $\begin{array}{c}34 \\
13 \\
6\end{array}$ \\
\hline $\begin{array}{l}\text { Evénements mondiaux } \\
\text { Attentats du } 11 \text { septembre } 2001 \\
\text { Progrès, technologie } \\
\text { Autre }\end{array}$ & $\begin{array}{l}2 \\
- \\
- \\
2\end{array}$ & $\begin{array}{c}\mathbf{1 0} \\
2 \\
2 \\
6\end{array}$ & $\begin{array}{l}3 \\
1 \\
- \\
3\end{array}$ & $\begin{array}{l}9 \\
3 \\
1 \\
5\end{array}$ \\
\hline $\begin{array}{l}\text { Changements économiques } \\
\text { Inflation et augmentation des prix } \\
\text { Autre }\end{array}$ & $\begin{array}{l}3 \\
3 \\
-\end{array}$ & $\begin{array}{l}5 \\
4 \\
1\end{array}$ & $\begin{array}{l}4 \\
4 \\
-\end{array}$ & $\begin{array}{l}5 \\
3 \\
2\end{array}$ \\
\hline $\begin{array}{l}\text { Guerres et politique indiennes } \\
\text { Indépendance et Cachemire (1947-48) } \\
\text { Assassinat d'Indira Gandhi (1984) } \\
\text { Anna Hazare et anticorruption (2011) } \\
\text { Election à la Lok Sabha (2014) } \\
\text { Autres guerres } \\
\text { Autre }\end{array}$ & $\begin{array}{l}6 \\
- \\
1 \\
1 \\
- \\
1 \\
3\end{array}$ & $\begin{array}{l}7 \\
- \\
1 \\
2 \\
1 \\
1 \\
2\end{array}$ & $\begin{array}{l}8 \\
1 \\
1 \\
2 \\
- \\
1 \\
3\end{array}$ & $\begin{array}{c}17 \\
2 \\
- \\
5 \\
2 \\
3 \\
4\end{array}$ \\
\hline $\begin{array}{l}\text { Attentats et émeutes en Inde } \\
\text { Pogroms interreligieux (1984) } \\
\text { Attentats à Ayodhya (1992) } \\
\text { Pogroms interreligieux (1992-93) } \\
\text { Pogroms interreligieux (2011) } \\
\text { Attentats en Inde en général } \\
\text { Attentats à Mumbai (2006) } \\
\text { Attentats à Mumbai (2008) } \\
\text { Attentats à Mumbai (2011) } \\
\text { Autre }\end{array}$ & $\begin{array}{c}36 \\
1 \\
- \\
13 \\
4 \\
4 \\
4 \\
6 \\
2 \\
2\end{array}$ & $\begin{array}{l}16 \\
- \\
1 \\
3 \\
- \\
1 \\
3 \\
5 \\
1 \\
2\end{array}$ & $\begin{array}{c}36 \\
1 \\
- \\
11 \\
4 \\
5 \\
4 \\
8 \\
2 \\
1\end{array}$ & $\begin{array}{l}20 \\
- \\
4 \\
2 \\
- \\
1 \\
5 \\
7 \\
- \\
1\end{array}$ \\
\hline
\end{tabular}

12 En gris les pourcentages où moins de cinq individus sont représentés. 


\begin{tabular}{l|cc|cc}
\hline Domaine & \multicolumn{2}{|c|}{ Femmes } & \multicolumn{2}{c}{ Hommes } \\
Type & Bandra & Santa Cruz & Bandra & Santa Cruz \\
\hline Evénements du quartier (Mumbai) & $\mathbf{1 0}$ & $\mathbf{9}$ & $\mathbf{1 0}$ & $\mathbf{6}$ \\
$\quad$ Incendies & 4 & - & 3 & - \\
Projet SRA & 2 & - & 2 & - \\
Violences et conflits divers & 2 & 1 & 3 & 1 \\
Effondrement d'un building (2014) & - & 5 & - & 3 \\
Autre & 2 & 3 & 2 & 2 \\
Autre événements en Inde & $\mathbf{5}$ & $\mathbf{2 8}$ & 7 & $\mathbf{9}$ \\
Viols et violences contre les femmes & 1 & 21 & - & 5 \\
Culture/sport & 1 & - & 3 & 1 \\
Autre & 3 & 7 & 4 & 3 \\
\hline
\end{tabular}

\section{La distribution des souvenirs sur l'échelle de l'Histoire}

Les figures 8.1 et 8.2 présentent la distribution des événements sociohistoriques cités à Bandra et à Santa Cruz, selon l'année de survenue du changement et la classe des répondants. Deux observations se lisent aisément sur ces graphiques, soulignant ce que le tableau 8.2 laissait entrevoir: les individus évoquent majoritairement des éléments qui se sont déroulés récemment, depuis l'ouverture économique de l'Inde (soit le début des années 1990). En outre, cette récence permet que toutes les classes d'âge mentionnent les mêmes souvenirs. Ainsi, les courbes se superposent presque parfaitement.

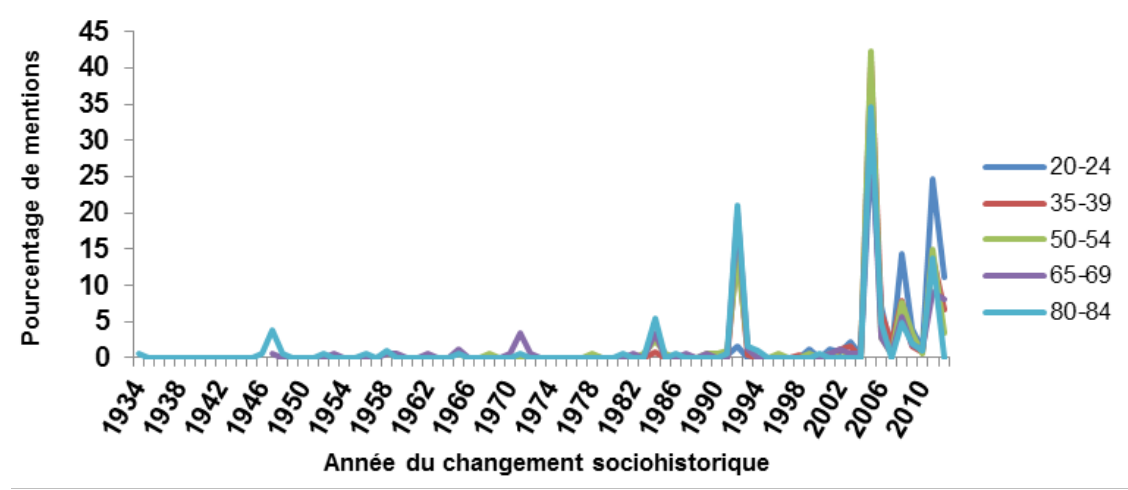

Figure 8.1: les changements sociohistoriques (\%) mentionnés à Bandra, selon l'année de survenue et la classe d'âge du répondant 


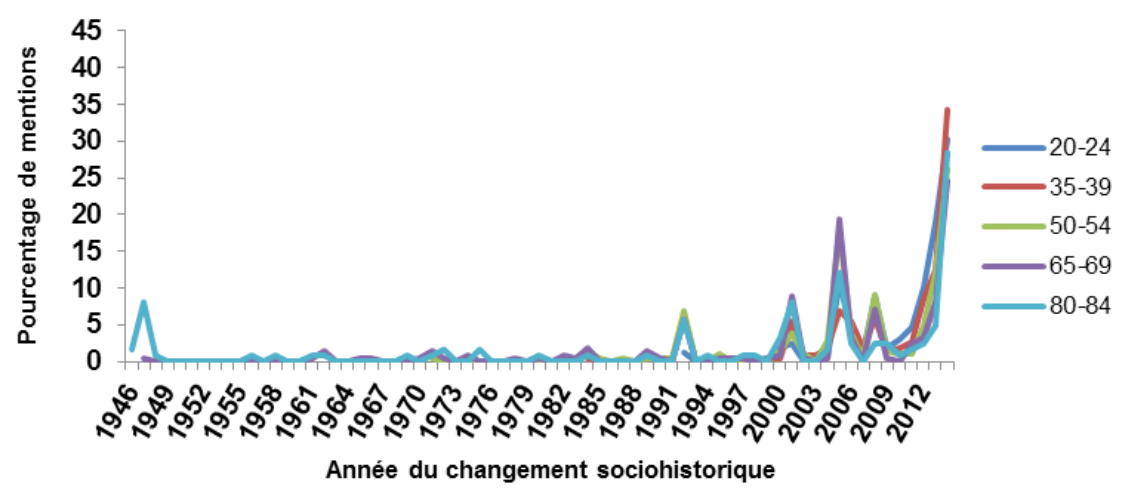

Figure 8.2: les changements sociohistoriques (\%) mentionnés à Santa Cruz, selon l'année de survenue et la classe d'âge du répondant

$* * *$

Ces premiers résultats confirment une représentation partagée de l'histoire autour d'événements violents, négatifs et nationaux. Le pourcentage élevé d'individus ayant mentionné les mêmes souvenirs (en particulier les inondations de 2005) est saisissant, et souligne la vulnérabilité du cadre de vie des répondants, surtout pour ceux résidant dans les bidonvilles (la section 8.3.1 approfondira ce constat). Autre syndrome commun aux habitants de Bandra (et dans une moindre mesure à ceux de Santa Cruz), le fait d'évoquer des souvenirs très localisés à Mumbai, voire directement dans certains quartiers de cette immense ville. Enfin, la quasi absence de mentions d'événements internationaux est surprenante et contrevient largement à d'autres résultats d'enquêtes, notamment en ce qui concerne le plus jeune groupe d'âge ${ }^{13}$ (Bergeron, 2015; Guichard \& Henríquez, 2011; Lalive d'Epinay et al., 2008, 2012), qui apparaît donc peu affecté par la mondialisation, en tous cas de ce point de vue spécifique.

Cette récence des événements mentionnés implique une concordance des souvenirs entre les différentes classes d'âge. A rebours, ceci démontre l'absence de mémoire générationnelle dans les réponses évoquées par les répondants. Cette homogénéité dans la vision de l'histoire est originale, presque inédite par rapport aux études empiriques menées dans d'autres

13 Il a en effet été constaté que les individus nés dans les années 1980 et après ont généralement une inclinaison à mentionner le 11 Septembre 2001 avant tout autre événement, y compris les faits nationaux (Bergeron, 2015; Guichard, 2015; Liu et al., 2009; Martenot \& Cavalli, 2014). 
parties du globe (Guichard, 2015; Lalive d'Epinay \& Cavalli, 2009; Lalive d'Epinay et al., 2008; Schuman \& Scott, 1989). Une source explicative se trouve possiblement dans l'un des moyens d'information le plus évident: la télévision. En effet, coïncidant avec les premiers événements mentionnés par toutes les cohortes au début des années 90, les médias télévisés indiens se sont largement démocratisés au cours des deux dernières décennies.

\subsubsection{Les médias, au centre des processus mémoriels}

L'existence de turbulences ayant marqué le pays ces dernières années (attentats, pogroms, catastrophes,...) peut en partie éclairer la récence des événements rapportés. Toutefois, cette explication à elle seule ne peut suffire, puisque les cohortes les plus âgées ont rencontré sans les évoquer d'autres événements, antérieurs, dont la portée est tout aussi voire plus - importante que les incidents de ces deux dernières décennies (Menon, 2011). Par ailleurs, les émeutes interreligieuses, les attentats et les guerres entre l'Inde et le Pakistan prennent leur source dans la colonisation et dans le conflit ayant conduit à la Partition: chaque nouvel élément aurait ainsi pu servir de rappel aux précédents (Schuman \& Corning, 2000; Schuman \& Rodgers, 2004). Pourtant, les classes d'âge ayant vécu ces années de violences dans leur jeunesse (les 80-84 ans pour la Partition et l'Indépendance, les 65-69 ans en ce qui concerne la seconde et la troisième guerre indo-pakistanaise) ne se focalisent guère sur ces souvenirs.

La concentration des souvenirs des cohortes autour des mêmes incidents peut être interprétée selon un autre angle, celui de la présence médiatique. En effet, la formation de la mémoire collective est fortement liée au rôle de la télévision (le média le plus populaire, plus facile d'accès que le journal par exemple (Sonwalkar, 2001)), que cela concerne des rappels de la vie quotidienne, ou des événements plus dramatiques et inattendus (Bourdon \& Kligler-Vilenchik, 2011).

\section{La présence de la télévision en Inde: un processus de diffusion long et controversé}

Introduite en Inde en 1959, la télévision est tout d'abord utilisée comme un moyen de propagande étatique, de construction de l'identité et de l'unité nationale, mais aussi d'information publique et d'éducation (Thussu, 
1999, 2013). Jusqu'en 1991, une unique chaîne gouvernementale (Doordarshan) détient le monopole (Rajagopal, 2001; Sonwalkar, 2001) et le nombre de foyers possédant un petit écran est très réduit (Roy, 2011). Il faut attendre la fin des années 1990 pour que l'accès à la télévision se démocratise, à tout le moins en ce qui concerne les habitants des bidonvilles et les classes moyennes inférieures des quartiers urbains (dans les campagnes, c'est encore une autre histoire).

Avant cela, l'accès à l'électricité et à un poste de télévision étaient difficiles. La figure 8.3 illustre la situation générale du pays (ménages ruraux et urbains, pauvres et riches confondus). L'enquête NFHS de 2005-06 a étudié de manière plus précise les foyers ayant une télévision. Il ressort qu'à Mumbai, l'écart dans l'accès aux médias télévisés a sensiblement diminué entre les niveaux socioéconomiques, puisque 78\% des ménages dans les bidonvilles ont un poste contre $89 \%$ des ménages résidant hors des slums (IIPS, 2007).

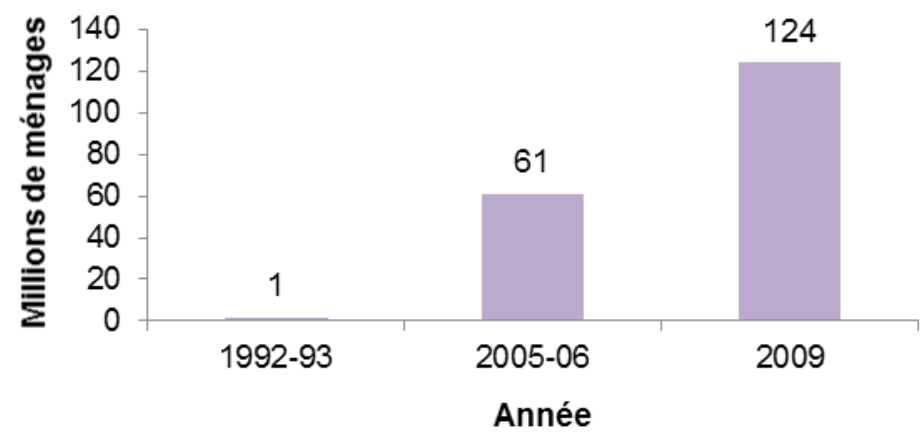

Figure 8.3: les ménages indiens possédant un poste de télévision (en millions) ${ }^{14}$ Source: Roy, 2011; Thussu, 2007.

A partir de l'ouverture économique, le monopole détenu par l'état indien saute et des chaînes privées étrangères entrent sur le marché. Ainsi, entre 1990 et 2012, l'Inde passe d'une unique chaîne de télévision à près de 600 (Thussu, 1999, 2007, 2013). Les chaînes indiennes doivent apprendre à se défendre dans un contexte de concurrence effrénée, notamment en

14 Données issues des enquêtes NFHS de 1992-93 et 2005-06, ainsi que du rapport 2010 sur les médias en Inde produit pas PricewaterhouseCoopers. 
proposant des émissions locales, dans des langues indigènes ${ }^{15}$ (Roy, 2011; Sonwalkar, 2001).

La fin du monopole signifie d'une part l'utilisation accrue de la télévision comme un moyen de diffusion des discours politiques, non contrôlés par l'état mais soumis aux volontés des entreprises privées et des lobbys (Roy, 2011; Sonwalkar, 2001). D'autre part, cela ouvre la possibilité aux journalistes de parler plus ouvertement de tous les sujets, de dénoncer les actions gouvernementales, etc. Dans la réalité cependant, c'est davantage une transposition des médias d'information vers des médias de divertissement qui s'opère (Johnson, 2001; Rajagopal, 2001; Thussu, 2007). Les nouvelles internationales, qui étaient déjà très peu diffusées à l'époque (seulement deux minutes par jour sur la chaîne Doordarshan), diminuent paradoxalement alors que le monopole étatique s'achève. Seuls les désastres naturels importants sont répercutés. En effet, afin d'être compétitives, les chaînes se focalisent sur les événements proches plutôt que distants, et sur des programmes récréatifs (Thussu, 2007).

\section{Les médias indiens dans la globalisation}

La globalisation tend à affecter le cadre social mémoriel de l'état-nation pour l'étendre à un ensemble transnational, avant tout par le biais des massmédias (Bisht, 2013). Ces derniers deviennent globaux (de mêmes chaînes couvrent plusieurs pays, plusieurs continents) et transmettent les mêmes informations, ce qui a un impact direct sur l'identité et la mémoire des individus (Bisht, 2013). Pourtant, en Inde, cette évolution est à nuancer.

D'un côté, les pays pauvres se servent souvent d'agences de presse occidentales pour dénicher les nouvelles à moindre coût (Archetti, 2008). D'un autre, les chaînes internationales présentes en Inde s'adaptent au pays (les journalistes sont indiens, parlent en hindi de thèmes indiens, etc.) et s'appliquent à produire une «globalisation à l'indienne» ou à donner une aura locale à la mondialisation (Bisht, 2013). Or, c'est en fait une occidentalisation cachée, au travers d'une assimilation des techniques de

15 Pendant longtemps, seul l'anglais fut utilisé dans les médias, langue qui demeure en Inde réservée aux élites et à la bureaucratie (Roy, 2011; Thussu, 2013). La libéralisation et l'arrivée de la presse internationale en 1991 se font, dans un premiers temps, toujours en anglais (Thussu, 1999), mais bientôt le hindi et les idiomes régionales sont introduits dans les médias, accroissant la diffusion des programmes TV vers les masses populaires (Rajagopal, 2001; Thussu, 2013). En parallèle, se constate une explosion du nombre de journaux en langues indigènes depuis la fin des années 1970, avec déjà 40\% de journaux rédigés en Hindi en 2002 (Jeffrey, 1993; Montaut, 2004). 
large audience utilisées dans le monde anglo-saxon, majoritairement à destination des classes moyennes urbaines (Thussu, 2007). Cet impérialisme médiatique, pur produit de l'Occident, se révèle en fait plus diversifié qu'il n'y parait (Sonwalkar, 2001).

Le pays a fonctionné comme nation souveraine (où le pouvoir appartenait complétement à l'Etat) de 1947 à 1990, les médias servant à asseoir cette idée et véhiculant un discours spatial pour diffuser le nationalisme ${ }^{16}$ (Asthana, 2013). Avec l'ouverture économique et le libéralisme, la nécessité de passer des droits étatiques vers des droits citoyens se fit sentir. Les médias ont dû faire évoluer leurs contenus, de la critique des responsabilités occidentales dans la période postcoloniale jusqu'à la promotion de l'ouverture économique (Asthana, 2013). Les larges mobilisations des classes moyennes dès les années 1980, opposées à l'état (et surtout à l'état d'urgence lancé par Indira Gandhi), encouragent la modification de l'attitude médiatique indienne qui, dès les années 1990, s'oriente vers la défense du nationalisme et du consumérisme ${ }^{17}$ (Chakravartty, 2004; Fernandes, 2000). La faveur du local face au global est utilisée pour mettre l'emphase sur la formation d'une identité nationale, pour renforcer le nationalisme initialement provoqué en réaction au colonialisme (Neyazi, 2010; Rajagopal, 2001; Thussu, 2007). Mais la promotion de cette identité n'est pas sans ambiguïté: sans se cacher, elle met en avant les intérêts de l'hindouisation que l'existence des minorités, notamment musulmanes, menacerait (Rajagopal, 2001).

La récence des souvenirs sociohistoriques rapportés par les habitants de Bandra et de Santa Cruz semble en partie imputable à une diffusion tardive de l'accès aux médias, en particulier à la télévision. Par ailleurs, la faible présence de nouvelles internationales dans les médias indiens

16 Le nationalisme a été construit pour restructurer les relations capitalistes internationales des pays économiquement moins développés (Asthana, 2013; Chatterjee, 1993). En Inde, les pratiques coloniales et l'expansion capitaliste ont toujours été liées. Les médias indiens ont été guidés par ces impératifs politiques: d'abord sous les Anglais, puis sous les nationalistes (qui se réclament de la culture indienne mais représentent les castes et les classes supérieures et développent des projets promouvant la nation, à l'image de la chaîne Doordarshan), enfin avec l'ouverture économique et l'arrivée des lobbys (qui amènent la privatisation des chaînes, la dérégulation des médias, etc.) (Asthana, 2013).

17 Par exemple, en «nationalisant» des produits étrangers pour encourager leur diffusion et leur consommation. 
explique que les mentions des répondants soient très nationales. S'ajoute au phénomène le développement d'un nationalisme renforcé par les vecteurs d'informations.

Le niveau d'éducation des individus n'est pas non plus à négliger comme piste explicative. Déjà, les différences de réponses entre les deux terrains d'enquête laissent entrevoir qu'un niveau socioéconomique plus élevé encourage une perception historique plus large, éloignée de soi, alors que la pauvreté implique un regard très proche du quotidien, des lieux connus et des collectivités avoisinantes. La récence et la proximité des événements historiques rapportés s'expliquent aussi par cela. Les sections suivantes proposent de reprendre les diverses catégories d'événements cités pour creuser davantage ce postulat, également au travers des raisons évoquées.

\subsection{Souvenirs de vulnérabilités environnementales et sociales}

Les inondations sont les plus dévastatrices des catastrophes naturelles en termes de pertes humaines et matérielles (Chan, Mitchell, Adekola, \& McDonald, 2012; Dewan, 2013). Il n'est pas inutile ici de rappeler que l'une des origines principales du concept de vulnérabilité est la démonstration du caractère non naturel de ces catastrophes (Oris et al., 2016). Les pays du Sud l'illustrent de manière évidente. Ils sont particulièrement affectés par ces drames, en raison de leur exposition au changement climatique, de leurs concentrations humaines, de choix politiques et économiques (tel que celui en faveur de la globalisation économique) ainsi que la pauvreté de leur état et de leur population (Chan et al., 2012). Les événements environnementaux, ou climatiques, ont un impact global sur les sociétés, mais aussi les individus et les ménages. Les inondations sont caractérisées par leur magnitude (taille et étendue), leur fréquence et leur durée, affectant largement le revenu par des dégâts matériels (Rayhan, 2008). Au sein de sociétés exposées aux désastres naturels, les personnes possédant le moins de ressources (qu'elles soient économique, sociale ou autre) sont particulièrement vulnérables: les bas revenus sont les premières victimes des catastrophes, avant tout parce qu'elles logent 
plus fréquemment dans des zones à risque (Rayhan, 2008; Zakour \& Gillespie, 2013).

En Asie du Sud, le nombre d'inondations est en augmentation depuis quelques années, en particulier en Inde (Chan et al., 2012). L'urbanisation est largement responsable de cette situation, les mégapoles du sud étant devenues plus menacées que les campagnes suite à l'augmentation de la population dans des espaces fragiles, à une construction généralement située sur les côtes ou dans des zones marécageuses et inondables, à une activité humaine non protégée (usines et espaces constructibles en expansion) (Rayhan, 2008). En outre, les bidonvilles et l'habitat sauvage créent des espaces où les eaux ne peuvent s'écouler, bloquant des drainages souvent défaillants (Dewan, 2013). Tous ces traits résonnent dans l'espace urbain de Mumbai (cf. chapitre 1).

\subsubsection{Précarité matérielle, vulnérabilités environnementales}

Le tableau 8.4, qui rassemble les résultats de plusieurs modèles de régressions logistiques portant sur la variable «avoir cité une catastrophe naturelle», démontre explicitement que de résider à Bandra induit une propension plus élevée à mentionner un tel événement. Avoir une famille à charge (soit appartenir aux groupes d'âge 35-39 ans et 50-54 ans) inclut également un risque statistiquement plus grand de citer le souvenir d'une catastrophe. Dans une moindre mesure, avoir brièvement eu accès à l'école accroit aussi ce risque.

Tableau 8.4: rapports de cotes issus de modèles de régression logistique sur la variable «avoir cité une catastrophe naturelle»

\begin{tabular}{|c|c|c|c|c|c|}
\hline & modèle 1 & modèle 2 & modèle 3 & modèle 4 & modèle 5 \\
\hline \multicolumn{6}{|c|}{ Lieu de résidence (référence $=$ Santa Cruz East) } \\
\hline Bandra East & $1.526 * * *$ & $1.531 * * *$ & $1.543 * * *$ & $1.462 * * *$ & $1.457 * * *$ \\
\hline \multicolumn{6}{|c|}{ Sexe (référence = hommes) } \\
\hline Femmes & & 0.953 & 0.952 & 0.927 & 0.930 \\
\hline \multicolumn{6}{|c|}{ Classe d'âge (référence $=20-24$ ans) } \\
\hline $35-39$ ans & & & $1.327^{*}$ & $1.275^{\circ}$ & $1.260^{\circ}$ \\
\hline $50-54$ ans & & & $1.461 * *$ & $1.354 *$ & $1.345^{*}$ \\
\hline $65-69$ ans & & & 1.158 & 1.050 & 1.075 \\
\hline $80-84$ ans & & & 1.209 & 1.085 & 1.112 \\
\hline
\end{tabular}




\begin{tabular}{|c|c|c|c|}
\hline modèle 2 & modèle 3 & modèle 4 & modèle 5 \\
\hline \multicolumn{4}{|l|}{ Education (référence = Supérieure) } \\
\hline Sans & & 1.154 & 1.181 \\
\hline Primaire & & $1.245^{\circ}$ & $1.260^{\circ}$ \\
\hline Secondaire & & 1.049 & 1.048 \\
\hline \multicolumn{4}{|l|}{ Santé auto-évaluée (référence $=$ Très bonne) } \\
\hline Bonne & & & 1.118 \\
\hline Moyenne & & & 0.981 \\
\hline Mauvaise & & & 0.946 \\
\hline
\end{tabular}

Seuils de significativité $:{ }^{\circ} \leq 0.1 ; * \leq 0.05 ; * * \leq .01 ; * * * \leq 0.001$.

Le nombre de mentions le permettant $(n=495)$, une seconde régression a été effectuée sur les évocations spécifiques de l'inondation de 2005 à Mumbai (voir tableau 8.5). Habiter dans un bidonville est encore plus déterminant du fait de citer ce souvenir que pour les catastrophes en général, corroborant les résultats descriptifs précédents (tableaux 8.2 et 8.3). L'âge n'est pas significatif et le niveau d'éducation apparaît à nouveau comme ayant un faible impact.

Tableau 8.5: rapports de cotes issus de modèles de régression logistique sur la variable «avoir cité l'inondation de 2005 à Mumbai»

\begin{tabular}{|c|c|c|c|c|}
\hline modèle 1 & modèle 2 & modèle 3 & modèle 4 & modèle 5 \\
\hline \multicolumn{5}{|c|}{ Lieu de résidence (référence $=$ Santa Cruz East) } \\
\hline Bandra East & $3.394 * * *$ & $3.425 * * *$ & $3.130 * * *$ & $3.081 * * *$ \\
\hline \multicolumn{5}{|l|}{ Sexe (référence $=$ hommes) } \\
\hline Femmes & 1.043 & 1.048 & 0.997 & 0.983 \\
\hline \multicolumn{5}{|c|}{ Classe d'âge (référence $=20-24$ ans) } \\
\hline $35-39$ ans & & $1.283^{\circ}$ & 1.207 & 1.159 \\
\hline $50-54$ ans & & $1.539 * *$ & $1.361^{\circ}$ & 1.293 \\
\hline $65-69$ ans & & 1.177 & 1.002 & 0.944 \\
\hline $80-84$ ans & & $1.322^{\circ}$ & 1.100 & 1.034 \\
\hline \multicolumn{5}{|c|}{ Education (référence $=$ Supérieure) } \\
\hline Sans & & & 1.231 & 1.221 \\
\hline Primaire & & & $1.420 *$ & $1.398 *$ \\
\hline Secondaire & & & 0.985 & 0.981 \\
\hline \multicolumn{5}{|c|}{ Santé auto-évaluée (référence $=$ Très bonne) } \\
\hline Bonne & & & & 1.236 \\
\hline Moyenne & & & & 1.294 \\
\hline Mauvaise & & & & 1.163 \\
\hline
\end{tabular}

Seuils de significativité : ${ }^{\circ} \leq 0.1 ; * \leq 0.05 ; * * 0.01 ; * * \leq 0.001$. 
Une analyse des raisons apportées au choix d'avoir cité l'inondation de 2005 a été effectuée. Celles-ci ont été subdivisées en trois catégories : description de l'événement, pertes financières, mise en situation.

La catégorie «description» rassemble $17 \%$ des raisons, et consiste en un aperçu général de l'événement. Si un impact est mentionné, il concerne la collectivité de manière indéterminée, ou le pays. Toutefois, ces souvenirs ne sont pas dénués d'émotion :

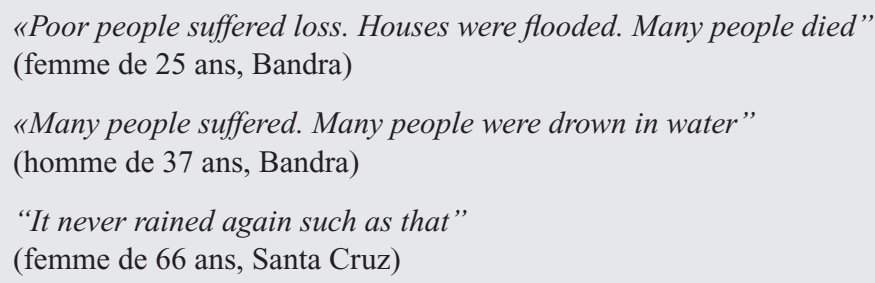

Le groupe «pertes financières» associe expressément des raisons évoquant des dommages matériels, sa maison dévastée, les frais engendrés par la catastrophe, etc. Plus de la moitié des explications du souvenir en font partie $(52 \%)$. Cette fois, elles contiennent toutes une conséquence concrète et directe pour le foyer, supposant des effets sur le long terme :

\footnotetext{
"I remember that incident because my house was flooded" (femme de 24 ans, Bandra)

"The house was full of water. There was the problem of food for some days" (homme de 50 ans, Santa Cruz)

"All material got rained. I took loan to repurchase material" (femme de 82 ans, Bandra)
}

La dernière catégorie «mise en situation», qui comprend $31 \%$ des raisons, se concentre autour des évocations précises du répondant ou de ses proches au moment de la catastrophe. Les heures passées sont décrites avec précision, les lieux et les situations rencontrés, les émotions ressenties alors. Sans forcément exprimer une tonalité négative (ce qui est davantage le cas du second critère), le degré de bouleversement et de traumatisme est malgré tout palpable, presque plus tangible que lors des mentions de pertes matérielles : 
"My daughter was out and water was flooded. Two persons brought her home" (femme de 80 ans, Bandra)

"My children were in school and husband was on job. There was no electricity. Mobile network went off. Relatives came to house. Because I prepared food in darkness",

(femme de 37 ans, Bandra)

"The river on the sides had very many dead bodies which I saw with my own eyes" (femme de 64 ans, Santa Cruz)

"Water level was till neck then to I did daring and reached home. My family was at home»

(homme de 40 ans, Bandra)

Les raisons pour avoir parlé de l'inondation de 2005 lorsque les répondants résident à Bandra sont majoritairement du type «pertes financières» (60\%), puis de manière plus réduite «mise en situation» (28\%); les descriptions générales représentent seulement $12 \%$ des raisons. Le contraste est évident avec les interviewés de Santa Cruz, qui partagent leurs explications presque équitablement entre «mise en situation» (42\%), «description» $(32 \%)$ et «pertes financières $(26 \%)$.

Les conséquences ont été directes pour les habitants des slums, qui ont perdu dans cette inondation leur maison, souvent le peu de biens qu'ils possédaient, et ont dû en supporter les coûts sur plusieurs années. Ceci se traduit par des raisons mettant en avant l'impact concret et négatif de cette catastrophe. Ils n'ont pas non plus oublié les circonstances dans lesquelles cette inondation est survenue, près d'un tiers des explicitations du souvenir exposant les faits et gestes du répondant ou de ses proches au moment du drame. A Santa Cruz par contre, davantage d'individus ont eu connaissance de cette inondation par les médias, ou en ont été témoin mais sans en payer directement le prix. Malgré tout, cela reste un traumatisme.

La répartition des raisons entre les sexes révèle des différences. Les femmes ont gardé mémoire surtout de la destruction de biens matériels et de leur foyer («pertes financières»: 58\%), ainsi que les souvenirs précis d'elles-mêmes ou de leurs proches dans la tragédie («mise en situation»: $30 \%$ ). De leur côté, les hommes présentent une répartition relativement plus égale entre «pertes financières» $(45 \%)$, «mise en situation» $(33 \%)$ et «description» $(22 \%)$. Les femmes, en charge de la gestion du foyer, ont 
enregistré les implications concrètes pour le ménage avant toute chose, ainsi que la peur ressentie face à la catastrophe.

La vulnérabilité ciblée par les mentions de l'inondation de 2005 est explicitement liée à l'environnement urbain, insalubre et précaire dans le cas des bidonvilles. Les répondants sont ramenés à leur condition première d'être humain fragile, sans protection face au déluge. Un second événement, cité avant tout par les individus résidant à Santa Cruz, souligne également une fragilité de la condition humaine, cette fois le fait d'être né femme dans une société inégalitaire, fortement patriarcale. Il s'agit des violences faites aux femmes, largement mises en lumière par les médias et les mouvements sociaux ces dernières années.

\subsubsection{Montée des violences contre les femmes}

Le tableau 8.6 démontre, au travers d'une série de modèles de régressions logistiques sur la variable «avoir cité les violences féminines», que le lieu de résidence et le sexe des répondants sont les principaux déterminants des mentions. Résider à Santa Cruz accroit explicitement le rappel, tout comme le fait d'être une femme.

La forte présence de ces souvenirs parmi les réponses des individus du quartier de classe moyenne inférieur s'explique en grande partie car les médias se sont mis massivement à parler des agressions suite au viol collectif et à l'assassinat de Jyoti Singh, en décembre 2012 à Delhi, soit 6 mois après le terrain effectué à Bandra. Les habitants des bidonvilles n'ont donc tout simplement pas pu citer cet incident dramatique. Il est aussi envisageable qu'un certain tabou autour des agressions règne dans les quartiers pauvres. En effet, si les bidonvilles sont des lieux où la promiscuité et les difficultés matérielles (devoir se rendre loin de chez soi pour aller aux toilettes par exemple) accroissent les risques d'insécurité pour les femmes (Ghosh, 2015; Pandey, Dutt, \& Banerjee, 2009), la crainte que la révélation d'un viol mette en péril la réputation de la victime et de sa famille entrave généralement la parole. Inversement, sans que leur base informative soit claire, les médias ont systématiquement associé les manifestations contre les viols depuis fin 2012 aux classes moyennes émergentes en Inde, n'acceptant plus ces attitudes et jugeant police et justice trop indulgentes envers les agresseurs. 
Tableau 8.6 rapports de cotes issus de modèles de régression logistique sur la variable «avoir cité les violences contre les femmes»

\begin{tabular}{|c|c|c|c|c|c|}
\hline & modèle 1 & modèle 2 & modèle 3 & modèle 4 & modèle 5 \\
\hline \multicolumn{6}{|c|}{ Lieu de résidence (référence $=$ Santa Cruz East) } \\
\hline Bandra East & $0.047 * * *$ & $0.043 * * *$ & $0.040 * * *$ & $0.048 * * *$ & $0.048 * * *$ \\
\hline \multicolumn{6}{|c|}{ Sexe (référence = hommes) } \\
\hline Femmes & & $4.624 * * *$ & $4.675^{* * *}$ & $4.963 * * *$ & $5.130 * * *$ \\
\hline \multicolumn{6}{|c|}{ Classe d'âge (référence $=20-24$ ans) } \\
\hline $35-39$ ans & & & 0.881 & 0.960 & 1.006 \\
\hline $50-54$ ans & & & $0.414^{* *}$ & $0.474 *$ & $0.499 *$ \\
\hline $65-69$ ans & & & $0.478 * *$ & $0.567^{\circ}$ & 0.609 \\
\hline $80-84$ ans & & & 0.715 & 0.912 & 0.997 \\
\hline \multicolumn{6}{|c|}{ Education (référence $=$ Supérieure) } \\
\hline Sans & & & & $0.388^{\circ}$ & 0.457 \\
\hline Primaire & & & & 0.798 & 0.853 \\
\hline Secondaire & & & & 0.838 & 0.869 \\
\hline \multicolumn{6}{|c|}{ Santé auto-évaluée (référence $=$ Très bonne) } \\
\hline Bonne & & & & & 0.808 \\
\hline Moyenne & & & & & 0.797 \\
\hline Mauvaise & & & & & 0.378 \\
\hline
\end{tabular}

Seuils de significativité : ${ }^{\circ} \leq 0.1 ; * \leq 0.05 ; * * \leq 0.01 ; * * \leq 0.001$.

Parmi les 128 mentions de violences à l'encontre des femmes, seules six d'entre elles font référence à des violences domestiques. Une analyse des raisons, à nouveau en trois catégories, permet d'éclairer les sentiments révélés derrière ces citations, généralement portées par une émotion forte.

Un premier codage rassemble $62 \%$ des raisons. Celles-ci sont des descriptifs de cas de viols ou de violences, parfois entendus dans les médias, d'autres fois dans la communauté. Globalement, ressort une prise de conscience de l'augmentation des violences :

\footnotetext{
"We get to see and hear this everywhere."

(femme de 38 ans, Santa Cruz)

"The repeated atrocities on women in Delhi are striking events"

(femme de 25 ans, Santa Cruz)

"She was a student who was raped brutally"

(homme de 36 ans, Santa Cruz)

"Nowadays we here only these news on TV and newspaper"

(femme de 21 ans, Santa Cruz)
} 
Le second groupe d'explications concerne un tiers d'entre elles (33\%) et se caractérise par la mise en avant de craintes pour les femmes en général, pour les proches du répondant, pour soi-même et sa condition de femme, ainsi qu'une dénonciation du manque de sécurité dans la société. L'émotion est évidente, ainsi qu'une certaine dose d'empathie :

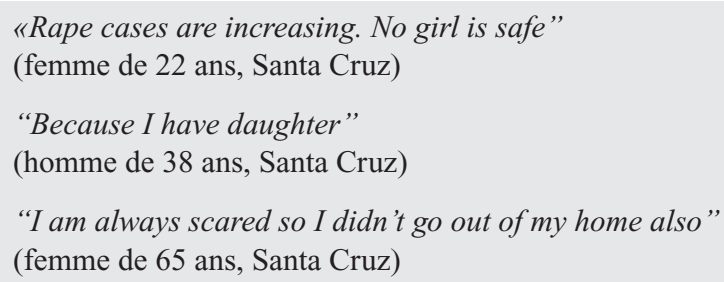

Finalement, la troisième catégorie contient $5 \%$ des raisons (soit six cas, dont trois à Bandra, où les habitants ne citent en tout que six violences féminines). Il s'agit des mentions où les répondants ont usé de leur propre expérience, ou de celle de leurs proches, pour justifier leur choix. Explicitement, ils évoquent le fait d'avoir été victime ou témoin de violences :

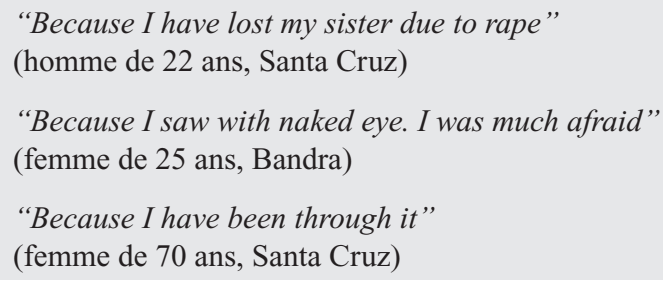

Au regard des raisons rapportées pour avoir mentionné ces violences, la forte représentation des femmes parmi les répondants les ayant évoquées s'explique aisément. Dans près de $40 \%$ des raisons, l'individu s'est placé au centre de la réflexion. Sinon, s'exprime un sentiment d'insécurité face à l'augmentation des violences, qui souligne une peur réelle de l'interviewée, généralement pour elle-même ou, dans le cas d'interviewés, pour leurs proches.

Notons l'absence totale d'évocation de la problématique des castes (et de la pauvreté) dans ces souvenirs. Pourtant, la volonté de contrôle des Dalits et des basses castes par le biais des violences est au cœur de cette réalité qui, si elle n'est effectivement que peu traduite dans les médias, est dénoncée par de nombreuses personnalités politiques et académiques (Channel 4: «Arundhati Roy speaks out against Indian rape culture», 2012; Record, 2013). 


\subsection{La nation indienne dans la mondialisation}

Les tableaux 8.2 et 8.3 l'on prouvé, dans les souvenirs des répondants l'histoire indienne est empreinte de terribles violences internes (guerres civiles, violences contre les minorités, ...) et externes (l'Indépendance, les guerres, ...). Violence politique, économique et sociale s'il en fut, la colonisation n'est pas si lointaine ; pourtant, ce sont davantage ses prolongements récents qui imprègnent les mémoires, en parallèle de nouveaux conflits suscités par la mondialisation et dont les origines sont toujours l'impérialisme occidental.

\subsubsection{Emeutes et pogroms : le poids de l'histoire politique et religieuse}

La Partition représente, pour les Indiens, le principal événement du $20^{\text {ème }}$ siècle, à l'image de la Première Guerre mondiale pour les Anglais ou de la Seconde Guerre mondiale pour les Français et les Japonais (Pandey, 1992). De nombreuses émeutes interreligieuses ont eu lieu depuis, pour lesquelles le rapprochement avec l'événement fondateur est clair et dont les premières victimes sont les membres de la forte minorité musulmane (Pandey, 1992). Mais la relation entre le passé et le présent n'est ni linéaire ni simple : la forme des pogroms et les raisons de leur survenue ont évolué depuis le début du $20^{\text {ème }}$ siècle.

Les premières émeutes datent de la fin du $19^{\text {ème }}$ siècle (Jaffrelot, 2010; Menon, 2011), alors que les colons anglais encouragent la distinction entre les religions, afin de procéder à une gestion administrative et un contrôle social (Baber, 2004; Robin, 2004). Jusqu'aux années 1960, les incidents de ce type restent rares, l'opposition réelle entre les groupes n'apparaît que dans les années 1980 (Jaffrelot, 2010; Robin, 2004). Depuis, une augmentation des pogroms et de leur brutalité se constate, dont les causes s'inscrivent dans une transformation du nationalisme indien (Robin, 2004).

Au moment de l'Indépendance, le nationalisme indien porte déjà les stigmates de l'exclusion, puisque les musulmans, les Dalits, les femmes, pour ne citer que ces trois groupes, n'accèdent pas au droit de citoyen à part entière (ce, malgré des lois prônant l'égalité et les non-discriminations) (Pandey, 2001). Avec les années et les mutations géopolitiques globales, ce nationalisme se renferme de plus en plus autour d'une seule définition, une 
seule culture, une seule religion (l'hindouisme), excluant et discriminant les individus situés hors de ces critères d'appartenance (Prakash, 2000).

Le développement de l'hindouisme nationaliste amène un discours de haine envers les musulmans, et un sentiment anti-minoritaire se construit qui ne trouve pas d'équivalent envers les autres minorités (sauf peut-être en ce qui concerne les femmes) (Appadurai, 2000). Loin de résulter d'une réelle opposition entre deux traditions, deux communautés, la violence et le racisme sont des instruments politiques (Baber, 2004; Jaffrelot, 2010; Robin, 2004; Sen \& Wagner, 2005).

Tableau 8.7: rapports de cotes issus de modèles de régression logistique sur la variable «avoir cité des émeutes interreligieuses»

\begin{tabular}{|c|c|c|c|c|c|}
\hline modèle 1 & modèle 2 & modèle 3 & modèle 4 & modèle 5 & modèle 6 \\
\hline \multicolumn{6}{|c|}{ Lieu de résidence (référence $=$ Santa Cruz East) } \\
\hline Bandra East $6.427 * * *$ & $6.386^{* * *}$ & $6.337 * * *$ & $5.311 * * *$ & $5.297 * * *$ & $5.302 * * *$ \\
\hline \multicolumn{6}{|c|}{ Sexe (référence $=$ hommes) } \\
\hline Femmes & 1.111 & 1.125 & 1.058 & 1.080 & 1.079 \\
\hline \multicolumn{6}{|c|}{ Classe d'âge (référence $=20-24$ ans) } \\
\hline $35-39$ ans & & $2.886^{* * *}$ & $2.966^{* * *}$ & $2.906^{* * *}$ & $2.933 * * *$ \\
\hline $50-54$ ans & & $2.531 * * *$ & $2.568 * * *$ & $2.503 * *$ & $2.482 * *$ \\
\hline $65-69$ ans & & $2.879 * * *$ & $3.214 * * *$ & $3.179 * * *$ & $3.086 * * *$ \\
\hline $80-84$ ans & & $4.843 * * *$ & $4.432 * * *$ & $4.599 * * *$ & $4.427 * * *$ \\
\hline \multicolumn{6}{|c|}{ Religion (référence $=$ Hindoue) } \\
\hline Musulmane & & & $2.130 * * *$ & $2.206^{* * *}$ & $2.263 * * *$ \\
\hline Autre & & & 0.737 & 0.728 & 0.743 \\
\hline \multicolumn{6}{|c|}{ Education (référence $=$ Supérieure) } \\
\hline Sans & & & & 0.773 & 0.782 \\
\hline Primaire & & & & 1.269 & 1.251 \\
\hline Secondaire & & & & 0.889 & 0.895 \\
\hline \multicolumn{6}{|c|}{ Santé auto-évaluée (référence $=$ Très bonne) } \\
\hline Bonne & & & & & 0.853 \\
\hline Moyenne & & & & & 1.057 \\
\hline Mauvaise & & & & & 0.701 \\
\hline
\end{tabular}

Seuils de significativité $:{ }^{\circ} \leq 0.1 ; * \leq 0.05 ; * * \leq 0.01 ; * * * 0.001$.

Les émeutes interreligieuses ont été particulièrement violentes à Mumbai entre décembre 1992 et janvier 1993 (les chiffres officiels rapportent 900 décès, mais il y en eut sans doute bien plus), créant de graves cassures entre hindous et musulmans, se reflétant dans des attitudes hostiles entre 
les communautés, des déplacements de population d'un quartier à un autre, etc. (Menon, 2011). C'est surtout dans les bidonvilles que les violences se concentrent, là où s'additionnent pauvreté, manque de travail et minorités défavorisées (comme les musulmans).

Ce constat se retrouve dans les réponses des répondants, en particuliers ceux interrogés à Bandra (voir section 8.2.1). Afin de le tester, le tableau 8.7 propose des régressions sur le fait de mentionner une émeute (243 mentions d'émeutes religieuses ont été faites, dont 209 à Bandra et 34 à Santa Cruz). Il apparaît sans surprise que de résider à Bandra augmente significativement la propension de mentionner un tel souvenir. La proximité des émeutes dans ces quartiers, ainsi que la répétition de leur survenue (le dernier pogrom ayant eu lieu en 2011) n'a pas permis aux habitants des slums d'oublier.

Avec l'avancée en âge, la remémoration est plus importante, jusqu' aux 80-84 ans (qui ont vécu les turbulences interreligieuses depuis l'époque de la Partition) qui sont ceux qui mentionnent le plus ces émeutes. Une variable indépendante concernant la religion du répondant a été ajoutée dans cette analyse. Elle démontre que les individus musulmans ont eu tendance à davantage évoquer les pogroms, surement parce que leur vie a été directement mise en danger dans ces moments.

Les raisons alléguées pour citer une émeute ont été codées en deux catégories. La première regroupe les explications générales ou mettant les événements en lien avec des choix politiques, soit des réflexions plutôt globales qui laissent transparaître des émotions et de la violence mais sans que l'individu ne se trouve au centre du souvenir. Dix-sept pourcents des raisons composent ce groupe, $11 \%$ de celles rapportées par les habitants des bidonvilles et $50 \%$ de celles évoquées par les résidents de Santa Cruz :

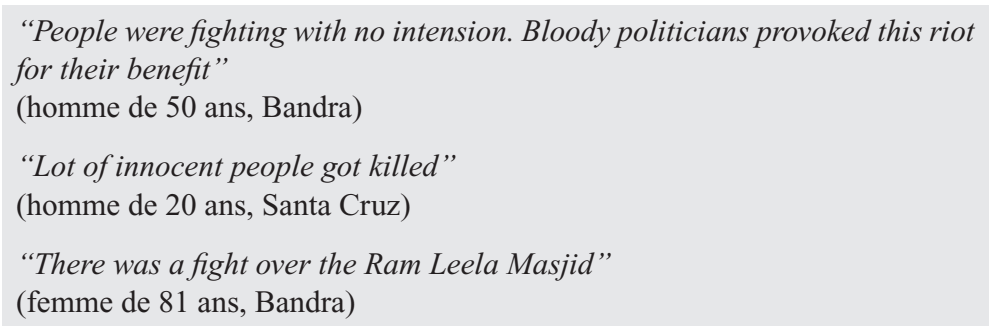

Le second type de raisons concerne cette fois des souvenirs précis, mettant en scène l'individu ou ses proches. Il est dominant, réunissant $83 \%$ des explications fournies, $89 \%$ de celles indiquées par les habitants des slums : 
"We were unable to go out of house. We were afraid"

(femme de 68 ans, Bandra)

"Because people were fighting with swords"

(femme de 40 ans, Bandra)

"That time we were worried for parents as we were away from them" (homme de 35 ans, Bandra)

"I had mutton shop and in front of the shop people put vehicles on fire" (homme de 82 ans, Bandra)

$\mathrm{Au}$ travers de ces raisons, il est évident que les répondants des bidonvilles ont davantage mentionné les émeutes interreligieuses car ils ont été bien plus concernés par ces événements, et ce de manière directe. Entre les religions, la différence n'est pas flagrante : la première catégorie rassemble 11\% des raisons évoquées par des hindous et $22 \%$ de celles mentionnées par des musulmans, tandis que la seconde réunit respectivement $89 \%$ et $78 \%$.

Si les émeutes ont ainsi secoué l'histoire indienne de sanglants épisodes, de nouvelles formes de violences sont apparues plus récemment. En 1993, alors que les émeutes font rage, Mumbai est pour la première fois le théâtre d'explosions meurtrières. Depuis, des attentats sont survenus à de nombreuses reprises (en 2006 et 2008 notamment, faisant à chaque fois près de 200 victimes).

\subsubsection{L'Inde mondialisée et les attentats terroristes}

Tout comme pour les pogroms, les religions sont brandies comme la source des violences et les musulmans montrés du doigt. Ainsi, en 2008 alors que dix hommes d'origine pakistanaise avaient tiré dans la foule à différents endroits de la ville, l'un deux (Ajmal Kasab) est pris vivant et sera condamné à mort en 2010. Les fustigations contre le Pakistan prennent de l'ampleur au cours du procès, largement médiatisé. Les musulmans doivent alors se justifier et montrer leur allégeance à la nation indienne (Menon, 2011). Pourtant, les discours raciaux et les injonctions à la haine proviennent des mêmes leaders politiques que pour les émeutes intercommunautaires $^{18}$ (Chakravartty, 2002; Rajagopal, 2001).

18 En outre, la récupération des attentats du 11 Septembre 2001 aux Etats-Unis a servi cette cause fondamentaliste hindoue. Les partis politiques ultra nationalistes ont su 
Dans les questionnaires, 349 mentions ont concerné des attentats, qu'ils aient eu lieu en Inde ou à Mumbai. Bien que mieux répartis entre les habitants de Bandra $(n=214)$ et de Santa Cruz $(n=135)$, un plus fort impact sur la mémoire des résidents des slums est à nouveau clair. Une série de régressions logistiques permet de confirmer ce résultat (voir tableau 8.8). Les hommes, les individus ayant atteint le niveau primaire et secondaire de scolarisation, ainsi que les cohortes les plus jeunes montrent également une plus grande propension au rappel de ces événements. Contrairement aux émeutes, les musulmans ont un peu moins de risque d'évoquer des attentats que les personnes d'autres confessions religieuses.

Tableau 8.8: rapports de cotes issus de modèles de régression logistique sur la variable «avoir cité des attentats en Inde»

\begin{tabular}{|c|c|c|c|c|c|}
\hline modèle 1 & modèle 2 & modèle 3 & modèle 4 & modèle 5 & modèle 6 \\
\hline \multicolumn{6}{|c|}{ Lieu de résidence (référence $=$ Santa Cruz East) } \\
\hline Bandra East $1.552 * * *$ & $1.579 * * *$ & $1.576 * * *$ & $1.630 * * *$ & $1.747 * * *$ & $1.722 * * *$ \\
\hline \multicolumn{6}{|c|}{ Sexe (référence $=$ hommes) } \\
\hline Femmes & $0.763 *$ & $0.741 * *$ & $0.758 *$ & $0.771 *$ & $0.751 *$ \\
\hline \multicolumn{6}{|c|}{ Classe d'âge (référence $=20-24$ ans) } \\
\hline $35-39$ ans & & 0.864 & 0.869 & 0.934 & 0.904 \\
\hline $50-54$ ans & & $0.685^{*}$ & $0.685^{*}$ & 0.775 & $0.735^{\circ}$ \\
\hline $65-69$ ans & & $0.470 * * *$ & $0.458 * * *$ & $0.519^{* * *}$ & $0.475^{* * *}$ \\
\hline $80-84$ ans & & $0.430 * * *$ & $0.443 * * *$ & $0.497 * *$ & $0.454 * * *$ \\
\hline \multicolumn{6}{|c|}{ Religion (référence $=$ Hindoue) } \\
\hline Musulmane & & & $0.793^{\circ}$ & $0.791^{\circ}$ & $0.793^{\circ}$ \\
\hline Autre & & & 1.277 & 1.262 & 1.266 \\
\hline \multicolumn{6}{|c|}{ Education (référence $=$ Supérieure) } \\
\hline Sans & & & & 0.869 & 0.848 \\
\hline Primaire & & & & $0.727^{\circ}$ & $0.708^{\circ}$ \\
\hline Secondaire & & & & $0.743 *$ & $0.738^{*}$ \\
\hline \multicolumn{6}{|c|}{ Santé auto-évaluée (référence = Très bonne) } \\
\hline Bonne & & & & & 1.121 \\
\hline Moyenne & & & & & 1.325 \\
\hline Mauvaise & & & & & 1.161 \\
\hline
\end{tabular}

Seuils de significativité : ${ }^{\circ} \leq 0.1 ; * \leq 0.05 ; * * \leq 0.01 ; * * \leq 0.001$.

utiliser l'exemple américain pour désigner un même ennemi, le terrorisme islamique, et ce faisant légitimer un certain nombre d'actions équivoques comme le conflit autour du Cachemire (Chakravartty, 2002). 
Les raisons pour avoir évoqué des attentats ont été divisées en deux catégories, proches de celles définies pour les pogroms. Dans ce cas, les résultats sont plutôt équilibrés. Ainsi, le premier code rassemble $47 \%$ des raisons et le second $53 \%$. Sous le premier label se trouve des descriptions générales des attentats, déplorer la mort d'innocents ou dénoncer la responsabilité de Kasab ou du gouvernement :

\author{
"Because many people died" \\ (homme de 39 ans, Bandra) \\ "Shown in TV again and again" \\ (homme de 52 ans, Santa Cruz) \\ "Kasab still not penalised. I am angry with us" \\ (femme de 20 ans, Bandra)
}

La seconde catégorie cible des raisons concrètes, dans lesquelles le répondant ou ses proches se situent. Un sentiment de peur, de traumatisme suite à ces journées d'effroi transparaît :

\author{
"It was horrible. I couldn't sleep that night" \\ (homme de 66 ans, Bandra) \\ "Because I was near that. We took people to Hospital" \\ (homme de 35 ans, Bandra) \\ "Because my brother was travelling in the same train" \\ (femme de 21 ans, Bandra) \\ "My friend died in this" \\ (homme de 35 ans, Santa Cruz) \\ "Because I left job after Bomb blast occurred in share market" \\ (femme de 50 ans, Santa Cruz)
}

Les individus sont généralement moins impliqués dans les souvenirs des attentats, qui ont touchés moins de personnes et ont été plus localisées que les émeutes. Par ailleurs, peu de divergences apparaissent entre les terrains d'enquête : les répondants de Bandra mentionnent une faible majorité de raisons $(57 \%)$ de la seconde catégorie, un pourcentage à peine plus réduit en ce qui concerne les habitants de Santa Cruz (47\%). Nonobstant, le caractère traumatique de ces violences est évident, et rappelle dans une certaine mesure le phénomène du 11 Septembre 2001, qui a frappé par sa violence et sa médiatisation, en ayant toutefois directement concerné peu 
de gens à l'échelle planétaire (Bergeron, 2015; Brown et al., 2009; Guichard, 2015). Que ce soit les jeunes groupes d'âge qui aient le plus cité les attentats en Inde ne fait que renforcer ce constat.

\subsection{Conclusion}

Plus encore que de retenir un fait appris, avoir vécu collectivement un événement d'ampleur - qu'il soit positif ou négatif -, offre un référent mémoriel commun aux individus (Guichard, 2015; Halbwachs, 2004 [1925]). L'expérience vécue, malgré tout ce qu'elle a de subjectif, rassemble et donne une identité au groupe (Thompson, 2012 [1963]; Zerubavel, 2012). Ce phénomène est indiscutable concernant l'inondation de 2005. Dans les deux enquêtes, les chiffres sont parlants : $57 \%$ des répondants de Bandra et 19\% de ceux de Santa Cruz en font le rappel, c'est un raz de marée mémoriel. L'analyse des raisons permet en outre de souligner que l'ampleur des conséquences de la catastrophe (endettement, pertes matérielles) et de la terreur ressentie sur le moment (perte du lien avec les proches, éloignement, etc.) expliquent ce résultat ainsi que la prévalence de souvenirs chez les pauvres. La vulnérabilité de l'habitat et des conditions de vie ne fait que croître face au désastre.

Les événements historiques mémorisés (guerres, terrorisme, catastrophes naturelles) s'enregistrent plus lorsque les événements sont directs, puissants et ont des impacts qui se prolongent dans le temps (Brown et al., 2009). Nos données corroborent ce constat, ainsi que celui d'une surreprésentation des événements violents, négatifs et nationaux dans les mémoires (Liu et al., 2012; Paez \& Liu, 2012; Pennebaker et al., 2006). En effet, bien que temporellement très récents, les principaux changements sociohistoriques rapportés par les répondants - exception fait de l'inondation de 2005 -, sont tous des conséquences directes du passé colonial et des guerres d'Indépendance qu'a connu l'Inde. Ceci se cristallise dans le souvenir plus prégnant parmi les populations les plus persécutées (les musulmans, les âgés) des émeutes religieuses.

Il a été souligné que les minorités étaient normalisées à suivre les mêmes constructions, notamment mémorielles, que le mainsteam conformiste indien (homme, hindou, Brahmane) (Pandey, 1992). Pourtant, la 
surreprésentation de souvenirs des pogroms parmi les musulmans atteste de l'existence d'une mémoire qui prend en compte la position de précarité. C'est également le cas des mentions nombreuses des violences contre les femmes, largement évoquées avant tout par les femmes elles-même. Le ressenti de la montée des violences et les perceptions de danger sont les principales raisons à ces citations, révélatrices d'un fort sentiment de vulnérabilité.

Tout comme le cours de vie est divisé en différentes étapes (enfance, âge adulte, retraite...), les événements sociohistoriques qui frappent les vies n'ont pas le même impact en fonction de l'âge des individus (Lalive d'Epinay et al., 2008; Martenot \& Cavalli, 2014; Schuman et al., 1998; Schuman \& Scott, 1989). Pourtant, dans nos résultats ressortent peu de divergences entre les groupes d'âge, qui rapportent tous des événements récents. L'effet de la modernisation et de la globalisation, qui s'est fait largement sentir dans des enquêtes effectuées dans d'autres contextes, n'est pas au rendez-vous. De même, les événements internationaux sont très peu représentés dans les mémoires, faits marquant en particulier chez les jeunes. Pour ces deux aspects (absence de divergence générationnelle et de changements internationaux), l'impact des médias indiens est sans doute à mettre en cause, démocratisés depuis peu et très centrés sur l'Inde. Néanmoins, il est possible d'extrapoler que les jeunes cohortes ont malgré tout leur propre façon de voir la globalisation, au travers des mentions importantes de «leur 11 Septembre 2001», leur pied dans la modernité et dans «le terrorisme»: les attentats de Mumbai en 2006 et 2008. 



\title{
Chapitre 9. Vulnérabilités multiples dans la perception du parcours de vie
}

\author{
«[...] toutes les conceptions soulignaient l'étroite \\ articulation entre les positions sociales et les \\ perceptions subjectives, entre les valeurs et les
}

conduites.»

(Martuccelli, 2009, p.18)

Ce chapitre précède et entame quelque peu la conclusion, afin d'adjoindre aux différents constats de vulnérabilités «objectives», soulignées dans les chapitres préalables, des analyses portant sur d'autres formes de vulnérabilités que l'on peut qualifier de subjectives ou recouvrant des aspects indirects. En effet, les vulnérabilités sont multiformes, surgissant tant dans le rapport des individus à l'histoire, à leur collectivité, qu'à eux-mêmes ; dans la capacité (et surtout l'incapacité) de choisir l'orientation des trajectoires de sa vie ; dans le stress, latent ou manifeste, qui pèse sur ces trajectoires.

Les chapitres antérieurs ont permis de poser un certain nombre de constats à propos des perceptions des composantes du parcours de vie, ainsi que du contexte qui les entoure, par des habitants de Mumbai. En particulier, l'aspect crucial de la famille et des contraintes socioéconomiques qui régissent les vies (chapitres 5 et 6), et l'impact considérable de la santé ainsi que la vulnérabilisation qu'une perte de celle-ci peut signifier (chapitre 7). De son côté, le chapitre 8 a pu souligner l'importance de l'environnement sociohistorique entourant les vies, et les diverses formes d'incertitudes et de risques macrosociaux qui marquent Mumbai depuis quelques décennies.

S'appuyant sur ces constats, la première partie du présent chapitre commence par se pencher sur la place des individus dans les différentes réponses à nos questionnaires. Dans quelle mesure les personnes se considèrent-elles au centre des événements personnels et sociohistoriques, plutôt que leur famille ou la collectivité ? Par la suite, c'est sur la part laissée à l'agency, à la capacité d'être acteur de sa vie, que l'on se penchera. 
Effectivement, en Inde aussi, les individus sont de plus en plus soumis à l'injonction de responsabilisation et de gestion de leur propre existence, chargés de dépasser leurs vulnérabilités en agissant eux-mêmes, en «se prenant en main» (Beck, 2001; Delory-Momberger, 2009; Misztal, 2011): mais les plus précaires en sont-ils capables? En ont-ils les ressources ou les capabilités?

Le deuxième axe de ce chapitre s'intéresse aux stress patent et latent associés aux tournants de l'existence. Comme nous l'avons détaillé en section 2.4.3, le stress est central pour expliquer le développement des vulnérabilités. Il provient en grande partie du changement présent dans l'événement, mais il s'avère que les tournants évoqués par les répondants à Mumbai prennent parfois d'autres formes inattendues (comme une situation de longue durée ou une absence de changement évoquées en réponse à la question sur les tournants de la vie). En outre, si les raisons de citer certains événements peuvent objectivement être qualifiées de négatives, et donc renvoyer à une perte de bien-être, d'autres révèlent un stress chronique ou encore la survenue d'un tournant en dehors du calendrier normatif tel que l'individu l'applique à lui-même (trop tôt ou trop tard).

Finalement cela nous conduit à questionner directement la dimension du bien-être telle qu'elle transparaît au travers des réponses aux trois volets du questionnaire. Simplement dit, peut-on percevoir sa vie de manière heureuse lorsque l'on est pauvre, malade ou encore une femme au sein d'une culture fortement patriarcale ? La dernière partie de ce chapitre souhaite répondre à ce questionnement un peu superficiel et néanmoins passionnant, touchant au cœur de la dimension de la subjectivité.

\subsection{L'incapacité d'être agent de sa vie comme marqueur de vulnérabilité}

Dans le monde occidental depuis la révolution industrielle, l'institutionnalisation des étapes de l'existence a, jusqu'aux années 1960, conduit les individus à connaître les mêmes transitions aux mêmes âges (Sapin et al., 2007). Par la suite, la modernité et les changements sociohistoriques qui s'y rapportent ont modifié les manières de fonctionner et de s'identifier 
des personnes, provoquant une individualisation des comportements et des normes (Bergeron, 2013; Kohli, 2007; Martuccelli, 2005; Mayer, 2004).

Dans le contexte indien, où les institutions formelles ne sont une réalité que pour une minorité de la population, ce sont les cadres normatifs traditionnels d'âge et de genre qui participent à l'homogénéisation des trajectoires. Les chapitres 4 à 7 ont empiriquement démontré que, grossièrement, les événements de la vie appréciés et mémorisés par des habitants de Mumbai restent sensiblement les mêmes à travers les âges, exception faite d'une certaine ouverture envers les transitions formatives pour les plus jeunes. Au-delà, les moments marquants de l'existence tournent autour de la construction de la famille (mariage et naissances), de la santé et du travail pour toutes les cohortes interrogées.

Néanmoins, dans une mégapole du Sud fortement impliquée dans l'économie globalisée, avec une présence généralisée des médias porteuse d'une certaine mondialisation de référents culturels (Appadurai, 2005; Thussu, 2013), il y a fort à parier que l'individualisation des parcours de vie perçue dans les pays occidentaux fasse son chemin, bien que possiblement sous une forme spécifique à la société en question. Sous l'optique de la perception des événements marquants de la vie et de l'histoire, ce phénomène pourrait se traduire par une centration accrue autour de soimême, de ses choix, de ses contraintes, une manifestation qui serait plus spécifique aux jeunes cohortes qu'aux anciennes, traduisant de la sorte l'inscription générationnelle d'une évolution macrosociale. Afin de questionner cette vision, nous avons opté pour l'utilisation des dimensions d'agency et d'individualisation, qui jouent alors le rôle de proxys pour évaluer cette centration autour de l'individu. La section qui suit a pour but de poser quelques jalons théoriques puis de les tester au regard des réponses apportées à nos questionnaires.

\subsubsection{Singularité des trajectoires et processus d'agency dans la seconde modernité}

Le passage à la seconde modernité (Bergeron, 2013; Giddens, 2004 [1994]; Lalive d'Epinay, 2012), survenu au cours de la deuxième moitié du $20^{\mathrm{ème}}$ siècle, se caractérise par une période de profonde transformation sociétale (voir section 2.2.2) porteuse de nouveaux risques et d'incertain pour les populations (Beck, 1992; Levy \& Bühlmann, 2016; Sørensen \& 
Christiansen, 2014). Certains auteurs soulignent que, contrairement aux siècles précédents, la précarité face aux aléas de l'existence est devenue universelle et la condition sociale ne définit plus la vulnérabilité, ce qui les amène à parler d'une disparition des classes sociales (voir section 2.4.2) (Beck, 2001 [1986]; Beck \& Beck-Gernsheim, 2002). Toutefois, de manière quelque peu paradoxale, ils reconnaissent que les risques ne se distribuent pas égalitairement au sein des sociétés, comme le prouve le renforcement des inégalités sociales (Beck, 1998; Sørensen \& Christiansen, 2014).

Dans une approche moins ciblée autour de la gestion des risques, différentes publications issues de la sociologie francophone se sont penchées sur le concept de modernité, pour le mettre en lien avec celui d'émergence de l'individu (Bergeron, 2013; Dubet \& Martuccelli, 1996; Le Bart, 2008; Martuccelli, 1999). Selon ces travaux, la modernité est vue comme «l'expérience de vivre dans un monde chaque fois plus étranger, [...]. L'individu ne reconnaît plus le monde qui l'entoure, davantage même : il ne cesse de questionner de façon existentielle (et non seulement conceptuelle) la nature du lien qui le relie à lui.» (Martuccelli, 2009, p. 18). Ce constat a soulevé la question de la place de la réflexivité (ou de la pensée réflexive) autour de l'identité des individus (de la capacité à se reconnaître) comme une forme de socialisation de soi vis-à-vis d'autrui (Bergeron, 2013; Dubar, 2010 [1991]; Goffman, 1975; Martuccelli, 2002). Ainsi, la modernité entre en opposition avec la période traditionnelle qui la précède, où le holisme l'emportait sur l'individualisme: l'individu moderne est plus individualiste que ses pairs des sociétés traditionnelles puisqu'il se centre avant tout sur lui-même (Delory-Momberger, 2009; Dubet \& Martuccelli, 1996).

Cette évolution sociétale a poussé les sociologues à comprendre les phénomènes sociaux contemporains en partant des personnes, parce qu'elles révèlent mieux la société, désormais, que les classes (de plus en plus hétérogènes) ou les institutions d'antan. Rejoignant un constat cher au paradigme du parcours de vie (Kohli, 2007; Mayer, 2004; Sapin et al., 2007), ces différentes approches rappellent qu'une individualisation de l'existence (ou une pluralisation des destinées) est à l'œuvre, devenant un cadre normatif renvoyant chacun à la responsabilité de l'échec ou de la réussite de sa propre vie (Beck, 2001 [1986]). 


\section{Individualisation des parcours : augmentation des choix ou des con- traintes?}

La singularisation des comportements et des parcours individuels, semblant s'émanciper des normes sociales et des positions de classe, s'affiche comme l'expression ultime de l'identité individuelle (Bergeron, 2013; Martuccelli, 2009). Pratiquement, elle signifie qu'à une même position sociale, un acteur peut connaître différents événements et faire différents choix relatifs à ses trajectoires de vie (Martuccelli, 2009). Ainsi, les individus apparaissent à la fois membres du groupe au sens large et affranchis de ses contraintes en ce qui concerne le déroulement de leur trajectoire personnelle, expliquant l'apparente disparition des classes sociales et de leur habitus.

Toutefois, cette autonomie est essentiellement subjective, car certaines pratiques sociales restent dominantes, définies par une stratification sociale toujours de mise. L'individu intériorise ces lois dans une socialisation qui, paradoxalement, encourage l'individualisation ${ }^{1}$. Dans une société qui se complexifie, toujours plus hétérogène en termes de cercles sociaux, l'individu moderne a l'injonction de se singulariser tout en respectant des codes sociaux liés à sa position (Martuccelli, 2005): il est donc loin d'être libre de ses choix. L'analyse selon les classes sociales ne doit donc pas être abandonnée, mais affinée par l'étude de l'individualisation des parcours, afin d'offrir une compréhension des évolutions historiques et des influences qu'elles exercent sur les êtres humains (Martuccelli, 2005). En d'autres mots, réaliser le vieux projet toujours actuel de lier biographies et histoire (Martuccelli, 2009; Mills, 1997 [1959])2.

1 «En raison de la diversité des sous-systèmes sociaux, les individus doivent acquérir des compétences multiples pour faire face à la diversité des actions qu'ils sont tenus d'accomplir. La socialisation conduit vers une individualisation croissante dans la mesure où chaque individu fait partie de cercles d'action différents [...]» (Dubet \& Martuccelli, 1996, p. 514).

2 «Si la notion d'individualisation est souvent employée pour décrire le processus de différenciation croissante des parcours personnels, et donc une réalité observable sur le plan empirique, elle se veut aussi, dans son acception proprement analytique, l'interprétation d'un processus spécifique à la seconde modernité, qui, à la suite d'une série de changements institutionnels, forge les individus en augmentant leurs capacités de réflexion. Autrement dit, et comme Beck le souligne si bien, la thèse de l'individualisation est inséparable de l'émergence d'un nouvel individualisme institutionnalisé. En revanche, l'individuation désigne un processus plus large puisque bien d'autres facteurs structurels, et non le seul travail des institutions au sens fort du terme, sont pris en compte. La dynamique de l'individuation s'efforce ainsi 
Même si les contraintes liées aux normes et à la stratification sociale demeurent, il n'en reste pas moins que donner un sens à son parcours de vie et en être l'acteur premier devient une priorité qui fragilise considérablement les individus (Ehrenberg, 2000 [1998]; Pinçon \& Pinçon-Charlot, 1999). La montée de l'individualisme institutionnel (Beck, 2001 [1986]) - issu de la mise en place des appareils éducatif, juridique, du marché de l'emploi, etc. - contraint les personnes à assumer leurs propres biographies. La complexification des vies s'apparente à une montée des tensions, notamment entre les générations ou cohortes. Il y a ambivalence : chaque événement vécu en appelle à une décision individuelle, mais dénote aussi d'un comportement social (soumis aux exigences des normes et des institutions) (Martuccelli, 2009). Par ailleurs, le risque dont Beck (2001 [1986]) parle s'exprime différemment au sein des diverses classes sociales et de manière individualisée. Ce qui conduit la plupart des individus à connaitre un sentiment de vulnérabilité parce que leur position n'est plus immuable, mais au contraire perpétuellement soumise au changement et à la détérioration (Martuccelli, 2009).

Finalement, l'individualisation sous-entend une nécessité d'autonomisation ou, dit autrement, une injonction contraignante à faire des choix et à assumer la responsabilité de ceux-ci. En demeurant les derniers régisseurs de leurs existences, capables de planifier et de choisir un certain nombre des événements qu'ils rencontrent (voir section 2.4.2) (Clausen, 1995; Elder, 1994; Marshall \& Clarke, 2010; Settersten, 2003), les individus développent réellement une autogestion (Kohli, 2007; Levy \& Bühlmann, 2016) et une réflexion qui leur permet de comprendre les normes alentour et de percevoir les échecs ou les réussites de leur propre parcours (Bergeron, 2013; Cavalli, 2007; Martuccelli, 2009). Ainsi, l'imbrication entre individualisation (injonction à diriger son parcours et en assumer les conséquences) et agency (être acteur de sa vie) est indéniable.

Or, la possibilité de choisir dépend de nombreux facteurs: les ressources à disposition, la liberté réelle d'en user, la latitude pour chacune et chacun de décider ce qui lui convient le mieux (Bonvin, 2005; Bonvin \& Farvaque, 2008; Sen, 1999). En l'absence de ces capabilités, l'agency n'est qu'illusion et la confrontation au réel peut s'avérer source de souffrances (stress, dépression, ...). Dans une société indienne où tant les traditions que les inégalités socioéconomiques sont prégnantes (cf. chapitre 1), il

d'interpréter à l'horizon d'une vie — ou d'une génération — les grandes transformations historiques d'une société.» (Martuccelli, 2009, p.22). 
convient de se demander quelle est la place laissée au choix des événements qui marquent les vies.

\subsubsection{La place de l'individu dans les souvenirs personnels et sociohis- toriques des habitants de Mumbai}

Pour percevoir l'individualisation et l'agency présents dans les réponses à nos trois principales questions, deux typologies ont été utilisées. Concernant la première dimension, la codification a eu pour objectif de déterminer si l'événement cité et la raison pour l'avoir mentionné mettaient ou non en scène le répondant. Selon que la question considérée portait sur la mémoire personnelle de l'individu (volet I et II) ou sa mémoire sociohistorique (volet III), les codes séparaient : respectivement la personne versus son entourage (proches, famille, amis, société), la personne et sa famille versus la société ou la communauté en général. Rappelons que les questions posées s'adressaient à l'interviewé, le choix d'évoquer des souvenirs (personnels ou sociohistoriques) ayant été directement vécu par autrui lui revenait donc.

Les variables indépendantes utilisées dans les analyses qui suivent sont à nouveau les cinq dimensions déjà utilisées dans les chapitres précédents : le groupe d'âge, le sexe, la santé auto-évaluée ainsi que deux variables socioéconomiques, le niveau d'éducation et le lieu de vie.

\section{Individualisation dans les changements sociohistoriques remémorés}

La question portant sur les souvenirs sociohistoriques laisse l'opportunité aux individus de sortir de leur existence pour évoquer des changements plus globaux, au niveau sociétal. Chercher l'individualisation dans les réponses à ce volet de l'étude revient à observer la capacité réflexive des individus, leur aptitude à posséder une vision large de la société, ou au contraire à souligner une compréhension du monde essentiellement centrée autour d'eux-mêmes. Il a déjà été constaté que les contenus des souvenirs sociohistoriques rapportés par les répondants sont très locaux, davantage lorsque leur niveau socioéconomique est bas et lorsqu'ils appartiennent à des groupes plus âgés (voir chapitre 8). Ce résultat se trouve-t-il confirmé lorsque l'on se penche sur l'identité de la (ou des) personne au centre de l'événement ou de ses conséquences? 
Encadré 1: Variable d'individualisation : qui est au centre de l'événement ou de ses conséquences?

La codification des changements sociohistoriques (CSH) et des raisons (RS)

$\underline{0=\text { soi ou sa famille proche }}$

CSH: "Hindu Muslim fights took place"

RS: "It was impossible to get out of the

house, there was a severe shortage of food

and water»

(femme de 70 ans, Bandra)

CSH: "There were floods and water logging"

RS: "I had water in my house."

(homme de 54 ans, Santa Cruz)

CSH: "Daily travel is a hardship"

RS: "I sometimes wonder as to where

all the public comes from"

(femme de 22 ans, Santa Cruz) $\underline{1=}$ autrui (en général, la société)

CSH: "Hindu-muslim Riots"

RS: "Our countries peace was destroyed. Many people were died"

(homme de 65 ans, Bandra)

CSH: "26th july 2005"

RS: "Many people suffered. Many people were drown in water"

(homme de 37 ans, Bandra)

CSH: "There are atrocities against women"

RS: "Because one knows about it in TV and news therein"

(femme de 53 ans, Santa Cruz)

Comme déjà indiqué, la typologie de la variable d'individualisation répartit les changements sociohistoriques et leurs raisons selon que l'individu, ou sa famille proche, se trouvent au sein de la mention (rapporter des souvenirs précis sur le moment, des conséquences immédiates, un impact sur des activités de la vie quotidienne, etc.), ou plutôt si la citation concerne la société plus largement (une identification de classe, des conséquences générales sociétales de l'événement, pas de vécu direct) (cf. encadré 1). Selon cette première subdivision, $47 \%$ des événements mettent l'individu ou ses proches au centre du souvenir, contre $53 \%$ évoquant autrui (seuls 12 changements n'ont pu être codés).

Le tableau 9.1 présente les résultats de plusieurs modèles de régressions logistiques binaires. Il ressort un effet important du lieu d'habitation et du sexe, un faible impact du niveau d'éducation, ainsi que de s'estimer dans un état de santé mitigé. Par contre, l'âge se révèle sans effet significatif, une fois contrôlée l'influence des autres variables.

Résider en bidonville est un facteur diminuant significativement la propension à évoquer un changement sociohistorique ayant une portée sociétale. Ceci corrobore le constat fait au chapitre précédent, qui soulignait les mentions très locales et proches des répondants dans les slums. De leur côté, les femmes sont aussi un peu plus centrées sur elles-mêmes que les hommes, exprimant sans doute en cela leur relégation dans l'espace privé, comparées au genre masculin. 
Posséder un niveau d'éducation primaire ou secondaire diminue légèrement l'évocation d'événements historiques liés à la collectivité par rapport au niveau universitaire et toutes choses égales par ailleurs. En d'autres mots, lorsque l'on contrôle pour l'âge, ceux qui ont un haut niveau de formation ont une mémoire sociohistorique moins centrée sur eux-mêmes. N'avoir que peu d'éducation scolaire, donc de connaissances historiques, signifie être plus touché par les événements de quartier plutôt que nationaux ou mondiaux. En ce sens, se mettre seul en tant qu'individu au centre du souvenir est plus aisé, alors que les personnes très formées ont une vision plus large, englobant la communauté.

Tableau 9.1: rapports de cotes issus de modèles de régression logistique sur la variable «avoir cité un changement sociohistorique mettant le répondant au centre»

\begin{tabular}{|c|c|c|c|c|c|}
\hline & modèle 1 & modèle 2 & modèle 3 & modèle 4 & modèle 5 \\
\hline \multicolumn{6}{|c|}{ Lieu de résidence (référence $=$ Santa Cruz East) } \\
\hline Bandra East & $4.200 * * *$ & $4.196 * * *$ & $4.229 * * *$ & $3.867 * * *$ & $3.866^{* * *}$ \\
\hline \multicolumn{6}{|c|}{ Sexe (référence = hommes) } \\
\hline Femmes & & $1.443 * * *$ & $1.461 * * *$ & $1.410 * * *$ & $1.350 * *$ \\
\hline \multicolumn{6}{|c|}{ Classe d'âge (référence $=20-24$ ans) } \\
\hline $35-39$ ans & & & 1.168 & 1.062 & 1.030 \\
\hline $50-54$ ans & & & 1.126 & 0.958 & 0.903 \\
\hline $65-69$ ans & & & $1.442 *$ & 1.200 & 1.050 \\
\hline $80-84$ ans & & & $1.858 * * *$ & $1.529 *$ & 1.330 \\
\hline \multicolumn{6}{|c|}{ Education (référence $=$ Supérieure) } \\
\hline Sans & & & & 1.295 & 1.204 \\
\hline Primaire & & & & $1.526^{* *}$ & $1.434 *$ \\
\hline Secondaire & & & & $1.248^{\circ}$ & 1.228 \\
\hline \multicolumn{6}{|c|}{ Santé auto-évaluée (référence = Très bonne) } \\
\hline Bonne & & & & & 1.014 \\
\hline Moyenne & & & & & $1.481^{*}$ \\
\hline Mauvaise & & & & & 1.297 \\
\hline
\end{tabular}

Seuils de significativité $:{ }^{\circ} \leq 0.1 ; * \leq 0.05 ; * * \leq 0.01 ; * * \leq 0.001$.

\section{Individualisation dans les changements personnels}

Dans les questions portant sur les événements personnels importants, qu'ils soient récents (volet I) ou concernent l'entièreté de l'existence (volet II), le questionnement autour de l'individualisation porte davantage 
sur l'importance du réseau des proches (des linked lives [Dannefer, 2003; Elder, 1998]) que sur la capacité de saisir la société dans son ensemble. La variable d'individualisation a dès lors été simplement codée selon que l'événement et la raison de l'évoquer correspondaient à l'individu directement, ou plutôt à un autre membre de la famille, à la communauté voire à la société (ces derniers cas de figures sont toutefois rares) ${ }^{3}$.

Tableau 9.2: rapports de cotes issus de modèles de régression logistique sur la variable «avoir cité un changement récent mettant le répondant au centre»

\begin{tabular}{|c|c|c|c|c|c|}
\hline & modèle 1 & modèle 2 & modèle 3 & modèle 4 & modèle 5 \\
\hline \multicolumn{6}{|c|}{ Lieu de résidence (référence $=$ Santa Cruz East) } \\
\hline Bandra East & 0.906 & 0.927 & 0.872 & 0.825 & 0.901 \\
\hline \multicolumn{6}{|c|}{ Sexe (référence $=$ hommes) } \\
\hline Femmes & & 0.865 & 0.910 & 0.875 & 0.915 \\
\hline \multicolumn{6}{|c|}{ Classe d'âge (référence $=20-24$ ans) } \\
\hline $35-39$ ans & & & $0.376^{* * *}$ & $0.366^{* * *}$ & $0.405^{* * *}$ \\
\hline $50-54$ ans & & & $0.259 * * *$ & $0.247 * * *$ & $0.284 * * *$ \\
\hline $65-69$ ans & & & $0.476^{* * *}$ & $0.465^{* * *}$ & $0.602 *$ \\
\hline $80-84$ ans & & & 0.867 & 0.789 & 1.058 \\
\hline \multicolumn{6}{|c|}{ Education (référence $=$ Supérieure) } \\
\hline Sans & & & & $1.652^{\circ}$ & $1.687^{\circ}$ \\
\hline Primaire & & & & 0.874 & 0.896 \\
\hline Secondaire & & & & 1.095 & 1.069 \\
\hline \multicolumn{6}{|c|}{ Santé auto-évaluée (référence = Très bonne) } \\
\hline Bonne & & & & & 0.769 \\
\hline Moyenne & & & & & $0.513 * *$ \\
\hline Mauvaise & & & & & $0.503 *$ \\
\hline
\end{tabular}

Seuils de significativité ${ }^{\circ} \leq 0.1 ; * \leq 0.05 ; * \leq 0.01 ; * * \leq 0.001$.

Le tableau 9.2 annonce les rapports de cotes de plusieurs modèles de régressions sur une variable ciblant la citation d'un changement récent mettant le répondant au centre du souvenir (soit $70 \%$ des changements récents, 10 seulement n'ayant pu être classés).

3 Il est arrivé que la codification ne soit pas si simple. Ainsi, lors de l'évocation de son propre mariage, il a été décidé d'attribuer le changement à la catégorie «ego» - bien que l'on sache que des implications sont aussi notables pour la famille du répondant -, alors que la citation de la naissance de son enfant a été attribuée à la catégorie plus large «famille et société», malgré l'évident impact sur la vie de l'interviewé. 
Alors que dans l'analyse précédente, sur les changements sociohistoriques, leur impact était inexistant ou peu clair, ce sont ici la classe d'âge et la santé auto-évaluée qui ressortent comme les principales variables explicatives. Les trentenaires (37\%), les quinquagénaires (46\%) et dans une moindre mesure les sexagénaires $(32 \%)$ ont une propension beaucoup plus affirmée à parler de leurs proches que les plus jeunes, qui apparaissent très centrés sur eux-mêmes (dans $83 \%$ des cas) autant que les octogénaires $(80 \%)$. Ce constat résonne fortement avec la théorie de l'individualisation en ce qui concerne la cohorte des 20-24 ans, mais doit être nuancé en considérant les réalités du parcours de vie. En effet, comme cela a été constaté aux chapitres 4 et 6 , la majorité des événements familiaux (mariages des enfants et naissances) ont été évoqués par des trentenaires ou des quinquagénaires, alors que la cohorte dans la vingtaine n'a pas encore connu ces événements démographiques. Il n'est donc pas réellement surprenant que ce soient également ces derniers qui mentionnent plus facilement des changements les concernant directement plutôt que leur famille ou amis.

Quant à la santé auto-évaluée, elle montre un phénomène auquel on ne s'attendait pas. En effet, ce sont ceux se considérant dans un état sanitaire moyen ou déplorable qui mentionnent près de deux fois plus d'événements concernant autrui, toutes choses égales par ailleurs. Cela illustre possiblement leur dépendance aux autres, à celles et ceux sur lesquels ils doivent s'appuyer pour survivre.

Dans le tableau 9.3, ce sont les résultats des mêmes analyses sur la variable d'individualisation, mais portant cette fois sur les grands tournants de l'existence, qui sont exposés ( $57 \%$ des tournants mettent l'individu au centre, contre $43 \%$ les proches et la communauté, et 20 événements n'ont pu être codés). Ils sont quelque peu différents par rapport à ceux issus des analyses sur les changements récents. En effet, toutes les variables, à l'exception du niveau d'éducation, sont significatives. Habiter à Santa Cruz accroit la propension à parler d'autrui. Être une femme signifie près de deux fois moins de chance de mentionner un événement portant sur sa propre vie. Cette différence n'était pas présente dans la question sur les changements récents. La responsabilité du foyer et de l'éducation des enfants incombant majoritairement aux femmes, ainsi que leur moindre accès au marché du travail ou à la scolarisation, expliquent largement ce constat qui émerge lorsque l'on explore la mémoire longue. D'ailleurs, l'effet d'âge, donc de durée à considérer, est fort. Toutes les cohortes montrent entre 1.5 et 2 fois plus de chance de parler d'un changement 
concernant autrui que les plus jeunes. Tout comme le fait de s'estimer en bonne, moyenne ou mauvaise santé, par rapport à se considérer en très bonne santé.

En somme, hormis la différence - somme toute peu marquée - entre le quartier de classes moyennes inférieures et de bidonvilles, tous ces résultats suggèrent que les individus en situation défavorables - femmes, âgés, affectés dans leur santé, et même ceux sans éducation - parlent plus des autres que d'eux-mêmes. Sans que l'approche quantitative puisse le prouver, cela suggère à nouveau la dépendance aux autres de ces êtres fragilisés par leur position sociale et la précarité de leurs ressources, qui ne leur laissent guère d'opportunité de s'offrir le luxe de l'individualisme.

A côté, semble se détacher une "génération de l'individualisme», qui réaffirme l'hypothèse d'une individualisation récente des parcours de vie dans une ville comme Mumbai, parmi les jeunes, mieux dotés en capital humain, plus dans des quartiers de classe moyenne inférieure que dans les bidonvilles et à condition d'être en bonne forme physique, ce qui tend à aller de pair avec la jeunesse.

Tableau 9.3: rapports de cotes issus de modèles de régression logistique sur la variable «avoir cité un tournant mettant le répondant au centre»

\begin{tabular}{|c|c|c|c|c|c|}
\hline & modèle 1 & modèle 2 & modèle 3 & modèle 4 & modèle 5 \\
\hline \multicolumn{6}{|c|}{ Lieu de résidence (référence $=$ Santa Cruz East) } \\
\hline Bandra East & 1.126 & $1.180^{\circ}$ & 1.158 & $1.231 *$ & $1.283^{*}$ \\
\hline \multicolumn{6}{|c|}{ Sexe (référence = hommes) } \\
\hline Femmes & & $0.482 * * *$ & $0.468 * * *$ & $0.487 * * *$ & $0.505^{* * *}$ \\
\hline \multicolumn{6}{|c|}{ Classe d'âge (référence $=20-24$ ans) } \\
\hline 35-39 ans & & & $0.548 * * *$ & $0.570 * * *$ & $0.636 * *$ \\
\hline $50-54$ ans & & & $0.465 * * *$ & $0.495 * * *$ & $0.560 * * *$ \\
\hline $65-69$ ans & & & $0.336 * * *$ & $0.367 * * *$ & $0.428 * * *$ \\
\hline $80-84$ ans & & & $0.456 * * *$ & $0.516 * * *$ & $0.614 * *$ \\
\hline \multicolumn{6}{|c|}{ Education (référence = Supérieure) } \\
\hline Sans & & & & 0.774 & 0.814 \\
\hline Primaire & & & & 0.891 & 0.930 \\
\hline Secondaire & & & & 0.979 & 1.011 \\
\hline \multicolumn{6}{|c|}{ Santé auto-évaluée (référence $=$ Très bonne) } \\
\hline Bonne & & & & & $0.555 * * *$ \\
\hline Moyenne & & & & & $0.555^{* * *}$ \\
\hline Mauvaise & & & & & $0.468 * *$ \\
\hline
\end{tabular}

Seuils de significativité ${ }^{\circ} \leq 0.1 ; * \leq 0.05 ; * * 0.01 ; * * * \leq 0.001$. 


\subsection{3 Être l'acteur des changements de sa vie : une capabilité réservée aux moins vulnérables?}

Pour compléter ces premiers résultats, nous nous sommes penchés sur l'aspect d'agency présent dans les événements personnels. Par définition, un changement sociohistorique est imprévu et ne découle pas de la décision d'un individu; il n'apparaît donc pas opportun de réaliser cette analyse sur le troisième volet du questionnaire. Les changements récents de la vie et les grands tournants de l'existence ont été classés selon qu'ils étaient explicitement subis ou choisis par la personne les ayant vécus ${ }^{4}$. Les cas douteux ont été mis de côté, mais sont assez peu nombreux $(6 \%$ des changements récents et $12 \%$ des tournants).

\section{L'agency dans les changements récents}

Le tableau 9.4 présente les résultats de plusieurs modèles de régressions logistiques binaires portant sur la variable dépendante : avoir cité un changement récent choisi. Seules $6 \%$ des réponses de ce type n'ont pu être classifiés. Parmi les autres, 39\% expriment un choix, 61\% rapportent un événement subi.

Comme dans l'analyse concernant l'individualisation, le lieu de résidence et le sexe ne montrent pas d'effet significatif. Inversement, appartenir au groupe d'âge le plus jeune offre un risque bien inférieur d'évoquer un événement subi par rapport aux autres cohortes et toutes choses égales par ailleurs. Au niveau de l'éducation, seul l'item primaire ressort comme ayant un impact statistiquement significatif sur la mention d'un changement récent choisi, mais la tendance générale est bien que les individus détenteurs d'un diplôme supérieur subissent moins la vie que les autres. Il en va de même pour les personnes qui se considèrent comme étant en très bonne santé.

Les souvenirs personnels récents choisis sont davantage évoqués par les plus jeunes, dont on sait par ailleurs qu'ils ont (eu) un meilleur accès à l'éducation que les cohortes suivantes, et qui bien souvent ne connaissent

4 Nous entendons par là le fait que cette personne ait eu un contrôle sur la survenue de l'événement, ou pas. Certains événements ne présentaient aucune difficulté de classement (les changements du domaine de la santé ont ainsi presque tous été classés comme subis, à l'exception des opérations volontaires par exemple), alors que d'autres étaient plus ambivalents. Par exemple, les mariages étaient par défaut placés dans la catégorie choisis (y compris les mariages arrangés), à moins qu'une absence de volonté (voire une opposition de l'individu) ne soit explicitement énoncée. 
pas encore les contraintes de la vie familiale. A l'inverse, les personnes âgées sont les plus tributaires des mentions de changements récents subis, et ce sans guère de doute en lien avec leur santé déclinante. Ce dernier résultat est en effet confirmé par ce qui ressort de la santé auto-évaluée.

Tableau 9.4: rapports de cotes issus de modèles de régression logistique sur la variable «avoir cité un changement récent choisi»

\begin{tabular}{|c|c|c|c|c|c|}
\hline & modèle 1 & modèle 2 & modèle 3 & modèle 4 & modèle 5 \\
\hline \multicolumn{6}{|c|}{ Lieu de résidence (référence $=$ Santa Cruz East) } \\
\hline Bandra East & 1.135 & 1.148 & 0.955 & 1.043 & 1.137 \\
\hline \multicolumn{6}{|c|}{ Sexe (référence = hommes) } \\
\hline Femmes & & 0.931 & 1.018 & 1.055 & 1.125 \\
\hline \multicolumn{6}{|c|}{ Classe d'âge (référence $=20-24$ ans) } \\
\hline 35-39 ans & & & $0.400 * * *$ & $0.449 * * *$ & $0.497 * *$ \\
\hline $50-54$ ans & & & $0.215 * * *$ & $0.254 * * *$ & $0.292 * * *$ \\
\hline $65-69$ ans & & & $0.099 * * *$ & $0.127 * * *$ & $0.171 * * *$ \\
\hline $80-84$ ans & & & $0.072 * * *$ & $0.091 * * *$ & $0.127 * * *$ \\
\hline \multicolumn{6}{|c|}{ Education (référence $=$ Supérieure) } \\
\hline Sans & & & & 0.864 & 0.980 \\
\hline Primaire & & & & $0.541^{*}$ & $0.583 *$ \\
\hline Secondaire & & & & 0.859 & 0.857 \\
\hline \multicolumn{6}{|c|}{ Santé auto-évaluée (référence = Très bonne) } \\
\hline Bonne & & & & & $0.664^{\circ}$ \\
\hline Moyenne & & & & & $0.470 * *$ \\
\hline Mauvaise & & & & & $0.251^{* *}$ \\
\hline
\end{tabular}

Seuils de significativité $:{ }^{\circ} \leq 0.1 ; * \leq 0.05 ; * * \leq 0.01 ; * * * \leq 0.001$.

\section{L'agency dans les grands tournants de l'existence}

Le tableau 9.5 expose les rapports de cotes de modèles de régression logistique sur la variable d'agency, effectués cette fois sur les tournants de l'existence rapportés par les répondants. A nouveau, 39\% des tournants sont choisis contre $61 \%$ subis ; $12 \%$ des événements ont été sortis de l'analyse car indéterminés.

De manière attendue, plus le répondant est âgé et plus sa trame de vie a été affectée par des événements subis. Mais cet effet d'âge est largement capturé par les variables ajoutées dans les modèles 4 et 5 . Ceci suggère qu'il ne s'agit pas tant d'un effet de cohorte, que l'on pourrait attribuer à des traits de modernisation récente de l'Inde, que de la distribution différenciée des ressources dans les différents groupes d'âges observés, en 
l'occurrence l'éducation et la santé. En effet, tous les stades de formation inférieurs au niveau tertiaire sont significativement plus à risque d'évoquer un tournant subi, de même que les personnes en mauvaise santé. Cette logique s'applique aussi à l'écart entre hommes et femmes.

De manière cohérente, même si la distance se réduit au fur et à mesure de l'addition de variables explicatives, les tournants de l'existence sur lesquels les individus n'ont pas eu de prise ont plus marqué la mémoire dans les bidonvilles de Bandra East que dans le quartier de classe moyenne inférieure, à Santa Cruz East.

Tableau 9.5: rapports de cotes issus de modèles de régression logistique sur la variable «avoir cité un tournant choisi»

\begin{tabular}{|c|c|c|c|c|c|}
\hline & modèle 1 & modèle 2 & modèle 3 & modèle 4 & modèle 5 \\
\hline \multicolumn{6}{|c|}{ Lieu de résidence (référence $=$ Santa Cruz East) } \\
\hline Bandra East & $0.568 * * *$ & $0.578 * * *$ & $0.564 * * *$ & $0.724 * *$ & $0.755^{*}$ \\
\hline \multicolumn{6}{|c|}{ Sexe (référence = hommes) } \\
\hline Femmes & & $0.747 * *$ & $0.724 * *$ & 0.869 & 0.915 \\
\hline \multicolumn{6}{|c|}{ Classe d'âge (référence $=20-24$ ans) } \\
\hline 35-39 ans & & & $0.699 *$ & 0.878 & 1.000 \\
\hline $50-54$ ans & & & $0.537 * * *$ & 0.804 & 0.929 \\
\hline $65-69$ ans & & & $0.342 * * *$ & $0.574 * *$ & $0.685^{\circ}$ \\
\hline $80-84$ ans & & & $0.344 * * *$ & $0.629 *$ & 0.768 \\
\hline \multicolumn{6}{|c|}{ Education (référence $=$ Supérieure) } \\
\hline Sans & & & & $0.351 * * *$ & $0.364 * * *$ \\
\hline Primaire & & & & $0.349 * * *$ & $0.362 * * *$ \\
\hline Secondaire & & & & $0.605^{* * *}$ & $0.617 * * *$ \\
\hline \multicolumn{6}{|c|}{ Santé auto-évaluée (référence = Très bonne) } \\
\hline Bonne & & & & & $0.595^{* *}$ \\
\hline Moyenne & & & & & $0.540 * *$ \\
\hline Mauvaise & & & & & $0.540 *$ \\
\hline
\end{tabular}

Seuils de significativité ${ }^{\circ} \leq 0.1 ; * \leq 0.05 ; * * \leq 0.01 ; * * \leq 0.001$.

$* * *$

Le tableau 9.6 offre un résumé comparatif des résultats sur l'individualisation et l'agency dans la mémoire courte (l'année écoulée) ou longue (l'ensemble de la vie). A l'exception de la question portant sur les événements récents, les résultats sur la variable d'individualisation montrent une différence notable liée au lieu de résidence. Les habitants des bidonvilles évoquent nettement plus d'événements sociohistoriques ou de tournants 
centrés sur eux-mêmes que les résidents d'un quartier de classe moyenne inférieure. Concernant la variable d'agency, les grandes transitions de la vie sont plus subies par les habitants de Bandra que par ceux de Santa Cruz, où se perçoit davantage la capacité de choix derrière les événements rapportés. En outre, ce sont aussi les individus avec une éducation tertiaire qui évoquent le plus de tournants choisis. Nous pouvons conclure que le niveau socioéconomique a un impact fondamental sur la capacité d'agency et de réflexivité sur les changements marquants de l'existence.

Tableau 9.6: résumé des modèles de régression concernant les variables d'individualisation et d'agency

\begin{tabular}{|c|c|c|c|c|c|}
\hline & $\begin{array}{l}\text { individ. } \\
\text { (volet III) }\end{array}$ & $\begin{array}{l}\text { individ. } \\
\text { (volet I) }\end{array}$ & $\begin{array}{l}\text { individ. } \\
\text { (volet II) }\end{array}$ & $\begin{array}{l}\text { agency } \\
\text { (volet I) }\end{array}$ & $\begin{array}{l}\text { agency } \\
\text { (volet II) }\end{array}$ \\
\hline \multicolumn{6}{|c|}{ Lieu de résidence (référence $=$ Santa Cruz East) } \\
\hline Bandra East & $>* * *$ & - & $>*$ & - & $<*$ \\
\hline \multicolumn{6}{|c|}{ Sexe (référence $=$ hommes) } \\
\hline Femmes & $>* * *$ & - & $<* * *$ & - & - \\
\hline \multicolumn{6}{|c|}{ Classe d'âge (référence $=20-24$ ans) } \\
\hline 35-39 ans & - & $<* * *$ & $<* *$ & $<* *$ & - \\
\hline $50-54$ ans & - & $<* * *$ & $<* * *$ & $<* * *$ & - \\
\hline $65-69$ ans & - & $<*$ & $<* * *$ & $<* * *$ & $<\circ$ \\
\hline $80-84$ ans & - & - & $<* *$ & $<* * *$ & - \\
\hline \multicolumn{6}{|c|}{ Education (référence $=$ Supérieure) } \\
\hline Sans & - & $>0$ & - & - & $<* * *$ \\
\hline Primaire & $>*$ & - & - & $<*$ & $<* * *$ \\
\hline Secondaire & - & - & - & - & $<* * *$ \\
\hline \multicolumn{6}{|c|}{ Santé auto-évaluée (référence $=$ Très bonne) } \\
\hline Bonne & - & - & $<* * *$ & $<$ & $<* *$ \\
\hline Moyenne & $>*$ & $<* *$ & $<* * *$ & $<* *$ & $<* *$ \\
\hline Mauvaise & - & $<*$ & $<* * *$ & $<* *$ & $<*$ \\
\hline
\end{tabular}

Seuils de significativité ${ }^{\circ} \leq 0.1 ; * \leq 0.05 ; * * \leq 0.01 ; * * * 0.001$.

Les variables démographiques d'âge et de sexe possèdent des impacts divers. La question du genre n'influence pas la citation d'un événement subi ; ce qui, dans le contexte indien, aurait pourtant été un résultat attendu. Inversement, être une femme incite à mentionner des tournants concernant ses proches (davantage que les hommes), alors qu'être un homme encourage à évoquer des changements sociohistoriques ayant une portée sociétale (plus que les femmes). En ce sens, les normes de genre concernant la sphère privée et la sphère publique sont respectées. 
Par ailleurs, un effet d'âge très net ressort en ce qui concerne les deux questions sur la vie personnelle et la centration sur soi-même : les jeunes dénotent d'une individualisation plus marquée que leurs prédécesseurs. A propos des changements récents, les répondants dans la vingtaine évoquent nettement plus d'événements choisis que les cohortes plus âgées.

Le manque de ressources et les conditions de vie plus défavorables limitent la réflexivité et l'agency des individus. La biographisation des parcours démontre donc une croissance de la vulnérabilité (Spini et al., 2017), qui se traduit par une augmentation des pressions et du stress (Oris et al., 2016; Pearlin, 1989; Spini et al., 2013). C'est précisément autour de la question du stress que porte la section qui suit.

\subsection{Stress et imprévisibilité dans les tournants de l'existence}

La section qui précède a mis l'accent sur l'individualisation et l'agency présentes dans les réponses des individus interrogés au cours des deux terrains d'enquête. Concrètement, les changements de l'existence, selon qu'ils sont choisis ou subis, de portée très individualiste ou plutôt sociétale, se traduisent par plus ou moins de stress, par une perte ou un gain de bienêtre pour les individus (Pearlin, 1989; Pearlin et al., 1981).

Une majorité des transitions étudiées en Occident se distingue par un caractère attendu dans la mesure où, bien qu'elles résultent de décisions individuelles, ces dernières sont le produit de décisions dictées par les normes sociales (Cavalli et Lalive d'Epinay, 2008; Hareven \& Masaoka, 1988). Cependant, de nombreux tournants divergent des codes établis et deviennent des moments particuliers d'exposition aux risques car ils impliquent une incertitude pour l'existence, en vertu de leur caractère négatif, imprévisible, irréversible, rapide ou au contraire graduel, trop précoce ou trop tardif, etc. (Bessin, 2009; Bessin et al., 2010; Grossetti, 2010; Hélardot, 2010; Hughes, 1996; Reese et Smyer, 1983).

Le stress issu des changements peut provenir de diverses origines: un événement critique entraînant un changement dans la vie de la personne (une transition); un stress chronique (un problème récurrent tel qu'une maladie); une difficulté quotidienne mineure; l'absence d'un événement attendu ou encore sa survenue hors-timing (Elder, 1975; Neugarten, 
Moore, \& Lowe, 1965; Spini et al., 2013). La non survenue d'un événement (comme, par exemple, ne pas pouvoir suivre d'études), empêchant la modification de la trajectoire, peut également être source d'une perte de bien-être (Hélardot, 2010; Leclerc-Olive, 1998, 2010). Néanmoins, l'impact de tels événements peut être médiatisé en fonction des ressources que les personnes ont à disposition pour y répondre, voire des moyens fournis par la société pour les mettre en œuvre (Pearlin, 1989; Sen, 1999). Dans cette configuration, le genre possède une influence importante, les femmes subissant davantage de contraintes, ce qui les rend plus à risque de subir du stress (Pearlin, 1989), ce dans la plupart des sociétés, notamment en Inde (McLeod \& Kessler, 1990; Pearlin et al., 2005).

En ce sens, le concept de transition est central dans la compréhension du stress qui influence les trajectoires individuelles, d'autant plus lorsque celles-ci ne sont pas normatives (Chiriboga, 1995). C'est pour cette raison que les sociologues qui s'intéressent au stress et à ses causes se sont dirigés vers l'étude des transitions (George, 1993; Pearlin, 1989). Dans la section qui suit, nous nous penchons exclusivement sur les grands tournants évoqués par les répondants, avant de prendre en compte les raisons de les citer, afin d'analyser le stress et l'imprévisibilité qu'ils peuvent receler.

\subsubsection{Vulnérabilité des tournants marquants de la vie : changements, états et carences}

Une typologie a été élaborée afin de prendre en compte la forme des grands tournants de la vie (volet II) rapportés par les répondants, en termes de degré de modification de la trajectoire. L'idée consiste à séparer les réponses - sans utiliser encore les raisons - comprenant un «changement» explicite, en opposition à celles qui évoquent une situation de vie ou un «état». Déjà, la forte présence de ces dernières surprend puisque la question portait explicitement sur des «événements» ou des «changements» de l'existence. Or, une partie des réponses fait sans l'ombre d'un doute référence à une temporalité longue, loin de correspondre à une bifurcation ou à une rupture. Dans ces mentions, aucune transformation du parcours de vie n'est discernable et la durée de l'événement cité est généralement longue (voir encadré 2). Plus que des tournants, ce sont en fait des étapes du parcours de vie qui sont citées. 


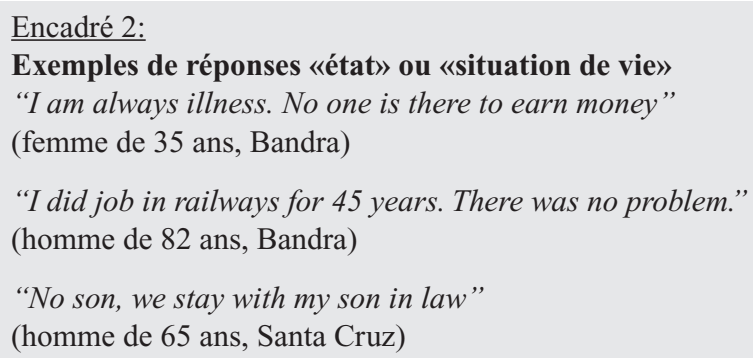

En outre, à ce stade est aussi apparue une nouvelle catégorie qui a dû être classifiée à part : la «non survenue» d'un changement souhaité ou attendu. Effectivement, un certain nombre des réponses données ne se réfère pas à un événement de la vie des personnes, mais explicitement à son absence (voir encadré 3). Mentionner ce qui n'a pas eu lieu manifeste le regret d'un déclencheur qui, lui, aurait pu signifier une évolution. Cette catégorie est particulière car se presse en toile de fond le changement qui, s'il était survenu, aurait modifié la trame de vie.

L'exercice de citer un moment charnière de la vie a quand même été entendu pour $82 \%$ des tournants mentionnés (79\% à Bandra et $85 \%$ à Santa Cruz $)^{5}$. En ce qui concerne l'évocation d'une «absence de tournant», elle rassemble $3 \%$ des réponses à ce second volet $(n=60)$, plus notablement à Bandra $(4 \% ; n=47)$ qu'à Santa Cruz $(1 \% ; n=13)$. Il paraît indiscutable que celle-ci s'ancre dans la condition vulnérable des individus. Les personnes interrogées ressentent la privation d'un élément comme ayant affecté durablement leur vie.

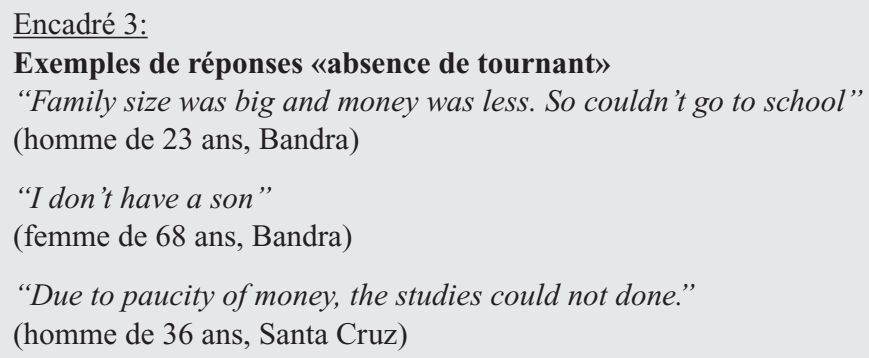

5 Par ailleurs, 90\% des individus ont cité au moins un tournant comportant une idée de changement. 
De leur côté, les mentions entrant dans la catégorie «état» représentent $15 \%$ des tournants, quasiment similaires entre les terrains d'enquête (17\% à Bandra et $14 \%$ à Santa Cruz). Mais ce sont près de $40 \%$ des répondants qui ont évoqué au moins une situation de vie plutôt qu'un tournant ${ }^{6}$. C'est d'autant plus remarquable que partout où l'enquête CEVI a été réalisée, en Europe et dans les Amériques, nulle part il n'a été utile de créer un code $a d$ hoc pour ce type de réponse, qui est donc une spécificité indienne. Ces déclarations peuvent être interprétées de deux manières : la perception par l'enquêté de son propre parcours de vie ne cible pas forcément des moments charnières mais davantage des périodes de stabilité, déterminantes dans des vies marquées par les aléas du quotidien. Ainsi, la situation précaire des existences et la variabilité de celles-ci se traduisent par une perception plus marquée des phases planes que des changements.

En second lieu, il est possible que, au moment de répondre à la question, les participants indiens n'aient pas nécessairement perçu les transitions mais aient pris aussi en compte les étapes durables de leur vie. Mauvaise interprétation de la question, ou révélation d'une perception singulière de sa vie construite par un contexte spécifique ? Nous penchons pour la seconde option car la traduction de la question dans les langues locales a été vérifiée et les enquêteurs formés. En outre, les raisons avancées pour justifier ces mentions sont cohérentes, comme nous le verrons plus précisément ci-dessous.

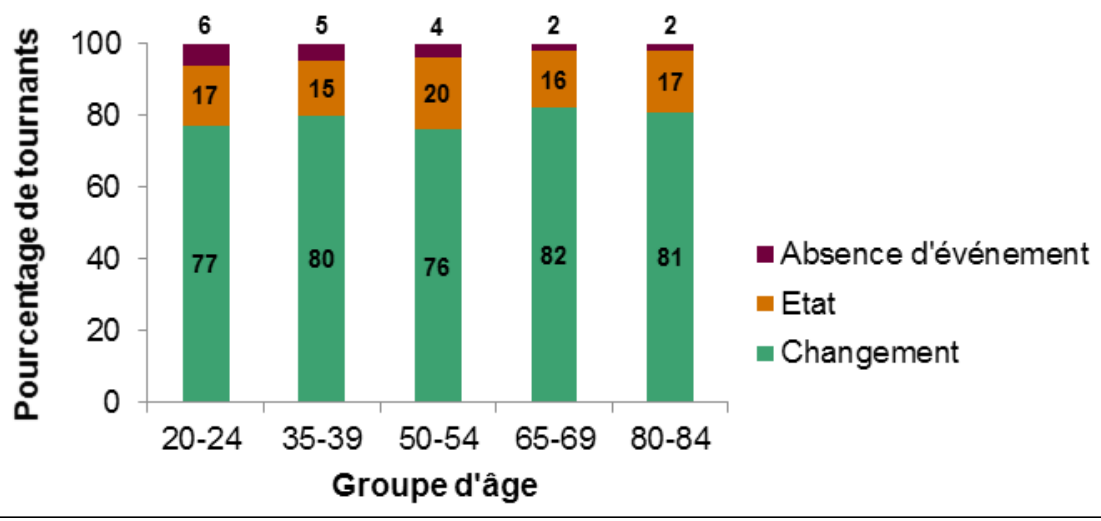

Figure 9.1: le pourcentage de tournants à Bandra, selon la classe d'âge

6 Seules 42 personnes parmi ces quelque 40\% mentionnent uniquement des états (soit $8 \%$ de l'échantillon total). 


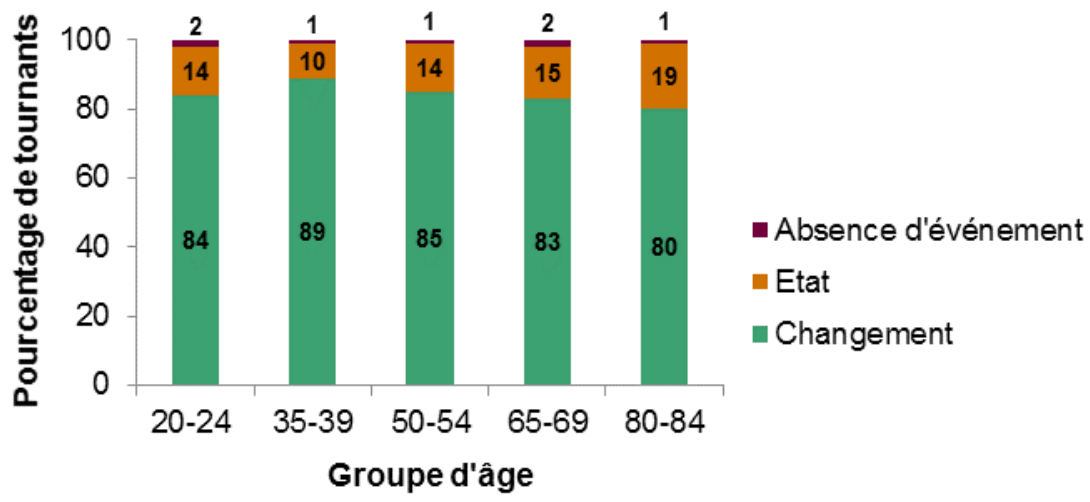

Figure 9.2: le pourcentage de tournants à Santa Cruz, selon la classe d'âge

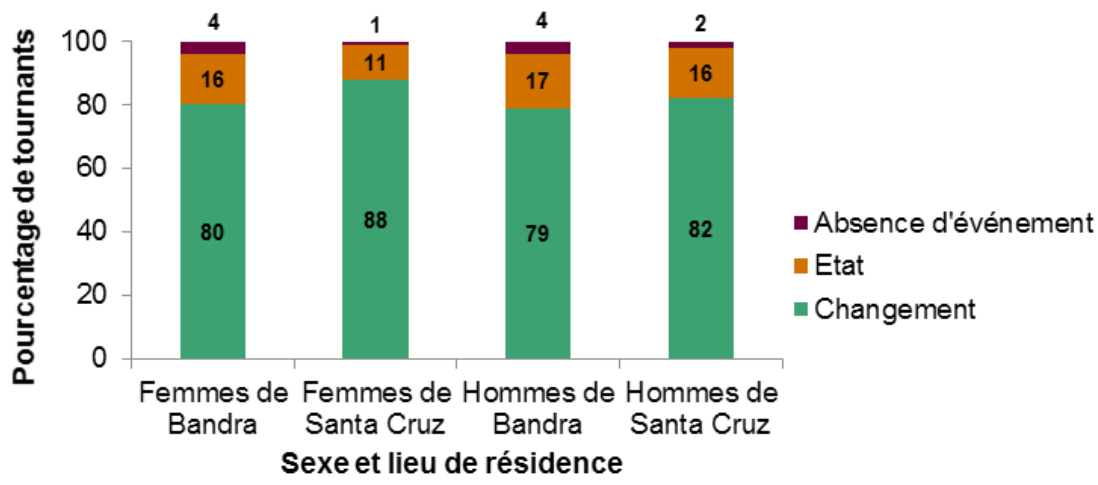

Figure 9.3: le pourcentage de tournants, selon le sexe et le lieu de vie

La proportion d'individus donnant des réponses contenant un «changement» ou un «état» est similaire entre les classes d'âge et le sexe (cf. figures 9.1 à 9.3). Les réponses «absence de tournant» sont prédominantes parmi les trois plus jeunes cohortes interrogées dans les bidonvilles, peutêtre davantage conscientes des privations qu'elles subissent, ou plus frustrées de les vivre dans le contexte d'un pays désormais en émergence. Mais il ne faut pas sur-interpréter cette distribution car globalement elles restent rares. L'écart le plus net est entre Bandra et Santa Cruz, les «absences de tournant» et surtout les «états» étant plus nombreux dans les bidonvilles. 
Lorsque cette nouvelle typologie de tournants est croisée avec le contenu rapporté (soit les domaines de la vie auquel ils appartiennent), des divergences importantes ressortent (figure 9.4).

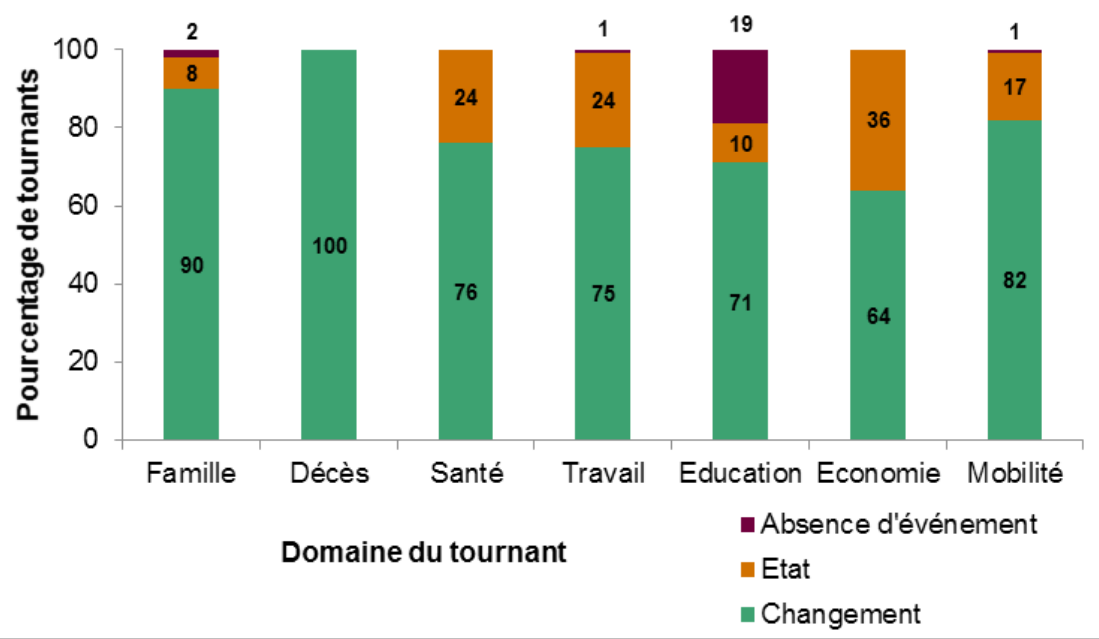

Figure 9.4: le pourcentage de tournants, selon les sept domaines les plus mentionnés

Les domaines famille et décès sont particulièrement représentés parmi les tournants au sens strict ( 90 et 100\%). Effectivement, autant les mariages, les naissances que la perte d'un proche sont situés à une date précise dans le temps ; de plus, il est facile de discerner un avant et un après. C'est également le cas des déménagements (domaine mobilité) (82\%). Concernant les états, ce sont davantage des situations de santé (comme une maladie chronique), professionnelles (des périodes d'emploi stable par exemple) ou économique (pauvreté ou au contraire stabilité financière) qui sont mentionnés (respectivement 24, 24 et 36\%). Enfin, l'absence de tournant concerne majoritairement le domaine de l'éducation (19\%): essentiellement la difficulté d'accès (pour des raisons structurelles - école trop éloignée, etc. - mais surtout économiques) à la scolarité. Dans une faible mesure, certaines situations familiales (la vaine attente d'une naissance notamment) ont également suscité l'évocation d'une carence de survenue d'un événement. 


\subsubsection{Les différentes formes de stress présentes dans les raisons de citer un tournant}

Pour affiner ces constats, une classification des raisons a été établie. Le résultat de cet exercice est ramassé en quatre catégories qui se comprennent ainsi (voir encadré 4): les raisons «positives» et «négatives» sont celles où est clairement évoquée une évaluation du changement de la part des individus. Appuyée sur les recherches intégrant les études de vulnérabilité et du parcours de vie (Spini et al., 2013), la catégorie «stress chronique» se réfère à l'évocation d'un stress persistant à la suite ou précédant le changement, l'état ou l'absence cité. Enfin, les raisons hors-timing définissent les cas où les individus se percevaient comme trop âgés ou trop jeunes au moment du tournant. A partir des justifications énoncées par les individus, la qualification du tournant a donc été déduite a posteriori.

\begin{tabular}{|c|c|}
\hline Encadré 4: & \\
\hline$\overline{\text { Exemples de raisons positives }}$ & Exemples de raisons négatives \\
\hline "Then I got job I was happy for that" & "Now I feel my life is wasted" \\
\hline (homme de 26 ans, Bandra) & (femme de 37 ans, Bandra) \\
\hline "I married the boy of my choice" & "He died suddenly so the family was disturbed" \\
\hline (femme de 22 ans, Santa Cruz) & (femme de 54 ans, Santa Cruz) \\
\hline $\begin{array}{l}\text { "To buy a car was my dream" } \\
\text { (homme de } 70 \text { ans, Santa Cruz) }\end{array}$ & $\begin{array}{l}\text { "I am very sad because I couldn't save her" } \\
\text { (homme de } 84 \text { ans, Bandra) }\end{array}$ \\
\hline Exemples de raisons stress chronique & Exemples de raisons hors-timing \\
\hline $\begin{array}{l}\text { "My education is incomplete" } \\
\text { (homme de } 50 \text { ans, Santa Cruz) }\end{array}$ & $\begin{array}{l}\text { "I delivered a baby boy after } 10 \text { years of marriage" } \\
\text { (femme de } 38 \text { ans, Bandra) }\end{array}$ \\
\hline "Because of poverty" & "I started doing job when I was in playing age" \\
\hline (homme de 39 ans, Bandra) & (homme de 65 ans, Bandra) \\
\hline «My responsibility increased» & «My marriage was late» \\
\hline (femme de 64 ans, Bandra) & (homme de 54 ans, Santa Cruz) \\
\hline
\end{tabular}

De manière marquante, les raisons positives arrivent en tête (43\%), suivies par le stress chronique (28\%), puis par les raisons négatives $(25 \%)$ et enfin celles que les individus ont évoquées lorsqu'ils ont jugé leur âge au moment de la survenue comme hors de la norme (4\%). Les études antérieures sur l'évaluation des transitions de la vie ont montré que le souvenir des tournants de la vie était surtout positif (Birren, \& Schroots, 2006). Même parmi les habitants de bidonvilles de Mumbai, cette tonalité est bien présente, ce qui est en soi remarquable; toutefois, cette 
appréciation heureuse est balancée par l'expression particulièrement forte des conditions de vie difficiles (contraintes chroniques), expression qui met en évidence la pression continue que vivent les individus. L'addition du stress et du clairement négatif grimpe à 53\% des raisons évoquées, soit davantage que le positif.

Le croisement de cette typologie avec les groupes d'âge (cf. figures 9.5 et 9.6) montre sans trop de surprise que les jeunes sont plus positifs que leurs aînés. Les personnes âgées sont davantage affectées par des transitions qui surviennent hors-timing, peut-être parce que les transformations dans le calendrier des étapes de vie qui se sont produites en Inde durant le cours de leur existence leur donne une plus grande sensibilité à cet aspect. Au début de la période adulte, les 35-39 ans mentionnent davantage de stress chronique, ce qui peut être lié au développement de leur vie familiale et aux responsabilités qui en découlent (éducation des enfants, épargne pour assurer les coûts d'un mariage, voire simplement parvenir à nourrir sa famille).

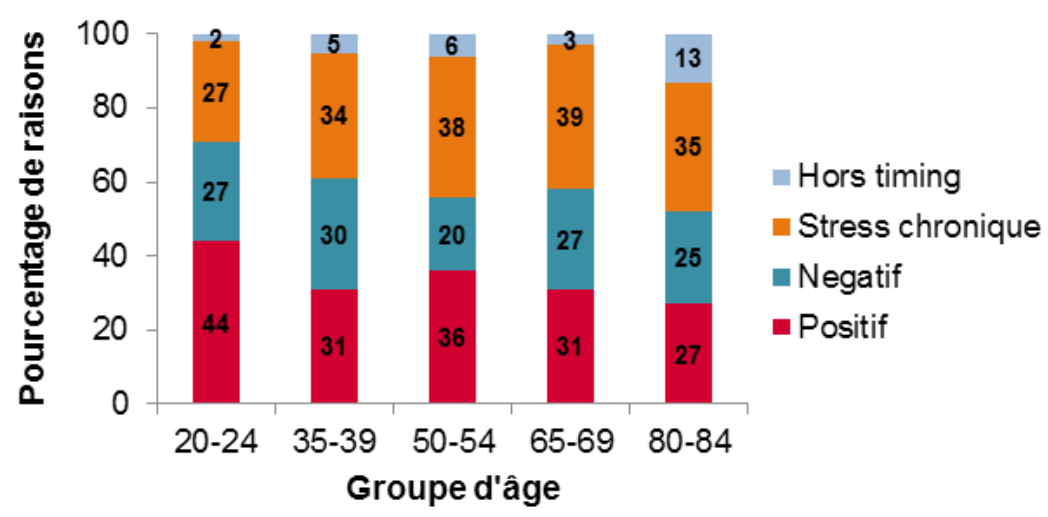

Figure 9.5: les raisons des tournants (\%) à Bandra, selon la classe d'âge 


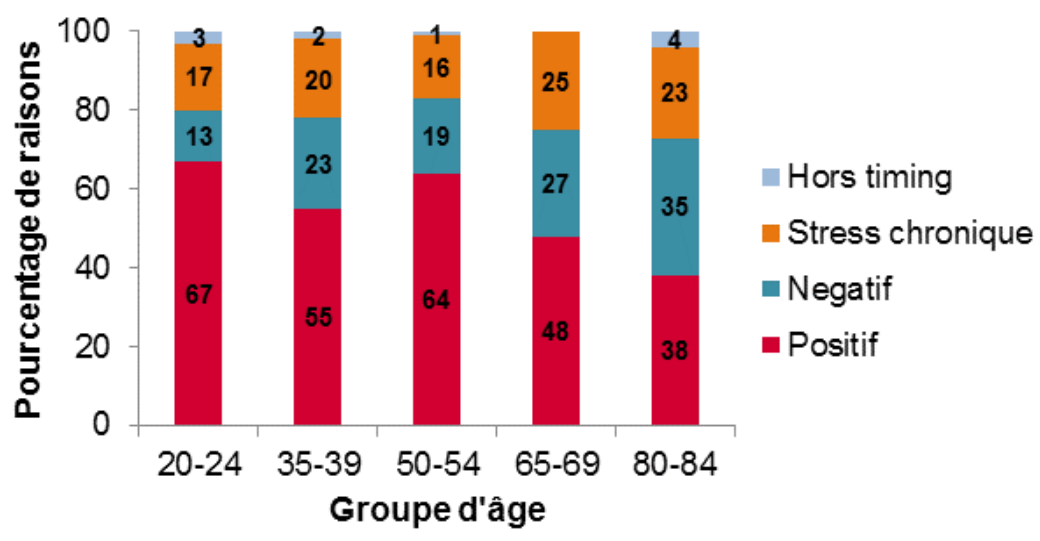

Figure 9.6: les raisons des tournants (\%) à Santa Cruz, selon la classe d'âge

Lorsque la typologie des raisons est croisée avec le sexe (voir figure 9.7), il ressort que les femmes de Bandra mentionnent davantage de raisons hors-timing. La pression pour une femme de trouver un époux et de pouvoir donner naissance à ses enfants (mâles) explique cette différence, comme nous le confirmera ci-dessous la figure 9.8. Mais une telle pression ne génère toutefois pas un écart de genre parmi les répondants de Santa Cruz, plus favorisés que ceux des bidonvilles. Toutefois, l'écart le plus important qui se manifeste entre les sexes correspond aux mentions de raisons positives, plus fréquentes à Santa Cruz et parmi les réponses faites par des hommes en général. Les femmes, elles, sont plus soumises au stress chronique, comme le sont les habitants de Bandra par rapport à ceux de Santa Cruz. Ceci nous ramène également à la théorie: dans les études menées sur le pourtour atlantique, les femmes évoquent en effet davantage de transitions négatives ou stressantes que les hommes (Kessler, \& McLeod, 1984), mais c'est encore plus prégnant dans le contexte de pauvreté de masse des bidonvilles de Mumbai. C'est sur elles que reposent en priorité et au quotidien la charge de nourrir, vêtir et éduquer, avec tous les tracas de la vie ménagère et des devoirs envers les autres, sous la tension constante de ressources faibles et souvent incertaines. 


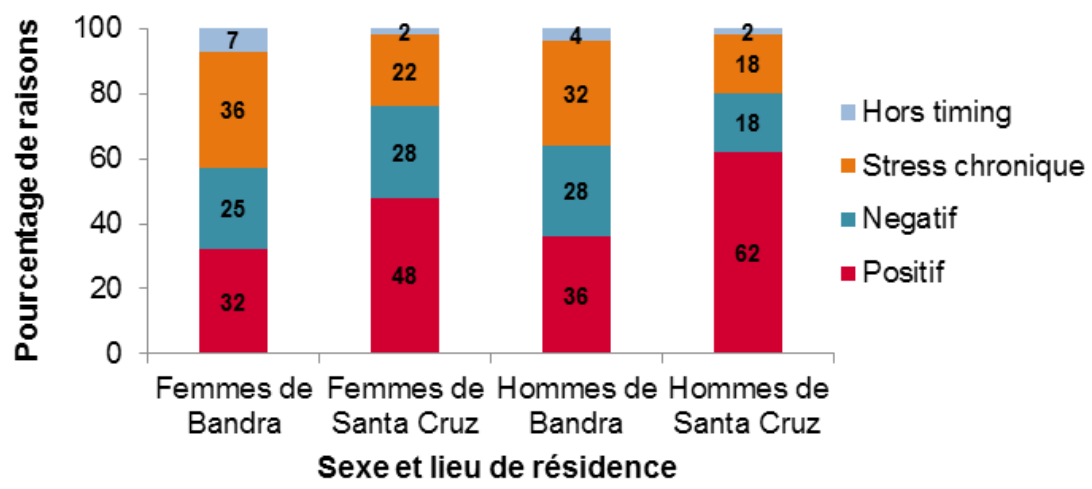

Figure 9.7: les raisons des tournants (\%), selon le sexe et le lieu de résidence

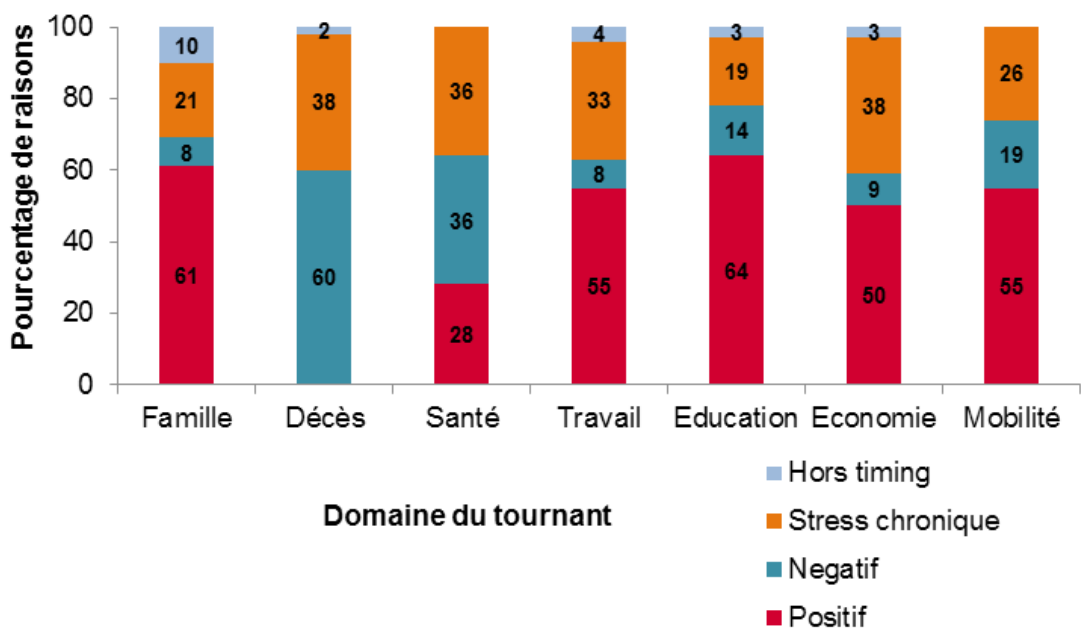

Figure 9.8: les raisons des tournants (\%), selon les sept domaines les plus mentionnés

La répartition des raisons des tournants selon leur valence ou leur degré de stress avec le domaine du tournant correspondant (voir figure 9.8) confirme qu'il y a des thématiques clairement associées à une évaluation négative (les décès, la santé), les sources de souvenirs heureux se trouvant dans la mémoire de la formation, de la famille, de la vie professionnelle, et de la mobilité. Même la moitié des tournants d'ordre économique renvoie à des opportunités positives. Le stress chronique se situe dans tous 
les domaines de la vie, mais il est particulièrement (un tiers ou plus de tournants) associé au décès d'un proche, aux fluctuations de l'économie, aux problèmes de santé et au travail (ou plutôt sa perte ou son absence). Quant aux raisons «hors-timing», elles se concentrent avant tout parmi les tournants familiaux, portant majoritairement sur des naissances trop tardives ou des mariages trop précoces.

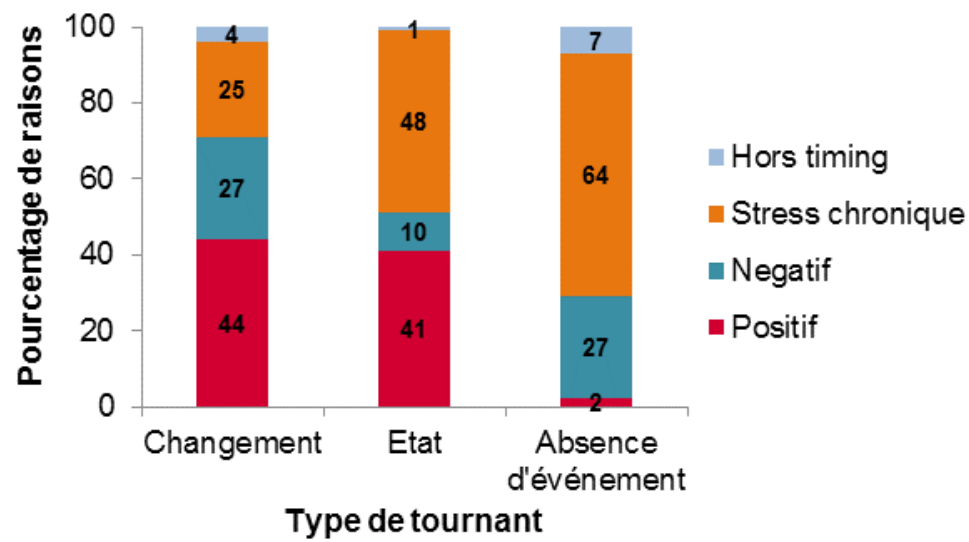

Figure 9.9: les raisons (\%) selon le type de tournant (changement, état, absence d'événement)

Le croisement des typologies des réponses et des raisons permet de discerner des liens entre ces deux classifications (voir figure 9.9). Premièrement, lorsque les individus ont répondu en citant un changement, les raisons se répartissent entre les différents types de raisons, avec une dominante négative puisque la somme des trois modalités négatif, stress et hors-timing monte à 56\%. De manière cohérente, les mentions d'état sont fortement justifiées par l'évocation d'une contrainte chronique (48\%). Comme nous en posions l'hypothèse, les personnes ciblant des étapes de la vie les caractérisent par un stress récurrent. Mais la nuance étant toujours présente, il est aussi vrai que $41 \%$ de ces états sont eux justifiés par une raison positive et correspondent plutôt à des périodes heureuses (ou protégées). Enfin, l'absence de tournant est largement liée aux raisons de type stress chronique (64\%), exprimant le regret qui s'étend sur la durée, d'une opportunité, d'une ressource perdue. 
Les manifestations de la vulnérabilité sont nombreuses dans nos résultats. L'apparition d'étapes marquantes de la vie ou d'absence de changement à la place de la mention d'une transition révèle elle-aussi une perception du parcours de vie marquée par la vulnérabilité. Ceci tant dans les réponses valorisant les moments stables, par opposition aux aléas quotidiens, que dans celles évoquant des périodes de privations qu'ont connu ou que connaissent les individus. L'appréciation des tournants au travers de la raison donnée par les répondants atteste quant à elles de la présence d'un nombre conséquent de changements négatifs et d'une forte imprégnation de la mémoire individuelle par le stress chronique. Plus déterminant encore, ce nombre est supérieur aux raisons positives exprimées par les individus, en opposition avec ce qui a été constaté jusqu'ici dans les enquêtes passées en Occident.

L'impact des structures et des dynamiques socioéconomiques apparaît d'autant plus déterminant que, inversement, divers éléments dans nos résultats concordent avec la théorie, actuellement essentiellement centrée sur les pays du Nord, ce qui doit nous amener à ne pas surestimer l'impact des distances culturelles. Premièrement, les décès sont effectivement des événements fortement rappelés par les individus, avec une présence marquée parmi les cohortes les plus âgées. Ceci, justifié par un nombre de pertes de contemporains accru en fin de vie, explique également les mémoires plus sombres des sexagénaires et octogénaires, ou tout au moins leur contenu plus pauvre en souvenirs heureux. Son corollaire, soit le fait que les jeunes évoquent davantage de tournants positifs, rejoint tout autant la théorie. Par ailleurs, les femmes citent effectivement, comme en Occident, plus de souvenirs possédant une connotation négative ou de stress latent que les hommes.

Il est probable que les mutations sociales, très rapides en Inde et plus encore à Mumbai, soient également des révélateurs des privations subies : les individus se comparent à la norme actuelle.

\subsection{Vulnérables et heureux ?}

Le bien-être, ou la perception subjective d'être heureux, se situait en filigrane derrière les analyses précédentes (dans les raisons positives de mentionner un tournant notamment). Dans la section qui suit, il sert de fil 
conducteur, afin de questionner directement l'aspect somme toute le plus subjectif de nos réponses : le bonheur est-il envisageable pour des existences fortement marquées par les privations en tous genres?

En premier lieu, nous utiliserons les réponses au premier volet du questionnaire, portant sur les événements de l'année écoulée. En effet, cet axe offre un avantage particulier, celui d'avoir demandé directement aux répondants de signaler s'ils considéraient l'événement mentionné comme un gain, une perte, ou aucun des deux. Par la suite, nous nous pencherons sur les grands événements de la vie et de l'histoire, codifiés a posteriori selon qu'ils apparaissaient comme positifs ou négatifs, afin de compléter ce tableau.

\subsubsection{Gains et pertes aux différents âges de la vie}

La psychologie développementale, dite du lifespan, s'intéresse au développement des individus sur la totalité de leur vie (Baltes, Staudinger, \& Lindenberger, 1999). Elle se focalise sur les périodes de stabilité et de changement à travers le parcours de vie et l'un de ses principaux apports est de considérer qu'à tous les âges de la vie, les trajectoires connaissent des transitions marqués par des événements dont la portée peut être positive (amenant un gain) ou négative (provoquant des pertes) (Baltes, 1987; Baltes, Reese, \& Lipsitt, 1980; Baltes et al., 1999; Perrig-Chiello \& Perrig, 2005; Sugarman, 2001). Ainsi, l'architecture des parcours de vie est issue d'une dynamique entre biologie et culture (Baltes, 1997; Baltes, Lindenberger, \& Staudinger, 2006), soulignant le caractère «multidimensionnel» (biologique, social, historique et culturel) et «multidirectionnel» (l'évaluation des changements n'est pas unidirectionnelle) du processus.

Se penchant sur les gains et les pertes de l'existence, les chercheurs du lifespan (Baltes, 1987; Baltes et al., 2006) ont établi qu'avec l'avancée en âge, les pertes estimées vont en augmentant, alors que les changements considérés comme des gains déclinent (voir figure 9.10). Autrement dit, le début de la vie jusqu'à l'âge adulte serait une période marquée par plus de gains, alors que la vieillesse se caractériserait par une prévalence des pertes (Uttal \& Perlmutter, 1989). Une mutation dans l'allocation des ressources, une optimisation sélective et une capacité adaptative des individus opèrent en fonction du ration gains/pertes (Baltes et al., 2006, 1999). 


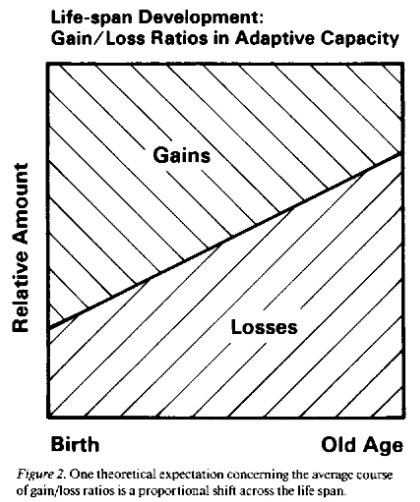

Figure 9.10: le rapport des gains et des pertes à travers le parcours de vie Source: Baltes, 1987, p.616

Toutefois, le développement humain se définit davantage par une dynamique d'équilibre entre gains et pertes, plutôt que par une chute linéaire des gains au profit des pertes. Ainsi, dans chaque changement de l'existence sont inclus tant des gains que des pertes (Baltes et al., 2006). Même si la vieillesse est associée à un déclin des ressources, des changements positifs y surviennent néanmoins (Guilley \& Lalive d'Épinay, 2008; Smith, 2003). Réussir le processus de développement vise donc à une maximisation des gains et une minimisation des pertes (Ludwig \& Chicherio, 2008; Smith, 2003).

Un problème subsiste quant à la définition de ce qui peut être considéré comme un gain ou une perte (Settersten, 1999), ainsi que l'interaction possible entre les deux. Notamment car la nature d'un gain ou d'une perte peut changer avec l'avancée en âge (Baltes \& Smith, 1999), mais aussi parce que les définitions varient selon que l'on s'intéresse à l'aspect objectif ou subjectif des attentes. Globalement, la nature des gains et des pertes "is conditioned by cultural and personal factors as well as by the position in the lifetime of an individual" (Baltes et al., 1999, p.482). Nonobstant, il n'y a pas nécessairement de causalité entre gains et pertes, et quand bien même elle existerait, la direction de celle-ci ne saurait être prédéterminée (Uttal \& Perlmutter, 1989).

Dans une perception subjective du parcours de vie, où les changements de la vie sont évalués comme des gains (événements de vie souhaités et/ ou bienvenus) ou des pertes (événements de vie indésirables) par l'acteur lui-même, peu d'études - à notre connaissance - ont empiriquement testé 
la répartition de l'évaluation des événements selon l'âge. Toutefois, Heckhausen, Dixon, \& Baltes (1989) ont interviewé 112 adultes allemands (de jeunes à âgés) sur la perception de 358 adjectifs décrivant les caractéristiques de personnalité, sociales et intellectuelles. Les participants ont été invités à évaluer chaque adjectif comme indésirable, neutre ou souhaitable - selon leur âge au moment de l'enquête, mais aussi aux âges antérieurs et supérieurs. Compte tenu du ratio gains/pertes, les résultats montrent un impact net de l'âge, avec des pertes de $<5 \%$ jusqu'à l'âge de 30 ans, atteignant $30 \%$ à l'âge de 70 ans et subissant une augmentation drastique par la suite (jusqu'à $80 \%$ des pertes à l'âge de 90 ans). Mais cette étude prouve aussi que des changements sont encore considérés comme des gains dans la vieillesse $(20 \%)$.

L'enquête de Heckhausen et al. (1989) a permis de confirmer empiriquement le modèle proposé par les chercheurs du lifespan (Baltes et al., 1999). Néanmoins, elle a été conduite en Allemagne (à nouveau dans l'espace atlantique) et tient peu compte d'autres facteurs que l'âge dans l'évaluation des adjectifs proposés (comme le niveau socioéconomique, le genre, etc.). Analyser les gains et les pertes des changements de l'année écoulée, subjectivement évalués par les individus les ayant vécus, prend donc tout son sens dans un pays du Sud et une mégapole affectée par les mutations économiques, qui plus est pour une population diversement marquée par les inégalités et les privations.

\section{La valence des changements récents évoqués à Mumbai}

La question portant sur les changements récents de la vie demandait une évaluation de ces mentions par le répondant, en termes de «gain», "perte», «ni l'un ni l'autre», «les deux». Une fois l'ambivalence ôtée (les options «ni l'un ni l'autre» et «les deux»), nous avons répartis la totalité des événements restant selon qu'ils appartiennent aux gains ou aux pertes.

Les domaines les plus mentionnés comme des gains par les répondants sont : la famille (27\%), le travail (19\%), la santé (16\%) et l'éducation $(12 \%)^{7}$. Une répartition plutôt égalitaire entre les principaux domaines de changements récents, soulignant que les gains peuvent provenir de différentes trajectoires. A l'inverse, les pertes se regroupent sous un domaine principal de mention : la santé $(63 \%)$. Cette dernière comprend : les maladies (31\%), le déclin graduel de la santé (11\%), les hospitalisations (8\%),

7 Dans une analyse plus fine des types de tournants, ressortent: le changement de travail ou la reprise du travail (12\%), les naissances (11\%), les mariages (11\%), l'amélioration de la santé $(8 \%)$ et la réussite scolaire $(7 \%)$. 
les accidents (7\%). Parmi les grands domaines, suivent ensuite l'économie $(8 \%)$, le travail $(8 \%)$ et les décès $(7 \%)$.

La figure 9.11 expose le ratio relatif des gains et des pertes selon les groupes d'âge, sans distinction entre les quartiers de résidence. En général, les résultats suivent la distribution théorique attendue et montrent une distribution claire tout au long de la vie : les jeunes ont tendance à mentionner plus de gains alors que les personnes âgées évoquent plus de pertes. L'inclinaison du graphique est régulière, montrant une diminution continue du nombre de gains pendant la durée de vie, tandis que les pertes augmentent. Cependant, la plus grande chute des gains semble se situer entre le début de l'âge adulte (20-24 ans) et les cohortes suivantes. La jeunesse serait-elle une période marquée par plus d'insouciance, même à Mumbai ?

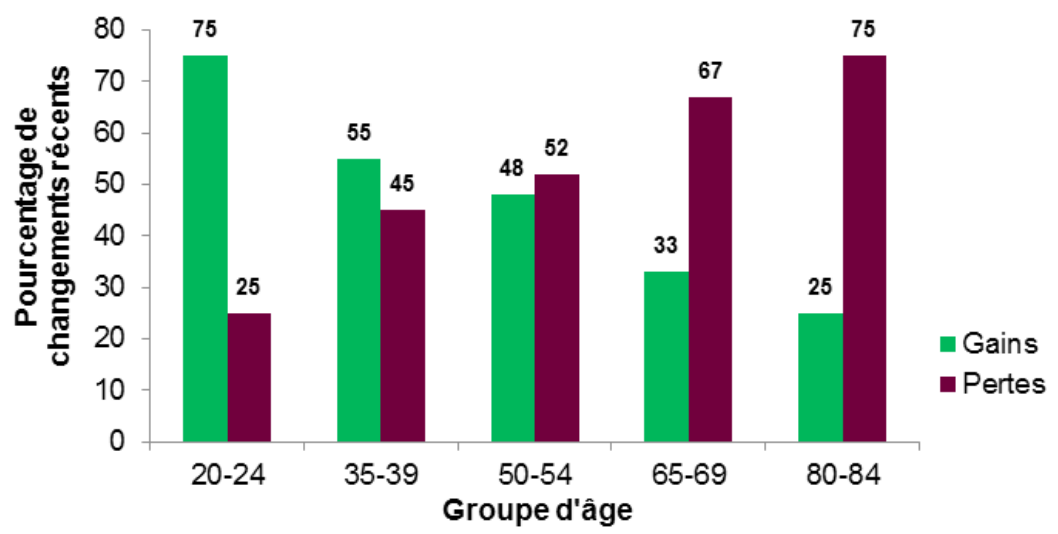

Figure 9.11: la répartition des gains et des pertes (\%) selon les groupes d'âge

Plusieurs modèles de régressions logistiques ont permis de détailler quels individus possèdent une propension à mentionner une perte (voir tableau 9.7). Trois variables se révèlent déterminantes pour expliquer cette propension : le groupe d'âge, le niveau d'éducation et la santé auto-évaluée. Le lieu de résidence ne s'avère pas une variable significativement déterminante dans l'explication des mentions de pertes parmi les changements de l'année écoulée.

Indiscutablement, il apparaît que plus l'individu est âgé, plus il évalue négativement les événements récents qu'il évoque. Ceci corrobore parfaitement la littérature, ainsi que nos analyses descriptives. De même, une santé estimée bonne (mais pas excellente), moyenne ou mauvaise a le même effet, ce qui n'est pas surprenant. 
De son côté, le niveau d'éducation permet de souligner un phénomène intéressant : les personnes qui ont atteint l'école primaire ont davantage tendance à évoquer des souvenirs récents négatifs que ceux ayant accompli des études supérieures (y compris universitaires), mais ce n'est pas le cas des illettrés. Avoir été à l'école, même peu de temps, semble permettre de saisir les implications de l'environnement et de sa condition socioéconomique sur sa vie, induisant qu'il existe une «acceptabilité sociale» à se plaindre qui n'existe pas chez les personnes exemptes d'éducation qui activeraient des mécanismes d'accommodation à leur misère (Bourdieu, 1998b). Ceci reste toutefois une hypothèse, qui n'est pas corroborée par la variable du lieu de résidence. En effet, aucune différence significative en fonction du lieu d'habitation ne ressort dans le fait de citer une perte.

Ces résultats ne sont ni simples ni univoques ; ils appellent un approfondissement des souvenirs évoqués. En effet, réussir à l'école, entrer à l'université, etc. sont des souvenirs qui ne peuvent être mentionnés que par ceux qui les ont vécus, et qui sont positifs. A l'inverse, ne pas pouvoir continuer ses études, comme cela a beaucoup été évoqué, représente un aspect négatif et contraint qui ne peut s'appliquer qu'aux individus appartenant à un niveau de scolarisation bas, voire inexistant.

Tableau 9.7: rapports de cotes issus de régressions logistiques sur la question des changements survenus au cours de la dernière année

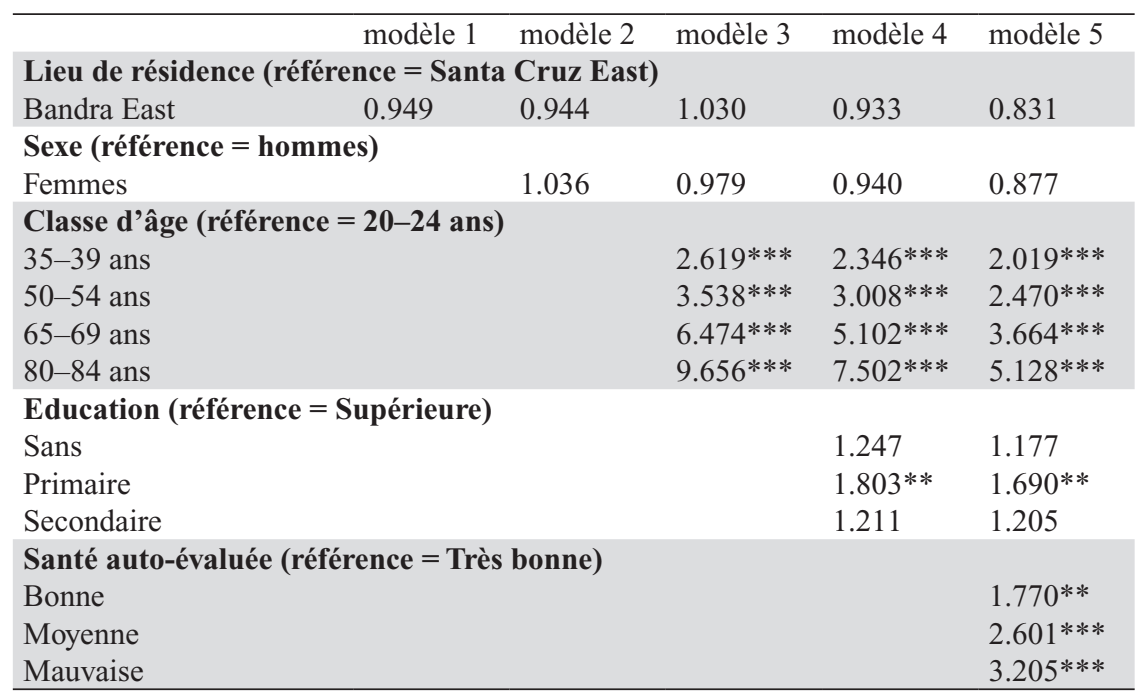

Seuils de significativité ${ }^{\circ} \leq 0.1 ; * \leq 0.05 ; * * 0.01 ; * * \leq 0.001$. 


\subsubsection{La valence des grands changements de la vie et de l'histoire}

Afin de considérer aussi les questions sur les principaux tournants de l'existence et de l'histoire, pour lesquelles les individus n'avaient pas été sollicités de donner une évaluation, nous avons choisi de passer par une reconstruction a posteriori de la valence du changement. Ainsi, la signification négative, positive ou ambivalente ${ }^{8}$ des tournants de la vie et des changements sociohistoriques a fondé notre codification. Bien souvent, la valeur de la mention était très marquée, mais cela n'a pas été toujours le cas : $38 \%$ des tournants étaient positifs, $45 \%$ négatifs et $17 \%$ ambivalents. Plus tranchés, les changements sociohistoriques étaient essentiellement négatifs (93\%), $4 \%$ positifs et $3 \%$ ambivalents.

Tableau 9.8: rapports de cotes issus de régressions logistiques sur la question des grands tournants de la vie

\begin{tabular}{|c|c|c|c|c|c|}
\hline & modèle 1 & modèle 2 & modèle 3 & modèle 4 & modèle 5 \\
\hline \multicolumn{6}{|c|}{ Lieu de résidence (référence $=$ Santa Cruz East) } \\
\hline Bandra East & $2.257 * * *$ & $2.207 * * *$ & $2.234 * * *$ & $1.663 * * *$ & $1.582 * * *$ \\
\hline \multicolumn{6}{|c|}{ Sexe (référence = hommes) } \\
\hline Femmes & & $1.525^{* * *}$ & $1.588 * * *$ & $1.283^{*}$ & $1.231 *$ \\
\hline \multicolumn{6}{|c|}{ Classe d'âge (référence $=20-24$ ans) } \\
\hline $35-39$ ans & & & $1.636^{* *}$ & 1.256 & 1.140 \\
\hline $50-54$ ans & & & $1.730 * * *$ & 1.100 & 0.984 \\
\hline $65-69$ ans & & & $2.308 * * *$ & 1.254 & 1.041 \\
\hline $80-84$ ans & & & $2.636 * * *$ & 1.253 & 1.033 \\
\hline \multicolumn{6}{|c|}{ Education (référence $=$ Supérieure) } \\
\hline Sans & & & & $3.874 * * *$ & $3.603 * * *$ \\
\hline Primaire & & & & $2.874 * * *$ & $2.732 * * *$ \\
\hline Secondaire & & & & $1.589 * * *$ & $1.557 * * *$ \\
\hline \multicolumn{6}{|c|}{ Santé auto-évaluée (référence $=$ Très bonne) } \\
\hline Bonne & & & & & $1.369^{\circ}$ \\
\hline Moyenne & & & & & $1.720 * *$ \\
\hline Mauvaise & & & & & $1.932 *$ \\
\hline
\end{tabular}

Seuils de significativité ${ }^{\circ} \leq 0.1 ; * \leq 0.05 ; * * 0.01 ; * * \leq 0.001$.

8 Les tournants ambivalents correspondent à un événement où ni le positif ni le négatif ne prime: par exemple «I got married» (femme de 52 ans, Bandra). Les changements sociohistoriques ambivalents représentent des événements neutres, ou qui ne révèlent pas d'avis tranché du répondant à leur encontre: par exemple «Mobile and internet and media in India. Mobile is useful. It is also bad" (homme de 53 ans, Santa Cruz). 
Dans les analyses qui suivent, les changements ambivalents ont été ôtés. Le tableau 9.8 se focalise sur la question touchant aux grands événements marquants de la vie. L'âge apparaît comme n'étant pas significatif. Par contre, habiter à Bandra accroît le risque d'évoquer un tournant négatif, tout comme être une femme. Par ailleurs, posséder un niveau d'éducation nul ou bas (primaire ou secondaire) augmente la propension à évoquer un tournant connoté négativement par rapport aux universitaires. Finalement, s'estimer dans une santé relative ou mauvaise est également un facteur explicatif d'avoir mentionné un tournant négatif.

Dans le tableau 9.9, les mêmes analyses des mentions négatives ont été faites, mais cette fois-ci portant sur les changements sociohistoriques. Ces derniers possèdent une teneur généralement sombre, mais elle a d'autant plus tendance à survenir lorsque le répondant réside à Santa Cruz. Le fait de n'avoir pas reçu (ou peu) d'éducation est également un facteur accroissant la propension à mentionner un événement négatif. Finalement, s'estimer en bonne ou moyenne santé (par rapport à avoir une santé très bonne) incite aussi à évoquer des événements négatifs.

Tableau 9.9: rapports de cotes issus de régressions logistiques sur la question des changements sociohistoriques

\begin{tabular}{|c|c|c|c|c|c|}
\hline & modèle 1 & modèle 2 & modèle 3 & modèle 4 & modèle 5 \\
\hline \multicolumn{6}{|c|}{ Lieu de résidence (référence $=$ Santa Cruz East) } \\
\hline Bandra East & 0.935 & 0.923 & 0.942 & $0.604 *$ & $0.568^{*}$ \\
\hline \multicolumn{6}{|c|}{ Sexe (référence = hommes) } \\
\hline Femmes & & $1.566^{*}$ & $1.579 *$ & 1.272 & 1.164 \\
\hline \multicolumn{6}{|c|}{ Classe d'âge (référence $=20-24$ ans) } \\
\hline $35-39$ ans & & & $1.698^{\circ}$ & 1.300 & 1.085 \\
\hline $50-54$ ans & & & $2.320^{*}$ & 1.358 & 1.072 \\
\hline $65-69$ ans & & & $2.024 *$ & 0.981 & 0.769 \\
\hline $80-84$ ans & & & $2.926 * *$ & 1.052 & 0.788 \\
\hline \multicolumn{6}{|c|}{ Education (référence $=$ Supérieure) } \\
\hline Sans & & & & $5.068 * *$ & $5.499 * *$ \\
\hline Primaire & & & & $6.713^{* * *}$ & $6.836^{* * *}$ \\
\hline Secondaire & & & & 1.293 & 1.310 \\
\hline \multicolumn{6}{|c|}{ Santé auto-évaluée (référence = Très bonne) } \\
\hline Bonne & & & & & $1.818^{*}$ \\
\hline Moyenne & & & & & $2.103^{\circ}$ \\
\hline Mauvaise & & & & & 1.053 \\
\hline
\end{tabular}

Seuils de significativité : ${ }^{\circ} \leq 0.1 ; * \leq 0.05 ; * * \leq 0.01 ; * * * 0.001$. 
Un point intéressant de cette analyse concerne le fait de mentionner davantage de changements négatifs si l'on réside à Santa Cruz qu'à Bandra. Par ailleurs, le fait de mentionner plus de changements historiques négatifs si l'on n'a pas ou peu d'éducation.

Alors que l'âge influence le fait de mentionner des pertes ou des gains parmi les changements de l'année écoulée, cette variable n'a plus d'impact sur la mention de tournants ou de changements sociohistoriques négatifs. Ce qui tend à prouver que la position dans le parcours de vie a une influence sur la valence des événements vécus directement, mais que, lorsque l'on raconte rétrospectivement les moments marquants de son existence, alors les variables socioéconomiques (lieu de résidence, niveau d'éducation et auto-évaluation de la santé) ainsi que le sexe sont bien plus déterminantes. Les inégalités issues du fait d'être pauvre, mal formés ou femme à l'heure actuelle dans une mégapole indienne apparaissent illustrées par cette propension à donner une tonalité négative à sa mémoire.

\subsection{Conclusion}

La mémoire mobilisée par les répondants renvoie à leur existence de façon dans l'ensemble réaliste, sans nécessairement se référer de manière préférentielle au souvenir des moments heureux, qui sont malgré tout également présents. Plusieurs constats peuvent être établis au travers de ces résultats. Le premier volet du questionnaire se rapporte directement aux événements vécus aux différentes étapes de la vie (les individus devant évoquer des éléments survenus dans la dernière année). Il ressort des analyses effectuées que la classe d'âge possède le principal impact sur le fait de mentionner un événement subi, négatif et concernant autrui plutôt que soi-même. Sous contrôle des autres variables socioéconomiques, être âgé accroit la potentialité de se souvenir de changements imposés et aux teintes sombres. Ceci est conforme à la théorie qui postule que les souvenirs des jeunes sont moins chargés du sentiment de perte.

La question sur les changements de l'entier de la vie fait davantage ressortir les inégalités socioéconomiques et socioculturelles. En effet, être peu ou pas du tout éduqué augmente fortement les risques d'évoquer des souvenirs négatifs et subis, par rapport à des individus ayant un niveau 
secondaire ou supérieur. L'inégalité sociale est également visible dans la propension à citer des événements subis et négatifs parmi les habitants des bidonvilles plutôt que parmi ceux résidant dans un quartier formel.

Dans les résultats portants sur les souvenirs sociohistoriques, les inégalités sociales ressortent à nouveau comme les variables explicatives les plus probantes. La valence négative des souvenirs s'explique ainsi largement par le niveau d'éducation. Les individus illettrés, mais plus encore les personnes qui ont été scolarisées sans dépasser le niveau de l'école primaire, évoquent des événements négatifs. Avoir atteint un premier stade d'éducation permet-il de saisir les disfonctionnements environnementaux et sociétaux avec une meilleure précision ? Les individus ontils davantage conscience de leur situation de privation ? Ou le fait d'être privé de base scolaire implique-t-il une vision plus centrée sur l'espace étroit de son quartier, de sa communauté, et un éloignement vis-à-vis de l'histoire et de l'environnement plus larges? 



\section{Conclusion}

Laisser la parole aux principaux concernés pour qu'ils rapportent leur propre perception de leur existence et de l'histoire vécue était le premier objectif de ce livre. Les enquêtes effectuées à Bandra et à Santa Cruz ont permis une telle approche, confiant l'interprétation des événements marquants de leur vie aux acteurs eux-mêmes. L'utilisation de données quantitatives et qualitatives liées l'une à l'autre a rempli pleinement son rôle, permettant de faire ressortir des tendances générales, des distributions, puis de creuser la diversité des appréhensions. Les résultats sont nombreux, évoluant entre similarités surprenantes, originalités significatives, absences révélatrices ; finalement, avant tout des constatations nuancées et des remises en question d'a priori.

Avant de passer aux résultats, un bref bilan des apports et des limites de notre étude comme outil méthodologique s'impose, dans une optique constructive de compréhension de la recherche. Entamée dans le chapitre 3, cette réflexion autour de la relation d'enquête et des contraintes sociales qui s'imposent aux répondants peut à présent être mise en regard de l'empirie.

\section{Repenser la relation d'enquête : les résistances et les interprétations de la part des répondants}

La présence et les marqueurs du statut de l'enquêteur influencent celui qu'il observe (Jodelet, 2003). Cela fait partie des interventions inéluctables d'éléments subjectifs au cours de la relation d'enquête (intérêts, préjugés, prise de position du chercheur d'une part, de l'autre le lien avec l'enquêté - conditions d'entretien, interprétation...) (de Sardan, 2000). C'est en particulier le cas pour des entretiens en «face-à-face» (Masson \& Haas, 2010). Lors de nos terrains d'enquête, il est possible qu'une relation de confiance ait pu s'établir (du fait par exemple que l'enquêteur provienne également des bidonvilles dans le cas de Bandra East) ou à l'inverse de défiance (par exemple dans le cas de Santa Cruz, avec l'impression d'être jugé par un intervieweur plus qualifié). En outre, ces relations sont biaisées par les rapports de pouvoir lié au genre, à l'âge, à la classe sociale et au non-dit puissant de la caste (respectivement de l'interviewé et de 
l'enquêteur). Nous l'avons noté, il fut plus malaisé pour les travailleuses du CSSC d'interviewer des hommes que des femmes.

Par ailleurs, un décalage peut exister entre l'objet questionné par l'enquêteur (ou le chercheur) et la compréhension qui en est tirée par le répondant (Bourdieu, 1998a). L'enquêteur pose les règles et possède bien souvent une position symboliquement supérieure. On ne peut maîtriser tous les effets de la relation: crainte des préjugés, effet de censure, fausse objectivation complaisante, illusion de neutralité (Bourdieu, 1998a). Il ne s'agit pas pour autant de sur-interpréter le rapport dominant-dominé, en sous-estimant les possibilités de «manipulations», de jeu de la part des enquêtés (de Sardan, 2000). C'est ainsi que dans les chapitres d'analyse (surtout le 7), nous nous sommes longuement demandée si le report de problèmes de santé à Bandra relevait du réel (relié aux conditions de vie dans les bidonvilles) ou d'un effet de désirabilité sociale (restituer aux enquêtrices - également assistantes sociales pour une organisation médicale - ce que le répondant croit qu'elles veulent entendre).

Des résistances surviennent aussi parfois lors d'un échange de parole avec l'interviewé. Par exemple, nous nous sommes aperçue que les individus avaient parfois déclaré des périodes (des étapes de leur existence, ou des situations d'une temporalité longue) plutôt que des changements (voir section 9.2). Ce constat tend à indiquer que le répondant négocie face à l'intervieweur et aux consignes d'enquête, et «impose» sa propre interprétation de sa vie dans la réponse rapportée. Dans le même ordre d'idée, raconter une absence de changement (i.e., ne pas avoir pu faire d'études), alors même que la question posée porte explicitement sur les tournants ou les changements de sa vie, démontre de la capacité d'appropriation des répondants du questionnement qui leur est (im)posé.

Dans la littérature (occidentale), de telles résistances ou altérations sont présentées comme issues du processus d'objectivation (une forme de justification du récit) et d'une «régionalisation» des représentations (Goffman, 1973). Ce dernier terme illustre le procédé par lequel, lorsque quelqu'un s'exprime, fait ou pense une chose, il ne le fait pas de la même manière selon la situation, la personne en face, le moment, ou le lieu où il se trouve (Blanchet \& Gotman, 2010). Or, selon les circonstances, certaines représentations ne peuvent être relatées. Les chapitres d'analyse ont ainsi révélé la quasi absence de mentions de dimensions absolument cruciales de la vie sociale indienne (i.e., la pauvreté, les castes et la religion), 
alors même que d'autres aspects émergent, telles les violences envers les femmes.

En outre, les raisons de rapporter un changement personnel ont parfois fait explicitement le rapprochement avec une survenue trop précoce ou trop tardive de celui-ci, au regard d'une certaine norme sociale (mentions hors-timing, voir section 9.2.2). Il est probable, et c'est ce que nous mettons en avant, que ce soit un décalage avec les normes actuelles d'une société indienne qui a beaucoup évolué en peu de temps qui soit souligné par ces propos. Toutefois, il est également raisonnable d'envisager que d'avoir été interrogés sous l'égide de l'Université de Genève, pour des chercheurs issus d'un pays du Nord, ait poussé quelques répondants à prendre plutôt en compte la distance existant avec notre société occidentale. Au sein de la relation d'enquête, la réponse donnée se serait ainsi adaptée aux chercheurs, basée sur ce que l'enquêté a interprété comme étant la réalité indienne pour des yeux occidentaux.

Tout ceci posé, il demeure que les questions soulevées dans les enquêtes CEVI présentent un avantage puisqu'il s'agit de parler d'un sujet que l'on maîtrise mieux que quiconque : sa propre existence. Il est malgré tout possible que la crainte d'un jugement de valeur ou celle de se tromper dans les informations demandées n'ait retenu le répondant sur une partie des sujets abordés (dans une forme de sélection), ou l'ait entraîné à ne pas parler de certaines dimensions (non-dits, silences,...) (Devereux, 2012 [1967]; Masson \& Haas, 2010).

\section{Revers de la médaille : les limites d'une démarche atypique}

Une première limite à ne pas négliger provient de la méthodologie des enquêtes. Si elle a offert l'opportunité de la simplicité et d'une passation aisée dans un contexte difficile d'accès, elle implique également un certain nombre de restrictions à ne pas négliger. Tout d'abord, se fonder sur des échantillons non-aléatoires, arbitrairement élaborés sur des critères d'âge et de sexe, limite nos constatations à la population observée et ne permet - théoriquement - pas d'en étendre les interprétations à la population en général. Nonobstant, au vu du contexte de nos enquêtes (particulièrement dans le cas des slums) et de la situation socioéconomique des habitants de la mégapole (dont une part considérable loge à même la rue et migre souvent), une telle représentativité aurait paru, de toute façon, illusoire.

Ensuite, cet échantillonnage repose sur des repères d'âge construits selon la compréhension occidentale des étapes de l'existence (jeunesse, 
âge adulte, vieillesse, dépendance). Cette décision a relevé exclusivement de notre volonté de correspondre aux critères de l'enquête CEVI, et de ce fait ne tient pas compte du contexte culturel différent dans lequel ont été passées les enquêtes indiennes. Avoir interrogé d'autres classes d'âge, notamment plus jeunes (étant donné que l'entrée dans l'âge adulte survient parfois très tôt), aurait sans doute apporté des éclairages supplémentaires. A l'inverse, les octogénaires interrogés constituent un groupe singulier, au vu de l'espérance de vie indienne. Nous y avons sélectionné des survivants avant tout, des personnes très âgées, certes, mais n'ayant probablement pas une connaissance exacte de leur date de naissance.

Par ailleurs, les classes moyennes inférieures et les habitants des bidonvilles représentent, les uns comme les autres, des populations plus hétérogènes en termes socioéconomiques que ce que leur caractérisation initiale pourrait donner à penser. Il a finalement été peu possible d'en tenir compte, malgré quelques questions complémentaires portant sur le niveau de vie. Seule l'éducation a été retenue comme indicateur de la variabilité au sein de ces groupes (et, dans une optique quelque peu différente, la santé auto-évaluée). Même le sous-échantillon SRA a été peu utilisé en tant que tel dans le second terrain d'enquête.

Enfin, il n'est pas inutile de rappeler que les terrains ont été réalisés sous la direction de chercheuses extérieures aux structures traditionnelles indiennes, religieuses et de caste, ainsi que de langue. Ainsi, il n'a pas été possible de correspondre directement avec les répondants, difficilement avec les enquêtrices et les enquêteurs. Néanmoins, les deux terrains ont été suivis au plus près ; pour ce faire, des intermédiaires ont été engagés et un travail considérable a été accompli, de prises de contact en amont et de renseignements sur les zones et les populations étudiées.

Une autre limite concerne le contenu des réponses. Malgré le potentiel de liberté que les questions ouvertes offrent, et peut-être à cause de lui, les résultats révèlent plusieurs carences d'information. Le questionnaire CEVI tel qu'il est construit permet une première approche efficace vers des personnes qui n'ont probablement jamais eu l'occasion de repenser leur vie de cette manière ; mais, simultanément, il soulève aussi de nombreuses interrogations, alors même que le format réduit des réponses ne laisse que peu de marge pour creuser. Qui plus est, travailler sur la mémoire signifie également affronter l'oubli. Or, la configuration subjective de l'étude ne permet nullement de saisir cet aspect, qui demeure une explication parmi d'autres de divers résultats (choix de ne pas parler, choix 
de mettre en avant d'autres éléments, etc.); il est pour autant très délicat pour la chercheuse de la convoquer, tant cela la place dans la conjecture. La subjectivité montre ici le défaut de ses qualités. Les non-dits (les sujets qui ne furent pas abordés par les répondants) représentent des obstacles devant lesquels les outils à disposition contraignent à reculer, mais qu'il serait passionnant de dépasser, par exemple au travers d'entretiens approfondis ou de focus groupes.

$$
* * *
$$

Il reste que les répondants ont amené nombre de pistes de réflexion quant à nos questions de recherche : quelle application des théories occidentales du parcours de vie dans le contexte culturel indien ? Comment les habitantes et les habitants de Mumbai, pauvres et moins pauvres, ont-ils perçus les évolutions de leurs trajectoires sous l'impulsion de l'ouverture économique et de la globalisation, face aux normes traditionnelles encore en vigueur? Comment se révèlent les stratifications d'âge et de sexe au travers des moments marquants du parcours de vie et de l'histoire vécue? Quelles formes peut prendre la vulnérabilité perceptible dans les événements remémorés, pour des individus vivant dans un pays toujours miné par la pauvreté de masse ?

Pour cette conclusion, trois axes structurants ont été définis : (1) l'impact de l'environnement sur le déroulement des vies humaines ; (2) les normes sociales, les diverses temporalités du parcours de vie et les tensions intergénérationnelles ; (3) les vulnérabilités présentes dans les mémoires, déjà apparentes sous les deux premiers axes mais sur lesquelles nous terminerons.

\section{Le temps et l'espace : des environnements qui vulnérabilisent}

Ce que le paradigme du parcours de vie a rapidement mit en évidence, est l'importance de comprendre le contexte dans lequel se déroulent les vies pour saisir les évolutions de ces dernières (Elder, 1994, 1999 [1974]; Guillaume, 2010; Lalive d'Epinay, Bickel, Cavalli, \& Spini, 2005; Mills, 1997 [1959]). A Mumbai, les quartiers sur lesquels nous nous sommes focalisés, et en particulier les slums, ont été le théâtre de nombreux 
épisodes historiques, souvent violents et négatifs, qui n'ont pas manqué d'imprégner la mémoire et l'identité des personnes y résidant.

\section{Un environnement à risque qui s'imprime dans les mémoires}

Vivre dans un habitat urbain précaire fragilise face aux désastres naturels (Chan et al., 2012; Dewan, 2013; Rayhan, 2008). Les mentions concernant l'inondation de 2005 à Mumbai confortent pleinement ce constat. D'abord, ressort la double discrimination des inscriptions de l'inégalité socioéconomique dans un espace urbain chaotique, puisque ce phénomène est plus faible parmi les réponses des résidents de Santa Cruz, moins nombreux à en parler car ayant vécu l'inondation avec plus de distance. Inversement, les citations de cette catastrophe ont été particulièrement importantes parmi les habitants des slums. Avec les raisons pour les avoir évoquées, elles soulignent la vulnérabilité humaine, l'effroi face au risque, mais aussi les conséquences directes de celles-ci en termes économiques pour des individus pauvres (pertes matérielles, endettement pour s'en relever, etc.).

Qu'un tel événement ait frappé à ce point les mémoires de personnes de tous les âges et des deux sexes révèle pour autant une perception très centrée sur sa propre existence, sur le local, ainsi qu'une absence de différence mémorielle entre les générations. Davantage que d'être formées par leur histoire, les cohortes apparaissent ici unies par le milieu partagé, toutes soumises aux risques engendrés par celui-ci et à la mémoire traumatique ainsi générée. Les bouleversements environnementaux qui frappent la communauté prennent une signification cruciale pour chaque personne, quel que soit et ait pu être son statut au sein de son foyer.

Les catastrophes ne sont pas les seuls changements sociohistoriques rappelés avant tout par les individus habitant dans un taudis de la mégapole. Les pogroms interreligieux, séquelles à la fois si lointaines et si actuelles de l'Indépendance et de la Partition de 1947, se sont déroulés majoritairement dans les bidonvilles de Mumbai, lieux où misère, mixité et proximité ont été utilisés pour promouvoir l'émergence de la violence. Ceci se retrouve dans les évocations nombreuses des altercations de 1984, 1992 et 2011 par les répondants de Bandra. Cette fois, une certaine distance s'opère entre les groupes d'âge, laissant entrevoir une brisure générationnelle : les jeunes dans la vingtaine mentionnent nettement moins ce souvenir. A l'autre bout de l'échelle d'âge, les octogénaires ont bien plus tendance à évoquer les émeutes, de par leur fragilité face aux violences mais 
aussi, sans doute, au rappel que cela suscite de la période tumultueuse de la Partition (que les plus âgés sont seuls à avoir vécu directement).

Autre indice d'écart générationnel, les 20-24 et les 35-39 ans interrogés à Bandra montrent une prévalence plus grande de mentions d'attentats en Inde. Alors que les racines historiques de cette violence politique proviennent du même terreau que les pogroms, les citations de ces attentats, surtout effectuées par des individus nés dans les années 1980 et 1990, interpellent par leur similarité avec le cas du 11 Septembre 2001 dans nombre de pays occidentaux (Bergeron, 2015; Guichard, 2015; Martenot \& Cavalli, 2014). Une marque de la globalisation des médias et des voies de communication, ciblant le terrorisme comme un mal faussement caractéristique du $21^{\mathrm{e} m e}$ siècle, semble se distinguer ici.

Ces événements sociohistoriques (inondation, émeutes, attentats) sont, nous l'avons répété, surtout l'apanage des réponses données par les habitants des taudis, et se recoupent en ce qu'ils ont tous été expérimentés de manière proche par les individus (ils se sont déroulés sur un périmètre rapproché des lieux de vie des répondants, impliquant directement des conséquences pour eux, etc.). Une quatrième forme de violence mentionnée dans le volet III de l'enquête révèle également un effet de proximité avec les répondants, apparue davantage parmi les réponses des résidents de Santa Cruz : il s'agit des agressions contre les femmes qui ont défrayé la chronique en Inde ces quelques dernières années. Précisément, cette récence explique en partie la faiblesse des évocations de tels souvenirs par les habitants des slums, l'engagement des classes moyennes indiennes contre ce fléau intervenant aussi, à l'inverse. Le point le plus important est néanmoins la fréquence de ces mentions parmi les femmes : elle reflète indubitablement une forme d'empathie, confirmée par les raisons (descriptions des violences transmises dans les médias, peur pour soi ou ses proches, etc.).

Ce que les changements sociohistoriques marquants rapportés par les individus soulignent est une surreprésentation des événements violents, négatifs et nationaux, corroborant ainsi les découvertes faites par d'autres études dans d'autres contextes (Guichard, 2015; Liu et al., 2012; Liu et al., 2005; Martenot \& Cavalli, 2014). Néanmoins, l'originalité de notre échantillon (d'une part provenant d'une culture distincte, et d'autre part d'un niveau socioéconomique parfois très bas) permet d'y ajouter une caractéristique supplémentaire : pour les personnes pauvres, possédant un accès réduit à la formation et aux médias, une proximité historique 
et géographique avec les événements historiques rappelés se remarque. Pour les habitants des slums, se projeter dans l'histoire ou concevoir les changements à l'échelle du pays ou du monde paraît moins évident. Leurs souvenirs illustrent davantage une prise en compte de la communauté proche, du quartier, et tendent à posséder une teinte plus sombre encore, que ce qui a été observé ailleurs.

\section{Insalubrité et pauvreté : les mentions de santé comme éclairage des inégalités}

L'environnement ne doit pas être compris uniquement comme le contexte historique encadrant les trajectoires dans des espaces singuliers, il représente également les conditions (sociales, économiques, urbanistiques) dans lesquelles celles-ci se déroulent. Parmi les événements personnels rapportés par les individus, les mentions de santé ont été largement mises en exergue. Plus que dans aucun autre pays où une enquête subjective sur la perception des moments forts de l'existence a été réalisée, les Indiennes et les Indiens vivant à Mumbai associent bonne, et surtout mauvaise santé aux changements marquants de leur vie. En particulier, les habitants des bidonvilles citent de tels événements, révélateurs de leur condition d'habitat insalubre, de leur manque d'accès aux soins et des changements sanitaires globaux dont ils sont les premières victimes dans un cadre urbain qui les expose à des risques nombreux.

Le multiple burden of disease apparaît donc, et ce dans la mémoire des pauvres. Il s'observe à l'échelle de l'échantillon (c'est-à-dire entre les groupes mais pas sur les personnes, les citations de plusieurs événements de santé par un même individu étant rares) : les jeunes mentionnent quelques maladies infectieuses, mais très vite ce sont les maladies chroniques qui ressortent, dès la trentaine. Le phénomène est plus faible parmi les habitants de Santa Cruz, qui sont davantage partagés entre problèmes chroniques et accidents, ces derniers événements faisant écho à la circulation dense et chaotique dans une mégapole émergente. La disparition des infections, les plus liées à la pauvreté, laisse percevoir les avantages de la vie urbaine, pour celles et ceux qui ont le double privilège d'habiter dans des espaces salubres et de posséder le minimum vital ou davantage. Les études menées dans d'autres villes des Suds confirment l'exposition particulière des classes moyennes aux accidents, alors même qu'elles bénéficient de la concentration en ville des institutions 
hospitalières auxquelles elles ont, elles, les moyens d'accéder (voir par exemple Rossier et al., 2016).

Les raisons pour évoquer un événement de santé sont surtout de deux ordres : les émotions ressenties et les conséquences économiques, pour la personne et le ménage. Alors que les premières sont nombreuses à être au centre des explications rapportées par les personnes âgées, les secondes sont présentes parmi les groupes d'âge plus jeunes, dans la vingtaine, ainsi que par les adultes dans la trentaine ou la cinquantaine, soulignant la pression liée aux responsabilités familiales. Avoir un ennui de santé, qui plus est lorsqu'il s'étend sur la durée, signifie une perte de revenu, des dépenses supplémentaires et parfois l'entrée sur le marché de l'emploi d'autres membres du foyer qui ne devraient pas (l'épouse), pas encore (les enfants) ou plus (les parents âgés) y être. Ces conséquences économiques et familiales sont davantage évoquées par les femmes, qui ont à charge le ménage et qui, si elles doivent travailler, cumulent leurs charges domestiques. Ce stress latent à propos des pressions familiales ressort à maintes occasions dans nos données, comme signal de l'existence de normes sociales liées aux puissantes institutions informelles que restent la famille, la religion, la culture.

\section{Evolution des normes sociales, enjeux des temporalités et écarts générationnels}

En Inde, les normes proviennent à la fois de structures anciennes (religion, familles, castes, patriarcat) et modernes (classes, âge), ainsi que de constructions ambivalentes (les genres sexués). Aucune véritable politique sociale universelle n'a encore participé à constituer la tripartition de l'existence constatée dans le monde occidental depuis la fin du $19^{\text {ème }}$. Dans ce contexte particulier, les événements personnels remémorés par les répondants montrent la persistance d'anciennes normes (notamment l'importance de la famille), un silence assourdissant sur d'autres normes informelles (religieuses et culturelles), les hiatus entre les configurations anciennes et les réalités modernes, et plus généralement l'impact du non-accès aux institutions formelles. 


\section{Les temporalités du parcours de vie en Inde : des moments plus chargés}

en événements où la vulnérabilité individuelle serait exacerbée?

Les contenus des changements personnels mémorisés par nos répondants ne font qu'une bipartition imparfaite des âges de la vie, comprenant une division entre la jeunesse (la période de formation) et l'âge adulte, avec dans ce dernier cas une porosité puisque c'est surtout la transition vers ce rôle (constitution de la famille, entrée sur le marché du travail, montée des responsabilités) qui ressort. Pour ce qui est de la troisième phase, la vieillesse, ce ne sont encore que des balbutiements, les contraintes économiques et la présence d'enfants étant encore les principales régisseuses de la possibilité (ou non) d'arrêter de travailler. Toutefois, l'idéal est effectivement d'obtenir ce temps soulagé des tracas de la survie.

Nonobstant ce qui vient d'être dit, pris globalement, les événements personnels remémorés montrent peu d'effet de réminiscence (reminiscence bump) durant l'entrée dans l'âge adulte. Les changements récents ne sont pas plus denses parmi les jeunes groupes d'âge, et les tournants de l'existence ne présentent qu'un léger pic de souvenirs entre 10 et 30 ans, à peine plus élevé au final que l'effet de récence. La mémoire personnelle suit la courbe mémorielle classique. Il n'y a qu'un léger décalage entre les quartiers, l'entrée dans l'âge adulte apparaissant plus précoce parmi les pauvres, confirmant ce que la littérature avait souligné (étudiant des cohortes disparates en termes socioéconomiques, Battagliola, Brown, \& Jaspard [1997] remarquent que les écarts d'entrée dans l'âge adulte se réduisent et retardent parmi les individus dotés en capitaux, alors qu'ils s'accroissent parmi les individus dépourvus d'atouts, souvent en rajeunissant, ce que Claire Bidart [2006b] confirme également). C'est alors que se dessinent de manière décisive des trajectoires de cumul des (dés)avantages.

Si cela vaut pour les individus du même âge, entre les cohortes la véritable divergence repose plutôt sur le contenu des événements rappelés. Les jeunes évoquent des capitaux plus diversifiés, des ressources et des trajectoires multiples alors que les autres classes d'âge se concentrent sur la famille et la santé. Si la vulnérabilité signifie vivre peu de changements (parce que l'on n'aurait pas les moyens de les réaliser), cela ne se confirme pas dans notre enquête. Par contre, l'importance des capitaux multiples (social, culturel, de santé et pas uniquement économique) est clairement perceptible dans les réponses fournies par nos répondants, illustrative d'une Inde urbain émergente où l'éducation devient petit à petit accessible 
à tous, où la santé est impactée par les modifications de style de vie, où la famille demeure la seule assurance sociale.

La société indienne étant régie par des normes informelles fortes, le besoin de régulations formelles pour les appuyer ne se fait pas sentir (Settersten, 2004). Au contraire, les individus ont conscience de ces normes renforcées par un mécanisme de contrôle social fait de prescriptions ainsi que de proscriptions - et s'autorégulent spontanément. Ceci se constate dans la convergence des événements qui marquent la vie des Indiennes et des Indiens, au niveau de leur contenu mais aussi des âges auxquels ceux-ci surviennent. Une sorte d'horloge sociale détermine ainsi les âges de la vie appropriés où fonder une famille, être indépendant financièrement, marier ses enfants, prendre soin de ses parents âgés, etc.

\section{L'institution familiale au centre des existences}

Le constat que la trajectoire familiale, celle qui engage directement l'individu ou qui le touche à travers ses proches, est identifiée comme centrale dans les trajectoires de vie perçues est attesté en Occident ou dans des aires occidentalisées comme l'Amérique du Sud (Bonvalet \& Lelièvre, 2012; Cavalli et al., 2006; Gastrón \& Lacasa, 2009). Il se retrouve également dans les réponses des habitants de Mumbai. Les mariages et les naissances sont les souvenirs les plus courants parmi les tournants de la vie rapportés par les individus, toutefois moins nombreux qu'attendu à en parler, au vu de la place qu'accorde la littérature à la famille au cœur de la société indienne et de ses valeurs.

Des marques de vulnérabilités liées aux anciennes normes (avoir eu un mariage trop précoce, avoir accepté un mariage arrangé, la préférence pour un fils) sont observables dans nos résultats, mais demeurent en infériorité numérique. La plupart des évocations laissent entendre une acceptation des normes de mariage et de naissance, la joie de vivre ces événements attendus et espérés.

Une part des changements familiaux remémorés concerne néanmoins des événements inattendus (un mariage trop jeune, par exemple) qui, dans un cadre comme l'Inde et pour des individus pauvres avant tout, prend une importance toute particulière. Ainsi, les décès sont évoqués beaucoup dans les deux quartiers, mais les pertes hors-timing (trop précoces) ressortent davantage parmi les réponses des habitants de Bandra (avoir perdu un enfant, ou un parent en bas âge). 
L'emphase est sur des continuités millénaires, la résistance du système des castes, de l'endogamie, du patriarcat, tendant à donner une image passablement monolithique des familles indiennes et de leur vie. Toutefois, les résultats dessinent un portrait à la fois réaliste et nuancé de cette réalité. Certes, les questions étudiées ici, même si elles remplissent plutôt leur fonction de levier, ne peuvent tout révéler, mais le jeu des dits et non-dits n'en reste pas moins instructif. Ainsi, la criante absence d'événements religieux ou de mentions de la caste, qui nous a surpris à bien des moments au cours de ce livre, semble indiquer pour part des évidences qui ne méritent pas d'être évoquées, et d'autre part des tensions, des tabous autour de notions encore existantes et toujours sources de discrimination.

Dans la religion hindoue, largement majoritaire en Inde, le mariage est strictement associé à l'endogamie de caste (Joshi, 2016). Généralement, les unions sont arrangées par les familles, qui choisissent les candidats et négocient une dot financée par la famille de l'épouse. Selon LoiselleLéonard (2001), à l'orée du $21^{\text {ème }}$ siècle, 95\% des mariages étaient encore arrangés et accompagnés d'une dot, alors même que cette dernière est sensée être interdite par la Constitution depuis 1961. Tant la dot et les violences qu'elle génère que la minorité de «mariages d'amour» exogames sont des objets médiatiques, mais à Bandra et Santa Cruz, ils sont à peine, voire pas du tout cités et, tout comme la religion et les castes, les arrangements et la dot restent essentiellement dans le non-dit. Il est difficile d'interpréter ce silence, mais il suggère une intériorisation si forte qu'elle va sans dire, ce qui nous ramène à l'ambivalence des témoignages collectés.

\section{Le choc des générations : une modernité à plusieurs vitesses}

$\mathrm{Au}$ fil du temps, les sociétés et les personnes qui les composent se transforment. Toutefois, ces individus n'ont pas tous la même durée de vie et si certains n'ont connu que des mutations récentes, d'autres ont traversé le temps : les âgés possèdent ainsi, par évidence, des référents différents de ceux des plus jeunes. Dans nos données, cette opposition s'observe à deux niveaux. Premièrement, de manière transversale, les membres d'une société ne sont pas égaux et une stratification d'âge apparaît, évidente en ce qui concerne l'éducation : quasi impossibilité pour les âgés d'avoir été à l'école (en leur temps) alors qu'une démocratisation relative ces dernières années a permis à presque tous les jeunes de suivre un cursus scolaire au moins minimal. 
En second, cette fois de manière longitudinale, les transformations sociétales influencent le regard analytique de tous les individus, quel que soit leur âge, mais les inégalités sautent sans doute davantage aux yeux des personnes ayant vécu le temps passé. Pour reprendre l'exemple indien de la scolarité, il est imaginable que d'être né au milieu du $20^{\text {ème }}$ siècle et de ne pas avoir pu aller à l'école (une discrimination qui touchait antérieurement l'essentiel du peuple) ait été plus ou moins toléré, alors qu'à l'heure actuelle (où la majorité des gens bénéficie de cet accès) cette situation devienne révélatrice d'une inégalité cruciale et insupportable. Dans les mémoires des répondants, ce phénomène se révèle par les mentions d'absence d'éducation et leurs raisons (regret de n'avoir pas pu continuer ses études), nombreuses parmi les jeunes dans la vingtaine, les trentenaires, voire les cinquantenaires.

Lorsqu'il a été demandé aux répondants leur perception concernant leur style de vie, proche de l'ancienne ou de la nouvelle génération, l'influence des normes est apparue déterminante, expliquant que la majorité des individus se sent en harmonie avec ses géniteurs. Toutefois, parmi les personnes âgées, la proportion ayant indiqué vivre selon les modes de vie de leur descendance est plus importante que chez les trentenaires ou les quinquagénaires, dénotant à la fois de la conscience d'un monde qui évolue et d'une relation de dépendance au moment où être actif n'est plus possible et où seuls les enfants garantissent la survie. Enfin, cela souligne aussi l'avancée de l'histoire, puisque la génération précédant les octogénaires dans nos enquêtes a connu un monde bien différent, sous la colonisation.

Ces vulnérabilités multiples prennent également leur source dans les bouleversements des relations familiales et traditionnelles issues de la modernité, porteuse d'améliorations futures mais qui brise les anciennes formes de solidarité et laisse, pour l'heure, les individus bien démunis (Saglio-Yatzimirsky, 2002).

\section{Questions de genre : être une femme dans l'Inde urbaine actuelle}

Dans les domaines économiques et professionnels, les recherches existantes soulignent la forte séparation des tâches selon le genre, la distinction persistante entre sphères publique et privée, l'une comme l'autre exprimant le manque d'indépendance féminine (Guérin, 2008). L'épouse est responsable de l'éducation de ses enfants, de la tenue du ménage et de son harmonie. Même au sein d'un foyer dont la gestion est reléguée aux membres féminins, l'égalité n'est pas obligatoirement de mise. Des 
marques de discriminations sont visibles au quotidien, par exemple lorsque les hommes mangent avant les femmes. Mais dans la sphère publique, les obligations financières reposent sur les épaules du mari, qui doit assurer la subsistance du ménage, même si, en pratique, ce sont souvent les femmes qui se chargent de l'organisation financière et de la comptabilité.

Plus spécifiquement, nos résultats confirment que la vie professionnelle, le monde économique, sont encore largement l'apanage - et la charge - des hommes, qui font l'objet d'une pression constante pour assurer leur fonction de breadwinner dans un contexte économique marqué, en particulier dans les bidonvilles, par l'économie informelle et le sous-emploi. Pour autant, le contraste est loin d'être absolu. Une proportion significative des femmes, aussi bien des classes pauvres que moyennes, s'engage dans l'espace public et se sent directement concernée par l'économie ou la qualité de l'éducation. Revers de la médaille, c'est bien souvent en lieu et place d'assurance maladie ou chômage que les épouses se trouvent contraintes de travailler, et les mentions de promotion ou de carrière sont très absentes des réponses fournies par les habitantes de Mumbai. Néanmoins, l'autonomie réduite, la subordination aux hommes de la famille, d'abord le père puis le mari, enfin les fils (Vella, 2003), ne ressortent pas de nos témoignages.

Seule, comme nous l'avons vu, la satisfaction face à la naissance de garçons exprime ouvertement la discrimination envers les femmes. Le reste se trouve dans le non-dit, ou dans les nombreux signes d'inégalités qui transparaissent en filigrane dans nos résultats. Ainsi, le stress familial et la dépendance au mari, les mariages trop précoces ou la pression pour devenir mère, les impacts de la virilocalité et de la vie dans un ménage élargi sont notamment apparus comme raisons pour évoquer des tournants familiaux (naissances, mariages, décès, ...).

\section{Vulnérabilités patentes, vulnérabilités cachées}

Comme la discussion des deux premiers axes l'a fait ressortir avec constance, les vulnérabilités sont partout; elles ressortent souvent avec force. Pour conclure cette conclusion, revenons explicitement sur leurs modalités et significations. 
En Inde, la globalisation ne permet pas de s'affranchir de la montée des risques nouveaux; l'absence de protection sociale, le maintien des inégalités reproduites par les structures en place (à l'heure actuelle, 100 personnes possèdent l'équivalent de $25 \%$ du PIB du pays [Roy, 2014]) et la pauvreté de masse créent une augmentation des fractures sociales et des vulnérabilités, ce malgré une diminution tendancielle de la misère en Inde. Un phénomène révélé par nos données est celui de la montée d'une vulnérabilité perçue au niveau personnel (dans les domaines de la santé, de l'éducation ou de la famille par exemple, mais aussi en ce qui concerne les événements sociohistoriques, dont la portée est généralement collective mais dont les raisons apportées sont souvent centrées sur l'individu), dans un monde qui connait déjà la vulnérabilité de groupe. Amartya Sen (1999) l'a souligné voici quelques temps: quand bien même le niveau de vie général d'une population augmente, ne s'observe pas nécessairement dans une même proportion un accroissement des libertés réelles de mener une vie conforme à ses valeurs.

\section{Le silence assourdissant de la pauvreté}

La pauvreté de masse est très peu évoquée par les répondants (qui ne parlent jamais de misère ni de dénuement, faisant seulement, parfois, quelques allusions à la difficulté de boucler les fins de mois). Elle est intériorisée, si évidente qu'elle va sans dire. De manière significative, les mentions de difficultés économiques ne ressortent guère qu'à Santa Cruz, parmi les classes moyennes inférieures; elles font souvent référence à l'inflation et à l'augmentation des prix qui font rage en Inde depuis quelques années. Pour les habitants des bidonvilles, les aspects économiques ne semblent pas marquer leur vie, malgré l'évidence ; il est plus juste de dire qu'ils n'émergent que peu d'un travail de sélection mémorielle. Lorsque c'est le cas, ils apparaissent plutôt sous forme de conséquences de tel ou tel événement sur leur existence quotidienne (nécessité de reprendre le travail, coût d'une dépense particulière comme un mariage, etc.). Nonobstant, la pauvreté est, nous l'avons vu, de manière récurrente au centre des raisons pour avoir cité un tournant, par exemple dans les dépenses liées aux problèmes médicaux, aux répercussions sur les autres membres du ménage en cas de décès, etc.

En cohérence, le soulagement d'avoir un travail est l'une des premières motivations des 20-24 et 35-39 ans vivant dans les bidonvilles pour évoquer leur situation professionnelle. Toutefois, le regret d'avoir 
débuté trop tôt sur le marché de l'emploi, impliquant la fin des études et plus globalement une entrée précoce dans l'âge adulte, est aussi présent parmi les répondants de Bandra, y compris les plus jeunes. De leur côté, les licenciements et le chômage apparaissent davantage parmi les réponses des quinquagénaires et des sexagénaires, ceux qui ont vécu la désindustrialisation de Mumbai dans les années 1980. Ces réponses soulignent la prégnance de l'incertitude au quotidien issue de l'informalité ainsi que, en contrepartie, l'importance au cours d'une vie des périodes de stabilité professionnelle. Parmi les répondants de classe moyenne inférieure, les citations de promotions, bien que peu nombreuses, laissent entrevoir une vision différente du travail, plus positive.

Dans les deux terrains d'enquête, l'éducation est un sujet qui compte, car chargé d'espoirs et de désespoirs. La partie positive (réussite scolaire, début des études supérieures, ...) est marquée, quel que soit le lieu de résidence. Néanmoins, les aspects négatifs de la formation (échec, impossibilité de continuer, ...) ressortent presque exclusivement chez les habitants des slums, qui sont ou ont été entravés dans leur accès aux études. Le manque est crucial, mentionné comme un regret surtout par les 20-24 et 35-39 ans résidant à Bandra. En outre, plus de personnes éduquées vivant dans ce quartier évoquent l'éducation, signe manifeste d'une vulnérabilité de classe, de laquelle les individus s'extirpent par la réussite individuelle.

\section{L'enchâssement de l'agency et de l'individualisation dans les stratifica- tions sociales}

Une focale sur les dimensions d'agency et d'individualisation présents dans les souvenirs des répondants a également permis de creuser des formes diverses et sous-jacentes de la vulnérabilité. Corroborant la littérature sur le sujet, nos données montrent qu'avoir un faible niveau d'éducation et résider dans les bidonvilles réduisent fortement la mention d'événements qui ont résulté de choix personnels. Bien qu'en Inde aussi, les individus soient soumis à l'injonction de réussir par eux-mêmes leur existence, leurs choix sont délimités par les ressources à disposition. Ceci se confirme en observant les différences selon la classe d'âge : les événements cités par les jeunes - au bénéfice de plusieurs capitaux - sont nettement plus le résultat du volontarisme des acteurs, tandis que leurs aînés évoquent des souvenirs davantage subis (l'impact de la santé se fait ici sentir, ce qui atteste d'un effet d'âge et pas juste de cohorte). 
Lorsque l'on se penche sur la personne concernée par le changement récent vécu, il en ressort que la position dans le parcours de vie a son importance : les jeunes et les très âgés se centrent sur eux-mêmes, alors que les individus en responsabilité d'une famille élargissent les événements mentionnés à d'autres proches. Parmi les tournants, les pauvres, les femmes et les moins éduqués se placent davantage au centre des événements évoqués. Un double constat s'impose : être indépendant, répondre à l'injonction d'individualité et d'agency prônée par la modernité dépend à la fois de la position dans les stratifications sociales (âge, sexe, niveau d'éducation...) et d'autre part des rôles (et donc des devoirs, des charges) que la personne doit assumer selon son âge.

\section{Les diverses manifestations du stress dans le parcours de vie}

Présentés dans le chapitre 9 de ce livre, quelques dimensions atypiques de la vulnérabilité ont encore émergé dans nos résultats. La première est illustrée par les répondants qui ont cité des états de vie, des situations étales sur la durée, alors même que la question appelait la mention de transitions, de tournants. Le contenu de ces réponses spontanées souligne clairement l'instabilité de l'existence vécue par ces individus pauvres, le sentiment d'insécurité qui en résulte. Il s'y ajoute le stress chronique qui s'exprime au travers de certaines raisons, illustrant les conditions de vie difficiles dans lesquelles les trajectoires se déploient. L'absence de tournant est une autre particularité des événements personnels rapportés par les répondants, se focalisant avant tout sur le non-accès aux études ou l'espérance déçue de voir arriver un enfant. Finalement, les raisons pour avoir cité un événement personnel se rapportant à une temporalité trop longue ou trop courte (hors-timing) dénotent elles-aussi d'une prise de conscience cruciale de vulnérabilités ayant impacté le parcours de vie sur sa longueur.

$* * *$

L'ambition de ce livre a été d'ouvrir un espace dédié à la voix des pauvres, de donner la parole à celles et ceux qui vivent un quotidien difficile et n'ont que trop rarement l'occasion de le partager. Plonger au cœur des bidonvilles, en s'appliquant à éviter tant le voyeurisme que l'apitoiement, a représenté le premier pas de cette entreprise. Les réponses fournies par les individus ont montré à la fois sincérité, dignité et pudeur à propos d'un monde en émulsion, fracturé et inégal. Elles ont permis de tenter une interprétation des parcours de vie en leur rendant, autant que la distance le permette, bonne justice. 



\section{Bibliographie}

Abbott, A. (1997). On the concept of turning point. Comparative Social Research, 16, 85-105.

Abbott, A. (2010). A propos du concept de turning point. In M. Bessin, C. Bidart, M. Grossetti (Eds.), Bifurcations. Les sciences sociales face aux ruptures et à l'événement (p.187-211). Paris: La Découverte.

Aeby, G. (2006). L'impact de l'histoire sur les mémoires individuelles (Mémoire de licence en Sociologie). Université de Genève, Genève.

Agarwala, R. (2008). Reshaping the social contract: emerging relations between the state and informal labor in India. Theory and Society, $37(4), 375-408$.

Allendorf, K. (2013). Going Nuclear? Family Structure and Young Women's Health in India, 1992-2006. Demography, 50(3), 853-880.

Alter, G., Neven, M., \& Oris, M. (2004). Mortality and Modernization in Sart, 1812-1900. In T. Bengtsson, C. Campbell (Eds.), Life Under Pressure: Mortality and Living Standards in Europe and Asia, 1700-1900 (p. 173-208). Cambridge: MIT Press.

Alter, G., \& Oris, M. (2008). Effects of inheritance and Environment on the Heights of Brothers in Nineteenth-Century Belgium. Human Nature, 19, 44-55.

Alwang, J., Siegel, P. B., \& Jorgensen, S. L. (2001). Vulnerablity: A View From Different Disciplines. Social Protection Discussion Paper Series, 115, World Bank.

Alwin, D. F., \& McCammon, R. J. (2007). Rethinking Generations. Research in Human Development, 4(3-4), 219-237.

Alwin, D. F., \& McCammon, R. J. (2004). Generations, Cohorts, and Social Change. In J. T. Mortimer, M. J. Shanahan (Eds.), Handbook of the Life Course (p. 23-49). US: Springer.

Alwin, D. F., \& Wray, L. A. (2005). A Life-Span Developmental Perspective on Social Status and Health. The Journals of Gerontology Series B: Psychological Sciences and Social Sciences, 60(2), 7-14.

Ambedkar, B. R. (1936) [1990]. The Annihilation of Caste. New Delhi: Arnold Publishers. 
Amossy, R. (2010). La présentation de soi. Paris: Presses universitaires de France.

Anand, G. (2011, juin). India Lacks 'Depth of Learning': OECD. Consulté à: http://blogs.wsj.com/indiarealtime/2011/06/14/india-lacks-depthof-learning-oecd/

Anderson, B. (2002). L'imaginaire national. Réflexions sur l'origine et l'essor du nationalisme. Paris: La Découverte.

Angueletou, A. (2007, septembre). Etalement urbain et périurbanisation des grandes métropoles indiennes, le cas de Mumbai. Présenté au Colloque étalement urbain et ville fragmentée à travers le monde, des théories aux faits. France: Orléans.

Antoine, P., Lelièvre, E., Collectif, \& Gourgeau, D. (2006). Etats flous et trajectoires complexes : Observation, modélisation, interprétation. Paris: INED.

Appadurai, A. (2000). Spectral Housing and Urban Cleansing: Notes on Millennial Mumbai. Public Culture, 12(3), 627-651.

Appadurai, A. (2005) [1996]. Après le colonialisme: les conséquences culturelles de la globalisation. Paris: Payot.

Archetti, C. (2008). News Coverage of 9/11 and the Demise of the Media Flows, Globalization and Localization Hypotheses. International Communication Gazette, 70(6), 463-485.

Arundhati Roy speaks out against Indian rape culture. (2012). Channel 4, Record 2013. Consulté à l'adresse https://www.channel4.com/news/ arundhati-roy-speaks-out-against-indian-rape-culture.

Assayag, J. (1999). Christophe Jaffrelot, La démocratie en Inde. Religion, caste et politique. L'Homme, 39(151), 312-315.

Assayag, J. (2005). La mondialisation vue d'ailleurs: l'Inde désorientée. Paris: Seuil.

Assmann, J., \& Czaplicka, J. (1995). Collective Memory and Cultural Identity. New German Critique, 65, 125-133.

Asthana, S. (2013). Broadcasting, space, and sovereignty in India. Media, Culture \& Society, 35(4), 516-534.

Atkinson, W. (2007). Beck, individualization and the death of class: a critique. The British Journal of Sociology, 58(3), 349-366.

Attané, I. (2005). En Chine, des millions de femmes «manquantes». Outre-Terre, 15(2), 471-479. 
Attias-Donfut, C. (1988). Sociologie des générations: l'empreinte du temps. Paris: Presses universitaires de France.

Attias-Donfut, C. (1991). Générations et âges de la vie. Paris: Presses universitaires de France.

Avison, W. R., \& Turner, R. J. (1988). Stressful Life Events and Depressive Symptoms: Disaggregating the Effects of Acute Stressors and Chronic Strains. Journal of Health and Social Behavior, 29(3), 253-264.

Baber, Z. (2004). 'Race', Religion and Riots: The 'Racialization' of Communal Identity and Conflict in India. Sociology, 38(4), 701-718.

Bairoch, P. (1997). Victoires et déboires. Histoire Economique et sociale du monde du XVe siècle à nos jours. Paris: Gallimard.

Balarajan, Y., Ramakrishnan, U., Özaltin, E., Shankar, A. H., \& Subramanian, S. (2011). Anaemia in low-income and middle-income countries. The Lancet, 378(9809), 2123-2135.

Baltes, P. B. (1987). Theoretical propositions of life-span developmental psychology: On the dynamics between growth and decline. Developmental Psychology, 23(5), 611-626.

Baltes, P. B. (1997). On the incomplete architecture of human ontogeny. Selection, optimization, and compensation as foundation of developmental psychology. American Psychologist, 4(52), 366-380.

Baltes, P. B., Lindenberger, U., \& Staudinger, U. M. (2006). Life-span Theory in Developmental Psychology. In W. Damon, R. M. Lerner (Eds.), Handbook of Child Psychology (p. 1029-1144). New York: Wiley.

Baltes, P. B., Reese, H. W., \& Lipsitt, L. P. (1980). Life-span developmental psychology. Annual Review of Psychology, 31, 65-110.

Baltes, P. B., \& Smith, J. (1999). Multilevel and Systemic Analyses of Old Age: Theoretical and Empirical Evidence for a Fourth Age. In V. L. Bengtson, K. W. Schaie (Eds.), Handbook of Theories of Aging (p. 153-173). New York: Springer.

Baltes, P. B., Staudinger, U. M., \& Lindenberger, U. (1999). Lifespan Psychology: Theory and Application to Intellectual Functioning. Annual Review of Psychology, 50, 471-507.

Banerjee, A., \& Piketty, T. (2005). Top Indian Incomes, 1922-2000. The World Bank Economic Review, 19(1), 1-20.

Banerjee, P. (2005). Femmes en Inde : législation et réalités. Diogène, 212, $107-127$. 
Banerjee, P. (2016). Burning Women: Widows, Witches, and Early Modern European Travelers in India. New York: Springer.

Banerjee, S., Chatterji, A., Chaudhry, L. N., Desai, M., Toor, S., \& Visweswaran, K. (2004). Engendering Violence: Boundaries, Histories, and the Everyday. Cultural Dynamics, 16(2-3), 125-139.

Banerjee-Guha, S. (2002). Shifting Cities: Urban Restructuring in Mumbai. Economic and Political Weekly, 37(2), 121-128.

Banerji, D. (1979). Place of the Indigenous and the Western Systems of Medicine in the Health Services of India. International Journal of Health Services, 9(3), 511-519.

Bartley, M. (1994). Unemployment and ill health: understanding the relationship. Journal of Epidemiology \& Community Health, 48(4), 333337.

Bartley, M. (2012). Explaining health inequality: Evidence from the UK. Social Science \& Medicine, 74(5), 658-660.

Bartley, M. (2016). Health Inequality: An Introduction to Concepts, Theories and Methods. New York: Wiley.

Bartley, M., Blane, D., \& Montgomery, S. (1997). Health and the life course: why safety nets matter. British Medical Journal, 314(7088), 1194-1196.

Basu, R., \& Thomas, M. D. (2009). Exploring women's daily lives and participation in the informal labour market in Mumbai, India. Gender \& Development, 17(2), 231-242.

Battagliola, F., Brown, E., \& Jaspard, M. (1997). Itinéraires de passage à l'âge adulte. Différences de sexe, différences de classe. Sociétés contemporaines, 25(1), 85-103.

Bautès, N., Saglio-Yatzimirsky, M.-C., \& Boissinot, E. (2011). Ressources foncières et pression immobilière à Mumbai (Inde). Revue Tiers Monde, 206, 55-74.

Beck, U. (1992). Risk Society: Towards a New Modernity. London: Sage.

Beck, U. (1998). Le conflit des deux modernités et la question de la disparition des solidarités. Lien social et Politiques, 39, 15-25.

Beck, U. (2001) [1986]. La société du risque. Paris: Aubier.

Beck, U. (2003). La société du risque globalisé revue sous l'angle de la menace terroriste. Cahiers internationaux de sociologie, 114(1), 27-33. 
Beck, U. (2007). Beyond class and nation: reframing social inequalities in a globalizing world. The British Journal of Sociology, 58(4), 679-705.

Beck, U., \& Beck-Gernsheim, E. (2002). Individualization: institutionalized individualism and its social and political consequences. London: Sage.

Bengtson, V. L., \& Allen, K. R. (1991). Life course perspective applied to the family. In P. Boss, W. J. Doherly, R. LaRossa, W. R. Schumm, S. K. Steinmetz (Eds.), Sourcebook of Family Theories and Methods: A contextual approach (p. 469-504). New York: Plenum.

Bengtson, V. L., \& Allen, K. R. (2009). The Life Course Perspective Applied to Families Over Time. In P. Boss, W. J. Doherty, R. LaRossa, W. R. Schumm, S. K. Steinmetz (Eds.), Sourcebook of Family Theories and Methods (p. 469-504). US: Springer.

Bengtsson, T. (2004). Living Standards and Economic Stress. In T. Bengtsson, C. Campbell (Eds.), Life Under Pressure: Mortality and Living Standards in Europe and Asia, 1700-1900 (p. 28-59). Cambridge: MIT Press.

Bengtsson, T., \& van Poppel, F. (2011). Socioeconomic inequalities in death from past to present: An introduction. Explorations in Economic History, 48(3), 343-356.

Bergeron, C. (2013). L'individualisation des épreuves de la vie dans la modernité avancée: une analyse selon la perspective du parcours de vie. Québec: Université de Laval.

Bergeron, C. (2015). Réception du 11 septembre 2001 au Québec. Persistance des mémoires nationales et émergence de mémoires globalisées. Transatlantica. Revue d'études américaines, 1.

Bergouignan, C. (2011). Recourir aux microsimulations pour étudier la mortalité de crise: illustration par la mortalité au Burundi en 1993. Cahiers québécois de démographie, 40(2), 299-329.

Bergouignan, C., \& Eljim, K. (2013, août). Le profil par sexe et âge de la mortalité violente liée aux conflits armés. Présenté à l'IUSSP International Population $27^{\mathrm{ème}}$ Conference. Busan, Corée.

Berntsen, D., \& Rubin, D. C. (2002). Emotionally charged autobiographical memories across the life span: The recall of happy, sad, traumatic and involuntary memories. Psychology and Aging, 17(4), 636-652. 
Berntsen, D., \& Rubin, D. C. (2004). Cultural life scripts structure recall from autobiographical memory. Memory \& Cognition, 32(3), 427442.

Berntsen, D., \& Rubin, D. C. (2006). The centrality of event scale: A measure of integrating a trauma into one's identity and its relation to post-traumatic stress disorder symptoms. Behaviour Research and Therapy, 44(2), 219-231.

Bertaux, D. (1980). L'approche biographique: sa validité méthodologique, ses potentialités. Cahiers internationaux de sociologie, 69, 197-225.

Bertaux, D. (1997). Les récits de vie: perspective ethnosociologique. Paris: Nathan.

Bertaux, D. (2014). Sept propriétés des récits de vie. In S. Ertul, J.-P. Melchior, C. Lalive d'Epinay (Eds.), Subjectivation et redéfinition identitaire: parcours sociaux et affirmation du sujet (p. 29-49). Rennes: Presses universitaires de Rennes.

Berthier, N. (2010). Les techniques d'enquête en sciences sociales. Méthodes et exercices corrigés (4e Ed.). Paris: Armand Colin.

Bertrand, A.-L., El Rassi, N., \& Weibel, V. (2011). Populations in the slums of Bandra East (Mumbai). A story of poverty and birth control. (Mémoire de master en Socioéconomie) Genève: Université de Genève.

Bessin, M. (1996). Les catégories d'âge face aux mutations temporelles de la société. Gérontologie et Société, 77, 45-57.

Bessin, M. (2009). Parcours de vie et temporalités biographiques: quelques éléments de problématique. Informations sociales, 6(156), 12-21.

Bessin, M., Bidart, C., \& Grossetti, M. (2010). Bifurcations: les sciences sociales face aux ruptures et à l'événement. Paris: La Découverte.

Béteille, A. (1974) [1969]. Social Inequality. UK: Penguin Education.

Bhagat, R. B. (2011). Migrants' (Denied) Right to the City. Urban Policies and the Right to the City in India: Rights, Responsibilities and Citizenship. UNESCO, New Delhi.

Bhagat, R. B., Guha, M., \& Chattopadhyay, A. (2006). Mumbai after 26/7 Deluge: Issues and Concerns in Urban Planning. Population and Environment, 27(4), 337-349.

Bhagat, R. B., \& Jones, G. W. (2015). Changement démographique et migration dans la métropole de Mumbai en Inde. In F. Gemenne, 
F. Polet (Eds.), Migrations internationales : un enjeu Nord-Sud? (p. 127-140). Alternatives Sud: Syllepse.

Bhagat, R. B., \& Mohanty, S. (2009). Emerging Pattern of Urbanization and the Contribution of Migration in Urban Growth in India. Asian Population Studies, 5(1), 5-20.

Bhowmik, S. K., \& More, N. (2001). Coping with Urban Poverty: Ex-Textile Mill Workers in Central Mumbai. Economic and Political Weekly, 36(52), 4822-4827.

Bi, M. (1981). Galbraith J. K. - Théorie de la pauvreté de masse. Population, 36(4), 966-966.

Bidart, C. (2005). Les temps de la vie et les cheminements vers l'âge adulte. Lien social et Politiques, 54, 51-63.

Bidart, C. (2006a). Crises, décisions et temporalités: autour des bifurcations biographiques. Cahiers internationaux de sociologie, 1(120), $29-57$.

Bidart, C. (2006b). Introduction : Les transitions vers l'âge adulte, différenciations sociales et culturelles. In C. Bidart (Ed.), Devenir adulte aujourd'hui : perspectives internationales (p. 9-19). Paris: L'Harmattan.

Bidart, C. (2010). Bifurcations biographiques et ingrédients de l'action. In M. Bessin, C. Bidart, M. Grossetti (Eds.), Bifurcations. Les sciences sociales face aux ruptures et à l'événement (p. 224-238). Paris: La Découverte.

Bidart, C., \& Brochier, D. (2010). Les bifurcations comme changements d'orientation dans un processus. In A. Mendez (Ed.), Processus : concepts et méthode pour l'analyse temporelle en sciences sociales (p. 171-190). Academia Bruylant: Intellection.

Bidart, C., \& Gosselin, C. (2014). Rythmes sociaux et interférences temporelles - Exploration de séquences biographiques de calendriers et de récits. Bulletin of Sociological Methodology, 124(1), 34-52.

Birren, J. E., \& Schroots, J. J. F. (2006). Autobiographical memory and the narrative self over the life span. In J. E. Birren, K. W. Schaie (Eds.), Handbook of the psychology of aging (6th edition) (p. 477-498). San Diego: Academic Press.

Bisht, P. (2013). The politics of cosmopolitan memory. Media, Culture \& Society, 35(1), 13-20. 
Blanchet, A., \& Gotman, A. (2010). L'entretien: L'enquête et ses méthodes. Paris: Armand Colin.

Blane, D., Smith, G. D., \& Bartley, M. (1993). Social selection: what does it contribute to social class differences in health? Sociology of Health \& Illness, 15(1), 1-15.

Bongaarts, J. (2001). Household size and composition in the developing world in the 1990s. Population Studies, 55(3), 263-279.

Bonvalet, C., \& Lelièvre, E. (2012). De la famille à l'entourage. L'enquête biographies et entourage. Paris: INED.

Bonvin, J.-M. (2005). La démocratie dans l'approche d'Amartya Sen. L'économie politique, 27(3), 24-37.

Bonvin, J.-M., \& Farvaque, N. (2008). Amartya Sen : Une politique de la liberté. Paris: Michalon.

Boquérat, G. (2002). Le Swadeshi à l'épreuve de l'ouverture. In F. Landy, B. Chaudhuri (Eds.), De la mondialisation au développement local en Inde: questions d'échelles (p. 27-42). Paris: CNRS.

Boucher, M. (2015). Sociologie des turbulences: penser les désordres des inégalités. Paris: L'Harmattan.

Bouffartigue, P., \& Busso, M. (2010). «Précarité», «informalité»: une perspective Nord-Sud pour penser les dynamiques des mondes du travail. Présenté à Congrès $6^{\text {ème }}$ de l'Association Latino Américaine de Sociologie du Travail, Mexique, Mexico.

Bourdelais, P. (2005). Qu'est-ce que la vulnérabilité? Annales de démographie historique, 110(2), 5-9.

Bourdieu, P. (1979). La distinction. Paris: Les Editions de Minuit.

Bourdieu, P. (1980). Le sens pratique. Paris: Les Editions de Minuit.

Bourdieu, P. (1982). Ce que parler veut dire: l'économie des échanges linguistiques. Paris: Fayard.

Bourdieu, P. (1984). The distinction: a social critique of the judgement. Cambridge: Harvard University Press.

Bourdieu, P. (1986). L'illusion biographique. Actes de la recherche en sciences sociales, 62(1), 69-72.

Bourdieu, P. (1994). Stratégies de reproduction et modes de domination. Actes de la recherche en sciences sociales, 105(1), 3-12.

Bourdieu, P. (1997). Le champ économique. Actes de la recherche en sciences sociales, 119(1), 48-66. 
Bourdieu, P. (1998a). Comprendre. In P. Bourdieu (Ed.), La misère du monde (p. 903-939). Paris: Seuil.

Bourdieu, P. (1998b). La misère du monde. Paris: Seuil.

Bourdieu, P. (2011). The Forms of Capital. (1986). In I. Szeman, T. Kaposy (Eds.), Cultural Theory: An Anthology. New York: Wiley.

Bourdon, J. (1992). Television and political memory. Media, Culture \& Society, 14(4), 541-560.

Bourdon, J., \& Kligler-Vilenchik, N. (2011). Together, nevertheless? Television memories in mainstream Jewish Israel. European Journal of Communication, 26(1), 33-47.

Bourgeois, I. (2013). Le droit international localement pour et par les femmes indiennes: la protection contre la violence domestique à Mumbai, Inde. (Mémoire de master en Droit international). Université de Montréal, Montréal.

Boutayeb, A. (2006). The double burden of communicable and non-communicable diseases in developing countries. Transactions of The Royal Society of Tropical Medicine and Hygiene, 100(3), 191-199.

Bresson, M., Géronimi, V., \& Pottier, N. (2013). La vulnérabilité: questions de recherche en sciences sociales. Fribourg: Editions Universitaires Fribourg.

Brown, N. R., Lee, P. J., Krslak, M., Conrad, F. G., G.B. Hansen, T., Havelka, J., \& Reddon, J. R. (2009). Living in History: How War, Terrorism, and Natural Disaster Affect the Organization of Autobiographical Memory. Psychological Science, 20(4), 399-405.

Brown, R., \& Kulik, J. (1977). Flashbulb memories. Cognition, 5(1), 73-99.

Buchholz, S., Hofäcker, D., Mills, M., Blossfeld, H.-P., Kurz, K., \& Hofmeister, H. (2009). Life Courses in the Globalization Process: The Development of Social Inequalities in Modern Societies. European Sociological Review, 25(1), 53-71.

Buckner, R. L., \& Wheeler, M. E. (2001). The cognitive neuroscience of remembering. Nature Reviews Neuroscience, 2(9), 624-634.

Burton, L. M., \& Whitfield, K. E. (2006). Health, Aging, and America's Poor: Ethnographic Insights on Family Co-morbidity and Cumulative Disadvantage. In J. Baars, D. Dannefer, C. Phillipson, A. Walker (Eds.), Aging, Globalization and Inequality: The New Critical Gerontology (p.215-230). Amityville: Routledge. 
Burton-Jeangros, C. (2009). Les inégalités face à la santé: l'impact des trajectoires familiales et professionnelles sur les hommes et les femmes. In M. Oris, E. Widmer, A. de Ribaupierre, D. Joye, D. Spini, G. Labouvie-Vief, J.-M. Falter (Eds.), Transitions dans les parcours de vie et construction des inégalités (p.273-296). Lausanne: Presses polytechniques et universitaires romandes.

Burton-Jeangros, C., Cullati, S., Sacker, A., \& Blane, D. (2015). A life course perspective on health trajectories and transitions. Cham: Springer.

Butalia, U. (2002). Les voix de la partition. Paris: Actes Sud.

Cain, L. D. (1964). The Life Course and Social Change. In R. E. L. Faris (Ed.), Handbook of Modern Sociology (p. 309-319). Chicago: Rand McNally.

Cain, L. D. (2009). Life Course and Social Structure. In W. R. Heinz, J. Huinink, A. Weymann (Eds.), The Life Course Reader (p. 31-63). Frankfurt: Campus.

Calves, A. E., Bozon, M., Diagne, A., \& Kuépié, M. (2006). Le passage à l'âge adulte: repenser la définition et l'analyse des «premières fois». In P. Antoine, E. Lelièvre (Eds.), Etats flous et trajectoires complexes. Observation, modélisation, interprétation (p.137-156). Paris: INED.

Campos, P. H. F., \& Lima, R. de C. P. (2017). Social positions and groups: New approximations between Pierre Bourdieu's sociology and social representation theory. Culture \& Psychology, 23(1), 38-51.

Cartier-Bresson, J. (2013). L'analyse économique des vulnérabilités microéconomiques dans les pays en développement. In M. Bresson, V. Géronimi, N. Pottier (Eds.), La vulnérabilité: questions de recherche en sciences sociales (p. 207-224). Fribourg: Editions Universitaires Fribourg.

Castel, R. (1994). La dynamique des processus de marginalisation : de la vulnérabilité à la désaffiliation. Cahiers de recherche sociologique, $22,11-27$.

Castel, R. (1999). Les métamorphoses de la question sociale. Paris: Folio.

Castel, R. (2013). Individus, risques et supports collectifs. Idées économiques et sociales, 171, 24-32.

Cavalli, S. (2007). Modèle de parcours de vie et individualisation. Gérontologie et société, 123(4), 55-69. 
Cavalli, S., Aeby, G., Battistini, M., Borloz, C., Bugnon, G., De Carlo, I., \& Rosenstein, E. (2006). Ages de la vie et changements perçus. Genève: Université de Genève.

Cavalli, S., \& Lalive d'Epinay, C. (2008). L'identification et l'évaluation des changements au cours de la vie adulte. Schweizerische Zeitschrift für Soziologie, 34(3), 453-472.

Cavalli, S., Lalive d'Epinay, C., Martenot, A., Borella, E., Brahy, R., Concha, V., Gastròn, L., Guichard, E., Henríquez, G., Oddone, M. J., Suárez, H. J., Vrancken, D. (2013). La perception des grands tournants de sa propre vie: une comparaison internationale. In N. Burnay, S. Ertul, J.-P. Melchior (Eds.), Parcours sociaux et nouveaux desseins temporels (p. 29-47). Louvain-la-Neuve: Academia-Bruylant.

Chadda, R. K., \& Deb, K. S. (2013). Indian family systems, collectivistic society and psychotherapy. Indian Journal of Psychiatry, 55(6), 299.

Chakravartty, P. (2002). Translating Terror in India. Television \& New Media, 3(2), 205-212.

Chakravartty, P. (2004). Telecom, National Development and the Indian State: a Postcolonial Critique. Media, Culture \& Society, 26(2), 227249.

Chalana, M. (2010). Slumdogs vs. Millionaires: Balancing Urban Informality and Global Modernity in Mumbai, India. Journal of Architectural Education, 63(2), 25-37.

Chambers, R. (2006). Vulnerability, Coping and Policy. IDS Bulletin, 37(4), 33-40.

Chan, F. K. S., Mitchell, G., Adekola, O., \& McDonald, A. (2012). Flood Risk in Asia's Urban Mega-deltas Drivers, Impacts and Response. Environment and Urbanization Asia, 3(1), 41-61.

Chandavarkar, R. (1994). The origins of industrial capitalism in India: business strategies and the working classes in Bombay, 1900-1940. New York: Cambridge university press.

Chasles, V. (2008). Femmes en Inde. L'information géographique, 72(1), $57-69$.

Chatterjee, P. (1993). The nation and its fragments: colonial and postcolonial histories. Princeton: Princeton University Press.

Chauvel, L. (1998). Le destin des générations. Paris: Presses universitaires de France. 
Chauvel, L. (2000). Les rapports entre les générations. In Y. Michaud (Ed.), Qu'est-ce que la société? Paris: Odile Jacob.

Chauvel, L. (2001). Le retour des classes sociales? Revue de l'OFCE, 79(4), 315-359.

Chauvin, S., \& Lemoine, F. (2005, avril). L'économie indienne: changements structurels et perspectives à long-terme. CEPII.

Chiriboga, D. A. (1995). Transitions. In G. L. Maddox (Ed.), The Encyclopedia of Aging (2nd edition) (p. 941-942). New York: Springer.

Chiriboga, D. A. (1996). In Search of Continuities and Discontinuities Across Time and Culture. In V. L. Bengtson (Ed.), Adulthood and Aging. Research on Continuities and Discontinuities (p. 173-203). New York: Springer.

Cingolani, P. (2013). La vulnérabilité à l'aune d'une critique de la vie quotidienne. In M. Bresson, V. Géronimi, N. Pottier (Eds.), La vulnérabilité: questions de recherche en sciences sociales (p. 165-176). Fribourg: Editions Universitaires Fribourg.

Clausen, J. A. (1995). American Lives: Looking Back at the Children of the Great Depression. US: University of California.

Connell, J., Dasgupta, B., Laishley, R., \& Lipton, M. (1976). Migration from rural areas: the evidence from village studies. Delhi: Oxford University Press.

Contractor, Q. (2012). Unwanted in My City - The Making of a Muslim slum in Mumbai. In L. Gayer, C. Jaffrelot (Eds.), Muslims in Indian Cities: Trajectories of Marginalisation (p. 23-42). Delhi: Oxford University Press.

Conway, M. A. (1997). The Inventory of Experience: Memory and Identity. In J. Pennebaker, D. Paez, B. Rime (Eds.), Collective memory of political events: social psychological perspectives (p. 21-46). New Jersey: L. Erlbaum.

Conway, M. A., \& Haque, S. (1999). Overshadowing the Reminiscence Bump: Memories of a Struggle for Independence. Journal of Adult Development, 6(1), 35-44.

Conway, M. A., \& Pleydell-Pearce, C. W. (2000). The construction of autobiographical memories in the self-memory system. Psychological Review, 107(2), 261-288.

Conway, M. A., Wang, Q., Hanyu, K., \& Haque, S. (2005). A cross-cultural investigation of autobiographical memory. On the universality and 
cultural variation of the reminiscence bump. Journal of Cross-Cultural Psychology, 36(6), 739-749.

Corbin, A. (1982). L'hygiène publique et les «excreta» de la ville préhaussmannienne. Ethnologie française, 12(2), 127-130.

Cordazzo, P., \& Fichet, B. (2013). Transition, passage en sciences sociales. Strasbourg: Néothèque.

Courgeau, D., \& Lelièvre, E. (1989). Analyse démographique des biographies. Paris: INED.

Crenner, E., Guérin, F., \& Houseaux, F. (2002). L'exploitation d'une enquête pilote en vue de l'amélioration d'une enquête innovante auprès des ménages: le cas de l'enquête Histoire de vie. INSEE-Méthodes: Actes des Journées de Méthodologie Statistique (p. 103-125).

Crompton, R., \& Le Feuvre, N. (1996). Paid Employment and the Changing System of Gender Relations: A Cross-National Comparison. Sociology, 30(3), 427-445.

Crystal, S. (2006). Dynamics of Late-Life Inequality: Modeling the Interplay of Health Disparities, Economic Resources, and Public Policies. In J. Baars, D. Dannefer, C. Phillipson, A. Walker (Eds.), Aging, Globalization and Inequality: The New Critical Gerontology (p. 205213). Amityville: Routledge.

Cullati, S. (2013). Factors of change in health trajectories: a life course approach. (Thèse de doctorat en Sociologie) Université de Genève, Genève.

Damon, J. (2014). L'exclusion. Paris: Presses universitaires de France.

Dannefer, D. (1987). Aging as intracohort differentiation: Accentuation, the Matthew effect, and the life course. Sociological Forum, 2(2), 211-236.

Dannefer, D. (2003a). Cumulative Advantage/Disadvantage and the Life Course: Cross-Fertilizing Age and Social Science Theory. The Journals of Gerontology Series B: Psychological Sciences and Social Sciences, 58(6), 327-337.

Dannefer, D. (2003b). Whose life course is it, anyway? Diversity and "linked lives" in global perspective. In R. A. Settersten (Ed.), Invitation to the life course: Toward new understandings of later life (p. 259-268). US: Baywood Publishing Company. 
Dannefer, D. (2004). Toward a Global Geography of the Life Course. In J. T. Mortimer, M. J. Shanahan (Eds.), Handbook of the Life Course (p. 647-660). New York: Springer.

Dannefer, D. (2006). Reciprocal Co-Optation: The Relationship of Critical Theory and Social Gerontology. In J. Baars, D. Dannefer, C. Phillipson, A. Walker (Eds.), Aging, Globalization and Inequality: The New Critical Gerontology (p. 103-120). Amityville: Routledge.

Dannefer, D., \& Kelley-Moore, J. A. (2008). Theorizing the Life Course: New Twists in the Paths. In V. L. Bengtson, D. Gans, N. M. Putney, M. Silverstein (Eds.), Handbook of Theories of Aging, Second Edition (p. 435-454). New York: Springer.

Das Gupta, M., Zhenghua, J., Bohua, L., Zhenming, X., Chung, W., \& Hwa-Ok, B. (2003). Why is Son preference so persistent in East and South Asia? A cross-country study of China, India and the Republic of Korea. The Journal of Development Studies, 40(2), 153-187.

Dasoki, N. (2017). Mémoire autobiographique et vieillissement: représentations des périodes heureuses et vulnérables. (Thèse de doctorat en Psycho-sociologie). Université de Lausanne, Lausanne.

D'Costa, W., \& Das, B. (2002). Life and living of the vulnerable in Ahmedabad City. In A. Kundu, D. Mahadevia (Eds.), Poverty and vulnerability in a globalising metropolis, Ahmedabad (p. 179-206). New Delhi: Manak Publications.

D'Cruz, P., \& Bharat, S. (2001). Beyond Joint and Nuclear: The Indian Family Revisited. Journal of Comparative Family Studies, 32(2), 167-194.

de Sardan, J.-P. O. (2000). Le «je» méthodologique: Implication et explicitation dans l'enquête de terrain. Revue française de sociologie, 41(3), 417-445.

Deaton, A., \& Drèze, J. (2009). Food and Nutrition in India: Facts and Interpretations. Economic and Political Weekly, 44(7), 42-65.

Dejouhanet, L. (2016). L'Inde, puissance en construction. Documentation photographique - Les dossiers, 8109.

Deliège, R. (2006). Le système indien des castes. Villeneuve d'Ascq: Presses universitaires du Septentrion.

Deliège, R. (2011). Caste, Class and Untouchability. In I. Clark-Decès (Ed.), A companion to the anthropology of India (p. 45-61). Chichester: Wiley-Blackwell. 
Delory-Momberger, C. (2009). La condition biographique : Essais sur le récit de soi dans la modernité avancée. Paris: Téraèdre.

Demiray, B., Gülgoz, S., \& Bluck, S. (2009). Examining the life story account of the reminiscence bump: Why we remember more from young adulthood. Memory, 17(7), 708-723.

Desai, A. R. (2000). Recent trends in Indian nationalism. Delhi: Popular Prakashan.

Desai, V. (1994). Migration and labour characteristics of slum dwellers in Bombay. Sociological Bulletin, 43(1), 50-77.

Deschamps, J.-C., Paez, D., \& Pennebaker, J. (2001). Mémoire collective des événements sociopolitiques et culturels: représentation sociale du passé à la fin du millenium. Psychologie et société, 2, 53-74.

Deschamps, J.-C., Paez, D., \& Pennebaker, J. (2002). Mémoire collective et Histoire à la fin du second millénaire. In S. Laurens, N. Roussiau (Eds.), La mémoire sociale: identités et représentations sociales (p. 245-257). Rennes: Presses universitaires de Rennes.

Deshingkar, P. (2008). Circular Internal Migration and Development in India. In J. DeWind, J. Holdaway (Eds.), Migration and Development Within and Across Borders: Research and Policy Perspectives on Internal and International Migration (p.161-188). Genève et New York: OIM, SSRC.

Devereux, G. (2012). De l'angoisse à la méthode dans les sciences du comportement. Paris: Flammarion.

Devriese, M. (1989). Approche sociologique de la génération. Vingtième Siècle. Revue d'histoire, 22, 11-16.

Dewan, A. (2013). Floods in a megacity geospatial approach on hazards, risk and vulnerability. Dordrecht: Springer.

Dimri, A. (2015). Household composition \& women empowerment: Living with in-laws in India. Revista de Economía Aplicada.

Doise, W. (1989). Attitudes et représentations sociales. In D. Jodelet (Ed.), Les représentations sociales (p. 220-238). Paris: Presses universitaires de France.

Doise, W., \& Lorenzi-Cioldi, F. (1989). Sociologues et psychologie sociale. Revue européenne des sciences sociales, 27(83), 147-196.

Draaisma, D. (2008) [2001]. Pourquoi la vie passe plus vite à mesure qu'on vieillit. Paris: Flammarion. 
Drèze, J., \& Sen, A. (2013). An uncertain glory: India and its contradictions. London: Allen Lane.

Dreze, J., \& Sen, A. (2013). Splendeur de l'Inde? Développement, démocratie et inégalités. Londres: Flammarion.

Dribe, M., Manfredini, M., \& Oris, M. (2014). The roads to reproduction. Comparing life course trajectories in preindustrial Eurasia. In C. Lundh, S. Kurosu (Eds.), Similarity in difference. Marriage in Europe and Asia, 1700-1900 (p. 85-116). Harvard: MIT Press.

Dubar, C. (2010) [1991]. La socialisation: Construction des identités sociales et professionnelles (4e Edition). Paris: Armand Colin.

Dubet, F., \& Martuccelli, D. (1996). Théories de la socialisation et définitions sociologiques de l'Ecole. Revue française de sociologie, 37(4), 511-535.

Dumont, L. (1959). Le renoncement dans les religions de l'Inde. Archives de sociologie des religions, 7, 45-69.

Dumont, L. (1966). Homo hierarchicus: Essai sur le système des castes. Paris: Gallimard.

Dupont, V. (2008). L'urbanisation en Inde : révision de quelques mythes et vrais défis. Rayonnement du CNRS, 47, 7-14.

Durkheim, E. (1898). Représentations individuelles et représentations collectives. Revue de Métaphysique et de Morale, 6(3), 273-302.

Dye, C., Trunz, B. B., Lönnroth, K., Roglic, G., \& Williams, B. G. (2011). Nutrition, Diabetes and Tuberculosis in the Epidemiological Transition. PLOS ONE, 6(6).

Dyson, T., Cassen, R., \& Visaria, L. (2004). Twenty-first century India: population, economy, human development, and the environment. Oxford: Oxford University Press.

Echanove, M., \& Srivastava, R. (2011). The high rise \& the slum: speculative urban development in Mumbai. In N. Brooks, K. Donaghy, G.-J. Knaap (Eds.), The Oxford Handbook of Urban Economics \& Planning (p. 789-813). Oxford: Oxford University Press.

Eggimann, M., \& Yannick, R. (2012). Women of the slums: Reproductive life courses in Bandra East, Mumbai. (Mémoire de master en Socioéconomie) Genève: Université de Genève.

Ehrenberg, A. (2000) [1998]. La Fatigue d'être soi. Dépression et société. Paris: Odile Jacob. 
Elder, G. H. (1975). Age Differentiation and the Life Course. Annual Review of Sociology, 1, 165-190.

Elder, G. H. (1994). Time, Human Agency, and Social Change: Perspectives on the Life Course. Social Psychology Quarterly, 57(1), 4-15.

Elder, G. H. (1998). The life course as developmental theory. Child Development, 69(1), 1-12.

Elder, G. H. (1999 [1974]). Children of the great depression: social change in life experience (25th anniversary ed). Boulder, Colorado: Westview Press.

Elder, G. H., \& Johnson, M. K. (2003). The life course and aging: Challenges, lessons, and new directions. In R. A. Settersten (Ed.), Invitation to the life course: Toward new understandings of later life (p. 49-81). US: Baywood Publishing Company.

Elder, G. H., \& George, L. K. (2016). Age, Cohorts, and the Life Course. In M. J. Shanahan, J. T. Mortimer, M. Kirkpatrick Johnson (Eds.), Handbook of the Life Course (p. 59-73). New York: Springer.

Elder, G. H., Shanahan, M. J., \& Jennings, J. A. (2015). Human Development in Time and Place. In R. M. Lerner (Ed.), Handbook of Child Psychology and Developmental Science (p. 6-54). New York: Wiley.

Elder, G. H. (1995). The life course paradigm: Social change and individual development. In P. Moen, G. H. Elder, K. Lüscher (Eds.), Examining lives in context: Perspectives on the ecology of human development (p. 101-139). Washington: American Psychological Association.

Elder, G. H. (2002). Historical times and lives: A journey through time and space. In E. Phelps, F. F. Furstenberg, A. Colby (Eds.), Looking at lives: American longitudinal studies of the twentieth century (p. 194218). New York: Russel Sage Foundation.

Elkouri, R. (2000). Pour le meilleur ou juste pour le pire? La Presse, 21.

Erikson, E. H. (1959). Identity and the life cycle: Selected papers. Psychological Issues, 1, 1-171.

Erikson, E. H. (1988). Youth: Fidelity and Diversity. Daedalus, 117(3), $1-24$.

Ertul, S., Melchior, J.-P., \& Lalive d'Epinay, C. (2014). Introduction. In S. Ertul, J.-P. Melchior, C. Lalive d'Epinay (Eds.), Subjectivation et redéfinition identitaire: parcours sociaux et affirmation du sujet (p. 15-25). Rennes: Presses universitaires de Rennes. 
Esping-Andersen, G. (1999). Social Foundations of Postindustrial Economies. Oxford: Oxford University Press.

Eyerman, R., \& Turner, B. S. (1998). Outline of a theory of generations. European Journal of Social Theory, 1(1), 91-106.

Felber, Y., \& Schmid, M. (2014). Sortir du slum: quelles voies et quels impacts? Une étude sur la relocalisation/réhabilitation des habitants des bidonvilles de Mumbai (Inde). (Mémoire de master en Socioéconomie). Université de Genève, Genève.

Fernandes, L. (2000). Nationalizing 'the global': media images, cultural politics and the middle class in India. Media, Culture \& Society, 22(5), 611-628.

Ferraro, K. F., \& Kelley-Moore, J. A. (2003). Cumulative Disadvantage and Health: Long-Term Consequences of Obesity? American sociological review, 68(5), 707-729.

Ferraro, K. F., Shippee, T. P., \& Schafer, M. H. (2009). Cumulative Inequality Theory for Research on Aging and the Life Course. In V. L. Bengtson, D. Gans, N. M. Putney, M. Silverstein (Eds.), Handbook of Theories of Aging (p. 435-454). New York: Springer.

Fijalkow, Y. (2013). Du territoire vulnérable au territoire qui protège. In M. Bresson, V. Géronimi, N. Pottier (Eds.), La vulnérabilité: questions de recherche en sciences sociales (p. 245-252). Fribourg: Editions Universitaires Fribourg.

Fink, G., Günther, I., \& Hill, K. (2016). Urban Mortality Transitions: The Role of Slums. In D. Ramiro Fariñas, M. Oris (Eds.), New Approaches to Death in Cities during the Health (p. 197-216). Switzerland: Springer.

Fiske, M., \& Chiriboga, D. A. (1990). Change and continuity in adult life. San Francisco: Jossey-Bass Publishers.

Folkman, S., Lazarus, R. S., Pimley, S., \& Novacek, J. (1987). Age Differences in Stress and Coping Processes. Psychology and Aging, 2(2), 171-184.

Galbraith, J. K. (1980) [1952]. A Theory of Price Control. Cambridge: Harvard University Press.

Garenne, M. (2016). Will Urban and Rural Mortality Converge in Africa? In D. Ramiro Fariñas, M. Oris (Eds.), New Approaches to Death in Cities during the Health (p. 181-196). Switzerland: Springer. 
Gastrón, L., \& Lacasa, D. (2009). La percepciòn de cambios en la vida de hombres y mujeres, segùn la edad. Poblaciòn y sociedad, 16, 3-28.

Gemenne, F. (2015). Migrations: réaligner les discours publics sur les réalités. In F. Gemenne, F. Polet (Eds.), Migrations internationales : un enjeu Nord-Sud? (p. 189-208). Alternatives Sud, Syllepse

George, L. K. (1993). Sociological Perspectives on Life Transitions. Annual Review of Sociology, 19, 353-373.

George, L. K. (1990). Social structure, social processes, and social-psychological states. In R. H. Binstock, L. K. George (Eds.), Handbook of Aging and the Social Sciences (p. 186-204). UK: Academic Press.

George, L. K. (2005). Socioeconomic Status and Health Across the Life Course: Progress and Prospects. The Journals of Gerontology Series B: Psychological Sciences and Social Sciences, 60(2), 135-139.

Geronimi, V. (2013). Indicateurs et mesures de la vulnérabilité macroéconomique. In M. Bresson, V. Géronimi, N. Pottier (Eds.), La vulnérabilité: questions de recherche en sciences sociales (p. 203-206). Fribourg: Editions Universitaires Fribourg.

Ghosh, S. (2015). The Political Economy of Domestic Violence in a Mumbai Slum: An Ethnographic Analysis. Journal of Interdisciplinary Economics, 27(2), 175-198.

Giddens, A. (1979). Central Problems in Social Theory: Action, Structure, and Contradiction in Social Analysis. US: University of California Press.

Giddens, A. (1982). Profiles and Critiques in Social Theory. Californie: University of California Press.

Giddens, A. (1986). Action, Subjectivity, and the Constitution of Meaning. Social Research, 53(3), 529-545.

Giddens, A. (1991). The consequences of modernity. Cambridge: Polity Press.

Giddens, A. (2004) [1994]. Les conséquences de la modernité. Paris: L'Harmattan.

Gilgenkrantz, S. (2007). La préférence masculine-Causes et conséquences démographiques. Médecine/sciences, 23(11), 1051-1056.

Girardin, M. (2017). Les configurations familiales aux dernières étapes de la vie. (Thèse de doctorat en Sociologie). Genève: Université de Genève. 
Glasner, T., van der Vaart, W., \& Belli, R. F. (2012). Calendar Interviewing and the Use of Landmark Events - Implications for Cross-cultural Surveys. Bulletin de Méthodologie Sociologique, 115(1), 45-52.

Glickman, L., Tanaka, J. S., \& Chan, E. (1991). Life events, chronic strain, and psychological distress: Longitudinal causal models. Journal of Community Psychology, 19(4), 283-305.

Glück, J., \& Bluck, S. (2007). Looking back across the life span: A life story account of the reminiscence bump. Memory \& Cognition, 35(8), 1928-1939.

Goffman, E. (1973). La mise en scène de la vie quotidienne : La présentation de soi. Paris: Les Editions de Minuit.

Goffman, E. (1975). Stigmate : Les usages sociaux des handicaps. Paris: Les Editions de Minuit.

Golaz, V., \& Lelièvre, E. (2012). L'entourage familial pendant l'enfance et l'adolescence, entre faits et perceptions. Population, 67(3), 491-515.

Grob, A., Krings, F., \& Bangerter, A. (2001). Life markers in biographical narratives of people from three cohorts: A life span perspective in its historical context. Human Development, 44, 171-190.

Grossetti, M. (2004). Sociologie de l'imprévisible: Dynamiques de l'activité et des formes sociales. Paris: Presses universitaires de France.

Grossetti, M. (2010). Imprévisibilités et irréversibilités: les composantes des bifurcations. In M. Bessin, C. Bidart, M. Grossetti (Eds.). Bifurcations. Les sciences sociales face aux ruptures et à l'événement (p. 147-159). Paris: La Découverte.

Guérin, I. (2008). L'argent des femmes pauvres: entre survie quotidienne, obligations familiales et normes sociales. Revue française de Socio-économie, 2(2), 59-78.

Guichard, E. (2015). Mémoires de crises en Amérique Latine: L'histoire vue par ses acteurs (Thèse de doctorat en Sociologie). Genève: Université de Genève.

Guichard, E., \& Henríquez, G. (2011). Memoria histórica en Chile: Una perspectiva intergeneracional para el caso del Concepción metropolitano. Revista Española de Investigaciones Sociológicas, 135(3), 3-26.

Guillaume, J.-F. (2010). Les parcours de vie, entre aspirations individuelles et contraintes structurelles. Informations sociales, 156, 22-30. 
Guilley, E., \& Lalive d'Epinay, C. (2008). The closing chapters of long lives: Results from the 10-year Swilsoo study on the Oldest Old. New York: Nova Science.

Guilmoto, C. Z. (1996). La population de 1'Inde: Evolution historique et tendances contemporaines. Historiens \& Géographes, 356, 301-330.

Guilmoto, C. Z. (2005). L'Inde et son milliard. Démographie en début de siècle. Laboratoire Population-Environnement-Développement, Université de Provence.

Guilmoto, C. Z. (2008). L'économique, le social et le spatial. Les trois dimensions de la surmasculinité juvénile en Inde. Population, 63(1), 93-122.

Guilmoto, C. Z. (2011). Demography for Anthropologists: Populations, Castes, and Classes. In I. Clark-Decès (Ed.), A companion to the anthropology of India (p. 25-44). Chichester: Wiley-Blackwell.

Gupta, B. (1994). Modernity and the Hindu joint family system: a problematic interaction. International Journal on World Peace, 11(4), $37-60$.

Haas, V., \& Jodelet, D. (2007). Pensée et mémoire sociales. In J. P. Pétard (Ed.), Psychologie sociale (p. 117-163). Paris: Bréal.

Haberfeld, Y., Menaria, R. K., Sahoo, B. B., \& Vyas, R. N. (1999). Seasonal migration of rural labor in India. Population Research and Policy Review, 18(5), 471-487.

Hagestad, G. O. (1990). Social perspectives in the life course. In R. H. Binstock, L. K. George (Eds.), Handbook of aging and social sciences (p. 142-159). New York: Academia Press.

Halbwachs, M. (1997) [1950]. La Mémoire Collective. Paris: Albin Michel. Halbwachs, M. (2004) [1925]. Les Cadres Sociaux de la mémoire. Paris: Albin Michel.

Harasty. (2002). Effets différenciés de la libéralisation des échanges sur le niveau de vie, du national au régional. In F. Landy, B. Chaudhuri (Eds.), De la mondialisation au développement local en Inde: questions d'échelles (p. 43-62). Paris: CNRS.

Hareven, T. K. (1986). Historical Changes in the Social Construction of the Life Course. Human Development, 29(3), 171-180.

Hareven, T. K. (1993). Family Time \& Industrial Time: The Relationship Between the Family and Work in a New England Industrial Community. US: University Press of America. 
Hareven, T. K. (1996). Aging and Generational Relations: Life-Course and Cross-Cultural Perspectives. US: Transaction Publishers.

Hareven, T. K., \& Masaoka, K. (1988). Turning Points and Transitions: Perceptions of the Life Course. Journal of Family History, 13(1), 271-289.

Heckhausen, J., Dixon, R. A., \& Baltes, P. B. (1989). Gains and losses in development throughout adulthood as perceived by different adult age groups. Developmental Psychology, 25(1), 109-121.

Heinz, W. R. (2003). Combining Methods in Life-Course Research: A Mixed Blessing? In W. Heinz, V. Marshall (Eds.), Social Dynamics of the Life Course: Transistions, Institutions and Interrelations (p. 73-90). New York: Aldyn De Gruyter.

Heinz, W. R., \& Krüger, H. (2001). Life Course: Innovations and Challenges for Social Research. Current Sociology, 49(2), 29-45.

Heinz, W. R., \& Marshall, V. V. W. (2003). Social Dynamics of the Life Course: Transitions, Institutions, and Interrelations. US: Transaction Publishers.

Hélardot, V. (2010). Vouloir ce qui arrive? Les bifurcations biographiques entre logiques structurelles et choix individuels. In M. Bessin, C. Bidart, M. Grossetti (Eds.), Bifurcations. Les sciences sociales face aux ruptures et à l'événement (p. 160-167). Paris: La Découverte.

Helfter, C. (2009). Cheminer en âge. Informations sociales, 156, 6-9.

Hendricks, J., \& Hatch, L. R. (2008). Theorizing Lifestyle: Exploring Agency and Structure in the Life Course. In V. L. Bengtson, D. Gans, N. M. Putney, M. Silverstein (Eds.), Handbook of Theories of Aging (p. 435-454). New York: Springer.

Henke, J., \& Schlaefli, K. (2009). Integrating Poverty Reduction, Primary Health Care and Women Empowerment: The Example of the Women of India Network in the Slums of Mumbai, India. (Mémoire de master en Socioéconomie). Genève: Université de Genève.

Heuzé, D. G. (2009). Complexité de l'informel. Réflexions à partir des transformations de l'industrie textile en Inde. Les mondes du travail, 9-10, 53-66.

Heuzé, D. G. (2012). L'hindouisme nationalisé et internationalisé. Revue Tiers Monde, 173, 99-126.

Heuzé, G. (1989). Monde ouvrier contemporain et mouvements sociaux. Revue Tiers Monde, 30(119), 613-633. 
Heuzé, G. (1991). Les anthropologues dans la tourmente : la controverse sur les quotas d'embauche en Inde. Journal des anthropologues, 43(1), 167-174.

Heuzé, G. (1997). L'évolution des familles de milieux populaires à Mumbai, Inde. Autrepart, 2, 15-31.

Heuzé, G. (1999). La classe moyenne ou l'enjeu mouvant de l'égalité : réflexion sur le cas indien. Journal des anthropologues. Association française des anthropologues, 77-78, 99-112.

Heuzé, G. (2001). Les conséquences sociales de la libéralisation en Inde. Revue Tiers Monde, 42(165), 33-59.

Hitlin, S., \& Elder, G. H. (2007). Time, Self, and the Curiously Abstract Concept of Agency. Sociological Theory, 25(2), 170-191.

Hogan, D. P., \& Astone, N. M. (1986). The Transition to Adulthood. Annual Review of Sociology, 12, 109-130.

Holmes, A., \& Conway, M. A. (1999). Generation identity and the reminiscence bump: Memory for public and private events. Journal of Adult Development, 6(1), 21-34.

Holmes, T. H., \& Rahe, R. H. (1967). The social readjustment rating scale. Journal of psychosomatic research, 11, 213-218.

Horiuchi, S. (2000). Demography: Greater lifetime expectations. Nature, 405(6788), 744-745.

Hughes, E. C. (1996). Carrières, cycles et tournants de l'existence. In E. C. Hughes, J.-M. Chapoulie (Eds.), Le regard sociologique: essais choisis (p. 165-173). Paris: Ecole des hautes études en sciences sociales.

Husain, Z., \& Ghosh, D. (2017). Analysis of Perceived Health Status Among Elderly in India: Gender and Positional Objectivity. In T. Samanta (Ed.), Cross-Cultural and Cross-Disciplinary Perspectives in Social Gerontology (p. 177-202). Singapore: Springer.

Ihle, A., Oris, M., Fagot, D., \& Kliegel, M. (2017). No cross-sectional evidence for an increased relation of cognitive and sensory abilities in old age. Aging \& Mental Health, 21(4), 409-415.

INED. (1982a). Les âges de la vie. Actes du colloque. Tome 1. Travaux et documents, Cahier n ${ }^{\circ}$ 66, Strasbourg: Presses universitaires de France.

INED. (1982b). Les âges de la vie. Actes du colloque. Tome 2. Travaux et documents, Cahier $\mathrm{n}^{\circ} 102$, Strasbourg: Presses universitaires de France. 
Inglehart, R. (2003). Human values and social change: findings from the values surveys. Leiden: International studies in sociology and social anthropology.

International Institute for Population Sciences (IIPS). (2007). National Family Health Survey (NFHS-3) 2005-06: India. Mumbai: IIPS \& Macro International.

International Institute for Population Sciences (IIPS). (2008). Youth in India: Situation and Needs 2006-2007. Mumbai: IIPS \& Population Council.

Iyer, A., Sen, G., \& George, A. (2007). The Dynamics of Gender and Class in Access to Health Care: Evidence from Rural Karnataka, India. International Journal of Health Services, 37(3), 537-554.

Jadhav, A., Sathyanarayana, K. M., Kuma, S., \& James, K. S. (2013). Living Arrangements of the Elderly in India: Who lives alone and what are the patterns of familial support. Présenté à l'IUSSP International Population $27^{\mathrm{èm}}$ Conference. Busan, Corée.

Jaffrelot, C. (1992). Le syncrétisme stratégique et la construction de l'identité nationaliste hindoue: l'identité comme produit de synthèse. Revue française de science politique, 42(4), 594-617.

Jaffrelot, C. (2010). Les émeutes entre hindous et musulmans : essai de hiérarchisation des facteurs culturels, économiques et politiques. $\mathrm{Cul}$ tures \& Conflits, 5.

Jaffrelot, C. (2014). L'Inde contemporaine. Paris: Fayard.

Jansari, A., \& Parkin, A. J. (1996). Things that go bump in your life: explaining the reminiscence bump in autobiographical memory. Psychology and Aging, 11(1), 85-91.

Janssen, S., Chessa, A., \& Murre, J. (2007). Temporal distribution of favourite books, movies, and records: differential encoding and re-sampling. Memory, 15(7), 755-767.

Janssen, S., Murre, J., \& Meeter, M. (2008). Reminiscence bump in memory for public events. European Journal of Cognitive Psychology, 20(4), 738-764.

Jaoul, N. (2007). Le point de vue des fonctionnaires Dalits sur les quotas d'embauche de la fonction publique. Droit et cultures. Revue internationale interdisciplinaire, 53, 63-87.

Jeffrey, R. (1993). Indian-Language Newspapers and Why They Grow. Economic and Political Weekly, 28(38), 2004-2011. 
Jennings, M. K. (1996). Political knowledge over time and across generations. Public Opinion Quarterly, 60(2), 228-252.

Jennings, M. K., \& Zhang, N. (2005). Generations, Political Status, and Collective Memories in the Chinese Countryside. The Journal of Politics, 67(4), 1164-1189.

Jodelet, D. (1989a). Les représentations sociales. Paris: Presses universitaires de France.

Jodelet, D. (1989b). Représentations sociales: un domaine en expansion. In D. Jodelet (Ed.), Les représentations sociales (p. 31-61). Paris: Presses universitaires de France.

Jodelet, D. (2003). Aperçus sur les méthodologies qualitatives. In S. Moscovici, F. Buschini (Ed.), Les Méthodes des sciences humaines (p. 139-162). Paris: Presses universitaires de France.

Jodelet, D. (2006). Place de l'expérience vécue dans le processus de formation des représentations sociales. In V. Haas (Ed.), Les savoirs $d u$ quotidien. Transmission, appropriations, représentations (p. 235255). Rennes: Presses universitaires de Rennes.

Jodelet, D. (2008). Le mouvement de retour vers le sujet et l'approche des représentations sociales. Connexions, 89, 25-46.

Johansson, S. R. (2000). Macro and micro perspectives on mortality history. Historical Methods: A Journal of Quantitative and Interdisciplinary History, 33(2), 59-72.

Johansson, S. R., \& Kasakoff, A. B. (2000). Mortality History and the Misleading Mean. Historical Methods: A Journal of Quantitative and Interdisciplinary History, 33(2), 56-58.

Johansson, S. R., \& Mosk, C. (1987). Exposure, Resistance and Life Expectancy: Disease and Death During the Economic Development of Japan, 1900-1960. Population Studies, 41(2), 207-235.

Johnson, K. (2001). Media and social change: the modernizing influences of television in rural India. Media, Culture \& Society, 23(2), 147-169.

Jones, G. W., \& Visaria, P. (1997). Urbanization in large developing countries. Oxford: Oxford University Press.

Joshi, D. S. C. (1999). Sociology of Migration and Kinship. Delhi: Anmol Publications.

Joshi, M. (2016). Femmes, de l'objet au sujet du mariage: discours sur le statut des épouses et les mariages mixtes en Inde. Revue Miroirs, 2(4), 100-113. 
Justino, P. (2007). Social security in developing countries: myth or necessity? Evidence from India. Journal of International Development, 19(3), 367-382.

Kadir, M. M., Fikree, F. F., Khan, A., \& Sajan, F. (2003). Do Mothers-inlaw matter? Family dynamics and fertility decision-making in urban squatter settlements of Karachi, Pakistan. Journal of Biosocial Science, 35(4), 545-558.

Kaufmann, J.-C. (2011). L'entretien compréhensif-L'enquête et ses méthodes. Paris: Armand Colin.

Kaviraj, S. (1990). On State, Society and Discourse in India. IDS Bulletin, 21(4), 10-15.

Kellerhals, J., \& Widmer, E. (2007). Familles en Suisse: les nouveaux liens. Lausanne: Presses polytechniques et universitaires romandes.

Kennedy, L. (2002). Développement endogène et mondialisation. Un district industriel au Tamil Nadu. In F. Landy, B. Chaudhuri (Ed.), De la mondialisation au développement local en Inde. Questions d'échelles (p. 99-120). Paris: CNRS.

Kertzer, D. I. (1983). Generation as a Sociological Problem. Annual Review of Sociology, 9, 125-149.

Kessler, R. C., \& McLeod, J. D. (1984). Sex Differences in Vulnerability to Undesirable Life Events. American Sociological Review, 49(5), 620-631.

Kohli, M. (1986). The world we forgot: a historical review of the life course in later life. In V. W. Marshall (Ed.), Later life: The social psychology of aging (p. 271-303). London: Sage.

Kohli, M. (1989). Le cours de vie comme institution sociale. Enquête. Archives de la revue Enquête, 5, 1-6.

Kohli, M. (2007). The Institutionalization of the Life Course: Looking Back to Look Ahead. Research in Human Development, 4(3-4), 253271.

Kroll, M., Bharucha, E., \& Kraas, F. (2014). Does rapid urbanization aggravate health disparities? Reflections on the epidemiological transition in Pune, India. Global Health Action, 7, 1-9.

Krüger, H. (2009). The Life-Course Regime: Ambiguities Between Interrelatedness and Individualization. In W. R. Heinz, J. Huinink, A. Weymann (Eds.), The Life Course Reader: individuals and societies across time (p. 121-138). Frankfurt: Campus. 
Krüger, H., \& Levy, R. (2001). Linking Life Courses, Work, and the Family: Theorizing a not so visible nexus between Women and Men. Cahiers canadiens de sociologie, 26(2), 145-166.

Kuate Defo, B. (2016). Estimating Area-Based Influences on Urban-Rural Inequalities in Health Outcomes: A Longitudinal Multilevel Epidemiological Investigation. In D. Ramiro Fariñas, M. Oris (Eds.), New Approaches to Death in Cities during the Health (p. 157-180). Switzerland: Springer.

Kuh, D., Ben-Shlomo, Y., Lynch, J., Hallqvist, J., \& Power, C. (2003). Life course epidemiology. Journal of Epidemiology and Community Health, 57(10), 778-783.

Kuhn, M. H. (1964). The Reference Group Reconsidered. Sociological Quarterly, 5(1), 5-19.

Kulkarni, V. S., Kulkarni, V. S., \& Gaiha, R. (2017). "Double Burden of Malnutrition": Reexamining the Coexistence of Undernutrition and Overweight Among Women in India. International Journal of Health Services, 47(1), 108-133.

Kundu, A., \& Mahadevia, D. (2002). Globalisation, poverty and vulnerability. In A. Kundu, D. Mahadevia (Eds.), Poverty and vulnerability in a globalising metropolis, Ahmedabad (p. 1-29). New Delhi: Manak Publications.

Kundu, A., \& Sarangi, N. (2007). Migration, Employment Status and Poverty: An Analysis across Urban Centres. Economic and Political Weekly, 42(4), 299-306.

Kundu, D. (2002). Provision of infrastructure and basic amenities. Analysing institutional vulnerability. In A. Kundu, D. Mahadevia (Eds.), Poverty and vulnerability in a globalising metropolis, Ahmedabad (p. 133-178). New Delhi: Manak Publications.

Laborde, C., Lelièvre, E., \& Vivier, G. (2012). Trajectoires et événements marquants, comment dire sa vie? Une analyse des faits et des perceptions biographiques. In Bonvalet C., Lelièvre E. (Eds.), De la famille à l'entourage. L'enquête Biographies et entourage (p. 419-434). Paris: INED.

Lalive d'Epinay, C. (2012). Les parcours de vie au temps de la globalisation. Un examen du «paradigme du parcours de vie». In V. Caradec, S. Ertul, J.-P. Melchior (Eds.), Les dynamiques des parcours sociaux. 
Temps, territoires, professions (p. 21-30). Rennes: Presses universitaires de Rennes.

Lalive d'Epinay, C., Bickel, J.-F., Cavalli, S., \& Spini, D. (2005). Le parcours de vie: émergence d'un paradigme interdisciplinaire. In Guillaume J.-F (Ed.), Parcours de vie. Regards croisés sur la construction des biographies contemporaines (p. 187-210). Liège: Université de Liège.

Lalive d'Epinay, C., \& Cavalli, S. (2007). Changements et tournants dans la seconde moitié de la vie. Gérontologie et Société, 121, 45-60.

Lalive d'Epinay, C., \& Cavalli, S. (2009). Mémoire de l'histoire et appartenance générationnelle des personnes âgées. Gérontologie et Société, 130, 127-144.

Lalive d'Epinay, C., \& Cavalli, S. (2013). Le quatrième âge ou la dernière étape de la vie. Lausanne: Presses polytechniques et universitaires romandes.

Lalive d'Epinay, C., Cavalli, S., Aeby, G., Gastrón, L., Oddone, M. J., Lynch, G., \& Lacasa, D. (2008). Générations et mémoire historique. Une comparaison internationale. In D. Vrancken, L. Thomsin (Eds.), Le social à l'épreuve des parcours de vie (p. 245-259). Louvain-laNeuve: Academia Bruylant.

Lalive d'Epinay, C., Concha, V., Gastrón, L., Guichard, E., Henriquez, G., Lynch, G., \& Oddone, M. J. (2012). Mondialisation et mémoires de l'histoire. Une comparaison internationale et intergénérationnelle. In R. Bourqia (Ed.), La sociologie et ses frontières (p. 119-134). Paris: L'Harmattan.

Landy, F. (2009). India, "Cultural Density" and the Model of Food Transition. Economic and Political Weekly, 44(20), 59-61.

Lani-Bayle, M., \& Mallet, M.-A. (2006). Evénements et formation de la personne: écarts internationaux et intergénérationnels. Paris: 1'Harmattan.

Laub, J., \& Sampson, R. (1993). Turning points in the life course: Why change matters to the study of crime. Criminology, 31(3), 301-325.

Lautier, B. (2013). Universalisation de la protection sociale et protection des plus vulnérables. Revue Tiers Monde, 214, 187-217.

Le Bart, C. (2008). L'individualisation. Paris: Presses de Sciences Po.

Leclerc-Olive, M. (1997). Le dire de l'événement (biographique). Villeneuve d'Ascq: Presses universitaires du Septentrion. 
Leclerc-Olive, M. (1998). Les figures du temps biographique. Cahiers internationaux de sociologie, 104, 97-120.

Leclerc-Olive, M. (2010). Enquêtes biographiques entre bifurcations et événements. Quelques réflexions épistémologiques. In M. Bessin, C. Bidart, M. Grossetti (Eds.), Bifurcations. Les sciences sociales face aux ruptures et à l'événement (p. 329-346). Paris: La Découverte.

Lee, J. Z., Campbell, C., \& Bengtsson, T. (2004). Agency and Demography: Eurasian Comparisons of Life under Pressure. In T. Bengtsson, C. Campbell (Eds.), Life Under Pressure: Mortality and Living Standards in Europe and Asia, 1700-1900 (p. 431-440). Cambridge: MIT Press.

Lelièvre, E. (2006). Ages, Life Courses, Life Event History Analysis. In C. Sauvain-Dugerdil, H. Leridon, N. Mascie-Taylor (Eds.), Human Clocks: The Bio-cultural Meanings of Age (p. 125-143). Bern: Peter Lang.

Lelièvre, E., \& Robette, N. (2015). Trajectoires perçues - Une approche quantitative des perceptions de bien-être. Bulletin of Sociological Methodology, 125(1), 25-45.

Lelièvre, E., \& Vivier, G. (2001). Evaluation d'une collecte à la croisée du quantitatif et de qualitatif. Population, 56(6), 1043-1073.

Le Monde.fr. (2014, 7 juillet). En Inde, 100 millions de personnes supplémentaires considérées comme pauvres.

Levy, R. (2009). Toward a Theory of Life Course Institutionalization. In W. R. Heinz, J. Huinink, A. Weymann (Eds.), The Life Course Reader: individuals and societies across time (p. 178-202). Frankfurt: Campus.

Levy, R. (2013). Analysis of life courses. A theoretical sketch. In R. Levy, E. Widmer (Eds.), Gendered life courses between standardization and individualization. A European approach applied to Switzerland (p. 13-36). Zurich: Lit Verlag.

Levy, R., \& Bühlmann, F. (2016). Towards a socio-structural framework for life course analysis. Advances in Life Course Research, 30, 30-42.

Levy, R., Gauthier, J.-A., \& Widmer, E. (2006). Entre contraintes institutionnelle et domestique: Les parcours de vie masculins et féminins en Suisse. Cahiers canadiens de sociologie, 31(4), 461-489. 
Levy, R., Ghisletta, P., Le Goff, J.-M., Spini, D., \& Widmer, E. (2005). Towards an Interdisciplinary Perspective on the Life Course. New York: Elsevier.

Link, B. G., \& Phelan, J. (1995). Social Conditions As Fundamental Causes of Disease. Journal of Health and Social Behavior, Extra Issue, 80-94.

Liu, J. H., Goldstein-Hawes, R., Hilton, D., Huang, L.-L., Gastardo-Conaco, C., Dresler-Hawke, E., Pittolo F., Hong Y.-Y., Ward C., Abraham S., Kashima Y., Kashima E., Ohashi M. M., Yuki M., \& Hidaka, Y. (2005). Social representations of events and people in world history across 12 cultures. Journal of Cross-Cultural Psychology, 36(2), 171-191.

Liu, J. H., \& László, J. (2007). A Narrative Theory of History and Identity. In G. Moloney, I. Walker (Eds.), Social Representations and Identity: Content, Process, and Power (p. 85-108). New York: Springer.

Liu, J. H., Paez, D., Hanke, K., Rosa, A., Hilton, D. J., Sibley, C. G., Cabecinhas R., Zaromb F., Garber I. E., Leong C.-H., Moloney G., Valchev V., Gastardo-Conaco C., Huang L.-L., Quek A.-H., Techio E., Sen R., van Osch Y., Muluk H., Wagner W., Wang F., Khan S.S., Licata L., Klein O., László J., Fülöp M., Cheung J.C.-k., Yue X., Ben Youssef S., Kim U., Park Y., Puch-Bouwman J., Hassall K., Adair J., Unik L., Spini D., Henchoz K., Böhm G., Selart M., Erb H.-P., Thoben D.F., Leone G., Mastrovito T., Atsumi T., \& Suwa, K. (2012). Cross-Cultural Dimensions of Meaning in the Evaluation of Events in World History? Journal of Cross-Cultural Psychology, 43(2), 251-272.

Liu, J. H., Paez, D., Slawuta, P., Cabecinhas, R., Techio, E., Kokdemir, D., Sen R., Vincze O., Muluk H., Wang F., \& Zlobina, A. (2009). Representing World History in the 21st Century The Impact of 9/11, the Iraq War, and the Nation-State on Dynamics of Collective Remembering. Journal of Cross-Cultural Psychology, 40(4), 667-692.

Loftus, E., \& Ketcham, K. (1994). The Myth of Repressed Memory. False Memories and Allegations of Sexual Abuse. New York: St Martin's Press.

Loiselle-Léonard, M. (2001). Mariage arrangé, dot et migration: une combinaison à risque pour une femme hindoue. Canadian Social Work Review, 18(2), 305-319. 
Longo, M.-E., Bourdon, S., Charbonneau, J., Kornig, C., \& Mora, V. (2013). Normes sociales et imprévisibilités biographiques. Agora débats/jeunesses, 65, 93-108.

Lowenthal, M. F., \& Chiriboga, D. A. (1975). Responses to Stress. In M. F. Lowenthal, M. Thurnher, D. A. Chiriboga (Eds.), Four stages of life (p. 146-162). San Francisco: Jossey-Bass.

Ludwig, C., \& Chicherio, C. (2008). Pertes et gains dans le développement adulte. Gérontologie et Société, 123, 109-134.

Lukacs, G. (1974) [1960]. Histoire et conscience de classe : Essais de dialectique marxiste. Paris: Les Editions de Minuit.

Macmillan, R. (2005). The structure of the life course: Standardized? Individualized? Differentiated. New York: Elsevier.

Mahadevia, D. (2001). Sustainable urban development in India: An inclusive perspective. Development in Practice, 11(2-3), 242-259.

Mahadevia, D. (2002). Changing economic scenario. Informalisation and Increased Vulnerability. In A. Kundu, D. Mahadevia (Eds.), Poverty and vulnerability in a globalising metropolis, Ahmedabad (p. 30-79). New Delhi: Manak Publications.

Mahadevia, D., Liu, Z., \& Yuan, X. (2012). Housing of Urban Migrants in India and China. Environment and Urbanization Asia, 3(1), 143-163. Mahadevia, D., \& Sarkar, S. (2012). Handbook of Urban Inequalities. Oxford: Oxford University Press.

Mallath, M. K., Taylor, D. G., Badwe, R. A., Rath, G. K., Shanta, V., Pramesh, C. S., Digumarti, R., Sebastian, P., Borthakur, B. B., Kalwar, A., Kapoor, S., Kumar, S., Gill, J. L., Kuriakose, M. A., Malhotra, H., Sharma, S. C., Shukla, S., Viswanath, L., Chacko, R. T., Pautu, J. L., Reddy, K. S., Sharma, K. S., Purushotham, A. D., \& Sullivan, R. (2014). The growing burden of cancer in India: epidemiology and social context. The Lancet Oncology, 15(6), 205-212.

Mandel, E. (2007) [1974]. Introduction au marxisme. Bruxelles: Formation Léon Lesoil.

Mannheim, K. (1990) [1928]. Le Problème des Générations. Paris: Nathan. Marchal, H., \& Stébé, J.-M. (2012). Les lieux des banlieues. Paris: Le Cavalier Bleu.

Marius-Gnanou, K. (2008). Debt bondage, seasonal migration and alternative issues : lessons from Tamil Nadu (India). Autrepart, 46, 127-142. 
Marjit, S., \& Kar, S. (2009). A Contemporary Perspective on the Informal Labour Market: Theory, Policy and the Indian Experience. Economic and Political Weekly, 44(14), 60-71.

Marmot, M. (2005). Social determinants of health inequalities. The Lancet, 365(9464), 1099-1104.

Marshall, V. W. (2011). Risk, vulnerability, and the life course. LIVES Working Papers, 1.

Marshall, V. W., \& Clarke, P. J. (2010). Agency and Social Structure in Aging and Life-course Research. In D. Dannefer, C. Philipson (Eds.), International Handbook of Social Gerontology (p. 294-305). US: Sage.

Marshall, V. W. (1983). Generations, Age Groups and Cohorts: Conceptual Distinctions. La Revue canadienne du vieillissement, 2(2), 51-62.

Marshall, V. W. (2005). Agency, Events, and Structure at the End of the Life Course. In R. Levy, P. Ghisletta, J.-M. Le Goff, D. Spini, E. Widmer (Eds.), Towards an interdisciplinary perspective on the life course (p. 57-91). US: Elsevier.

Marshall, V. W., \& Mueller, M. M. (2003). Theoretical Roots of the LifeCourse Perspective. In W. R. Heinz, V. W. Marshall (Eds.), Social dynamics of the life course: transitions, institutions, and interrelations (p. 3-32). New York: Aldine de Gruyter.

Martenot, A., \& Cavalli, S. (2014). Histoire et parcours de vie: la perception des changements sociohistoriques. Temporalités, 20, 1-18.

Martin, C. (2013). Penser la vulnérabilité. Les apports de Robert Castel. Alter: Journal Européen de Recherche sur le Handicap, 7(4), 293298.

Martuccelli, D. (1999). Sociologie de la modernité: l'itinéraire du XXe siècle. Paris: Gallimard.

Martuccelli, D. (2002). Grammaires de l'individu. Paris: Gallimard.

Martuccelli, D. (2005). Les trois voies de l'individu sociologique. Espaces Temps.net, 8, 1-12.

Martuccelli, D. (2009). Qu'est-ce qu'une sociologie de l'individu moderne ? Pour quoi, pour qui, comment? Sociologie et sociétés, 41(1), $15-33$.

Marx, K. (1993) [1867]. Le Capital, Livre III. UK: Penguin Classics.

Marx, K. (2009) [1867]. Le Capital. Livre I. Paris: Presses universitaires de France. 
Marx, K., \& Engels, F. (1999) [1847]. Manifeste du Parti communiste. Paris: Flammarion.

Marx, K., \& Engels, F. (2014). L'idéologie allemande. Paris: Sociales Editions.

Masson, E., \& Haas, V. (2010). Dire et taire : l'intersubjectivité dans l'entretien de recherche. Bulletin de psychologie, 505(1), 5-13.

Mauger, G. (1995). Jeunesse : l'âge des classements. Recherches et Prévisions, 40(1), 19-36.

Mauger, G. (2015). Âges et générations. Paris: La Découverte.

Mayer, K. U. (2001). The paradox of global social change and national path dependencies : Life course patterns in advanced societies. In A. Woodward, M. Kohli (Eds.), Inclusions and exclusions in European societies (p. 89-110). London: Routledge.

Mayer, K. U. (2004). Whose Lives? How History, Societies, and Institutions Define and Shape Life Courses. Research in Human Development, 1(3), 161-187.

Mayer, K. U. (2015). An Observatory for Life Courses: Populations, Countries, Institutions, and History. Research in Human Development, 12(3-4), 196-201.

Mayer, K. U., \& Schoepflin, U. (1989). The State and the Life Course. Annual Review of Sociology, 15, 187-209.

Mayer, K. U., \& Schoepflin, U. (2009). The State and the Life Course. In W. R. Heinz, J. Huinink, A. Weymann (Eds.), The Life Course Reader: individuals and societies across time (p. 121-138). Frankfurt: Campus.

Mazuy, M., \& Lelièvre, E. (2005). Déclarer ses enfants, déclarer ses conjoints. In C. Lefèvre, A. Filhon (Eds.), Histoires de familles, histoires familiales (p. 573-588). Paris: INED.

McFarlane, C. (2012). Rethinking Informality: Politics, Crisis, and the City. Planning Theory \& Practice, 13(1), 89-108.

McKeown, T. (1980) [1976]. The Role of Medicine: Dream, Mirage, or Nemesis. Princeton: Princeton University Press.

McLeod, J. D. (1996). Life Event. In J. E. Birren (Ed.), Encyclopedia of Gerontology (p.667-670). San Diego: Academic Press.

McLeod, J. D., \& Kessler, R. C. (1990). Socioeconomic Status Differences in Vulnerability to Undesirable Life Events. Journal of Health and Social Behavior, 31(2), 162-172. 
Mehta, S. (2006). Maximum City. Bombay lost and found. New York: Buchet-Chastel.

Menon, M. (2011). Riots and After in Mumbai : Chronicles of Truth and Reconciliation. Thousand Oaks: Sage.

Merton, R. K. (1968). The Matthew Effect in Science. Science, 159(3810), $56-63$.

Merton, R. K. (1988). The Matthew Effect in Science, II: Cumulative Advantage and the Symbolism of Intellectual Property. Isis, 79(4), 606-623.

Meslé, F. (2004). Allongement de la vie et évolution des pathologies. Gérontologie et société, 108, 15-34.

Meslé, F., \& Vallin, J. (2002). La transition sanitaire: tendances et perspectives. In G. Caselli, J. Vallin, G. Wunsch (Eds.), Démographie : analyse et synthèse. Les déterminants de la mortalité (p. 439-461). Paris: INED.

Mills, C. W. (1997) [1959]. L’imagination Sociologique. Paris: La Découverte.

Mills, M., \& Blossfeld, H.-P. (2003). Globalization, uncertainty and changes in early life courses. Zeitschrift Für Erziehungswissenschaft, $6(2), 188-218$.

Misztal, B. A. (2011). The challenges of vulnerability: in search of strategies for a less vulnerable social life. Basingstoke: Palgrave Macmillan.

Moffitt, T. E., \& Caspi, A. (2001). Childhood predictors differentiate lifecourse persistent and adolescence-limited antisocial pathways among males and females. Development and Psychopathology, 13(2), 355375.

Mohanty, S. K., \& Ram, F. (2011). Spatial Pattern of Poverty Reduction and Fertility Transition in India. Population Review, 50(1), 62-78.

Montaut, A. (2004). L'anglais en Inde et la place de l'élite dans le projet national. Hérodote, 115, 63-89.

Moscovici, S. (1961). La psychanalyse, son image et son public: étude sur la réprésentation sociale de la psychanalyse. Paris: Presses universitaires de France.

Moscovici, S. (1989). Des représentations collectives aux représentations sociales: éléments pour une histoire. In D. Jodelet (Ed.), Les 
représentations sociales (p. 61-86). Paris: Presses universitaires de France.

Moscovici, S. (1996). Préface. In C. Herzlich (Ed.), Santé et maladie : Analyse d'une représentation sociale (p. 7-12). Paris: EHESS.

Moscovici, S. (2000). Social Representations: Explorations in Social Psychology. Cambridge: Polity Press.

Moscovici, S. (2001). Pourquoi l'étude des représentations sociales en psychologie? Psychologie et société, 4, 7-27.

Moscovici, S., \& Buschini, F. (2003). Les Méthodes des sciences humaines. Paris: Presses universitaires de France.

Moss, N. E. (2002). Gender equity and socioeconomic inequality: a framework for the patterning of women's health. Social Science \& Medicine, 54(5), 649-661.

Mullatti, L. (1995). Families in India: Beliefs and Realities. Journal of Comparative Family Studies, 26(1), 11-25.

Mundu, G. B., \& Bhagat, R. B. (2008). Slum Conditions in Mumbai with Reference to the Access of Civic Amenities. IIPS ENVIS center, 5(1).

Municipal Corporation of Greater Mumbai. (2009). Mumbai Human Development Report. Mumbai: UNDP.

Murthy, S. L. (2012). Land Security and the Challenges of Realizing the Human Right to Water and Sanitation in the Slums of Mumbai, India. Health and Human Rights, 14(2).

Mushtaq, M. U. (2009). Public health in British India: A brief account of the history of medical services and disease prevention in colonial India. Indian Journal of Community Medicine, 34(1), 6.

Nainan, N. (2008). Building Boomers and Fragmentation of Space in Mumbai. Economic and Political Weekly, 43(21), 29-34.

Nangia, S., \& Thorat, S. (2000). Slum in a metropolis: the living environment. Delhi: Shipra Publications.

Narayan, D., Patel, R., Schafft, K., Rademacher, A., \& Koch-Schulte, S. (2000). Voices of the Poor: Can Anyone Hear Us? World Bank Publications.

Narayan, L. (2014). Urbanisation, urban poverty and slums in India: a socio-economic study of selected slums in Faridabad, India. Saarbrücken: Lambert Academic Publishing. 
Neugarten, B. L., \& Hagestad, G. O. (1976). Age and the life course. In R. Binstock, E. Shanas (Ed.), Handbook of Aging and the Social Sciences (p. 35-55). New York: Van Nostrand Reinhol.

Neugarten, B. L., Moore, J. W., \& Lowe, J. C. (1965). Age Norms, Age Constraints, and Adult Socialization. American Journal of Sociology, 70(6), 710-717.

Neyazi, T. A. (2010). Cultural imperialism or vernacular modernity? Hindi newspaper in a globalizing India. Media, Culture \& Society, 32(6), 907-924.

Nijman, J. (2015). India's Urban Future: Views From the Slum. American Behavioral Scientist, 59(3), 406-423.

Nora, P. (1989). Between Memory and History: Les Lieux de Mémoire. Representations, 26, 7-24.

Nugent, R. (2008). Chronic Diseases in Developing Countries. Annals of the New York Academy of Sciences, 1136(1), 70-79.

Oddone, M. J., \& Lynch, G. (2008). Las memorias de los hechos socio-históricos en el curso de la vida. Revista Argentina de Sociologia, 6(10), 121-142.

Oddone, M. J., \& Lynch, G. (2010). Memoria e impacto de la crisis de 2001 en las biografías individuales: Argentina en el cambio de siglo. Societal and Political Psychology International Review, 1(2), 37-53.

Ofshe, R., \& Watters, E. (1994). Making Monsters: False memories, psychotherapy, and sexual hysteria. New York: Charles Scribner.

Okamoto, Y. (1992). Health care for the elderly in Japan: medicine and welfare in an aging society facing a crisis in long term care. British Medical Journal, 305(6850), 403-405.

Omran, A. R. (2005). The Epidemiologic Transition: A Theory of the Epidemiology of Population Change. Milbank Quarterly, 83(4), 731-757.

OMS. (2008). Combler le fossé en une génération. Instaurer l'équité en santé en agissant sur les déterminants sociaux de la santé. Organisation mondiale de la santé.

O'Rand, A. M. (1996). The Precious and the Precocious: Understanding Cumulative Disadvantage and Cumulative Advantage Over the Life Course. The Gerontologist, 36(2), 230-238.

O'Rand, A. M. (2004). The Future of the Life Course: Late Modernity and Life Course Risks. In J. T. Mortimer, M. J. Shanahan (Eds.), Handbook of the Life Course (p. 693-702). New York: Springer. 
Oris, M., Gabriel, R., Ritschard, G., \& Kliegel, M. (2017). Long lives and old age poverty: social stratification and life-course institutionalization in Switzerland. Research in Human Development, 14(1), 68-87.

Oris, M., \& Ramiro Fariñas, D. (2016). New Approaches to Death in Cities During the Health Transition: An Introduction. In D. Ramiro Fariñas, M. Oris (Eds.), New Approaches to Death in Cities during the Health (p. 1-16). Switzerland: Springer.

Oris, M., Roberts, C., Joye, D., \& Ernst Stähli, M. (2016). Surveying human vulnerabilities across the life course. Heidelberg: Springer.

Oris, M., Widmer, E., de Ribaupierre, A., Joye, D., Spini, D., Labouvie-Vief, G., \& Falter, J.-M. (2009). Transitions dans les parcours de vie et construction des inégalités. Lausanne: Presses polytechniques et universitaires romandes.

Osmani, S., \& Sen, A. (2003). The hidden penalties of gender inequality: fetal origins of ill-health. Economics \& Human Biology, 1(1), 105121.

Paez, D., \& Liu, J. (2009). Collective Memory of Conflicts. In D. Bar-Tal (Ed.), Intergroup Conflicts and their Resolution: Social Psychological Perspective. New York: Psychology Press.

Paez, D., \& Liu, J. (2012). Collective Remembering. In D. J. Christie (Ed.), The Encyclopedia of Peace Psychology (p. 365-369). Oxford: Blackwell Publishing.

Pandey, G. (1992). In Defense of the Fragment: Writing about Hindu-Muslim Riots in India Today. Representations, 37, 27-55.

Pandey, G. (2001). Remembering partition: violence, nationalism and history in India. Cambridge: Cambridge University Press.

Pandey, G. K., Dutt, D., \& Banerjee, B. (2009). Partner and Relationship Factors in Domestic Violence: Perspectives of Women From a Slum in Calcutta, India. Journal of Interpersonal Violence, 24(7), 1175-1191. Pearlin, L. I. (1989). The Sociological Study of Stress. Journal of Health and Social Behavior, 30(3), 241-256.

Pearlin, L. I., Menaghan, E. G., Lieberman, M. A., \& Mullan, J. T. (1981). The Stress Process. Journal of Health and Social Behavior, 22(4), 337-356.

Pearlin, L. I., Schieman, S., Fazio, E. M., \& Meersman, S. C. (2005). Stress, Health, and the Life Course: Some Conceptual Perspectives. Journal of Health and Social Behavior, 46(2), 205-219. 
Pennebaker, J., Paez, D., \& Deschamps, J.-C. (2006). The social psychology of history. Defining the most important events of the last 10, 100, and 1000 years. Psicología Política, 32, 15-32.

Pennebaker, J., Paez, D., \& Rimé, B. (1997). Collective memory of political events: social psychological perspectives. Mahwah: Erlbaum.

Perlman, J. (2010). Favela: Four Decades of Living on the Edge in Rio de Janeiro. Oxford: Oxford University Press.

Perrig-Chiello, P., \& Perrig, W. J. (2005). The impact of personality and living context on remembering biographical transitions. In R. Levy, P. Ghisletta, J.-M. L. Goff, D. Spini, E. Widmer (Eds.), Towards an Interdisciplinary Perspective on the Life Course (p. 217-235). US: Elsevier.

Phelan, J. C., Link, B. G., Diez-Roux, A., Kawachi, I., \& Levin, B. (2004). "Fundamental Causes" of Social Inequalities in Mortality: A Test of the Theory. Journal of Health and Social Behavior, 45(3), 265-285.

Phelan, J. C., Link, B. G., \& Tehranifar, P. (2010). Social Conditions as Fundamental Causes of Health Inequalities: Theory, Evidence, and Policy Implications. Journal of Health and Social Behavior, 51(1), 28-40.

Piketty, T. (2013). Le Capital au XXIe siècle. Paris: Seuil.

Pinçon, M., \& Pinçon-Charlot, M. (1999). Ehrenberg Alain, La fatigue d'être soi. Dépression et société. Revue française de sociologie, 40(4), $778-780$.

Piraud, A. (2012). Réalisation d'une enquête sociologique dans des quartiers informels: Mumbai: rapport de stage. (Mémoire de master en Socioéconomie) Genève: Université de Genève.

Pison, G. (2015). Tous les pays du monde (2015). Population \& Sociétés, $525,1-4$.

Portes, A. (1998). Social Capital: Its Origins and Applications in Modern Sociology. Annual Review of Sociology, 24, 1-24.

Portes, A. (2000). The Two Meanings of Social Capital. Sociological Forum, 15(1), 1-12.

Prakash, G. (2000). The Impossibility of Subaltern History. Nepantla: Views from South, 1(2), 287-294.

Preston, S. H. (2013) [1976]. Mortality Patterns in National Populations: With Special Reference to Recorded Causes of Death. Elsevier.

Prévert, J. (1946) [1976]. Paroles. Paris: Gallimard. 
Price, D. J. de Solla. (1965). Networks of Scientific Papers. Science, 149(3683), 510-515.

PricewaterhouseCoopers. (2010). Indian entertainment and media outlook $2010(1-152)$.

Pryer, J. (1989). When Breadwinners fall III: Preliminary Findings from a Case Study in Bangladesh. IDS Bulletin, 20(2), 49-57.

Quigley, M. A. (2006). Commentary: Shifting burden of disease - epidemiological transition in India. International Journal of Epidemiology, 35(6), 1530-1531.

Racine, J. (2009). L'Inde et l'Asie: nouveaux équilibres, nouvel ordre mondial. Paris: CNRS.

Radkar, A., Kanitkar, T., \& Talwalkar, M. (2010). Epidemiological Transition in Urban Maharashtra. Economic and Political Weekly, 45(39), 23-27.

Rajagopal, A. (2001). Politics After Television: Hindu Nationalism and the Reshaping of the Public in India. Cambridge University Press.

Ram, U., Mohanty, S. K., Singh, A., \& Ram, F. (2010). Youth in India: Situation and Needs 2006-2007. Working Paper, 396. Mumbai: International Institute for Population Sciences.

Ramaiah, A. (1992). Identifying Other Backward Classes. Economic and Political Weekly, 27(23), 1203-1207.

Ramaraj, R., \& Alpert, J. S. (2008). Indian Poverty and Cardiovascular Disease. The American Journal of Cardiology, 102(1), 102-106.

Rayhan, I. (2008). Assessing household vulnerability and coping strategies to floods: a comparative study of flooded and non-flooded areas in Bangladesh, 2005. Göttingen: Cuvillier Verlag.

Razavi, S. (2007). The political and social economy of care in a development context. Conceptual issues, research questions and policy options. Gender and Development, 3. United Nations Research Institute for Social Development.

Record. (2013, septembre). Violence against women in India: culture, institutions and inequality. Consulté le 5 juin 2017, à l'adresse https:// www.newsrecord.co/violence-against-women-in-india-culture-institutions-and-inequality/

Reese, H. W., \& Smyer, M. A. (1983). The Dimensionalization of Life Events. In E. J. Callahan, K. A. McCluskey (Eds.), Life-Span 
Developmental Psychology. Nonnormative life events (p. 1-34). New York: Academic Press.

Ricoeur, P. (2003). La Mémoire, l'Histoire, l'Oubli. Paris: Seuil.

Riley, M. W. (1971). Social Gerontology and the Age Stratification of Society. The Gerontologist, 11(1), 79-87.

Riley, M. W. (1987). On the Significance of Age in Sociology. American Sociological Review, 52(1), 1-14.

Riley, M. W., Johnson, M., \& Foner, A. (1972). A sociology of age stratification. New York: Sage.

Riley, M. W., \& Riley, J. W. (2000). Age Integration Conceptual and Historical Background. The Gerontologist, 40(3), 266-270.

Risbud, N. (2003). Urban Slums Reports: The case of Mumbai, India. Case Studies for the Global Report on Human Settlements. UN Habitat.

Risbud, N. (2009). The Poor and Morphology of Cities. In India Urban Poverty Report (p. 177-198). Oxford: Oxford University Press.

Robin, C. (2004). Emeutes ethno-religieuses en Inde. Revue Projet, 4, $32-35$.

Rogaly, B., Biswas, J., Coppard, D., Rafique, A., Rana, K., \& Sengupta, A. (2001). Seasonal Migration, Social Change and Migrants' Rights: Lessons from West Bengal. Economic and Political Weekly, 36(49), 4547-4559.

Rönkä, A., Oravala, S., \& Pulkkinen, L. (2003). Turning Points in Adults' Lives: The Effects of Gender and the Amount of Choice. Journal of Adult Development, 10(3), 203-215.

Rossier, C., Soura, A. B., Lankoande, B., \& Roch, M. M. (2016). Health Disparities on the Periphery of Ouagadougou. In D. Ramiro Fariñas, M. Oris (Eds.), New Approaches to Death in Cities during the Health (p. 217-241). Switzerland: Springer.

Rossignon, F., \& Gaillard, J. (2013). The migrant population in the slums of Mumbai: Fortunate or wretched? (Mémoire de master en Socioéconomie) Genève: Université de Genève.

Rowntree, B. S. (1971) [1901]. Poverty; a study of town life. New York: Fertig.

Roy, A. (1999). Le Coût de la vie. Paris: Gallimard.

Roy, A. (2011). Slumdog Cities: Rethinking Subaltern Urbanism. International Journal of Urban and Regional Research, 35(2), 223-238.

Roy, A. (2014). Capitalism A Ghost Story. Chicago: Haymarket. 
Roy, A., \& Demanuelli, C. (2015). La honte de l'Inde. Le Débat, 184, $125-138$.

Roy, S. (2011). Television news and democratic change in India. Media, Culture \& Society, 33(5), 761-777.

Rubin, D. C. (1982). On the retention function for autobiographical memory. Journal of Verbal Learning and Verbal Behavior, 21, 21-38.

Rubin, D. C. (1995). Remembering your past. Studies in autobiographical memory. Cambridge: Cambridge University Press.

Rubin, D. C., Schulkind, M., \& Rahhal, T. (1999). A study of Gender Differences in Autobiographical Memory: Broken Down by Age and Sex. Journal of Adult Development, 6(1), 61-71.

Rubin, D. C., Wetzler, S. E., \& Nebes, R. D. (1986). Autobiographical memory across the lifespan. In D. C. Rubin (Ed.), Autobiographical memory (p. 57-74). Cambridge: Cambridge University Press.

Ryder, N. B. (1965). The Cohort as a Concept in the Study of Social Change. American Sociological Review, 30(6), 843-861.

Saglio, M.-C. (2001). Mumbai : mutations spatiales d'une métropole en expansion. Mappemonde, 62, 26-31.

Saglio-Yatzimirsky, M.-C. (1999). La pauvreté en Inde : une question de castes? Cultures \& Conflits, 35.

Saglio-Yatzimirsky, M.-C. (2002). Intouchable Bombay: le bidonville des travailleurs du cuir. Paris: CNRS.

Saglio-Yatzimirsky, M.-C. (2013). Dharavi. From Mega-Slum to Urban Paradigm. New Delhi: Routledge.

Sakthivel, S., \& Joddar, P. (2006). Unorganised Sector Workforce in India: Trends, Patterns and Social Security Coverage. Economic and Political Weekly, 41(21), 2107-2114.

Sapin, M., Spini, D., \& Widmer, E. (2007). Les parcours de vie : de l'adolescence au grand âge. Lausanne: Presses polytechniques et universitaires romandes.

Sauvain-Dugerdil, C. (2014). Cibler les inégalités: Contribution de l'approche des Capabilités aux études de la population. African Population Studies, 28(2), 732-743.

Schacter, D. (1996). A la recherche de la mémoire. Le passé, l'esprit et le cerveau. Paris: De Boeck.

Schaie, K. W., \& Abeles, R. P. (2008). Social Structures and Aging Individuals: Continuing Challenges. US: Springer. 
Schrauf, R. W., \& Rubin, D. C. (1998). Bilingual Autobiographical Memory in Order Adult Immigrants: A Test of Cognitive Explanations of the Reminiscence Bump and the Linguistic Encoding of Memories. Journal of Memory and Language, 39(3), 437-457.

Schuman, H., Akiyama, H., \& Knaüper, B. (1998). Collective memories of Germans and Japanese about the past half-century. Memory, 6(4), 427-454.

Schuman, H., \& Corning, A. (2000). Collective Knowledge of Public Events: The Soviet Era from the Great Purge to Glasnost. American Journal of Sociology, 105(4), 913-956.

Schuman, H., \& Corning, A. (2012). Generational Memory and the Critical Period Evidence for National and World Events. Public Opinion Quarterly, 76(1), 1-31.

Schuman, H., \& Rodgers, W. L. (2004). Cohorts, chronology, and collective memories. Public Opinion Quarterly, 68(2), 217-254.

Schuman, H., \& Scott, J. (1989). Generations and collective memories. American Sociological Review, 54(3), 359-381.

Scott, J., \& Zac, L. (1993). Collective memories in Britain and the United States. Public Opinion Quarterly, 57(3), 315-331.

Sears, D. O., \& Valentino, N. A. (1997). Politics matters: Political events as catalysts for preadult socialization. American Political Science Review, 91(1), 45-65.

Sen, A. (1981). Poverty and Famines: An Essay on Entitlement and Deprivation. Oxford: Oxford University Press.

Sen, A. (1988). The Standard of Living. In A. Sen, G. Hawthorn (Eds.), The Standard of Living (p. 1-38). Cambridge: Cambridge University Press.

Sen, A. (1992). Inequality Reexamined. Oxford: Oxford University Press.

Sen, A. (1993). Positional Objectivity. Philosophy \& Public Affairs, 22(2), 126-145.

Sen, A. (1999). Development as Freedom. Oxford: Oxford University Press.

Sen, A. (2007). L'Inde. Histoire, culture et identité. Paris: Odile Jacob.

Sen, A. (2009). The Idea of Justice. Harvard: Harvard University Press.

Sen, A., \& Hawthorn, G. (1987). The Standard of Living. Cambridge University Press. 
Sen, R. (2003). We the Billion: A Social Psychological Perspective on India's Population. New Delhi: Sage Publications.

Sen, R., \& Wagner, W. (2005). History, Emotions and Hetero-Referential Representations in Inter-Group Conflict: The Example of HinduMuslim Relations in India. Papers on Social Representations, 14, 2-23.

Settersten, R. A., \& Gannon, L. (2005). Structure, Agency, and the Space Between: On the Challenges and Contradictions of a Blended View of the Life Course. Advances in Life Course Research, 10, 35-55.

Settersten, R. A. (1999). Lives in time and place: The problems and promises of developmental science. Amityville: Baywood.

Settersten, R. A. (2002). Socialization and the life course: New frontiers in theory and research. Advances in Life Course Research, 7, 13-40.

Settersten, R. A. (Ed.). (2003). Invitation to the Life Course: Toward New Understandings of Later Life. Amityville: Baywood.

Settersten, R. A. (2004). Age Structuring and the Rhythm of the Life Course. In Handbook of the Life Course (p. 81-98). Jeylan Mortimer, Michael Shanahan.

Settersten, R. A. \& Hagestad, G. O. (1996). What's the Latest? II. Cultural Age Deadlines for Educational and Work Transitions. The Gerontologist, 36(5), 602-613.

Settersten, R. A., \& Mayer, K. U. (1997). The measurement of age, age structuring, and the life course. Annual Review of Sociology, 23, 23361.

Settersten, R. A., \& Martin, L. (2002). The imprint of time: Historical experiences in the lives of mature adults. In R.A. Settersten, T.J. Owens (Ed.), New frontiers in socialization (p. 471-497). Oxford: Elsevier.

Settersten, R. A., \& Owens, T. J. (Ed.). (2002). New Frontiers in Socialization. Oxford: Elsevier.

Shah, A. M. (1996). Is the Joint Household Disintegrating? Economic and Political Weekly, 31(9), 537-542.

Shanahan, M. J. (2000). Pathways to Adulthood in Changing Societies: Variability and Mechanisms in Life Course Perspective. Annual Review of Sociology, 26, 667-692. 
Shanahan, M. J., Elder, G. H., \& Miech, R. A. (1997). History and Agency in Men's Lives: Pathways to Achievement in Cohort Perspective. Sociology of Education, 70(1), 54-67.

Sharangpani, M. (2010). Browsing for Bridegrooms: Matchmaking and Modernity in Mumbai. Indian Journal of Gender Studies, 17(2), 249276.

Sharma, H. K., Tripathi, B. M., \& Pelto, P. J. (2010). The Evolution of Alcohol Use in India. AIDS and Behavior, 14(1), 8-17.

Shetty, P. S. (2002). Nutrition transition in India. Public Health Nutrition, $5(1), 175-182$.

Shukla, A. (2007). Key Public Health Challenges in India: a Social Medicine Perspective. Social Medicine, 2(1), 1-7.

Siddiqui, R. N., \& Pandey, J. (2003). Coping with Environmental Stressors by Urban Slum Dwellers. Environment and Behavior, 35(5), 589-604.

Singh, R. B., Pella, D., Mechirova, V., Kartikey, K., Demeester, F., Tomar, R. S., Beegom, R., Mehta, A. S., Gupta, S. B., De, A. K., Neki, N. S., Haque, M., Nayse, J., Singh, S., Thakur, A. S., Rastogi, S. S., Singh, K., \& Krishna, A. (2007). Prevalence of obesity, physical inactivity and undernutrition, a triple burden of diseases during transition in a developing economy. Acta Cardiologica, 62(2), 119-127.

Singh, S. (2015). The Emergence of the Migrant as a Problem Figure in Contemporary Mumbai: Chronicles of Violence and Issues of Justice. Calcutta: Mahanirban Research Group.

Small, M. L. (2011). How to Conduct a Mixed Methods Study: Recent Trends in a Rapidly Growing Literature. Annual Review of Sociology, $37,57-86$.

Smith, J. (2003). The Gain-Loss Dynamic in Lifespan Development: Implications for Change in Self and Personality During Old and Very Old Age. In U. M. Staudinger, U. E. R. Lindenberger (Eds.), Understanding Human Development (p.112-124). Boston: Kluwer Academic.

Smith, K. R. (2000). National burden of disease in India from indoor air pollution. Proceedings of the National Academy of Sciences, 97(24), 13286-13293.

Solomon, B. B. (1976). Black Empowerment: Social Work in Oppressed Communities. New York: Columbia University Press 
Sonwalkar, P. (2001). India: Makings of Little Cultural/Media Imperialism? International Communication Gazette, 63(6), 505-519.

Sørensen, G., Gupta, P. C., \& Pednekar, M. S. (2005). Social Disparities in Tobacco Use in Mumbai, India: The Roles of Occupation, Education, and Gender. American Journal of Public Health, 95(6), 1003-1008.

Sørensen, M. P., \& Christiansen, A. (2014). Ulrich Beck: An Introduction to the Theory of Second Modernity and the Risk Society. In U. Beck (Ed.), Ulrich Beck (p. 7-13). US: Springer.

Spini, D., Bernardi, L., \& Oris, M. (2017). Vulnerability Across the Life Course. Research in Human Development, 14(1), 1-4.

Spini, D., Hanappi, D., Bernardi, L., Oris, M., \& Bickel, J.-F. (2013). Vulnerability across the life course: A theoretical framework and research directions. LIVES Working Papers, 27.

Spoorenberg, T. (2005). 30 years of Demography India: Content analysis of the back volumes. Demography India, 34(2), 151-165.

Srinath Reddy, K., Shah, B., Varghese, C., \& Ramadoss, A. (2005). Responding to the threat of chronic diseases in India. The Lancet, 366(9498), 1744-1749.

Srinivas, M. N. (1974). The Caste System in India. In A. Béteille (Ed.), Social Inequality (p. 265-272). UK: Penguin Education.

Srivastava, R., \& Bhattacharyya, S. (2003). Globalisation, reforms and internal labour mobility: analysis of recent Indian trends. Labour and Development, 9(2), 31-55.

Srivastava, R., \& Echanove, M. (2015). Translating the City. Interdisciplinarity in urban studies. In S. Vincent-Geslin, Y. Pedrazzini, H. Adly, Y. Zorro (Eds.), Translating the City. Interdisciplinarity in urban studies (p. 95-114). Lausanne: Presse polytechniques et universitaires romandes.

Subbaraman, R., O’brien, J., Shitole, T., Shitole, S., Sawant, K., Bloom, D. E., \& Patil-Deshmukh, A. (2012). Off the map: the health and social implications of being a non-notified slum in India. Environment and Urbanization, 24(2), 643-663.

Sugarman, L. (2001). Life-Span Development. Frameworks, accounts and strategies. New York: Taylor \& Francis.

Tabutin, D., \& Bartiaux, F. (1986). Structures familiales et structures sociales dans le Tiers-Monde. Présenté à Les familles d'aujourd'hui, AIDELF. Suisse, Genève. 
Techio, E., Bobowik, M., Páez, D., Cabecinhas, R., Liu, J., Zubieta, E., \& Espinosa, A. (2010). Representaciones sociales de la historia, guerra y politica en America Latina, Europa y Africa. Revista de Psicología Social, 25(1), 11-26.

Thoits, P. A. (2006). Personal Agency in the Stress Process. Journal of Health and Social Behavior, 47(4), 309-323.

Tholomier, A. (2017). Vivre et survivre au grand âge: enjeux des inégalités sociales et de santé au sein des générations qui ont traversé le 20ème siècle. (Thèse de doctorat en Sociologie). Genève: Université de Genève.

Thomas, H. (2008). Vulnérabilité, fragilité, précarité, résilience, etc. Recueil Alexandries.

Thomas, H. (2010). Les vulnérables: la démocratie contre les pauvres. Bellecombe-en-Bauges: du Croquant.

Thomas, W. I., \& Znaniecki, F. (1918) [1974]. The Polish Peasant in Europe and America. Urbana: University of Illinois Press.

Thompson, E.-P. (2012) [1963]. La formation de la classe ouvrière anglaise. Paris: Points.

Thornberry, T. P., Freeman-Gallant, A., Lizotte, A. J., Krohn, M. D., \& Smith, C. A. (2003). Linked Lives: The Intergenerational Transmission of Antisocial Behavior. Journal of Abnormal Child Psychology, 31(2), 171-184.

Thussu, D. K. (1999). Privatizing the airwaves: the impact of globalization on broadcasting in India. Media, Culture \& Society, 21(1), 125-131

Thussu, D. K. (2007). The 'Murdochization' of news? The case of Star TV in India. Media, Culture \& Society, 29(4), 593-611.

Thussu, D. K. (2013). India in the international media sphere. Media, Culture \& Society, 35(1), 156-162.

Tichit, C., \& Lelièvre, E. (2006). Analyse du passage à l'âge adulte à l'épreuve de l'approche biographique : seuils, transition ou observation incertaine? In P. Antoine, E. Lelièvre (Eds.), Etats flous et trajectoires complexes. Observation, modélisation, interprétation (p. 197218). Paris: INED.

Todaro, M. (1980). Internal Migration in Developing Countries: A Survey. In R. A. Easterlin (Ed.), Population and Economic Change in Developing Countries (p. 361-402). Chicago: University of Chicago Press. 
Touraine, A. (2003). Les méthodes de la sociologie. In S. Moscovici, F. Buschini (Eds.), Les Méthodes des sciences humaines (p. 113-138). Paris: Presses universitaires de France.

Touraine, A. (2007). Penser autrement. Paris: Fayard.

Trivedi, I. (2014). India in Love: Marriage and Sexuality in the 21st Century. New Delhi: Aleph Book.

Uhlenberg, P., \& Mueller, M. (2003). Family Context and Individual Weil-Being. In J. T. Mortimer, M. J. Shanahan (Eds.), Handbook of the Life Course (p. 123-148). US: Springer.

UN-Habitat. (2006). State of the world's cities 2006/2007. UN-Habitat. Unni, J. (2009). The Unorganized Sector and Urban Poverty: Issues of Livelihood. In India Urban Poverty Report (p. 177-198). Oxford: Oxford University Press.

Upadhyay, P. R. (2012). An overview of the burden of non-communicable diseases in India. Iranian Journal of Public Health, 41(3), 1-8.

Uttal, D. H., \& Perlmutter, M. (1989). Toward a Broader Conceptualization of Development: The Role of Gains and Losses across the Life Span. Developmental Review, 9, 101-132.

van de Velde, C. (2015). Sociologie des âges de la vie. Paris: Armand Colin.

Vella, S. (2003). Ethique et pratiques reproductives: les techniques de sélection sexuelle en Inde. Autrepart, 28(4), 147-163.

Verd, J. M., \& López, M. (2011). The Rewards of a Qualitative Approach to Life-Course Research. The Example of the Effects of Social Protection Policies on Career Paths. Qualitative Social Research, 12(3).

Véron, J. (1987). L'urbanisation indienne (1901-1981). Population, 42(3), $485-502$.

Véron, J. (2006). L'urbanisation du monde. Paris: La Découverte.

Véron, J. (2007). La moitié de la population mondiale vit en ville. Population \& Sociétés, 435, 1-4.

Véron, J. (2008). La démographie de l'Asie du sud des années 1950 aux années 2000. Synthèse des changements et bilan statistique. Population, 63(1), 7-92.

Véron, J., \& Nanda, A. K. (2011). Recensement de l'Inde de 2011: 181 millions d'habitants de plus en dix ans. Population et sociétés, 478, $3-6$. 
Vincent-Geslin, S., Pedrazzini, Y., Adly, H., \& Zorro, Y. (2015). Translating the City. Interdisciplinarity in urban studies. Lausanne: Presse polytechniques et universitaires romandes.

Vlahov, D., Freudenberg, N., Proietti, F., Ompad, D., Quinn, A., Nandi, V., \& Galea, S. (2007). Urban as a Determinant of Health. Journal of Urban Health, 84(1), 16-26.

Vrancken, D., \& Thomsin, L. (2008). Le social à l'épreuve des parcours de vie. Louvain-la-Neuve: Academia Bruylant.

Weber, M. (2003) [1978]. Economie et société, tome 1 : Les Catégories de la sociologie. Paris: Pocket.

Weymann, A. (2009). The Life Course, Institutions, and Life Course Policy. In W. R. Heinz, J. Huinink, A. Weymann (Eds.), The Life Course Reader: individuals and societies across time (p. 139-158). Frankfurt: Campus.

WHO. (2015). World Health Statistics 2015. Part II Global Health Indicators. World Health Organization.

Woelfel, J., \& Haller, A. O. (1971). Significant Others, The Self-Reflexive Act and the Attitude Formation Process. American Sociological Review, 36(1), 74-87.

Wright, E. O. (2000). Working-Class Power, Capitalist-Class Interests, and Class Compromise. American Journal of Sociology, 105(4), 957-1002.

Wright, E. O. (Ed.). (2005). Approaches to Class Analysis. Cambridge: Cambridge University Press.

Yesudian, P. P. (2009). Changing workforce of Mumbai: a critical analysis. Présenté à XXXVIe Congrès international de la population. Maroc, Marrakech.

Zakour, M. J., \& Gillespie, D. F. (2013). Community Disaster Vulnerability. New York: Springer.

Zappi, S. (2013, février 7). L'«empowerment», nouvel horizon de la politique de la ville. Le Monde.fr.

Zerubavel, E. (2003). Time Maps: Collective Memory and the Social Shape of the Past. Chicago: University of Chicago Press.

Zimmer, Z., \& Kwong, J. (2003). Family size and support of older adults in urban and rural China: Current effects and future implications. Demography, 40(1), 23-44. 


\section{Annexe 1: le questionnaire CEVI en anglais}

(Questionnaire en anglais, adapté et passé à Santa Cruz en 2014)

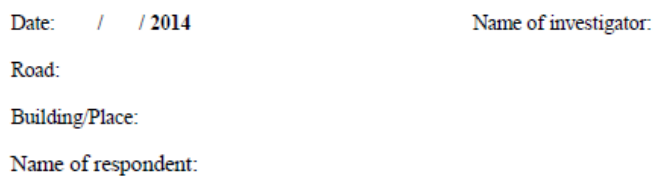

\section{I) RECENT CHANGES IN THE LIFE}

During the last year (from $1^{\text {st }}$ March 2013 until today), did any significant change occur in your life?

- Yes $\square$
- No $\square$

If yes, please describe briefly each change and evaluate it.

- Change 1:

Gain $\square$; Loss $\square$; Both $\square$; Neither $\square$; Don't know $\square$

- Change 2:

Gain $\square$; Loss $\square$; Both $\square$; Neither $\square$; Don't know $\square$

- Change 3:

Gain $\square$; Loss $\square$; Both $\square$; Neither $\square$; Don't know $\square$

- Other important changes:

Gain $\square$; Loss $\square$; Both $\square$; Neither $\square$; Don't know $\square$ 


\section{II) MAIN TURNING POINTS OF YOUR LIFE}

If you think about your whole life, which were the main turning points, these moments which marked a significant change in your life?

(You may answer up to four turning points)

\section{Description:}

Why was this turning point significant?

Year(s) Your age at that moment:

Place

2. Description:

Why was this turning point significant?

Year(s): Your age at that moment:

Place

3. Description:

Why was this turning point significant?

Year(s): Your age at that moment:

Place

\section{Description:}

Why was this turning point significant?

Year(s): Your age at that moment:

Place 


\section{III) SOCIO-HISTORICAL CHANGES AND EVENTS}

Let us now consider the main changes and events which occurred in your country and in the world during your life. What are the ones which most struck you?

(You may answer up to four events)

1. Description:

Why did this event strike you?

Year(s): ....... Your age at that moment: ....... Place of the event:

\section{Description:}

Why did this event strike you?

Year(s): ....... Your age at that moment: ..... Place of the event:

\section{Description}

Why did this event strike you?

Year(s): ....... Your age at that moment: ...... Place of the event:

\section{Description:}

Why did this event strike you?

Year(s): ....... Your age at that moment: ...... Place of the event: 
MORE INFORMATION

Sex: $\quad$ female $\square \quad$ - male $\square$

Year of birth:

19

Age:

Nationality:

Place of birth:

(State)

Civil status: - single

Type of place of birth: - big city

- married

- medium city

- divorced, separated

- small city

- widow(er)

- village

Do you have children ? - yes $\square$ - no $\square$ number:

Who do you live with (several possible answers)?

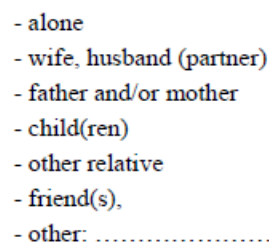

Educational level : $\quad$ - primary school - secondary school - junior college - university

What is now your professional situation (main activity)?

$$
\begin{aligned}
& \text { - student } \\
& \text { - employed } \\
& \text { - househusband/wife } \\
& \text { - unemployed } \\
& \text { - retired } \\
& \text { - other: }
\end{aligned}
$$


How do you estimate your current health?

$$
\begin{aligned}
& \text { - very good } \\
& \text { - good } \\
& \text { - neither good nor bad } \\
& \text { - bad } \\
& \text { - very bad }
\end{aligned}
$$

What is your household monthly income?

$$
\begin{aligned}
& -<3 \text { 3'699 Rs } \\
& - \text { 3'700 - 7'999 Rs } \\
& - \text { 8'000 - 14'999 Rs } \\
& -15^{\prime} 000-29^{\prime} 999 \mathrm{Rs} \\
& -30^{\prime} 000-49^{\prime} 999 \mathrm{Rs} \\
& ->50 \text { '000 Rs }
\end{aligned}
$$

Do you own a...?

- Air-conditioner (AC)
- computer
- internet access
- Indian toilet
- English toilet
- fridge
- TV/radio
- mobile phone
- car
- printer
- liquid petroleum gas (LPG)
- compress natural gas (CNG)

What is your religion?

$$
\begin{aligned}
& \text { - Hindu } \\
& \text { - Muslim } \\
& \text { - Christian } \\
& \text { - Buddhist } \\
& \text { - other: ... }
\end{aligned}
$$


According to you, is your lifestyle similar to the lifestyle of :

$$
\begin{aligned}
& \text { - your parents } \\
& \text { - your children (if you have) } \\
& \text { - neither }
\end{aligned}
$$

Why?

Thank you! 


\section{Annexe 2: le questionnaire CEVI en marathi}

(Questionnaire en marathi (langue du Maharastra), passé à Bandra en 2012)

$$
\begin{aligned}
& \text { नाव } \\
& \text { Date: }
\end{aligned}
$$

UNIVERSITY OF GENEV

CENTER FOR INTERDISCIPLINARY GERONTOLOGY

$$
\text { अयुष्यमरानीत्म बदल आणि घटना }
$$

1) काही काकापुर्वीचे आयुष्यातील बदल

गेल्या वर्षभरान ( डिसेंबर 1,2010 पासून आजपर्यं) तमत्या

आयुष्यान कही महत्वाचा वा विशेष बदल घडला आहे का?

होय - $\square$

नाही

जर हो, कृपया बदल संगा आणि स्पष्ट करा

-बढल्भ 1:

फायदा $\square$; नोटा $\square$; दोनी०; दोनी $\square$; मासित
नाही नाही

- बदल 2

फायदा $\square$; नोटा $\square$; छोन्ही $\square$; दौन्ही $\square$; माहित
नाही नाही

- बदल 3

फायदा $\square$; तोला $\square$; दोनी $\square$; दोन्ही $\square$; माहित
नाही नाही

आणखी महत्वाचे बदल :

कायदा $\square$; नौब $\square$; दोन्ही $\square$; दोनी म; माहित
नाही नाही

CEVI - 1 
II) तुमच्या आयुष्यानील महत्वाचा टप्या

जर नुम्ही तुमच्या पुर्ण आयुष्याचा विचार केलान, तर तुमच्या जीवनात महत्वचि टप्पे, जीवनात महत्वाचे बदल घउवणारे क्षण / प्रसंग/घटना कोणत्या आहेत?

1. वर्णन

हा टप्या का महत्वाचा ओहे ?

बर्ष (वर्ष) ….....तुमचे नेव्दाचे वय क्...... किकाज

2. वर्णन

हा टापा का महत्वाचा आहे?

वर्ष(वर्ष):.......तुमच ते द्राचे वय :....... बिकाण

3.. वर्णन

हा टथ्पा का मदत्वाचा ओ?

वर्ष(वर्षे):......तुमचे तैव्हाचे वय :..... हिकाण

4. वर्णन

हा टप्पा का महत्वाचा आहे?

वर्ष (वर्षे) ........ तुमचे नैष्टचे वय :.......िकिका

CEVI - 2 
III) सामाजिक-ऐतिहासिक बदल आणि घटना आना आपण तुमच्या आयुष्यभरमघ्ये तुमच्या देशान व जगान घडल्येसे महत्वाचे बदल व घटनांचा विचार करूया. तुम्हाला उल्लेखनिय/धक्कादायक वारणाख्या घटना कोणत्या आहेत ?

1.. तुम्ही चार घटना सांगू शंकता) वर्णन

नुम्हाल्या हिच घटना का आठवल्यीं ?

वर्ष(वर्ष) ……... तुमचे ने व्हाच वय घटनास्थक क

2. वर्णन

तुम्कात्या हीच घटना का आग्वली?

वर्ष(वर्ष) .......... तुमचे तेवाचे वय ........ घटनास्थल

3.. वर्णन :

तुम्हाला हीच घटना का आव्वली

वर्ष(वर्ष): ........तुमचे नेव्हाचे बय .......छटना:स्थल

4.. वर्णन

तुम्काला हीच घटना का आठवर्ती ?

बर्ष (वर्षे.......... तुमचे नेव्दाचें वय :

घर नार्थल्ब.

CEVI - 3 


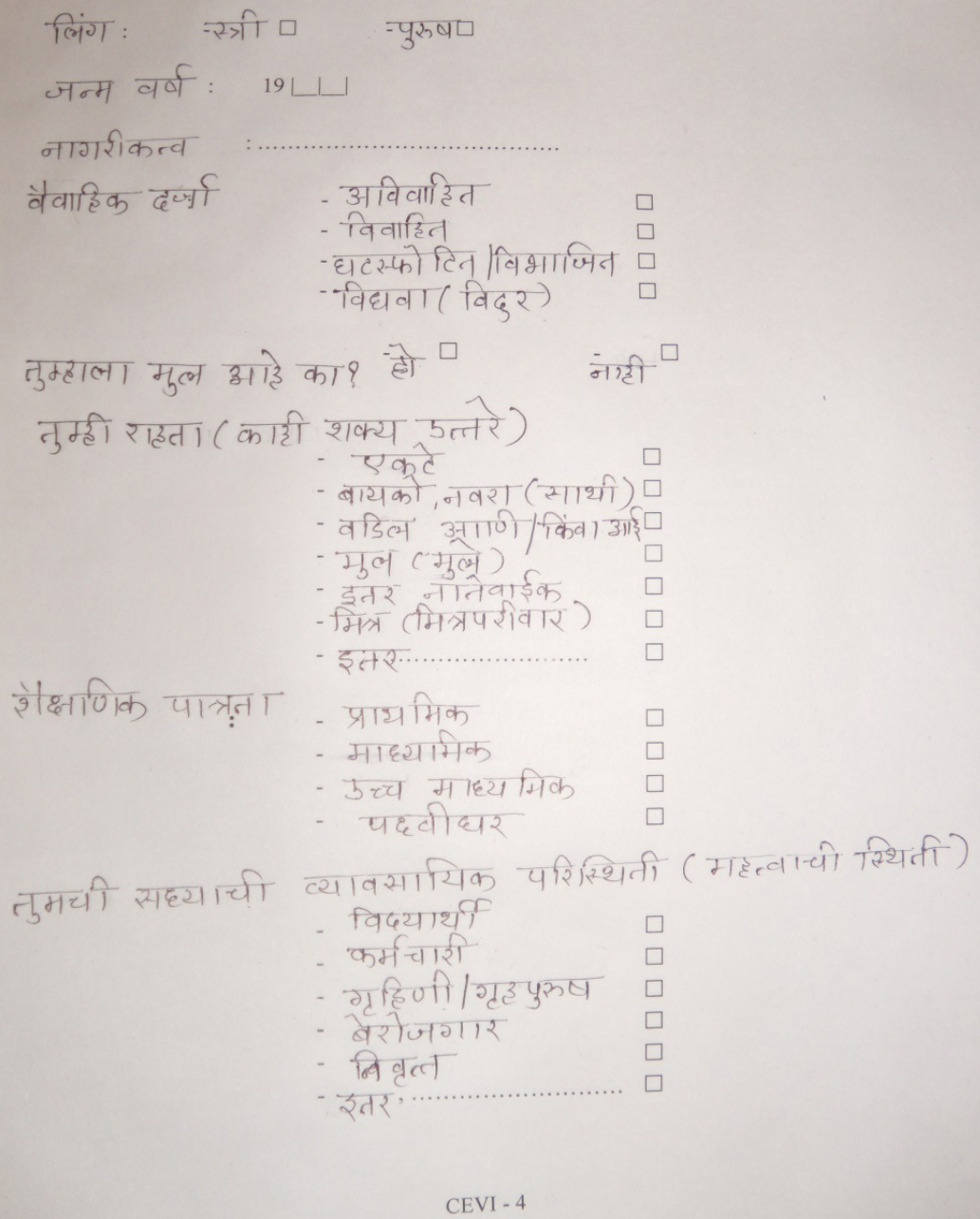


तुमच्या सध्याच्या आरोग्याचे कसे वर्णन कराल?

: यूप चांगली
चोंगली
समाधनकारक
खूप वाईट
वोईट




\section{Annexe 3: tableau descriptif des capitaux culturels et économiques $(\%)^{1}$}

\begin{tabular}{|c|c|c|c|c|c|c|c|c|c|c|c|c|}
\hline \multirow{2}{*}{ Changements récents } & \multicolumn{2}{|c|}{\begin{tabular}{|c|} 
Total \\
$\mathrm{BA}^{2} \mathrm{SC}^{3}$ \\
\end{tabular}} & \multicolumn{2}{|c|}{\begin{tabular}{|l}
$20-24$ \\
BA
\end{tabular}} & \multicolumn{2}{|c|}{$\begin{array}{c}\text { 35-39 } \\
\text { BA SC }\end{array}$} & \multicolumn{2}{|c|}{$\begin{array}{c}\mathbf{5 0}-\mathbf{5 4} \\
\text { BA SC }\end{array}$} & \multicolumn{2}{|c|}{$\begin{array}{c}65-69 \\
\text { BA SC }\end{array}$} & \multicolumn{2}{|c|}{$\begin{array}{r}80-84 \\
\text { BA SC }\end{array}$} \\
\hline & & & & & & & & & & & & \\
\hline 1) Capital culturel & 9 & 12 & 30 & 30 & 5 & 9 & 4 & 11 & 3 & 8 & 1 & 5 \\
\hline Education & 4 & 5 & 17 & 22 & 1 & 4 & 2 & - & - & 1 & - & - \\
\hline Début & 1 & 1 & 4 & 2 & - & 2 & - & - & - & 1 & - & - \\
\hline Réussite & 2 & 2 & 7 & 11 & - & - & - & - & - & - & - & - \\
\hline Dévelop & 2 & 3 & 6 & 5 & 1 & 1 & 1 & 3 & 1 & 2 & 1 & 1 \\
\hline Voyage & 1 & 1 & 2 & 2 & 1 & 1 & 1 & - & 2 & 3 & - & 1 \\
\hline Activité & 2 & 3 & 5 & 1 & 2 & 3 & - & 8 & - & 2 & - & 3 \\
\hline Sport & - & 2 & 1 & - & - & 3 & - & 4 & - & 2 & - & 3 \\
\hline 2) Capital écon & 26 & 30 & 31 & 32 & 39 & 46 & 27 & 35 & 17 & 22 & 5 & 6 \\
\hline Economie & 7 & 8 & 4 & 9 & 12 & 10 & 7 & 11 & 7 & 6 & 1 & - \\
\hline Travail & 13 & 11 & 23 & 17 & 15 & 22 & 15 & 4 & 7 & 8 & - & 3 \\
\hline Reprise/nouveau & 7 & 4 & 12 & 10 & 9 & 7 & 5 & 2 & 4 & 1 & - & 3 \\
\hline Chômage & 3 & 1 & 6 & 1 & 2 & 2 & 2 & 1 & 2 & 2 & - & - \\
\hline Promot & 2 & 3 & 1 & 3 & 3 & 8 & 5 & - & - & 4 & - & - \\
\hline Mobilité & 2 & 2 & 2 & 2 & 1 & 4 & 2 & 1 & 2 & 2 & 4 & - \\
\hline Dans l'Etat/Mumbai & 1 & 2 & 1 & 2 & - & 4 & 1 & 1 & 1 & 2 & 3 & - \\
\hline Autre & 4 & 9 & 2 & 4 & 11 & 10 & 3 & 19 & 1 & 6 & - & 3 \\
\hline Tournants & & & & & & & & & & & & \\
\hline 1) Capital cu & 11 & 11 & 31 & 31 & 12 & 9 & 6 & 5 & 4 & 5 & 3 & 12 \\
\hline Education & 9 & 7 & 25 & 23 & 9 & 7 & 6 & 1 & 2 & 3 & 3 & 5 \\
\hline Début & 1 & 1 & 3 & 3 & - & - & - & - & - & - & - & - \\
\hline Réussite & 2 & 3 & 10 & 11 & 2 & 5 & - & - & - & 1 & - & 2 \\
\hline Pas d'étude & 2 & - & 3 & 1 & 4 & 1 & 2 & - & 1 & - & 1 & - \\
\hline Echec & 1 & - & 2 & 1 & - & - & 1 & - & - & - & - & - \\
\hline
\end{tabular}

En gris les pourcentages où moins de cinq individus sont représentés.

$2 \quad \mathrm{BA}=$ Bandra

$3 \quad \mathrm{SC}=$ Santa Cruz 


\begin{tabular}{l|cc|cc|cc|cc|cc|cc} 
Développement personnel & $\mathbf{1}$ & $\mathbf{2}$ & $\mathbf{2}$ & $\mathbf{3}$ & $\mathbf{2}$ & $\mathbf{2}$ & - & $\mathbf{2}$ & $\mathbf{1}$ & $\mathbf{2}$ & $\mathbf{1}$ & $\mathbf{4}$ \\
Voyage & - & - & $\mathbf{1}$ & $\mathbf{1}$ & - & - & - & $\mathbf{1}$ & - & - & - & $\mathbf{1}$ \\
Activité & $\mathbf{1}$ & $\mathbf{2}$ & $\mathbf{3}$ & $\mathbf{4}$ & $\mathbf{1}$ & - & - & $\mathbf{1}$ & $\mathbf{1}$ & - & - & $\mathbf{2}$ \\
$\quad$ Sport & - & 1 & - & 3 & - & - & - & 1 & - & - & - & - \\
2) Capital économique & $\mathbf{2 5}$ & $\mathbf{3 1}$ & $\mathbf{2 2}$ & $\mathbf{2 2}$ & $\mathbf{2 4}$ & $\mathbf{2 7}$ & $\mathbf{3 3}$ & $\mathbf{3 9}$ & $\mathbf{2 3}$ & $\mathbf{3 3}$ & $\mathbf{3 0}$ & $\mathbf{2 9}$ \\
Economie & $\mathbf{3}$ & $\mathbf{6}$ & $\mathbf{2}$ & $\mathbf{4}$ & $\mathbf{4}$ & $\mathbf{5}$ & $\mathbf{5}$ & $\mathbf{1 0}$ & $\mathbf{3}$ & $\mathbf{4}$ & $\mathbf{2}$ & $\mathbf{3}$ \\
Travail & $\mathbf{1 4}$ & $\mathbf{1 6}$ & $\mathbf{1 3}$ & $\mathbf{1 1}$ & $\mathbf{1 4}$ & $\mathbf{1 0}$ & $\mathbf{1 7}$ & $\mathbf{1 8}$ & $\mathbf{1 3}$ & $\mathbf{2 1}$ & $\mathbf{1 6}$ & $\mathbf{1 9}$ \\
$\quad$ Premier emploi & 4 & 3 & 4 & 4 & 5 & 2 & 3 & 5 & 1 & 3 & 6 & 4 \\
Travail & 4 & 4 & 4 & 4 & 5 & 2 & 5 & 5 & 2 & 5 & 5 & 5 \\
Reprise/nouveau & 2 & 4 & 1 & 1 & 2 & 3 & 4 & 5 & 3 & 6 & 1 & 6 \\
Chomage & 3 & 1 & 2 & - & 1 & - & 4 & 1 & 4 & - & 2 & 2 \\
Promotion & - & 2 & - & 2 & - & 2 & - & 1 & 1 & 2 & - & 1 \\
Mobilité & $\mathbf{5}$ & $\mathbf{6}$ & $\mathbf{5}$ & $\mathbf{3}$ & $\mathbf{4}$ & $\mathbf{9}$ & $\mathbf{8}$ & $\mathbf{7}$ & $\mathbf{3}$ & $\mathbf{7}$ & $\mathbf{7}$ & $\mathbf{2}$ \\
Dans l'Etat & 3 & 1 & 2 & 1 & 3 & - & 2 & 1 & 1 & 3 & 4 & 1 \\
$\quad$ Dans Mumbai & 1 & 3 & 1 & 1 & 1 & 5 & 1 & 5 & 1 & 2 & 1 & - \\
Autre & $\mathbf{3}$ & $\mathbf{3}$ & $\mathbf{2}$ & $\mathbf{4}$ & $\mathbf{2}$ & $\mathbf{3}$ & $\mathbf{4}$ & $\mathbf{4}$ & $\mathbf{4}$ & $\mathbf{1}$ & $\mathbf{5}$ & $\mathbf{5}$ \\
\hline
\end{tabular}




\section{Annexe 4: les changements récents et les tournants concernant la famille $(\%)^{1}$}

\begin{tabular}{|c|c|c|c|c|c|c|c|c|c|c|c|c|}
\hline \multirow{4}{*}{ Changements récents } & \multirow{2}{*}{\multicolumn{2}{|c|}{\begin{tabular}{|c|}
\multicolumn{2}{c}{ Total } \\
$\mathrm{BA}^{2} \mathrm{SC}^{3}$ \\
\end{tabular}}} & \multicolumn{2}{|c|}{$20-24$} & \multicolumn{2}{|c|}{ 35-39 } & \multicolumn{2}{|c|}{$50-54$} & \multicolumn{2}{|c|}{$65-69$} & \multicolumn{2}{|c|}{$80-84$} \\
\hline & & & BA & $\mathrm{SC}$ & BA & $\mathrm{SC}$ & BA & $\mathrm{SC}$ & BA & $\mathrm{SC}$ & BA & $\mathrm{SC}$ \\
\hline & & & & & & & & & & & & \\
\hline & 17 & 13 & 23 & 20 & 14 & 16 & 23 & 12 & 14 & 7 & 9 & 8 \\
\hline Entrée en couple & 1 & 1 & 1 & 6 & 1 & - & 2 & - & - & - & - & - \\
\hline Mariage & 7 & 4 & 6 & 5 & 5 & 6 & 10 & 5 & 8 & 3 & 6 & - \\
\hline Naissance & 7 & 5 & 10 & 5 & 7 & 7 & 8 & 3 & 4 & 3 & 2 & 5 \\
\hline Divorce & - & 1 & - & 2 & - & - & - & - & 1 & - & 1 & - \\
\hline Autre $^{4}$ & 2 & 2 & 6 & 2 & 1 & 3 & 3 & 4 & 1 & 1 & - & 3 \\
\hline Décès & 4 & 4 & 2 & 2 & 4 & 2 & 2 & 6 & 7 & 5 & 4 & 4 \\
\hline Tournants & & & & & & & & & & & & \\
\hline Famille & 27 & 24 & 19 & 22 & 31 & 33 & 23 & 23 & 28 & 20 & 31 & 20 \\
\hline Entrée en couple & - & 1 & 1 & 3 & - & - & - & 1 & - & - & - & - \\
\hline Mariage & 14 & 13 & 7 & 12 & 15 & 16 & 13 & 13 & 14 & 11 & 19 & 13 \\
\hline Naissance & 7 & 7 & 6 & 4 & 11 & 13 & 5 & 5 & 7 & 5 & 8 & 2 \\
\hline Divorce & 1 & - & 2 & 1 & 1 & - & - & - & 1 & - & - & - \\
\hline Autre ${ }^{5}$ & 5 & 3 & 3 & 2 & 4 & 4 & 5 & 4 & 6 & 4 & 4 & 5 \\
\hline Décès & 15 & 12 & 7 & 7 & 12 & 10 & 13 & 9 & 23 & 17 & 19 & 18 \\
\hline
\end{tabular}

En gris les pourcentages où moins de cinq individus sont représentés.

$2 \quad \mathrm{BA}=$ Bandra

$3 \quad \mathrm{SC}=$ Santa Cruz

4 La catégorie "autre" regroupe avant tout: divers problèmes familiaux conflictuels, les mentions d'anniversaire de proches, être heureux/satisfait de sa vie de famille, la responsabilité du ménage, le fait de n'avoir pas pu se marier ou pas pu avoir d'enfant.

5 Dans cette catégorie "autre" se retrouvent essentiellement: les citations de plusieurs types d'événements familiaux (naissances et mariage des enfants par exemple), les conflits ou à l'inverse le fait d'être heureux/de se soutenir en famille (ou dans la belle-famille), le devoir de se charger de l'éducation des enfants ou de la responsabilité (sans précision) du ménage, le fait de n'avoir pas pu se marier ou pas pu avoir d'enfant. 


\section{Annexe 5: avoir mentionné un événement de santé}

Tableau 1: rapports de cotes issus de modèles de régression logistique sur la variable «avoir mentionné au moins un changement récent de santé»

\begin{tabular}{|c|c|c|c|c|c|}
\hline & modèle & modèle 2 & modèle 3 & modèle 4 & modèle 5 \\
\hline \multicolumn{6}{|c|}{ Lieu de résidence (référence $=$ Santa Cruz East) } \\
\hline Bandra East & $1.316^{*}$ & $1.310^{*}$ & $1.343^{*}$ & $1.309^{\circ}$ & 1.270 \\
\hline \multicolumn{6}{|c|}{ Sexe (référence $=$ hommes) } \\
\hline Femmes & & 1.086 & 1.157 & 1.181 & 1.160 \\
\hline \multicolumn{6}{|c|}{ Classe d'âge (référence $=20-24$ ans) } \\
\hline $35-39$ ans & & & $2.762 * * *$ & $2.374 * *$ & $2.257 * *$ \\
\hline $50-54$ ans & & & $2.810 * * *$ & $2.242 * *$ & $2.113^{*}$ \\
\hline $65-69$ ans & & & $7.784 * * *$ & $6.480 * * *$ & $5.653 * * *$ \\
\hline $80-84$ ans & & & $8.261 * * *$ & $7.270 * * *$ & $6.262 * * *$ \\
\hline \multicolumn{6}{|c|}{ Education (référence $=$ Supérieure) } \\
\hline Sans & & & & 1.300 & 1.082 \\
\hline Primaire & & & & $1.863 * *$ & $1.732 *$ \\
\hline Secondaire & & & & $2.330 * * *$ & $2.248 * * *$ \\
\hline \multicolumn{6}{|c|}{ Santé auto-évaluée (référence $=$ Très bonne) } \\
\hline Bonne & & & & & 1.459 \\
\hline Moyenne & & & & & 1.474 \\
\hline Mauvaise & & & & & $3.959^{* *}$ \\
\hline
\end{tabular}

Seuils de significativité: ${ }^{\circ} \leq 0.1 ; * \leq 0.05 ; * * \leq 0.01 ; * * * \leq 0.001$. 
Tableau 2: rapports de cotes issus de modèles de régression logistique sur la variable «avoir mentionné au moins un tournant de santé»

\begin{tabular}{|c|c|c|c|c|c|}
\hline & modèle & modèle 2 & modèle 3 & modèle 4 & modèle 5 \\
\hline \multicolumn{6}{|c|}{ Lieu de résidence (référence $=$ Santa Cruz East) } \\
\hline Bandra East & $1.309^{\circ}$ & $1.334 *$ & $1.376^{*}$ & $1.300^{\circ}$ & 1.250 \\
\hline \multicolumn{6}{|c|}{ Sexe (référence = hommes) } \\
\hline Femmes & & $0.738^{*}$ & $0.744^{*}$ & $0.735^{*}$ & $0.709 *$ \\
\hline \multicolumn{6}{|c|}{ Classe d'âge (référence $=20-24$ ans) } \\
\hline 35-39 ans & & & 1.135 & 0.964 & 0.886 \\
\hline $50-54$ ans & & & $1.457^{\circ}$ & 1.164 & 1.054 \\
\hline $65-69$ ans & & & $2.191 * * *$ & $1.821 *$ & $1.562^{\circ}$ \\
\hline $80-84$ ans & & & 1.201 & 1.010 & 0.847 \\
\hline \multicolumn{6}{|c|}{ Education (référence $=$ Supérieure) } \\
\hline Sans & & & & 1.588 & 1.430 \\
\hline Primaire & & & & $1.515^{\circ}$ & 1.439 \\
\hline Secondaire & & & & $2.391 * * *$ & $2.322 * * *$ \\
\hline \multicolumn{6}{|c|}{ Santé auto-évaluée (référence $=$ Très bonne) } \\
\hline Bonne & & & & & $1.686^{*}$ \\
\hline Moyenne & & & & & $1.622^{\circ}$ \\
\hline Mauvaise & & & & & $2.995 * *$ \\
\hline
\end{tabular}

Seuils de significativité: ${ }^{\circ} \leq 0.1{ }^{*} \leq 0.05 ; * * \leq 0.01 ; * * \leq 0.001$. 


\title{
Population, Family, and Society Population, Famille et Société
}

\author{
Edited by / Edité par \\ Michel Oris
}

Cette collection a pour ambition d'accueillir et de promouvoir le dialogue entre les démographes et les spécialistes de la famille, dialogue qui renouvelle profondément tant l'histoire sociale que la sociologie contemporaine. Animée par un réseau international qui s'appuie sur le Laboratoire de Démographie et d'EFtudes Familiales de I'Université de Genève, la collection est largement ouverte et veut refléter les dynamiques de recherche les plus récentes. Elle privilégie les perspectives comparatives, internationales, ainsi que les approches interdisciplinaires, celles qui mêlent les apports de l'histoire, de l'économie, de la statistique, de la sociologie, de la géographie, de la démographie, de l'anthropologie culturelle, etc. L'innovation méthodologique, dans les domaines du qualitatif aussi bien que du quantitatif, qui permet de refonder les problématiques et d'articuler de nouvelles questions, est particulièrement saluée. La collection accueille aussi bien des contributions individuelles que collectives. Dans le premier groupe se rangent les monographies ou travaux de synthèse issus du milieu scientifique suisse et international, en ce compris les meilleures thèses de doctorat. Le second groupe réunit des recueils d'articles organisés autour d'un thème qui émerge dans le débat scientifique, et qui requiert le croisement de regards venus de multiples horizons disciplinaires et/ou géographiques.

La collection accueille des ouvrages en langue française, anglaise et allemande.

Vol. 1 Frans van Poppel, Michel Oris \& James Lee (eds). The Road to Independence. Leaving Home in Western and Eastern Societies, 16th-20th centuries. 2004. ISBN 3-906770-61-3 / US-ISBN 0-8204-5949-6

Vol. 2 Guy Brunet, Michel Oris \& Alain Bideau (éds). Les minorités. Une démographie culturelle et politique, XVIIle-XXe siècles / Minorities. A Cultural and Political Demography, 18th-20th centuries. 2004. ISBN 3-03910-220-6 / US-ISBN 0-8204-6874-6

Vol. 3 Erwin Zimmermann \& Robin Tillmann (éds/Hrsg.). Vivre en Suisse 1999-2000. Une année dans la vie des ménages et familles en Suisse / Leben in der Schweiz 1999-2000. Ein Jahr im Leben der Schweizer Familien und Haushalte. 2004. ISBN 3-03910-370-9

Vol. 4 Jean-Marie Le Goff, Claudine Sauvain-Dugerdil, Clémentine Rossier \& Josette Coenen-Huther. Maternité et parcours de vie. L'enfant a-til toujours une place dans les projets des femmes en Suisse? 2005. ISBN 3-03910-666-X 
Vol. 5 Claudine Sauvain-Dugerdil, Henri Leridon \& Nicholas Mascie-Taylor (eds). Human Clocks.

The Bio-Cultural Meanings of Age.

2006. ISBN 3-03910-785-2 / US-ISBN 0-8204-7570-X

Vol. 6 Michel Oris, Guy Brunet, Eric Widmer \& Alain Bideau (éds). Les fratries. Une démographie sociale de la germanité.

2007. ISBN 978-3-03911-255-5

Vol. 7 Angélique Janssens (ed.). Gendering the Fertility Decline in the Western World.

2007. ISBN 978-3-03911-311-8

Vol. 8 Laurence Leitenberg. La population juive des villes d'Europe. Croissance et répartition, 1750-1930. 2008. ISBN 978-3-03911-478-8

Vol. 9 Eric D. Widmer \& Riitta Jallinoja (eds). Beyond the Nuclear Family: Families in a Configurational Perspective.

2008. ISBN 978-3-03911-704-8

Vol. 10 Antoinette Fauve-Chamoux and Emiko Ochiai (eds).

The Stem Family in Eurasian Perspective. Revisiting House Societies, 17th-20th centuries. 2009. ISBN 978-3-03911-739-0

Vol. 11 Michel Oris, Guy Brunet, Virginie De Luca Barrusse \& Danielle Gauvreau (éds.).

Une démographie au féminin - A Female Demography. Risques et opportunités dans le parcours de vie - Risks and Chances in the Life Course.

2009. ISBN 978-3-03911-738-3

Vol. 12 Reto Schumacher

Structures et comportements en transition. La reproduction démographique à Genève au 19 siècle. 2010. ISBN 978-3-0343-0302-6

Vol. 13 Laurent Heyberger

L'histoire anthropométrique.

2011. ISBN 978-3-0343-0586-0

Vol. 14 Irenka Krone-Germann

Part-Time Employment in Switzerland. Relevance, Impact and Challenges.

2011. ISBN 978-3-0343-0614-0

Vol. 15 Guy Brunet

Vie et mort dans la Dombes des étangs aux XVIII et XIXe siècles.

2011. ISBN 978-3-0343-0669-0

Vol. 16 Mariorie Bourdelais

La Nouvelle-Orléans: croissance démographique, intégrations urbaine et sociale (1803-1860).

2012. ISBN 978-3-0343-1200-4 
Vol. 17 Virginie De Luca Barrusse

Population en danger ! La lutte contre les fléaux sociaux sous la Troisième République.

2013. ISBN 978-3-0343-1430-5

Vol. 18 Angélique Janssens

Labouring Lives. Women, work and the demographic transition in the Netherlands, 1880-1960.

2014. ISBN 978-3-0343-1571-5

Vol. 19 Guy Brunet (éd.)

Mariage et métissage dans les sociétés coloniales. Amériques, Afrique et lles de l'Océan Indien (XVIe-XXe siècles).

Marriage and misgeneration in colonial societies. Americas, Africa and islands of the Indian ocean (XV/th-XXth centuries).

2015. ISBN 978-3-0343-1605-7

Vol. 20 Sylvie Burgnard

Produire, diffuser et contester les savoirs sur le sexe. Une sociohistoire de la sexualité dans la

Genève des années 1970.

2015. ISBN 978-3-0343-1537-1

Vol. 21 Mikołai Szottysek

Rethinking East-Central Europe: family systems and co-residence in the Polish-Lithuanian Commonwealth

Vol. 1: Contexts and analyses

Vol. 2: Data quality assessments, documentation, and bibliography.

2015. ISBN 978-3-03911-781-9

Vol. 22 Fabrice Boudjaaba, Christine Dousset \& Sylvie Mouysset (éd.)

Frères et sceurs du Moyen Âge à nos jours.

2016. ISBN 978-3-03431468-8

Vol. 23 Marc Debuisson

Régimes démographiques et structures socio-économiques. Les communautés villageoises de la province de Namur durant la première moitié du 198 siècle.

2015. ISBN 978-3-0343-1671-2

Vol. 24 Jonathan Zufferey

Pourquoi les migrants vivent-ils plus longtemps? Les inégalités face à la mort en Suisse

(1990-2008).

2017. $978-3-0343-2106-8$

Vol. 25 Dionigi Albera, Luigi Lorenzetti, Jon Mathieu (eds.)

Reframing the History of Family and Kinship: From the Alps towards Europe.

2016. 978-3-0343-2127-3 
Vol. 26 Walter Letsch

Demographic Aspects of the Early Modern Times. The Example of the Zurich Countryside in a

European Perspective.

2017. 978-3-0343-2973-6

Vol. 27 Nathalie Burnay et Cornelia Hummel (éds.)

Vieillissement et classes sociales.

2017. 978-3-0343-3022-0

Vol. 28 Oreste Foppiani \& Oana Scarlatescu (eds.)

Family, Separation and Migration: An Evolution-Involution of the Global Refugee Crisis.

2017. 978-3-0343-3026-8

Vol. 29 Forthcoming

Vol. 30 Guy Brunet \& Kamel Kateb

L'Algérie des Européens au XIXe siècle. Naissance d'une population et transformation d'une société 2018. 978-3-0343-3629-1

Vol. 31 Paul-André Rosental

Population, the state, and national grandeur. Demography as political science in modern France 2018. 978-3-0343-3081-7

Vol. 32 Paulo de Teodoro Matos, Helena Da Silva, José Miguel Sardica (eds)

War Hecatomb. International Effects on Public Health, Demography and Mentalities

in the 20th Century

2018. 978-3-0343-3736-6

Vol. 33 Aude Martenot

Parcours de vie et mémoires de pauvres. Changements personnels et sociohistoriques dans les bidonvilles de Mumbai.

2019. 978-3-0343-3828-8 